\title{
NOTISE
}

SRTIONS OF THIS REPOHT ARE ILLEABLE.

it has been reproduced from the best ayailable copy to permit tire srocusest

\section{Compact Reversed-Field Pinch Reactors (CRFPR): Preliminary Engineering Considerations}

\author{
R. L. Hagenson* \\ R. A. Krakowski \\ C. G. Bathke \\ A. L. Miller \\ M. J. Embrechts** \\ N. M. Schnurr \\ M. E. Battat \\ R. J. LaBauve \\ J. W. Davidson
}

\section{DISCLAIMER}

\begin{abstract}
This report was prepared as an account of work sponsored by an agency of the United States Government. Neither the United States Government nor any agency thereof, nor any of their employees, makes any warranty, express or implied, or assumes any legal liability or responsibility for the accuracy, completeness, or usefulness of any information, apparatus, product, or process disclosed, or represents that its use would not infringe privately owned rights. Reference herein to any specific commercial product, process, or service by trade name, trademark, manufacturer, or otherwise does not necessarily constitute or imply its endorsement, recommendation, or favoring by the United States Government or any agency thereof. The views and opinions of authors expressed herein do not necessarily state or roflect those of the United States Government or any agency thereof.
\end{abstract}


TABLE OF CONTENTS

PAGE

I. EXECUTIVE SUMMARY . . . . . . . . . . . . . . . . . . . I

A. Introduction . . . . . . . . . . . . . . . . . . . . 1

B. Background . . . . . . . . . . . . . . . . . . 3

C. Scope of Study ...................... . 6

D. The Reversed-Field Pinch Confinement Princlple . . . . . . . 9

E. Reactor Design Overview . . . . . . . . . . . . . . 16

F. Technology Assessment . . . . . . . . . . . . . . . . . 22

G. Conclusions . . . . . . . . . . . . . . . . . 27

II. BACKGROUND . . . . . . . . . . . . . . . . . . . . . . . . 29

A. Status of Experimental and Reactor Studies . . . . . . . . . . . 29

B. RatLonale for High-Power-Density Fusion . . . . . . . . . . 38

C. Simplified Reactor Model . . . . . . . . . . . . . . . . 46

D. The Reversed-Field Pinch . . . . . . . . . . . . . 61

1. Confinement Principle and Application to

Reactor Studies .. . . . . . . . . . . . 61

2. Reactor Design-Point Update and Sensitivity . . . . . . 68

III. PRELIMINARY ENGINEERING DESIGN. . . . . . . . . . . . . . . 77

A. Design Overview . . . . . . . . . . . . . . . . . . 77

B. Neutronics . . . . . . . . . . . . . . . . . . 84

1. Calculational procedure . . . . . . . . . . . . . 84

2. Computational Results . . . . . . . . . . . . . 86

a. Parametric Studies . . . . . . . . . . . . 86

b. The Canonical Blanket. . . . . . . . . . . . . 91

C. Materials . . . . . . . . . . . . . . . . . . 100

1. Overview . . . . . . . . . . . . . . . 100

2. Materials Selection . . . . . . . . . . . . 103

a. First Wall . . . . . . . . . . . . . . 103

b. Blanket/Shield . . . . . . . . . . . . 108

c. Magnets ..................... 109

3. Radiation Effects . . . . . . . . . . . . . . 109

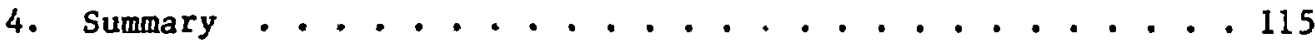


D. In-Vacuum Components (F1rst Wall and Limiters) . . . . . . . 117

1. General Thermal-Mechanical Considerations . . . . . . 117

2. Thermal-Hydraulic Analysis . . . . . . . . . . . . 122

a. First Wall . . . . . . . . . . . . . . 122

b. Pumped Limiter. . . . . . . . . . . . . . . 130

E. Blanket . . . . . . . . . . . . . . . . . . . 134

1. General Considerations. . . . . . . . . . . . . 134

2. Thermal-Hydraulic Analysis. . . . . . . . . . . . . 138

a. Calculation of Blanket Pumping Power . . . . . . 139

b. Results . . . . . . . . . . . . . . . . . 142

3. Thermal Analysis of Shield. . . . . . . . . . . . 146

4. First-Wa11/Blanket/Shield Integration . . . . . . . . . 148

F. Magnets . . . . . . . . . . . . . . . . . . . 150

1. Toroidal-Field-Cotl System. . . . . . . . . . . . 151

2. The Poloidal-Field-Coll System . . . . . . . . . . 158

a. Startup Operations. . . . . . . . . . . . 160

b. PFC (OHC + EFC) Conductor Locations . . . . . . . . 167

Ohmic-Heating Coil (OHC) . . . . . . . . . . 168

Equilbrium-Field Coil (EFC) . . . . . . . . . . 170

Combined OHC/EFC Magnet lc Properties . . . . . . . 172

c. PFC (OHC + EFC) Circuit Analysis . . . . . . . 173

d. Resistive PFC Numerical Circuit Analysis . . . . . 179

G. Plasma and Circuit Simulation . . . . . . . . . . . . . 188

H. Particle Control by Pumped Limiters . . . . . . . . . . . 208

1. Scrapeoff and Limfter Model . . . . . . . . . . . 209

2. Parametric Results . . . . . . . . . . . . 214

I. Magnet1c Divertor . . . . . . . . . . . . . . . . 232

J. Vacuum and Fueling . . . . . . . . . . . . . . . . . 236

K. Current Drive... . . . . . . . . . . . . . . 249 
L. Fusion-Power-Core Integration . . . . . . . . . . . . 261

1. Power-Plant Energy Balance Integration . . . . . . . . 261

2. Thermal-Hydraulic Design Incegration . . . . . . . . . 265

a. Thermal-Conversion Efficiency . . . . . . . . 266

b. Thermal-Hydraulics Design Parametics Study . . . . 269 Limiter Analysis. . . . . . . . . . . . . 270 First-Wa11/Second-Wall Analysis . . . . . . . . 271 Shield Analysis . . . . . . . . . . . . 271 Pressurized-Water Manifold/Header Design . . . . . 271 Blanket Analysis . . . . . . . . . . . . 272 Cycle Efficiency . . . . . . . . . . . . 272

c. Thermal-Hydraulics Design Parametrics Study Results . 272

3. Mechanical Design Integration . . . . . . . . . . . 281 IV. ASSESSMENT . . . . . . . . . . . . . . . . . . . . . 301

A. Physics Assessment . . . . . . . . . . . . . . . . . 301

B. Technology Assessment . . . . . . . . . . . . . 303

1. Plasma Heating System . . . . . . . . . . . . . 303

2. Particle Control Systems . . . . . . . . . . . . 304

a. Fueling . . . . . . . . . . . . . . 304

b. Particle Removal . . . . . . . . . . . . 305

c. Vacuum Pumping . . . . . . . . . . . . 306

3. Plasma Current-Drive System . . . . . . . . . . 307

4. Nuclear Systems . . . . . . . . . . . . . . 308

a. In-Vacuum Components . . . . . . . . . . 308

b. Blanket ................... . . 310

c. Shield....................... 312

d. Afterheat and Radwaste . . . . . . . . . . 313

5. Magnet Systems . . . . . . . . . . . . . . 315

a. Torolda1-Field Colls . . . . . . . . . . 315

b. Pololdal-Field Colls . . . . . . . . . . 316

6. Electrical and Mechanical Systems . . . . . . . . 316

a. Energy Transfer and Storage (ETS) . . . . . . . . 317

b. FPC Structural Support, Assembly, and Maintenance. . . 317

7. Tritium Systems . . . . . . . . . . . . . 318

8. Balance-of-Plant systems . . . . . . . . . . . 318 
C. Economic Assesssment . . . . . . . . . . . . . . . . 320

1. Parametric Systems Model . . . . . . . . . . . . . 320

2. CRFPR Cost Tradeoffs................... . . 321

D. Conclustons ....................... . . 332

REFERENCES . . . . . . . . . . . . . . . . . . . . . . . 334

ACRONYMS AND NOTATION . . . . . . . . . . . . . . . . . . . . . 366 ACKNOWLEDGEMENTS . . . . . . . . . . . . . . . . . . . . . . 348 APPENDICES . . . . . . . . . . . . . . . . . . . . . . . . 349

APpendix A. CRFPR BURN MODEL AND REACTOR DESIGN CODE . • . • • • . 349

A.1. Computer Model for Burn Simulation . . . . . . 351

A.2. Fokker-Planck Slowing-Down Calculation . . . . 355

A.3. Plasma Powers and Proffle Averages . . . . . . 357

a. Heating by Energetic Particle . . . . . . 358

b. Ohmic Heating . . . . . . . . . . . 359

c. Radiation Power ............ . 359

d. Transport . . . . . . . . . . . 361

A.4. Magnetic-Field, Density, and Temperature Profiles • 363

A.5. Equilibrium ald Stability . . . . . . . . . 369

A.6. F1rst-Wall The:ma1-Mechanical Response . . . . . 371

A.7. Reactor Energy Balance . . . . . . . . . . 372

Appendix B. TABLE OF CRFPR DESIGN PARAMETERS . . . . . • . . • . 377

Appendix C. TWO DIMENSIONAL NEUTRONICS ANALYSES . . . . . . . . . 387

C.1. Introduction . . . . . . . . . . . . 387

C.2. Two-Dimensional Model . . . . . . . . . . 388

C.3. Neutronics Calculations . . . . . . . . . 388

a. Codes . . . . . . . . . . . . . . 388

b. Cross Sections.............. 393

c. Calculational Specifications... . . . . 393

C.4. Parametric Results . . . . . . . . . . . 394

a. Reference (Canonical) FW/B/S Model . . . . . 394

b. Modifled Reference (Carionical) FW/B/S Model . . 400

c.5. Conclusions and Recommendations . . . . . . . 403 
R. I. Hagenson, R. A. Krakowski, C. G. Bathke, R. L. Miller, M. J. Embrechts, N. M. Schnurr, M. E. Battat, R. J. LaBauve, and J. W. Davidson

\section{ABSTRACT}

The unique confinement physics of the Reversed-Field Plinch (RFP) projects to a compact, high-power-density fusion reactor that promises a significant reduction in the cost of electricity. The compact reactor also promises a factor-of-two reduction in the fraction of total cost devoted to the reactor plant equipment [1.e., fusion power core (FPC) plus support systems]. In addition to operational and developmental benefits, these physically smaller systems can operate economically over a range of tota: power output. After giving an extended background and rationale for the compact fusion approaches, key FPC subsystems for the Compact RFP Reactor (CRFPR) are developed, designed, and liategrated for a minimum-cost, 1000-MWe(net) system. Both the problems and promise of the compact, high-power-density fusion reactor are quantitatively evaluated on the basis of this conceptual design. The material presented in this report both forms a framework for a broader, more expanded conceptual design as well as suggests directions and emphases for related research and development.

\section{EXECUTIVE SUMMARY}

\section{A. Introduction}

The cost-opitimized Compact Reversed-Field Pinch Reactor (CRFPR) design suggested by this study would operate with a fusion-power-core (FPC, i.e., first wall, blanket, shield, and colls) power density (MWt $/ \mathrm{m}^{3}$ ) and mass utilization (tonne/MWt) that are comparable to fissior power plants. These two measures of FPC performance couple with appropriately modified values for plant availability and operating cost to give a cost of electricity [COE (mills/kWeh)] that is 
1.6-2.0 times less than that being projected for other approaches to fusion powe:; a similar basis is used in evaluating these cost differences, which are significant. Eurthermore, the fraction of the total plant cost required for the reactor plant equipment (i.e., FPC and support systems) can be reduced from the 50-70\% generally considered necessary for fuston to $\sim 30 \%$ for the compact systems; the influence of plasma physics in the overall cost equation for fusion is reduced, thereby diminishing the risk of development and deploymeat. After the background and rationale for compact fusion reactors are reviewed, preliminary designs and related technology assessments of crucial FPC engineering subsystems are presented. Physics and engineering models for the RFP are developed, many for the first time, to describe parametrically key FPC subsystems required for all aspects of plasma initiation, startup, sustenance, and plasma power conversion. Once defined, these subsystems are Integrated into a cost-optimized FPC design that is used for a physics, technology, and economic assessment while simultaneously providing a basis for future, more comprehensive power-plant design studies. The material contained herein, therefore, is both summary and interim in form and is intended to provide a framework for future work. 
The development and eventual commercialization of magnetic fusion energy (MFE) is pursued in the U. S. through two co-mainline concepts, the tokamak and the tandem mirror, with a number of promising but less-developed approaches being investigated as alternative or supporting fusion concepts (AFCs). The engineering development needs for the mainline tokamak have been quantified by detailed conceptual design studies of both first-generation engineering experiments ${ }^{1,2}$ and commercial power eactors. 3 To a lesser extent, but nevertheless at a significant level of effort and conceptual design detail, are studies of the Tandem Mirror Reactor (TMR) ${ }^{4-6}$ as well as nearer-term engineering devices $^{7,9}$ based on the tandem-mirror confinement principle. Complementing and supporting both the tokamak and tandem-mirror mainline approaches are the AFCs. The status of reactor designs for tokamaks, tandem mirrors, and AFCs has been summarized quantitatively. ${ }^{9}, 10 \mathrm{~A}$ qualitative assessment of the engineering and technology needs of the major AFCs has also been presented recently. Il

A generic category termed "compact" or "high-power-density" approaches to fusion has been identified, 10-13 into which is placed the Compact Reversed-Field Pinch Reactor (CRFPR), 14-17 the reactor embodiment of the Ohmically-Heated Toroidal Experiment (OHTE), 18 high-field tokamaks, 9,19-22 and certain subelements of the Compact Toroids (CT, i.e., spheromaks, field-reversed configurations, field-reversed mirrors, etc.). $23-30$ The word "compact" as used here describes approaches that would operate explicitly with increased engineering or system power density compared to the low power density and large physical size projected for the mainline fusion concepts. Small plant capacity [i.e., MWe(net)], although an attractive trait for first-of-a-kind systems, is not necessarily implied by "compact". While the combination of compactness and smallness in capacity is possible, economies of scale generally dictate higher unit costs (i.e., $\$ / \mathrm{kWe}$ or mills/kWeh) for systems with lower capacity.

Concern over the dominance in mass and cost of the fusion power core [FPC, 1.e., first wall/blanket/shield/coils (FW/B/S/C)] for many fusion appruaches $1-10$ has led to consideration of compact options. This concern about the large size, low power density, and physical complexity of fusion is most succinctly summarized in Fig. I.B-1, which gives on a scale of toroidal major radius cross sections of the FPCs being projected for a number of fusion reactor concepts. Although the system power densities for other fission systems are less than for the Pressurized-Water (fission) Reactor (4-5 MW/m ${ }^{3}$ for an Advanced Gas Reactor, 
2-3 MW/m ${ }^{3}$ for a Liquid-Metal Fast Breeder Reactor), most fusion systems depicted on Fig. I.B-1 project power densities that are below these values. General improvements to this situation being sought through the compact, high-power-density options include a) power densities within the FPC approaching those of fission reactors (i.e., 10-30 times greater than promised by most fusion reactor conceptual designs); b) projected costs that are relatively insensitive to large changes in unit costs $(\$ / \mathrm{kg})$ used to estimate FPC and associated reactor plant equipment costs; c) considerably reduced size and mass of the FPC with potential for "block" (i.e., single or few-piece) installation and maintenance; and $d$ ) the potential for rapid, minimum-cost development and deployment, which generally implies rapid progress along cruclal learning curves. The compact approach is not necessarily limited to a specific confinement scheme; just as the RFP has a low-power-density reactor
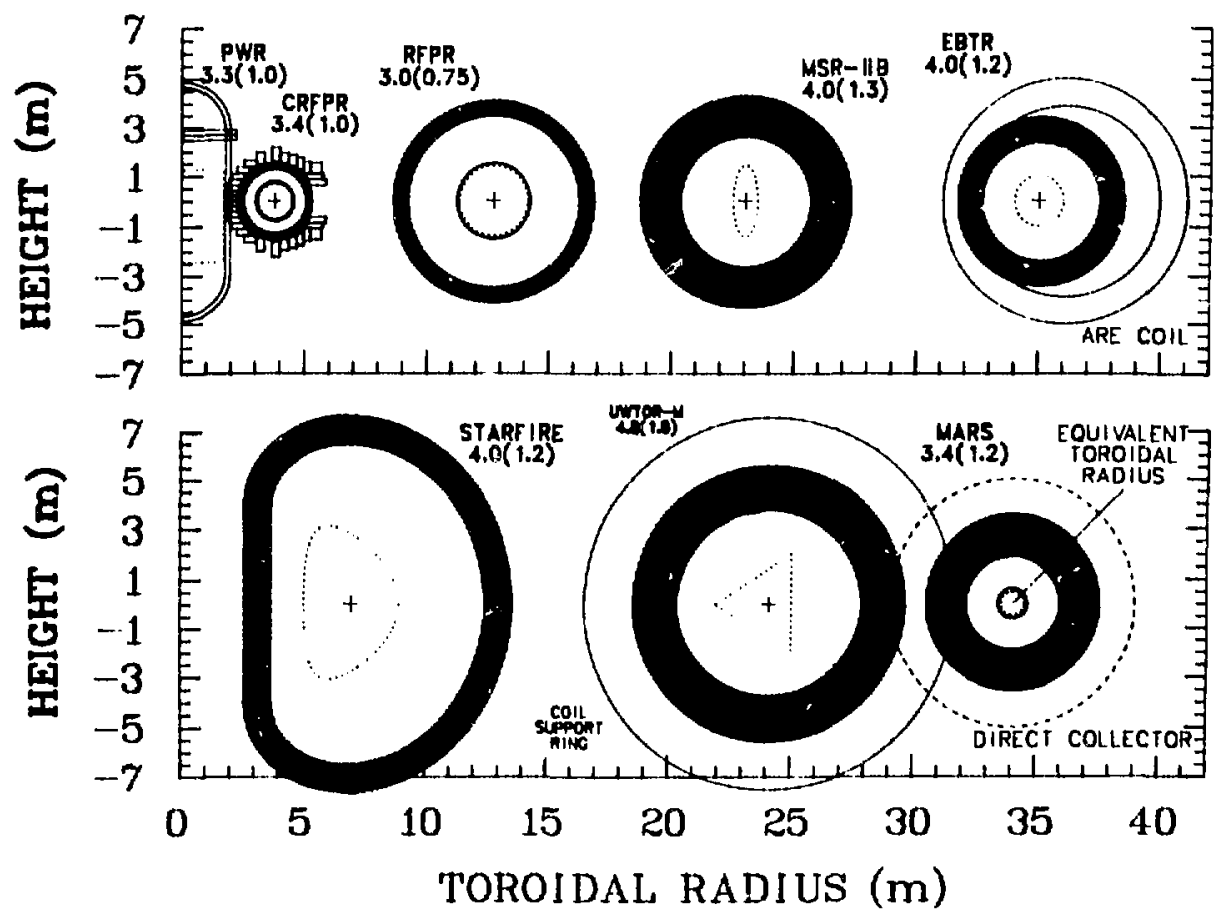

Fig. I.B-1. Relative sizes of mainline and alternative magnetic fusion power reactors. With the exception of the compact RFP reactor (CRFPR), all systems propose the use of large superconducting magnet coils. Thermal (net electrical) powers are indicated in GWt(GWe) units, and FPC powcr densities are also shown. In presenting the (1inear) MARS tandem-mirror design, its length has been reduced by $2 \pi$ to generate an effective major radius for purposes of comparison only. 
embodiment, 31 it is possible to envisage compact reactor options for the tokamak, 1 the stellarator/torsatron/heliotron ( $/ \mathrm{T} / \mathrm{H}), 32$ and certain $\mathrm{CT}$ configurations. 24,25

The compact option for fusion power will require the extension of existing technologies to accommodate high heat flux, higher power density, and in some instances higher magnetic fields required to operate with FPC power densities in the 5-15 MWt $/ \mathrm{m}^{3}$ range rather than in the $0.3-0.5 \mathrm{MWt} / \mathrm{m}^{3}$ range being predicted for most other approaches (Fig. I.B-1). Ultimately, however, any comprehensive analysis of the "optimum" fusion power system must be based on the cost of electricity, COE(mills/kWeh), which accurately reflects the cost of achievi.g engineering feasibility (1.e., design complexity and degree of needed design conservatism), operation and maintenance costs (i.e., FPC "burnup"), costs of environment and safety restrictions, construction time, and plant availability. Hence, system power density is one important input to the equation for a competitive fusion power plant. The present study of the compact RFp reactor addresses this problem on the basis of these aforementioned broader 1ssues. 
C. Scope of Study

Althcugh considerable insight into the engineering and economic constraints of high-power-density fusion has developed from past parametric studies, 15 it nevertheless becomes necessary to perform specific conceptual engineering design studies on key FPC subsystems in order to advance the design credibility. This "framework" study develops models needed to describe all key aspects of the FPC performance for the Reversed-Field Pinch (RFP) and, then on the basis of parametric analyses using these models, develops an integrated and optimized FPC conceptual design. of necessity, each subsystem model is simplified and will certainly be subjected to future upgrading. The FPC neutronics model and materials options couple to the unique features of the RFP confinement physics to form a starting point for this framework study. Design models and results for the following FPC subsystems are then developed, forming the central effort of this study.

- First Wall

- Blanket

- Magnets

- Plasma and Circuit Simulation

- Limiters

- Magnetic Divertors

- Vacuum and Fueling

- Current Drive

The simplified neutronics and materials models (1.e., one-dimensional, steady-state, constant properties) that serve as a basis for key design choices w111 require iteration and redesign as the system layout evolves, particularly with respect to blanket/shield performance (1.e., peak temperatures, stresses, two-dimensional effects on tritium-breeding ratio, coolant/manifold/header placement and size, etc.). In some cases, design iteration to a fully self-consistent integrated FPC is not within the scope of this study. This framework study concludes with an ergonic, thermal-hydraulic, and mechanical integration of the complete FPC, the results of which are reported in this Executive Summary (Sec. I.E.). An assessment of physics, technology, and economics for this high-power-density FPC is then given (Sec. I.F.). The 
results presented herein are intended to provide a data base for a comprehensive power-plant design study some time in the future.

This framework study focuses almost completely on the physics, technolngy, and economics of the FPC, with little consideration being given to FPC support systems or to the balance of plant (BOP). General ground rules and approach that guided this study are given below.

- When applicable and adequate, models and results emerging from mainline reactor studies are used, with a major effort being placed on specific RFP issues.

- Parameterization of key physics and technological results is stressed, with the selection of a specific or "canonical" design point being made only when cholces are needed to advance or focus the design for purposes of assessment.

- Key plasma parameters are selected on the basis of economic and operational considerations while simultaneously assuring that the resulting design point can be reasonably matched with existing or developing RFP physics results (transport, beta, profiles, impurity control, current drive, field ripple constraints, field reversal conditions, current risetimes, ohmic neating, etc.).

- All reactor designs considered are based on ohmic heating by plasma currents to DT ignition using resistive copper magnets to induce currents and the RFP dynamo to generate large toroidal fields within the plasma from a relative low bias field initially introduced into the torus.

- Key FPC subsystems are designed on the basis cf very long-pulse or steady-state plasma operation using current-drive and impurity-control concepts that are specific to RFPs.

- The FPC design is focused on (poloida1) pumped-limiter impurity control, but examination of (toroidal) magnetic divertors is initiated, in both cases taking advantage when possible of the advanced state of these areas for the tokamak mainline.

- The impact on cost and plant operations is considered for a range of capacities and power densities (neutron first-wall loaiing), but a 1000-MWe(net) power plant is selected to facilitate comparisons with the mainline reactor designs, both past and present, as well as with reactor concepts being proposed for key alternative fusion concepts.

- A neutron first-wall loading $\left(\leqslant 20 \mathrm{MW} / \mathrm{m}^{2}\right)$ is selected that minimizes COE and FPC mass while simultaneously assuring fundamental limits of heat-transfer and stress are not violated.

- As for the mainline tokanak, sputter erosion is recognized as a potentially serious lifetime determinant, but solutions are assumed to be found that will allow a high proportion of the plasma energy to be shed by radiation. 
- Neutron radiation 1 ifetimes are assumed to be in the range of 10-15 MWyr $/ \mathrm{m}^{2}\left(\sim 10 \mathrm{dpa} / \mathrm{MWyr}^{2} / \mathrm{m}^{2}\right)$.

- In spite of marginal economic benefits accrued by increasing neutron first-wall. loadings beyond $\sim 10 \mathrm{MW} / \mathrm{m}^{2}$, the minimum-COE design point at $20 \mathrm{MW} / \mathrm{m}^{2}$ is retained by this framework study to minimize the FPC mass for purposes of utilizing single- or few-piece batch maintenance schemes.

- A commonality with the tokamak in cost data base and methodology is stressed, as well as the level of allowed physics assumption/extrapolation and technological innovation, to assure meaningful and (when possible) quantitative intercomparison and assessment of design end products. 
The RFP is a toroidal axisymmetric device in which the primary confinement field, ${ }^{B}{ }_{\theta}$, is poloidal and is generated by a toroldal current, $I_{\phi}$, flowing in the plasma. 33-35 The RFP plasma supports a toroidal bias field, $B_{\phi}$, to stabilize sausage $(m=0)$ and elliptical $(m-2)$ distortions. Grossly unstable MHD modes with wavelengths longer than the minor radius, $r_{w}$, of an electrically conducting shell are stabillzed by the shell on a short time scale and by feedback coils for longer times. If the toroidal bias field is slightly reversed near the plasma edge, $r_{p} \leq r_{W}$, the resulting magnetic shear in the plasma-edge region is sufficient to stabilize local pressure-driven and currentdriven instabilities. This stabilization occurs at relatively high values of the normalized plasma pressure, $\beta \equiv 2 \mathrm{nk}_{B} \mathrm{~T} /\left(\mathrm{B}^{2} / 2 \mu_{0}\right)$. A sample profile for a stable RFP is illustrated in Fig. I.D-1, which also gives a comparison with a tokamak.

The existence of the RFP profiles shown in Fig. I.D-1 would seem unlikely because of the probable annihilation of opposing toroidal flux at the field reversal surface, as is illustrated graphically in the lower right quadrant of Fig. I.D-1. Experiments during the previous 25 years, however, have shown that a self-reversed field and a minimum-energy plasma are generated even if such a field is not externally imposed. Recent theories of relaxed energy states has led to a substantial increase in the fundamental appreciation of this nearminimum-energy, field-reversed state. Given any arbitrary dissipation mechanism operative within a plasma surrounded by a flux-conserving shell, that plasma w11 relax through resistive processes to a minimum-energy, force-free state. The key descriptive parameters in the RFP theory are the pinch parameter, $\theta$, and the reversal parameter, $F$, which are defined as $\theta=B_{\theta}\left(r_{w}\right) /\left\langle B_{\phi}\right\rangle$ and $F=B_{\phi}\left(r_{w}\right) /\left\langle B_{\phi}\right\rangle$, where $\left\langle B_{\phi}\right\rangle=\left\langle 2 / r_{w}{ }^{2} ; \int_{0}^{r_{w}} B_{\phi} r d r\right.$ is the average toroidal field within the conducting-shell radius, $r_{w}$; Fig. I.D-1 illustrates graphically the meaning of $F$ and $\theta$. The primary attraction of the self-generating RFP as a reactor becomes obvious from Fig. I.D-1: a) high plasma current from ohmic heating; b) high fields in the plasma but low fields and currents at the magnet coils. The RFP is also an inherently high-beta configuration with the potential for a unique, low-frequency current drive for steady-state operation.

The locus of minimum-energy RFP states, as described in an $F-\theta$ phase space, Is shown in Fig. I.D-2; both the analytic, $\beta=0$ state and the experimental, finite- $\beta$ states are shown. The experimental F- $\theta$ traces shown in Fig. I.D-2 

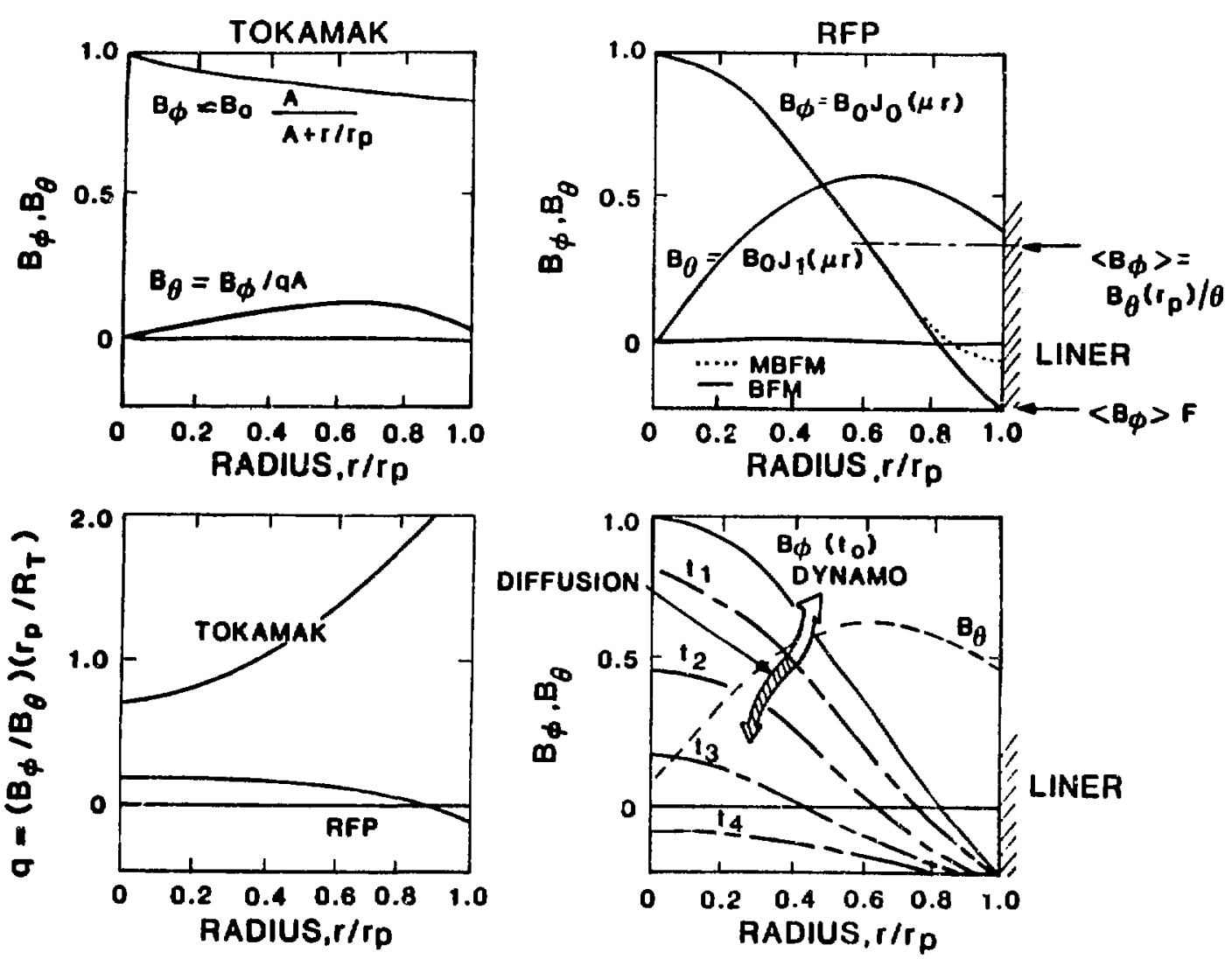

Fig. 1.D-1. Sample magnetic-field and $q=\left(B_{\phi} / B_{\theta}\right)\left(r_{p} / R_{T}\right)$ minor radial profiles for the RFP and the tokamak. Shown also are graphic definitions of the RFP pinch parameter, $\theta$, and reversal parameter, $F$.

illustrate the plasma drifting toward and residing within a specific region of F- $\theta$ space. This desired RFP state corresponds to $F<0$ and $1.2<\theta<1.6$. Both the relaxation mechanism and associated time constants for the self-sustained toroidal-field reversal are not easily explained in terms of commonly understood plasma processes. In spite of this lack of theoretical understanding, howevex, strong experimental evidence exists for a plasma regeneration or "dynamo" mechanism that continually maintains the minimum-energy RFP state. Either turbulence or resistive MHD instabilities drive the plasma dynamo action, wherein toroidal plasma currents are converted to the toroidal 


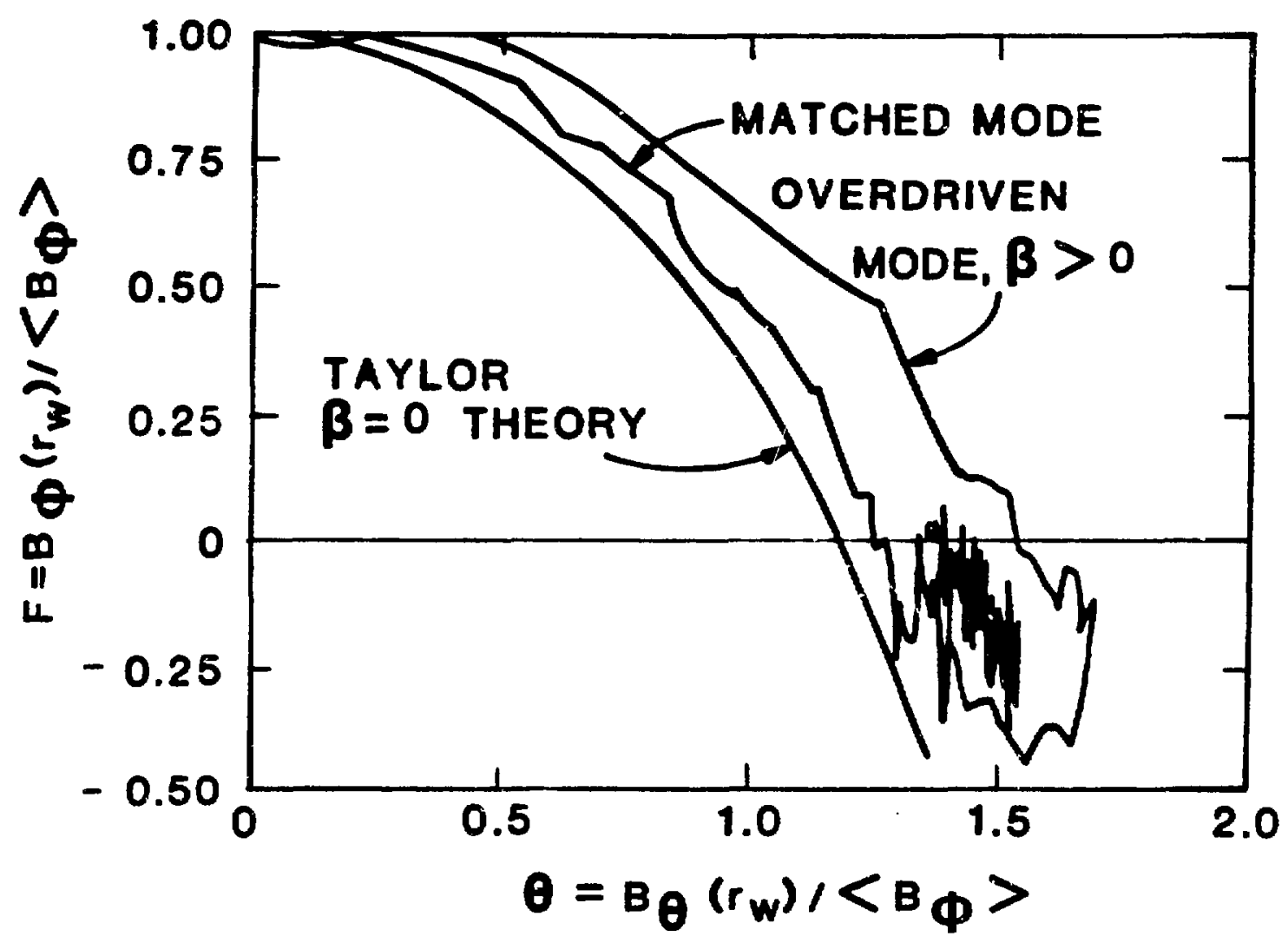

Fig. I.D-2. The F- $\theta$ diagram showing two experimental traces and the tendency for the plasma to seek and reside within the RFP minimum-energy state. The tokamak-like state corresponds to $(F=1, \theta \simeq 1 / q A)$, whereas the spheromak state resides around $F \simeq 0$.

flux needed for maintenance of the toroidal field reversal depicted on Fig. I.B-1 and experimently shown on FIg. I.D-2.

The relationship between $F$ and $A$, the tokamak safety factor, $q \equiv\left(r_{p} / R_{T}\right)\left(B_{\phi} / B_{\theta}\right)$, and beta points out the essential differences between the RFP and the tokamak reactor. First, one definition of the total average beta for both RFPs and tokamaks is $\langle\beta\rangle=2 \mu_{o}\langle p\rangle /\left(B_{\theta}^{2}+B_{\phi}^{2}\right)$, which in terms of the pololdal beta, $\beta_{\theta}$, is $\langle\beta\rangle=\beta_{\theta} /\left[1+\left(B_{\phi} / B_{\theta}\right)^{2}\right]=\beta_{\theta} /\left[1+(F / \theta)^{2}\right]$. Proper accounting of profile effects can introduce a factor of $\sim 2$ into these simplified relationships between $\langle\beta\rangle$ and $\beta_{\theta}$ for both the RFP and tokamak. The tokamak-like minimum-energy state would correspond to the region on Fig. I.D-2 defined by $F \rightarrow 1$ and $\theta=F / A q=1 / A q$, where $A=R_{T} / r_{p}$ is the plasma aspect 
ratio; for the tokamak, $\langle\beta\rangle=\beta_{\theta} /\left[1+(q A)^{2}\right]$. The spheromak minimum-energy state corresponds to $F \rightarrow 0$ on Fig. I.D-2. For the RFP state, $|F / \theta| \simeq 0.2 / 1.5=0.13$ and $\langle\beta\rangle \simeq \beta_{\theta}$ (actually $\sim \beta_{\theta} / 2$, accounting for profiles), whereas for the tokamak $F / \theta=q A \simeq 8-10$, with $\langle\beta\rangle$ being much less than $\beta_{\theta} \simeq 1-2$. As a maximum for the tokamak, $\beta_{\theta} \leq A$ to avoid the ideal kink stability ind, therefore, $\langle\beta\rangle \leq A /\left[1+(\mathrm{Aq})^{2}\right] \simeq 1 / \mathrm{Aq}^{2}$.

The Impact of these basic differences on the level of ohmic-heating allowed in resistive coils for systems that can operate stably with low values of $|F / \theta|$ compared to those with large $|\mathrm{F} / \theta|$ values (1.e., the tokamak) is significant. Specifically, the ohmic losses incurred in the toroidal-field coils relative to those in the poloidal-coll system for both RFPs and tokamaks are nominally proportional to $\sim 1+|F / \theta|$. In addition, lower ohmic losses occur in the RFP pololdal colls because of the higher aspect ratio and better inductive coupling between that coil set and plasma. The lower flelds and currents in external conductors for the RFP allow the efficlent use of resistive copper (or possibly aluminum) coils, significant reduction in blanket/shield thickness, and the possibility for significant reductions in FPC nass and complexity. Furthermore, the close electrical coupling between poloidal and toroidal circuits through the RFP dynamo can be used to establish a noninductive current drive for RFPs that may allow steady-state plasma operation using low-frequency and low-power rectifying technology ( $F-\theta$ pumping). For these reasons as well as good experimental progress, the RFP represents an attractlve candidate with which to examine the technical and economic feasibility of compact, high-power-density Euston reactors.

The present status of the RFP physics is depicted on Fig. I.D-3 in terms of a) the usual $n \tau_{\mathrm{E}}$ versus $\mathrm{T}$ meast ement of performance and; $b$ ) the ohmic scaling of $\tau_{E} \propto I_{\phi}^{V_{p}^{2}}$ with plasma current, $I_{\phi}$, and plasma minor radius, $r_{p}$. Theoretically, $v \simeq 3 / 2$, whereas experimentally $v=1$; the extension of this RFP ohmic scaling to the compact reactor is also depicted on Fig. I.D-3.

Experimentally derived poloidal betas of $\beta_{\theta} \sim 0.1-0.15$ are typically measured on the Los Alamos 2T-40M experiment. 35 Neutral-beam-heated tokamak experiments have exhibited a degradation in the energy confinement time as the tokamak beta, in response to neutral-beam heating, is increased according to $\tau_{E} \propto 1 / \beta^{2}$ [Appendix A.3.d.]. The poloidal beta used for the reactor is based on the experimental value of $\beta_{\theta} \sim 0.13$, and, if the internal plasma heating (alpha-particle power) forces $\beta_{\theta}$ above 0.13 , the energy confinement time is 
decreased according to $\tau_{E} \propto 1 / \beta$. The basic conclusions emerging from present-day RFP experiments are summarized below.

- Classical plasma resistivity is observed In experiment, the higher voltages required to drive current in RFPs relative to tokamaks being a result of geometric factors (i.e., higher pitch) rather than "annomalous" resistivity need to drive current against added $\vec{v} \times \vec{B}$ electric fields being generated by local, stochastic plasina motion.

- The plasma dynamo action is verified but not theoretically understood, with the dynamo dissipating little energy beyond that required by classical ohmic losses.

- Separate oscillation of poloidal and toroidal circuits result in the plasma moving predictably along the F- $\theta$ diagram, giving strong promise for $F-\theta$ pumping current drive. F- $\theta$ pumping for steady-state, ignited current drive should be efficient if the plasma dynamo action occurs with a nearly classical electrical resistivity.

- Ignition by ohmic heating is consistent with classical plasma resistivity and is a strong possibility in the RFP.

- The energy confinement time, $\tau_{E}(\mathrm{OPT})$, required for an economically optimized reactor relative to an ohmic dissipation t.tme, $\tau_{E}$ (OHMIC), and relative to some measure of Alcator scaling, $\tau_{E}$ (ALC), is given by $\tau_{E}(\mathrm{OHMIC}) / \tau_{E}(\mathrm{OPT})>20-50$ and $\tau_{E}(\mathrm{ALC}) / \tau_{E}(\mathrm{OPT})>5-10$, respectively, showing wide safety margins for ohmic ignition in RFPs.

In summary, these unique characteristics of a dynamo-sustained RFP (or spheromak) promise equally unique and attractive leactor advantages. The specific reactor implications of the $q<I$ RFP confinement system based on the above physics indications are

- Relatively free choice of plasma aspect ratio.

- Economically high betas.

- Possibility of high values of $\beta B^{2}$ at the plasma and correspondingly increased plasma power density for a given field at coll. 


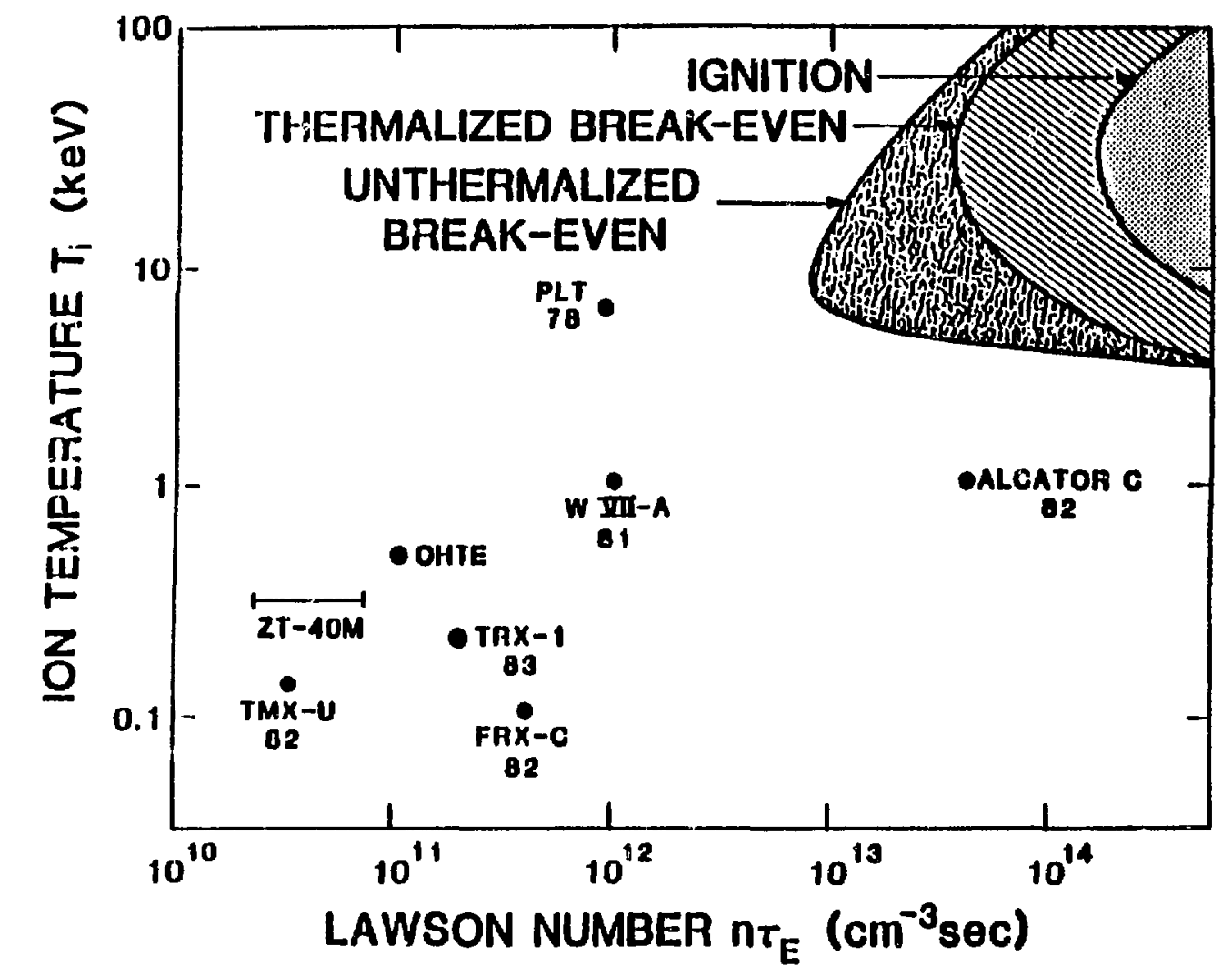

Fig. I.D-3A. Measure of device performance in relationship to ignition and reactor goals.

- Internal plasma currents are sufficlent for ohmic heating to ignition without auxiliary heating and with low-to-moderate flelds and currents in the colls.

- Efflcient plasma/coil electrical coupling, resulting in

- ${ }_{\theta}\left(r_{p}\right)$ dominates and, hence, the fields and currents at the poloidal field colls are low.

- $B_{\phi}\left(r_{p}\right)$ near zero, resulting in low flelds and currents at toroldal-field colls.

- Use of resistive colls is possible while maintaining efficlent reactor performance.

- Potential for efficlent steady-state current drive through " $F-\theta$ pumping" with low-frequency, smallamplitude oscillations of TFCs and PFCs. The ohmicheating-coll current can then be reduced to zero. 


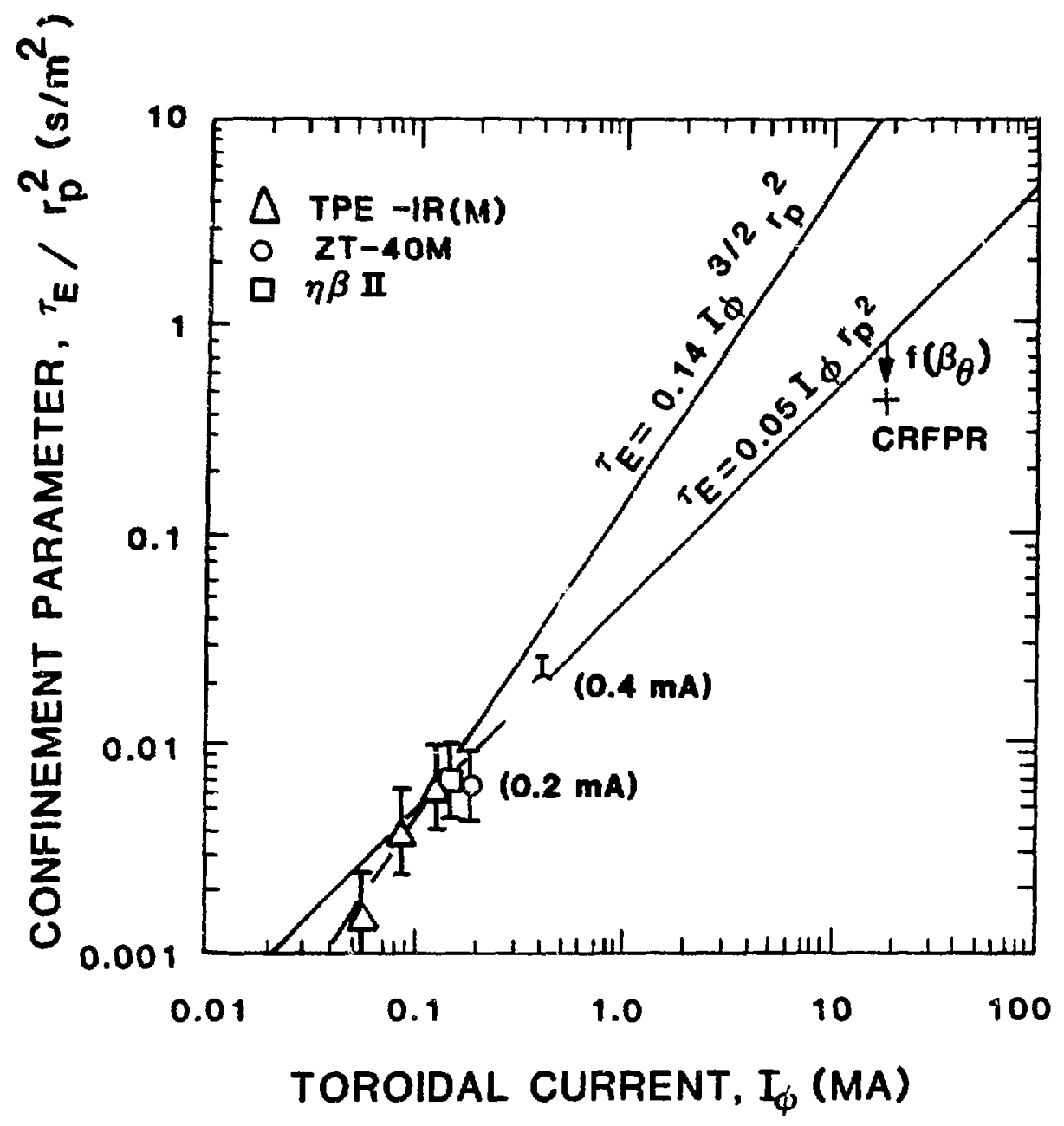

Fig. I.D-3B. Scaling of RFP confinement with toroidal current, $I_{\phi}$, and plasma radius. $r_{p}$, needed to take present experimental results to reactor conditions. 


\section{E. Reactor Design Overview}

The Compact Reversed-Field Pinch Reactor (CRFPR) study has focused on the FPC physics and technology needs for a nominal 1000-MWe(net) power plant. This toroldal reactor is based on a deuterium-tritium fuel cycle. Major features of this design are steady-state and ignited operation, ohmic heating to ignition, pumped-1imiter impurity control, pressurized-water cooling of the high-heat-flux, copper-alloy first-wall and limiter systems, self-cooled lead-lithium ( $\mathrm{Pb}_{83} \mathrm{Li}_{17}$, designated throughout this report as "PbLi") tritium-breeding blanket using ferritic steel (HT-9) structure, use of water-cooled copper colls positioned outside the blanket/shield system, and fully remote block maintenance of a single-piece $(\sim 300$ tonne less $\sim 800$ tonne of coolant), first-wall/blanket/ shield/toroldal-field-coll assembly.

Figure I.E-1 gives a cross-sectional view of the FPC, showing that this $1000-M W e$ (net) system is not much larger than TFTR $^{36}$ and requires an energy confinement time that differs little from 1-MA TFTR operation (0.25-0.30 s). An isometric sketch of one of 24 toroidal sectors is shown in Fig. I.E-2. sach of

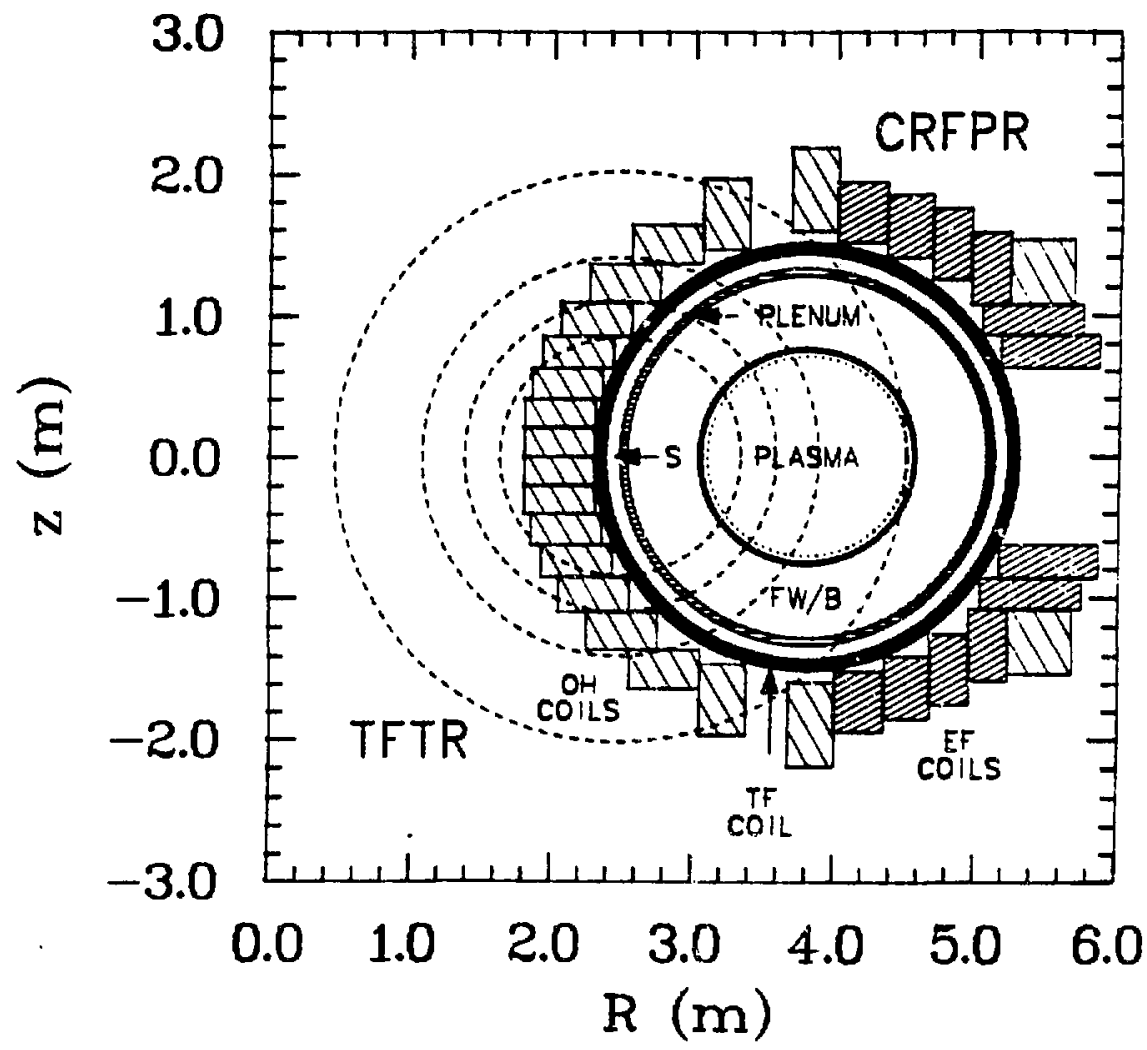

Fig. I.E-1. Cross-sectional view of the 1000-MWe(net) CRFPR in comparison with the TFTR tokamak experiment ${ }^{36}$ (poloidal-field colls not shown). 


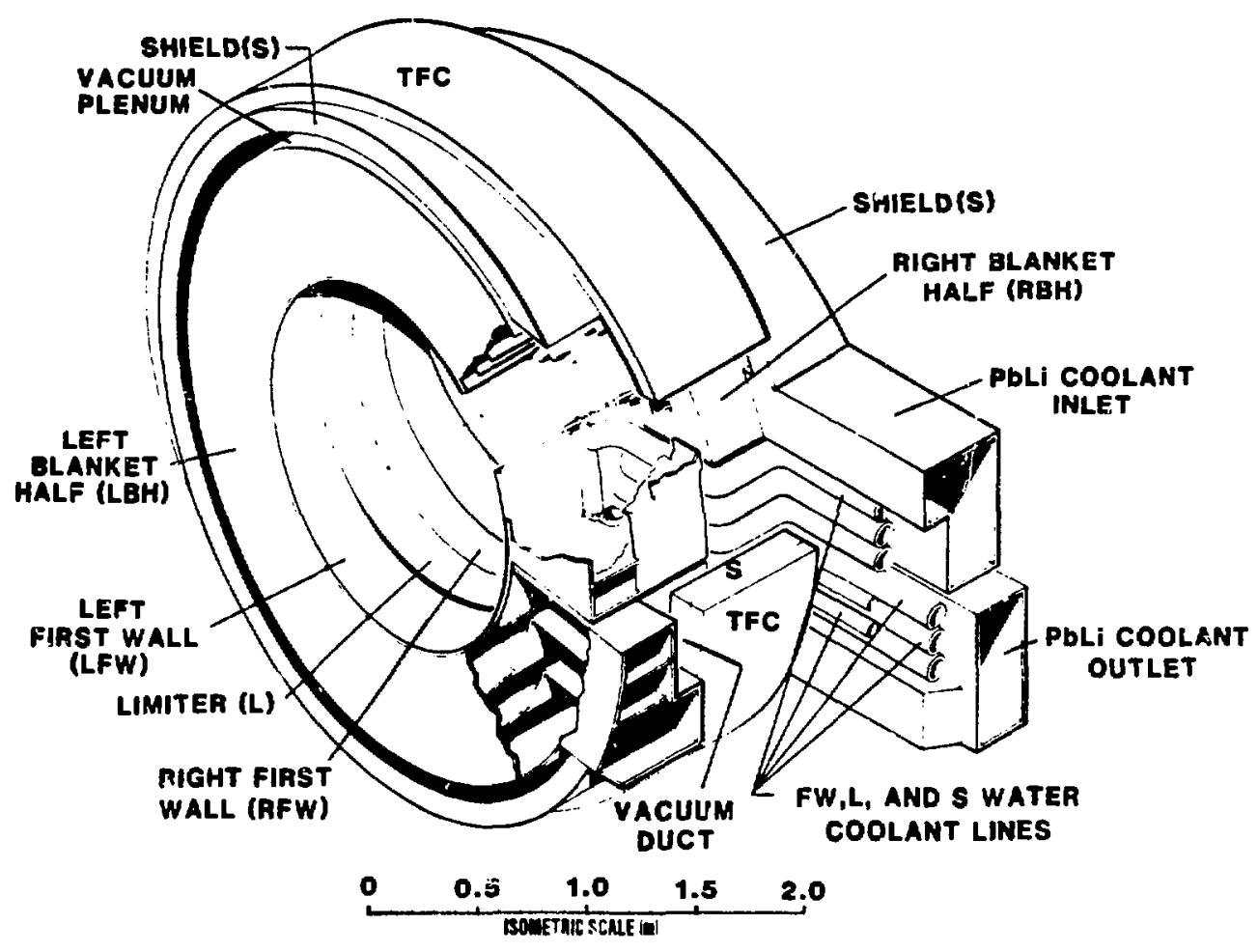

Fig. I.E-2. Isometric view of one of 24 toroidal sections for a 1000-MWe(net) CRFPR. Off-site, single-untt construction of the first-wall/ blanket/shield/toroidal-field-coll torus ( 300 tonne total without coolants) and single-piece maintenance/repair scheme is envisaged for this high-power-density design. Poloidal-field colls are not shown.

these toroldal sectors could be fabricated, assembled into the 300-tonne torus, and tested (electrically, under vaccum, and thermal-hydraulically) off site prior to shipment to and installation at the operating power-plant site. The high neutron first-wall loading of $\sim 20 \mathrm{MW} / \mathrm{m}^{2}$ would give a one-year chronological life for a radiation lifetime of $15 \mathrm{MWyr} / \mathrm{m}^{2}$, which for the 45.2-tonne first-wall/blanket systems corresponds to a mass usage of 17.9 $\mathrm{kg} / \mathrm{MWtyr} \quad\left(\sim 20,000 \mathrm{MWtd} /\right.$ tonne, somewhat less than $\mathrm{vo}_{2}$ fissile fuel burnup in an LWR) for this 3365-MWt plant operating at a $75 \%$ plant factor. Table I. $\bar{L}-\mathrm{I}$ summartzes the majer CRFPR design parameters. 
MAJOR CRFPR DESIGN PARAMETERS

\begin{tabular}{|c|c|}
\hline Net electrical power (MWe) & 1000 . \\
\hline Gross electrical power (MWe) & 1227. \\
\hline Total thermal power (MHt) & 3365. \\
\hline Gross power-conversion efficiency $(\%)$ & 36.5 \\
\hline Overall plant availability $(\%)$ & 75. \\
\hline Major radius (m) & 3.8 \\
\hline Plasma radius (average)(m) & 0.71 \\
\hline Plasma volume $\left(\mathrm{m}^{3}\right)$ & 37.8 \\
\hline Neutron wall loading $\left(\mathrm{MW} / \mathrm{m}^{2}\right.$, & 19.5 \\
\hline Number of toroidal sectors & $24 \cdot(a)$ \\
\hline Maximum field at magnet $(T)$ & $4 \cdot 5(b)$ \\
\hline Field at plasma axis (T) & 9.5 \\
\hline Field at plasma edge (T) & 5.2 \\
\hline Average poloidal beta & 0.23 \\
\hline Average total beta & 0.12 \\
\hline Average DT ion density $\left(10^{20 / m^{3}}\right)$ & 6.6 \\
\hline Average DT fon temperature (keV) & 10.0 \\
\hline Plasma burn mode & Continuous/ignited \\
\hline Plasma heating method (startup) & Onmic (246 Webers) \\
\hline Plasma impurity-control method & Pumped limiter \\
\hline First-wall/limiter structural materials & MZC copper alloy \\
\hline Blanket/shield structural material & HT-9 ferritic steel \\
\hline Tritium-breeding medium & PbLi \\
\hline Primary coolant & PbLi \\
\hline Thermal-conversion method & Dual-cycle, steam \\
\hline
\end{tabular}

\footnotetext{
(a) For off-site fabrication purposes only, single-piece or batch FPC maintenance is envisaged for this system thet weighs $\sim 300$ tonne (first wall, blanket, shield, toroidal-field coils), to which is added a separate 800-tonne poloidal-field coil set and $\sim 800$ tonne of PbLi coolant.

(b) At the OHC during the burn, 9.2 'T during startup.
} 
The design parameters listed in Table $\mathrm{I} . \mathrm{E}-\mathrm{I}$ reflect the results of design guidelines (Sec. I.C.) that targeted the FPC power densities $\left(\sim 11.8 \mathrm{MW} / \mathrm{m}^{3}\right)$ and physical sizes ( 0.33 tonne/MWt, $\left.285 \mathrm{~m}^{3}\right)$ at values at which pressurized-water fission reactors (PWRs) presently operate; the "fission power cores" (j.e.e, volume enclosed by primary pressure vesse1) of Advanced Gas Reactors and Liquid-Metal Fast Breeder Reactors operate at engineering power densitles that are 3-5 times less than for a PWR but are nevertheless much higher than values projected for most superconducting fusion reactors. Although the CRFPR power density is targeted at the PWR, considerable flexiblilty exists for systems of either reduced power density or reduced plant capacity (and neutron first-wall loading) while maintaining favorable projections for energy cost. The cost projections are summarized in Table I.F-I, which also gives comparisons with other fusion systems on a relatively common basis. An isometric sketch of the FPC is shown in Fig. I.E-3 which shows access being made at the outboard equatorlal plane for all coolant(s), vacuum, and electrical lines. The top half of the PFC set ( 400-tonne total) would be lifted vertically in two sections, exposing the $\sim$ 300-tonne first-wall/blanket/shield/TFC unit for removal as a single assembly during the annual maintenance period.

The costing results given on Table I.F-I for the CRFPR represents a complex tradeoff between physics ( $\tau_{E} \propto I_{\phi} \gamma_{p}^{2}$ scaling with $\nu<3 / 2$ a variable), technology (neutron and heat loadings, power density, etc.), economics (plant factor, economy of scale, operating cost, etc.), and safety (afterheat, stored field energy, etc.). The operating and design margins between physics, technology, and economics have been charted on Fig. I.E-4, and a comfortable operating regime is indicated for a range of net electric output, $P_{T}$, neutron first-wall loadings, $I_{w}$, physical size as measured by plasma minor radius, $r_{p}$, and plasma confinement parameter, $\nu$. The $I_{W} / P_{E} / \tau_{E}(\nu) / C O E$ tradeoffs depicted on Fig. I.E-4 identify broad design margins between physics (confinement), technology (1.e., high heat/particle-flux surfaces, high-power-density blankets, batch versus patch maintenance, off-site versus on-site monolithic FPC construction, etc.), and economics (including plant avallability, operating costs, recirculating power, etc. The design point summarized on Table I.E-I is also shown on Fig. I.E-4, and the implications of decreasing both the neutron first-wall loading, net electric power, and system size ( $r_{p}$, with the minimum-COE designs being relatively independent of $A=R_{T} / r_{p}$ ) and power density are clearly shown, as constrained by the confinement parameter $v$. Although the increased COE 
resulting from Increased physical size and reduced FPC power density is not great for the ranges examined, the desire to maintain a single- or few-piece FPC malntenance scheme makes operating near the (shallow) COE minimum desirable. For systems with power density and first-wall loadings below those considered on Fig. I.t-4, a breakpolnt occurs beyond which superconducting RFPs become more attractive; the exact position of this breakpoint remains to be determined, however.

The thrust of this framework study has been the definition of the technology and cost margins depicted in Fig. I.E-4 through conceptual design of a specific FPC design point. On the basis of these studies and conceptual designs generated, it is generally concluded that the RFP offers sufficiently broad physical, technical, and economic margins to promise a unique, realistic, robust, and "forgiving" approach to magnetic fusion energy. This framework study has also identifled a number of technology problem and development areas, which are summarized in the following section.

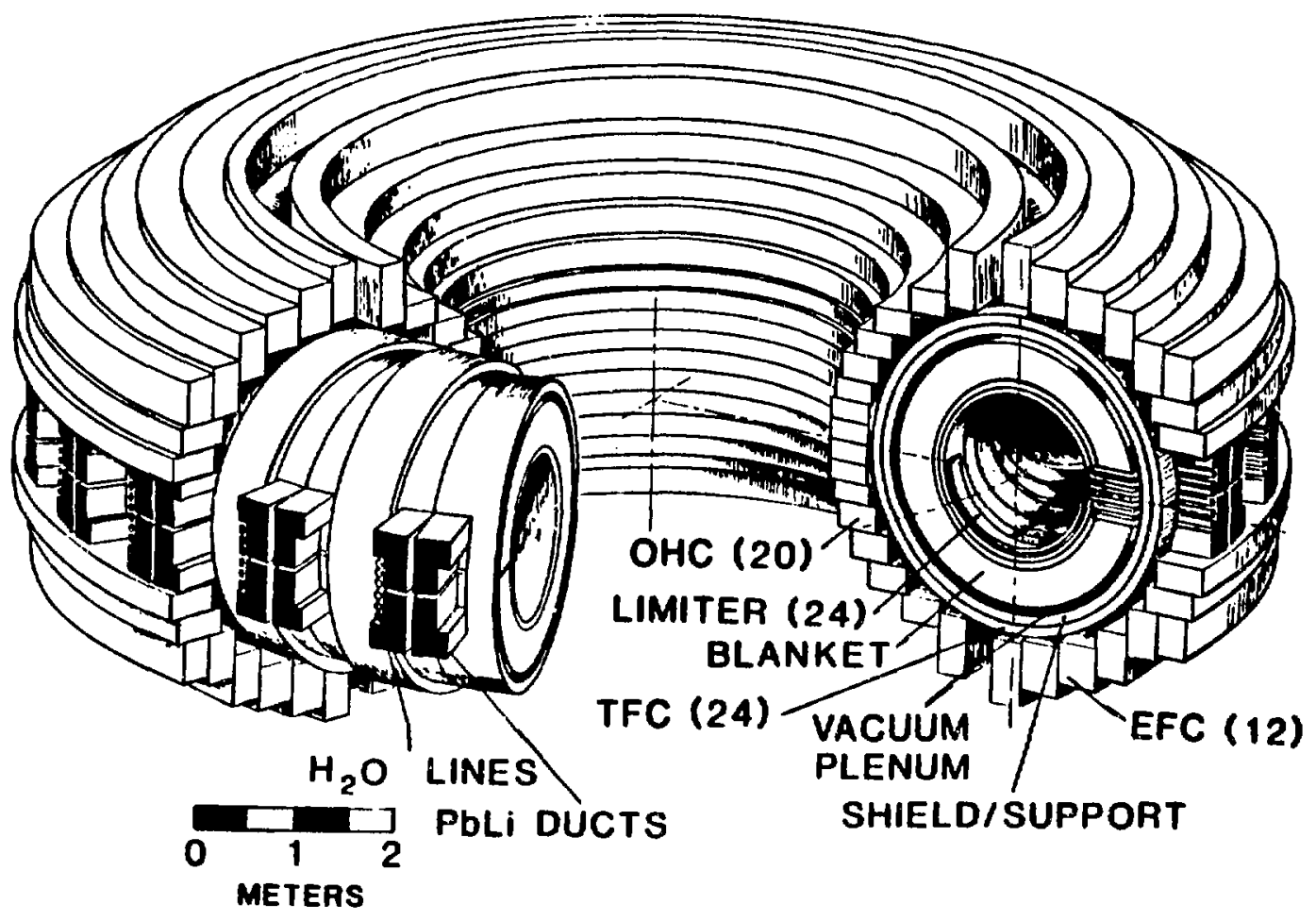

Fig. I.E-3. Isometric view of CRFPR fusion power core. 


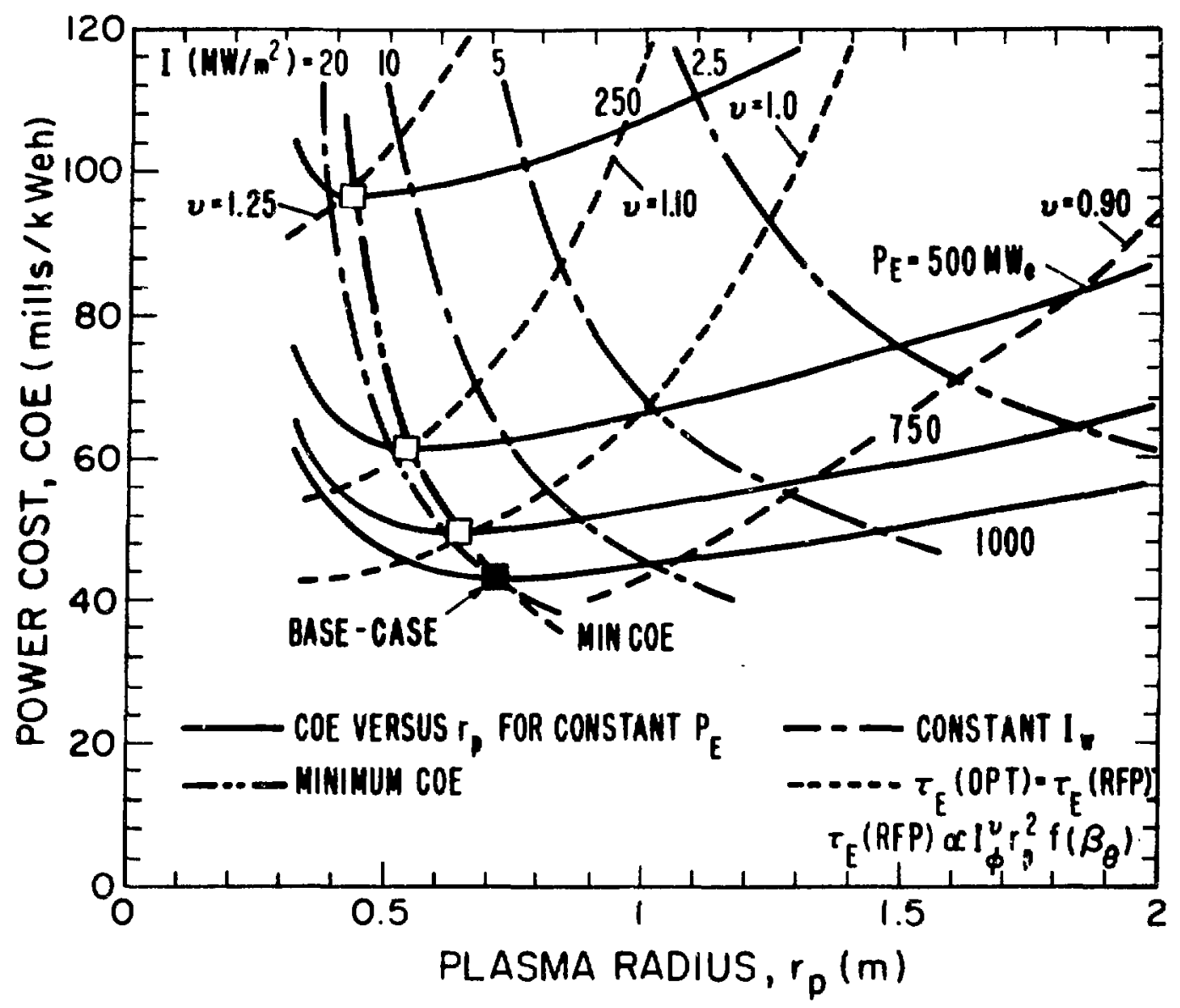

F1g. I.E-4. Dependence of $C O E$ on $r_{p}$ for a range of $P_{E}$ values. Also shown are lines of constant first-wall neutron loading, $I_{w}$. The locus of points where the confinement time dictated by economics equals the RFP physics confinement time of the form $\tau_{E}(R F P) \propto I_{\phi}{ }_{\phi}^{2}{ }_{p}^{2} f\left(\beta_{\theta}\right)$ is also shown for a range of current exponents, v. 
Generic technology requirements for compact reactors have been reviewed previously. 37 The design approach taken for the CRFPR allowed by the unique RFP physicsl principles projects a uniq'e cost advantage (Table I.F-I). Moreimportantly, however, is the prom a of fusion systems of smaller size and capacity that couple the real physics uncertainties more ioosely to the overall cost equation for fusion power; a more robust and resilient design results that is capable of an early and cost-effective proof of fiscal and technical risk. This design resilience permits the recovery of an economically and technically interesting end product in the advent that less-optimistic results emerge from the physics program. This asset is particularly important for a costly

TABLE I.F-I

COST COMPARISON NORMALIZED TO TDC (1980 Dollars)

$\underline{\text { ACCOUNT }}$ STARFIRE $^{3} \quad$ MARS $^{5}$

CREPR

20. Land and Land Rights

21. Structure and Site

0.19

0.21

0.34

22. Reactor Plant Equipment (RPE)

20.09

10.59

26. 07

22.1.1. First-Wal1/Blanket (FW/B)

56.00

64.07

33.37

22.1.2. Shield (S)

4.77

2.33

1.54
22.1.3. Col1 (C)
$F P C=F W / B+S+C$

10.78

2.46

9.90

16.06

3.20

25.48

20.85

4.74

23. Turbine Plant Equipment

14.47

11.35

23.15

24. Electric Plant Equipment

6.77

6.78

12.62

25. Miscellaneous Plant Equipment

2.37

1.41

4.36

26. Special Materials

0.014

5.29

0.026

90. Total Direct Costs (TDC)

100.

100.

100 .

99. Total Costs

185.23

174.12

173.00

Unit Direct Cost, UDC( $\$ / \mathrm{kWl})$

1439.

1480. (a)

959.

Cost of Electricity, COE(mills/kWeh) 67.1

Net Electric Power, $P_{E}$ (MWe)

1200

50 (a)

46.2

1202

1000

\footnotetext{
(a) Taken from $1966 \$ / \mathrm{kWe}$ and $67 \mathrm{mills} / \mathrm{kWeh}$ in 1983 to 1980 using $10 \%$ per year over 3 years to provide a common basis of comparison. 
development program that remains in the early stages of progress toward commercialization.

The CRFPR design differs considerably from supercunducting fusion reactors. The projected technology requirements correspondingly differ primarily because of

- Plasma Power Density: Increased plasma power density, which is proportional to $\beta^{2} B^{4}$, where $B$ is the confining magnetic $f$ field at the plasma surface and $\beta$ is the ratio of average plasma pressure to magnetic-field pressure at the plasma surface.

- Neutron First-Wall Loading: Increased first-wall neutron current $\left(15-20 \mathrm{MW} / \mathrm{m}^{2}\right)$ and surface heat flux $\left(4-5 \mathrm{MW} / \mathrm{m}^{2}\right.$, maximum, for uniform heat deposition onto the first wall).

- Blanket Power Density: Increased peak $\left(\gtrsim 200 \mathrm{MW} / \mathrm{m}^{3}\right)$ and average $\left(230 \mathrm{MW} / \mathrm{m}^{3}\right)$ power density within a tritium-breeding blanket.

- Radiation-Hardened Resistive Coils: Increased radiation and heat fluxes at resistive magnet coils in systems designed to operate at most with only a thin heat-recovering/tritiumbreeding blanket placed between the coil and the plasma.

This assessment of rechnological needs for the CRFPR design summarized on Table I.E-I is presented according to the three major FPC subdivisions: Plasma Engineering Systems (heating, particle control, etc.), Nuclear Systems (first wal1, blanket, shield), and Magnet Systems. In addition, Tritium Systems and Electrical/Mechanical Systems represent key elements of the reactor, which at the level of this study have not received detailed attention.

Compact reactors generally would operate with somewhat increased plasma densities, which impacts certain Plasma Engineering Systems. Refueling, impurity control, and ash removal requirements, therefore, may be more demanding in that the power and mass flow rates remain essentially unchanged for the same power output, but the plasma surface area is considerably reduced. The higher plasma density may also lead to more-difficult $r$ current-drive requirements for steady-state operation; the potential for low-frequency "F- $\theta$ pumping" that is unique to the RFP (and spheromak) confinement, however, represents $a$ new and potentially attractive means to drive steady-state plasma currents. Plasma heating by ohmic dissipation is unsurpassed in terms of efficiency and deliverable power density given the increased plasma current density allowed by 
the RFP. Because of the higher first-wall thermal loadings, a heat-fluxconcentrating limiter does not appear possible for the compact systems, and a sizeable portion ( $>40 \%$ ) of the first wall must function as a limiter if a magnetic divertor is not used. The orientation of the RFP for poloidal limitors, however, is favorable for accommodating large limiter coverages. Generally, the in-vacuum components represent a development program that is not unlike that envisaged by the mainline tokamak high-heat-flux program. Strengthened copper alloy appears particularly suited for this first-wall (limter) application, although control of sputter erosion for these high-heat-flux surfaces represents a crucial problem, as it does for fusion in general. Since both limiter area and energy carried from the plasma by particles are the same for the low-power-density STARFIRE and the CRFPR, the wall erosion problem should be similar. Although only beginning to be explored, the use of toroidal-field divertors for impurity control looks particularly attractive from the viewpoints of an open coil topology and the use of the minority toroldal fleld, both of which are mutually excluded in the tokamak.

In the sense that the increased power denstty within the tritium-breeding blankets all but eliminates solid breeders and helium coolant from consideration, the choices for the Nuclear Systems are appreciably narrowed. Self-cooled, liquid-metal breeders are particularly attractive for these high-power-density blankets, particularly in view of the lower flelds and shortened flow paths offered by the CRFPR, thereby allowing higher cooling rates for the same MHD pump power and pressure drop. Liquid-metal-cooled first walls and limiters do not appear possible, however, because of the higher heat rating, and an appreciable fraction $(\sim 40 \%)$ of the fusion power is delivered from the FPC by pressurized water. The design of an efficient dual-media thermal cycle becomes important, as it is for MARS ${ }^{5}$, since this quantity of high-quality heat emerging in the coolant water is far too great for use only as feedwater heat. Although the resistive-coil CRFPR requires the minimum breeding-blanket thickness $(\sim 0.5 \mathrm{~m})$, some nuclear shielding $(\sim 0.1 \mathrm{~m})$ is required to assure $>99 \%$ energy recovery. The increased albedo provided by this shield is also important to maintain an acceptable tritium-breeding ratio; the coolant and vacuum ducting becomes a larger fraction of the blanket volume in that the FPC has been reduced in size, but the power, burn rate, DT/ash/impurity flow rate, and heat-removal requirements remain unchanged. Hence, the tendency to increase structure near the first-wall region because of current-drive and 
thermal-hydraulic needs must be carefully balanced with the neutronics performance of a thin blanket/shield (reflector) system. This "real estate" issue strongly impacts assembly and blanket performance and requires considerably more study.

A potentially more difficult requirement for the DT-fueled rompact systems is the need for rellable core-cooling capabllity because of the higher afterheat power density, particularly in the first-wall and limiter region. This enhanced afterheat power density results from the higher overall operating first-wall loading and blanket power density. It should be noted that, unlike for fission reactors, the afterheat problem is not viewed as a safety issue for fusion, but instead represents a hazard only to the FPC and the plant investment should local damage occur because of loss of cooling capability. Passive afterheat control is possible only for lower-power-density, more-expensive FPCs, and the chotce between FPC efflciency varsus security represents an economic rather than an environmental tradeoff. This issue is also important and deserves considerably more study.

The technology requirements for the Magnet Systems are significantly less difficult because of the absence of superconducting magnets and the low steady-state magnetic flelds. The stored energy in these magnet systems is $\sim 50$ times less than for a siperconducting system, representing a significant cost savings and potentially increased safety margin. The maintenance procedure envisaged for the compact reactor, because of the significant reductions in FPC physical size and mass, makes possible consideration of "block" maintenance, wherein the complete reactor torus is removed for maintenance and repair operations external to the reactor cavity with a more rapid replacement by a fresh pre-tested unit prowising shorter downtimes and more reliable restarts. The reduced role of the toroidal-field colls $(\sim 0.4 \mathrm{~T})$ and the efficient use of the dominant poloidal-field colls ( 1 MA/GJ for STARFIRE compared to $>10 \mathrm{MA} / \mathrm{GJ}$ for CRFPR) allows physical decoupling of the two coll sets and the option for a unique Installation and maintenance/repair scheme. The use of a torofdal divertor for impurity/ash control, however, will complicate somewhat the toroidal-field-coil set and enlarge the poloidal-fleld-coll set. Magnetic divertors probably will render single-plece maintenance impractical; nevertheless, the advantages of few-piece FPC maintenance and a system of considerably reduced size are expected to be retained. 
A conceptual design of the F- $\theta$ pumping current-drive colls has not been made, which in all likelihood will be separate from the main confining coils and Idealiy would be located closer to the plasma for frequencles above $\sim 50-100 \mathrm{~Hz}$. A cruclal tradeoff between tritium breeding and reactive power remains to be examined in that copper alloy positioned at the first wall adversely impacts the tritium-breeding ratio for this PbLi blanket. If the current-drive frequency can be reduced to a few $\mathrm{Hz}$, however, it becomes feasible + oscillate the main TFC and PFC sets. At an even earlier stage of understanding is the design and Imparic of active feedback colls that are thought necessary to sustain electrically the conducting shell at the first-wall location; the liquid-metal blanket to a great extent should serve the function of a conducting shell for considerable times, however. Generally, the copper-alloy colls represent nearly state-of-the-art systems from the viewpoint of delivering the required fields on the required timescale, with the radiation hardening afforded by the use of Inorganic (powered-Mgo) Insulation presenting a modest development item. 
The general status of fusion reactor designs in relationship to present and projected near-term experiments is given in Sec. II.A. Section II.B. develops the rationale, incentives, and distncentive for high-power-density fuston options. After the RFP confinement principle is briefly summarized, the compact RFP reactor parametrlc design point origlnally suggested in Ref. 15 is further optimized and summarized in Sec. II.C. This most recent design point serves as the model used for the preliminary FPC design and integration developed in Sec. III. and assessed In Sec. IV.

A. Status of Experimental and Reactor Studies

Table II.A-I summarizes the experimental results and achievements emerging from the mainline and key alternative fusion programs. Although physics energy break-even and eventual ignition in a deuterium-tritium (DT) plasma represent major near-term and practically achievable goals, these conditions are likely to be demonstrated in devices that in terms of total plasma kinetic energy generate plasmas that are far from the requirements projected for commerclal power: reactors. This "stored-energy gap" between the anticipated and significant physics breakthrough and the achievement of commercial fuston is best illustrated by plotting in Fig. II.A-l the confineruent parameter, $n \tau_{E}$, againist the total kinetic energy stored in the plasma, $E_{p}$. This plot also includes a number of engineering test and commercial reactors projected both for the mainline and key alternative confinement concepts. Given steady progress and successes in achieving adequate confinement at reactor-like plasma densities and temperatures, the stored-energy gap existing between experiments and Fusion Engineering Devices (FED), as well as between FED-like devices and commercial reactors, represents a need for significant technology development. Generally, machine costs are expected to scale directly with the stored energy and size. 3ridging the stored-energy gap between FED and the commercial reactors becomes a concern in view of the already high costs projected for the former.

The key plasma, fusion-power-core (FPC), and power-plant parameters resulting from recent reactor design studies are summarized on Table II.A-II, which includes the Modular Stellarator Reactor (MSR), 51 the STARFIRE commerctal tokamak reactor, ${ }^{3}$ the EBT reactor (EBTR), 52 the RFP reactor (RFPR), 53 and the tandem-mirror reactor (MARS). 5,6 Given continued steady progress, improved plasma confinement to the point where plasma ignition occurs appears as an attainable goal. The extension of these desirable circumstances to the 
TABLE II.A-I

SUMMARY OF KEY PARAMEIERS OF SOME PRESENT MAGNETIC CONFINEMENT EXPERIMENTS

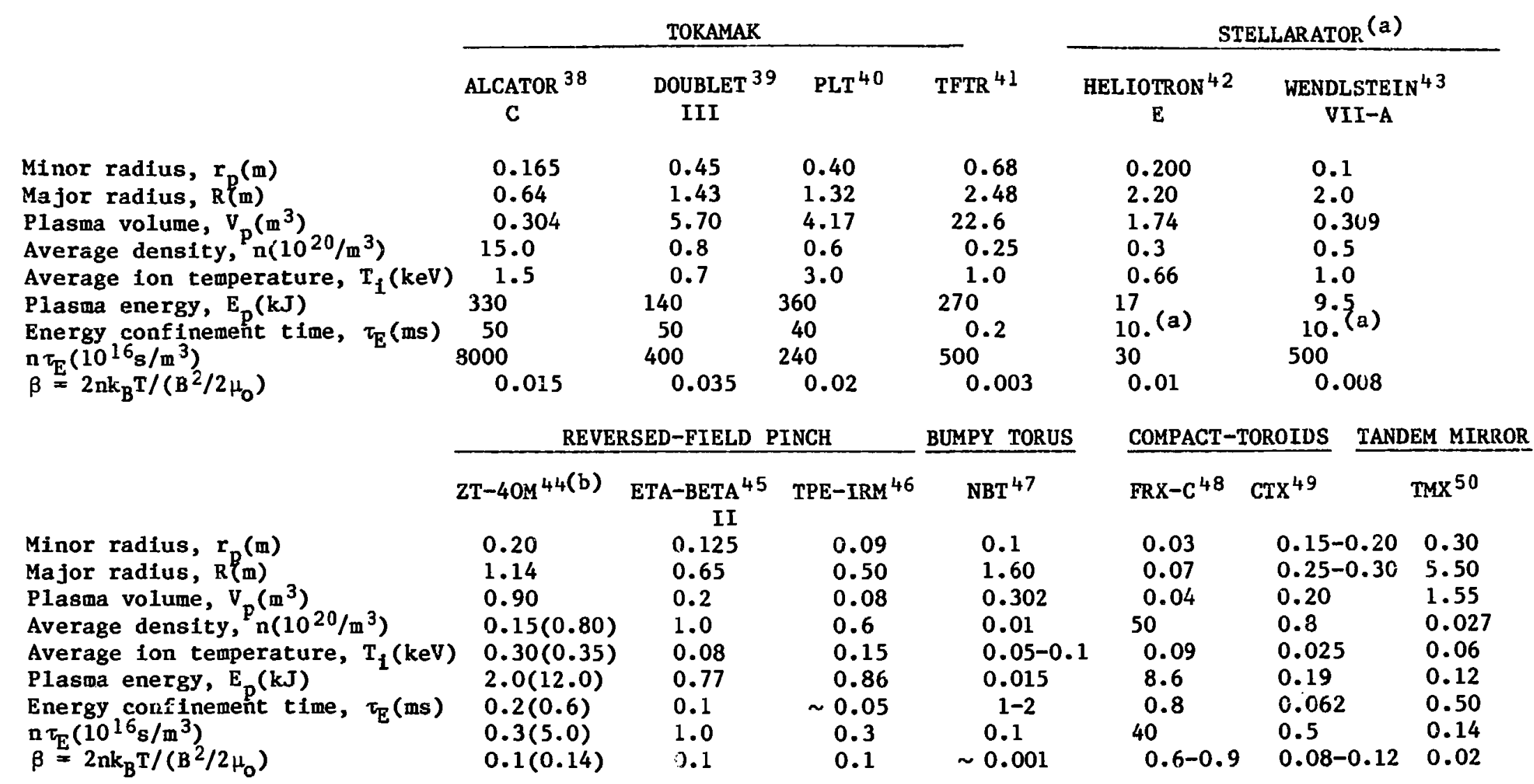

(a) Correcting for radiation gives $\tau_{E}=35 \mathrm{~ms}$ for $\mathrm{W}-\mathrm{VIIA}$ and $40 \mathrm{~ms}$ for Heliotron-E.

(b) Value outside parentheses correspond to $0.2-\mathrm{MA}$ operation, those within are for recent 0.4-MA discharges. 


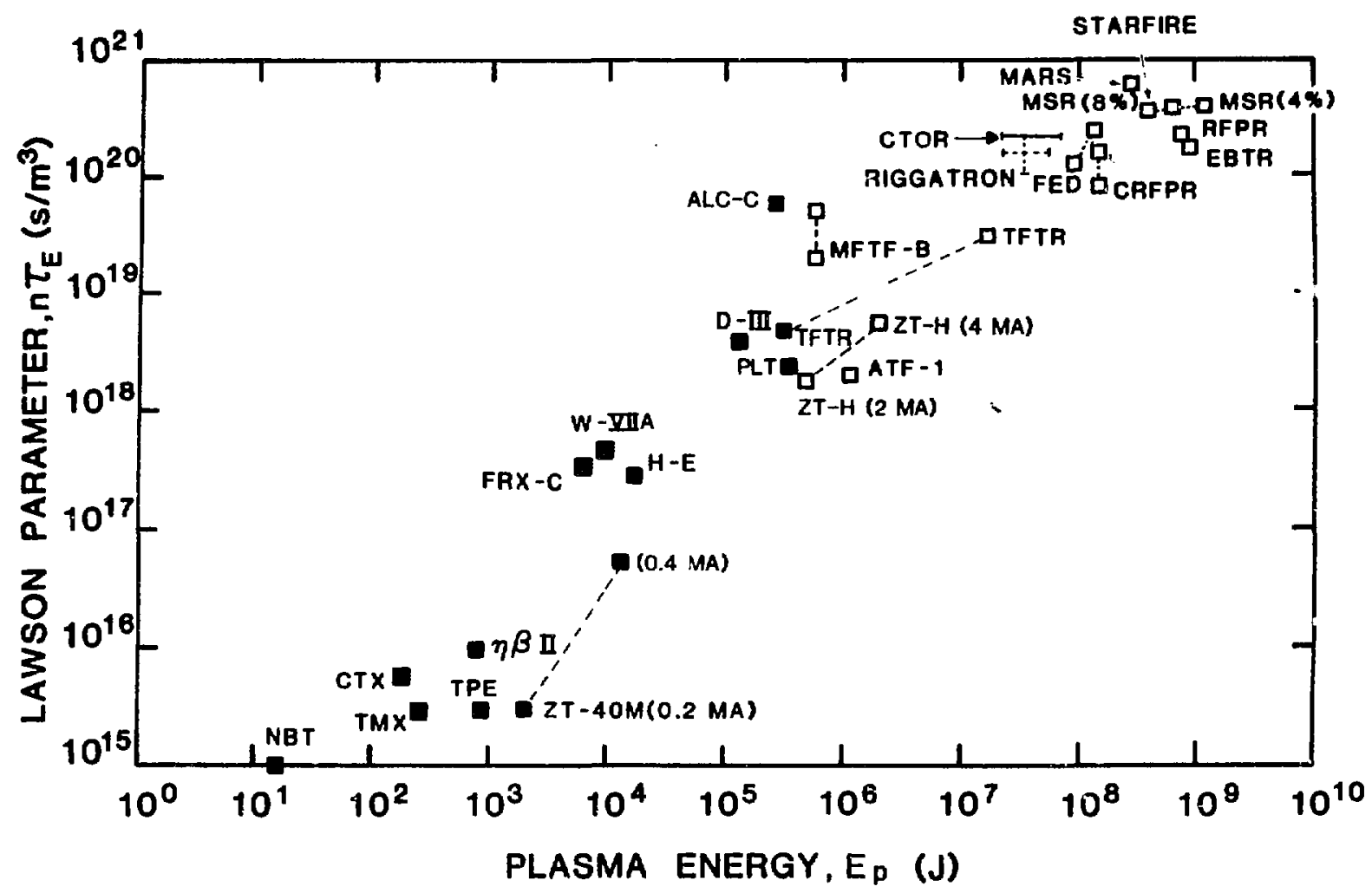

FIg. II.A-1. Achieved, projected, and reactor $n \tau_{\mathrm{E}}$ values plotted versus total kinetic energy stored in the plasma, $E_{p}$. Solid squares Indicate achieved parameters and open squares indicate design goals.

additional 100- to 1000-fold increase in stored plasma energy required for the commerclal reactors summarized in Table II.A-II, however, will require major increases in cost, physics performance, and technology development. The smaller, and presently lower-performance, Alternative Fusion Concepts (AFCs) depicted on FIg. II.A-1 may lead directly to a competitive, less-costly commerclal reactor; compact reactor embodiments that require an order-ofmagnitude lower value of $E_{p}$ may allow commercial fusion power to be achleved with considerably reduced development requirements.

With the exception of the CRFPR, all the reactor designs summarized on Table II.A-II would use large superconducting magnets, store large quantities of magnetic-field energy, and have system power densities (1.e., ratio of useful thermal power to FPC volume) that are 10-30 times below those for conventional fission reactors. Concurrently, these systems require large tonnages of high- 
TABLE II.A-II

SUMMARY OF KEY PARAMETERS FOR A RANGE OF DT FUSION REACTOR CONCEPTS(e)

\begin{tabular}{|c|c|c|c|c|c|c|c|}
\hline Device & MSR 51 & STARFIRE 3 & EBTR $^{52}$ & RFPR 53 & MARS 5,6 & CRFPR 15 & PWR $^{54}$ \\
\hline Design date & 1981 & 1980 & 1980 & 1979 & 1983 & 1982 & 1980 \\
\hline Plasma radius (m) & 0.81 & 2.38 & 1.0 & 1.2 & 0.49 & 0.71 & $\therefore$ \\
\hline Major radius (m) & 23.0 & 7.0 & 35.0 & 12.7 & $131^{(a)}$ & 4.3 & $-\infty$ \\
\hline Plasma volume $\left(\mathrm{m}^{3}\right)$ & 298 & 781 & 691 & 361 & 99 & 42.7 & - \\
\hline Average density $\left(10^{20} / \mathrm{m}^{3}\right)$ & 3.64 & 0.81 & 0.95 & 2.00 & 3.3 & 3.4 & - \\
\hline Temperature (kev) & 8.0 & 22 & 29 & 15 & 28 & 20 & -- \\
\hline Plasma energy (GJ) & 0.21 & 0.67 & 0.91 & 0.81 & $0.44^{(a)}$ & 0.14 & -- \\
\hline Field energy (GJ) & 109 & 61 & 131 & 14.7 & 60 & 1.65 & -- \\
\hline Lawson parameter $\left(10^{20} \mathrm{~s} / \mathrm{m}^{3}\right)$ & 3.7 & 3.0 & 1.7 & 2.0 & 6.6 & $0.7 s$ & -- \\
\hline Average beta & 0.08 & 0.067 & 0.17 & 0.30 & 0.28 & 0.20 & -- \\
\hline DT plasma power density $\left(\mathrm{MW} / \mathrm{m}^{3}\right)$ & 14.8 & 4.5 & 4.1 & 7.0 & 26.3 & 72.4 & 90 \\
\hline Magnetic field (T) & 6.56 & 5.8 & $5.0 / 2.25$ & 3.3 & 4.7 (b) & 5.2 & - \\
\hline Neutron current $\left(\mathrm{MW} / \mathrm{m}^{2}\right)$ & 1.9 & 3.6 & 1.4 & 2.7 & 4.3 & 19.5 & - \\
\hline Thermal power (MWt) & 4000 & 4033 & 4028 & 3000 & 3400 & 3350 & $\sim 3300$ \\
\hline Net power (MWe) & 1302 & 1200 & 1214 & 750 & $1202^{(c)}$ & 1006 & 1000 \\
\hline $\begin{array}{l}\text { System power density }\left(\mathrm{MWt} / \mathrm{m}^{3}\right) \\
\text { Mass utilization (tonne/MWt) }\end{array}$ & $\begin{array}{l}0.60 \\
6.6\end{array}$ & $\begin{array}{l}0.50 \\
5.7\end{array}$ & $\begin{array}{l}0.24 \\
10.85\end{array}$ & $\begin{array}{l}0.50 \\
3.7\end{array}$ & 0.29 & 15 & $19.8(7.5)^{(d)}$ \\
\hline $\begin{array}{l}\text { Mass utilization (tonne/MWt) } \\
\text { Thermal conversion efficiency }\end{array}$ & $\begin{array}{l}0.6 \\
0.35\end{array}$ & $\begin{array}{l}5.7 \\
0.35\end{array}$ & $\begin{array}{l}10.85 \\
0.35\end{array}$ & $\begin{array}{l}3.7 \\
0.30\end{array}$ & $\begin{array}{l}6.8 \\
0.43\end{array}$ & 0.37 & $\begin{array}{l}0.33 \\
0.33\end{array}$ \\
\hline $\begin{array}{l}\text { Thermal conversion efficiency } \\
\text { Recirculating power fraction }\end{array}$ & 0.07 & 0.167 & 0.15 & 0.17 & $\begin{array}{l}0.43 \\
0.22\end{array}$ & $\begin{array}{l}0.35 \\
0.14\end{array}$ & 0.33 \\
\hline Net plant efficiency & 0.33 & 0.30 & 0.30 & 0.25 & 0.33 & 0.30 & - \\
\hline Unit direct cost $(\$ / \mathrm{kWe})(\mathrm{g})$ & 1265 & 1438 & 1737 & 1104 & $1480^{(f)}$ & 863 & 900 \\
\hline Construction time (years) & 10 & 6 & 5 & 10 & 8 & 5 & $8-10$ \\
\hline "Then-current" (dafe) & 1991 & 11986 & 1985 & 1988 & 1988 & 1986 & 1983 \\
\hline $\operatorname{COE}(\operatorname{mills} / \mathrm{kWeh})(\mathrm{g})$ & 78 & 67 & 72 & 66 & $50^{(F)}$ & 41 & 40 \\
\hline
\end{tabular}

\footnotetext{
(a) Central cell.

(b) Central ce11, $24 \mathrm{~T}$ peak at mirror throat.

(c) Based on $2800 \mathrm{MW}$ of neutron power, which is multiplied in the blanket by 1.37 and an alpha-particle power of 700-MW (3500-MW fusion power), which is directly converted with an efficiency of 0.5 .

(d) Including steam-generator volume.

(e) More detailed physics, FPC and cost comparison between the tokamak (STARFIRE and MkIIb), tandem mirror (MARS), and GPFPR can be Eound in Table II.C-I of Sec. II.B.

(f) Reference 6 values are 1966 \$/kWe and $67 \mathrm{mills} / \mathrm{kWeh}$ in 1983 dollars. These values are brought to the common-base 1980 dollars using a $10 \%$ deflation rate.

${ }^{(g)}$ All costs based on 1980 dollars.
} 
technology FPC mass per unit of thermal power (1.e., mass utilization in Table II.A-II). Significant reductions in FPC mass utilization, stored plasma and magnetic-field energies, and projected unit costs are major improvements promised by the compact approaches. The compact systems are nearly an order-of-magnitude closer to comercialization as measured on Fig. II.A-1 by $E_{p}$, operate with low-technology copper (nonsuperconductiug) magnets, and provide a system with FPC power densities and mass utilizations that are closer to values deemed economic in contemporary fission power plants. The range of sizes projected for commercial fusion reactors, including the CRFPR and a PWR, is illustrated in Fig. II.A-2; this figure is a graphical display of the contents of Table II.A-II.
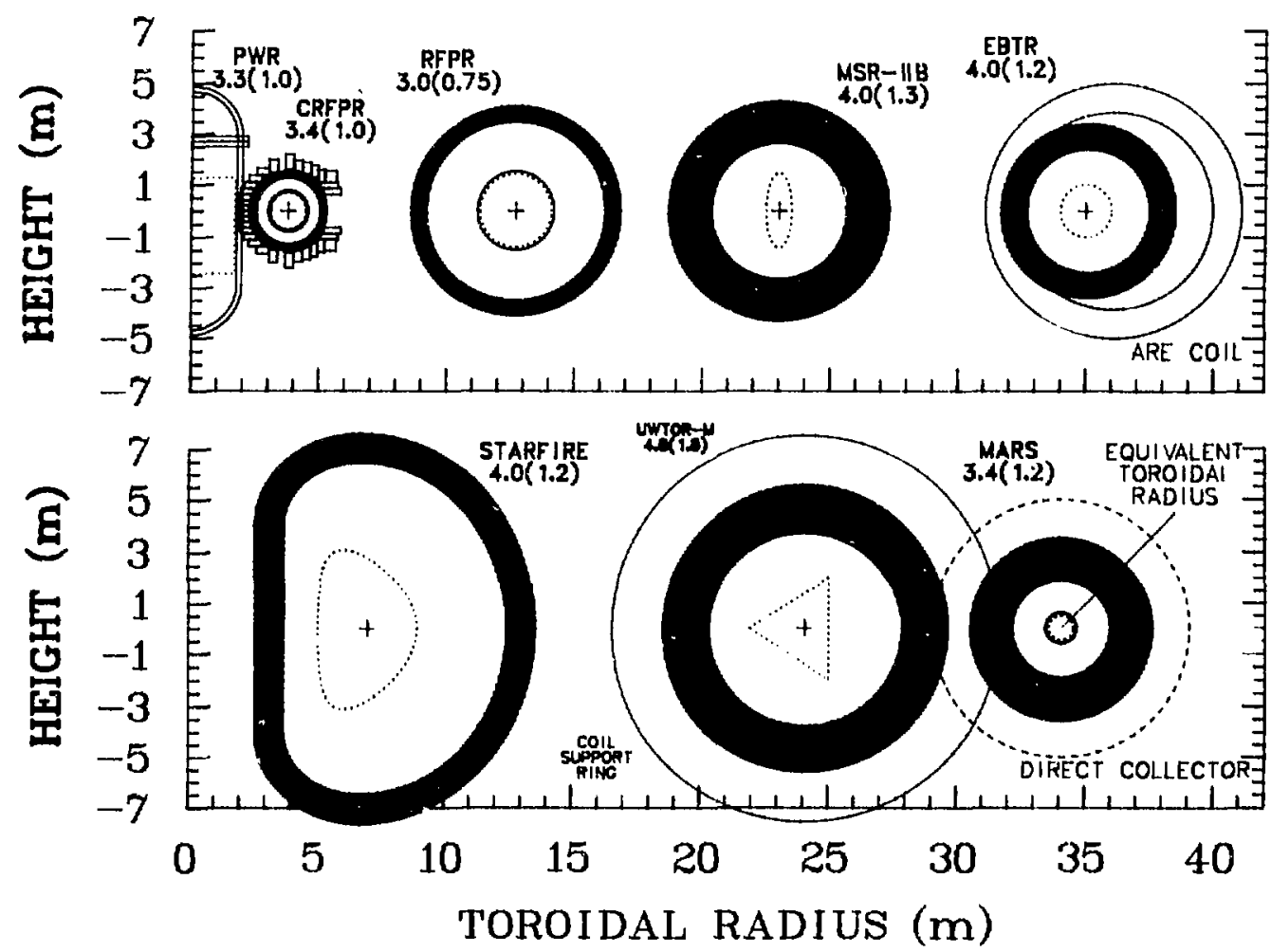

Fig. II.A-2. Relative sizes of malnline and alternative nagnetic fusion power reactors. With the exception of the compact $R F P$ reactor (CRFPR), all systems propose the use of large superconducting magnets. Thermal (net electrical) powers are indicated in GWt(GWe) units, and system power density is also shown. In presentation of the (linear) MARS tandem-mirror design, its length has been reduced by $2 \pi$ to generate an effective major radius for furposes of comparison only. 
In addition to the CRFPR, high-power-density versions of the tokamak (Riggatron ${ }^{9}, 2^{0}$ ) and the Ohmically-Heated Toroidal Experiment (OHTE ${ }^{13}$ ) have been proposed. Table II.Á-III summarizes key reactor parameters for the CRFPR, OHTE, and Riggatron in comparison to the superconducting STARFIRE tokamak reactor. Intercomparisons and preliminary technology assessments for these compact systems relative to the co-mainline fusion approaches can be found in Refs. 11 and 37.

A plot of the system power density versus the mass utilization projections given on both Tables II.A-II and II.A-III results in Fig. II.A-3. Shown also are the system power densities for the Magnox, 55 Superphenix (SP) Liquid-Metal Fast-Breeder Reactor (LMFBR), 56 Advanced Gas Reactors (AGR) ${ }^{57}$, and the aforementioned Pressurized-Water Reactor (PWR $)^{54,58}$ with both the reactor primary pressure vessel and the steam-generator (SG) power densities for the PWR indicated. The SP system power density referred to the "hot sodium pool" is most appropriately compared to the AGR and PWR cases, since use of the full volume of reactor vessel in this case also includes the intermediate heat exchangers (IHXs) and primary coolant pumps. The system power density projected for the gas-cooled Magnox reactors is very low; these older systems are economic today simply because the original investments for these older systems have long been paid. Hence, system power densities for present fission power systems range from 2-15 MW/m 3 , which typically is an order of magnitude above the system power densities being projected for most fusion concepts. The compact fusion concepts are attempting to operate with system power densities that are more in line with what is deemed economic for a range of fission power systems.

It is well recognized that system power density is but one of several figures of merit useful in projecting the competitiveness of a power system. Any comprehensive analysts of an optimum power system ultimately must be based on the cost of electricity, $\operatorname{COE}(\mathrm{mills} / \mathrm{kWeh})$. The $\operatorname{COE}$ should accurately reflect the cost of achieving a feasible engineering system (1.e., design complexity and degree of needed design conservatism), cost of operations and maintenance (i.e., FPC "burnup"), cost of environmental and safety restrictions (including resource utilization, afterheat and stored-magnetic-energy control, and long-term radwaste storage), construction time (on-site versus off-site fabrication), and plant avallability (1.e., mean-time-to-fallure versus "mean-time-to-repair). Generally, the system power density, mass utilization, or neutron first-wall loading reflect one important input into the COE equations through the unit 
SUMMARY OF KEY PARAMETERS $F^{-}$COMPACT HIGH-POWER-DENSITY TOROIDAL FUSION REAC'.JLS COMPARED TO STARFIRE

\begin{tabular}{|c|c|c|c|c|}
\hline & STARFIRE ${ }^{3}$ & CRFPR $^{15}$ & OHTE $^{18}$ & RIGGATRON 9,20 \\
\hline Plasma radius (m) & 2.38 & 0.71 & 0.67 & 0.32 \\
\hline Major radius (m) & 7.0 & 4.3 & 5.91 & 0.80 \\
\hline Plasma volume $\left(\mathrm{m}^{3}\right)$ & 781. & 42.7 & 52.1 & 2.0 \\
\hline Average density $\left(10^{20} / \mathrm{m}^{3}\right)$ & 0.8 & 3.4 & 10.0 & $20-30$ \\
\hline Average temperature (kev) & 22 & $20^{(a)}$ & $5-6$ & $12-20$ \\
\hline Average beta & 0.067 & 0.12 & 0.22 & 0.20 \\
\hline Plasma power density $\left(\mathrm{MW} / \mathrm{m}^{3}\right)$ & 4.5 & 72.4 & 64.0 & 500 . \\
\hline Plasma current (MA) & 10.1 & 18.5 & 12.4 & $3-4$ \\
\hline Plasma current density $\left(\mathrm{MA} / \mathrm{m}^{2}\right)$ & 0.57 & 11.7 & 8.8 & $7.2-9.6$ \\
\hline Magnetic field (T) & 5.8 & $5.2^{(b)}$ & $11.2(f)$ & $10 .-16 .(h)$ \\
\hline Neutron current $\left(M W / \mathrm{m}^{2}\right)$ & 3.6 & 19.5 & 19.5 & 68.4 \\
\hline Thermal power ${ }^{(c)}$ (MWt) & 4033. & 3350. & 2740. & 1325 . \\
\hline Net power (MWe) & 1200. & 1000 & 904. & 355. \\
\hline System power density $(\mathrm{d})\left(\mathrm{MWt} / \mathrm{m}^{3}\right)$ & 0.50 & 15.0 & 3.2 & $5.2^{(1)}$ \\
\hline Mass utilization $(e)$ (tonne/MWt) & 5.7 & 0.37 & $1.45(g)$ & 0.28 \\
\hline Thermal conversion efficiency & 0.35 & 0.35 & 0.40 & 0.41 \\
\hline Recirculating power fraction & 0.167 & 0.15 & 0.35 & 0.33 \\
\hline Net plant effictency & 0.30 & 0.30 & 0.24 & 0.27 \\
\hline
\end{tabular}

(a) Flat temperature profile, $\mathrm{J}_{0}^{2}(\mu \mathrm{r})$ density profile.

(b) Peak pololdal (confining) fields at plasma edge. Toroidal field at plasma axis is $9.5 \mathrm{~T}$, toroidal field at the TFC is $0.72 \mathrm{~T}$, and the maximum poloidal field is $4.5 \mathrm{~T}$ at the PFC. Meximum field at the back-biased oHC during startup is $9.12 \mathrm{~T}$.

(c) Total useful thermal power.

(d) Based on volume enclosed by and including the coils and total thermal power.

(e) Based on total thermal power and total mass of $\mathrm{FW} / \mathrm{B} / \mathrm{S} / \mathrm{C}$.

(f) Peak fields at Ohmic-heating coil during initiation of discharge.

$(\mathrm{g})_{0 \mathrm{f}}$ the 5500 tonne for $\mathrm{FW} / \mathrm{B} / \mathrm{S} / \mathrm{C}$, this particularly heavy (PbLi) blanket weighs 3200 tonne. An unusually heavy $\mathrm{OHC}$ is also used to minimize losses during startup.

(h) Peak fleld prior to ignition, with the field subsequently being decreased during the burn.

(i) The power density within volume defined only by the Riggatron coil set is $160 \mathrm{MWt} / \mathrm{m}^{3}$. 


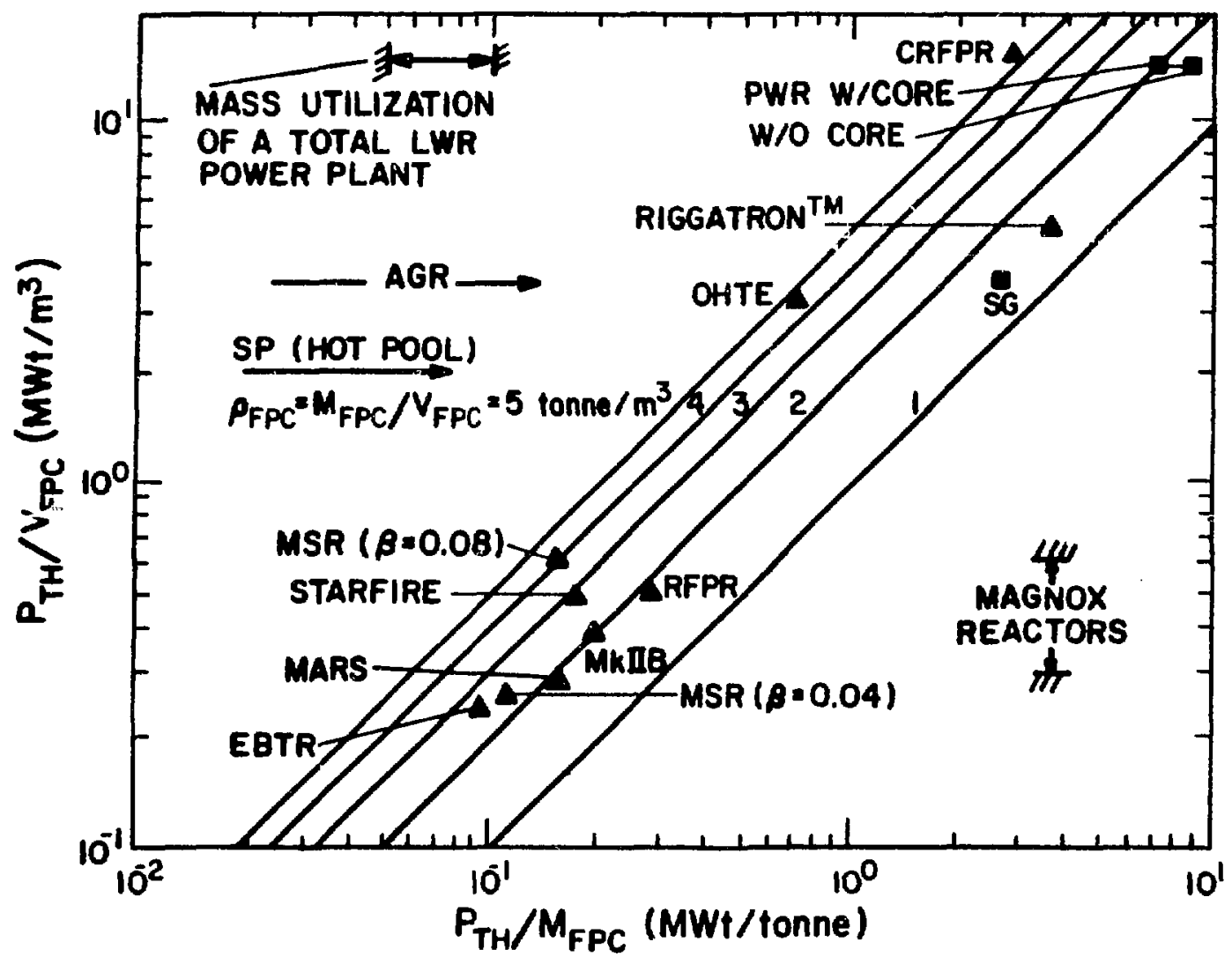

Fig. II.A-3. Display of system power density versus inverse of the mass utilization for a number of fusion reactor projections and a comparison with a number of fission reactor systems.

direct cost, UDC ( $\$ / \mathrm{kWe})$, which for a given net power output typically equals the sum of a fixed cost and a cost that varies inversely with the FPC power density or neutron first-wall loading. The system tradeoffs that enter into the determination of COE are fully incorporated into the CRFPR design-point determination and are explored more quantitatively in the following Secs. II.B. and II.C. 
In summary, the present status and planned near-term progress projects confinement parameters, $n \tau_{E}$, that should be adequate for a self-sustained Ignition in a DT plasma. This signiflcant achlevement, however, will occur in a plasma of energy content that remains many orders of magnitude below the requirements for a commercial power reactor. Generally, efficient confinement of hot and dense plasma does not appear as an insurmountable barrier to commerclal fusion power; Instead, the technology and cost required to contain such plastias with reactor-relevant total energy contents and total power appear as pacing items. In this context, certain AFC concepts, although less developed at present, may offer attractive routes to commercial fusion power by allowing viable reactors with reduced total plasma energy, physical size, complexity, and associated confinement technology. 
B. Rationale for High-Power-Density Tusion

On the basis of the summary of experimental status and reactor projections given In Fig. II.A-I, the compact, high-power-densicy approaches can reduce the stored plasma energy required for commercial fusion by an order of magnitude while simultaneously giving enhanced system power rensity and FPC mass utilization (Table II.A-II). Ultimately, however, the decision on an optimal system power density must be made on the basis of economics. This subsection develops further the rationale for compact systems on the basis of cost projections made for fusion.

The direct costs of building a fission or fusion reactor can be divided into two major components: the Reactor-Plant-Equipment (RPE) cost and the Balance-of-Plant (BOP) cost. The FPC consists primarily of the first wall, blanket, shield, magnets, and associated support systems, which for some concepts can represent an important contributor to the RPE cost. Other RPE items include main heat-transport systems, auxiliary heating equipment, vacuum, cryogenic, and tritium systems. The BOP consists of most subsystems outside the secondary contalnment, such as the secondary heat-transport system, heat exchangers and steam generators, turbines, electrical generators, auxillary systems, and buildings.

The RPE cost for fission reactors is approximately $25 \%$ of the plant total direct cost (TDC), the BOP accounting for the remaining $75 \%$. Studies of most fusion reactors project RPE costs that range from 50 to $75 \%$ of the TDC. The BOP costs for a fission and fusion plant of the same total electrical power output are expected to be similar. Hence, TDC estimates for fusion reactors predict higher values than for fission power plants because of high RPE costs related primarily to expensive ( $1 . \mathrm{e}$, massive, high-technology) FPCs. It is noted that fusion reactors capable of direct-energy conversion, such as the tandem mirror, attain higher overall energy conversion efficiencies and, therefore, project smaller BOP costs for the same total electrical power. In the case of the tandem mirror, however, this advantage is eroded somewhat by the higher recirculating power requirements. Furthermore, the TDC for the tandem mirror will be smaller only for sufficiently low cost of the direct-energy convertors. It is also noted that systems with unattractively high recirculating power fractions will require larger BOPs and associated costs; for such systems the RPE/BOP cost ratio will be artificially depressed, not because of low RPE, but because of abnormally high BOP costs. 
A summary of RPE/TDC values based on the recent fusion reactor design studies summarized in Table II.A-II is given in Fig. II.B-1; the dominance of the RPE costs for both mainline and major alternative fusion concepts is indicated. The Unit Direct Costs (UDC $=T D C / P_{E}$, where $P_{E}$ is the net elecric power) plotted on FIg. II.B-1 as a function of the ratio RPE/TDC use nominal values of $\sim 900 \$ / \mathrm{kWe}$ and 0.25 for UDC and RPE/TDC, respectively, to normalize the fusion projections to LWR fission power. The ratio of TDC for fusion relative to fission can then be determined analytically under the assumption that the BOP costs for similar fusion and fission power plants are nominally equivalent; this "analytic" curve of $R_{D C}=\left(\right.$ UDC) FUSION $_{\text {(UDC) }}$ FISSION is also given on Fig. II.B-1. Assuming that the fusion system can expend more on capital investment by taking advantage of a nil fuel cost, this tradeoff of fuel

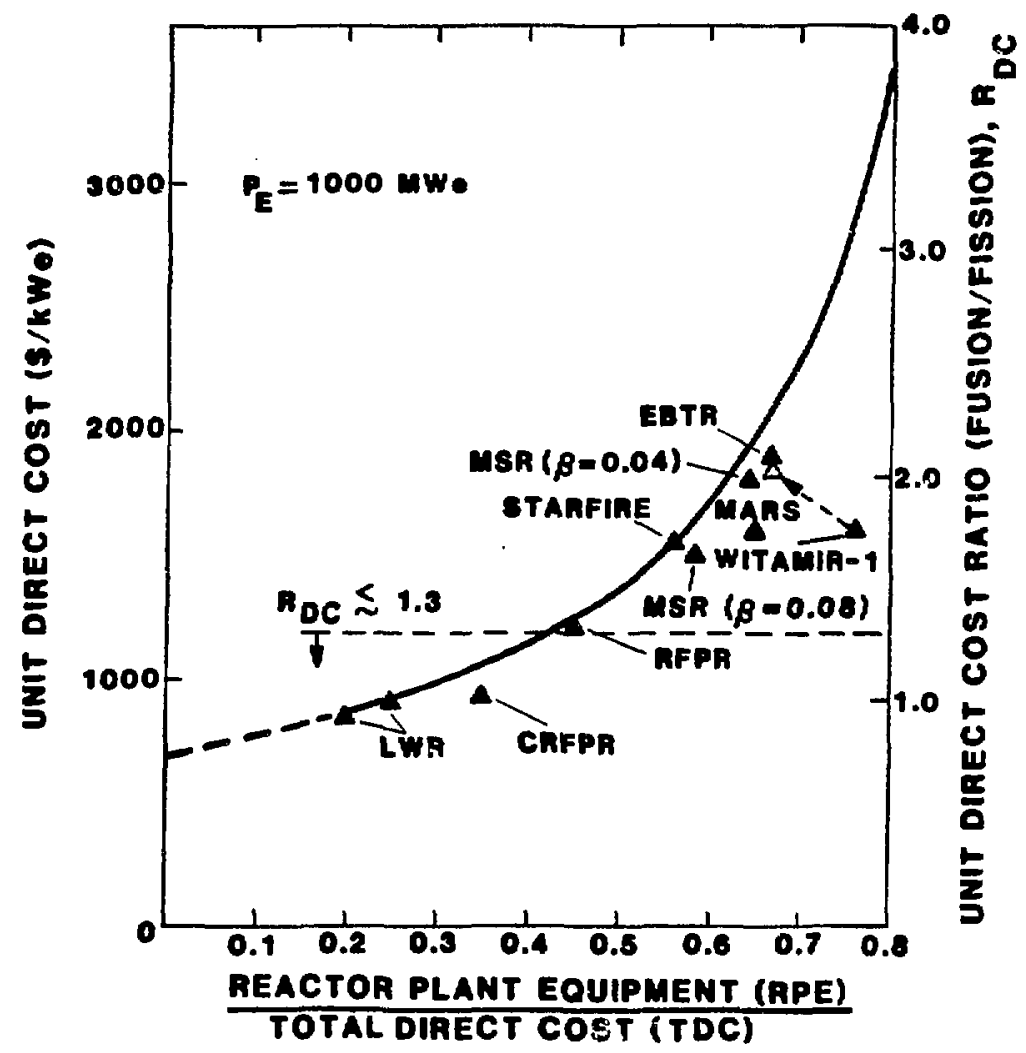

Fig. II.B-1. Plot of UDC versus RPE/TDC for a range of fusion reactor designs. Normalizing these costs to the LWR (UDC $\simeq 900 \$ / \mathrm{kWe}, \mathrm{RPE} / \mathrm{TDC} \simeq$ 0.25 ), the "analytic" curve of $\mathrm{R}_{\mathrm{DC}}=$ (UDC) FUSION $/$ (UDC) FISSION is also shown as a function of RPE TDC under the assumption of nearly equal BOP cost for comparable fusion and fission power plants. 
for capital cost becomes marginal for $R_{D C}$ values in excess of $\sim 1.3$ if the fuel cost for fission nominally comprises $1 / 4-1 / 3$ of the energy cost. Generally, operation in the RPE/TDC $\leq 0.3$, low-economic-leverage regime will require the FPC to be a less-dominant component of the TDC. For reasonable unit costs $(\$ / \mathrm{kg})$ of fabricated, high-technology components that compose the FPC and associated support systems, this criterion can be met only by decreased FPC mass utilization (tonne/MWt) or increased system power density; more-compact systems will be required.

The FPC mass per unit of generated thermal power (i.e., FPC mass utilization) for most fusion-power-plant designs is projected to lle in the range 5-10 tonne/MWt, compared to 0.3 tonne/MWt for LWRs (Table II.A-II and Fig. II.A-3). The mass utilization for the LWR is computed as the ratio of mass of the primary containment vessel (less the fuel) divided by the total thermal power generated. The mass utilization must be used carefully as a comparative measure of system performance; clearly, such comparisons imply a monotonic relutionship between total mass and cost. Systems that propose FPCs composed of large masses of inexpensive and easily replaced/reused coolant (i.e., PbLi) or concrete should appropriately compensate the mass utilizations (e.g., use mass of drained blanket). It is also noted that the mass of an entire LWR fission power plant, exclustve of concrete but including all reinforcing bar, is in the rauge 10-15 tonne/MWt, which in some cases is approached by the FPC mass utilization alone projected for certain fusion systems. Figure II.B-2 graphicaliy summarizes the FPC mass utilizations that have been predicted for a range of commercial fusion reactor designs; an average FPC cust of $\sim 30 \$ / \mathrm{kg}$ is indicated. The effect of doubling the unit cost of the FPC on the UDC is readily seen by doubling the slope of the dashed line on Fig. II.B-2; a $\sim 60 \$ / \mathrm{kg}$ FPC unit cost could rapidly catapult high-mass-utilizations systems out of economic contention.

The RPE for the compact RFP reactor is projected to be only $\sim 1 / 3$ of the TDC because the FPC and associated support equipment are smaller and less costly than the other fusion systems of simflar electrical output; the FPC mass utilization is similarly closer to the comparable Eigure ( 0.3 tonne/MWt) for LWR fission power. The direct cost of the compact fusion reactor, therefore, is expected to be only slightly higher than the cost of an LWR fission reactor. More importantly, however, the total cost of systems with RPE/TDC $\leq 1 / 3$ will be less sensitive to physics and technology uncertainties associated with the 


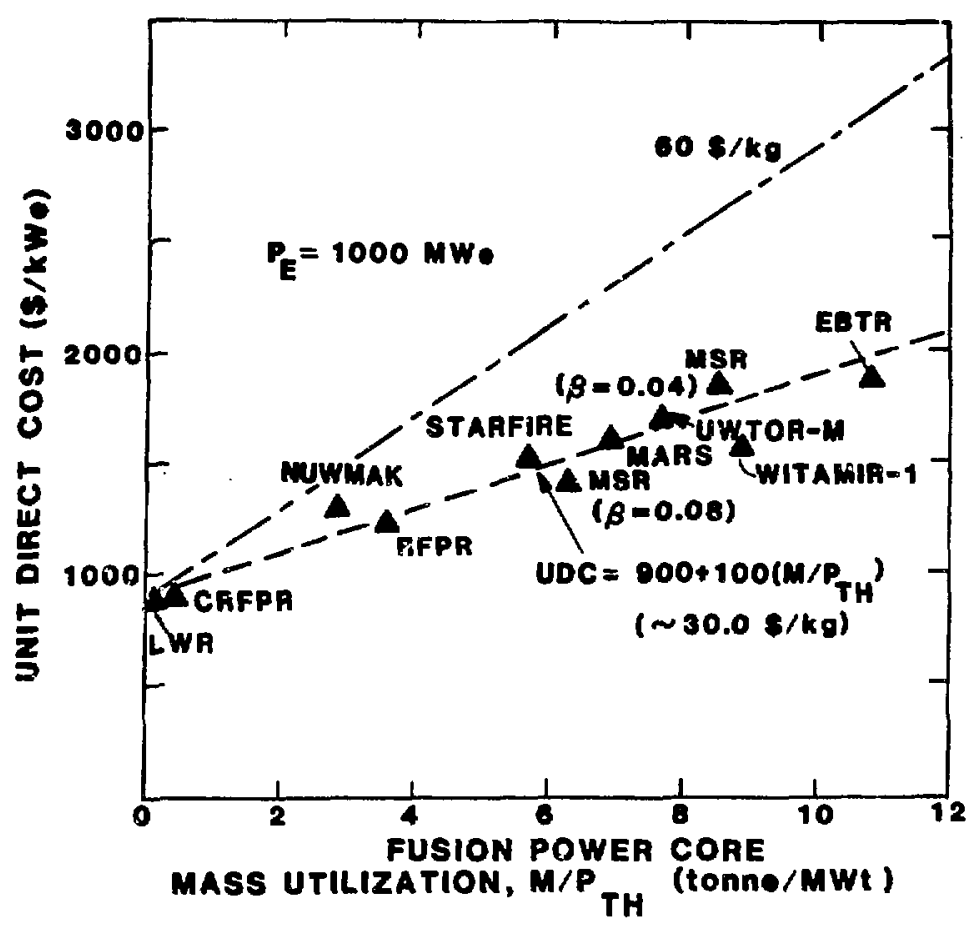

Fig. II.B-2. Correlation of the UDC projected for a number of fusion reactor designs with the FPC mass utilization. Increasing the FPC unit cost from $30 \$ / \mathrm{kg}$ to $60 \$ / \mathrm{kg}$ doubles the slope of the dashed line.

assumed plasma performance and FPC operation; both significantly effect plant performance and cost, which in turn can lead to appreciable costing uncertainty and potentially significant overruns. The criticism that absolute values of project cost should not be taken too seriously at this early stage of fusion power development has some legitimacy. Relative cost comparisons, however, are meaningful and useful. The uncertainty in predicting plasma performance and the dominance of the plasma performance in the overall cost equation for fusion represents a cause for concern. Rare are the examples where the projections of advanced technologies, be they energy or weapons systems, have proved to be either technically or economically conservative. 59

The direct capital cost represents only one component used in deriving the overall cost of electricity (COE) for a power plant. Figure II.B-3 graphically summarizes all major cost components and indicates the combination of these components to determine the COE. Also indicated on Fig. II.B-3 are elements and 


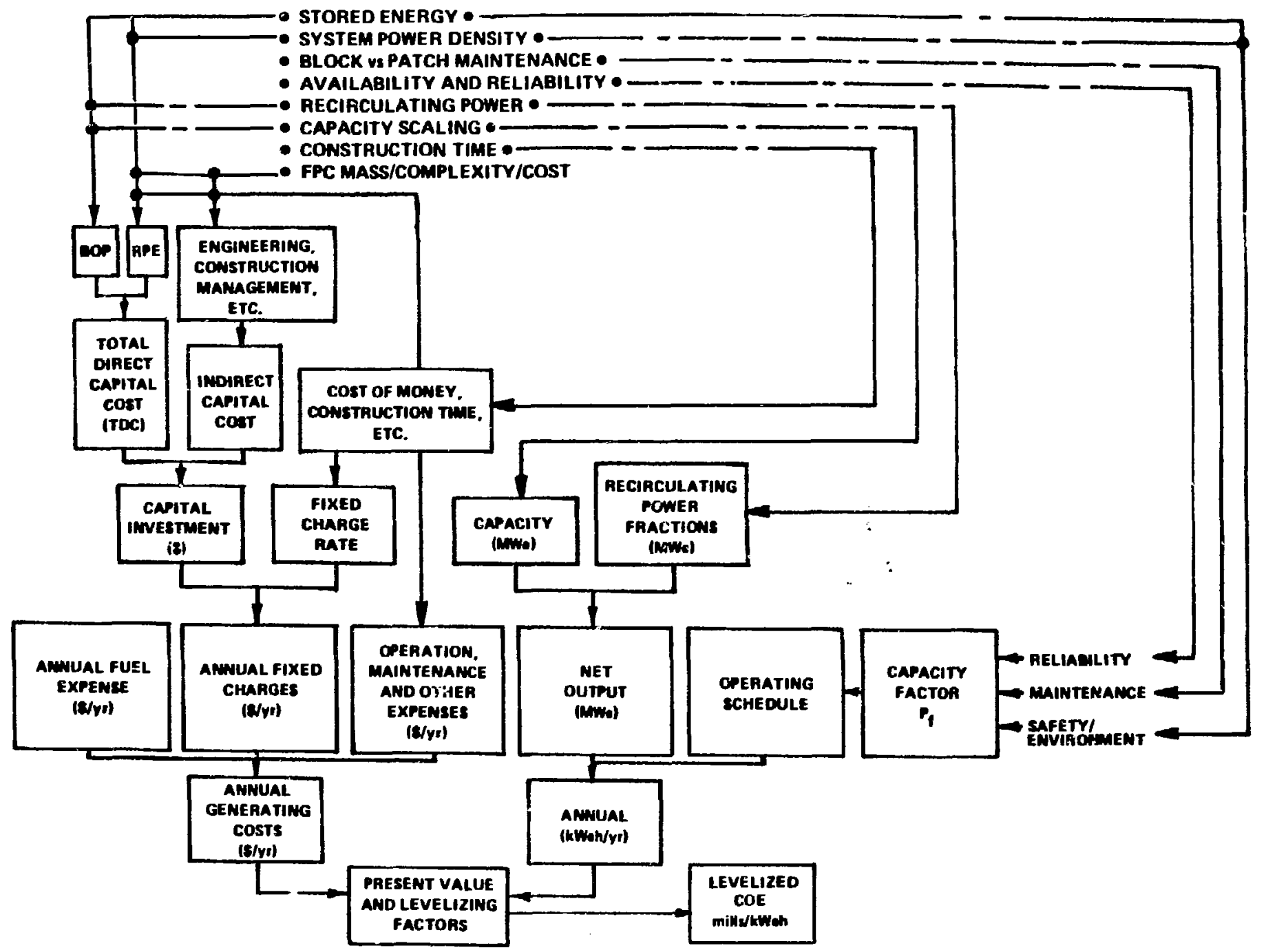

Fig. II.B-3. Logic diagram illustrating the means by which the levelized generating cost of electricity (COE) is compuced. Also shown at the top are key influences that may favorably or unfavorably impact the COE when considerations of compactness ur tigh power density are taken into account. 
tradeoffs that may impact favorably or unfavorably on the COE when compact versus conventional options are compared. The annual fixed charges for conventional and compact fusion reactors will be approximately proportional to the TDC because the Indirect capital cost is nominally the same percentage of the TDC for both compact and conventional reactors, and the fixed charge rate will be the same unless, for example, the compact reactor requires less time to construct and is more amenable to mass production methods. Fuel expenses will be equal for the same fusion power, and operation and maintenance (08M) costs are expected to be approximately equal for the same plant electrical capacity. The D\&M costs will vary if the costs of replacing the FPC differ; both conventional and compact reactors, however, require replacement of approximately equal masses of material per unit time $(\sim 200-400$ tonne/yr) for the same $\mathrm{FW} / \mathrm{B}$ lifetime $\left(\sim 15 \mathrm{MWyr} / \mathrm{m}^{2}\right)$. Although FPC 1ifetimes of 10-15 MWyr $/ \mathrm{m}^{2}$ are projected for stainless-steel first walls and blankets, transmutation-related resistivity increases in first-wall coils (1.e., for Riggatron 9,20 and OHTE 18 reactors) may reduce lifetimes to values as low as $5 \mathrm{MWyr} / \mathrm{m}^{2}$. The annual generating cost for a compact fusion reactor in some cases, therefore, can be lower than for a superconducting fusion reactor primarily belause of the lower RPE cost. The annual energy output ( $\mathrm{kWeh} / \mathrm{yr}$ ) for fusion reactors of equal capacity may not be equal because the recirculating power fractions and the capacity factors may be different. For instance, the compact tokamak (Riggatron) and the OHTE reactors may have higher recirculating power requirements and shorter radiation lifetimes because of the first-wall coil position.

Compact fusion reaciors are also expected to be higher-stress devices relative to other fusion reactors because of higher power densities, thermal loads, and neutron flukes (and higher magnetic fields at the coll for the Riggatron). Measured in terms of power density and surface heat fluxes, however, this operating condition is similar to the standard operating conditions encountered in LWR fission systems, albeit fusion in terms of load peaking has a more-difficult cooling geometry. Furthermore, operating at the higher-stress state should not reduce the plant capacity factor if equal engineering design criteria are used for compact and conventional reactors, although a higher cost for the former is expected. Because of the significantly reduced mass utilization, the compact systems can operate with a total FPC mass that is sufficlently small ( 1100-tonne for CRFPR) to allow total "block" (i.e., single-piece) maintenance of the FPC, with the attendant possibility for 
rapid change out, replacement, and system restart. Nevertheless, potentlal exists for a lower plant factor relative to the low-power-density fusion systems, this lower plant factor possibly diminishing the promise of reduced $\operatorname{COE}$ anticipated as a result of reduced TDC and construction time. Design-specific rellability analyses remain to be performed on a comparative basis; an lmportant tradeoff exists between a possible decrease in the mean-time-to-fallure and mean-time-to-repair related to increased power density in smaller, more Integrated systems.

Lastly, other less-quantiflable measures of techulcal and economic feasibility for an emerging energy technology can be formulated. The recent economic problems faced by large fission power plants60-62 point to a potentially important lesson for fusion. Over the past decades, fission power plants have Increased in size, reflecting primarily increased centralized demand, the availability of large turbine/generator units, percefved advantages of economies of scale, and the need to maintaln a competitive edge over fossil energy sources. Unfortunately, the construction costs for fission power relentlessly increased, this costs insidiously tracking increases in unit size, system complexity, construction time, prices of goods and services, and licensing.regulatory constraints. The increased size and complexity for fission power prolonged the "one-of-a-kind" phase, standardization became less avallable, outages were longer, repalr tlme was extended, and few repairs could be made with "off-the-shelf" Inventory items. The corrective measures presently being suggested for the fission industry generaliy call for sma11, less-complex reactor systems that promise to be economic because of the eroding economies of scale. Standardization and off-site factory construction is emphasized, and flexibility in sizing is deslgned to track more closely the (declining) rate of market growth, the market speccrum, and changing interest rates, escalation, and minimum coverage ratios.63-66 This approach is sald to result in a shortened and more-predictable construction schedule, with key nuclear systems being built and qualified by a small cadre of englneers and techniclans at a controlled, off-site factory, with the bulk of the nonnuclear BOP construction being performed or a more rapid and less-costly schedule and location. Based on this evolving picture for fission, no reason can be Identified that would cause these problems to be less severe for fusion. These problems may in fact be worse since the approaches to fusion embodied In Table II.A-II generally offer a power system that has 
- 10-30 times less power density.

- 10 times more mass utilization of systems that can be immensely more complex.

- RPE dominating the cost equation, with RPE being most sensitive to yet-to-be-resolved plasma performance.

- costs tending to optimize at large total power outputs.

- complex front-1ine support systems that must operate in a strong radiation field.

- slow and expensive migration along crucial learning curvas toward standardized reactor systems.

By focusing more onto fusion approaches that a) allow engineering tests to be performed on end-product-relevant devices, b) allow more options to be examined that are economic of time and dollars, and c) allow early demonstration of both financial and technical risk, fusion can become a serious energy option sooner. The compact, high-power-density approach offers this promise of $a$ bolder, more-aggressive development program that can lead to an end product with enhanced potentlal for market penetration. The compact systems in many respects can provide a more robust, "forgiving" approach to fusion power. 


\section{Simplified Reactor Model}

The heuristic arguments made in the last section have been guided by the results of detalled, parametric systems studies (Table II.A-III). This section uses a simplified global model to examine generic physics/engineering/economic issues related to the compact fusion systems. Of necessity the simplified physics and costing models adopted/extended for this anelysis are limited and, therefore, must be applied cautiously. A more-exact analysis that is specific to the RFP is given in Sec. IV.C.

The constraints imposed by plasma and particle physics on the selection of a reactor design can generally be classified according to the following areas:

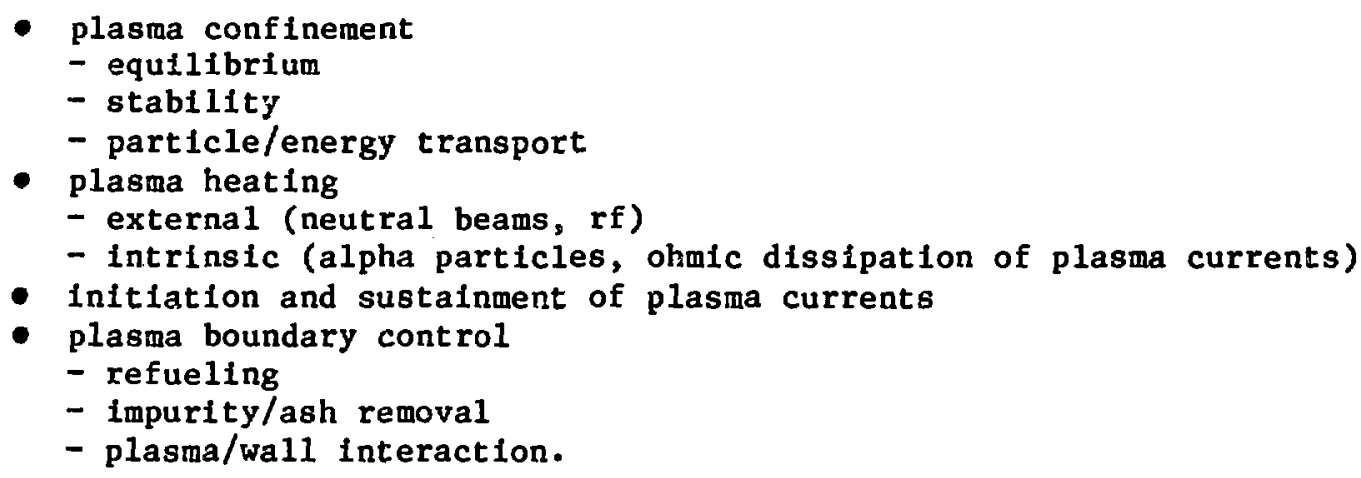

In practice, the physics associated with each of these important areas plays a secondary role in the selection of a reactor design point. 67 Ignition is specified for a global, steady-state plasma, which together with pressure balance determines the relationship between first-wall neutron (and heat) loading and plasma minor radius, $r_{p}$; the confinement $t$ ime, $\tau_{E}$, the $\beta B^{2}$ product evaluated at the plasma, system power density, $P_{T H} / V_{F P C}$, and total thermal power, $P_{T H}$, for a given aspect ratio, $A=R_{T} / \dot{r}_{p}$, then result. Figure II.C-l gives a schematic view of this idealized reactor model. For a blanket energy multiplication $M_{N} \approx 1.17\left(20 \mathrm{MeV} /\right.$ fusion), $\langle\sigma v\rangle / \mathrm{T}^{2} \simeq 1.1(10)^{-24} \mathrm{~m}^{3} / \mathrm{s} \mathrm{keV}^{2}, \mathrm{n} \tau_{\mathrm{E}}=$ $2(10)^{20} \mathrm{~s} / \mathrm{m}^{2}$, and $\mathrm{T}=15 \mathrm{keV}$, pressure balance, the specification of ignition, total thermal power, $P_{T H}$, the system power density, and a sample confinement (e.g., Alcator-1ike) scaling, respectively, give the following relationships:

$$
\begin{aligned}
& I_{w}\left(M W / m^{2}\right)=0.48 r_{p}\left(\beta B^{2}\right)^{2} \\
& I_{W}=\left(2.80 / \tau_{E}^{2}\right) r_{p}
\end{aligned}
$$




$$
\begin{aligned}
& I_{w}=\left[2\left(P_{T H} / V_{F P C}\right)(\Delta b+\delta) /\left(M_{N}+1 / 4\right)\right](1+x)^{2} / 4 x \\
& I_{w} r_{p}^{2}=\left(P_{T H} / A\right) /\left[(2 \pi)^{2}\left(M_{N}+1 / 4\right)\right] \\
& r_{p} \beta B{ }^{2}=2.42 / f_{A}^{1 / 2},
\end{aligned}
$$

where the blanket and shield thickness is $\Delta \mathrm{b}=\Delta \mathrm{b}_{\mathrm{BLK}}+\Delta \mathrm{b}_{\mathrm{SLD}}, \delta$ is the coll thickness, and $x=r_{p} /(\Delta b+\delta)$. Equation (3) results from the following definition of system power density:

$$
\begin{aligned}
& \frac{P_{T H}}{V_{F P C}}=\left(M_{N}+1 / 4\right) I_{w} \frac{A_{F W}}{V_{F P C}} \\
& \frac{V_{F P C}}{A_{F W}}=\frac{r_{w}}{2}\left\{1+\frac{\Delta b+\delta}{r_{w}}\right\}^{2} \geq 2 r_{w},
\end{aligned}
$$

where, as shown In FIg. II.C-1, the maximum FPC wer density for a given first-wa11 neutron loading occurs when $\left.x=1 ;{ }^{\prime}{ }_{H} / V_{F P C}\right)_{M A X}, I_{w}, M_{N}=I_{w}\left(M_{N}+\right.$ $1 / 4) /[2(\Delta b+\delta)]$. Shown also on EIg. II.C-1 are various reactor design polnts summarlzed on Table II.A-II. With the exception of the MARS design polnt, which has a considerable power flow out the open ends and minimizes the end-plug technology by minimizing the plasma radius, most of the closed systems tend to select a plasma radius based on power density and first-wall loading considerations rather than directly on the basis of plasma confinement o: transport. The ratio of FPC power density, ${ }_{\mathrm{TH}} / \mathrm{V}_{\mathrm{FPC}}$, to plasma fusion power density, $P_{F} / V_{P}$, where $P_{F}$ is the neutron plus alpha-particle power and $V_{P}$ is the plasma volume, is also shown on F1g. II.C-1; $\left(\mathrm{P}_{\mathrm{TH}} / \mathrm{V}_{\mathrm{FPC}}\right) /\left(\mathrm{P}_{\mathrm{F}} / \mathrm{V}_{\mathrm{P}}\right)=$ $(4 / 5)\left(M_{N}+1 / 4\right) x^{2} /(1+x)^{2}$. Depending on $M_{N}$, the plasma power denstty is reduced by 3-5 to give the FPC power density.

In deriving the expression for the maximum power density $\left(x_{w} \simeq \Delta b+\delta\right), I_{w}$, $\Delta \mathrm{b}$, and $\delta$ are held constant, ignoring the relatively weak interdependence between $\Delta b, I_{w}, r_{w}, M_{N}$, and the desire to achleve a given radiation and heating level at the coll position. The coefficients appearing in Eqs. (1) through (4) depend somewhat on assumed plasma temperature and density profiles as these quantities determine $n \tau_{E}$ and average fusion reactivity. The last expression 

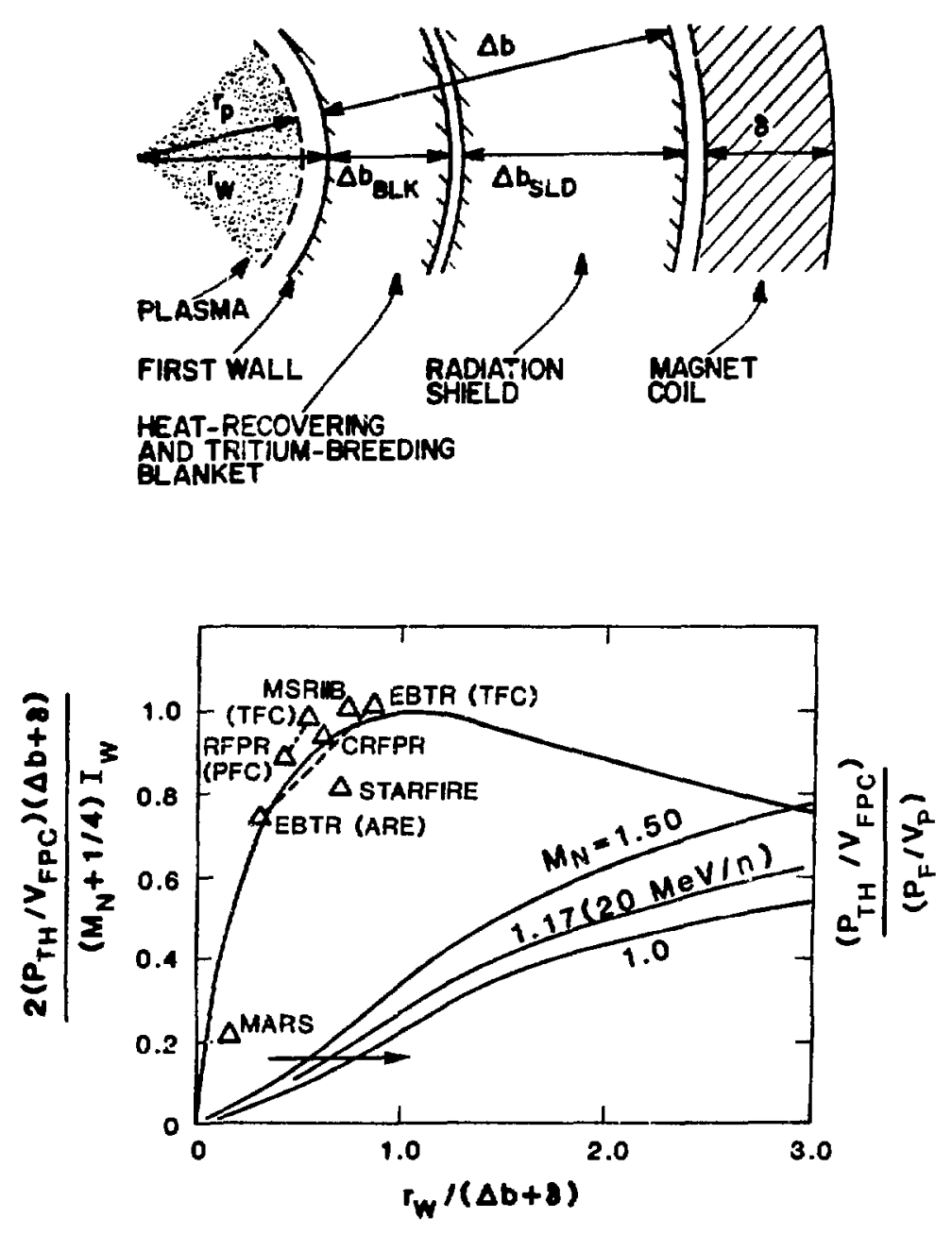

Fig. II.C-1. Radial build of a generic fusion reactor and the dependence of system power density on geometry. Shown also are the results from key reactor design studies (Table II.A-II).

[Eq. (5)] gives Alcator transport scaling for calibration purposes, where $\mathrm{F}_{A} \equiv$ $\tau_{E} / 5(10)^{-21}$ nre ${ }_{p}$; the confinement time required for a given $I_{w}$ and $r_{p}$ is given by Eq. (2) and must be matched by the physics scaling, be it Alcator or something else. Hence, the basic reactor characteristics are determined by a physics base that is no more profound than that required to assure a global energy and pressure balance on an idealized, average-property, steady-state, ignited plasma with the neutron first-wall loading being chosen on the basis of technology (coll fields, heat fluxes, etc.) and economics (i.e., power density, plant availability, etc.). These expressions are plotted in Fig. II.C-2, wherein 
Eqs. (1) and (2) are the dot-dashed lines (respective values of $\beta B^{2}$ and $\tau_{\mathrm{E}}$ are indicated), the long-dashed curves give the total power per ur:t aspect ratio, $\mathrm{P}_{\mathrm{TH}} / \mathrm{A}$, and the solid curves give the relationship between $I_{w}$ and $r_{p}$ for a given system power density. In order to evaluate Eq. (3) graphically, specific values of $\Delta b+\delta$ had to be assumed. Figure II.C-2 has been generated for a typical superconducting-coil case $(\Delta \mathrm{b}+\delta>3 \mathrm{~m})$ and the minimum $B / \mathrm{s}$ case allowed for exoblanket, resistive copper colls $(\Delta \mathrm{b}+\delta \simeq 1 \mathrm{~m})$.

Selection of a specific region of the $I_{w}: r_{p}:\left(P_{T H} / V_{F P C}\right): \beta B{ }^{2}: \tau_{E}$ hyperspace described on Fig. II.C-2 must be made on the basis of economic and physics judgments. Economics will generally specify an optimal system power density, $\mathrm{P}_{\mathrm{TH}} / \mathrm{V}_{\mathrm{FPC}}$, or first-wall neutron loading, $I_{w}$; phystcs will constrain the choice of $\tau_{\mathrm{E}}$ and the assoclated scaling with the plasma system variables; and a combination of physics and engineering will determine the all-important $\beta B 2$ product. Assumptions that underlie the results depicted in Fig. II.C-2 may shift the curves and regions defined thereby, these assumptions being summarized below.

- Plasma and first-wall minor radil are taken as equal, $r_{p}=$ $r_{w}$; the dilution of system power density by a scrapeoff region is not taken into account.

- Profile effects (1.e., peaked versus flat pressure profiles) can result in factors of two differences in fusion plasma power density for a given $\beta B^{2}$; flat profiles have been assumed.

- The field used in the product $\beta B^{2}$ refers to the field at the plasma surface, with the toroldal field at the coll roughly being increased to $B_{C} \simeq B /(1-2 / A)$ or more if ifinite cnils and field ripple are taken into account. This constraint, of course, applies only to those systems confined primarily by toroidal fleld; the main confining fleld in an RFP is poloidal and decreases from the plasma surface to the coll.

- The blanket energy multiplication, ${ }^{M}$, can vary over the range 1.1 to 1.5 , depending on materials selection.

- Implicit in Eq. (3) is the assumption of a fixed value of $\Delta \mathrm{b}+\delta$. Increased shlelding will be needed to protect the colls at higher first-wall neutron loadings, nominal values being selected for the purposes of Fig. II.C-2. 

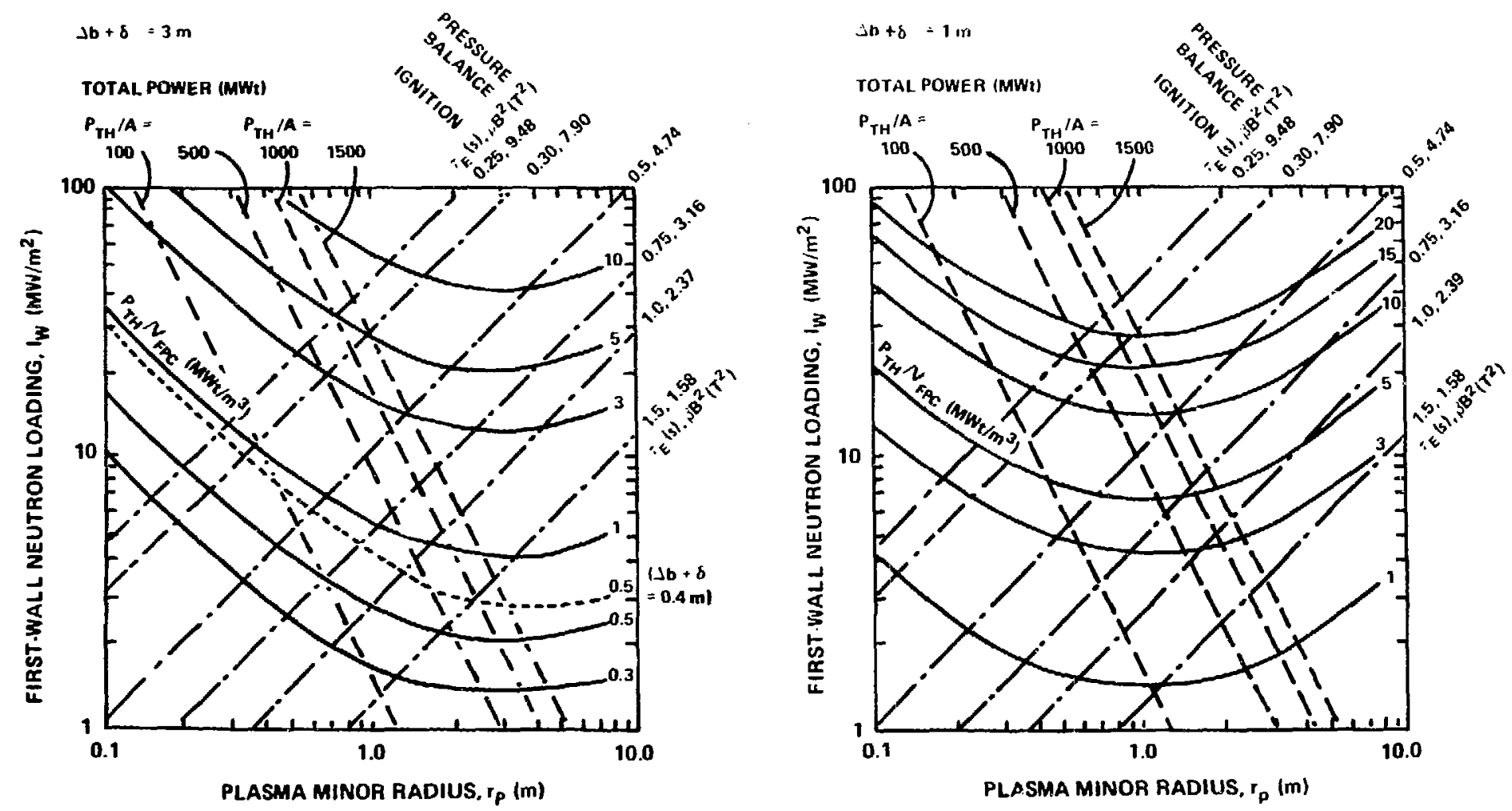

Fig. II.C-2. Dependence of $I_{w}$ versus $r_{p}$ as determined by pressure balance [Eq. (1)], ignition condition [Eq. (2)], economics [Eq. (3)], and power balance [Eq. (4)]. Expresised in terms of $I_{w}$ and $r_{p}$, the Alcator scaling [Eq. (5)] becomes $I_{w} r_{p}=2.8 / f_{A}$, which could also be plotted as straight lines on these figures. 
- The use of noncircular plasmas to achieve high-beta values obviously departs from the model used to generate Fig. II.C-2. In addition, other beta scalings (e.g., $\beta^{\prime} A=$ constant) can be applied to further constrain the problem, with the $\beta^{\prime} A \simeq$ constant condition actually giving a unique aspect ratio for maximum FPC power density.

Inclusion of these and other constraints and physics adds more realism to this simpliffed "systems model," with a considerable loss in transparency. In spite of these shortcomings, the present model nevertheless provides a relatively realistic description of a broad range of reactor options. Systems of low-to-moderate aspect ratio $(A=3-5)$ that are dominated by toroidal magnetic fleld generally cannot provide more than 5-6 $\mathrm{T}$ at the plasma if $10-$ to 12-T limits are imposed at superconducting magnets. Given beta limits in the range 0.05 to 0.10 , this limits the plasma pressure parameter to $\beta \mathrm{B}^{2} \leq 2.5 \mathrm{~T}^{2}$. For power plants of total thermal output $\mathbf{P}_{\mathrm{TH}} \leq 4000 \mathrm{MWt}$, most of these devices share the lower regions of Fig. II.C-2 in the narrow region defined by $\mathbf{P}_{\mathrm{TH}} / \mathrm{A}=500$ and $1500 \mathrm{MWt}$ contours. These systems generally are physics (i.e., beta) and engineering (i.e., field) limited in that better economics more than likely would result for higher values of $\mathrm{P}_{\mathrm{TH}} / \mathrm{N}_{\mathrm{FPC}}$ if this region could be obtained through higher $\beta B^{2}$ values.

At the othar end of the spectrum are the higher-power-density systems, which attain FPC power densities in the range above $5 \mathrm{MWt} / \mathrm{m}^{3}$; for these systems $\beta \mathrm{B}^{2}$ values above $\sim 5 \mathrm{~T}^{2}$ are required, with design points again generally residing within the band defined by $\mathrm{P}_{\mathrm{TH}} / \mathrm{A}=500$ and $1500 \mathrm{MWt}$. These designs, however, clustered in the upper regions of Fig. II.C-2. Generally, those confinement schemes capahle of compact, high-power-density operation use a thin blanket/shield of proportions requiring normal, water-cooled copper $(\Delta \mathrm{b}+\delta<1 \mathrm{~m})$ rather superconducting $(\Delta \mathrm{b}+\delta>3 \mathrm{~m})$ coils; also, unless highly stressed coils can be accommodated close to the plasma, confinement by poloidal rather than toroidal fields is preferred to achieve the increased $\beta B^{2}$ values (5-6 $\mathrm{T}^{2}$ ) required for high-power-density operation while minimizing ohmic losses in the copper coils.

The RFP allows access to both of the aforementioned reactor regimes: normal-coil, high-power-density 15,16 or superconducting-coil lower-powerdensity ${ }^{31}$ FPCs. In the case of the RFP, the pressure balance given by Eq. (1) can be expressed in terms of the toroidal plasma current, $I_{\phi}$, and the poloidal beta, $\beta_{\theta}$. In this case Eq. (1) becomes 


$$
\begin{aligned}
I_{w}\left(M W / m^{2}\right) & =7.67(10)^{-4} \frac{\beta_{\theta}^{2} I_{\phi}^{4}}{r_{p}^{3}} \\
& =0.086 \beta_{\theta}^{2} j_{\phi}^{2} r_{p}^{5},
\end{aligned}
$$

where $I_{\phi}$ has the units of $M A$ and $j_{\phi}\left(\mathrm{MA} / \mathrm{m}^{2}\right)$ is the plasma current density. This relationship can also be plotted in Fig. II.C-2 and indicates that for either standard or compact reactor embodiments, $\beta_{\theta}^{1 / 2} I_{\phi}$ in a narrow range about 9 MA w111 be required $\left(\sim 20 \mathrm{MA}\right.$ for $\left.\beta_{\theta} \approx 0.2\right)$.

Although this simplifled systems model is not Intended to foreclose the compact option for conflgurations that are domlnated by large toroldal fields, certain problems become apparent when attempts are made to increase power density in systems dominated by toroidal field. Generally, increased FPC power density and reduced FPC mass utilization are obtained by increasing $I_{w} \approx 0.48\left(\beta B^{2}\right)^{2} r_{p}$ and decreasing $\Delta b+\delta$, the latter accomplished by reduced shield thickness and the use of resistive copper colls. The Increased first-wall heat load becomes comparable to that considered acceptable for the limiters of the low-power-density systems, 3 with the total first-wall area for the former being comparable to the limiter area in the latter. Accompanying the increased first-wall neutron loading with reduced $B / S$ thickness and fixed total power is an increase in blanket power density that may require flowing liquid-metal coolant/breeder. Agaln, if the magnetic flelds within the blanket are too large, the MHD pressure drop for a flowing liquid-metal coolant, $\triangle \mathrm{P}_{\mathrm{MHD}} \propto l \mathrm{~B}^{2} \mathrm{v}$, can become prohibitive; even though the total flow path, $l, 1$ s reduced for a compact systiem, the higher blanket power density will require a high flow velocity, $v$, to maintain structural temperature and corrosion 1 imits, this also contributing to increased pumping power. Hence, because of plasma power density (Increased $\beta B^{2}$ with reduced $r_{p}$ ), MHD pumping power and coolant pressure, and magnet etress and ohmic losses, systems that are not dominated by toroidal fleld are preferred for compact, high-power-density operation. Given that these physics and engineering constraints can be met, plant and electricity costs will ultimately dictate the design region of interest on Fig. II.C-2. 
Before this region in the $I_{w}-r_{p}$ phase space where an economically attractive reactor might lie can be determined, a detalled costing tradeoff study must be made. ${ }^{15} \mathrm{~A}$ parameterized form of the costing algorithm described in Ref. 15 is used here to fllustrate the essential elements of the process used for design-point selection. Again, while this simplifled costing model reproduces the results from detailed designs, it must nevertheless be used cautiously when extended into reglons beyond those for which "calibrations" exist. The present model also fixes the recirculating power fraction, which can be significantly influenced by MHD-pumping and ohmic-coil power requirements.

Analytically fitting the tabular costing formulae used in Ref. 15, which in turn have been adopted from the detalled STARFIRE costing data base, 3 the following expression for the unit direct cost, UDC $(\$ / \mathrm{kHe})$, results:

$$
\begin{aligned}
& (1-\varepsilon) U D C / 1.17=F+\frac{C}{\left(\mathrm{P}_{\mathrm{TH}} / \mathrm{V}_{\mathrm{FPC}}\right)} \\
& \mathrm{F}=\frac{460 .}{\mathrm{P}_{\mathrm{ET}} 0.62}+\frac{44.3}{\eta_{\mathrm{TH}}}+\frac{14.2}{\eta_{\mathrm{TH}} 0.8_{\mathrm{P}_{\mathrm{ET}}} 0.2} \\
& \mathrm{C}=\frac{1000 \mathrm{c}_{\mathrm{FPC}}}{\eta_{\mathrm{TH}}},
\end{aligned}
$$

where $\varepsilon$ is the recirculating power fraction, $\eta_{\mathrm{TH}}$ is the thermal conversion efficiency, $P_{E T}(G W e)=\eta_{T H} P_{T H}$ is the total electric power, and $c_{F P C}\left(M \$ / m^{3}\right)$ is the volumetric cost of the FPC. The quantity F represents the flxed-cost component, whereas $C$ relates to those costs that depend on FPC volume and, hence, system power density, $\mathrm{P}_{\mathrm{TH}} / \mathrm{V}_{\mathrm{FPC}}$. The factor 1.17 in $\mathrm{Eq} \cdot(8 \mathrm{~A})$ accounts for $15 \%$ contingency and $2 \%$ spare-parts costs that are included in UDC. Equations (8) can be expressed in terms of $I_{W}\left(\mathrm{MW} / \mathrm{m}^{2}\right)$ given the ratio $A_{F W} / V_{F P C}$ of first-wall area to FPC volume [Eq. (6B)]. Hence, defining a FPC cost per unit FW area, $c_{F W}\left(M \$ / m^{2}\right) \equiv\left(V_{F P C} / A_{F W}\right) c_{F P C}$, the following expression for the UDC, emerges: 


$$
\begin{aligned}
\operatorname{UDC}(\$ / \mathrm{kWe})= & \frac{538.2}{\mathrm{P}_{\mathrm{E}}^{0.62}(1-\varepsilon)^{0.38}}+\frac{16.6}{\eta_{\mathrm{HH}}^{0.8}(1-\varepsilon)^{0.8} \mathrm{P}_{\mathrm{E}}^{0.2}} \\
& +\left[51.8+\frac{1170 \mathrm{c}_{\mathrm{FW}}}{\left(\mathrm{M}_{\mathrm{N}}+1 / 4\right) \mathrm{I}_{\mathrm{W}}}\right] \frac{1}{(1-\varepsilon) \eta_{\mathrm{TH}}} .
\end{aligned}
$$

It is noted that the FPC unit mass costs are $\mathrm{c}_{\mathrm{FPC}}(\$ / \mathrm{kg})=10^{6} \mathrm{c}_{\mathrm{FPC}}\left(\mathrm{MS} / \mathrm{m}{ }^{3}\right) / \mathrm{p}_{\mathrm{FPC}}=$ $10^{6} \mathrm{C}_{\mathrm{FW}}\left(\mathrm{MS} / \mathrm{m}^{2}\right)\left(\mathrm{A}_{\mathrm{FW}} / \mathrm{V}_{\mathrm{FPC}}\right) / \rho_{\mathrm{FPC}}$, in which $\rho_{\mathrm{FPC}} \simeq 2-3000 \mathrm{~kg} / \mathrm{m}^{3}$ is a typical FPC "smear" density, as is illustrated in Fig. II.A-3. The FPC unit mass costs range from 10-60 $\$ / \mathrm{kg}$, depending on the specific component, with an average viqlue of $\sim 30 \$ / \mathrm{kg}$ emerging from a composite of all major designs reported to date (Fig. II.B-2).

In order to convert the UDC into a COE(mills/kWeh), the annual Return On Investment (ROI) and operating costs must be specified as well as the fraction of the year, $p_{F}$, that the plant is capable of operating at full power. Hence, if the $\mathrm{FW} / \mathrm{B}$ costs a fraction $f_{F W B}$ of the total direct costs and $\left(I_{W} \tau\right)$ is the FW/B lifetime measured in inits of $\mathrm{MWyr} / \mathrm{m}^{2}$, the $\operatorname{COE}$ is given by

$$
\operatorname{COE}(\mathrm{mi} 11 \mathrm{~s} / \mathrm{kWeh})=\frac{\mathrm{UDC}}{8.76}\left[\mathrm{f}_{\mathrm{FWB}} \frac{\mathrm{I}_{\mathrm{w}}}{\left(\mathrm{I}_{\mathrm{w}} \tau\right)}+\mathrm{f}_{\mathrm{IE}} \frac{(\mathrm{ROI}+0 \& \mathrm{M})}{\mathrm{P}_{\mathrm{f}}}\right] .
$$

The quantity $f_{I E}$ gives the factor by which the costs must be increased to account for interest and escalacion during construction and other indirect costs. Using the standardized guideline given in Ref. $68, \mathrm{f}_{\mathrm{IE}}=1.73$, which for ROI $=0.15 / \mathrm{yr}$, and for operating and maintenance costs of $O \delta M=0.02 / \mathrm{yr}$, the annual pay rate amounts to $0.29 / \mathrm{p}_{\mathrm{f}}$. To this is added the annual rate at which payment is made for FW/B replacement, which is called out separately in Eq. (10).

The algorithm described in Ref. 3 for the dependence of the plant factor on $I_{W}$ is used, wherein 28 days/year are given to scheduled FW/B maintenance if the first-wall chronologial life, $\left(I_{W} r\right) / I_{W}$, exceeds one year; the annual maintenance time is increased above 28 days/year in proportion to $p_{f} I_{W} /\left(I_{W} \tau\right)$ if the FW/B 
must be replaced more frequently than once a year. This procedure along with the FW/B replacement cost givas the major economic disincentives from high $I_{w}$ values. The following expression gives the above-described dependence of $p_{f}$ on $I_{w}:$

$$
\mathrm{p}_{\mathrm{f}}=\left\{\begin{array}{c}
0.76 \quad \text { if } \mathrm{I}_{\mathrm{w}} /\left(\mathrm{I}_{\mathrm{w}} \tau\right)<1.32 \mathrm{yr}^{-1} \\
\frac{0.835}{1+0.0707 \mathrm{I}_{\mathrm{w}} /\left(\mathrm{I}_{\mathrm{w}} \tau\right)}
\end{array}\right.
$$

Before Eqs. (9), (10), and (11) can be evaluated, typical values for the composite costs, $c_{F P C}\left(M \$ / m^{3}\right), c_{F W}\left(M \$ / m^{2}\right)$, or $c_{F P C}(\$ / k g)$ must be chosen. Table II.C-I gives in three parts a summary of physics, economics, and FPC parameters for the STARFIRE ${ }^{3}$ and Culham MkIIB tokamak ${ }^{69}$, the CRFPR, 15 and the tandern-mirror MARS 5,6 reactor designs. The composite costs for each of these designs are summarized as part of Table I.C-IC.

Equations (9), (10), and (11) have been evaluated on Fig. II.C-3 using the composite FPC unit costs ( $37 \$ / \mathrm{kg}$ or $0.20 \mathrm{M} / \mathrm{m}^{3}$ ) 1isted for the CRFPR in Ref. 15 and Table II.C-IB. For all cases, the recirculating power fraction was fixed at $\varepsilon=0.15$, an assumption that must be examined by a detalled systems code. 15 For the case with longer first-wall lifetimes $\left[\left(I_{w} \tau\right)=10-15 \mathrm{MWyr} / \mathrm{m}^{2}\right]$, a sha1low cost minimum occurs at $I_{W} \simeq 20 \mathrm{MW} / \mathrm{m}^{2}$, this minimum shifting to low first-wall loadings as $\left(I_{w} \tau\right)$ is decreased. The shape of this minimum and the shift is a result of the $p_{f}$ algorithm described by Eq. (11), which discriminates against FPC replacements more frequently than nnce a year. The dot-dashed curve in Fig. II.C-3 shows a case similar to the STARFIRE design ${ }^{3}$ as well as the effects of Increasing the unit FPC costs from the $19-\$ / \mathrm{kg}$ value given in Ref. 3 (Table II.C-IB) to the $37-\$ / \mathrm{kg}$ values us.t for the CRFPR (1.e., $f_{\text {FWB }}$ increases from 0.05 to 0.10 and $c_{F W}$ increases from $0.66 \mathrm{Ms} / \mathrm{m}^{2}$ to $1.29 \mathrm{M \$} / \mathrm{m}^{2}$ ). It should be noted that such an increase in $c_{\text {FPC }}(M \$ / \mathrm{kg})$ for STARFIRE may not be justified since the massive shield required to protect the superconducting magnets is a low-cost item, probably justifiying a lower FPC unit cost compared to the CRFPR. 
TABLE II.C-IA

PLASMA PHYSICS/ENGINEERING PARAMETERS

PARAMETERS

Major toroldal radius, $R_{T}(m)$

Aspect ratio, A

Plasma elongation, $k$

Plasma triangularity, d

Average plasma minor radius, $r_{p}(m)$

Plasma volume, $v_{p}\left(m^{3}\right)$

Average beta, $\langle\beta\rangle$

Magnetic field at plasma, $B_{0}(T)$

Safety factor at Iimiter, $q_{a}$

Plasma toroidal current, $I_{\phi}(\mathrm{MA})$

Plasma current density, $I_{\phi} / \pi r_{p}{ }^{2}\left(\mathrm{MA} / \mathrm{m}^{2}\right)$

Average electron temperature, $T_{\mathbf{e}}(\mathrm{keV})$

Average ion temperature, $T_{i}(k \cdot e V)$

Average electron density, $n_{e}\left(10^{20} / m^{3}\right)$

Average fusion power, $P_{f}(M W)$

Average plasma power density, $\mathrm{P}_{\mathrm{f}} / \mathrm{V}_{\mathrm{p}}$

Average neutron $\mathrm{FW}$ loading, $I_{W}\left(\mathrm{MW} / \mathrm{m}^{2}\right)$

Burn-time/off-time

STARFIRE

7.0

MkIIB 69

6.7

$\frac{\text { MARS } 5,6}{131(a)}$

CRFPR

3.8

3.6

3.5

NA

5.3

1.6

1.75

NA

1.0

0.5

0.

NA

0 .

2.38

2.51

0.49

0.71

781 .

836.

98.8

37.8

0.067

0.092

0.28

$0.12^{(b)}$

5.8

4.0

4.7

$0.40^{(c)}$

5.1

2.5

NA

0.02

10.1

10.2

NA

18.4

0.54

0.51

NA

11.8

17.3

12.0

24.0

10.0

$24 \cdot 1$

12.0

28.0

10.0

0.81

1.50

3.3

6.55

3510 .

2992.

2600.

$3.60^{\circ}$

26.3

2732.0

4.49

3.6

3.2

4.3

72.5

20.

19.5

$\infty$

$\infty$

$\infty$

(a) Central-cell length.

(b) Pololdal beta, $\beta_{\theta}=0.23$.

(c) Toroidal field at plasma edge, tocoldal field on-axis is $9.5 \mathrm{~T}$, and polotdal fteld at plasma edge is $5.2 \mathrm{~T}$.

For the FW/B design life of $15 \mathrm{MWyr} / \mathrm{m}^{2}$, lower-cost systems at higher first-wall loadings are in principle possible for STARFIRE if the engineering and physics constraints would allow the increased $\beta B^{2}$ while maintaining the total power constant. The difficulties in both plasma and magnet performance encountered when attempting to access high values of $I_{W}$ and $P_{T H} / V_{F P C}$ for otherwise constant-power STARFIRE-1ike systems are better illustrated by the following example. Since the net electrical power is given by $P_{E}=(2 \pi)^{2}\left(M_{N}+1 / 4\right) \eta_{p} A I_{w} r_{W}^{2}$ (re: typical purameters given in Table II.C-I), an Increase from $3.6 \mathrm{MW} / \mathrm{m}^{2}$ for STARFIRE to $20 \mathrm{MW} / \mathrm{m}^{2}$ requires $r_{w}$ to be decreased from $2.83 \mathrm{~m}$ to $1.20 \mathrm{~m}$, keeping $A=R_{T} / r_{w}$ and $P_{E}$ constant. Since the blanket/shield thickness cannot be decreased (actually, $\Delta b$ must be increased 
TABLE II.C-IB

FUS ION-POWER-CORE (FPC) PERFORMANCE COMPARISONS(a)

\begin{tabular}{|c|c|c|c|c|}
\hline PARAMETER & STARFIRE ${ }^{3}$ & MkIIB 69 & MARS $^{5}, 6$ & $\operatorname{CRFPR}^{(e)}$ \\
\hline ross thermal po & $\overline{4000}$ & $\overline{3261}$ & $\overline{3400}$ & 3365 \\
\hline Blanket energy mu & 1.14 & 1.14 & 1.36 & 1.28 \\
\hline $\begin{array}{l}\text { Thermal conversion efficlency, } \eta_{\mathrm{TH}} \\
\text { Recirculating power fraction, } \varepsilon\end{array}$ & $\begin{array}{l}0.36 \\
0.1857\end{array}$ & $\begin{array}{l}0.37 \\
0.08\end{array}$ & $\begin{array}{l}0.427(0.343) \\
0.22\end{array}$ & $\begin{array}{l}0.365 \\
0.185\end{array}$ \\
\hline Plant efficiency, $n_{D}=n_{T H}(1-\varepsilon)$ & 0.30 & 0.34 & 0.33 & 0.30 \\
\hline et electrical po & 1200. & 1200. & 1202. & 1000 \\
\hline lominal B/S thick & 2.5 & & 0.84 & 0.60 \\
\hline Tominal coll thic & 1.6 & $\{3.2$ & 1.62 & 0.60 \\
\hline $\begin{array}{l}\text { FPC volume, } V_{F P C}\left(m^{3}\right) \\
\text { F1rst-wa11 area, } A_{F W}\left(m^{2}\right)\end{array}$ & $\begin{array}{l}8110(6630) \\
780\end{array}$ & $\begin{array}{l}8000(4401) \\
716\end{array}$ & $\begin{array}{l}11645 \\
494\end{array}$ & $\begin{array}{l}285 \\
112\end{array}$ \\
\hline $\begin{array}{l}\text { FPC volume/surface, } v_{\mathrm{FPC}} / \mathrm{A}_{\mathrm{FW}} \\
\text { FPC cost (MS) }\end{array}$ & $\begin{array}{l}10.4(8.50) \\
440.1 / 363.3\end{array}$ & $11.2(6.15)$ & 23.6 & $\begin{array}{l}2.55 \\
45.6\end{array}$ \\
\hline $\begin{array}{l}\text { - FW/B } \\
\text { - Shield }\end{array}$ & $\begin{array}{l}82.4 \\
186.1 / 109.3\end{array}$ & $\begin{array}{l}204.5[ \\
137.2[\end{array}$ & $\begin{array}{l}54 . \text { (a) } \\
56 .\end{array}$ & 14.8 \\
\hline - Colls & 171 & 377. & 371 . & 30.8 \\
\hline $\begin{array}{l}\text { FPC unit cost, } c_{F} \\
\text { FPC volumetric } c_{C}\end{array}$ & $19.0 / 22.0$ & 41.5 & 20.6 & 39.5 \\
\hline $\begin{array}{l}\text { FPC volumetric cos } \\
\text { FPC area cost, c } \\
\text { Cost figures of } \mathrm{me}\end{array}$ & $\begin{array}{l}0.053 / 0.059 \\
0.66 / 0.51\end{array}$ & $\begin{array}{l}0.16[0.11] \\
1.00[0.66]\end{array}$ & $\begin{array}{l}0.041 \\
0.97\end{array}$ & 0.13 \\
\hline - $\mathrm{RPE} / \mathrm{TDC}$ & 0.56 & 0.72 & 0.64 & 0.36 \\
\hline - $\mathrm{FPC} / \mathrm{TDC}$ & $0.26 / 0$ & 0.23 & 0.27 & 0.06 \\
\hline - $(\mathrm{EW} / \mathrm{B}) / \mathrm{TDC}$ & 0.050 & 0.067 & 0.030 & 0.017 \\
\hline System minor ra & & & & \\
\hline$r_{S_{s}}=\left[V_{F P f} / 2 \pi^{2} R_{T}\right.$ & $7.66(6.70)$ & $7.78(5.77)$ & 4.2 & 1.95 \\
\hline $\begin{array}{l}\text { Plasima chamber volume, } V_{P C}\left(\mathrm{~m}^{3}\right) \\
\text { First-wall radius, } r_{1}(\mathrm{~m})\end{array}$ & $1106(5$ & $970(83$ & 148 . & 42. \\
\hline $\begin{array}{l}\text { First-wall radius, } r_{w}(m) \\
F W \text { neutron loading, } I_{w}\left(M W / m^{2}\right)\end{array}$ & $\begin{array}{l}2.83 \\
3.6\end{array}$ & $\begin{array}{l}2.71 \\
3.2\end{array}$ & $\begin{array}{l}0.60 \\
4.3\end{array}$ & $\begin{array}{l}0.15 \\
19.5\end{array}$ \\
\hline Power density, $P_{T K}$ & $0.50<0.6$ & $0.41(0.74)$ & 0.29 & 11.8 \\
\hline FPC mass, MFC (tonne) & $\begin{array}{l}23174 / 16496 \\
1574\end{array}$ & $\begin{array}{l}17330 \\
4700\end{array}$ & $\begin{array}{l}23308 \\
194(3185)(b)\end{array}$ & $\begin{array}{l}1105 \\
45(845)(\end{array}$ \\
\hline $\begin{array}{l}\text { - Shield } \\
\text { - Colls }\end{array}$ & $\begin{array}{l}13360 / 6682 \\
8240\end{array}$ & $\begin{array}{l}3650 \\
8980\end{array}$ & $\begin{array}{l}2980(724)(d) \\
8509(3496)(d)\end{array}$ & $\begin{array}{l}189 \\
870(c)\end{array}$ \\
\hline Spectfic mass, $N$ & $29.7 / 21.2$ & 24.2 & 47.2 & 9.9 \\
\hline $\begin{array}{l}\text { Mass utilization } \mathrm{MPC}^{-} \ddot{\mathrm{P}}_{\mathrm{TH}} \\
\text { (tonne/MWt) } \\
\mathrm{FPC} \text { denstty, } \mathrm{M} / \mathrm{V}_{\mathrm{O}}\end{array}$ & $\begin{array}{l}5.7 / 4.1 \\
2.86 / 2.4\end{array}$ & $\begin{array}{l}5.3 \\
2.17\end{array}$ & $\begin{array}{l}6.8 \\
2.0\end{array}$ & 0.33 \\
\hline FPC density, $M_{\text {FPC }} / V_{\text {FPC }}$ & $2.86 / 2.49$ & 2.11 & 2.0 & 3.9 \\
\hline
\end{tabular}

(a) Values In () based on toroldal volume, otherwise volume of central column included. Values to $r i g h t$ of $/$ do not include vacuum-pumping ducts and ports. Values in [] give $\$ / \&$ conversion in 1977 followed by $\$$ inflation from 1977 to 1980; otherwise the conversion/inflation order is reversed.

(b) Drained (undrained).

(c) 72.8-tonne TFCs, 394-tonne OHC, 404-tonne EFC.

(d) Central cell (end plug).

(e) Cost figures based on Ref. 15 analysis. 
COST COMPARISON (NORMALIZED TO TDC) (1980 Dollars)

\begin{tabular}{|c|c|c|c|c|c|}
\hline & ACCOUNT & STARFIRE $^{3}$ & MkIIB ${ }^{69}$ & MARS 5,6 & CRFPR \\
\hline 20. & Land and Land Rights & 0.19 & -- & 0.21 & 0.34 \\
\hline 21. & Structure and Site Facilities & 20.09 & 12.48 & 10.59 & 26.07 \\
\hline \multirow[t]{5}{*}{22.} & Reactor Plant Equipment (RPE) & 56.00 & 72.04 & 64.07 & 3.37 \\
\hline & 22.1.1 First-Wal1/Blanket ( & $(\mathrm{FW} / \mathrm{B}) 4.77$ & 6.66 & 2.33 & 1.54 \\
\hline & 22.1 .2 Shield (S) & 10.78 & 4.47 & 2.46 & -- \\
\hline & $22.1 .3 \operatorname{Cot1}(\mathrm{C})$ & 9.90 & 12.30 & 16.06 & 3.20 \\
\hline & $F P C=F W / B+S+C$ & 25.48 & 23.43 & 20.85 & 4.74 \\
\hline 3. & Turbine Plant Equipment & 14.47 & 10.88 & 11.65 & 23.15 \\
\hline 24. & Electric Plant Equipment & 6.77 & 3.29 & 6.78 & 12.62 \\
\hline 25. & Miscellaneous Plant Equipment & 2.37 & 1.27 & 1.41 & 4.36 \\
\hline 26. & Special Materials & 0.014 & -- & 5.26 & 0.026 \\
\hline 90. & Total Direct Costs (TDC) & 100 & 100 & 100 & 100 \\
\hline \multirow[t]{4}{*}{99.} & Total Costs & 185.23 & 200.26 & 174.12 & 173.00 \\
\hline & Unit Direct Cost, UDC(\$/kWe) & 1439 & \multicolumn{2}{|c|}{$2556 \cdot[1685]^{(a)}{ }_{1480 .(b)}$} & 959. \\
\hline & Cost of Electricity, COE(mills/ & /kWe) 67.1 & - & $50 .(b)$ & 46.2 \\
\hline & Net Electric Power, $\mathrm{P}_{E}$ (MWe) & 1200 & 1200 & 1202 & 1000 \\
\hline
\end{tabular}

(a) Value in bracket gives $\$ / \mathcal{L}$ conversion in 1977 followed by $\$$ inflation from 1977 to 1980; otherwise the conversion/inflation order is reversed.

(b) Based on $1966 \$ / \mathrm{kWe}$ and $67 \mathrm{mills} / \mathrm{kWeh}$ In 1983 dollars and a $10 \%$ per year deflation factor.

somewhat to hold the same radiation/heat flux of the superconducting magnets), the STARFIRE design moves farther from the optimum power density as $x=r_{w} /(\Delta b+\delta)$ decreases from 0.69 to $0.29\left[x=1\right.$ maximizes $\left(P_{T H} / V_{F F C}\right) / I_{w}$ or $A_{F W} / V_{F P C}$ for a fixed $M_{N}, E q \cdot(3)$ and Fig. II.A-3]. Since $I_{w}=0.48\left(\beta B^{2}\right)^{2} r_{w}$ and $I_{w} r_{w}^{2}$ is held constant, then $\beta B^{2} \propto I_{w}^{3 / 4}$. Hence, for the same on-axis field, presuming the colls are already at a peak field of $11-12 \mathrm{~T}$, the $\beta \propto \mathrm{I}_{w} 3 / 4$ scaling dictates that beta would have to increase by a factor of 3.62 to $\beta \simeq 0.24$ compared to the design value of 0.067 ; for any realistic pressure proflle, an average beta of $>0.24$ would result in a nearly unity beta on axis. Nevertheless, glven that such improved plasma performance were possible, the 


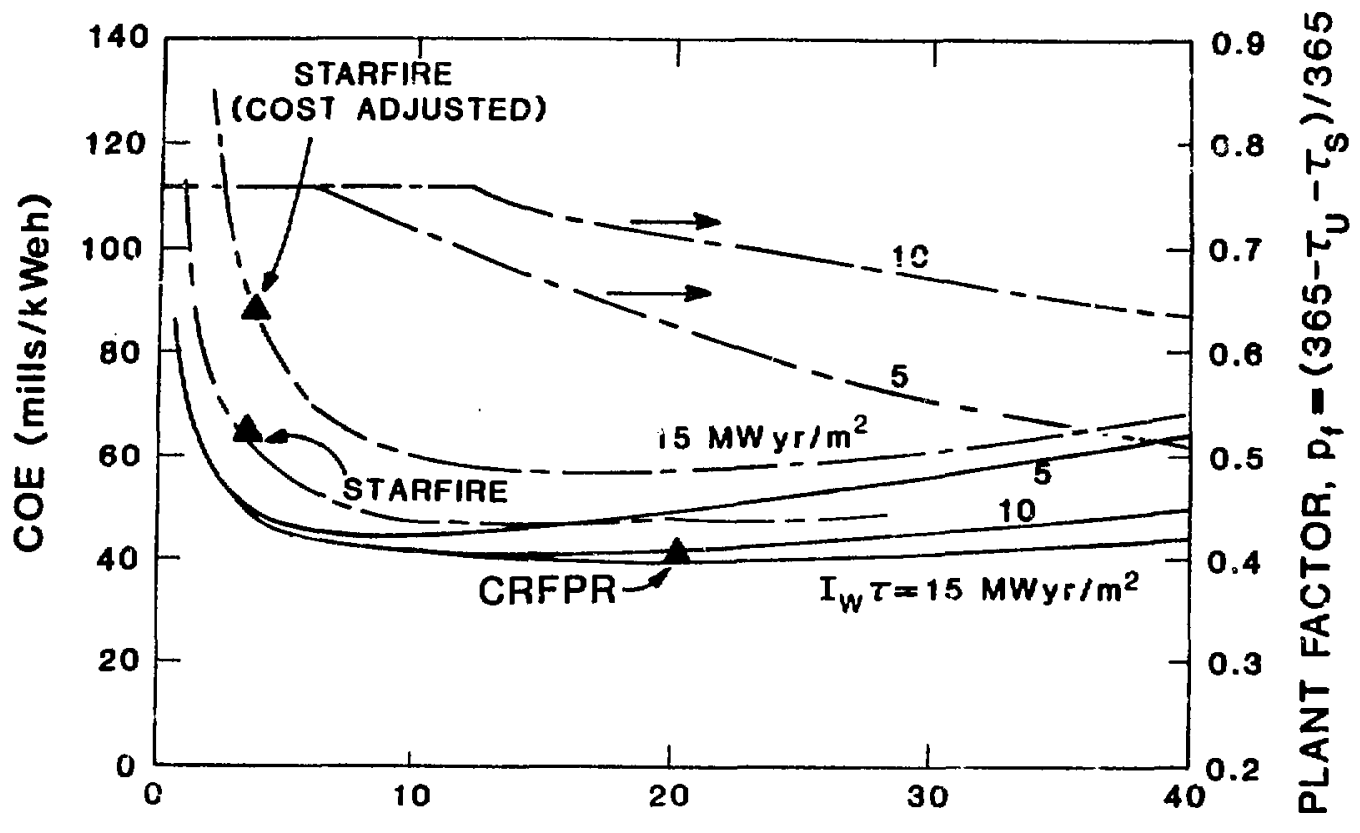

FIRST-WALL NEUTRON LOADING, $I_{W}\left(M W / m^{2}\right)$

Fig. II.C-3. Dependence of $\mathrm{COE}$ as UDC on $I_{W}$ for a range of first-wall lifetimes and for various FPC costs indicated. Also shown are the plant factors used to compute $\mathrm{COE}, \mathrm{P}_{\mathrm{f}}$ having a major influence on COE.

system power density would be increased in proportion to $I_{w} x /(1+x)^{2}$, which gives a factor of 3.9 , leading to $\mathrm{P}_{\mathrm{TH}} / \mathrm{V}_{\mathrm{FPC}} \simeq 2.0 \mathrm{MW} / \mathrm{m}^{3}$ for STARFLRE. Although such increases are important, significant increases in system power density are achievable also by reducing $\Delta b+\delta$, pointing toward a need for confinement systems that can operate efficiently (i.e., $\varepsilon \leqslant 0.15-0.20$ ) with normal copper magnets.

The compact, high-power-density region is technically available to RFPs even for betas far below the $\beta_{\theta}=0.2$ values used here. For this reason that region on Fig. II.C-2 where $I_{w}=10-20 \mathrm{MW} / \mathrm{m}^{2}$ and $r_{p}=0.6-0.8 \mathrm{~m}$ appears as most interesting frow the viewpoint of economic fusion power. The physics needed to achive plasmas in this region for the recirculating power fraction assumed for resistive-coil systems (i.e., $\Delta b+\delta \simeq r_{w}=0.7-1.0$ ) are largely avallable to the RFP configuration. 
Lastly, it is noted that although little cost penalty is indicated if $I_{w}$ closer to $10 \mathrm{MW} / \mathrm{m}^{2}$ rather than $20 \mathrm{MW} / \mathrm{m}^{2}$ is selected, if $\rho_{\mathrm{FPC}} \simeq 5$ tonne/m ${ }^{3}$, then $I_{W} \leq 20 \mathrm{~kW} / \mathrm{m}^{3}$ would be required to maintain the total FPC mass below $\sim 1000$ tonne; the high range of first-wall neutron loadings may be desirable from a block malntenance viewpolnt, which in turn has economic implication not directly reflected by the costing models. These tradeoffs between the increased $I_{W}$, reduced mean-time-to-fall, reduced FPC mass, and reduced mean-time-to-repair remain a topic of future study. The reduction in plant factor, $P_{f}$, as $I_{w}$ is increased is shown In FIg. II.C-3 for the simple algorithm previously described. clearly, the above-mentioned tradeoffs as well as a more realistic means to mode? $p_{f}$ deserve considerabiy more attention, as does the dependence of $\left(I_{w} \tau\right)$ on $I_{W^{*}}$ Section IV.C. describes the results of a more-comprehensive systems model designed specifically to examine these tradeoffs for the RFP. 
1. Confinement Principle and Application to Reactor Studies. The RFP is a toroldal axisymmetric device in which the primary confinement fleld, ${ }^{B}{ }_{\theta}$ is pololdal and is generated by a toroldal current, $I_{\phi}$, flowing in the plasma. 33,35 The RFP plasma supports a toroldal blas field, $B_{\phi}$, to stabilize sausage $(m=0)$ and elliptical $(m=2)$ distortions. Grossly unstable MHD modes with wavelengths longer than the minor radius, $r_{w}$, of an electrically conducting shell are stabilizedized by the shell on a short time scale and by feedback coils for longer times. If the toroldal bias field is slightly reversed near the plasma edge, $r_{p} \leq r_{w}$, the resulting magnetic shear in the plasma-edge region is sufficient to stabilize local pressure-driven and current-driven instabilities, as specified by the Mercler criterion. ${ }^{3}$ This stabilization occurs at relatively high values of the normalized plasiea pressure, $\beta=2 \mathrm{nk}_{B} \mathrm{~T} /\left(\mathrm{B} / 2 \mu_{0}\right)$. $A$ sample stable RFP profile is illustrated in Fig. II.D-1, which also gives a comparison with a tokamak.

The mere existence of the RFP profiles shown in Fig. II.D-l would seem unlikely because of the probable annihilation of opposing toroldal flux at the fleld reversal surface. Experiments during the previous 25 years have shown, ${ }^{33}, 35$ however, that a self-reversed fleld and a minimum-energy state are generated even if such a field is not externally imposed. A theory ${ }^{70}$ of relaxed energy states has led to a substantial increase in the fundamental appreciation of this near-minimum-energy, fleld-reversed state. Given any arbitrary dissipation mechanism, the Taylor theory predicts that a plasma surrounded by a flux-conserving shell will relax to a minimum-energy, force-free state. The key descriptive parameters in the Taylor theory are the pinch parameter, $\theta$, and the reversal parameter, $F$, which are defined as $\theta=B_{\theta}\left(r_{w}\right) /\left\langle B_{\phi}\right\rangle$ and $F=B_{\phi}\left(r_{w}\right) /\left\langle B_{\phi}\right\rangle$, where $\left\langle B_{\phi}\right\rangle=\left(2 / r_{w}{ }^{2}\right) \int_{0}^{r_{w}} B_{\phi} r d r$ is the average toroldal field within the conducting shell of radius, $r_{w^{*}}$ Appendix A gives a more detalled description of the Taylor minimum-energy state and the relationship between profiles, the parameters $\theta$ and $F$, and RFP burn physics.

The locus of minimum-energy states, as described in an $F-\theta$ phase space, is shown in Fig. II.D-2; botin the analytic $(\beta=0)$ Taylor state and the experimental, finite- $\beta$ states are shown. The experimental F- $\theta$ traces illustrated in Fig. II.D-2 show the tendency of the plasma to drift toward and reside within a specific region of $F-\theta$ space. This destred RFP state corresponds to $\mathrm{F}<0$ and $1.2<\theta<1.6$. Finite- $\beta$ RFP states have been observed 

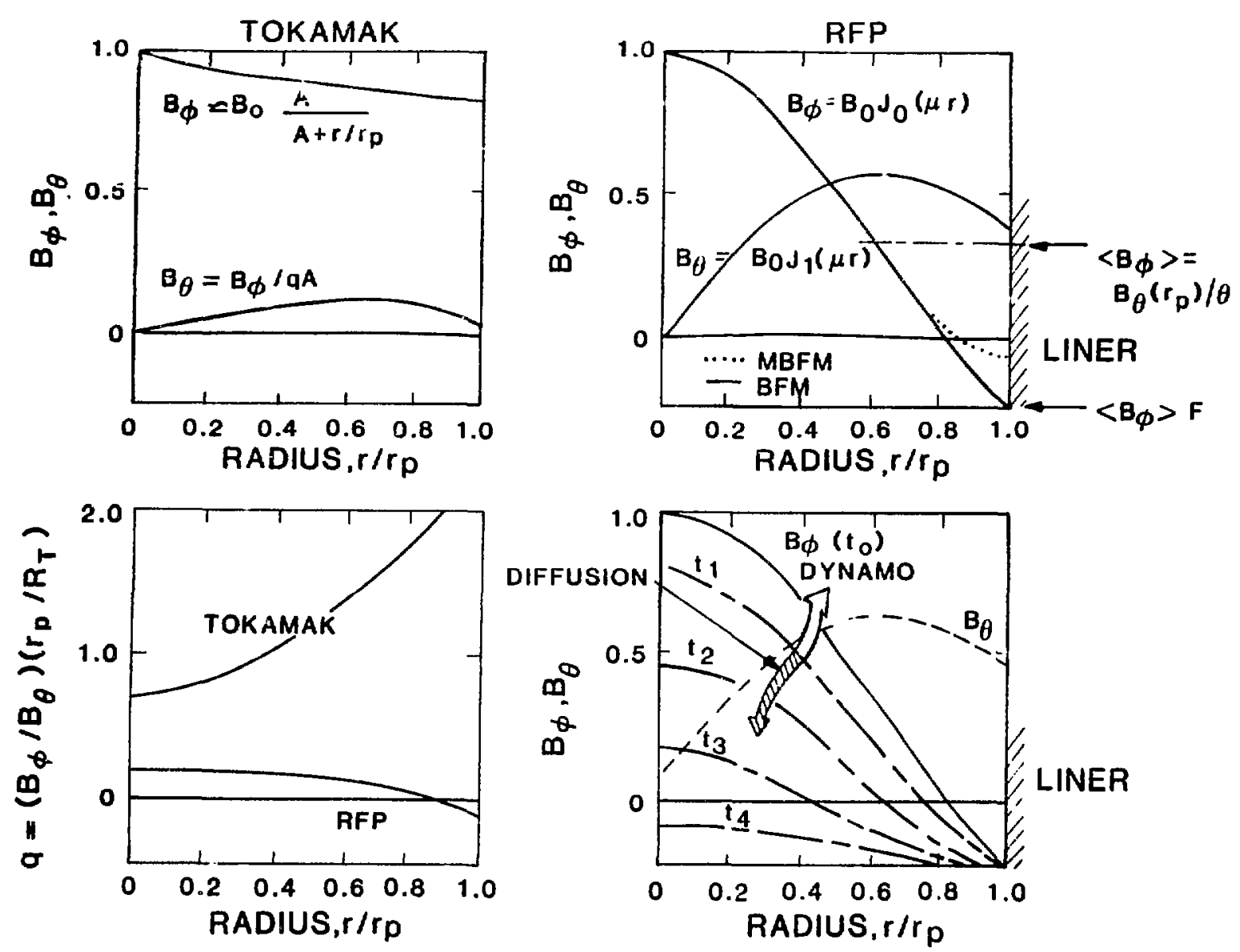

Fig. II.D-1. Sample magnetic-field and $q=\left(B_{\phi} / B_{\theta}\right)\left(r_{p} / R_{T}\right)$ minor radial profiles for the RFP and the tokamak.

both experimentally $44-46,71-72$ and numerically 73 for higher values of $\theta$, although the Taylor theory predicts an ultimate relaxation to the minimum-energy states shown in Fig. II.D-2 and Appendix A. Both the relaxation mechanism and associated time constants for the self-sustained toroidal-field reversal are not well understood in terms of fundamental plasma processes in spite of the strong experimental evidence for the existence of a plasma regeneration or "dynamo" mechanism that continually maintains the minimum-energy RFP state. Generally, 73-76 elther turbulence or resistive MHD instabllities drive a plasma dynamo action, wherein toroidal plasma currents are converted to the toroldal flux needed for malntenance of the toroidal-field reversal. For the purposes of RFP reactor studies, $15-16,31$ an efficient dynamo is enforced onto the plasma burn models both during startup and burn sustenance (Sec. III.G.) by requiring the F- $\theta$ constraint (Fig. II.D-2). Evidence fo: nearly classical resistivity in 


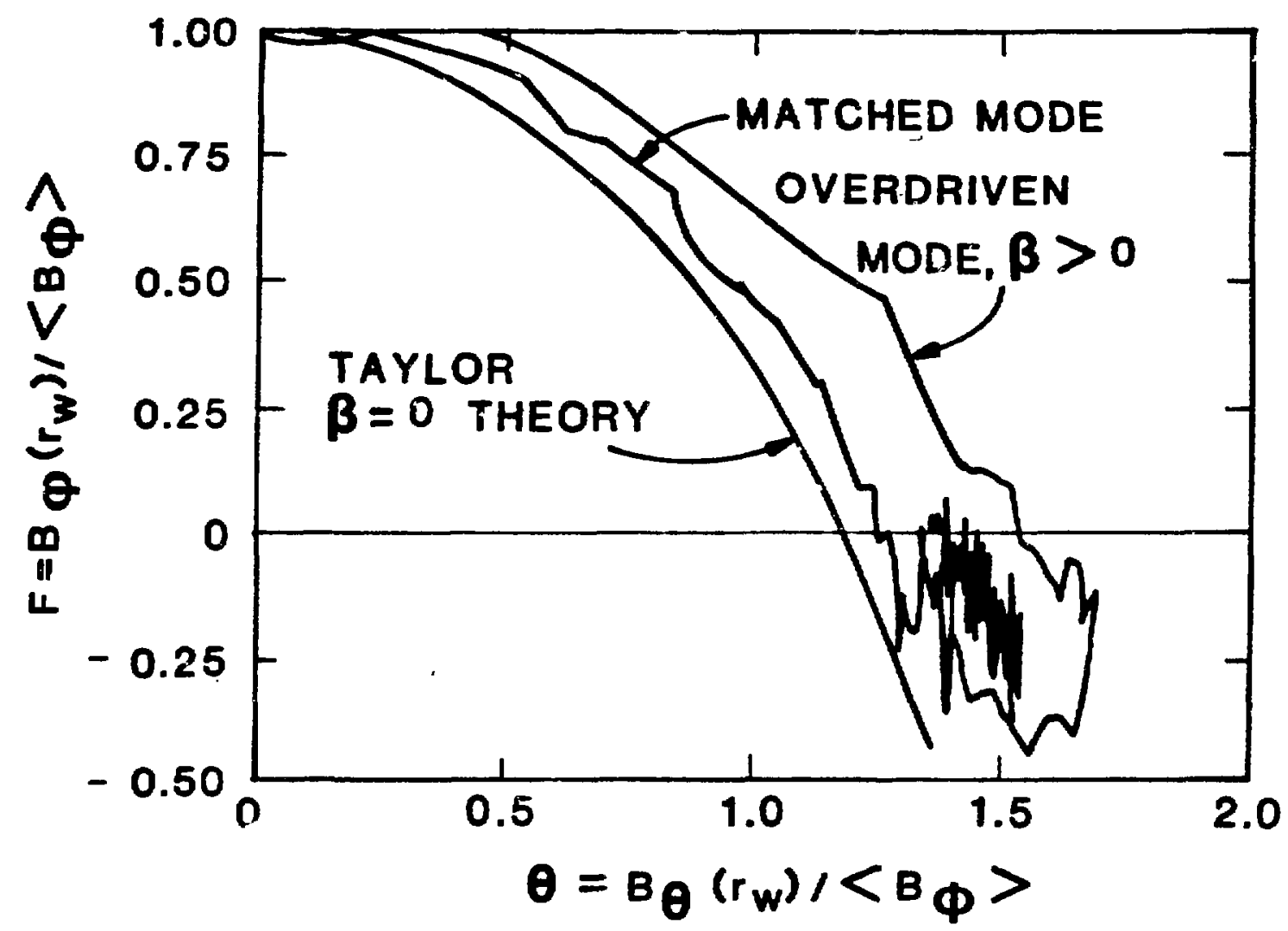

Fig. II.D-2. The Taylor $F-\theta$ diagram showing two experimental traces and the tendency for the plasma to seek and reside within the RFP minimumenergy state.

RFP plasmas exists, 77 giving a strong indication of an eificient plasma dynamo action. Unlike the tokamak, the close electrical coupling between the poloidal and toroidal circuits through the RFP plasma vis a vis the F- $\theta$ diagram. This coupling also provides a means to drive toroidal current noninductively $78-81$ at low frequency ( $50 \mathrm{~Hz}$ for the reactor, Sec. III.K.), preltminary experimental evidence 69 in support of these ideas recently being obtained.

The relationship between $F$ and $\theta$, the tokamak safety factor, $q \equiv\left(r_{p} / R_{T}\right)\left(B_{\phi} / B_{\theta}\right)$, and beta points out the essential differences between the RFP and the tokamak reactor. First, one definition of the total average beta for both RFPs and tokamaks is $\langle\beta\rangle=2 \mu_{0}\langle p\rangle /\left(B_{\theta}^{2}+B_{\phi}^{2}\right)$, which in terms of the poloidal beta, $\beta_{\theta}$, is $\langle\beta\rangle=f_{\theta} /\left[1+\left(B_{\phi} / B_{\theta}\right)^{2}\right]=\beta_{\theta} /\left[1+(F / \theta)^{2}\right]$. The tokamak-1ike minimum-energy state would correspond to the region where $F \rightarrow 1$ and $\theta=\mathrm{F} / \mathrm{Aq} \rightarrow 1 / \mathrm{Aq}$, where $A=R_{T} / r_{p}$ is the plasma aspect ratio; for the tokamak, $\langle\beta\rangle=\beta_{\theta} /\left[1+(q A)^{2}\right]$. It is noted that the spheromak state corresponds to $F \rightarrow 0$ 
on the F- $\theta$ diagram. For the RFP state, $|F / \theta|=0.2 / 1.5=0.13$ and $\langle\beta\rangle=\beta_{\theta}$, whereas for the tokamak $F / \theta=q A=8-10$, with $\langle\beta\rangle$ being much less than $\beta_{\theta}=1-2$. As a maximum for the tokamak, $\beta_{\theta} \leq A$ to avold the ideal kink stability and, therefore, $\langle\beta\rangle \leq A /\left[1+(\mathrm{Aq})^{2}\right]=1 / \mathrm{Aq}^{2}$. The Impact of these bastc differences on the level of ohmic heating allowed in resistive colls for systems that can operate stably with low values of $|\mathrm{F} / \theta|$, compared to those having large $|\mathrm{F} / \theta|$ values (1.e., the tokamak), is shown in Ref. 15. Specifically, the ohmic losses Incurred in the coroldal-field coils relative to those in the poloidal-coll system for both RFPs and tokamaks are nominally proportional to $\sim+|F / \theta|$. In addition, lower ohmic losses occur in the RFP pololdal colls because of the higher aspect ratio and better inductive coupling between the coll set and plasma. Efficient use of resistive copper colls becomes much more a practical matter for the RFY compared to the tokamak.

Application of the $\mathrm{F}-\theta$ constraint to the reactor infers the existence of a plasma dynamo; the crucial issue of enhanced plasma tramsport associated with the RFP dynamo remains. A transport-based confinement time scaling is not yet available for RFPs. By equating ohmic heating in the plasma, $\eta j^{2}$, to an energy loss rate of unspecifted mechanism, $3 \mathrm{nk}_{\mathrm{B}} \mathrm{T} / \tau_{\mathrm{E}}$, an "ohmic" scaling results, $\tau_{E}$ (OHMIC) $\propto \beta_{\theta} T^{3 / 2} r_{p}^{2} / z_{\text {eff }}$, when the plasma resistivity, $\eta$, is assumed to be classical and pressure balance is invoked. Figure II.D-3 compares this ohmic scaling with a number of key RFP experiments. The effects of flat and $J_{o}(\mu r)$ temperature profile are shown, where the $\beta=0$ Taylor theory gives $n(r) T(r) \propto J_{0}^{2}(\mu r)$ and $J_{\mathcal{O}}(\mu r)$ is the tenth-order Bessel function of the first kind (Appendix A, Sec. A.4.). It is noted that the centerline plasma temperature, $T(0)$, is used in Fig. II.D-3 rather than the density-weighted average temperature, $T$, commoniy used in the reactor burn siaulations (Sec. III.G.). An expression for $\tau_{E}$ (OHMIC) used to generate the constant-T curve 15 also shown. The weak dependence of the coefficlent $\tau_{E}$ (OHMIC) $/\left(\beta_{\theta} T(0)^{3 / 2} r_{p}^{2} / z_{E F F}\right)$ on $z_{E F F}$ is indicated on Fig. II.D-3, a nominal beta of $\beta_{\theta}=0.2$ being used to show this dependence. Whatever the actual transport mechanism, that process is effectively assumed to limit the beta to a specific value. If the $\mathrm{T}(\mathrm{r}) \propto \mathrm{J}_{\mathrm{o}}(\mu \mathrm{r})$ profiles are used, tre expression given in FIg. II.D-3 for $\tau_{E}$ (OHMIC) is reduced by $\sim 17.6\left(1-\beta_{\theta}\right) /\left(2-\beta_{\theta}\right) \simeq 7-8$. The previously reported CRF'RR destgn points 15 are also shown on Fig. II.D-3. Generally, the close agreement between experiment and "theory" is taken as an 


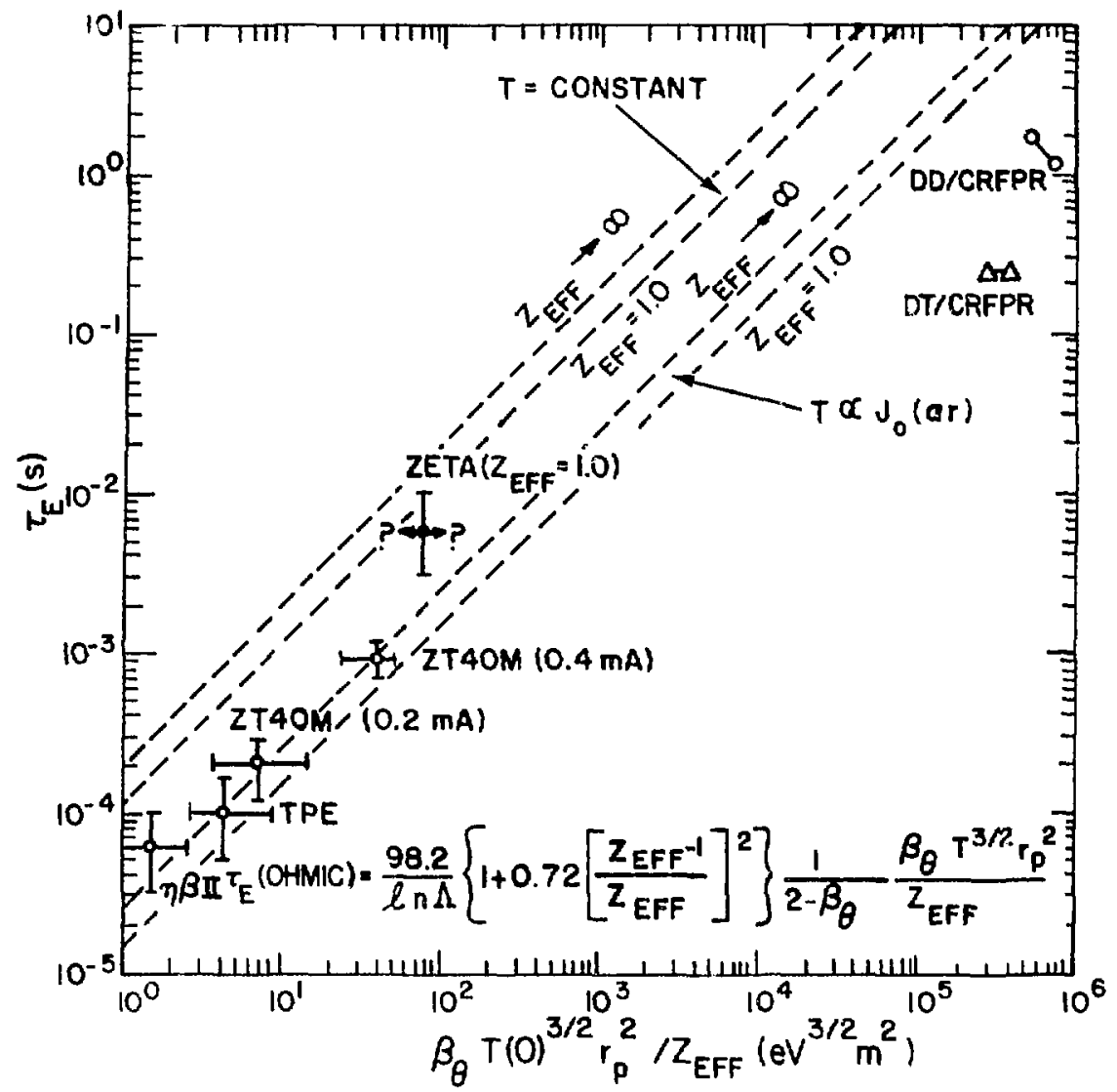

Fig. II.D-3. Comparison of experimental confinement times from a number of RFP devices with $\tau_{E}$ (OHMIC), the latter being obtained by equating ohmic-heating power density to transport losses, using pressure balance, and assuming stability-limited constant beta. The expression given for $\tau_{E}$ (OHMIC) pertains to a flat temperature profile, the $J g(\mu r)$ temperature profile case being reduced by a factor of $\left.\sim 17.691-\beta_{\theta}\right) /\left(2-\beta_{\theta}\right)=7-8$.

Indication that the plasma dynamo action malntaining the RFP profiles requires little energy beyond that dissipated by a nearly classical plasma resistivity.

Finally, a more direct, but empirical, expression of the scaling of confinement time emerging from small, ohmically heated experiments is desired. It is recalled that the combination of pressure balance $\mid T \propto \beta_{\theta} I_{\phi}\left(I_{\phi} / N\right)$, plasma energy balance $\left(n T / \tau_{E} \propto \eta j^{2}\right)$, and classical resistivity $\left(\eta \propto 1 / T^{3 / 2}\right)$ predicts that $\tau_{E} / x_{p}{ }^{2} \propto \beta_{\theta}^{5 / 2}\left(I_{\phi} / N\right)^{3 / 2} I_{\phi}{ }^{3 / 2}$. Figure II.D-4 illustrates this scaling from present experimental results to compact RFP reactor projections. The reactor burn simulations given in Sec. III.G. utilize a scaling of this form, as 
callbrated with existing experimental results using the more pessimistic $\tau_{E} \simeq 0.05 I_{\phi} r_{p}{ }^{2} f\left(\beta_{\theta}\right)$ scaling curve.

In summary, the basic conclusions emerging from RFP experiment and the implications for reactor projection are given below.

- Classical plasma resistivity is observed in experiment, the higher voltages required to drive current in RFPs relative to tokamaks being a result of geometric factors (i.e., higher pitch) rather than "anomalous" resistivity need to drive current against added $\vec{v} \times \vec{B}$ electric fields being generated by local, stochastic plasma motion.

- The plasma dynamo action is verified but not theoretically understood, with the dynamo dissipating 1 ittle energy beyond that required by classical ohmic losses.

- Separate oscillation of poloidal and toroldal circuits result in the plasma moving predictably along the $F-\theta$ diagram, giving strong promise for " $F-\theta$ pumping" current drive. F- $\theta$ pumping for steady-state, ignited current drive should be efficient if the plasma dynamo action occurs with a nearly classical electrical resistivity.

- Ignition by ohmic heating is consistent with classical plasma resistivity and is a strong possibility in the RFP.

- The energy confinement time, $\tau_{\mathrm{E}}(\mathrm{OPT})$, required for an economically optimized reactor relative to an ohmic dissipation time, $\tau_{E}$ (OHMIC), and relative to some measure of Alcator scaling, $\tau_{E}(A L C)$, is given by $\tau_{E}(O H M I C) / \tau_{E}$ (OPT) $>20-60$ and $\tau_{E}(A L C) / \tau_{E}(O P T)>5-10$, respectively, showing wide safety margins for ohmic ignition in RFPs.

Lastly, the reactor implications of the $q<1 \mathrm{kFP}$ confinement system are summarized below.

- Relatively free choice of plasma aspect ratio, $R_{T} / r_{p}$.

- Economically high betas. 


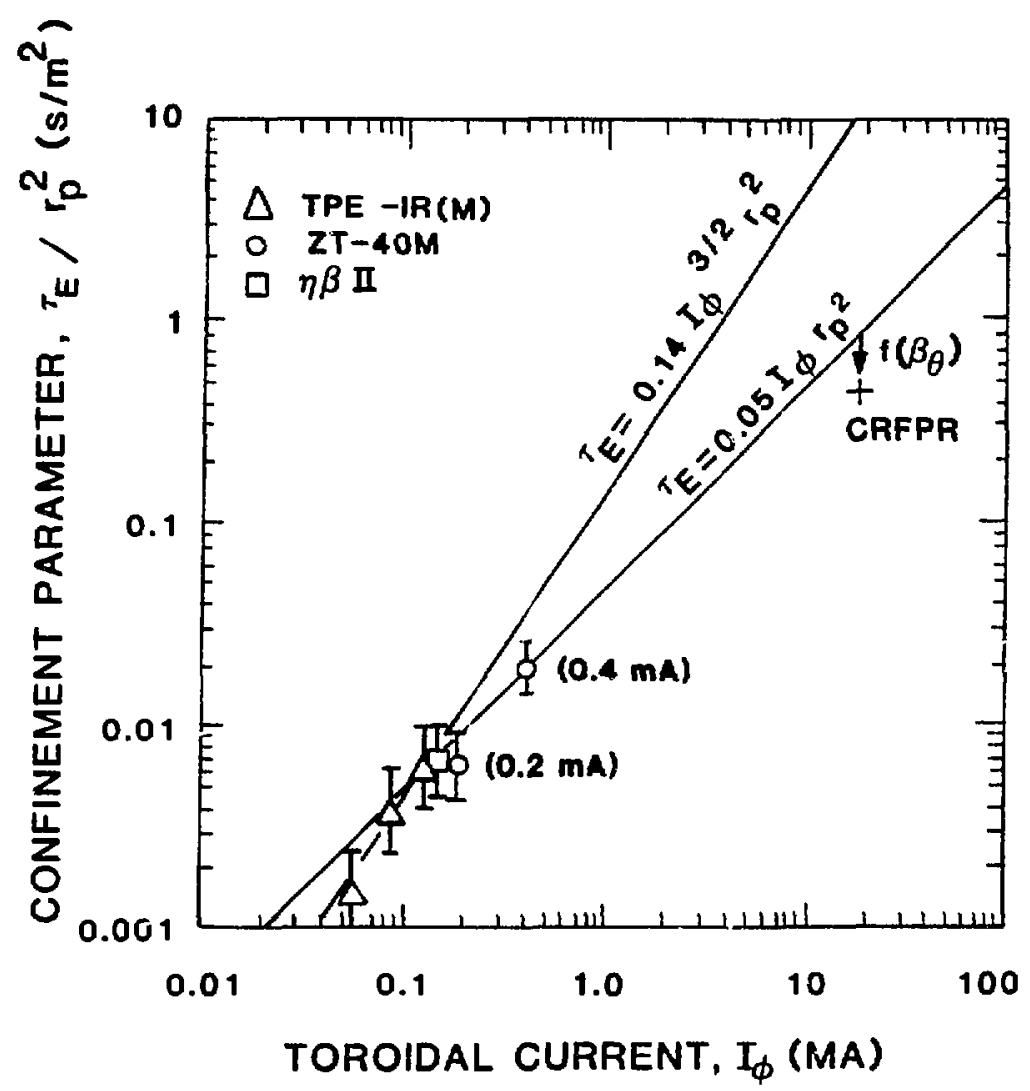

Fig. II.D-4. Scaling of RFP confinement with toroidal current needed to project present experimental results to reactor conditions.

- Possibility of high values of $\beta B^{2}$ at the plasma and correspondingly increased plasma power density for a given field at coil.

- Internal plasma currents are sufficient for ohmic heating to ignition without auxiliary heating and with low-to-moderate fields at the coils.

- Efficient plasma/coil electrical coupling results in

- ${ }^{B}$ dominates low PFC fields and currents.

- B ${ }_{\phi}$ near zero, low TFC fields and currents.

- Elimination of superconducting colls in favor of resistive coils is possible, while maintaining efficient reactor performance. 
- Potential for efficient steady-state current drive through modest oscillations of TFC and PFC voltages ("F- $\theta$ pumping"). With the possibility of reducing the OHC current to zero and with the inherently low TFC currents, a reactor configuration results in which external currents are flowing only in the EFCs.

2. Reactor Design-Point Update and Sensitivity. A unifled parametric systems model was used to examine a fully cost-optimized RFP reactor design. ${ }^{15} \mathrm{~A}$ $1000-\mathrm{MWe}$ (net) base case was selected and subjected to a parametric analysis that varted:

- plasma transport, $\tau_{E}$.

- beta, $\beta_{\theta^{*}}$

- blanket/shield thickness, $\Delta$ b.

- plasma density and temperature profiles.

- normal versus superconducting colls.

- DT versus catalyzed-DD fuel.

- net electric power, $P_{E}(M W e)$.

- First-wall/blanket radiation Iifetime, $I_{w} \tau\left(M W y r / m^{2}\right)$.

The plasma transport scaling was varied to give a design point that minimized the cost of electricity; the resulting value of confinement time, $\tau_{\mathrm{E}}$ (OPT), for a DT-fueled system amounted to $\sim 1 / 8$ the prediction of empirical (Alcator) tokamak transport. Table II.D-I lists parameters for both the DT and catalyzed-DD versions of the designs reported in Ref. 15. Table II.D-I also iacludes a "degraded" DD/CRFPR design that would operate with an average first-wall thermal flux equal to that predicted for a fully cost-optimlzed DT/CRFPR design. These results and associated discussions and interpretations have been presented in Ref. 15. The related assessment of engineering technology for the DT/CRFPR focused onto the following four major technological issues: 
- High-heat-flux first walls $\left(4-5 \mathrm{MW} / \mathrm{m}^{2}\right)$ similar to other MFE requirements, sputtering/erosion and particle control beling mäin concern and unknown.

- High-power-density $\left(30 \mathrm{MW} / \mathrm{m}^{3}\right.$ average, $200 \mathrm{MW} / \mathrm{m}^{3}$ peak) breeding blanket similar to LWR core and may preclude solid breeders, self-cooled 11quid-metal blankets appearing particularly attractive.

- High-radiation-flux resistlve magnets positioned outside a thin tritium-breeding/heat-recovering blanket.

- Long-pulsed versus steady-state operation a "soft" Issue frem viewpoint of energy balance, iechnology, and cost, this issue being dorinated by unresolved means for

- impurity control (full-wall poloidal pumped limiters or a magnetic divertor)

- steady-state current drive (F- $\theta$ pumping).

Table II.D-II summarizes in more detal137 the major issues and problems envisaged for compact fusion reactors, with an emphasis being placed here on the DT/CRFPR.

The DT/CRFPR design point reported in Ref. 1.5 and Table II.D-I has since been modified as a result of a subsequent conceptual engineering study periormed on key FPC systems. Engineering design studies that form the basis for this design-point readjustment are reported in Sec. III. Major changes include Increasing the blanket and shield thickness from $\Delta b=0.4 \mathrm{~m}$ to $0.6 \mathrm{~m}$ and an Increase in blanket energy multiplication from the $M_{N}=1.1$ value assumed in Ref. 15 to 1.3 computed for the $\Delta \mathrm{b}=0.5-\mathrm{m}$-thick PbLi blanket (Sec. III.B.). Table II.D-III gives the updated parameters for the DT-fueled CRFPR; generally, the increased losses and reduced power density accompanying an increased $\Delta b$ is soratensated by the increased $M_{N}$; the revised CRFPR design, therefore, represents a small perturbation from that originally reported in Ref. 15.

The revised, fully-optimized design point reported in Table II.D-III has been subjected to further parametric sensitivity study wherein both the economy of scale and the influence of assumed $F W / B$ lifetime was investigated. Figure II.D-5 compares this reactor cross section with chat for STARFIRE and a PWR, whereas Fig. II.D-6 gives the economy of scale for the Table II.D-III design point, comparisons with the STARFIRE ${ }^{3}$ and EBTR 52 designs also being shown. An economy-of-scale curve was determined 52 also for EBTR and is included on Fig. II.D-6, emphasizing, however, that the neoclassical EBT transport used 
in the Ref. 52 study did not admit to designs with small plant capacity at any cost.

The dependence of $\mathrm{COE}$ on assumed FW/B lifetime and plasma beta for the DT/CRFPR design given on Table II.D-III is depicted on Fig. II.D-7. The $I_{w} \tau=$ $15 \mathrm{MWyr} / \mathrm{m}^{2}\left(\mathrm{I}_{\mathrm{W}} \simeq 19.5 \mathrm{MW} / \mathrm{m}^{2}\right)$ design polnt can operate with a factor of $\sim 2$ reduction in FW/B lifetime (and first-wall neutron loading) without large increases in $C O E$; the degraded $I_{w}=8-10 \mathrm{MW} / \mathrm{m}^{2}$ system, however, requires a FPC mass that increases in mass by 1240 to $\sim 2200$ tonnes, which is still a considerable reduction compared to the more-conventional superconducting approaches (re: Table II.C-I). The abllity to utilize "block" (single-piece) maintenance approaches, however, is compromised by the more-massive FPC designs. The effect of decreased beta Erom the $\beta_{\theta}=0.2$ design value on $\operatorname{COE}$ is also depicted on Fig. II.D-7, showing that decreases by over a factor of two are possible without serious increases in cost. The decreased $\beta_{\theta}$ is compensated by increased $I_{\phi}$ and $\beta_{\theta}$, maintaining an economic value of $\beta_{\theta}{ }_{\theta}^{2}$ at a cost of increased coll losses but not overstressing the colls.

Given credible extensions of physics and engineering needed to achieve the kind of compactness represented by the design suggested in Table II.D-III, the key question that remains is "can the sustem total reliability, breakdown Erequency, repair/maintenance time, and operating cost (i.e.,FPC mass throughput) be designed within reasonable capital cost to provide a total plant factor that gives a competitive COE?" This report begins to establish a basis for answering this important question, although significant advances in theoretical and experimental understanding will be needed to assess fully this 1ssue. The following Sec. III. gives preliminary results of a more-detailed computational basis with which to assess the potentlal for extending both physles and engineering to achieve the kind of compact fusion suggested in Table II.D-III. Only better models based on actual operating experience, however, will provide a definitive answer to the last question; the material presented in Sec. III. is one step toward answering this crucial question. Modifications to the base-case parameters summarized in Table II.D-III will occur as more design realism is injected into this study; Sec. III.L. performs an energy, thermal-hydraulic, and mechanical integration of the FPC based on the models and results reported in other subsections in Sec. III. The comprehensive design table civen in Appendix B reflects key power-plant parameters that have been subjected to this kind of re-evaluation and integration. 
PARAMETER SUMMARY FOR INTERIM 1000-MWe CRFPR DESIGNS (Ref. 15)

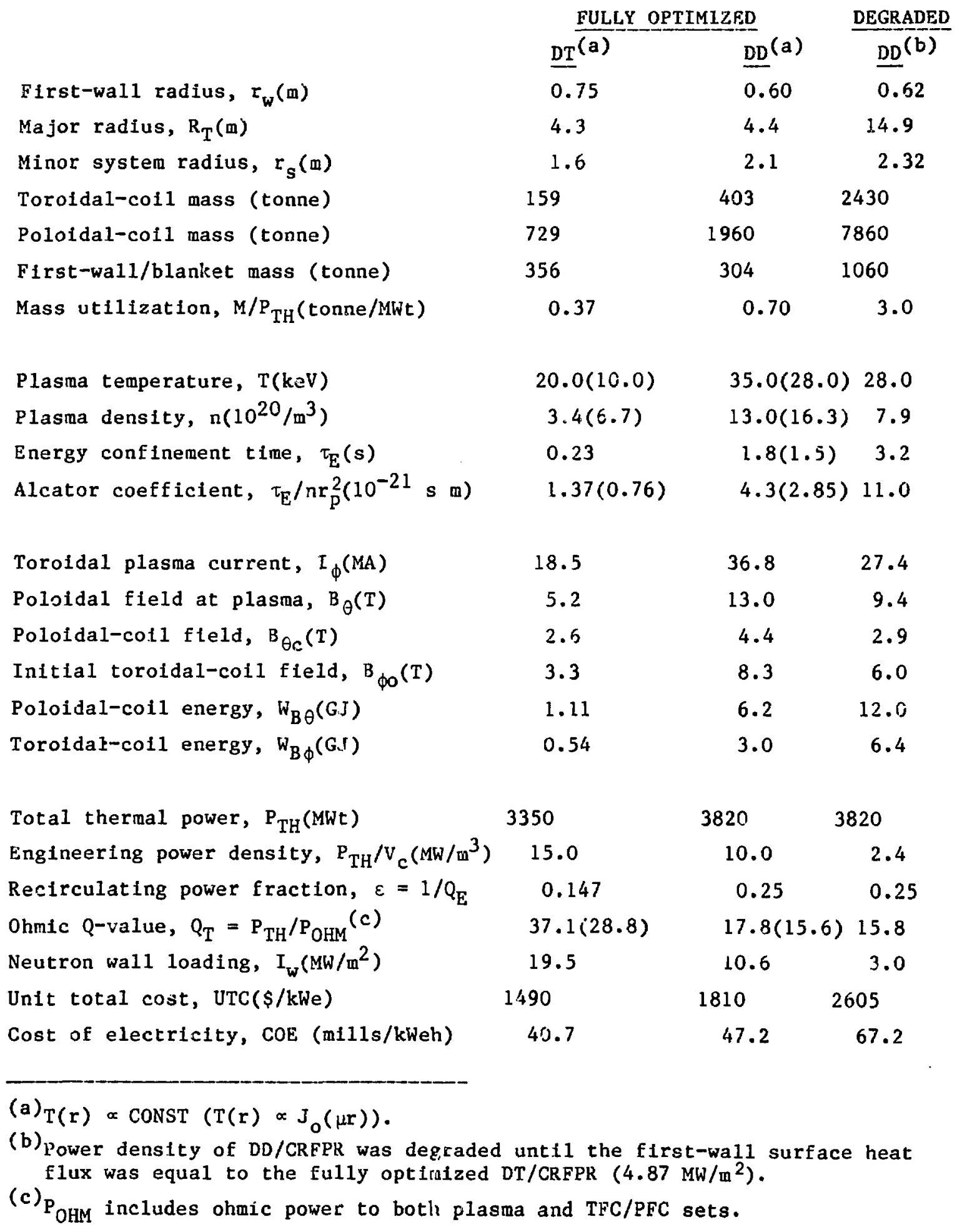




\section{COMPACT FUSION REACTOR ISSUES/PROBLEMS}

- FW heat flux and sputtering rates

- heat fluxes slmilar for other Eusion systems (i.e., neutrai-beam grids and dumps, limiters, rf tubes)

- integrated sputtering same as other fusion approaches

- less tolerance for peaking factors

- FW/B burnup rate

- replacement frequency greater

- burnup rate similar to other approaches (200-300 tonne/yr)

- FW plasma stabilization and active feedback

- ZT-40M conducting shell disappears on a time scale that is short compared to plasma discharge time

- passive role of $\mathrm{FW} / \mathrm{B}$ as a shell similar to tokamak

- slow-feedback requirement similar to tokamak

- both passive shell and active feedback requirements not well understood

- Long-pulsed versus steady-state operation

- no energy-balance problem as long as burn time exceeds field energy replacement time ( $>1 \mathrm{~s})$

- F- $\theta$ pumping unique RFP option for steady-state current. drive operating at low frequency $(50-60 \mathrm{~Hz})$

- both pololdal pumped limiters and toroidal magnetic divertors are impurity-ccntrol options

- Confinement time magnitude and scaling

- experimental $\tau_{E}$ dictated by need to shed ohmic-heating power with classical resisivity at a beta limit

- $\tau_{E} \propto I_{\phi} r_{p}{ }^{2}$ takes $Z T-40 M$ results to 1 gnition

- ohmic-heating scaling predicts $\tau_{\mathrm{E}} \propto \beta_{\theta} \mathrm{T}^{3 / 2} \mathrm{r}_{\mathrm{p}}{ }^{2} \propto$ $\beta_{\theta^{I}}{ }^{3 / 2} r_{p}^{2}$

- detailed $\mathrm{p}$ nowledge of $\tau_{\mathrm{E}}$ scaling is not necessary to predict an economic reactor with $\tau_{\mathrm{E}}(\mathrm{OPT}) \simeq \tau_{\mathrm{E}}$ (ALC)/8 and $\tau_{E}(O P T) \simeq \tau_{E}(O H M I C) / 50$.

- Startup power, magnetic flux requirement, and startup FW heat loads

- reactor would begin with a low-current RFP and slowly ramp current to maintain startup power $\leq 100-200$ MWe

- use dynamo action to build up $B_{\phi}$ th:ough PFC circult to minimize an already small TFC set

- startup from grid may be possible ( $>200 \mathrm{MWe}$ )

- FW heat load during startup will always be less than during burn period

- Disruptions and current terminations

- present evidence Indicates current termination caused by edge-plasma density starvation

- controllable by edge refueling 


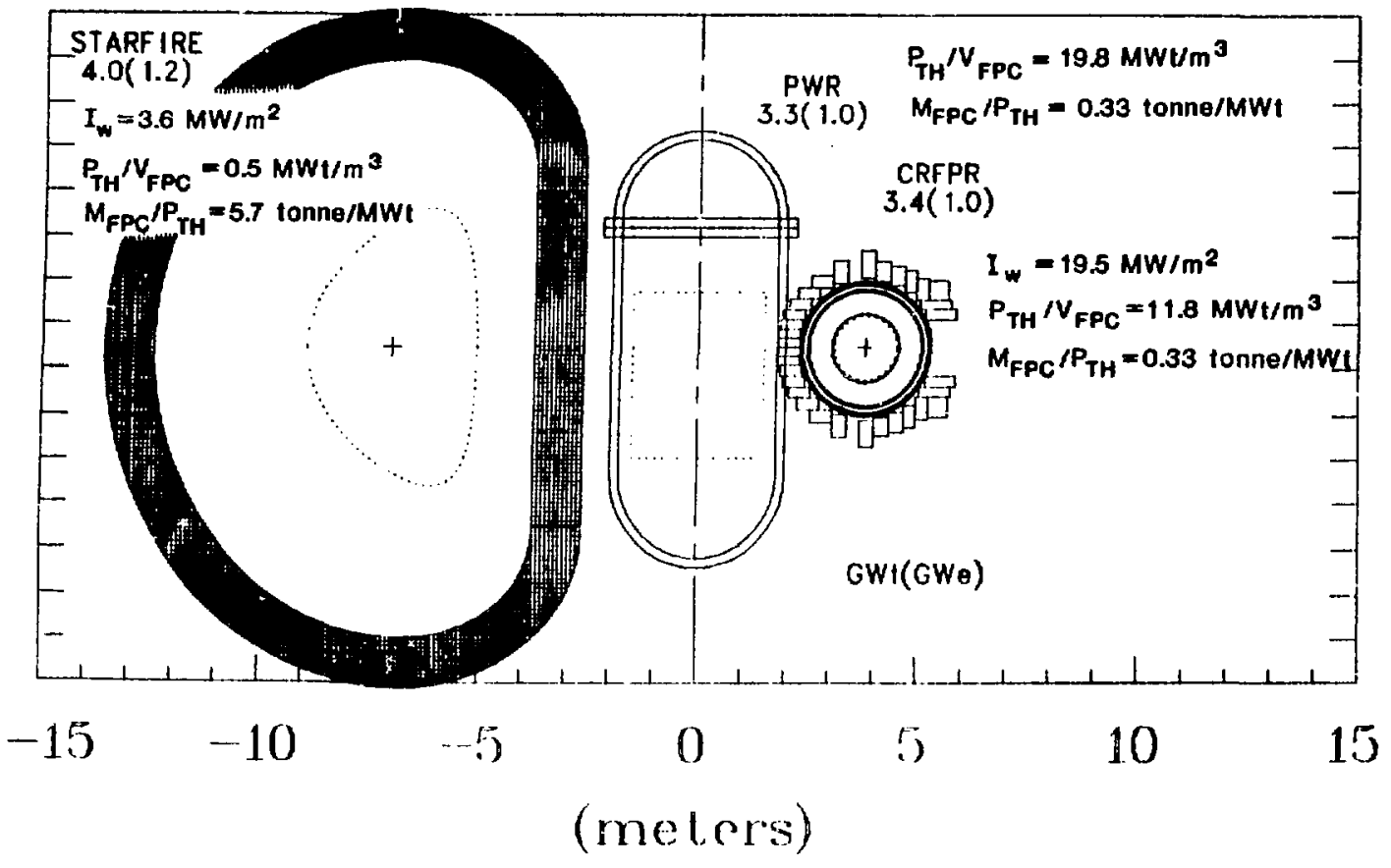

Fig. II.D-5. Cross-section comparison between CRFPR, STARFIRE, and a FWR.

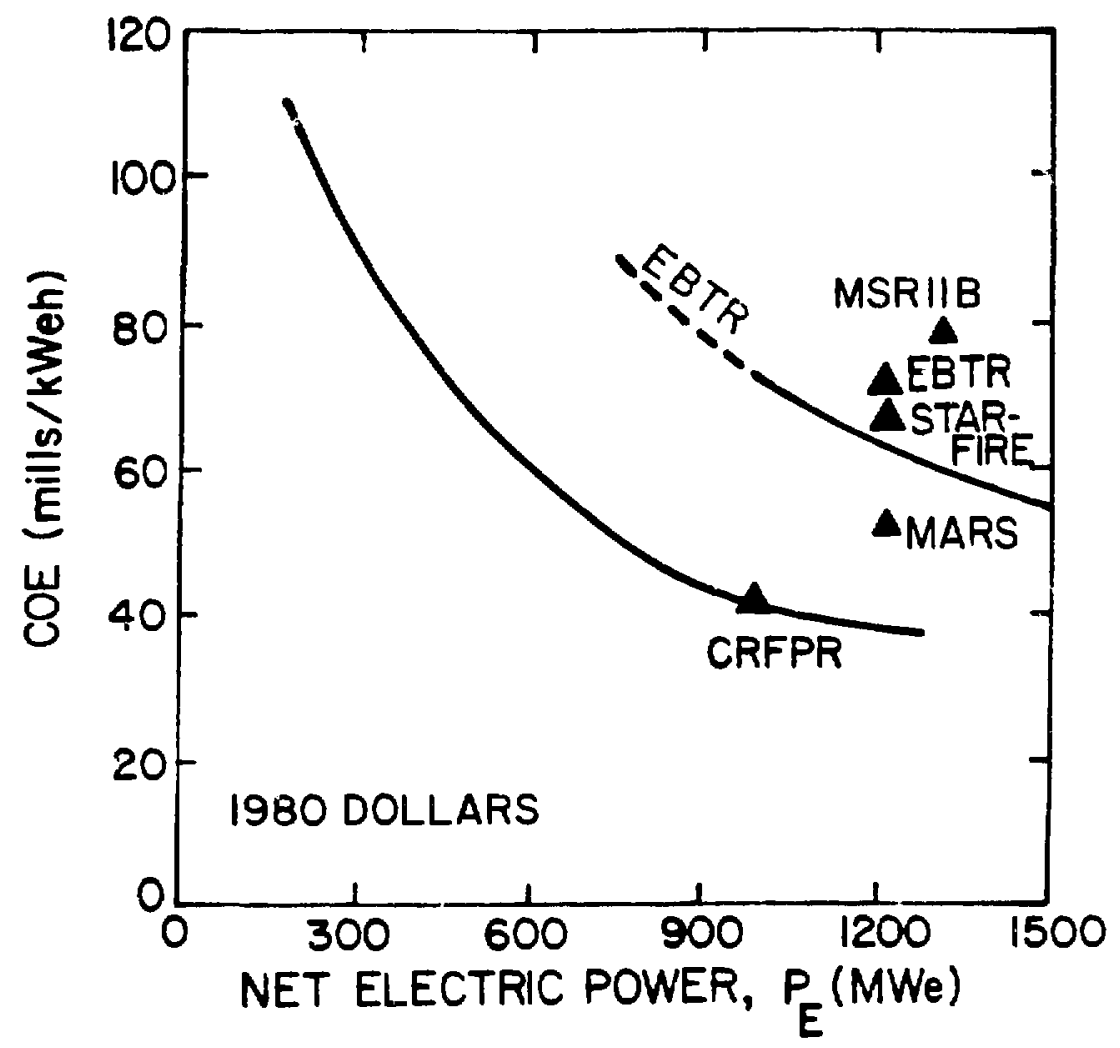

Fig. II.D-6. Economy of scale for CRFPR design point given in Table II.D-III, showing comparison with the STARFIRE tokamak ${ }^{3}$ and EBTR. 52 


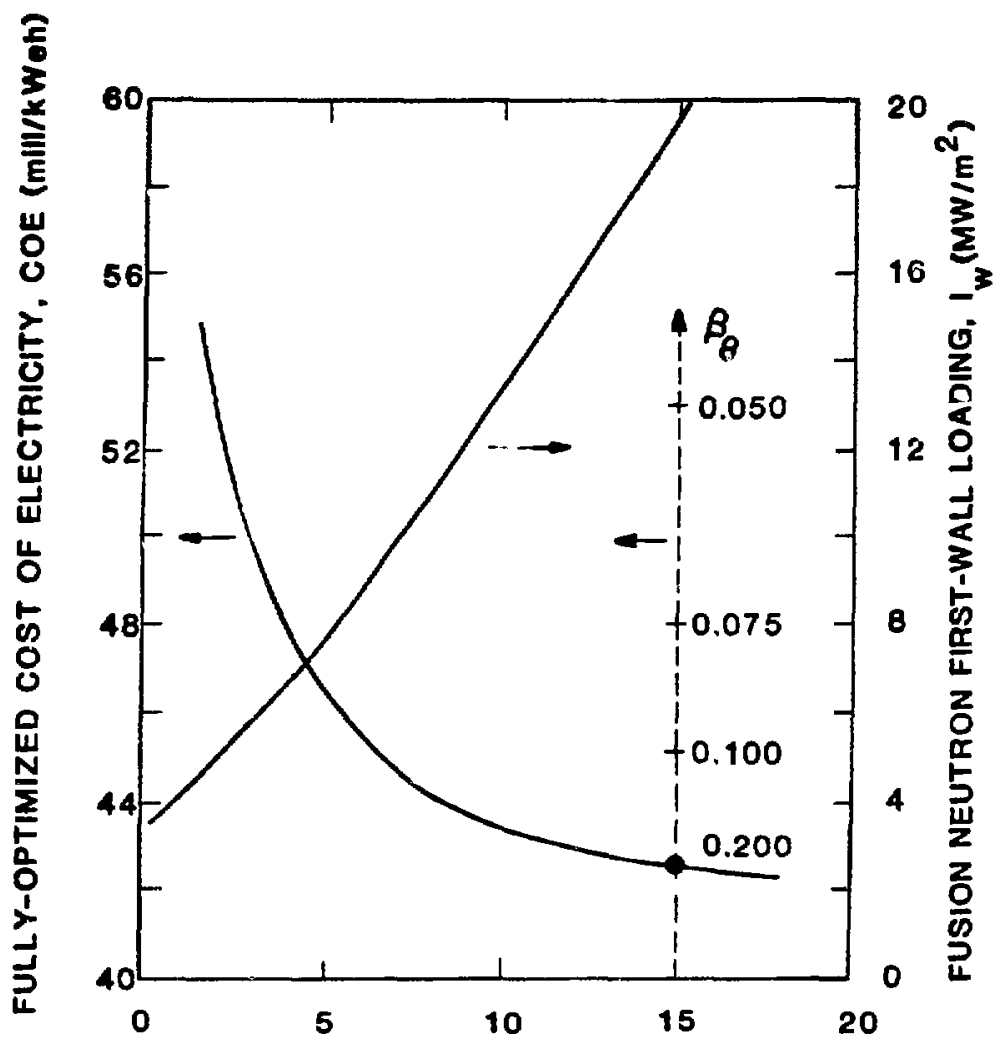

FIRST-WALL LIFETIME, I $T$ (MWyr/m²)

Fig. II.D-7. Dependence of the Table II.D-III design point in the assumed firstwall lifetime. 
KEY PHYSICS AND ENGINEERING PARAMETERS FOR THE $\mathrm{P}_{\mathrm{E}}=1000-\mathrm{MWe}$,

MINIMUM-COE CASES GIVEN IN TABLE II.D-IA UEDATED FOR MORE REALISTIC ENGINEERING DESIGN

Minor plasma cadius (m)

PLASMA

Major plasma radius (m)

Plasma aspect ratio

Plasma current (MA)

Toroidal current density (MA/m $\left.{ }^{2}\right)$

Plasma density $\left(10^{20} / \mathrm{m}^{3}\right)$

Plasma temperature ( $\mathrm{keV}$ )

Lawson parameter $\left(10^{20} \mathrm{~s} / \mathrm{m}^{3}\right)$

Energy confinement time ( $s$ )

Poloidal beta

Theta parameter

Reversal parameter

Plasma/first-wall radius ratio

Plasma Ohmic dissipation during burn (MW)

Coil thickness (m) POLOIDAL-FIELD QUANTITIES

Average minor radius of coil (m)

Mass of coil (tonne)

Magnetic-field level at the coil ( $T$ )

Magnetic-field level at the plasma surface

Poloidal-coil current (MA)

Poloidal-current denstty (MA/m ${ }^{2}$ )

Maximum energy stored in coil (MJ)

Ohmic dissipation during burn (MW)

Coil thickness (m)

TOROIDAL-FIELD QUANTITIES

Average minor radius of coll (m)

Mass of coil (tonne)

Initial toroidal bias field ( $T$ )

Reversed toroidal field during the burn ( $T$ )

Maximum energy stored in the coil (MJ)

Toroidal current density $\left(\mathrm{MA} / \mathrm{m}^{2}\right)$

Ohmic dissipation during burn (MW)

Ohmic Q-value

ENG INEERING SUMMARY

Total thermal power (MWt)

$14.1-\mathrm{MeV}$ neutron loading ( $\mathrm{MW} / \mathrm{m}^{2}$ )

14.1-MeV blanket multiplication

First-indll radius ( $\mathbb{B}$ )

Minor radius of system (m)

Fusion-power-core mass (Lonne)

System power density ( $\mathrm{MWt} / \mathrm{m}^{3}$ )

Mass utilization (tonne/MWt)

ianket thickness (m)

Mass of first wall/blanket (tonne)

rerage blanket power density $\left(\mathrm{MWt} / \mathrm{m}^{3}\right)$

\begin{tabular}{|c|c|}
\hline REF. 15 DESIGN & UPDATE (a) \\
\hline 0.71 & 0.71 \\
\hline 4.30 & 3.79 \\
\hline 6.06 & 5.37 \\
\hline 18.5 & 18.4 \\
\hline 11.7 & 11.8 \\
\hline $3.37(6.74)$ & $3.39(6.56)$ \\
\hline $20.0(10.0)$ & $20.0(10.0)$ \\
\hline $0.79(1.57)$ & $0.79(1.51)$ \\
\hline 0.23 & 0.23 \\
\hline 0.20 & 0.20 \\
\hline 1.45 & 1.45 \\
\hline-0.20 & -0.20 \\
\hline 0.95 & 0.95 \\
\hline $2.59(28.6)$ & $2.29(25.3)$ \\
\hline 0.37 & 0.36 \\
\hline 1.44 & 1.62 \\
\hline 729. & 703. \\
\hline 2.58 & 2.28 \\
\hline 5.21 & 5.22 \\
\hline 18.7 & 22.4 \\
\hline 5.54 & 5.06 \\
\hline 1113. & 1111. \\
\hline 72.0 & 83.0 \\
\hline 0.10 & 0.17 \\
\hline 1.20 & 1.39 \\
\hline 159. & 149. \\
\hline 3.33 & 3.34 \\
\hline 0.68 & 0.68 \\
\hline 539. & 642 . \\
\hline 5.54 & 6.06 \\
\hline 15.71 & 17.61 \\
\hline $37.11(28.8)$ & $32.92(27.5)$ \\
\hline $3350 .(3450)$. & $3389 .(3460)$. \\
\hline $19.5(20)$. & $19.6(20)$. \\
\hline 1.10 & 1.30 \\
\hline 0.75 & 0.75 \\
\hline 1.62 & 1.80 \\
\hline 1243. & 1368. \\
\hline 15.00 & 14.00 \\
\hline 0.37 & 0.40 \\
\hline 0.40 & 0.60 \\
\hline 355. & 516. \\
\hline 51.92 & 36.1 \\
\hline
\end{tabular}

(a) Based on flat plasma temperature profile, values in parentheses correspond to $J_{0}(\mu r)$ temperature profiles. 
COSTING SUMMARY FOR THE $P_{E}=1000$-MWe MINIMUM-COE CASES

GIVEN TABLE III.D-I URDATED FOR MORE REALISTIC ENGINEERING DESIGN

ACCOUNT

TJMBER

20. Land and Land Rights

21. Structures and Site Facilities

22. Reactor Plant Equipment

23. Turbine Plant Equipment

24. Electric Plant Equipment

25. Miscellaneous Plant Equipment

26. Special Materials

90. Direct Cost

91. Construction Facilities, Equipment, and Services (10\%)

92. Engineering and Construction Management Services $(8 \%)$

93. Other Costs (5\%)

94. Interest Diring Construction (10\%/yr)

95. Escalation During Construction ( $5 \% / \mathrm{yr})$

99. Total Cost
MILLION DOLLARS (1980)

REF.15 DESIGN UPDATE

$\begin{array}{cc}3.3 & 3.3 \\ 167 . & 250 .(\mathrm{a}) \\ 311 . & 320 . \\ 220 . & 222 . \\ 121 . & 121 . \\ 41.8 & 41.8 \\ 0.25 & 0.25 \\ 863 . & 959 .\end{array}$

86.3

95.9

69.1

43.2

267.

165.

1493.

3350 .

1172 .

3389.

Thermal power (MWt)

1000 .

Net electric power (MWe)

Engineering Q-value

Plant factor

Unit base cost ( $\$ / \mathrm{kWe})$

Unit total cost ( $\$ / \mathrm{kWe}$ )

Unit direct cost ( $\$ / \mathrm{kWe}$ )

Capital return $15 \%$ (m111s/kWeh)

Operating $2 \%$ (m111s/kWeh)

Replacement (mills/kweh)

Power cost (m111s/kWeh)

Construction time (yr)

Interest

Escalation
6.80

0.76

1062.

1493.

863.

33.7

4.08

$2.90^{(b)}$

40.7

5.0

0.251

0.155

\section{7}

48.0

296.

183.

1658 .
1000 .

\subsection{8}

0.76

1180.

1658 .

959 .

37.4

4.53

46.2

5.0

0.251

0.155

$4.24^{(b)}$

(a) The algorithum used is Ref. 15 for the reactor building size was based on the torus size and underestimated the total reactor building size (steam generators, pumps, tritium cleanup, lay-down areas, etc.). Based on scaling from the STARFIRE design ${ }^{3}$ and LWR experlence, a fixed volume of $120,000 \mathrm{~m}^{3}$ is added to the reactor hall volume as previously calculated to give the volume of the reactor building. The destgn is not of sufficient detall to allow a scaling of the reactor building size with total power, but the volume used conservatively applies to a $1000-\mathrm{MWe}$ (net) system.

(b) Most reactoi designs include replacement cost as part of the above $2 \%$ operating cost. 
III. PRELIMINARY ENGINEERING DESIGN

Although considerable insight deveioped from past and ongoing studies has been applied to establish realistic engineering constraints for the parametric systems analysis leading to the CRFPR design-point update given in Table II.D-III, it nevertheless becomes necessary to perform specific conceptual engineering design studies on key fusion-power-core (FPC) subsystems in order to advance the design credibility. The approach adopted for this study emphasizes FPC integration and, therefore, develops models for each FPC subsystem used to meet this goal. After presentacion of a design overview in Sec. III.A., the FW/B/S/C neutronics model and results in Sec. III.B., and a materiais overview in Sec. III.C., the following FPC subsystem design models and results are given:

- First Wall (Sec. III.D.)

- Blanket (Sec. III.E.)

- Magnets (Sec. III.F.)

- Plasma and Circuit Simulation (Sec. III.G.)

- Limiters (Sec. III.H.)

- Magnetic Divertors (Sec. III.I.)

- Vacuum (Sec. III.J.)

- Current Drive (Sec. III.K.)

- Fusion-Power-Ccre Integration (Sec. III.L.).

\section{A. Design Overview}

The Compact Reversed-Field Pinch Reactor (CRFPR) study has focused completely on the physics and technology needs of the fusion power core (FPC) for a nominal 1000-MWe(net) power plant. This toroidal reactor is based on a deuterfum-tritium fuel cycle. Major features of this design are steady-state and Ignited operation, heating to ignition by ohmic dissipation of intrinsic plasma currents, pumped-limiter impurity control, pressurized-water cooilng of the high-heat-flux copper-alloy first-wall and limiter systems, self-cooled lead-1ithium $\mathrm{Pb}_{83} \mathrm{Li}_{17}$ (referred to hereafter as "PbLi") blanket using ferritic steel (HT-9) structure, water-cooled copper colls positioned outside the blanket/shield system, and fully remote maintenance of a single-piece, 300-tonne, first-wall/blanket/shield/toroldal-field-coil assembly. 
Figure III.A-1 gives a cruos-sectional view of the FPC, showing that this 1000-MWe(net) system is not much larger than Tokamak Fusion Test Reactor (TFTR) ${ }^{36}$ and requires an energy conflnement time that differs little from 1-MA TFTR operation $(0.25-0.30 \mathrm{~s})$. An isometric sketch of one of 24 toroidal sections is shown in Fig. III.A-2. Each of these toroidal sectors would be fabricated, assembled into the 300-tonne torus, and tested (electrically, under vacuum, and thermal-hydraulically) off-site, prior to shipment to and installation at the operating power-plant site. The high neutron first-wall loading of $20 \mathrm{MW} / \mathrm{m}^{2}$ would give a one-year chronological life for a radiation Iffetime of $15 \mathrm{MWyr} / \mathrm{m}^{2}$, which for the 45.2-tonne first-wall/blanket systems corresponds to a mass usage of $17.9 \mathrm{~kg} / \mathrm{MWtyr}(\sim 20,000 \mathrm{MWtd} /$ tonne, somewhat less than fissile-fuel burnup in an LWR) for this 3365-MWt plant at a $75 \%$ plant

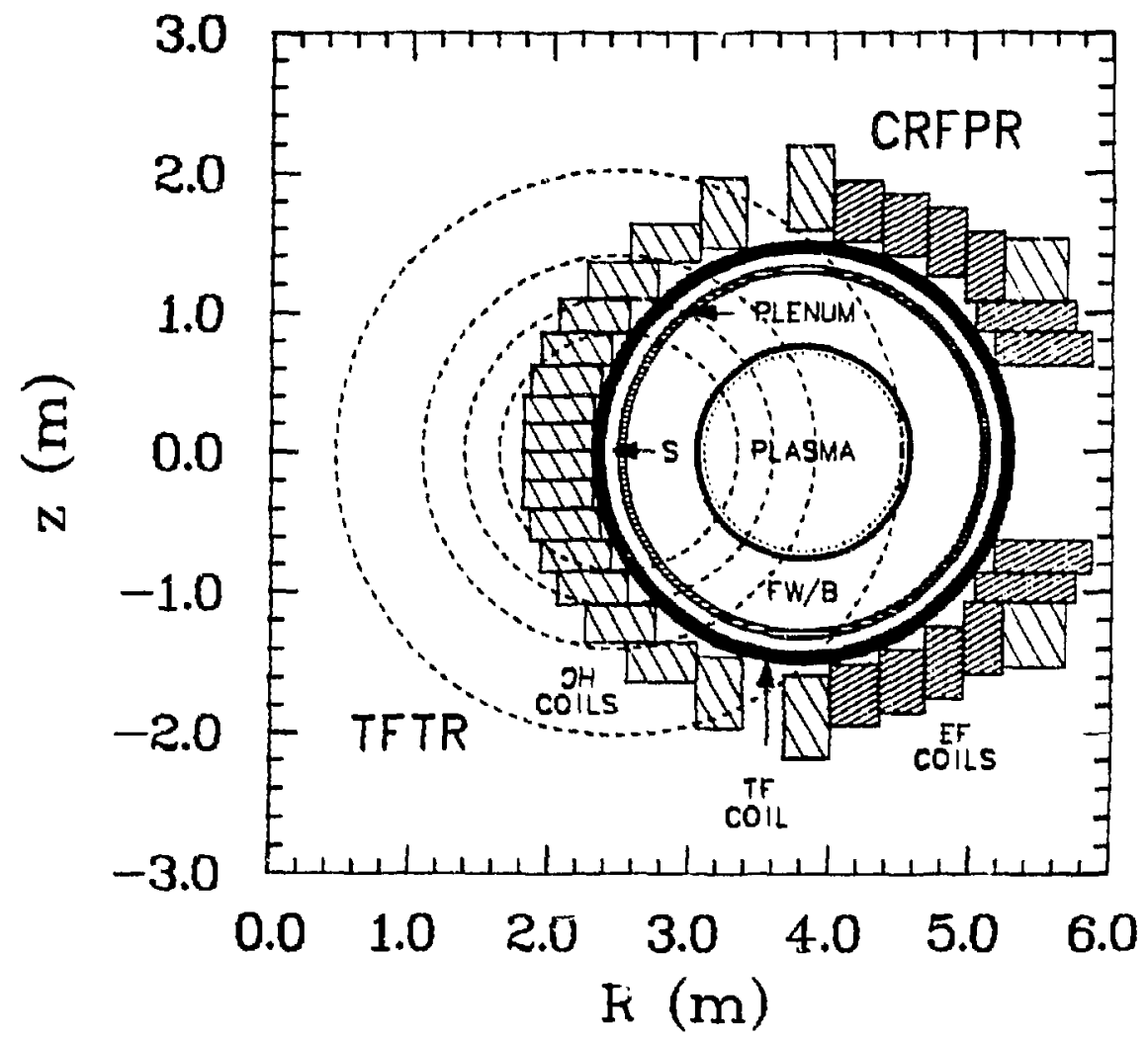

F18. III.A-1. Cross-sectlonal view of the 1000-MWe(net) CRFPR compared to TFTR $^{36}$ (poloidal-field colls not shown). 
factor. Table III.A-I summarizes the major CRFPR design parameters, which are presented in more detail in Appendix $B$. These parameters reflect the results of design guidelines that targeted the CRFPR at system power densities $\left(\sim 11.8 \mathrm{MW} / \mathrm{m}^{3}\right)$ and physical sizes $\left(0.33\right.$ tonne $\left./ \mathrm{MWt}, 285 \mathrm{~m}^{3}\right)$ at which pressurized-water (fission) reactor's (PWRs) presently operate; considerable flexibility exists for systems of either reduced power density or reduced plant capacity (and neutron first-wall loading) while malntaining favorable energy cost projections (Sec. IV.C.). The cost projections are summarized in Table III.A-II, which also gives comparisons with other fusion systems on a relatively common basis. The design approach taken for the CRFPR allowed by the unique RFP physics principles projects a (relative) cost advantage. More
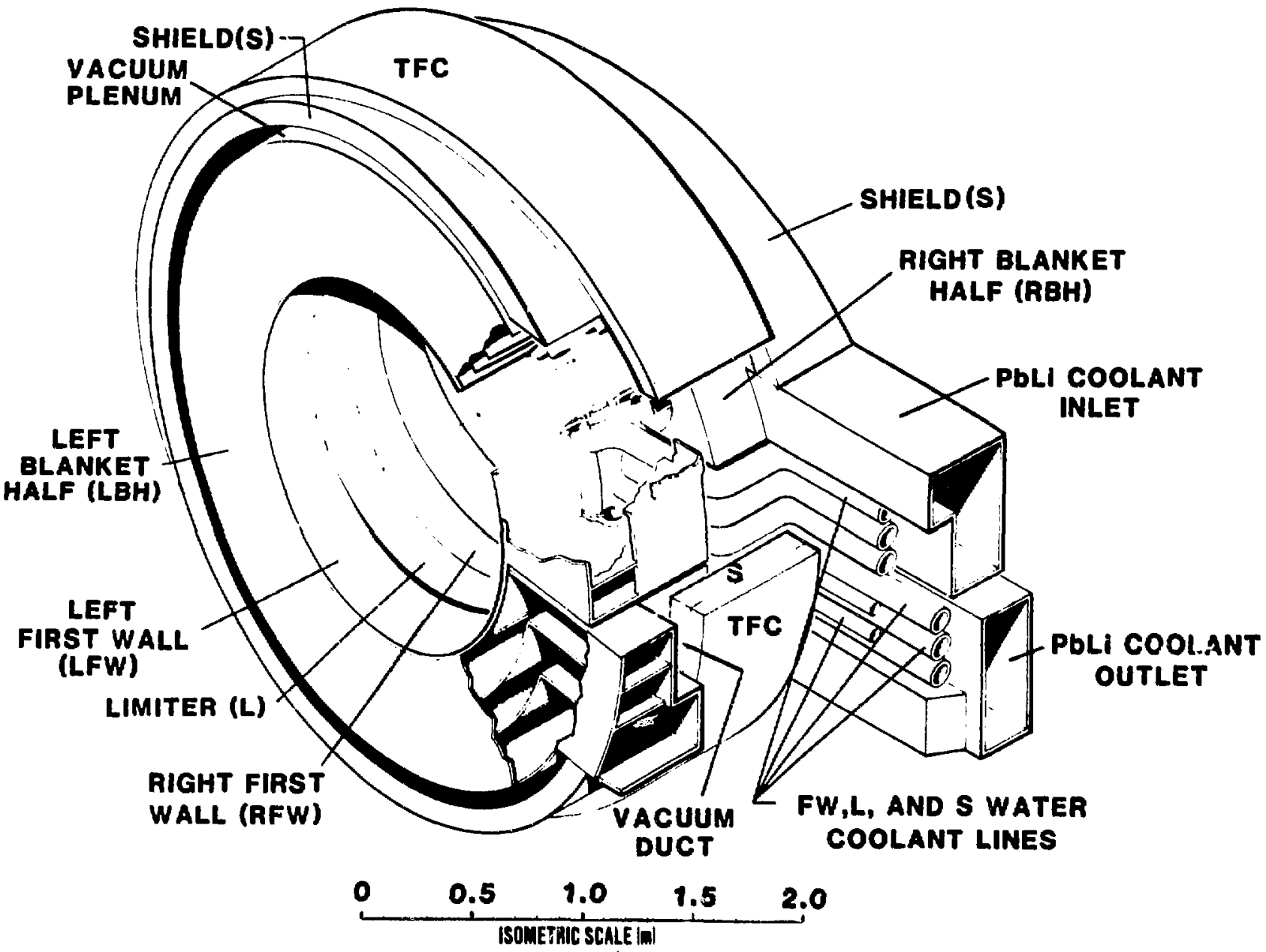

Fig. III.A-2, Isometric view of one of 24 toroidal sections for a 1000-MWe(net) CRFPR. Off-site, single-unit construction of the first-wa11/blanket/shield/toroldal-field-coll FPC ( 300 tonne total without coolants) and single-plece maintenence/repair scheme is envisaged for this high-power-density design. 
CRFPR MAJOR DESIGN PARAMETERS

\author{
Net electrical power (MH) \\ Gross electrical power (MW) \\ Total thermal power (MW) \\ Gross power-conversion efficiency (\%) \\ Overall plant availability (\%) \\ Major radius (m) \\ Plasma radius (average)(m) \\ Plasma volume $\left(\mathrm{m}^{3}\right)$ \\ Number of toroldal sectors \\ Maximum field at magnet $(T)(a)$ \\ Fleld at plasma axis $(T)$ \\ Field at plasma edge (T) \\ Average poloidal beta \\ Average total beta \\ Average DT Ion density $\left(10^{\left.20 / \mathrm{m}^{3}\right)}\right.$ \\ Average DT Ion temperature (keV) \\ Plasma burn mode \\ Plasma heating method (startup) \\ Plasma Impurity-control method \\ First-wa11/1imter structural materials \\ Blanket/shield structur ${ }^{\sim}$ materlal \\ Neutron wall loading $\left(\mathrm{MW} / \mathrm{m}^{2}\right)$ \\ Tritium-breeding medium \\ Primary coolant \\ Thermal conversion method
}

1000 .

1227 .

3365 .

36.5

75.

3.8

0.71

37.8

24.

4.5

9.5

5.2

0.23

0.12

6.6

10.0

Continuous/ignited

Ohmic (246 Wb)

Pumped limiter

MZC copper alloy

HT-9 ferritic steel

20.

Pbli

PbLi

Dual-cycle, steam

(a) During burn, 9.2 T during startup, OHC.

importantly, however, is the promise of a smaller fusion system that couples to real physics uncertainties more loosely than to the overall cost equation for fusion power; a more robust and resilient design results that is capable of early and cost-effestive proof of fiscal and technical risk. 
COST COMPARISON (NORMALIZED TO TDC) (1980 Dollars)

ACCOUNT

20. Land and Land Rights

21. Structure and Site

22. Reactor Plant Equipment (RPE)

22.1.1. First-Wa11/Blanket (FW/B)

22.1.2. Shleld (S)

22.1.3. Coil (C)

$\mathrm{FPC}=\mathrm{FW} / \mathrm{B}+\mathrm{S}+\mathrm{C}$

23. Turbine Plant Equipment

24. Electric Plant Equipment

25. Miscellaneous Plant Equipment

26. Special Materials

90. Total Direct Costs (TDC)

99. Total Costs

Unit Direct Cost, UDC ( $\$ / \mathrm{kWe})$

Cost of Electricity, $\operatorname{COE}(\mathrm{mills} / \mathrm{kWeh})$

Net Electric Power, $\mathrm{P}_{\mathrm{E}}$ (MWe)

\begin{abstract}
STARFIRE $^{3}$
\end{abstract}
$\underline{M k I I B} \underline{69}$

0.19

$-$

20.09

12.48

56.00

4.77

10.78

9.90

25.48

14.47

72.04

6.66

4.47

12.30

23.43

10.88

6.77

3.29

2. 37

1.27

0.014

100.

185.20

1439

67.0

1200

100.

200.26

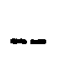

1200

MARS $5,6 \quad$ CRFPR

0.21

0.34

10.59

26.07

64.06

33.37

2.33

1.54

2.46

16.06

3.20

20.85

4.74

11.45

23.15

6.78

12.62

1.41

4.36

5.29

0.026

100.

100.

2556 [1685]

123.50

173.00 (a) 1480 . (b) 959

50. (b) 46.2

1202

1000

(a) Value in bracket gives $\$ / \mathcal{L}$ conversion in 1977 followed by $\$$ inflation from 1977 to 1980; otherwise the conversion/inflation order is reversed. Based on $1966 \$ / \mathrm{kWe}$ and $65 \mathrm{mills} / \mathrm{kWeh}$ in 1983 advising a $10 \%$ per year deflation factor.

(b) Based on $1966 \mathrm{~s} / \mathrm{kWe}$ and $67 \mathrm{mills} / \mathrm{kWeh}$ in 1983 dollars and a $10 \%$ per year deflation factor.

The CRFPR design differs considerably from superconducting fusion reactors. The profected technology requirements correspondingly differ primarily because of : 
- Increased plasma power density, which is proportional to $\beta^{2} B^{4}$, where $B$ is the confining magnetic field at the plasma and $\beta$ is the ratio of average plasma pressure to magneticfield pressure at the plasma surface.

- Increased first-wall neutron current $\left(15-20 \mathrm{MW} / \mathrm{m}^{2}\right)$ and surface heat flux $\left(4-5 \mathrm{MW} / \mathrm{m}^{2}\right.$, maximum, for uniform heat deposition onto the first wall).

- Increased peak $\left(>200 \mathrm{MW} / \mathrm{m}^{3}\right)$ and average $\left(\underset{\sim}{2} 30 \mathrm{MW} / \mathrm{m}^{3}\right)$ power density within a tritium-breeding blanket.

- Increased radiation and heat fluxes at resistive magnet coils in systems designed to operate at most with only a thin heat-recovering/tritium-breeding blanket placed between the coil and the plasma.

A summary of the key technology needs for the CRFPK is given in Sec. IV. This summary is presented according to the three major FPC subdivisions: Plasma Englneering systems (heating, particle control, etc.), Nuclear Systems (first wall, blanker, shleld), and Magnet Sy-' ms. In addition, Tritium Systems and Electrical/Mechanical systems represent key elements of the reactor, which at the level of this study have not recelved detalled attention. A number of the entries in the technology assessment given in Sec. IV. have been established Erom analyses performed in other, related studies. Compact reactors generally would operate with somewhat increased plasma densities. Refueling, impurity control, and ash removal requirements, therefore, may be more demanding. The higher plasma density may also lead to more-difficult $r$ current-drive requirements for steady-state operation. The potentlal for low-frequency "F- $\theta$ pumplng"7B-BI that is untque to the RFP (and spheromak) confinement, however, represents a new and potentially attractive means to drive steady-state plasma currents using the RFP dynamo action to sustain this unique, minfmum-energy magnetic configuration. The first-wall specific power loads for compact reactors are higher than projected for other MFE systems, which also leads to higher blanket power densitiea. Tho primary difference in magnet technology required of the CRFPR is reflected by the use of lower-field, copper-alloy coils that store considerably less total energy than the high-field superconducting colls envisaged for most other approaches.

The requirements for the Plasma Engineering Systems do not significantly differ between long-pulsed and steady-state operation except possibly for ash removal and impurity control; fueling should be similar for a $30-$ to 100- $s$ burn as for a truly steady-state burn, but the latter mode may require a magnetic 
divertor for ash removal and impurity control. Because of the higher first-wall thermal loadings, a heat-flux-concentrating limiter is not possible for the compact systems, and a sizable portion $(\sim 40 \%)$ of the first wall will have to serve the limiter function if a divertor is not used. The particle load and associated sputtering problem for these limiters do not differ from that for the lower-power-density systems, however. A potentially more-difflcult safety requirement for the DT-fueled compact systems is related primarily to the need for more-reliable off-power cooling capability because of the higher afterheat power density in the $\mathrm{FW} / \mathrm{B}$, this enhanced afterheat power density resulting from the higher overall operating blanket power density. The radwaste associated with the FW/B will be generated in more concentrated form, although the total radwaste generation will be the same for all systems for the same materials and ful1-power exposures. Maintenance of acceptable tritium-breeding rargins in the thin, high-power-density blankets presents a greater design challenge for the compact system, in that competition for this valuable "real estate" by vacuum ducts and coolant breeders/manifolds becomes more crucial. Because of the significant reductions in physical size and mass, the maintenance procedure envisaged for the compact reactor can consider "block" maintenance, wherein the coinplete FPC is removed for maintenance and repaix operations external to the reactor cavity, with a more rapid replacement by a fresh, pretested unit promising shorter downtimes and more reliable restarts. 
The starting point for the assessment of nuclear systems is the results of neutronics computations. These calculations are based on a scaled layout of the 1000-MWe(net) DT/CRFPR design and the corresponding key physics and engineering parameters reported in Ref. 15, as summarlzed in Sec. II.D.2. and Table II.D-III. The Initlal neutronics parameter studies were made using this one-dimensional reactor model to perform a preliminary assessment of materials and thermal-hydraulic needs. Specifically, the effects of varying the blanket thickness and material composition were examined. Following this parameter survey, a "canonical" neutronlcs model for the first wall, blanket, shleld, and coils is defined. This study emphasized the minimum blanket thickness within the constraints of efficient heat recovery and tritium breeding. All shielding f.nctions for the exoblanket colls were provided by the "blanket," although a thin, nonbreeding outer region was eventually identified as a shield operating at or near the blanket temperature.

The primary goals of this study were to examine key engineering considerations for key FPC subsystems, to generate and test models for these subsystems, to provide a preliminary integration of those subsystems, and generally to construct a framework for a future, more-comprehensive conceptual design. In a sense, this study begins and ends with the neutronics analysis. The FPC design integration has been made on the basis of one-dimensional neutronics calculations reported in this section. The multidimensional nature of the FPC destgn that emerged from this study will require minor design modifications and iteration. Preliminary, two-dimensional neutronics sensitlvity studies are reported in Appendix $C_{s}$ and these suggested changes will serve as a point of departure for future work.

1. Calculational Procedure. Neutronics calculations were performed using the one-dimenslonal ONEDANT discrete-ordinates transport code ${ }^{82}$ in the $\mathrm{S}_{8} \mathrm{P}_{3}$ approximation. The XSLIBA multigroup, coupled cross-section set ( 30 neutron and 12 gamma-ray groups) was used. This library for 35 elements and isotopes was derived using ENDF/B-IV data. 83 Data for kerma factors, dpa, and $H$ and He production were retrieved from the KERMA8 file. Activation cross sections were prepared from data contained in the GAMMON activation 1Lbrary, 84 An infinite cylinder model was used; all input and output quantities are normalized to a unit length of major circumference. Definitions of key quantities used in the 
neutronics parametrics study are given below; the quantity $P_{X X}$ is a linear heating rate $(\mathrm{MW} / \mathrm{m})$.

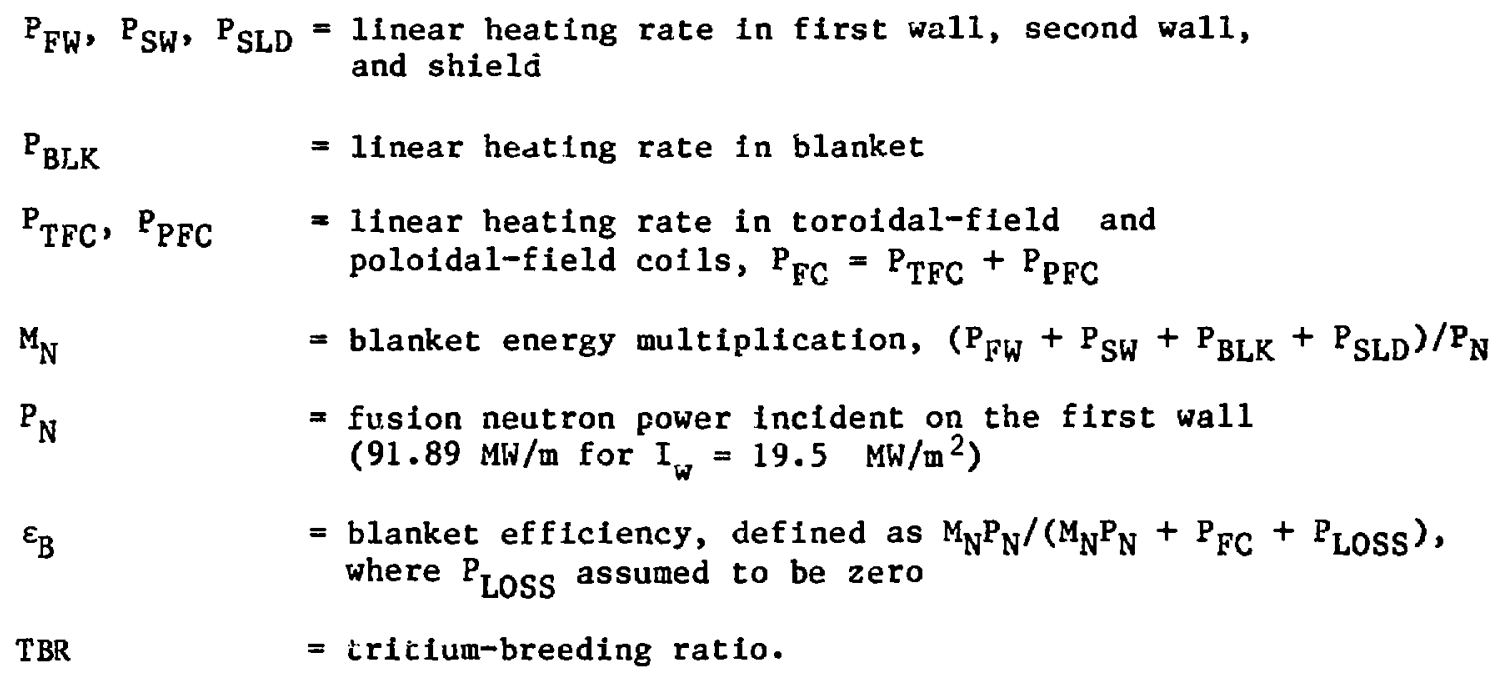

Figure III.B-1 displays the energy flow in the FW/B/C systems; interpretations of quantities adopted as measures of the blanket performance are also given.

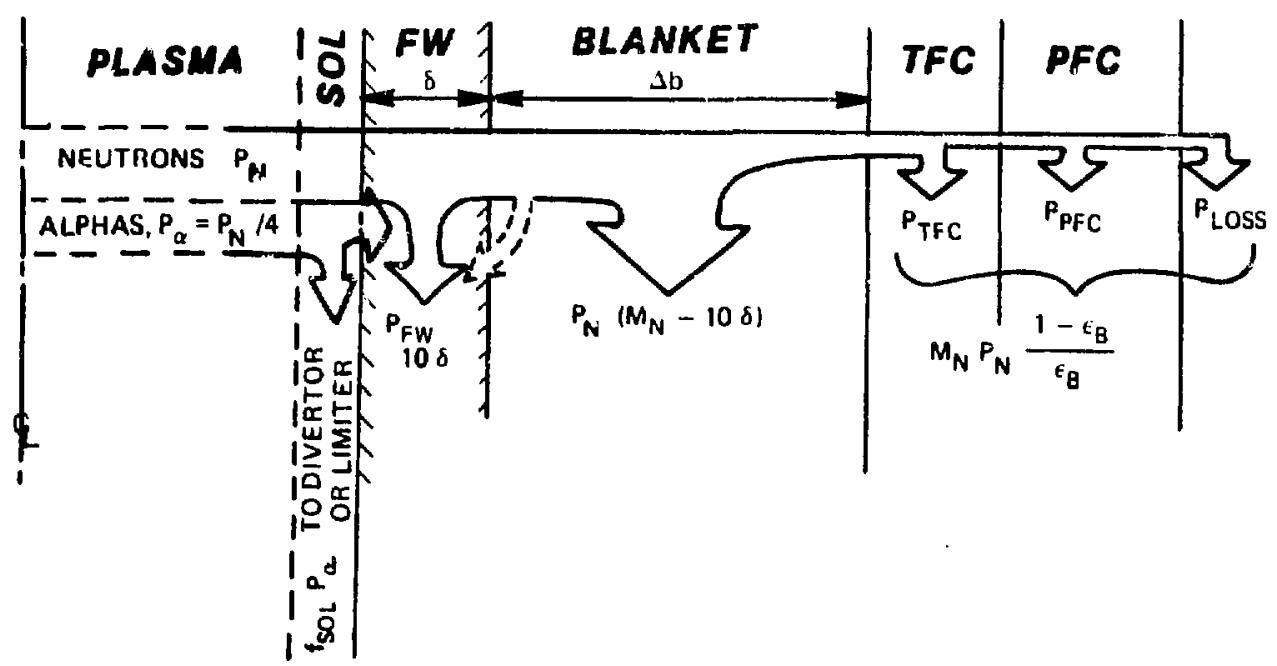

Fig. III.B-1. First-wall/blanket (FW/B) energy flows and efficiency parameters. The thin outboard shield eventually added to the FW/B system is not shown. 


\section{Computational Resules}

a. Parametric Studies. The materlal compositions and reference geometry used for the parametric studies are shown in FIg. III.B-2. All calculations are normalized to the desl.gn first-wall loading $\left(19.5 \mathrm{MW} / \mathrm{m}^{2}\right)$. The $\mathrm{Pb}_{83} \mathrm{LI}_{17}$ eutectic ${ }^{85,86}$ (PbLi) in the blanket consists of ${ }^{6} \mathrm{LI}$ enrichment at $60 \%$.

The effect of varying bianket thickness on selected neutronics parameters was first determined. The shield thickness (Fig. III.B-2) was fixed at $5 \mathrm{~mm}$; a blanket composed of $90 \mathrm{v} / 0 \mathrm{PbLI}$ and $10 \mathrm{v} / 0$ primary candidate alloy stainless steel (PCASS) was assumed. Although the neutronics results used in this study are based on PCASS, more-recent studies 5,87 indicate corrosion in PbLI may severely limit the operating temperature, interfaclal temperatures as low as $500^{\circ} \mathrm{C}$ being set by a $\sim 1-\mu \mathrm{m} / \mathrm{yr}$ limit on corrosion rate. At present HT-9 ferritic alloy may be preferred. The PCASS alloy, nevertheless, is retained for the purposes of the neutronics study.

Results are tabulated in Table III.B-I, with selected results being plotted in Fig. III.B-3. It is seen that $M_{N}, \varepsilon_{B}$, and $T B R$ increase as the blanket thickness is increased and, as expected, $\mathrm{P}_{\mathrm{TFC}}^{\mathrm{N}}$ and $\mathrm{P}_{\mathrm{PFC}}^{\mathrm{N}}$ decrease as the blanket is thickened. The blanket efficiency is limited to about $\varepsilon_{B} \simeq 0.94$ for the conf'guration examined unless thicker blankets and the attendant decrease in

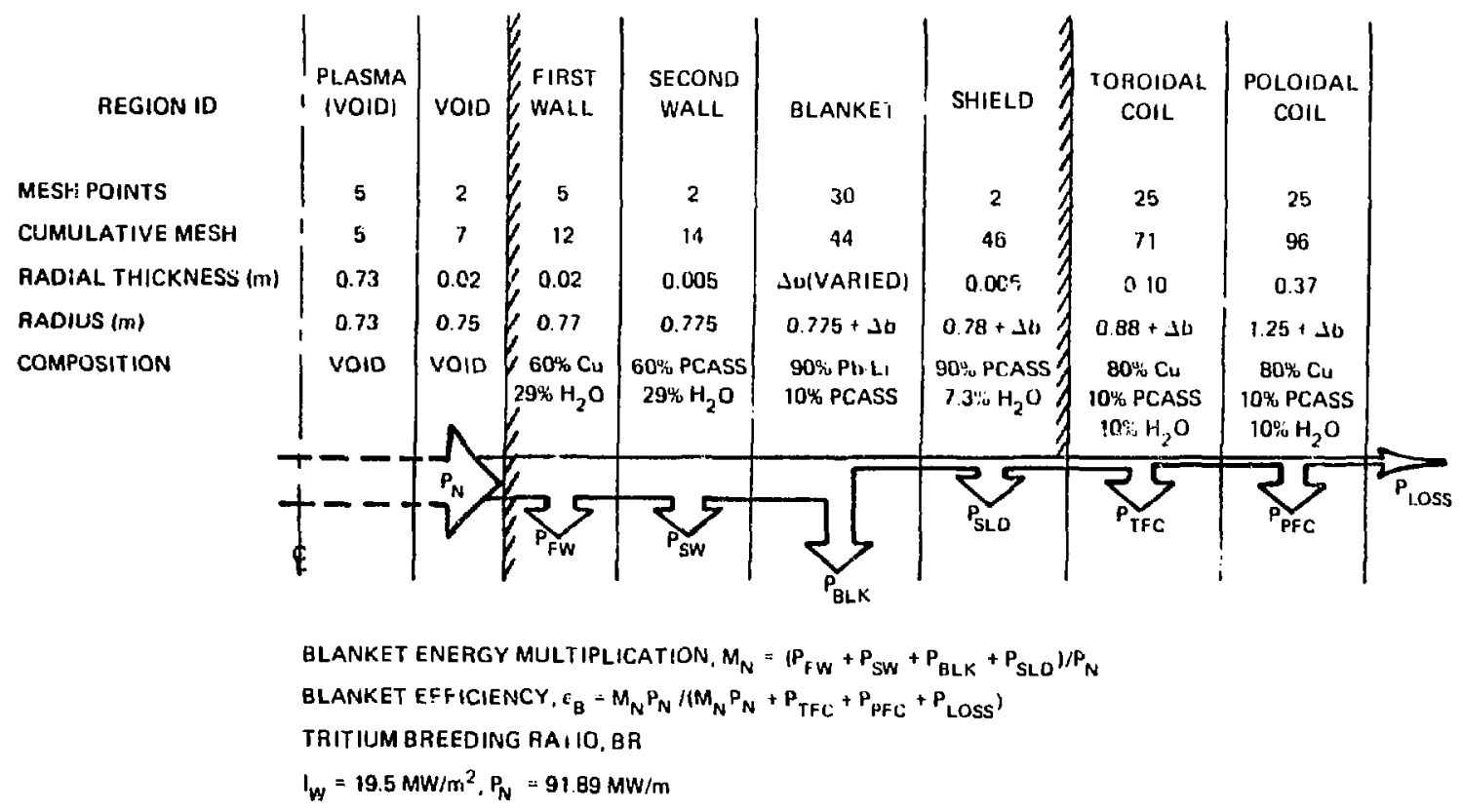

Fig. III.B-2. CRFPR neutronics model showing linear power $f$ lows and a moredetalled array of key regions. 
EFFECT OF VARYING BLANKET THICKNESS ON

CRFPR NEUTRONICS PARAMETERS(a)

Bianket thickness, $\triangle \mathrm{b}_{\mathrm{BLK}}(\mathrm{m})$

Power on first wall, $\mathrm{P}_{\mathrm{N}}(\mathrm{MW} / \mathrm{m})$

Total power, $\mathrm{P}_{\mathrm{BLK}}(\mathrm{MW} / \mathrm{m})$

Power in coils, $\mathrm{P}_{\mathrm{TFC}}^{\mathrm{N}}+\mathrm{P}_{\mathrm{PFC}}^{\mathrm{N}}(\mathrm{MW} / \mathrm{m})$

Blanket energy multiplication, $M_{N}$

Blanket efficiency, $\varepsilon_{B}$

Tritium-breeding ratio, TBR

First-wa11 nuclear heating

\begin{tabular}{cccc}
0.3 & 0.4 & -0.5 & 0.6 \\
\hline 91.89 & 91.89 & 91.89 & 91.89 \\
130.07 & 127.48 & 125.50 & 124.06 \\
24.37 & 16.19 & 10.68 & 6.97 \\
1.15 & 1.21 & 1.25 & 1.27 \\
0.813 & 0.873 & 0.915 & 0.944 \\
0.920 & 1.036 & 1.120 & 1.180
\end{tabular}

20.74
7.60

13.14

20.90

7.64

13.26

20.96

7.66

13.30

20.98

7.66

13.32

Toroidal-coll nuclear heating
- TotaI, P $\mathrm{TFC}$
14.95
10.09
6.77
0.52
4.48
- Neutron
1.61
1.00
6.15
0.38
13.34
9.09
$\therefore 10$

Poloidal-coil nuclear heating
- Tota1, P $\mathrm{PFC}$
9.42
6.10
3.91
2.48
- Neutron
0.51
0.30
0.17
(i. 10
- Gamma-zay
8.91
5.80
3.74
2.38

Maxinum dpa/yr in copper

- TrC

- PFC

Maximum He appm/yr in copper

- TFC

- PFC

Maximum $\mathrm{H}$ appm/yr in copper

- TFC

- $\mathrm{PFC}$

$$
26.00
$$

4.96
8.75

1.51

$$
1.51
$$

2.73$$
5.07
$$

0.84

$\begin{array}{rrrr}21.10 & 6.83 & 2.24 & 0.73 \\ 3.44 & 1.13 & 0.37 & 0.12\end{array}$

$$
99.9
$$

15.50
36.70

5.57

13.70

2.02

5.16

0.75

\footnotetext{
(a) Blanket is 90 v/0 $\operatorname{PbLi}\left(60 \% \sigma_{\mathrm{Li}}\right)+10 \mathrm{v} / 0$ PCASS, with the shield thickness fixed at $5 \mathrm{~mm}$.
} Exxed at 5 ano 

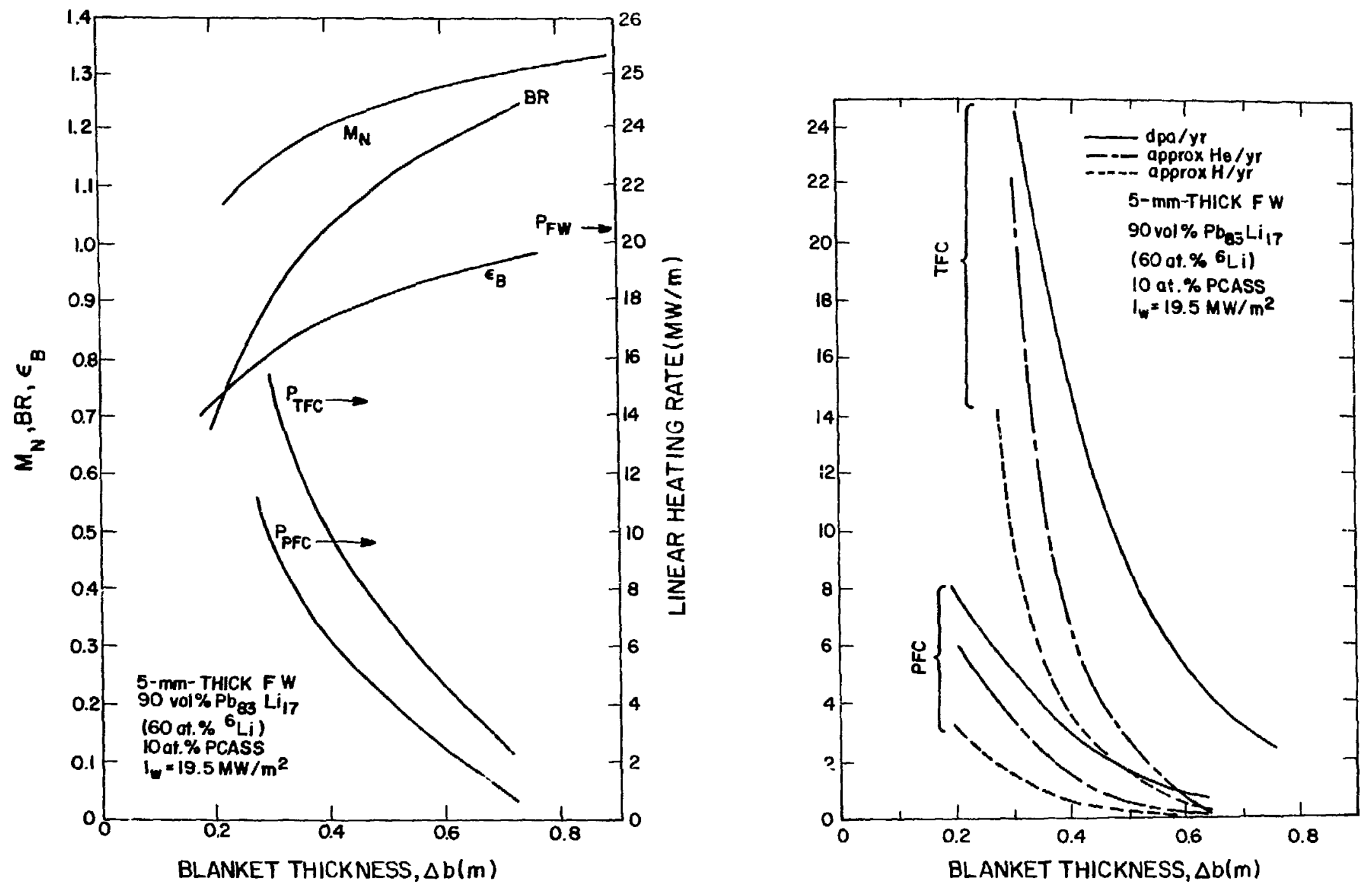

Fig. III.B-3. Effect of varying PbLi blanket thickness on energy multiplication, $M_{N}$, tritium-breeding ratio, TBR, blanket efficiency, $\varepsilon_{B}$, coil heating, and coil radiation responses. 
system power density are adopted. This tradeoff is further studied; an Increased shield thtckness to $\sim 100 \mathrm{~mm}$ increases $\varepsilon_{\mathrm{B}}$ to 0.985 .

The ONEDANT transport calculations were next made for a $0.5-m$-thick blanket with shield thicknesses ranging from 5 to $125 \mathrm{~mm}$; a 90/10 PbLi/PCASS mixture was again specifled for the blanket. These results are plotted in Fig. III.B-4 and summarized in Table III.B-II. Also shown in Table III.B-II are the results for an $80 / 20$ PbLi/PCASS with increased shield thicknesses. The variations of $M_{N}$, $\varepsilon_{B}$, and TBR are not dramatic for the range of shfeld thickness examined, but $P_{\text {TFC }}^{N}$ and $P{ }_{\text {PFC }}^{N}$ vary within factors of 2 to 4 . Comparing results for the $90 / 10$

\section{TABLE III.B-II}

EFFECT OF VARYING SHIELD THICKNESS ON NEUTRONICS PARAMETERS FOR A $\Delta \mathrm{b}_{\mathrm{BLK}}=0.5-\mathrm{m}$ BLANKET WITH 90/10 PbL1/PCASS. (a)

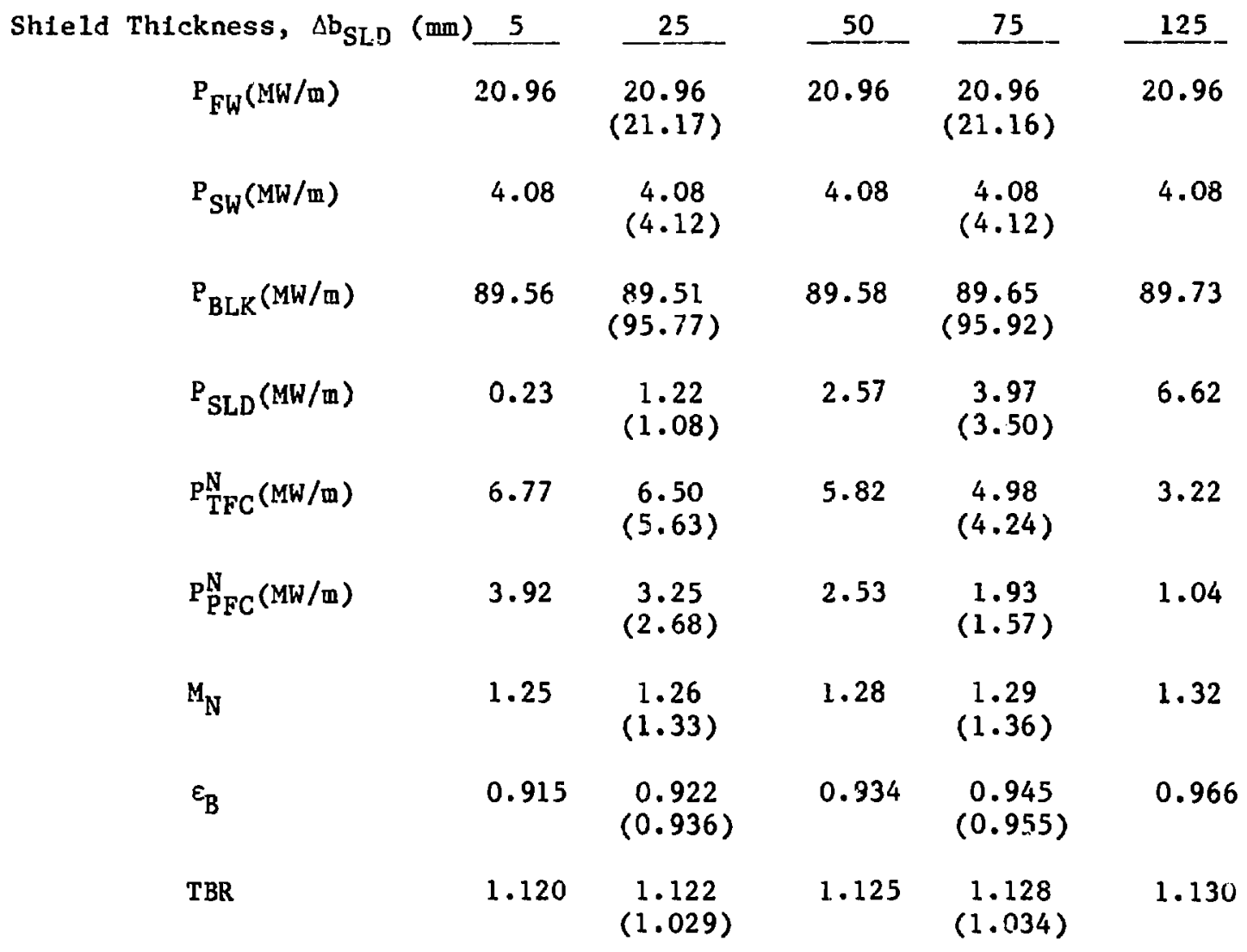

(a) F1rst-wall neutron loading $I_{w}=19.5 \mathrm{MW} / \mathrm{m}^{2}$, results for $80 / 20$ PbLf/PCASS blanket are shown in parentheses. 


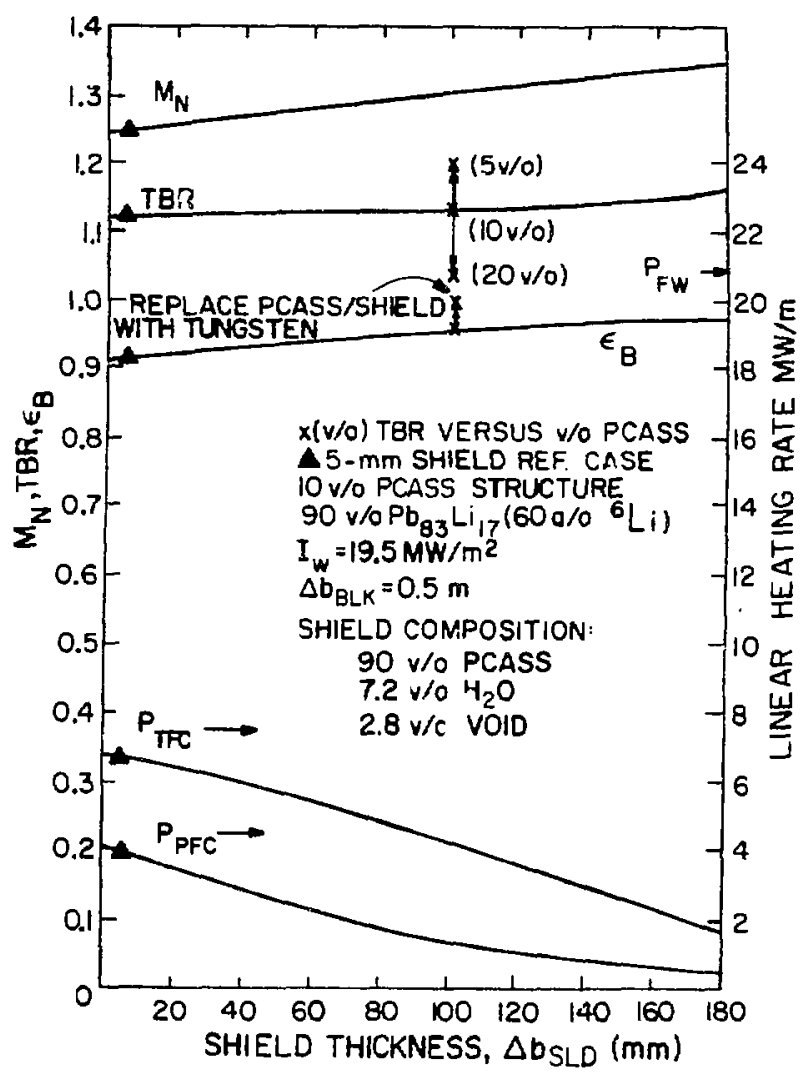

Fig. III.B-4. Effect on key measures of blanket performance on Increased th1rdwall shield thickness for a ilxed blanket $\Delta \mathrm{b}=0.5 \mathrm{~m}$.

and $80 / 20$ PbLi/PCASS blankets shows that $M_{N}$ and $\varepsilon_{B}$ are higher for the $80 / 20$ case, but the breeding ratio is $\sim 10 \%$ lower.

The results reported to this polnt used a 5-mm-thlck shleld material composed of $90 \mathrm{v} / 0$ PCASS and $7.26 \mathrm{v} / 0$ water $\left(10 \mathrm{v} / 0\right.$ water at $\left.0.726 \mathrm{~g} / \mathrm{cm}^{3}\right)$. If the shleld is made more absorbent of neutrons, the blanket efficiency, $\varepsilon_{B}$, would increase and the coll heating rates would decrease. A $0.1-m$-thick third wall consisting of varying proportions of tungsten, boron carbide, PCASS, and water coolant, therefore, was considered. In addition, the 90/10 PbL1/PCASS blanket used previously was replaced by a structurally more optimal $95 / 5$ ratio. Calculational results, assuming a homogeneous distribution of these materials, are shown in Table III.B-III. The results indicate a blanket efficiency of $\varepsilon_{B} \simeq 0.98$ for the $W / B_{4} C$ and $W$ shield cases. The breeding ratio, however, is lower than that for the $90 \mathrm{v} / 0$ PCASS configuration. 
b. The Canonical Blanket. On the basis of parametric studies described above, a "canonical" $\mathrm{FW} / \mathrm{B} / \mathrm{S} / \mathrm{C}$ design was adopted as a starting point for further calculations of this thin-blanket/copper-coll concept. The material and geometric specifications for this design are shown in Table III.B-IV and Fig. III.B-5. The important features of the canonical FPC neutronics design are a) a 0.5-m-thick blanket with 95/5 PbLi/PCASS and b) a 0.1-m-thick shield composed of 25-mm-thick alternating layers of $\mathrm{B}_{4} \mathrm{C}$ and tungsten metal. Water coolant was provided for this region, although flowing PbLi coolant is also possible. Thicknesses and material compositions of the other regions are those used in the previous studies. Key results for this canonical blanket are tabulated in Table III.B-V.

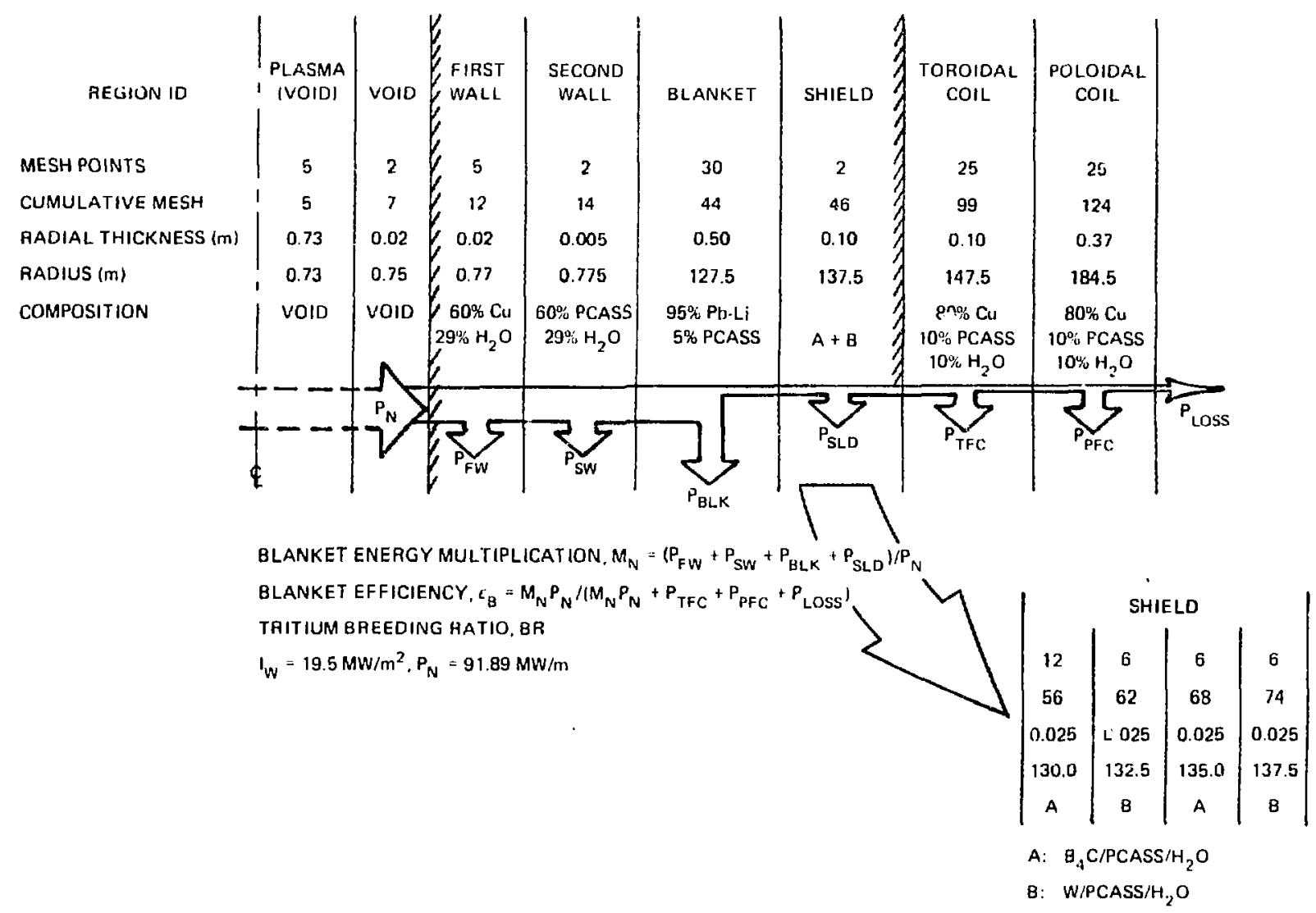

Fig. III.B-5. Canonical FPC neutronics model based on an infinite cylinder geometry. Shield materials: Region A - $\mathrm{B}_{4} \mathrm{C} / \mathrm{SS} / \mathrm{H}_{2} \mathrm{O}$; Region B $\mathrm{W} / \mathrm{SS} / \mathrm{H}_{2} \mathrm{O}$. 
Table III.B-VI lists the maximum and average volumetric heating rates and the maximum (annual) neutron fluences ( $>0.1 \mathrm{MeV}$ ). The heating rate increases from 4.1 to $17.7 \mathrm{MW} / \mathrm{m}^{3}$ across the blanket/shield interface. For the shield/TFC interface, the heating rate decreases from 6.6 to $1.9 \mathrm{MW} / \mathrm{m}^{3}$.

Quantitative estimates were made for the transmutation of copper to nickel and $z$ inc in the first wall and colls. The reactions of interest and the halflives of the radioactive products are listed below and summarized in Fig. III. B -6 .

Nicke1-producing reactions:

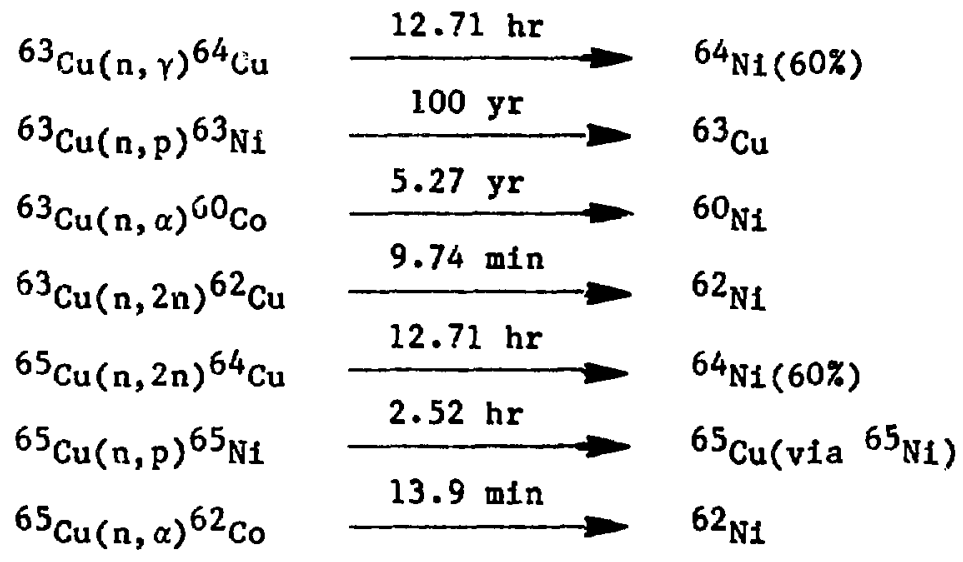

Zinc-producing reactions:

$$
\begin{array}{ll}
{ }^{63} \mathrm{Cu}(\mathrm{n}, \gamma){ }^{64} \mathrm{Cu} & \frac{12.71 \mathrm{hr}}{12.71 \mathrm{hr}} \rightarrow{ }^{64} \mathrm{Zn}(40 \%) \\
{ }^{65} \mathrm{Cu}(\mathrm{n}, 2 \mathrm{n}){ }^{64} \mathrm{Cu} & \frac{12.76 \mathrm{~min}}{5.10 \mathrm{~m}(40 \%)} \\
{ }^{65} \mathrm{Cu}(\mathrm{n}, \gamma){ }^{66} \mathrm{Cu} &
\end{array}
$$

Assuming a one-year irradiation time and using the appropriate production rates and decay constants, the concentration buildup of nickel and $z$ inc in the first wall and colls can be estimated. It is convenient to express the transmutation densities as fractions of the copper atom densities in the target material. Results of these calculations are summarized in Table III.B-VII. Table III.B-VIII gives the production of $63 \mathrm{~N} 1$ and ${ }^{60} \mathrm{Co}$ from activation of FW, TFC, and PFC copper after one year of continuous Irradiation for $I_{w}=19.5 \mathrm{MW} / \mathrm{m}^{2}$. 
TABLE II I - B-III

EFFECT OF VARYING SHIELD MATERIALS FOR A $\triangle \mathrm{b}_{\mathrm{SLD}}=100-\mathrm{mm}$-THICK SHIELD AND A $\triangle b_{\text {BLK }}=0.5-m m$-THICK BLANKET WITH 90 v/o PbLi and 5 v/0 PCASS. (a)

\begin{tabular}{lccrr} 
THIRD-WALL & $\begin{array}{c}40 \% \mathrm{~W}+40 \% \mathrm{~B}_{4} \mathrm{C} \\
\mathrm{MATERIAL}\end{array}$ & $80 \% \mathrm{~W}+10 \% \mathrm{~B}_{4} \mathrm{C}$ & $90 \% \mathrm{~W}$ & $90 \%$ PCASS \\
\cline { 2 - 4 } & 20.81 & 20.82 & 20.82 & 20.82 \\
$\mathrm{P}_{\mathrm{FW}}(\mathrm{MW} / \mathrm{m})$ & 4.07 & 4.07 & 4.08 & 4.08 \\
$\mathrm{P}_{\mathrm{SW}}(\mathrm{MW} / \mathrm{m})$ & 86.72 & 87.32 & 87.60 & 89.34 \\
$\mathrm{P}_{\mathrm{BLK}}(\mathrm{MW} / \mathrm{m})$ & 6.21 & 8.25 & 9.31 & 5.78 \\
$\mathrm{P}_{\mathrm{SLD}}(\mathrm{MW} / \mathrm{m})$ & 1.16 & 1.63 & 1.93 & 4.38 \\
$\mathrm{P}_{\mathrm{TFC}}^{\mathrm{N}}(\mathrm{MW} / \mathrm{m})$ & 0.59 & 0.75 & 0.85 & 1.64 \\
$\mathrm{P}_{\mathrm{PFC}}^{\mathrm{N}}(\mathrm{MW} / \mathrm{m})$ & 119.56 & 122.84 & 124.59 & 126.04 \\
$\mathrm{P}_{\mathrm{TOT}}(\mathrm{MW} / \mathrm{m})$ & 1.282 & 1.311 & 1.326 & 1.306 \\
$\mathrm{M}_{\mathrm{N}}$ & 0.985 & 0.981 & 0.978 & 0.952 \\
$\varepsilon_{\mathrm{B}}$ & 1.11 & 1.13 & 1.13 & 1.18 \\
$\mathrm{TBR}$ & & & &
\end{tabular}

Maximum dpa/yr

- TFC

1.180

1.450

1.620

2.890

- PFC

0.024

0.226

0.245

0.492

Maximum He appm/yr

$\begin{array}{lllll}- \text { TFC } & 0.532 & 0.386 & 0.355 & 0.599 \\ \text { - PFC } & 0.091 & 0.069 & 0.064 & 0.103\end{array}$

Maximum $\mathrm{H}$ appm/yr

$\begin{array}{lllll}- \text { TFC } & 2.61 & 1.900 & 1.760 & 3.540 \\ - \text { PFC } & 0.429 & 0.324 & 0.302 & 0.551\end{array}$

(a) The shield coolant is $10 \mathrm{v} / 0$ water at density of $0.726 \mathrm{~g} / \mathrm{cm}^{3}$.

Table III.B-VIII gives a finer breakdown of the first-wall transmutation rate expressed per unit $14.1-\mathrm{MeV}$ neutron first-wa11 loading. Although $\mathrm{zn}$ is formed primarily by nonthreshold $(n, \gamma)$ reactions, the build-in of $N i$ is almost equally divided between threshold and nonthreshold reactions, with the former dominating somewhat. Hence, reductions in the Ni production rate for this 20-mm-thick copper first wall by alterations in the blanket composition and shifting to a softer neutron spectrum should not be significant. It is also noted that uncertainties are associated with the ${ }^{65} \mathrm{Cu}(n, \gamma)$ cross sections. 
TABLE IIL.B-IV

MATERIAL COMPOSITIONS FOR THE CANONICAL BLANKET MODEL

PRIMARY MATERIALS

$\mathrm{Cu}$

$\mathrm{H}_{2} \mathrm{O}$

$\mathrm{Pb}_{83} \mathrm{Li}_{17}$

PCASS

$\mathrm{B}_{4} \mathrm{C}$

$\mathrm{W}$

\section{MIXTURES}

First wall

Second wall

Blanket

Shield (case A)

Shield (case B)

TFC and PFC coils

\section{DENSITY}

$8.96 \mathrm{~g} / \mathrm{cm}^{3}$

$1.00 \mathrm{~g} / \mathrm{cm}^{3}$

$9.40 \mathrm{~g} / \mathrm{cm}^{3}$

$7.86 \mathrm{~g} / \mathrm{cm}^{3}$

$60 \mathrm{v} / \mathrm{OCu}$

$60 \mathrm{v} / \mathrm{O}$ PCASS

95 v/o PbLi

$80 \mathrm{v} / 0 \mathrm{~B}_{4} \mathrm{C}$

10 v/O PCASS

$80 \mathrm{v} / 0 \mathrm{~W}$

$10 \mathrm{v} / 0$ PCASS

$10 \mathrm{v} / 0$ PCASS

$10 \mathrm{v} / \mathrm{O} \mathrm{H}_{2} \mathrm{O}$
COMPOSITION

$60 \%$ enriched in ${ }^{6} \mathrm{Li}$

$66.7 \mathrm{w} / \mathrm{O} \mathrm{Fe} \quad 13.7 \mathrm{w} / \mathrm{O} \mathrm{Cr}$

$2.0 \mathrm{w} / 0 \mathrm{Mo} \quad 15.6 \mathrm{w} / 0 \mathrm{NI}$

$2.0 \mathrm{w} / \mathrm{O} \mathrm{Mn}$
$29 \mathrm{v} / \mathrm{O} \mathrm{H}_{2} \mathrm{O}$

$\left(40 \mathrm{v} / 0,0.726 \mathrm{~g} / \mathrm{cm}^{3}\right)(a)$

$29 \mathrm{v} / \mathrm{O} \mathrm{H}_{2} \mathrm{O}$

$\left(40 \mathrm{v} / 0,0.726 \mathrm{~g} / \mathrm{cm}^{3}\right)$

5 v/O PCASS

$7.26 \mathrm{v} / \mathrm{o} \mathrm{H} \mathrm{H}_{2} \mathrm{O}$

$\left(10 \mathrm{v} / 0,0.726 \mathrm{~g} / \mathrm{cm}^{3}\right)$

$7.26 \mathrm{v} / \mathrm{O} \mathrm{H} \mathrm{H}_{2} \mathrm{O}$

$\left(10 \mathrm{v} / \mathrm{o}, 0.726 \mathrm{~g} / \mathrm{cm}^{3}\right)$

$80 \mathrm{v} / \mathrm{o} \mathrm{Cu}$

\section{$\overline{(a)}$ Volume percent at temperature.}

Although preliminary and approximate (1.e., homogenized, one-dimensional, parametric, interpolative), the neutronic results summarized herein are sufficient to provide a good indication of $\mathrm{FW} / \mathrm{B} / \mathrm{S} / \mathrm{C}$ radiation responses and thermohydraulic requirements. The increases needed in both $\Delta b=\Delta b_{B L K}+\Delta b_{S L D}$ $(0.4 \rightarrow 0.6 \mathrm{~m})$ and $\mathrm{M}_{\mathrm{N}}(1.1 \rightarrow 1.3)$ represent the major adjustments made to the preliminary design point suggested earlier in Ref. 15. and Table II.D-III. Table III.B-IX summarizes the concentration of major long-lived activation for the FW, TFC, and PFC. The results summarized in Tables III.B-V through III.B-IX represent the key neutronic parameters for the canonical blanket used in subsequent analyses leading to the FPC design integration. As will be shown, 
SUMMARY OF PARAMETERS FOR CANONICAL BLANKET MODEL(a)

WITH A $\Delta b_{B L K}=0.5-m-T H I C K$ BLANKET (95/5 PbLi/PCASS) AND A $\triangle b_{S L D}=0.1-m-T H I C K$ SHIELD WITH ALTERNATING LAYERS OF $B_{4} \mathrm{C}$ AND TUNSSTEN

$\begin{array}{lc}\mathrm{P}_{\text {EW }}(\mathrm{MW} / \mathrm{m}) & 20.80 \\ \mathrm{P}_{\mathrm{SW}}(\mathrm{MW} / \mathrm{m}) & 4.08 \\ \mathrm{P}_{\mathrm{BLK}}(\mathrm{MW} / \mathrm{m}) & 86.45 \\ \mathrm{P}_{\mathrm{SLD}}(\mathrm{MW} / \mathrm{m}) & 6.02 \\ \mathrm{P}_{\mathrm{TFC}}^{\mathrm{N}}(\mathrm{MW} / \mathrm{m}) & 1.26 \\ \mathrm{P}_{\mathrm{PFC}}^{\mathrm{N}}(\mathrm{MW} / \mathrm{m}) & 0.61 \\ \mathrm{P}_{\mathrm{TOT}}(\mathrm{MW} / \mathrm{m}) & 119.22 \\ { }_{\mathrm{M}} & 1.278 \\ \varepsilon_{\mathrm{B}} & 0.985 \\ \mathrm{TBR} & 1.11\end{array}$

Peak dpa/yr

- First wall 310.

- TFC 1.220

- PFC 0.208

Peak He appm/yr

- First wall 1750.

- TFC 0.524

- PFC 0.091

Peak $\mathrm{H}$ appm/yr

- First-wall 5020 .

- TFC 2.580

- PFC 0.430

(a) Based on a fusion neutron first-wall loading of $I_{w}=19.5 \mathrm{MW} / \mathrm{m}^{2}$.

subsequent analyses should be based on two-dimenstonal analyses that more accurately model the effects of pumped limiters, vacuum ducts, and coolant manifolds/headers on the key tlanket performance parameters $\left(M_{N}, \varepsilon_{B}\right.$, and TBR for a given $\Delta b$ ). Extensive two-dimensional modeling, however, was not within the scope of this study, although the results given in Appendix $C$ give a clear direction for future wor $s$ and FW/B/C designi options. 


\section{TABLE III.B-VI}

VOLUMETRIC HEATING RATES AND NEUTRON FLUENCES

FOR STEADY-STATE OPERATION OF THE

CANCNICAL BLANKET MODEL $\left(I_{\mathrm{w}}=19.5 \mathrm{MW} / \mathrm{m}^{2}\right)$

\begin{tabular}{lccc}
\multicolumn{1}{c}{ REGION } & $\begin{array}{c}\text { MAXIMUM POWER } \\
\text { DENSITY }\left(\mathrm{MW} / \mathrm{m}^{3}\right)\end{array}$ & $\begin{array}{c}\text { AVERAGE POWER } \\
\text { DENSITY }\left(\mathrm{MW} / \mathrm{m}^{3}\right)\end{array}$ & $\begin{array}{c}\text { MAXIMUM FPY }(\mathrm{a}) \text { NEUTRON } \\
\text { FLUENCE }\left(10^{27} \mathrm{n} / \mathrm{m}^{2}\right)\end{array}$ \\
\cline { 2 - 3 } First Wall & 239.0 & 218.0 & 2.1 \\
Second Wall & 173.0 & 168.0 & 2.0 \\
Blanket & 255.0 & 26.9 & 2.0 \\
Shield & 15.0 & 7.24 & 0.17 \\
TFC & 1.94 & 1.41 & 0.024 \\
PFC & 0.80 & 0.16 & 0.004
\end{tabular}

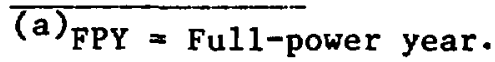

A question commonly asked is the effect of a thick copper first wall on the blanket performance. To address this issue ONEDANT calculations in cylindrical geometry were made to determine tritium-breeding ratios as a function of copper first-wall thickness. The reference case (Table III.B-V) was used: 20-mm-thick first wall consisting of $60 \mathrm{v} / \mathrm{O} \mathrm{Cu}$ and $29 \mathrm{v} / \mathrm{o} \mathrm{H}_{2} \mathrm{O}$; 5-mm-thick second wall contains $60 \mathrm{v} / 0$ PCASS and $29 \mathrm{v} / 0 \mathrm{H}_{2} \mathrm{O}$. Results of the calculations are given in Fig. III.B-7. The breeding ratio decreases monotonically with increasing wall thickness; the decrease is about $15 \%$ over the range of first-wall thicknesses from 0 to $20 \mathrm{~mm}$. This behavior can be explained in terms of the neutron cross sections for $\mathrm{Cu}$ and $\mathrm{Pb}$ at $14 \mathrm{MeV}$. These cross sections are as follows:

$\begin{array}{lcc} & \text { Copper } & \text { Lead } \\ \text { Total(barns) } & 3.0 & 5.4 \\ \text { Elastic } & 1.4 & 2.9 \\ \text { Nonelastic } & 1.6 & 2.5 \\ (n, 2 n) & 0.6 & 2.2\end{array}$

The $(n, 2 n)$ cross section is much higher for $\mathrm{Pb}$ compared to $\mathrm{Cu}$. Also, the $(n, 2 n)$ cross section for $\mathrm{Pb}$ is $88 \%$ of the nonelastic value, so that the competition from other nonelastic processes is small. The $(n, 2 n)$ and nonelastic cross sections for $\mathrm{Cu}$ are 0.6 and $1.6 \mathrm{~b}$, respectively, and the competition from other nonelastic reactions is much greater. The lead is a better neutron multiplier 
NICKEL AND ZINC PRODUCTION FROM NEUTRON TRANSMUTATLON OF COPPER FW AND COILS AFTER ONE FULL-POWER YEAR $\left(I_{w}=19.5 \mathrm{MW} / \mathrm{m}^{2}\right)$

$\begin{array}{llll} & \text { FIRST WALL } & \text { TFC } & \text { PFC } \\ \text { Average Ni/Cu } & 4.77(10)^{-2} & 1.79(10)^{-4} & 0.18(10)^{-4} \\ \text { Maximum } \mathrm{Ni} / \mathrm{Cu} & 5.52(10)^{-2} & 2.24(10)^{-4} & 0.99(10)^{-4} \\ \text { Average } \mathrm{Zn} / \mathrm{Cu} & 2.63(10)^{-2} & 0.18(10)^{-4} & 0.18(10)^{-4}\end{array}$

TABLE IIL.B-VIII

FIRST-WALL TRANSMUTATION RATES EXPRESSED IN UNITS OF ATOMIC \% PER YEAR PER $\mathrm{MW} / \mathrm{m}^{2}$ OF $14.1-\mathrm{MeV}$ NEUTRONS
Average $\mathrm{Ni} / \mathrm{Cu}$
0.2448
${ }^{64} \mathrm{Ni}$ from ${ }^{63} \mathrm{Cu}(\mathrm{n}, \gamma)$
0.1097
$60_{\mathrm{N} \perp}$ from ${ }^{63} \mathrm{Cu}(\mathrm{n}, \alpha)$
0.0004
${ }^{62} \mathrm{Ni}$ from ${ }^{63} \mathrm{Cu}(\mathrm{n}, 2 \mathrm{n})$
0.0743
${ }^{64} \mathrm{~N} 1$ from ${ }^{65} \mathrm{Cu}(\mathrm{n}, 2 \mathrm{n})$
0.0373
$62 \mathrm{Ni}$ from ${ }^{65} \mathrm{Cu}(\mathrm{n}, \alpha)$
0.0025
${ }^{63} \mathrm{N1}$ from ${ }^{63} \mathrm{Cu}(\mathrm{n}, \mathrm{p})$
0.0206
Average $\mathrm{Zn} / \mathrm{Cu}$
$0.13,0$
${ }^{64} \mathrm{Zn}$ from ${ }^{63} \mathrm{Cu}(\mathrm{n}, \gamma)$
0.0735
${ }^{64} \mathrm{zn}$ from ${ }^{65} \mathrm{Cu}(\mathrm{n}, 2 \mathrm{n})$
0.0246
${ }^{65} \mathrm{Zn}$ from ${ }^{65} \mathrm{Cu}(\mathrm{n}, \gamma)$
0.0369
Average $(\mathrm{Ni}+\mathrm{Zn}) / \mathrm{Cu}$
0.3798

than $\mathrm{Cu}$ at $14 \mathrm{MeV}$, causing the response shown in Fig. III.B-7. Several other cases were run to show that the water in the first and second walls is not responsible for the observed behavior; these cases are summarized in Table III.B-X. On the basis of these results, a clear incentive exists to operate with thinner copper first walls, particularly as breeding becomes difficult for more-realistic blanket designs (Sec. III.L.3., Appendix C.), 


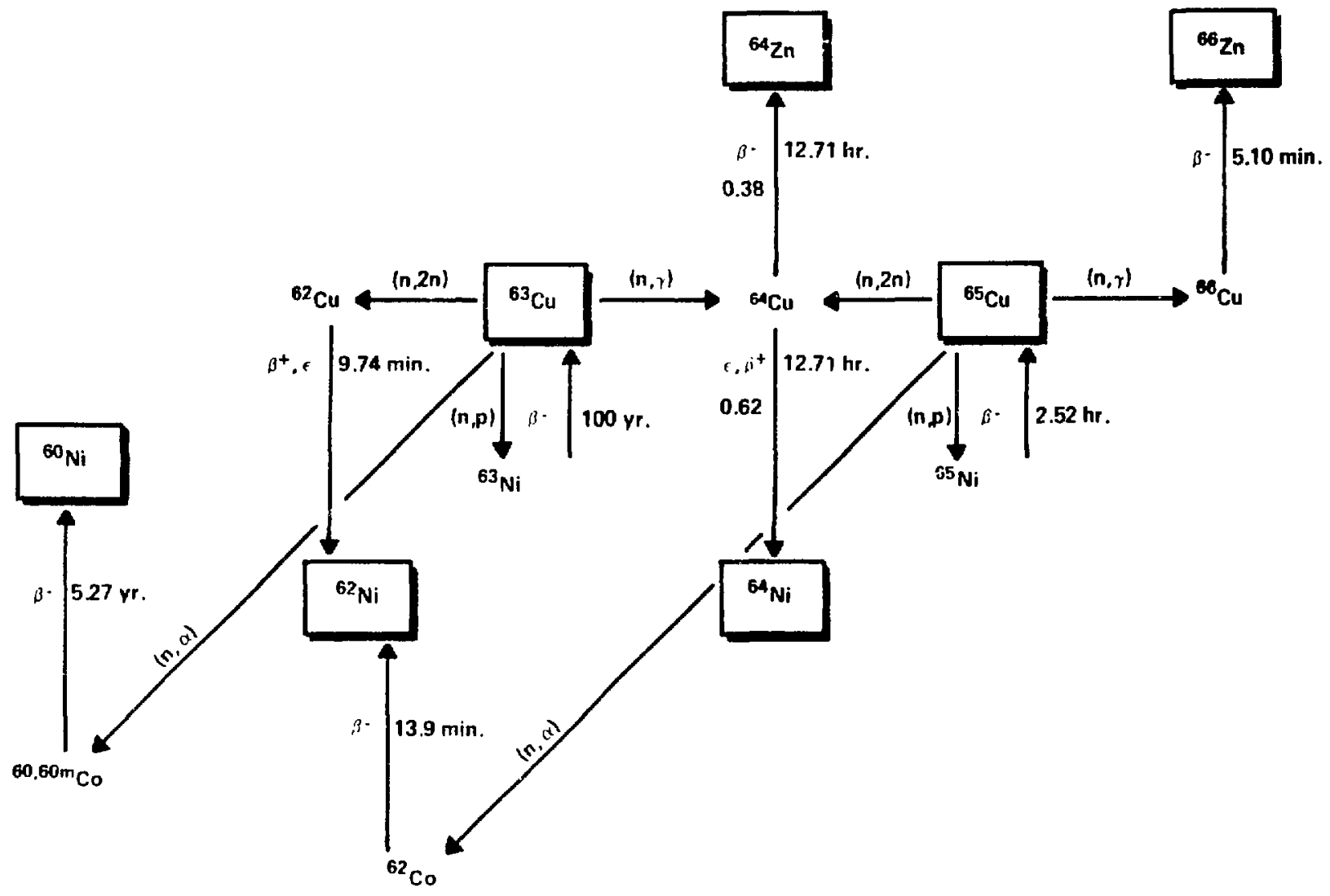

Fig. IIL.B-6. Copper activation chains (isotopes in boxes are stable).

TABLE IIL.B-IX

$6{ }^{3} \mathrm{NI}$ AND ${ }^{60} \mathrm{Co}$ PRODUCTION FROM NEUTRON ACTIVATLON OF COPPER $\left({ }^{63} \mathrm{Cu}\right)$ AFTER ON FULL-POWER YEAR $\left(I_{\mathrm{w}}=19.5 \mathrm{MW} / \mathrm{m}^{2}\right)$

CONCENTRATION

Average ${ }^{63} \mathrm{Ni} / \mathrm{Cu}$

Maximum $63_{\mathrm{iN} / \mathrm{Cu}}$

Average ${ }^{60} \mathrm{Co} / \mathrm{Cu}$

Maximum ${ }^{60} \mathrm{Co} / \mathrm{Cu}$

Average ${ }^{63} \mathrm{Ni} / 6{ }^{\circ} \mathrm{Co}$

Maximum $63_{\mathrm{Ni}} /{ }^{60} \mathrm{Co}$
FIRST WALI,

$$
4.02(10)^{-3}
$$

$4.72(10)^{-3}$

$1.07(10)^{-3}$

$1.30(10)^{-3}$

3.76

3.631
6.07

TFC

$1.18(10)^{-6}$

$2.39(10)^{-6}$

$1.96(10)^{-7}$

$3.94(10)^{-7}$

6.02

5.81
5.71

PEC

$6.68(10)^{-8}$

$4.00(10)^{-7}$

$1.17(10)^{-8}$

$6.89(10)^{-8}$ 


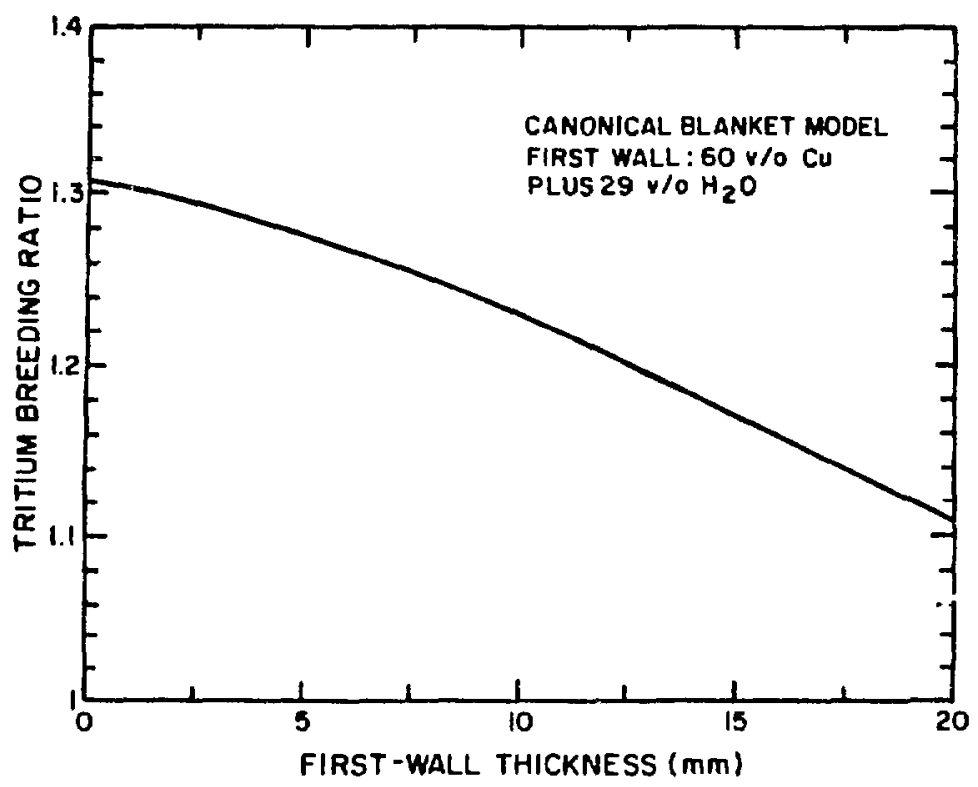

Fig. III.B-7. Effect of copper first-wall thickness on tritium-breeding ratio for the canonical design summarized in Table. III.B-V. The reference first-wall thickness is $20 \mathrm{~mm}$.

TABLE IIL.B-X

EFFECT OF COPPER FIRST-WALI, THICKNESSES AND COOLAN'T-WATER FRACTION ON CRFPR BREEDING RATIOS

First-Wa11

Thickness (mm)

Case 1

1.308

1.276

1.299

1.171

1.108 (a)

15

20

\section{Case 2}

$-$

1.238

-

1.181

\section{Case 3}

\section{Case 4}

Case 5

$-$

$-$

1.231

1.120
$-$

1.238

1.192
$-$

1.269

$-$

1.169

(a) Canonical blanket design

Case 1. Canonical blanket mode1 (Table III.B-V)

Case 2. Water removed from first wall

Case 3. Water removed from second wall

Case 4. Water removed from first and second walls

Case 5. Second wall replaced by vold. 
C. Materials Issues

This section summarizes the considerations used to select candidate first-wall ( $\mathrm{FW}$, blanket (B), shleld (S), and coll (C) materials. An overview of material requirements, thermal-mechanical constraints for the FW/B materials selection, and a synopsis of anticipated radiation effects are given.

l. Overview. The interdependence between plasma physics/engineering, reactor design, and materials science/engineering needed to achieve economic, commerclally attractive fusion power has been highlighted by a number of overview papers dealing with first walls, 88 blankets, 89 materials needs for specific devices,90,91 the world-wide materials programs addressing these needs. 92,93 The degree to which material performance requirements are altered by the needs of compact fusion reactors has been addressed recent $1 y^{20,94}$ as have the material needs for copper colls operating in a strong radiation field.95 Given acceptable plasma physics and confinement geometry, the viability of an economic compact fusion reactor will be determined by the achievable FPC engineered lifetime as measured both in terms of real time (t.e., maintenance interval and period) and radiation fluence (i.e., total amount of energy generated per mass of FPC consumed). The essential elements of this cructal question are summarized in Table III.C-I and center on a materials theme. Four major determinants of FPC lifetime are Identifled. By application of similar design and tailure criteria to all fusion approaches and assumption of negligible rate effects of radiation in changing materials properties, the FPC lifetime determinants for compact systems can be reduced from four to two: reactor operations and component geometry (size).

The major change in reactor operating conditions for the higher-power-density options is the increased heat/particle fluxes; designing to the same fallure criteria, however, should eliminate these differences, albeit potentially at a higher cost. The increased flexiblitity of reactor operations afforded by smaller EPCs, particularly with respect to the last point in Table III.C-I listed undez component geometry, potentlally can offset the added cost of designing for a more highly stressed reactor operating condition in order to assure that each unit mass of FPC delivers the economically necessary amount of energy within its lifetime. This potential for reduced mean-time-tofailure coupled with a potentially reduced mean-time-to-repair, both combinirg to give acceptable or even improved plant availability factors, is highly specific to system design (size, complexity, component geometry, etc.) and 
SUMMARY OF FPC LIFETIME DETERMINANTS

- Reactor Operating Conditions

- tirst-wall neutron loading

- jolumetric heating

-- damage rates (dpa/yr, He appm/yr, H appm/yr, burnup)

- plasma energy rejection

-- particle fluxes to in-vacuum components (DT, neutrals, He, impurities)

- di..y cycle

- coolant (kind and temperature/pressure)

- Material Properties

- thermal (heat capacity, conductivity, expansivity)

- mechanical (Young's modulus, uitimate and yield stresses, uniform elongation, total elongation, fracture toughness, creep, fatigue, crack growth, swelling)

- electrical (conductivity)

- nuclear (alloying constituents, transmutations, gas production, dpa, radioactivity, afterheat)

- surfaces (sputtering, adsorption, gas recycle, electron emission)

- Component Geometry and Constraints

- stress and temperature distributions

- component interactions/interplay

- size and degree precheck/shakeóown allowed, quality assurance, replacement/repair time

- Design and Failure Criteria

- elastic deformation and elastic instability

- plastic deformation and plastic instabilicy (incremental collapse/ratcheting)

- brittle fracture

- stress rupture/creep deformation

- high-strain/low-cycle fatigue and creep/fatigue interaction

- stress corrosion

- swelling and differential volume change

- undesirable changes in material properties (embrittlement, ductile-to-brittle transition temperature, electrical resistivity)

operation (pulsed nature, heat loads, etc.). Conslderably more work is required to relate more quantitatively the $\mathrm{COE}$ and the system power density through plant availability and operating/maintenance costs. Generally, however, the key elements of the FPC Iifetime issue (Table III.C-I) either may be comon to all fusion approaches, or the impact of more severe reactor operation may be reduced by a reactor geometry (size) that is more amenable for rapid repair. 
The key material issues and needs for fusion in general can be divided according to the following three FPC subsystems:

- In-vacuum Components (IVC) 88

- first wall

- Iimiter

- divertor (channels, plates)

- colls (feedback, current drive)

- antennae

- rf windows

- Blanket/Shield (B/S; 89

- breeder

- coolant

- structure

- multiplier

- reflector/moderator

- tritium tarrier

- ducts (rf, beams, fueling, vacuum, coolants)

- Magnet Colls (C) 95,96

- conductor (supercondictor versus resistive)

- insulator (organic versus inorganic)

- structure

- coolant [He(l) versus water]

- kinds (TF, PF, OH, EF, active feedback, passive shell, current drive)

The majority of the materials input to this study, as applied to the above three major subsystems, has been taken from the previously cited literature in confunction with the quantified needs of the CRFPR, as summarized below.

- high-heat-flux surfaces $\left(>5 \mathrm{MW} / \mathrm{m}^{2}\right)$

- high-power-density blanket $\left(>200 \mathrm{MW} / \mathrm{m}^{3}\right)$

- radiation-resistant, copper/inorganic-insulator coils $\left(>5(10)^{4} \mathrm{r} / \mathrm{s}\right)$

Ongoing, unffying studies of the kind reported in Ref. 87 will prove invaluable in providing a supporting data base in this area. 


\section{Materials Selection}

a. First Wall. The chotce of $\mathrm{FW} / \mathrm{B}$ materials used for the canonical blanket neutronics model described in Sec. ICI.B. (Fig. III.B-5) is dictater primarily by considerations of surface heat fluxes and volumetric power density. The selection of the cooling medium and the structural material for the first wall particularly is a critical step in the design. Many alternatives exist for withstanding high thermal and neutron fluxes and removing the associated heat. 88,97-99 Subcooled water was chosen as the coolant, and a high-strength, high-conductivity copper alloy was sflected as the structural material for the first wall and limiter. The MZC copper alloy 100 is a high-strength material with $0.06 \%$ magnesium, $0.15 \%$ zirconium, and $0.4 \%$ chromium by weight. As a heat-transfer fluid, water is unequaled. The use of subcooled, high-pressure water provides an additional margin of safety before burnout conditions are reached. These factors, along with the design heat-transfer coefficients and erosion, are discussed in Ref. 101 and serve as a starting point for the present design.

Bulk radiation effects and interactions between the plasma and the first wall must be accommodated by design and FPC operating mode. For example, the transmutation of copper to nickel (Table III.B-VII) reduces the thermal conductivity of the first wall while simultaneously increasling the electrical resistivity. The effect of the changing alloy struc"ure on first wall and coll performance is addressed later in this subsection. Additionally, the copper alloys have high radioactivity and afterheat at reactor shutdown. The activity of most copper alloys within one year is reduced to the point where the long-term radwaste problem is not severe. ${ }^{1 / 2}$ The afterheat at the first-wall and initer, particularly for high wall loadings, must be accomodated by either passive or active auxiliary cooling in order to minimize the chances for structural damage immediately after a shutdown.

Although copper alloys are rarely considered as structural materials for fusion reactors, they have attracted recent attention $97,98,102,103$ for use in higher-power-density systems. The criteria for selecting first-wall materials have been summarized 88 and in order of priority include resistance to radiation damage and surface effects (sputter anc chemical erosion), compatibility with coolant and with hydrogen isotopes, desirable mechanical and thermal properties (especially in the irradiated condition), and miscellaneous factors generally related to resource, avallability, and cost. A commonly accepted method for 
applying all of these criteria self-consistently to the selection process is not available, although several attempts along these lines have been made.98,99 Reactor system definition, design criteria, and materials requirements are mutually dependent and will be subject to change as the science and technology of fuston power systems evolves. High thermal conductivity is important in high-power-density systems to minimize the temperature gradients at high heat fluxes, thereby reducing therual cyclic fatigue. High strength at operating temperature and resistance to areep deformation are also important.

It is not possible to determine the relative irradiation performance of copper alloys with respect to other candidate materials, which include stainless steel, vanadium, nioblum, molybdenum, titanium, and aluminum alloys. The available data base summarized in Refs. 102-104 does not preclude copper alloy as a structural/heat-transfer material in fusion systems. The MZC copper alloy demonstrates all of the desired qualities in addition to being produced in commercial quantities, easily fabricated into the desired shapes, and relatively low cost. The latter items are favorable in 1ight of the probable need for frequent replacement of fusion reactor first walls, no matter what the material, particularly if the irradiation life is substantially less than the 10-15 MWyr/m ${ }^{2}$ presently assumed for the primary candidate alloy, stainless steel (PCASS). Copper alloys should be about as compatible with water, helium, and hydrogen as austenitic stainless steel.

A quantitative comparison between MZC copper alloy and other metals and alloys on a thermomechanical basis is useful to substantiate this matertal choice for the high-heat-flux CRFPR first wall. If the maximum temperature in the first wall or limiter is required not to exceed one-half the melting temperature, which is an optimistic criterion selected only for purposes of alloy comparison, then the maximum heat flux for the assumed conditions is only a function of the thickness, $\delta$, between the plasma and the first coolant. The results of this analysis for several representative metals are shown in Fig. III.C-1. When compared on this basis, the MZC copper alloy is superior to most common engineering metals and alloys, reflecting a favorable combination of thermal conductivity and melting point. Molybdenum and titanium alloys are also superior by this measure, but these alloys are considerably more costly. Furthermore, molybdenum is difficult to fabricate if proper precautions are not taken. Titanium, like molybdenum, is subject to hydrogen embrittlement. 


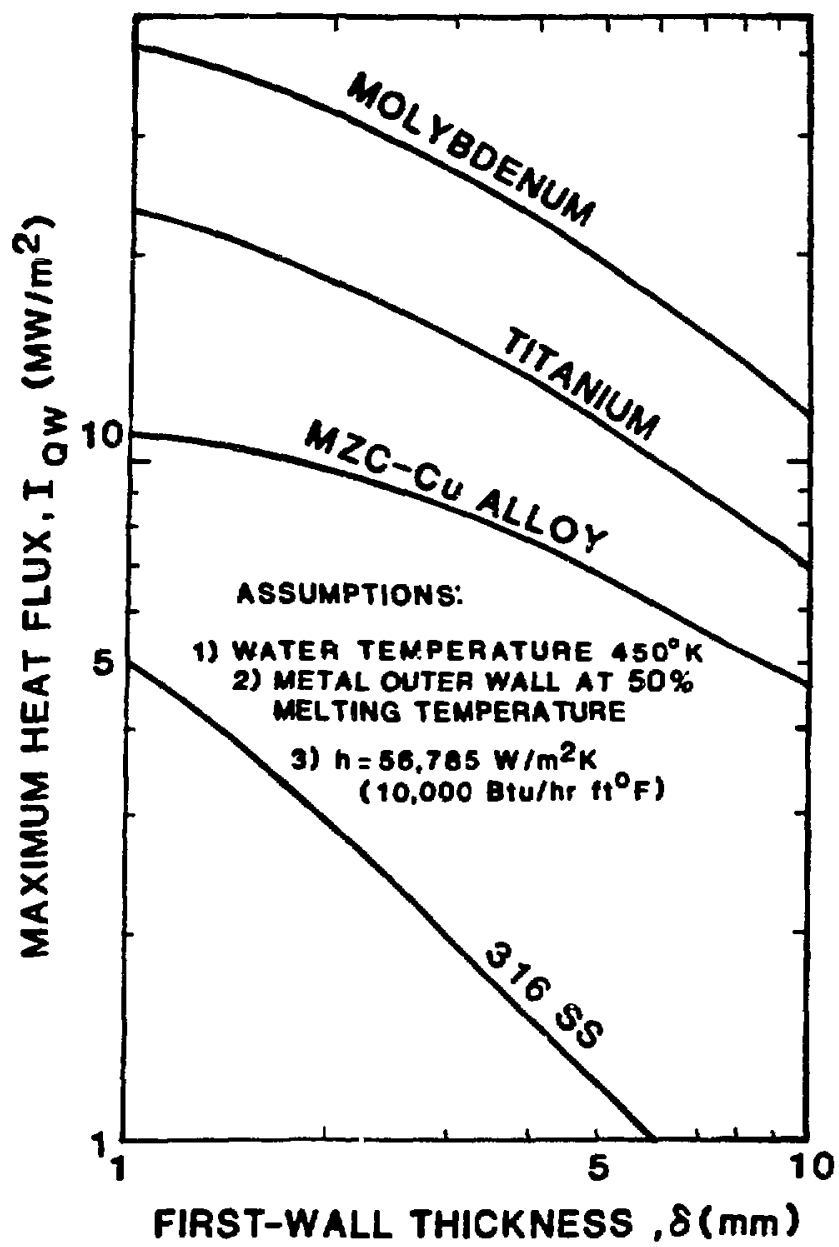

Fig. III.C-1. Heat flux for outer tube wall to achieve $50 \%$ of the melting temperature as a function of tube-wall thickness.

A measure of the ability of a material to withstand thermal stress is $g$ iven by the thermal stress parameter, $M(W / m)$ :

$$
\mathrm{M}=\frac{2 \sigma_{\mathrm{Y}} \mathrm{k}(1-v)}{\alpha \widehat{\mathrm{E}}}=\frac{\sigma_{\mathrm{Y}}}{\sigma_{\mathrm{TH}}} I_{\mathrm{QW}} \delta \text {. }
$$

In this expression, $\sigma_{\mathrm{Y}}$ is the yield strength, $k$ is the thermal conductivity, $v$ is Poisson"s ratio, $\alpha$ is the coefficient of thermal expansion, $E$ is Young "s modulus, $\sigma_{\mathrm{TH}}$ is the thermal stress, $\mathrm{I}_{\mathrm{QW}}$ is the surface heat flux, and $\delta$ is the first-wall thickness. This parameter is applicable to thin-walled tubes. The 
ratio $\sigma_{\mathrm{Y}} / \sigma_{\mathrm{TH}}$ represents a structural safety factor. If the same safety factor and the same tube-wall thickness is used for all materials, then $M$ is directly proportional to the raximum heat $f$ lux that the material can tolerate. Figure III.C-2 plote $\mathrm{M}\left(\sigma_{\mathrm{TH}}=\sigma_{\mathrm{Y}}\right)$ and illustrates that the MZC copper alloy compares favorably with other commonly employed alloys. The superior tantalum-tungsten alloy is expected to have problems related to cost, fabrication, neutronics, and corrosion.

The satisfactory thermal-mechanical ranking of the MZC copper alloy, along with low cost and ease of fabrication when compared to more exotic competitors, led to its adoption as the candidate first-wall material for the CRFPR. Fallure of the high-strength copper alloys to meet other design criteria leads to either

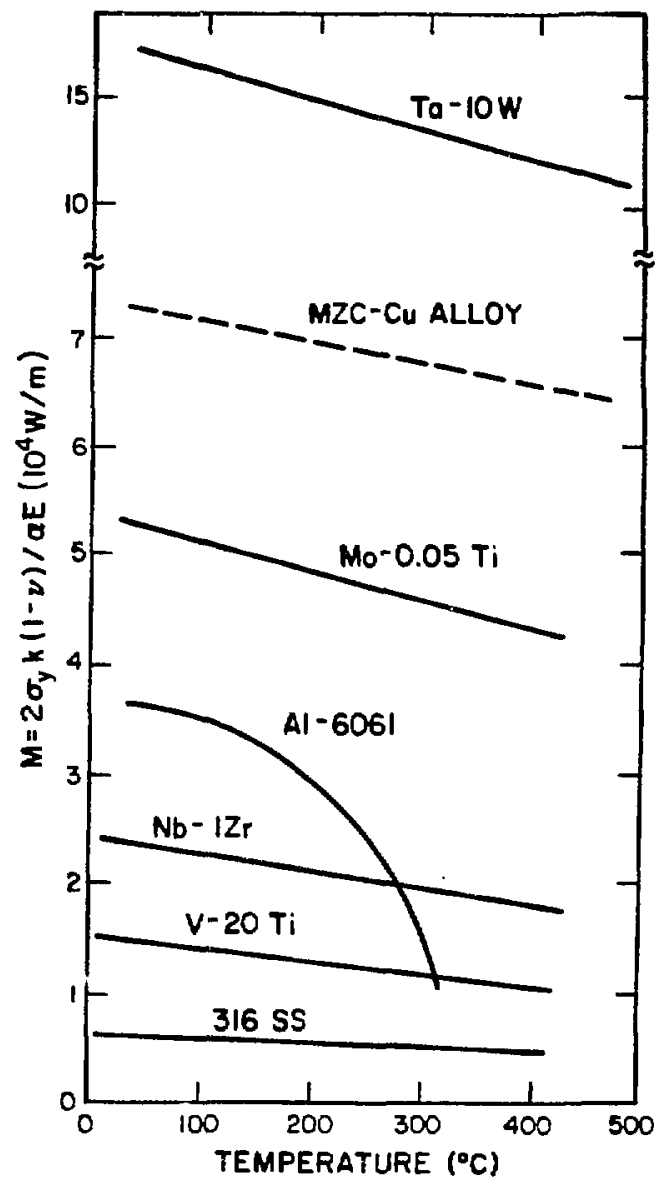

Fig. III.C-2, Stress parameter as a function of temperature for a range of alloys. This figure has been taken from Ref. 88 with the addition of the dotted curve for MZC copper alloy, based on properties reported in Ref. 100 . 
the cholce of a more exotic alloy or a reduced first-wall thermal loading. Property changes related to radiation effects, including sputtering, and nuclear transmutation represent other cruclal selection criteria remaining to be evaluated.

The response of IVC surfaces to high heat and particle fluxes is undoubtedly the primary engineering problem for both compact and other approaches to fusion. The partition of energy between particles versus photons, as well as between $\mathrm{FW}$, limiter, and/or divertor, further defines this crucial uncertainty. The major materials questions for the IVCs are

- Removal of both surface $\left(<\mathrm{I}_{\mathrm{W}} / 4 \mathrm{MW} / \mathrm{m}^{2}\right)$ and volumetric $\left(\sim 10 \mathrm{I}_{\mathrm{W}} \mathrm{MW} / \mathrm{m}^{3}\right)$ heat loads within acceptable temperature, stress, pumping power, coolant pressures, and critical-heat-flux limits.

- Sputter erosion and redeposition rates for $\mathrm{FW}$ and 1 imiter surfaces.

- Long-term (swelling, creep, embrittlement, alloy changes, etc.) and short-term (thermal conductivity changes, hydrogen permeation and recycle, erc.) radiation effects.

Although copper sputters at about triple the rate of PCASS, the active sputter-erosion rate depends on poorly understood edge-plasma and redeposition conditions. From the viewpoint of first-wall survivability, these problems are not unique to or more severe for the compact reactors. Aside from differences in basic plasma processes that may result when differences of $\sim$ 3-4 in average plasma density are taken into account, the ratio of particle flux to neutron current incident onto a first wall frou an ignited DT plasma should be similar for both compact and other systems, thereby decoupling. somewhat the first-wall erosion problem froin the issue of device compactness; the compact FPC simply achieves both neutron (dpa) and exosion ( $\mathrm{mm}$ ) lifetime "fluences" in an expected shorter chronological lifetime, but only after generating a similar total quantity of fusion energy for nominally a similar expenditure of first-wall/blanket mass and cost.

The long-term effects of radiation on copper alloy are not well understood either experimentally or theoretically. An estimate of the effects of neutron irradiation on a copper-alloy first wall, and possibly an inorganic electrical 
Insulation if first-wall colls or electrical breaks are required, has been summarized in Ref. 37 and more recently for the first-wall copper-coll insert proposed in MARS. 105

b. Blanket/Shield. The blanket and shleld thickness for the compact reactor approaches is reduced to the minimum required for adequate tritium breeding and thermal energy recovery. The mintmum-thickness (optimized) blanket/shleld can also provide appreciably different opitins for FPC installation and maintenance schemes if the mass and volume are sufficiently reduced. Magnet shielding in the usual sense is not envisaged; instead a thin $(0.10-\mathrm{m})$ outer region of the $0.5-\mathrm{m}-$ thick blanket may contain a mixture of $\mathrm{B}_{4} \mathrm{C}$ and a dense, high-Z material (1.e., steel, tungsten) operated at or near the blanket temper: :ure (Table III.B-III).

For first-wall neutron loadings in the $15-20 \mathrm{MW} / \mathrm{al}^{2}$ range, the local power density becomes comparable with that in the fuel of an LWR $\left(>200 \mathrm{MW} / \mathrm{m}^{3}\right)$, with the average blanket power density also being compattble to that of an LWR core, $\left(30-50 \mathrm{MW} / \mathrm{m}^{3}\right)$. At these power densities ceramic tritium breeders cooled by pressurized helium gas or water become less attractive. Because of the low lithlum inventory, reduced fire hazard, and unique combiration of breeder/coolant/multiplier functions, the low-melting $\left(235^{\circ} \mathrm{C}\right)$ lead-1ithium compound $\mathrm{Pb}_{83} \mathrm{LI}_{17}$, referred to hereafter as "PbL1," has emerged as a popular cholce for standard 106 and high-power-density ${ }^{86}$ blankets. Use of a second flutd $\left(\mathrm{H}_{2} \mathrm{O}\right.$, He) to $\mathrm{cool}$ a stagnant liquid-metal blanket requires a large number of high-pressure coolant tubes, making tritium breeding more difficult and reducing B/S rellability.

The self-cooled PbLI blankets, however, must design around the inevitable MHD pressure drop. Confinement systems with magnetic topologies that require liquid-metal coolant to flow long distances across strong magnetic flelds may be forced either to coat coolant ducts with electrical insulators 86 or to reduce the MHD pressure drop siaply by limiting the coolant flow velocity and thereby

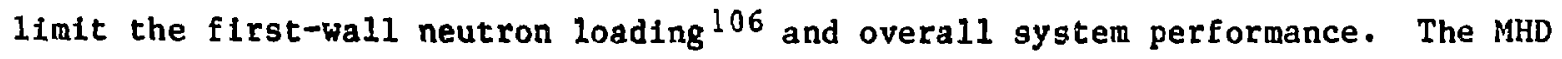
pressure drop mantests itself both in large pumping powers (>1-2\% of thermal power removed) and the need for pressurized liquid-metal blankets ( $>1-2 \mathrm{MPa})$, which in turn causes increased membrane stresses in an already corrosive and chemically reactive blanket. The high power density for the PbLi-cooled CRFPR blanket, as described in Sec. III.E., can be achieved with winimal pumping power without recourse to the use of electrically insulated coolant ducts 
because of the unique, low-field poloidal topology; the blanket overpressure actually becomes a limiting factor for the CRFPR design.

The MHD pressure drop that accompanies the need for a flow velocity that is sufficlent to achieve a given liquid-metal/structural metal interfacial temperature, as established for a given alloy by corrosion limite, may or may not be greater for the higher-power-density blankets; the higher coolant velocities, $v$, can be achieved with smaller total flow lengths, $\ell$, for efther increased or decreased field, $B$, depending on plasma beta and confinement geometry (i.e., $\triangle \mathrm{P}_{\mathrm{MHD}} \propto \mathrm{B}^{2} \mathbf{v}$ for a given coolant duct configuration). The coupling of $\mathrm{B} / \mathrm{S}$ power density, MHD pressure and pumping power, and corrosion (temperature) limits, therefore, is strongly dependent on design and concept. Furthermore, the basis upon which specific corrosion, and, hence, MHD pressure and pumping-power, limits are established for a given structural alloy requires further examination (i.e., duct wall thinning, alloy embrittlement, down-stream plugging, radioactivity transport, etc.). The materials problems related to corrosion (particularly for ceramic coatings), tritium recovery, and tritium barriers for the compact reactors remain similar to those for other systems using similar blankets. The acceleration of stress corrosion cracking by the addition of small amounts of water to these liquid-metal systems renains a particularly critical concern, however.

c. Magnets. Water-cooled copper colls located outside the thin, $\sim 0.6-\mathrm{m}$ high-power-density blanket will be required to operate in a strong radiation field (Sec.III.B.). Radiation-resistant inorganic electrical insulation will be required. Either insulator coatings would be plasma-sprayed onto preformed copper conductors, or a powdered insulation (i.e., $\mathrm{MgO}$ or $\mathrm{MgAl}_{2} \mathrm{O}_{4}$ ) would be coextruded with conductor and coolant tube, the latter method being used in the fabrication of radiation-hardened colls for use in high-energy particle accelerators. 107,108 The requirement for first-wall (feedback or current-drive) coils should be similar to the requirements envisaged for the MARS hybrid-magnet insert 105,109 or for the less severe cokamak conditions anticipated at inblanket equilibrium-fleld coils. The crucial issue of radiation damage to either exoblanket or $\mathrm{FW}$ coll is discussed in the following subsection.

3. Radiation Effects. The PbLi-cooled FPC design proposed for the CRFPR uses a 20-mm-thick copper-alloy first-wall and exoblanket colls. The results of neutronic computations given in Sec. III.B. based on this $\sim 0.6-$ m-thick canonical blanket/shield (Fig. III.B-5) can be used to assess systems that have 
TABLE III.C-II

IMPLICATIONS OF RADIATION EFFECTS FOR

COMPACT REACTOR "MODEL" FW/B/C (FIG. III.B-5)

- FIRST WALl $\left(\mathrm{Cu} / \mathrm{H}_{2} \mathrm{O}\right)$

- 14.1-MeV neutron current, $J_{w}\left(n / m^{2} s\right)=4.45(10)^{17} I_{w} 8.9(10)^{18}$

- Neutron flux, $\phi_{w}\left(\mathrm{n} / \mathrm{m}^{2} s\right) \approx 3.3(10)^{18} I_{w}$

- Total FPY $(a)$ fluence, $\phi_{w} \tau\left(n / m^{2}\right)=1.05(10)^{26} I_{w} \quad 2.1(10)^{27}$

- Radiation dose rate, $R$ neutrons, $R_{n}(\mathrm{rad} / \mathrm{s})=8.2(10)^{4} I_{w}$ gammas, $R_{\gamma}(\mathrm{rad} / \mathrm{s})=1.3(10)^{5} \mathrm{I}_{\mathrm{w}}$

- Peak (average) dpa/yr $\simeq 16 \mathrm{I}$

- Peak (average) He appm/yr $\simeq 90 I_{w}$

- Peak (average) $H$ appm/yr $=260 I_{w}$

- Average transmutation rates

$\mathrm{N1} / \mathrm{Cu}(\% / \mathrm{Yr})=0.25 \mathrm{I}_{\mathrm{w}} \quad 4.9$ $\mathrm{Zn} / \mathrm{Cu}(\% / \mathrm{yr})=0.14 \mathrm{I}_{\mathrm{w}}^{\mathrm{w}} \quad 2.7$

- Heat flux, $I_{Q}\left(M W / m^{2}\right) \leqslant I_{w} / 4 \quad 5.0$

- Average power density, $Q_{w}\left(M W / \mathrm{m}^{3}\right) \simeq 11 I_{w} \quad 224$

- BLANKET/SHIELD $\left(\triangle \mathrm{b}=0.6 \mathrm{~m}, \mathrm{PbL} i / \mathrm{PCASS} / \mathrm{B}_{4} \mathrm{C} / \mathrm{W}\right)$

- Peak power density, $Q_{B}\left(M N / m^{3}\right)=13 I_{W}$

- Average power density, $\left\langle Q_{B}\right\rangle\left(\mathrm{MH} / \mathrm{m}^{3}\right)=1.4 I_{w}$

- Average PCASS dpa/yr $=23^{\mathrm{I}} \mathrm{I}$

- Average PCASS He appm/yr $=y_{8} I_{w}$

- Average PCASS $\mathrm{H}$ appm/yr $=27 \mathrm{I}_{\mathrm{w}}$

$1.64(10)^{6}$

$2.67(10)^{6}$

$318(282)$

$1795(1480)$

$5149(4370)$

262 ( $P b L i$ coolant)

28

47

155

533

- EXOBLANKET (TFC) COIL (Cu/PCASS $/ \mathrm{H}_{2} \mathrm{O}$ )

- Peak neutron flux, $\phi_{C}\left(n / m^{2} s\right)=4.0(10)^{16} I_{w} \quad 7.9(10)^{17}$ neutrons, $R_{n}(\mathrm{rad} / \mathrm{s})=1.3(10)^{2} I_{w}$
gammas, $R_{\gamma}(\mathrm{rad} / \mathrm{s})=1.1(10)^{3} I_{w}$

- Peak Cu dpa $Y_{\text {yr }}=0.063 I_{\text {. }}$

- Peak Cu He appm/yr $=0.027 I_{w}$

- Peak Cu H appm/yr $=0.13 \mathrm{I}_{\mathrm{w}}$

- Peak PCASS dpa/yr $=0.004 \mathrm{I}_{\mathrm{H}}$

- Peak PCASS He appm/yr $=0.003 \mathrm{I}_{\mathrm{w}}$

- Peak PCASS H appm/yr $=0.113 \mathrm{I}_{\mathrm{w}}$

- Average Cu transmatation rates

$2.56(10)^{3}$

$2.26(10)^{4}$

1.25

0.537

2.65

0.801

0.61

2.25

$\mathrm{Ni} / \mathrm{Cu}(\% / \mathrm{yr})=9.2(10)^{-4} \mathrm{I}_{\mathrm{w}} \quad 0.0184$

$\mathrm{Zn} / \mathrm{Cu}(\% / \mathrm{yr})=9.3(10)^{-3} \mathrm{I}_{\mathrm{w}} \quad 0.0019$

- Peak power density, $Q_{c}\left(M W / m^{3}\right)=0.1 I_{w} \quad 2.0$ (nuclear)

0.8 (ohmic)

$\overline{(a)}$ FPY $=$ Ful1-power year.

either first-wal1 18,19 or exoblanket colls. Table III.C-II summarizes the key neutronic responses per unit of first-wall neutron loading as well as responses that would be typical of a $20-\mathrm{MW} / \mathrm{m}^{2}$ neutron loading. These results can be used 
to project the FPC performance in terms of pacing materials technology issues if sufficlent radiation effects information were available. Present understanding of the copper alloys and inorganic insulators, however, permits only inferences to be made. For instance, the swelling of candidate electrical insulator materials, $110 \mathrm{MgO}$ and $\mathrm{MgAl}_{2} \mathrm{O}_{4}$, has been measured after near-room-temperature Irradiation to fluences of $2.1(10)^{26} \mathrm{n} / \mathrm{m}^{2}$ and neutron energies above $0.1 \mathrm{MeV}$ $\left(2.8\right.$ and $0.8 \mathrm{v} / 0$, respectively). For a first-wall neutron loading of $20 \mathrm{MH} / \mathrm{m}^{2}$ extrapolation would predict swelling of $11 \mathrm{v} / 0$ per year for $\mathrm{MgAl}_{2} \mathrm{O}_{4}$ at the $\mathrm{fi}$. wall if saturation did not occur. For the same material and first-wall fll. Interposition of a $0.6-\mathrm{m}-$ thick $\mathrm{PbLi}$ blanket would reduce the predicted insulator swelling to $0.09 \mathrm{v} / 0$ per year. Swelling saturation is likely, however, and would greatly decrease the high-fluence swelling while possibly increasing this value somewhat at low fluence.

The electrical conductivity of many inorganic electrical insulators under irradiation is increased by roughly an order of magnitude for every order of magnitude increase in dose rate. 110 For the relatively low-voltage applications envisaged for the CRFPR, however, the increased leakage current and ohmic loss should be tolerable in rost cases, although more-detailed coll designs are required to assess fully this potential problem. For sufficiently high voltages () $700 \mathrm{~V}$ ) and instantaneous radiation dose rates $\left(>10^{4} \mathrm{~Gy} / \mathrm{s}=10^{6} \mathrm{rad} / \mathrm{s}\right)$, thermal runaway through ohmic heating potentially can be destructive to electrical insulators; ${ }^{i l l}$ these conditions generally apply near the first wall and for relatively high-field, actively driven coils. 105

Increases in the electrical resistivity of first-wall or exoblanket copper colls are anticipated from the introduction of point lactice defects (i.e., vacancies and interstitials), dislocations, voids, trismutation-induced impurities, and magnetoresistivity effects. The contribution of point defects to the enhanced electrical resistivity of copper is expected to saturate at $\sim 0.0034 \mu \Omega-m$ at $310 \mathrm{~K} .112$ This contribution to the increased resistivity will saturate at a considerably lower value at elevated temperatures because of the reduction in point-defect content. Since the starting resistivity is $\sim 0.02-0.04 \mu \Omega-m$, the effect of point defects on increased resistivity should be smal1.

A high dislocation density in the copper conductor may result fiom plastic deformation or from the formation of radiation-indused dislocation loops. Even for a density of $10^{16}$ dislocations/m ${ }^{2}$, which 1 s unlikely to be sustained at 
operating first-wall temperatures, the resistivity would be increased by only a few percent.113 The resistivity contribution from this source, therefore, is expected to be insignificant. Furthermore, volds or large defect aggregates should not have an important effect on resistivity. Similarly, the resistivity contribution from magnetoresistivity for magnetic fields in the range 2-3 $T$ and temperatures of $400-600 \mathrm{~K}$ is estimated to be a few percent at most and more likely will be less than one percent.

The high first-wall neutron flux will. generate a significant concentration of metallic impurities through $(n, p),(n, \gamma)$, and $(n, 2 n)$ reactions (Sec. III.B.). Similarly, transmutation of exoblanket coll conductor will also occur but at a considerably reduced level. Both $\mathrm{N} 1$ and $\mathrm{Zn}$ impurities will be generated (Table III.B-VII). Assuming the formation of only the former element, which will have the greater effect on the electrical resistivity, the predicted resistivity increase will be slightly exaggerated. The rate of impurity formation would be $\sim 5.0 \%$ per year at the first wall and is reduced to $0.02 \%$ per year outside a $0.6-\mathrm{m}$-thick $\mathrm{B} / \mathrm{S}$ for a first-wall neutron loadius of $20 \mathrm{MW} / \mathrm{m}^{2}$. Using two sets of data for Cl:-N1 alloy, 114 the (average) resistivity increases at a first-wall or exoblanket coll would be $100-2.00 \%$ per year and $0.7-1.4 \%$ per year, respectively. Both the insulator swelling and transmutation-related resistivity increases in a first-wall copper coil will require coll changeouts more frequently than once a year ( 3 times a year for OHTE ${ }^{18,86}$ and 12 times a year for Riggatron ${ }^{20}$ ). The dramatic decrease in radiation effects when a $\sim 0.6-\mathrm{m}$-thick $\mathrm{B} / \mathrm{S}$ is interposed between the plasma and coil points to significant benefits of locating even a thin $(0.10-0.15 \mathrm{~m})$ neucron-absorbing/moderating region between the plasma and the coil; the OHTE reactor design 18 in fact is pursuing this approach.

Since thermal conduction in copper takes place primarily by the motion of electrons, an increase in electrical resistivity w111 also result in a decrease in thermal conductivity. To a first approximation the changes in electrical and thermal resistivities may be assumed to be proportional 115 (Wledimann-Franz law). Consequently, changes in the thermal propertles of a copper-alloy first wall are expected over the FW/B lifetime, perhaps leading to higher temperatures, greater thermal gradients, and increased stresses as end of life is approached. The key transmutation products are $\mathrm{Ni}$ and $\mathrm{Zn}$, and $\mathrm{Co}$ to a lesse:: extent. The increased electrical resistivity 114 for additions of $N i$ to copper amounts to $\rho / \rho_{0} \simeq 1+0.8 f_{N 1}$ for $f_{N i} \leqslant 20$ wt $\%$. The effectiveness of $Z n$ In 
Increasing $\rho$ is 0.32 times that of $\mathrm{Ni}$ (1.e., $\rho / \rho_{0} \approx 1+0.26 \mathrm{f}_{\mathrm{Zn}}$; the $\mathrm{N} 1$ and $\mathrm{Zn}$ impurtites bulld into pure copper at rates of 0.24 and 0.13 w/o per year, respectively, for each untt of fusion-neutron first-wall loading, (Table III.j-VIII). Although the effectiveness of Co in increasing pure-copper resistivity is 5.2 times that of $\mathrm{Ni}$, Co is generated only at $4 \%$ the $\mathrm{Ni}$ rate and, therefore, can be ignored as a contribution to resistivity increase. Taking the upper value for the $\mathrm{N} / \mathrm{Zn}$ generation rate and weighting in accordance to the relative effect on resistivity increase gives the following approximate expression for $\rho$ as a function of $I_{w}\left(M W / m^{2}\right)$ and exposure time, $\tau(y r)$ :

$$
\rho \simeq \rho_{0}\left[1+0.23 I_{W} \tau\right]
$$

The Weldemann-Franz law, 115 (1.e., $k \rho / T \simeq 2.5(11)^{-8} \mathrm{~W}$ ohm $/ \mathrm{K}^{2}$ ) can be used to relate the increased resistivity, - to the decreased thermal conductivity. Hence,

$$
k=k_{0} /\left[1+0.23 I_{w} \tau\right]
$$

The most immediate result of a lower value of $k$ is a higher temperature drop through the coolant-tube wall, leading to increased thermal stresses and cyclic strain. For the base-case value of $I_{w} \tau=15 \mathrm{MWyr} / \mathrm{m}^{2}$, a decrease in $\mathrm{k}$ by as much as a factor of four may be possible, this value representing an upper value depending on whether the transmutation products added to thr crystal matrix are incorporated at grain boundaries or form a second phase.

Electrolytic tough-pitch copper (standard electrical wire grade) contains $\mathrm{Cu}_{2} \mathrm{O}$. Heating of this metal in hydrogen above $\sim 775 \mathrm{~K}$ results in internal formation of steam which causes embrittlement.116 Maximum first-wall temperatures envisaged for most compact systems are sufficiently low to avold this problem by the generation of molecular hydrogen. The presence of atomic hydrogen 1sotopes at the first-wall surface and the generation of transmutationinduced hydrogen within the lattice (Table III.C-II), however, may result in embrittlement at the operating temperature. It may be desirable to specify oxygen-free high-conductivity copper for this application, although this would 
not be fully consistent with the use of a dispersion-hardened, high-strength copper alloy.

Irradiation-induced swelling of copper occurs in the temperature range between $\sim 500$ and $825 \mathrm{~K}$, depending on the bombarding particle, damage rate, damage level, and gas content of the metal. 117 For "gassy" copper subjected to neutron irradiation at damage rates of $6(10)^{-7} \mathrm{dpa} / \mathrm{s}(18.8 \mathrm{dpa} / \mathrm{yr})$, the swelling range is shifted to $\sim 500-775 \mathrm{~K}$, with a maximum occuring at $625 \mathrm{~K}$. The Initial neutron-induced swelling rate at $775 \mathrm{~K}$ corresponds to $\sim 0.4 \mathrm{v} / \mathrm{o}$ per dpa.98 A copper first wall may have a high gas content because of transmutation-induced $\mathrm{H}$ and $\mathrm{He}$ and is expected to experience a damage rate that is greater than this value. A high inftial swelling rate, therefore, is Indicated, but saturation may occur, and the effects of alloying on swelling are not known. Possible constraint by a stainless-steel structure backing the copper first wall may also be considered. Temperature and neutron flux differences between Inner and outer first-wa11 surfaces, as we11 as throughout the steel blanket structure, will also result in spatial varlations of the swelling rate through the thickness. Some variation may also result from different displacement and gas generation rates at inner and outer surfaces. These separate effects remain to be integrated into a composite estimate of first-wall life based on a detailed engineering design. Uitimately, reliable materials models of an Integrated nature must be used to set the first-wall and blanket operating temperature. Little or no data are available for copper on the effect of alloying, neutron fluence (above $\sim 1 \mathrm{dpa}$ ), and temperature except for pure copper for damage levels < $1 \mathrm{dpa}$. Irradiation tests for these alloys in a fast spectrum, however, should be available soon.

Irradiation damage often results in strengthening and embrittlement of metals as a consequence of microstructural changes. Copper is strengthened by irradiation, at least up to $411 \mathrm{~K}, 116$ and, although experimental results showing embrittlement or decreased stress-rupture lifetime for this metal are not avallable, these effects are likely consequences of the formation of a damage microstructure. At temperatures below approximately half the melting polnt and in fast-neutron fluxes greater than $10^{17} \mathrm{n} / \mathrm{m}^{2} \mathrm{~s}$, metals typically show an enhanced creep rate compared to that observed for the unirradiated material. 118 This enhanced creep results from the generation of point defects during irradiation. Since a copper first wall most desirably would operate from $40 \%$ to $45 \%$ the absolute melting temperature in a high fast-neutron $f 1 u x$, 
accelerated creep can be expected. The interaction of enhanced creep and embrittlement processes in a high-radiation-flux first wall is largely unknown, however, particularly for solution-hardened alloys. Age-hardened copper alloys such as MZC, however, may overage or the alloying element may dissolve under irradiation; generally, dispersion-hardened alloys may exhibit greater radiation stability in this respect, although the impact of atomic hydrogen within the intrix on alloy stability is not known.

4. Summary. The compact reactor approaches will require materials development that in some areas differ in emphasis from the mainilne approaches. The greatest need for materials development rests with the high-heat-flux IVCs (FW, 1imiters, divertors). Given that IVCs can be designed and operated with 4-5 MW/m ${ }^{2}$ average heat fluxes, the critical areas reduce to a) the partition of radiation versus particle flux incident upon IVC surfaces; b) the associated sputter-erosion rate; c) the redeposition processes (location and integrity); and d) the impact on the overall plasma performance of potentially large transfers of impurities around the system. The problems related to sputter erosion, however, in magnitude and kind, are not unique to compact reactors. Even if sputtering rates are increased for the compact systems, given similar plasma and edge-plasma physics, the amount of sputter per neutron fluence $\left[\mathrm{mm} /\left(\mathrm{MWyr} / \mathrm{m}^{2}\right)\right.$ ] should be independent of the concept and simply becomes a matter of "fluence." Hence, the potentially unique materials problems for compact systems are related to the need to understand and control the bulk mechanical radiation damage properties of the new materials (copper or vanadiun alloys) required to deal with the increased heat fluxes. Even then, such materials may be used in pumper limiters and/or divertor plates for the larger superconducting fusion systems.

The compact reactor option narrows the many B/S choices listed in Ref. 87 to a few concepts that can operate at local and average power densities considered economically necessary for other nuclear power systems. The functional integration and design simplicity of the self-cooled PbLi blanket gives a strong attraction to this $B / S$ concept for application to compact, highpower-density fusion reactors. Extension of liquid metals for first-wall cooling, the level of MHD pressure drop and pumping power incurred within the blanket, and the impact of blanket structure corrosion limits on flow geometry and alloy selection, however, are sensitive to both design and confinement concept. The magnet development required to produce relatively small, 
radiation-hardened resistive colls appears to be we11 advanced, 107,108 albelt on a reduced scale. Generally, for both $B / S$ and magnets, the materials requirements for the compact options appear no more difficult, and in many respects may be easier, than the malnilne program needs. An increased emphasis, however, must be placed on understanding creep, fatigue, fatigue-creep Interaction, alloy stability, and coolant-alloy interaction for the high-heatflux materials. 
D. In-Vacuum Components (First Wall and Limiters)

1. General Thermal-Mechanical Considerat1ons. Section III.C.1. describes the first-wall materials selection criteria based primarily on generalized thermal-mechanical considerations. The feastbility of the compact RFP reactor depends on a practical first-wall and limiter design that is capable of withstanding the neutron, thermal, and particle fluxes from the high-powerdensity plasma. Most of the neutron flux passes through the first wall and is absorbed in a lithium-bearing blanket, but the thermal and particle fluxes are absorbed entirely by the first-wall and limiter surfaces. In addition, for every $1 \mathrm{MW} / \mathrm{m}^{2}$ of fusion neutrons incident upon the first wall, typically $\sim 10 \mathrm{MW} / \mathrm{m}^{3}$ will be deposited by neutron/gamma-ray volumetric heating within the first-wall structure; this heat also must be removed by the First-wall and limiter coolant circults. The first-wall thermal-mechanical response was judged early in the CRFPR study as a limiting engineering constraint. The work reported in Ré̃. 101 was performed early in this study to provide key thermohydraulic and fatigue input to the CRFPR design-point determination. It was on the basis of cost estimates (Ref. 15 and Sec. IV.C.), supported by these early thermal-mechanical analyses, that the $I_{W}=20-\mathrm{MW} / \mathrm{m}^{2}$ design point was selected.

Sections III.H. and III.I. give preliminary results of the more-serious but related problem of particle control. In addition to determining the heat load on the first wall and other in-vacuum-component (IVC) surfaces, the unresolved issue of particle control also impinges directly on the length and form of the thermonuclear burn cycle (Sec. III.G.); these factors in turn determine peak-to-average heat loads, cycle frequency and durations, and ultimately the cyclic fatigue lifetimes for the cruclal first-wallilimiter system. This section describes a thermal-hydraulic analysis for a first wall subjected to the full, average heat load emanating from the plasma. The analyses presented herein are also applied to a sample limiter surface suggested by the analyses given in Sec. III.H. Because of the anticlpated high first-wall coverage, the limiter surface would operate somewhat above the average flrst-wall heat load. Section III.H., particularly, focuses on particle-control aspects of the pumped $11 \mathrm{miter}$ and an estimate of limiter shape for a given design heat flux. The power split between first-wall, limiter, blanket, and other systems, and the means by which these various power streams 
are integrated and used to drive an effictent thermal cycle is described in Sec.III.L.

The first-wall thermal-mechantcal study reported in Ref. 101 conservatively adopted the burn cycle developed for the earlier superconducting RFP reactor 53 design, which also used a copper-alloy first wall. This latter burn cycle was adjusted for the higher steady-state flrst-wall loading gredicted by the earlier CRFPR parametric study.15 The first wall for this pulsed-burn case would be exposed to a time-averaged thermal flux of $I_{\mathrm{QW}}=4.9 \mathrm{MW} / \mathrm{m}^{2}$ with a transient in the cycle of $8.9 \mathrm{MW} / \mathrm{m}^{2}$. The peak-to-average heat flux can be considerably reduced, although the more-severe conditions examined in Ref. 101 admitted a fatigue life for a water-cooled, copper-alloy first wall that was $10^{6}$ cycles in one year of operation. To give some perspective to the heat-flux requirements projected CRFPR, a compilation of heat fluxes normally encountered in science and Industry as well as for specific fusion applications is given in Fig. III.D-1. The average heat fluxes for this CRFPR design are somewhat higher than in typical process equipment, but these heat ratings are approximately two orders of magnitude less than the highest rates reported in the 1iterature. For cyclic batch-burn operation, the service life based on radiation damage limits in the $I_{w} \simeq 20-\mathrm{MW} / \mathrm{m}^{2}$ case is projected to be also on the order of one year. The CRFPR operation is presently envisaged to be longer pulsed, if not steady state, the issue of steady-state current drive being addressed in Sec. III.K.

The criteria used in Sec. III.C.2.a. for materials selection does not specify a design. To carry this design further, the selection of the principal design variables (i.e., the coolant-channel dimensions, the wall thickness, and the configuration of a heat-transfer module) must be made. A simple coolanttube geometry is assumed. The primary (tensile) hoop stress, $\sigma_{p}$, in a tube subjected to internal coolant pressure, $P_{C}$, is given by

$$
\frac{\sigma_{p}}{\mathbf{P}_{c}}=\frac{\xi+1}{\xi-1} \text {, }
$$

where $\xi=(1+2 \delta / d)^{2}$ is the square of the ratio of outer tube diameter to inner tube diameter, $d$, and $\delta$ is the tube-wall thickness. The secondary thermal stress for a temperature difference $\Delta \mathrm{T}_{\mathrm{w}}$ across the tube wall is given by 


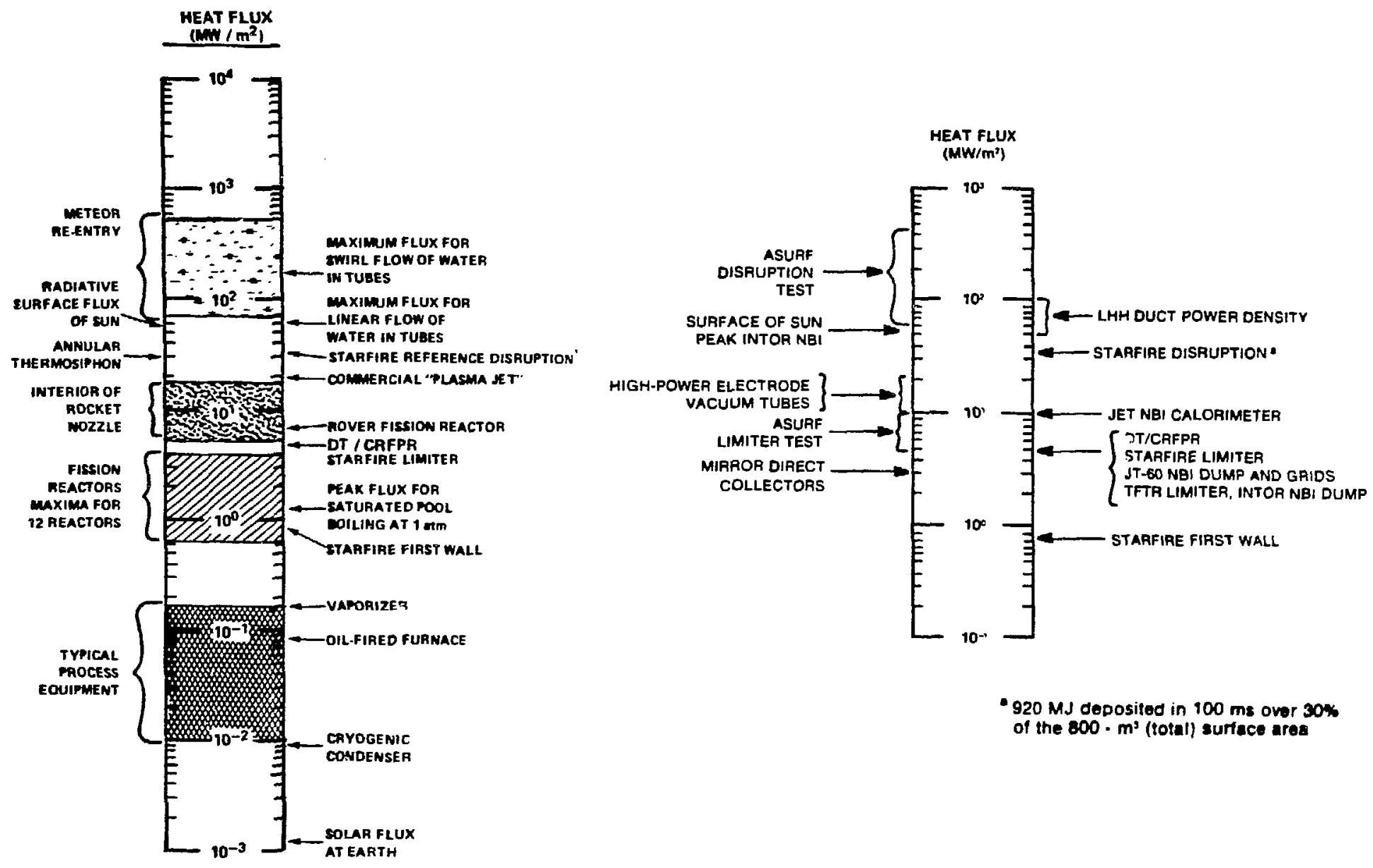

Fig. III.D-1. Comparison of heat fluxes for MFE and other systems. This figure extends a previous compilation, 119 where the magnitude of the heat flux is that transferred to the coolant. The compilation does not consider the enormous transient heat fluxes that can be generated for brief periods, for example, in shock tubes, but the heat is not transferred to a coolant. 


$$
\begin{aligned}
& \alpha_{\mathrm{TH}}=\frac{\alpha E \Delta T_{w}}{2(1-v)} f(\xi) \\
& f(\xi)=2 \frac{1-(\ln \xi) /(\xi-1)}{\ln \xi} .
\end{aligned}
$$

Combining the secondary (thermal) and primary (pressure) stresses for a thinwall tube leads to the following expression for the total stress, $\sigma=\sigma_{\mathrm{p}}+\sigma_{\mathrm{TH}}$ :

$$
\begin{aligned}
\sigma & =\frac{P_{c} d}{2 \delta}+\frac{\alpha E I_{Q w} \delta}{2 k(1-v)} \\
& =\frac{P_{c} d}{2 \delta}+\frac{\sigma_{Y} I_{Q w} \delta}{M},
\end{aligned}
$$

where $M=2 \sigma_{\mathrm{Y}} \mathrm{k}(1-\nu) / \alpha \mathrm{E}$ is the thermal stress parameter given in Fig. III.C-2, $\sigma_{\mathrm{Y}}$ is the yield scress, $I_{Q w}$ is the heat flux, and $\xi \rightarrow 1$ for the approximation of a thin-walled tube.

As an example, the nominal stress in a 6-mm-diameter MZC copper-alloy tube is given as a function of $\delta$ and $I_{Q w}$ in Fig. III.D-2 for an internal coolant pressure $P=15.2 \mathrm{MPa}(2200 \mathrm{psia})$; these conditions correspond to the baseline case developed in Sec. III.L. To minimize the combined thermal and pressure stress, an optimum wall thickness of $\sim 1.0-1.5 \mathrm{~mm}$ is needed. For tube walls of this thickness range, the nominal stress is $75 \mathrm{MPa}$, which is about a factor of five less than the reported 100 tensile strength for the MZC copper alloy at $763 \mathrm{~K}\left(750^{\circ} \mathrm{F}\right)$. These preliminary structural considerations do not constitute a complete failure analysis and must be refined to account for nonuniformity of heat flux, axial loading, creep, radiation effects, and (most crucially) sputter erosion.

More-detailed time/space-dependent thermal-mechanical analysis of this first-wall configuration 101 indicates even for short-pulsed $(\sim 26 \mathrm{~s})$ operation with peak-to-average heat-flux ratios of $\sim 2$ that the MZC copper is capable of $10^{6}$ cycles, which corresponds to a design life of approximately one year with a $\sim 75-80 \%$ duty factor. The effects of radiation damage, power density nonuniformities, and the use of discrete limiters, however, remain to be 120 


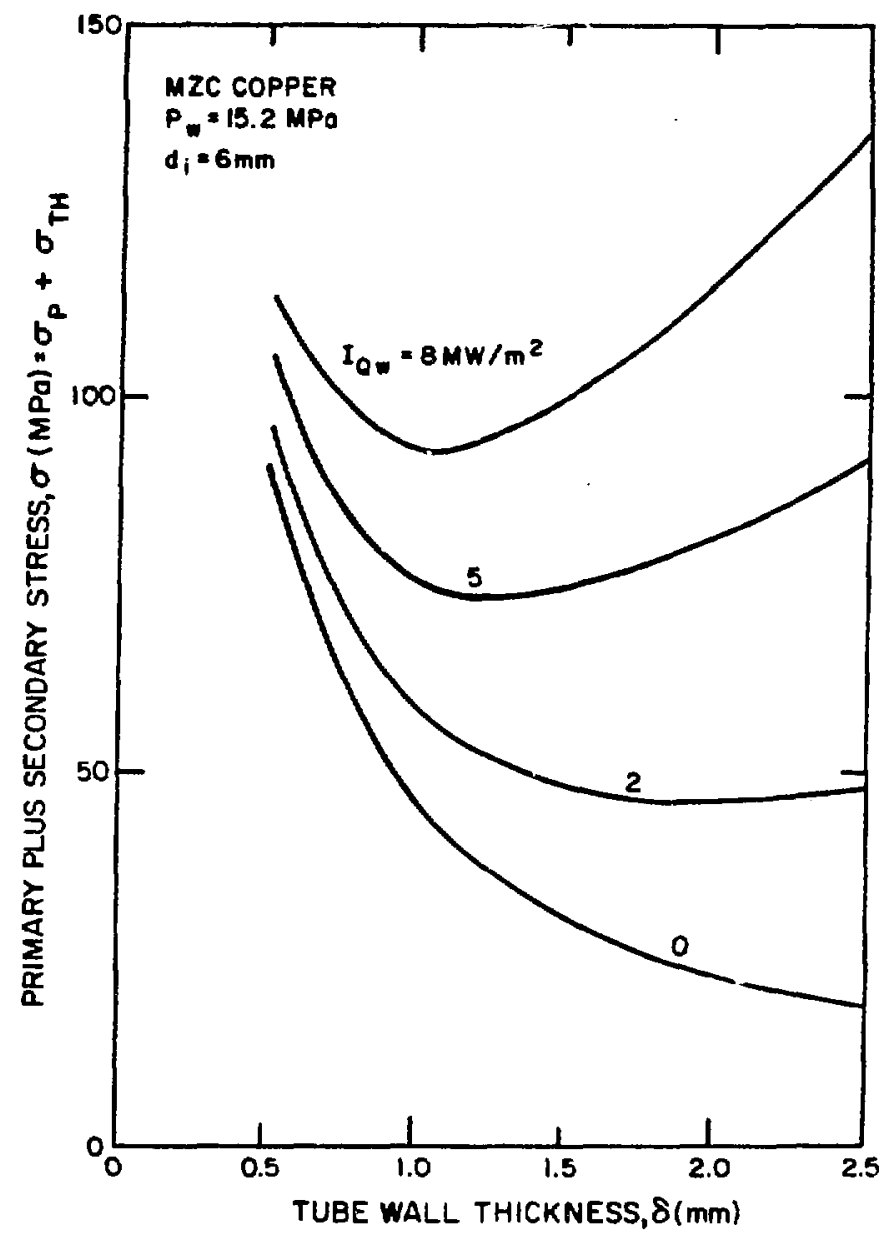

Fig. III.D-2. Stress in 6-mm-diameter MZC copper tube with 15.2-MPa (2200 psia) internal pressure and surface heat flux given by $I_{0 w^{*}}$ The minima in the curves indicate an optimum design for first-wall thicknesses in the range $1.0-1.5 \mathrm{~mm}$.

determined. It is noted, however, that a one-year fatigue life corresponds to a $14.1-\mathrm{MeV}$ neutron fluence of $15 \mathrm{MWyr} / \mathrm{m}^{2}$ for the design heat loads used in the Ref. 101 analysis.

As indicated in Sec. III.C., the thermal conductivity, $k$, of copper at this level of radiation may be reduced by a factor of $\sim 2-3$ over a $\sim 15-\mathrm{MWyr} / \mathrm{m}^{2} 11 \mathrm{fe}$. If the first-wa11 thickness is at the minimum stress point indicated on FIg. III.D-2 given by 


$$
\begin{aligned}
& \delta_{\mathrm{MIN}} \approx \sqrt{\frac{\mathrm{P}_{c^{d}}}{2} \frac{M}{\sigma_{\mathrm{Y}} \mathrm{Q}_{\mathrm{Q}}}} \\
& \sigma_{\mathrm{MIN}}=2 \sqrt{\frac{\mathrm{P}_{\mathrm{c}}^{\mathrm{d}} \sigma_{\mathrm{Y}} \mathrm{I}_{\mathrm{QW}}}{M}},
\end{aligned}
$$

a decrease by a factor of $\sim 2$ in $M \propto k$ will result in a thermal stress increase by $\sim 40 \%$ to $\sim 106 \mathrm{MPa}$, which is still a factor of two below the tensile strength of MZC copper alloy. The transmutations-related changes in key thermal-physical parameters for the $\mathrm{FW}$ advanced alloys, nevertheless, remain a concern.

2. Thermal-Hydraulic Analyses. This section describes the methodology used to estimate the first-wall and limiter coolant needs and limitations. Section III.H. gives the model used to estimate the limiter parameters. The integration of the first-wall, limiter, and blanket coolant circuits into the power cycle and consideration of related synergistic effects are addressed in Sec. III.L.

a. First Wal1. A sketch of a proposed first-wall configuration is shown in Fig. III.D-3. Several other coolant-channel configurations were considered: circular tubes, rectangular channels, and embossed panels. The nominal 20-mm thickness of the FW provides an eletrical shell time of $\sim 10 \mathrm{~ms}$, although the precise function of this shell beyond the theoretical predictions of the Taylor theory ${ }^{33}$ is not well understood, particularly in view of the thick liquid-metal blanket that surrounds the plasma. The effects of this thick first-wall structure on the tritium-breeding ratio is shown in Fig. III.B-7. The neutronic sensitivity studies performed in Appendix $C$ to estimate two-dimensional effects Indicate the desirability to reduce this first-wall thickness to $\sim 5 \mathrm{~mm}$. The $D$-shaped coolant tube was selected based on considerations of mechanical stress and ease of fabrication. High-pressure copling water flows through the D-shaped coolant tubes and absorbs the conductive, convective, and radiative heat flux emanating from the plasma that does not impinge on or under the limiter. In addit:on, nuclear heating within the first-wall material is transferred to this first-wall coolant. The coolant-channel dimensions were selected on the basis of a comprehensive parametric study (Sec. III.L.2.) that optimized on pumplng-power for fixed flow conditions (i.e., subcooling, velocity, stress, 


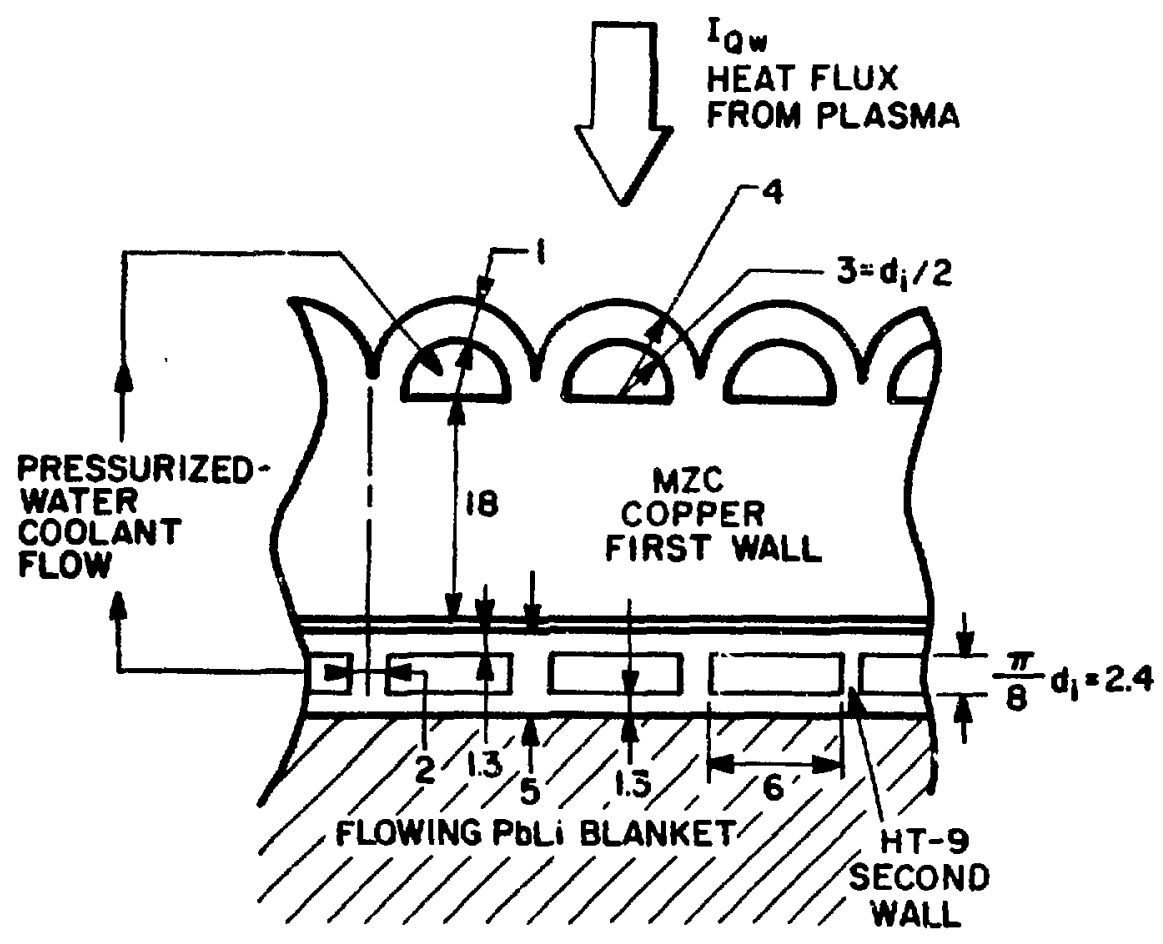

ALLDIMENSIONS IN mm

Fig. III.D-3. Proposed first-wall/second-wall (FW/SW) configuration.

etc.). This optimization is relatively independent of the actual first-wall thickness.

The direction of first-wall coolant flow can be either toroldal or pololdal. The toroldal direction was selected because of a simpler manifold design (Sec. III.L.3.). This first-wall coolant flow is in the opposite direction as that of the PbLI blanket coolant, which as shown in Sec. III.E. Is primarily poloidal in order to minimize MHD pressure drops and pumping power. The first-wall cooling water flows through the first-wall cooling tubes and returns through a similar tube array in the second wall (SW, Fig. III.D-3), where nuclear heating within the second-wall and conductive heat flow from the PbL1 blanket (back leakage) are absorbed. The water-cooled second wall was deemed necessary to hold temperature limits in the blanket structure set by PbLi/HT-9 corrosion limits. The toroldal FW/SW coolant flow is divided into $N \simeq 24$ toroldal segments for purposes of coolant manifolding and headering. 
A thermal-hydraulic analysis is performed for steady-state operation. Design variables include inside tube disineter, $d$, tube-wall thickness, $\delta$, and inlet and exit coolant temperatures. Results of the neutronics calculations (Sec. III.G.) and the thermal analysis of the PbLi blanket (Sec. III.E.) are used to determine the rate of heat transfer to the FW/SW coolant water. The mass flow rate in each coolant tube is then computed for a given tube geometry and inlet/exit water temperatures. A thermal analysis is performed for both the FW and SW coolant tubes to determine the temperature and strass distribution wittin the material. The pressure drop over the tube length and the total pumping-powex requirements are also computed for use in the integrated reactor energy balance (Sec. III.L.2.).

The total energy absorption rate for the first-wall and limiter coolant water is given by the sum of the alpha-particle and ohmic powers from the plasma, $\mathrm{P}_{\alpha}+\mathrm{P}_{\Omega}$, the neutron/gamma-ray energy generation within the first-wall and second-wall materials, $P_{F W}+P_{S W}$, and the convective heat transferred from the hotter PbLi blanket to the FW/SW system, $P_{B L}$. The plasma heat flux is obtalned from a plasma transport model (Sec. III.G.), the neutron/gamma-ray energy generation rates are avallable from the neutronics analysis (Sec. III.B.), and the convection loss to the FW/SW from the PbLi blanket is estimated as part of the blanket thermal analysis (Sec. III.E.). The alphaparticle and ohmic-heating powers are $P_{\alpha}=546.5 \mathrm{MW}$ and $P_{\Omega}=25.3 \mathrm{MW}$. From the neutronics analysis, $P_{F W}=495.1 \mathrm{MW}$ and $P_{S W}=97.1 \mathrm{MW}$. The blanket thermal-hydraulic analysis gives $P_{B L} \approx 96 \mathrm{MW}$ for a total of $1260.0 \mathrm{MW}$ delivered to the FW/SW water coolant, which amounts to $37.4 \%$ of the total thermal power generated belng delivered to the FW/SW (Includes limiter) coolant circuit. The impact of the power split between FW/SW, limiter, blankt,, and shield on the overall thermal efficlency thermal-cycle design is discussed in Sec. III.L.1.

The neutronics analysis (Sec. III.B.) is based on the assumption of a uniform (homogenized) first wall (FIg. III.B-5). In the actual design, pumped limiters may be incorporated. The limiters would be constructed of the same material and would have a similar effective thickness as the first wall. The rates of energy absorption by the first wall and limiter will also be proportional to the respective projected surface areas since the limiter design heat flux $\left(6 \mathrm{MW} / \mathrm{m}^{2}\right)$ is approximately that of the first wall $\left(5 \mathrm{MW} / \mathrm{m}^{2}\right)$. The analysis described in Sec. III.H. Indicates that the limiters will cover approximately $38 \%$ of the first wall. 
of the total power being lost directly from the steady-state plasma $\left(\mathrm{P}_{\alpha}+\mathrm{P}_{\Omega}=571.8 \mathrm{MW}\right), \quad \mathrm{f}_{\mathrm{RAD}}=0.9$ is assumed to be radiated, and the remaining $10 \%$ is lost as convective/conduction losses. This plasma transport loss, $P_{T R}=\left(1-f_{R A D}\right)\left(P_{\alpha}+P_{\Omega}\right)=57.2 \mathrm{MW}$, is concentrated almost entirely on the limiter surface. The limiter also receives $38 \%$ (limiter coverage fraction) of the neutron absorption and plasma radiative losses, which amounts to $0.38[495.1+0.9(546.5+25.3)]=383.6 \mathrm{MW}$. The limiter, therefore, receives a total of $440.8 \mathrm{MW}$ and the FW/SW system receives $819.1 \mathrm{MW}$. Details of this split and the integration of all power-producing systems are described in Sec. III.L.1.

A numerical analysis was used for the thermal-hydraulic design of the first and second walls. The inside diameter of the D-tubes, $d$, the inlet water temperature, $T_{w i}$, and the inlet water pressure, $P_{W}$, are treated as design variables. The stress model described in Sec. III.D.1. was used to calculate an appropriate tube-wall thickness, $\delta$, for the specified values of $d$ and $P_{w}$. The number 0 : first-wall and second-wall tubes is then determined from the following expression:

$$
\mathrm{N}_{\mathrm{T}}=2 \mathrm{~N}\left[2 \pi \mathrm{r}_{\mathrm{w}} /(\mathrm{d}+2 \delta)\right]
$$

where $N=24$ is the number of toroidal reactor segments and $r_{w}$ is the minor radius of the first wall. The factor 2 is included in Eq. (5) because the manifold pipe supplying the $\mathrm{FW} / \mathrm{SW}$ circuit in a given toroidal segment is positioned in the middle of that segment and supplies cooling tubes on both sides. The length of an average cooling tube is given by

$$
\ell=2 \pi R_{T} / 2 N
$$

An additional first-wall design variable is the outlet water temperature, $\mathrm{T}_{c o}$. Given a value for that parameter, the mass flow rate, $\dot{\mathrm{m}}_{\mathrm{w}}$, and velocity, $v$, for a single tube are computed from

$$
\dot{m}_{w}=Q_{w} /\left(N_{T} \Delta i\right)
$$

where $Q_{W}$ is the total cooling rate required for the first wall (i.e., all 
surface and volumetric heating), $\Delta i$ is the increase in enthalpy of the cooling water, and

$$
v=8 \dot{\mathrm{m}}_{\mathrm{w}} /\left(\pi \mathrm{d} \mathrm{p}_{\mathrm{c}}^{2}\right) \text {, }
$$

where $\rho_{c}$ is the average density of the cooling water.

The inside tube-wall temperature at the exit for a first-wall tube is given by

$$
T_{w i}=T_{C o}+q_{w i} / h
$$

where $q_{W i}$ is the heat flux at the inner surface of the tube wall and $h$ is the local film coeficient. The heat flux $q_{w 1}$ is determined by dividing $Q_{w}$ by the total effective area of the Inside surface of the tubes, and $h$ is calculated from the following expression: 119

$$
h=0.0155 \frac{k_{c}}{d_{H}} \operatorname{Pr}^{0.5} \operatorname{Re}^{0.83},
$$

where $k_{c}$ is the thermal conductivity of water, $d_{H}$ is the hydraulic diameter of the tubes, $\mathrm{Pr}$ is the Prandt 1 number, and $\mathrm{Re}$ is the Reynolds number.

The first-wall surface absorbs radiation at a rate of $I_{Q w}=4.59 \mathrm{MW} / \mathrm{m}^{2}$ (1.e., $\mathrm{f}_{\mathrm{RAD}}$ or $90 \%$ of $\mathrm{P}_{\alpha}+\mathrm{P}_{\Omega}$ divided by $\mathrm{A}_{F W}$ ) based on the projected wall area $\left[A_{F W}=(2 \pi)^{2} r_{w} R_{T}=111.8 \mathrm{~m}^{2}\right]$. The curved cylindrical surface (Fig. III.D-3) Increases the effective area by a factor of $\pi / 2$, giving an actual heat $f l u x$ through the tube surface of $I_{Q w}^{\prime}=2.92 \mathrm{MW} / \mathrm{m}^{2}$. The neutron/gamma-ray energy generation within the first wall is $Q_{N}=218.0 \mathrm{MW} / \mathrm{m}^{3}$ (Table III.B-VI). The temperature at the outside surface of the tubes (maximum material temperature) is calculated on the basis of one-dimensional steady-state thermal conduction, giving

$$
T_{w o}=T_{w 1}+Q_{N}\left(\delta / h+\delta^{2} / 2 k\right)+I_{Q w}^{\prime}(1 / h+\delta / k),
$$

where $k$ is the thermal conductivity of the MZC copper alloy and $T_{w}$ is given by Eq. (9). 
The following conditions are selected to be satisfied for an acceptable FW/SW/11miter design.

- The fluid velocity must not be large enough to cause excessive erosion. A value of $10 \mathrm{~m} / \mathrm{s}$ is selected as a nominal upper $11 \mathrm{mit}$.

- The maximum temperature at the inside surface of the tubes, $T_{w y}$, must be less than the saturation temperature of water by some selected amount. A difference of $10 \mathrm{~K}$ is selected for destgn purposes.

- The maximum material temperature, $T_{\text {wo }}$, must be less than a specified value. One-half the melt.ing temperature is selected as the upper limit.

The second condition listed above is more conservative than the condition ir a typical PWR, where the wall temperature can be above the sacuration temperature in some regtons, and subcooled nucleate boliting is allowed. If one or more of these conditions is not satisfied, the outlet coolant-water temperature, $T_{\text {co, is }}$ adjusted. Lowering the outlet coolant temperature will decrease $T_{w i}$ ind $T_{w o}$, but $v$ and the pumping power will increase. If adjusting the outlet coolant temperature does not yield an acceptable solution, one or more of the other design variables is adjusted. The pressure drop for the first-wall coolant tubes is given by

$$
\Delta P_{1}=f_{F} \frac{\ell}{d_{H}} \frac{\rho_{c} v^{2}}{2},
$$

where the Fanning friction factor is computed from the following relationship:

$$
f_{F}=0.184 / \operatorname{Re}^{0.2}
$$

The equations used to calculate thermal-hydraulic conditions for the second wall are the same as those used for the first wall except for the cooling rates and heat fluxes. The inlet coolant temperature for the second wall is the first-wall outlet temperature, and the mass flow rates and tube diameters are the same as those for the first wall since the first-wall coolant is returned through the second wall. The coolant temperatures are slightly higher in the second wall, but the heat fluxes are low and the maximum inside and outside wall temperatures are generally lower than those in the first wall. 
The mechanical loading applied to the FW/SW coolant tubes arises primirily from the internal coolant pressure, with a small stress being gerierated by the internal temperature gradient. Calculat lons have been performed for a range of coolant pressures from 8.3-15.2 MPa (1200-2200 psi). The maximum teisile (hoop) stress caused by the water coolant at $15.2 \mathrm{MPa}(2200 \mathrm{psi})$ for a tube with a 6-mm Inside diameter and a 1-mm-thick wall is only $45.6 \mathrm{MPa}$ (6600 psi), which is less than $20 \%$ of the tensile strength of MZC copper at $600 \mathrm{~K}$. A thermaI cyclic fatigue analysis 101 was performed for a pulsed mode of operation and indicated that the coolant tubes can easily withstand $10^{6}$ cycles under conditions that are 2-3 times more severe than long-pulsed or steady-state operation, as measured by the peak heat flux. The present design is based on steady-state operation using "F- $\theta$ pumping" current drive.

The coolant pressure and outlet water temperature were chosen to prevent subcooled-flow bolling. The adequacy of the margin between the local peak fluid temperature and the saturation temperature is a matter of judgment that should be based on a more-refined analysis and experiments at high transient heat fluxes. The first-wall design is not sufficiently advanced to allow heat transfer in the inlet and outlet headers to be considered. Careful attention to regions where the flow turns from axial to radial will be needed to prevent localized bolling. Table III.D-I gives the thermal-hydre lic design parameters

TABLE III.D-I

FIRST-WALL THERMAL-HYDRAULIC DESIGN PARAMETERS

Heat Flux and Total Power

- Heat flux $\left(\mathrm{MW} / \mathrm{m}^{2}\right)$

5.0

- Volumetric heat generation $\left(\mathrm{MW} / \mathrm{m}^{3}\right)$

222 .

- Power absorbed by first-wall coolant (MW)

819

- Percent of total thermal output

Geometry

- Number of first-wall sectors

- Number of channels per first-wall sector

- Channel length (m)

0.50

- Channel radius (mm)

3.0 ("D" shaped)

- Channel wall thickness (mm)

1.0 
that have been adopted for the $\mathrm{FW} / \mathrm{SW}$ system. The optimized FW/SW thermal-hydraulic parameters can be Eound in Table III.L-IV.

The turbulent, forced-convection correlation [Eq. (10)] predicts conservative heat-transfer coefflcients for the first-wall coolant. This correlation is valid for fully developed velocity profiles and thermal boundary layers in uniformly heated tubes under steady-state conditions. Because the heat flux is nonuniform around the tube, the Nusselt number will vary clrcumferentially. According to an analysis that includes effects of circumferential tube-wall heat conduction, 120 the local Nusselt number at the apex of the tube may be significantly less than that used in the present design. A more-detalled analysis includes both radial and circumferential conduction 121 and shows that neglect of radial conduction is not important and supports the decrease in heat transfer at the tube apex. Based on experimental results, 122 Ref. 121 cautions that the circumferentlal variation in the local heat-transfer coefficient is less pronounced than theoretical analyses predict, and the decrease in heat transfer mentioned above may be too severe a penalty.

If the first-wall heat transfer should require augmentatiou while maintaining an acceptable pressure drop, at least two methods for lmprovement can be envisaged. Extended surfaces or "fins" are considered an established technology as is the use of swirl-flow devices and twisted-tape inserts. Fine spiral grooves macilined on the inner tube surface achieve the benefits of both swirl and extended surfaces. Other variations are possible and are commercially available. The precise benefit in applications where the high-Reynolds-number flow is aonuniform, transient, and high flux, however, must be determined experimentally; a factormof-two reduction in the film temperature drop does not seem unreasonable, however. Some of the highest steady-state heat fluxes to flowing water ever measured $123\left(173 \mathrm{MW} / \mathrm{m}^{2}\right)$ were obtained with swirl flow in short smooth tubes.

Fouling of the surface at the tube/water interface will lead to reduced heat-transfer efficiency and higher copper temperatures. Many vartables affect fouling, including time in service, tube surface material, temperatures of the tube surface and the water, and the water velocity. Careful control of water chemistry can greatly reduce the effects of fouling. The operating parameter range (Table III.L-IV) expected for the FW/SW system generally is in a range where foullng should be low. 
At high flow velocities, the possibility of fluid erosion of the copper coolant passages must be considered. For pure water with low solids content and no cavitation, erosion should not be a problem. The flow passage geometry must carefully avoid low-pressure regions that can lead to cavitation. Corrosion of the copper wall by the water coolant in the one-year design life should be less than $0.1 \mathrm{~mm} ;{ }^{99}$ the effects of radiation and radiolytic decomposition of the water coolant on corrosion rate, however, remains to be assessed.

Although advanced by most standards of contemporary industrial heat-transfer systems, a water-cooled, copper-alloy first wall should not present a serious development problem from the viewpoint of thermal stress and heat transfer. The stress-optimal FW thickness interposed between the coolant channel and the plasma $(1.0-1.5 \mathrm{~mm})$ does present a serlous concern from the viewpoint of sputter-erosion life if a substantial fraction of the plasma transport incident upon the first wall appears as energetic ions or chargeexchanged neutrals. An accurate assessment of this crucial $\mathrm{FW}$ issue cannot be made until a better understanding of plasma-edge effects and the effectiveness of Impurity-control schemes (Sec. III.H. and ILI.I.) is obtained.

b. Pumped Limiter. The cooling requirement for the limiter is similar to that described for the first wall in that the heat flux and energy generation rate are similar for each because of the high fraction of radiated energy $\left(f_{R A D}=0.9\right)$ and high limiter coverage fraction $\left[l_{L} /\left(l_{L}+l_{W}\right) \neq 0.38\right]$. The MZC copper alloy is also proposed for use in the pumped limiter. The orientation of the limiter relative to the first wall is poloidal (Fig. III.E-6). The computation of the contour of the limiter surface facing the plasma is also discussed in Sec. III.H. The thlckness of the limiter is made as small as possible consistent with the Inclusion of coolant-water channels of sufficlent size to provide adequate cooling at acceptable cwolant velocities and pumping power while maximizing the limiter-slot area for purposes of particle pumping.

A thermal-hydraulic analysis similar to that used for the first-wall coolant tubes was used along with a structural analysis to determine suitable coolant-channel dimensions. The key design parameters are coolant flow rate, coolant inlet temperature, channel height and width, thickness of material between the channels and the 11miter surface, and spacing between coolant channels. Optimal design requires the highest possible outlet coolant temperature that is compatible with maintaining the limiter at an acceptable operating temperature. The coolant-channel configuration proposed for the 
limiter is shown in Fig. III.D-4. Cooling rates, heat fluxes, and geometric parameters for the limiters are summarized in Table III.D-II. The thermal-hydraulic analysis was made for various inlet water temperatures; pressures, and flow rates. Results of those calculations and a preliminary 1imiter design are given in Sec. III.L.2. (Table III.L-III).

A critical cooling problem occurs at the limiter tip. As discussed in Sec. III.H., the surface of the limiter facing the plasma receives both a radiation $f l u x$ and a particle flux. This surface is shaped so that the combined energy flux incident on the limiter surface in the perpendicular direction does not exceed a design limit equal to $q_{D}=6 \mathrm{MW} / \mathrm{m}^{2}$. Equations (5) and (6) in Sec. III H.1. describe the limiter shape as determined by the solution to the edge-plasma transport equations and this $q_{D}$ constralnt. The point where the scrapeoff particle energy flux in the toroidal or z-direction falls below $q_{D}$ determines the toroidal extent of the limiter, $z= \pm l_{L} / 2$, which in turn determines the radial extent, $x=\delta^{\prime}$, where the $x-z$ coordinate system (Fig. III.D-4) is defined by the limiter leading edge (plasma radius, $r_{p}$ ) for $x=0$ and the toroidal center, $z=0$. The distance $r_{w}-r_{p}-\delta^{\prime}$ determines the limiter-slot (radial) thickness available for pumping as well as the fraction, $f_{p}$, of the particles diffusing in the scrapeoff that enter the liaiter slot. The resulting thickness $\delta^{\circ}$, as seen from Fig. III.D-4, is not sufficient to support cooling channels, and structure must be added to the limiter underside for this purpose. The amount of added structure, $6^{\prime \prime}$, is shown in Fig. III.D-4, which gives a view of the limiter tip as a mocked-up infinite element mesh for purposes of a thermal analysis by the computer code AYER. 124

As Indicated in Fig. III.H-4, the particle energy flux, $q_{z}$, falls off nearly exponentially with distance in the scrapeoff; with an e-folding distance given approximately by $\lambda \simeq 3 \mathrm{~mm}$, where $D$ is the scrapeoff particle Alffusivity. The total heat fluxes incident onto the limiter leading edge and tip are shown In Fig. III.D-4, which is used to perform the limiter thermal analysis.

The two-dimensional finite-element AYER 124 code was used to compute tie steady-state temperature distributions in the MZC copper-alloy 11miter. Table III.E-I gives the thermal physical properties used, and Fig. III.D-4 gives the geometry and temperature distributions. The pressurized-water coolant flowing at the limiter tip through the 0.8 - by $4.0-\mathrm{mm}$ channels has a temperature of $520 \mathrm{~K}$ and a flow velocity of $10 \mathrm{~m} / \mathrm{s}$, which for this $\mathrm{Re} \simeq 10^{5}$ flow glves a local film coefficient of $94,000 \mathrm{~W} / \mathrm{m}^{2} \mathrm{~K}$ using $\mathrm{Eq} \cdot(10)$. The temperature at the 


\section{PUMPED-LIMITER THERMOHYDRAULIC DESIGN PARAMETERS}

Heat Flux and Total Power

- Heat flux (MW/m $\left.{ }^{2}\right)$

$$
6.0
$$

- Volumetric heat generation $\left(\mathrm{MW} / \mathrm{m}^{3}\right)$

222.

- Power absorbed by limiter coolant (MW)

396.

- Percent of total thermal output

Geometry

- Number of limiters

24.

- Number of channels for one side of a limiter 930 .

- Channel length (m)

0.38

- Channel width (mm)

4.0

- Channel tisight (m)

0.8

limiter tip is within limits established by creep limits for this alloy. The temperature contours at the limiter leading edge reflect the $q_{D}=6 \mathrm{MW} / \mathrm{m}^{2} \mathrm{des} / \mathrm{gn}$ heat flux, while the contours reflect heat flow from the limiter tip to the bulk Iimfter material. These calculations include the nuclear heating within the IImiter, which accounts only for $\sim 16 \%$ of the total energy received by the limiter blade and $3 \%$ of the energy transferred through the leading edge to the coolant. The overall limiter power balance, including nuclear heating and coolant temperature change, is treated in Sec. III.L.2. as part of the overa11 FPC thermal-mechanical design integration. Although the limiter temperatures are well within an acceptable range for the configuration shown, the relatively thin sections required allow little design margin for significant net sputter erosion. Analysis of low-z coatings on tiles and the improvements needed to better analyze these important plasma/wall interctions, including self-consistent calculation of sputtering rates, redeposition, and radiation fractions, represent ar important area of future work. 


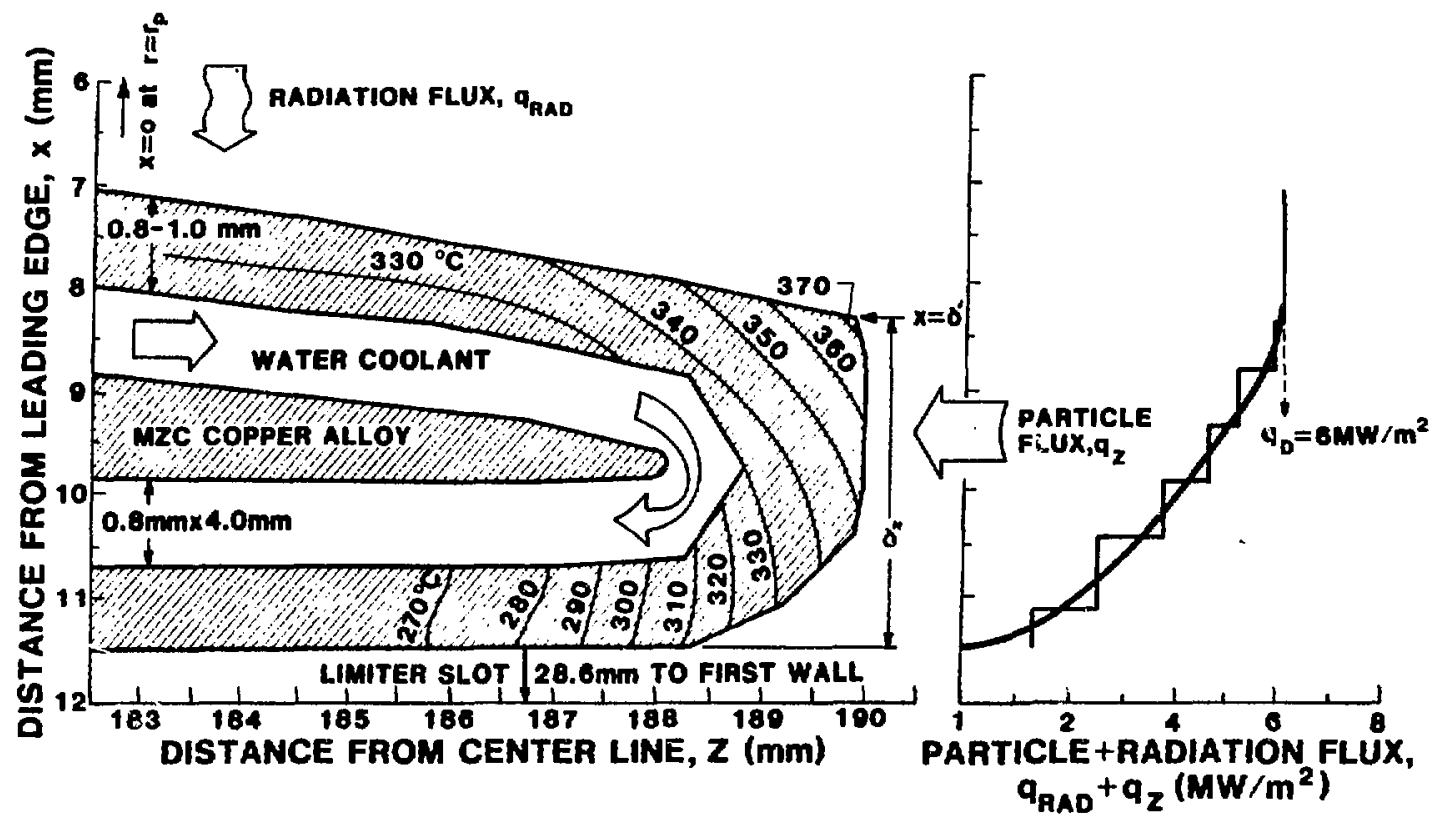

Fig. III.D-4. Limiter-tip temperature and heat-flux distribution based on desIgn developed in Sec. III.H. 
This section describes the thermohydraulic analysis and proposes an engineering design for the blanket. In addition to preliminary thermohydraulic analyses based on a simplified blanket model, the issues of assembly, maintenance, vacuum ducting, and coolant manifolding play an important role in this preliminary assessment. The results of this blanket scoping study are integrated with the first-wall scoping study (Sec.III.D.), the magnet design (Sec. III.F.), particle control (Sec. III.H.), and vacuum (Sec. III.J.) systems to provide an estimate of the integrated FPC (Sec. III.L.). The overall steampower cycle and integrated system energy balance based on these scoping calculations are also described in Sec. III.L. as part of the preliminary FPC integration.

1. General Considerations. The average power density in the PbLI blanket is expected to be $\sim 30 \mathrm{MW} / \mathrm{m}^{3}$, with the maximum occurring within the copper-alloy first wall $\left(240 \mathrm{MW} / \mathrm{m}^{3}\right)$. The range of power densities in which other conventional and advanced engineerlng systems operate is shown schematically on Fig. III.E-1. Although considerably above the blanket power densities envisaged for most fusion reactor concepts, the CRFPR blanket power density does not differ greatly from other nonfusion energy sources. A survey was conducted of the various first-wall/blanket concepts that have been previously proposed for fusion reactor application.3,4-7,86,125-126 Those concepts were critically evaluated and various aspects of those deslgns have been adapted for the present design. Because of the higher local power density, liquid-lithium metal or eutectic coolants seem particularly attractive, although certain of the solid breeder materials have not been completely eliminated from consideration for operation at somewhat lower power density.

The materlals used in the FW/B system provide structural, tritium-breeding, neutron-multiplying, shielding, and coolant functions. Ideally, materials selection is based on physical, thermal, neutronic, and radiation properties as well as on considerations of chemical compatibility, cost, fabricability, and resource. Ongoing work 87,125 is quantifing and ranking the full range of $\mathrm{FW} / \mathrm{B}$ concepts and materials combinations.

Generally, the blanket structural material must have good strength characteristics, good resistance to radiation damage, and acceptable compatibility with the blanket coolant. Surveys of potential structural blanket materials 3,87 considered austenitic steels, ferritic steels, nickel, titanium, 


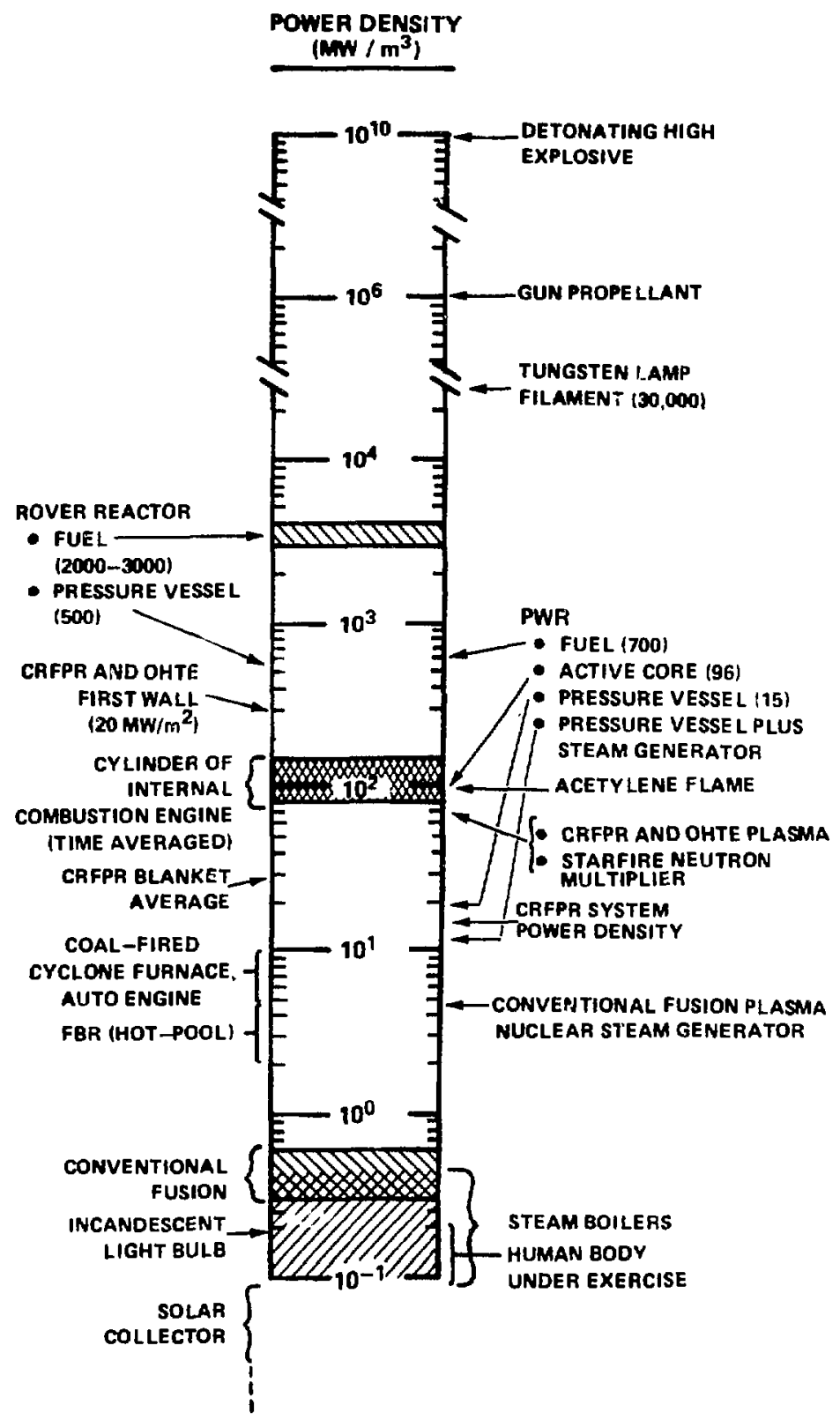

Fig. III.E-1. Range of power densities for a number of power systems showing the relationship to the CRFPR and other fusion systems.

vanadium, and niobium alloys. A titanium-modified stainless steel was initia.lly selected for the blanket channels and structure. This material has the same thermophysical and unirradiated mechanical properties as type 316 stainless steel but exhibits reduced swelling and embrittlement after irradiation. Considerations of Increased radiation life as well as PbLI corrosion limits and 
the desire to maintain high coolant temperature more recently points to the use of a ferritic steel alloy, HT-9. Table III.E-I gives selected properties for both 316 stainless steel and HT-9 ferritic alloy.

Both solid and liquid forms of lithlum have been considered for the tritium breeder. A solid breeder material requires the deposited energy to be conducted to tubes or channels carrying pressurized water or helium. A liquid breeder can either be stagnant or be circulated through heat exchangers and transfer the

TABLE III.E-I

SELECTED PROPERTIES OF TYPE 316 STAINLESS STEEL, HT-9 FERRITIC STEEL, MZC COPPER ALLOY, and PbLI COOLANT/BREEDER

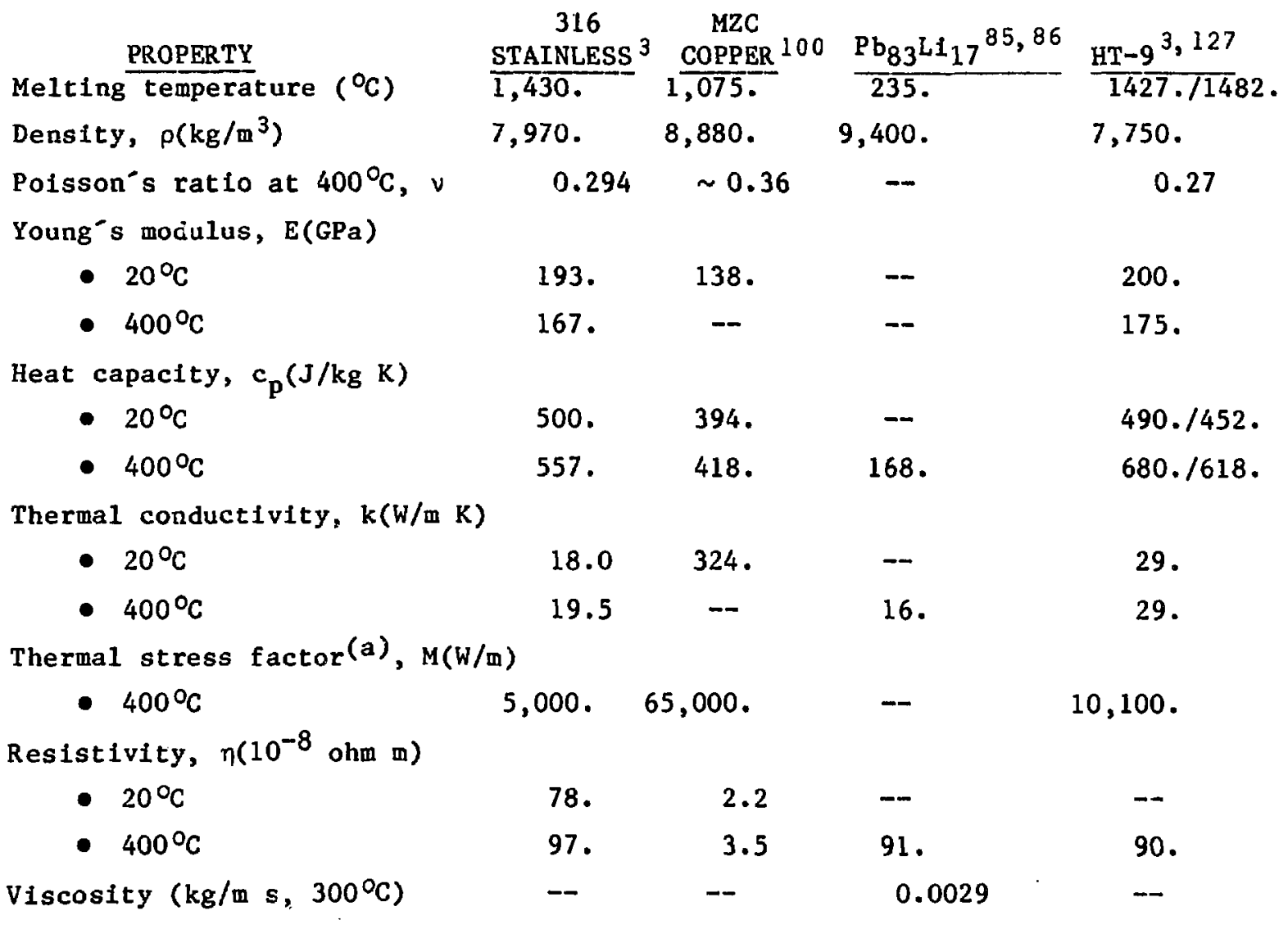

(a) Figure of merit $M=2 \sigma_{\mathrm{Y} k}(1-v) / \alpha E$ is shown in Fig. III.C-2. Higher values imply better thermal stress characteristics. If $\mathrm{I}_{\mathrm{OW}}\left(\mathrm{MW} / \mathrm{m}^{2}\right)$ is the sready-state heat flux through a slab of thickness $\delta$ and the associated thermal stress is $\sigma_{\mathrm{TH}}$, then $\mathrm{M}=\mathrm{I}_{\mathrm{Qw}} \delta / \mathrm{f}_{\mathrm{y}}$, where $\mathrm{f}_{\mathrm{y}} \equiv \sigma_{\mathrm{TH}} / \sigma_{\mathrm{Y}}$. 
energy from the blanket to a steam power cycle. A thermal analysis was performed to assess the feasibility of using $\mathrm{LiO}_{2}$ peliets surrounding tubes carrying pressurized water as a coolant at the local power density envisaged for the CRFPR. This study determined the maximum allowaile coolant-tube spacing as a function of the effective (porosity-dependent) thermal conductivity of the $\mathrm{LIO}_{2}$ and energy-generation rate within the packed bed for given values of water inlet temperature, coolant-tube size, water flow rate, and a maximum allowable $\mathrm{LiO}_{2}$ temperature $(943 \mathrm{~K})$. The results of this scoping study showed that the fraction of blanket volume occupled by the coolant water and tubes could be as high as $40 \%$ near the first wall, giving a significant reduction in the tritiumbreeding ratio. In addition, the removal of energy from the blanket at temperatures less than $573 \mathrm{~K}$ would result in a relatively low (Rankine cycle) thermal-cc.version efficiency. Hence, the use of pressurized-water-cooled, solid-breeder blankets of the kind studied in Refs. 3 and 53 does not seem feasible for the blanket power densities envisaged for the present design.

The other breeding material considered was the liquid $\mathrm{Pb}_{83} \mathrm{Li}_{17}$ eutectic, herein after simply referred to as "PbLi." This material can also be used as a coolant. Flowing liquid-metal coolants as used in most fusion reactor blanket concepts have problems related to potentially large pumping power and/or coolant pressures required to overcome MHD forces. The magnetic fields in the CRFPR blanket are both lower and more favorably orfented to reduce these problems. A thermal-hydraulic analysis presented later in this section shows that pumping power can be held to acceptable levels with careful design, with blanket pressurization being the primary design concern (Sec. III.L.2.).

Chemical corrosion and compatibility of PbLi with structural materials at elevated temperatures were addressed first in Ref. 85, which concluded that dissolution attack by lead is reasonably slow for stainless steel at temperatures up to $773 \mathrm{~K}$, even in uninhibited lead. Although experimental data on the reactivity of $\mathrm{PbLi}$ and stainless steel are not sufficient for an accurate determination of a maximum operating temperature, it appears that for a blanket lifetime of about one year temperatures of PbLi in contact with stainless steel as high as $773 \mathrm{~K}$ may be acceptable. More-recent work ${ }^{126}$ however, suggests coolant/structural interfacial temperatures that are $\sim 50 \mathrm{~K}$ below the values recommended in Ref. 85. Either reduced plant efficiency or an alloy substitution (i.e., ferritic HT-9) may be required. On the other hand, corrosion-related structural temperatures are usually set by mass transport and 
the potentlal for redeposition in cooler regions of the liquid-metal loop rather than by concerns for structural thinning and weakening. These corrosion 1irits, therefore, are dependent upon design cholce related to flow configurations, surface areas, and whether cold-trapping or corrosion inhibitors can be used. Until these issues are resolved, maximum structural temperatures are held to $\sim 500^{\circ} \mathrm{C}$, which for the CRFPR design requires a water-cooled "second wall" and a back leakage of higher-quality heat from the PbLi circuit to the somewhat cooler pressurized-water coolant.

The neutronics calculations given in Sec. III.B. Indicate the desirability of a thin shield positioned outboard of the blanket and constructed of a material with a high neutron absorption and reflection. The materials selected are boron carbide and tungsten, both also being cooled either by flowing PbLi or pressurized water. Since this thin $(0.1-\mathrm{m})$ high-temperature shield makes a major contribution to both the mass and cost of the blanket, an incentive exists to find a better composite for this location to achleve the desfred blanket energy recovery efficiency $\left(\varepsilon_{B} \simeq 0.985\right)$. Postdesign studies given in Appendix $C$ also indicate the desirability to enhance the reflector rather than the shielding properties of this system and point toward the use of more steel and/or tungsten and no boron carbide. Figure III.B-5 gives the CRFPR blanket used to assess both neutronic and thermohydraulic performance. As is shown in Sec.III.L., a water-cooled shield is selected, this shield also serving an important structural function as well as defining the vacuum ducting.

2. Thermal-Hydraulic Analysis. The proposed self-cooled PbLi/steel blanket can assume a number of configurations that are best adapted to the CRFPR magnetics toplogy. Four configurations that have been analyzed are illustrated in Fig. III.E-2, all adopting flow in the pololdal direction. A fifth configuration, simflar to configuration three with the flow in the toroidal direction, was also examined.

An MHD flow model was used to estimate the MHD pressure drop and p.jing power for specifled values of inlet and exit fluid temperature and for given channel dimensions. The calculational procedure is described below, which is then followed by parametric results and comparisons. Finaliy, a configuration is selected for the FPC integration studies (Sec. III.L.), which also served as an example for the hydraulic analysis (Table III.L-V). The blanket therma1hydraulic studies must be made 1teratively with both the magnetic (Sec. III.F.) 
FIRST CONFIGURATION:

SERIES CONNECTED

CHANNELS

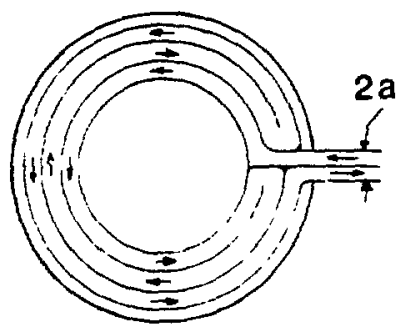

THIRD CONFIGURATION:

PARALLEL CHANNELS, DIVIDED MANIFOLD

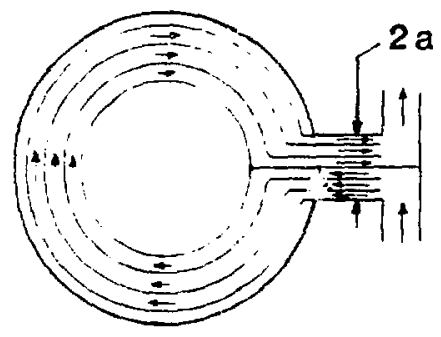

SECOND CONFIGURATION:

PARALLEL CHANNELS,

UNDIVIDED MANIFOLD

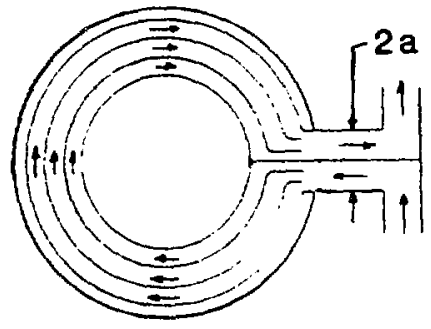

FOURTH CONFIGURATION:

SPLIT CHANNELS,

DIVIDED MANIFOLD

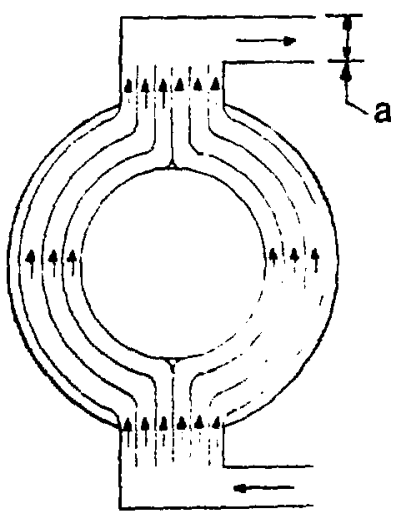

Fig. III.E-2. Blanket flow configurations considered for the high-powerdensity, flowing-Pbli/steel blanket. All cross sections indicate poloidal flow with the major axis of the torus to the left. All coolant ducting to and from the blanket is located at the lowfleld, accessible outboard side of the torus.

and particle-control/vacuum (Sec. III.G.) studies; the material contained herein represents a first step in that iterative design process.

a. Calculation of Blanket Pumping Power. Eddy currents are Induced when an electrically conducting fluid of viscosity $\eta$ and electrical conductivity $\sigma$ flows between parallel plates of separation 2 a with a velocity $u$ perpendicular to a magnetic field. These eddy currents interact with the magnetic field, and a pressure gradient $d P_{M} / d x$ results. 


$$
\frac{\mathrm{dP}_{M}}{\mathrm{dx}}=-\frac{\pi \mathrm{u}}{\mathrm{a}^{2}}\left[\frac{\mathrm{H}^{2} \tanh \mathrm{H}}{\mathrm{H}-\tanh \mathrm{H}}+\frac{\mathrm{H}^{2} \mathrm{C}}{1+\mathrm{C}}\right]
$$

where $\mathrm{H}=\mathrm{aB}(\sigma / \eta)^{1 / 2}$ is the Hartmann number, $B$ is the strength of the transverse magnetic-field, $C=\alpha_{w} t_{w} / \sigma$ a is the ratio of conductivity-thickness products of the flowing fluid, $\sigma a$, and the channel walls, $\sigma_{w} t_{w}$. If $\dot{m}_{p}=P_{B L K} / c_{p} \Delta T$ is the mass flow rate and $\Delta P$ is the pressure drop over the entire channel length, the pumping power is given by $\dot{m}_{p} \Delta P / \rho$, where $\rho$ is the fluld density and $P_{B L K}$ is the power carried through a temperature difference $\Delta \mathrm{T}$ by the fluld of heat capacity $c_{p}$.

The thermal power, $\mathrm{P}_{\mathrm{BLK}}$, is computed by Integration of the power distribution obtained from the neutronics analysis (Sec. III.B.) over the radial width of a given coolant channel. If the 1nlet and exit coolant temperatures are specified, then the mass flow rate is determined. For a specific flow channel geometry, magnetic-field configuration, and fluld properties, Eq. (1) is integrated to give the $M H D$ pressure drop $\Delta P_{M}$. In order to perform this integration, the pololdal-field and toroldal-field profiles within the blanket and (outboard) coolant-ducting regions must be estimated. Referring to Fig. III.E-3, the spatial variation of the poloidal and toroldal fields within the blanket (1.e., $r_{w} \leq r \leq r_{w}+\Delta b$ ) and $\operatorname{coll}\left(1 . e ., r_{w}+\Delta b \leq r \leq r_{c \theta}+\delta_{c \theta} / 2\right)$ regions are approximated by

$$
\begin{aligned}
& B_{\phi}= \begin{cases}\frac{1.52}{R}=\frac{1.52}{R_{T}+r \cos \theta}\left(r_{w} \leqslant r<r_{c \phi}+\varepsilon_{c \phi} / 2\right) \\
0 . \quad\left(r>r_{c \phi}+\delta_{c \phi} / 2\right)\end{cases} \\
& { }^{B}{ }_{\theta}=\frac{4.25}{r}-0.8 r-0.2\left(r_{w}<r<r_{c \theta}+\delta_{c \theta} / 2\right) \text {. }
\end{aligned}
$$

The viscous or hydraulic pressure drop, $\Delta P_{H} \simeq f_{F}(1 / 8 a) p u^{2}$ must be added to the magnetic pressure drop, $\Delta P_{M}$, whlch is computed by integrating Eq. ( 1 ), using Eqs. (2) and (3). The friction factor, $f_{F}$, is computed for a fluid of density $\rho$ and veloctty $u$ flowing through a channel of width $a$ and length $\ell$ [Sec. III.D., Eq. (13)]. With the total pressure drop given by $\Delta P=\Delta P_{H}+\Delta P_{M}$, the Ideal 


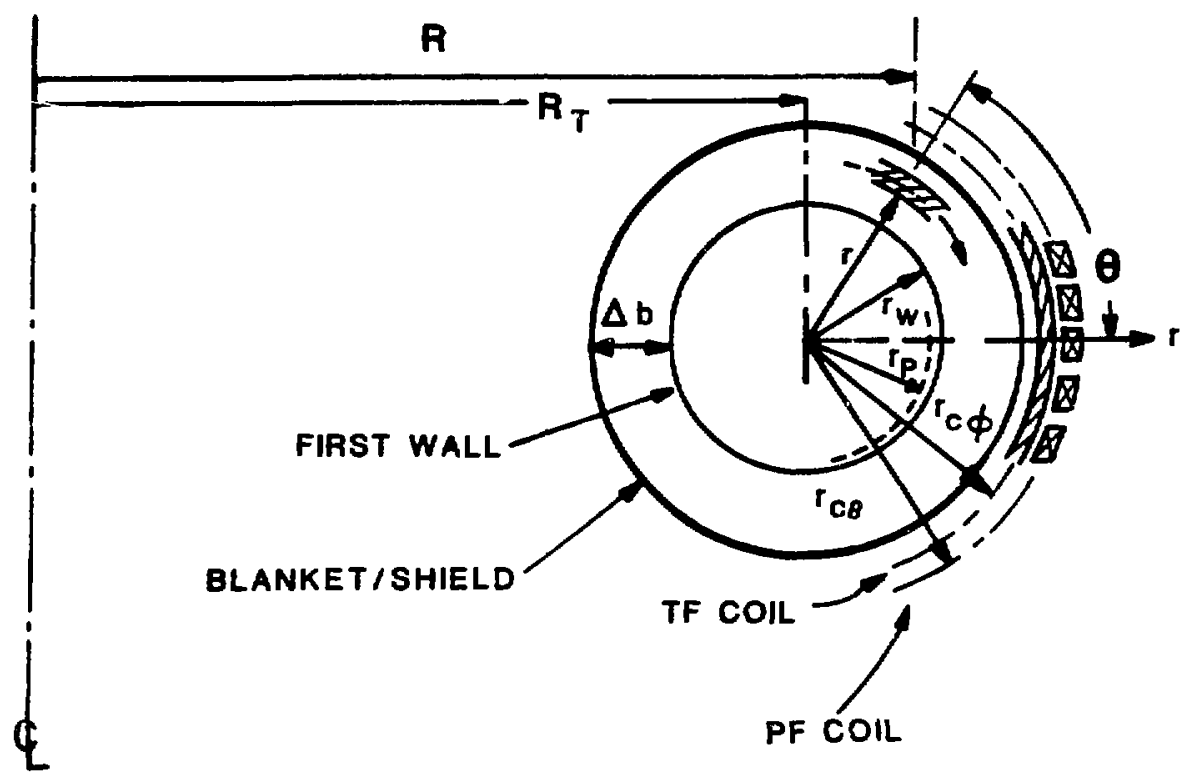

Fig. III.E-3. Geometry used to estimate MHD pressure drop for flowing PbLi coolant in the RFP magnetic topology.

pumping power, $\mathrm{m}_{\mathrm{p}} \Delta \mathrm{P} / \rho$, is computed for the range of flow configurations depicted in Fig. III.E-2. The actual pumping power is the ideal value multiplied by the

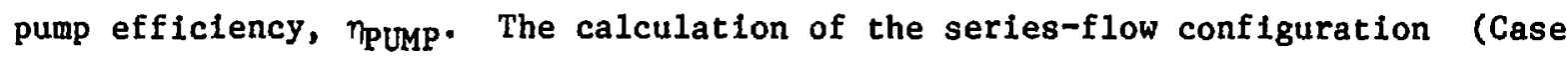
\#1, F18. III.E-2) proceeds with the mass flow rate computed from $\dot{m}_{p}=P_{B L K} /\left(c_{p} \Delta T\right)$. The average fluid velocity is calculated from $u=m \dot{m}_{c s}$, where $A_{c s}=(2 a)\left(2 \pi R_{T}\right)$ is the average cross sectlonal area for the flow, $R_{T}=$ $3.8 \mathrm{~m}$ is the mafor radius of the torus, and $a$ is the half-width of the channel. The magnetic-field pressure drop is estimated from Eq. (1), assuming that the pressure vartes linearly with distance along the channel. For flow in the poloidal direction, the toroldal fteld, $B_{\phi}$ is the transverse magnetic field. The half-width of the channel is $a=\left[\Delta b-(N-1) t_{W}\right] / 2 N$, where $\Delta b=0.5$ m is the blanket thickness, $t_{w}$ is the channel wall thickness, and $N$ is the number of channe1 sections. 
For flow in the radial direction, the transverse magnetic field, $B_{\theta}$, varies with distance in accordance with Eq. (3). An integrated average value of $B_{\theta}$ is determined for use in Eq. (1). The flow length in this case is the distance from the midline of the Inside channel to the outside of the blanket plus the length of the inlet or exit channel. After estimation of the hydraulic pressure drop, $\Delta P_{H}$, the total pumping power is computed based on the total pressure drop $\Delta \mathrm{P}=\Delta \mathrm{P}_{\mathrm{H}}+\Delta \mathrm{P}_{\mathrm{M}}$.

The analysis of the paralle1-flow configurations (configurations 非 and 非 In Fig. III.E-2) is somewhat more complex because the thickness of the channe1s must be selected such that the pressure drop and temperature increase are equal for al1 channels. Because the governing equations are nonlinear, an iterative procedure was used. The method included the following steps:

- Select Initial values of half-thickness for each channel, $\alpha_{1}$

- Determine the cooling rate required for each channel by multiplying the local volumeixic energy-generation rate by the enclosed volume.

- Calculate the mass flow rate, $\dot{\mathrm{m}}_{\mathrm{pI}}=\mathrm{P}_{\mathrm{BLK}} /\left(c_{\mathrm{p}} \Delta \mathrm{T}\right)$, for each channe1.

- Compute magnetic and hydraulic pressure drops, as discussed for the case of series flow. Add these to determine the total pressure drop for each channel, $\Delta \mathrm{P}_{i}$.

- If al1 $\Delta P_{1}$ are not equal, a new estimate of channel halfthtckness is computed from $\alpha_{i}=\alpha_{1}\left(\Delta P_{1} /\langle\Delta P\rangle\right)^{1 / 2}$, where $\langle\Delta P\rangle$ $=(1 / \mathrm{N}) \sum \Delta \mathrm{P}_{1}$.

- Repeat steps unt $11 \Delta \mathrm{P}_{1}$ is within $0.1 \%$ of $\langle\Delta \mathrm{P}\rangle$ for al1 channels.

The pumping power is then calculated for each channel and subsequently summed to determine the total pumping-power requitrement.

b. Results. For all. cases, the electrical conductivity ratio, $C=\sigma_{w} t_{w} / \sigma a$, was computed for an uncoated or "non-sandwiched" coolant duct; the potential for lowered pumping power and increased fabrication and compatibility problems that attends the coating or sandwiching of the coolant-shanne1 walls with electrical insulators was not considered. The first configuration 
described in Fig. III.E-2 (1.e., series flow) gave unacceptably high pumping power fractions (i.e., >5\%) and, therefore, was eliminated. The fourth configuration was also set aside because of practical problems associated with design of the inlet/outlet manifolds. Results for configurations $\# 2$ and $\# 3$ are shown in Fig. III.E-4 for $N=3$ channels. Calculations for four and five channels did not show a suffictently decreased pumping power to justify the added design complexity and increased structure. A finer resolution of a greater number of poloidal channels at the inboard, higher-power-density regions may give a better solution of the structural temperature control, however, and represents an area of future work. Within the constraints of the present study, however, configuration $\left.\right|_{2}$ is preferable from the standpoint of pumping power.

The model used to estimate $\Delta \mathrm{P}_{\mathrm{M}}$ does not account for the temperature variation in the transverse direction within the PbLi coolant. This variation is of little significance for a highly turbulent flow in which mixing causes a

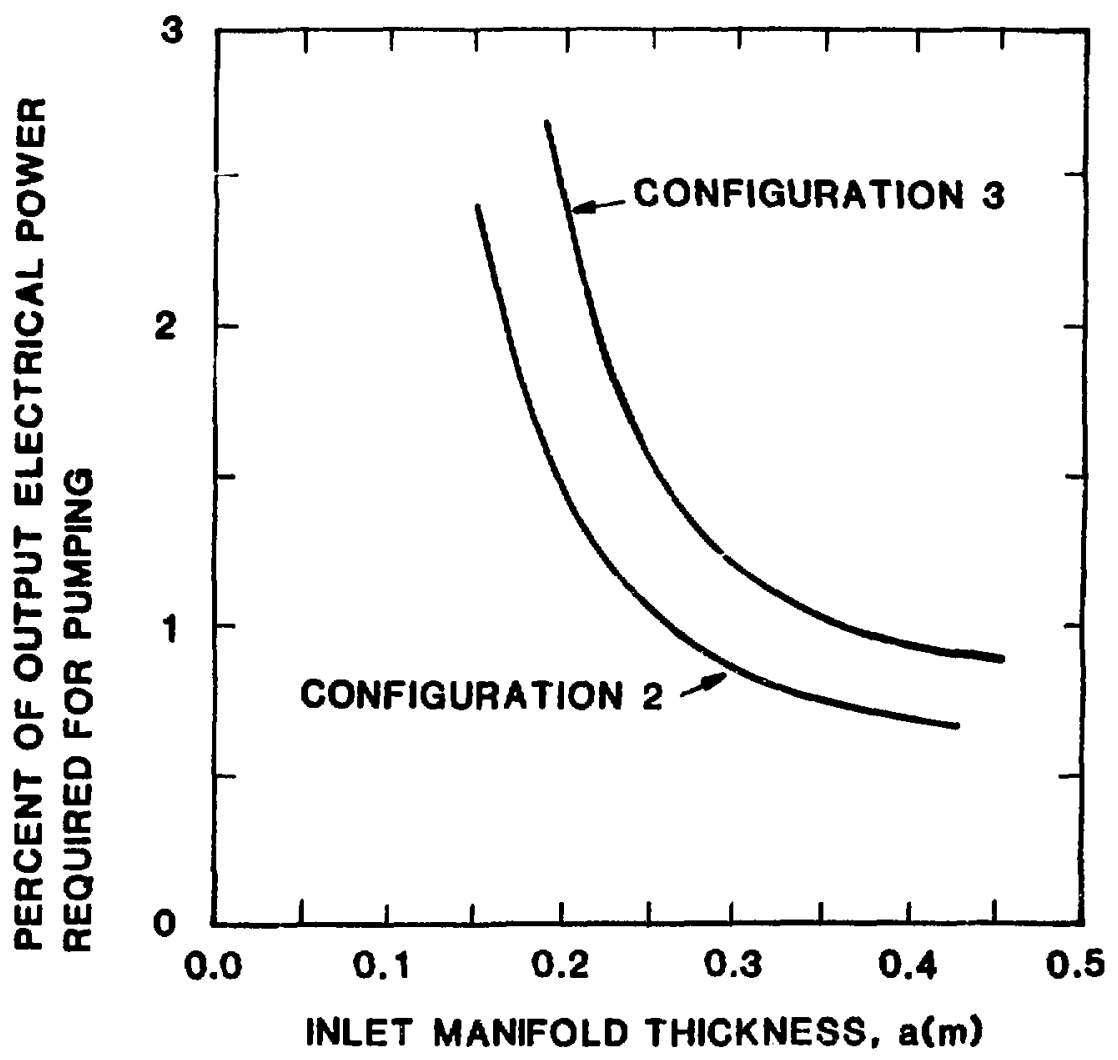

F1g. III.E-4. Pumping power as a function of manifold thickness (height) for the paralle1-flow configurations 2 and 3 shown in Fig. III.E-2. 
relatively uniform temperature proffle. The presence of magnetic flelds, however, greatly suppresses turbulence, and the flow is expected to be laminar for the flow rates and channel dimenslons used here. The rate of energy-generation within the blanket decreases exponentially with distance 1nto the blanket, so that the much higher energy-generation rates near the first wall will result in a highly nonuniform transverse temperature distribution. It is necessary, therefore, to ensure that $\mathrm{PbL1}$ temperatures in the vicinity of the structural steel do not exceed a specified (corrosion-related) 11mit. This 11mit Imposes a major design constraint and was accepted for design study, although the presence of flow jets and other energy-distributing phenomena may alleviate the impact of the constraint on the thertual cycle efficiency (i.e., decrease the power delivered to the lower-temperature second-wall water coolant). Furthermore, the corrosion-rate limits used to establish structural temperatures are generally based on coolant-duct plugging rather than structural thinning constraints, the former being sensitive to the specific size (surface area) and configuration. Hence, the key driving function for this blanket design that leads to adaption of a water-cooled structural wall on the inboard side of the blanket can very well be changed by more detailed design, and a number of reasons for eliminating this complication can be cited.

The interfacial temperature was investigated with the two-dimensional, time-dependent, finite-element, heat-transfer code.124 Laminar flow of PbLi through a channel of spesified thickness was analyzed for the flow rates estimated in the previous calculations using the energy absorption rates obtained from the neutronics analysis (Sec. III.B.). Heat flow through a thermal resistance to water at $473 \mathrm{~K}$ was used for the boundary condition at the second wall. The results of the thermal analysis on the blanket are shown in Fig. III.E-5 for channels of thickness 0.15 and $0.25 \mathrm{~m}$. The thickness of the channel nearest the first wall should be limited to approximately $0.15 \mathrm{~m}$ in order to maintain the structural temperature below $773 \mathrm{~K}$. As noted above, a number of other means for a) maintaining this structure temperature limit or b) allowing it to increase somewhat can be envisaged. Until better design and/or data bases are available, however, the water-cooled second wall is adapted for this design (Fig. III.D-3).

Figure III.E-4 shows a decrease in pumping power with increasing manifold thickness. The channel helght for the analysis shown in Fig. III.E-4 assumes the manifold covers the entire outboard circumference of the torus. 


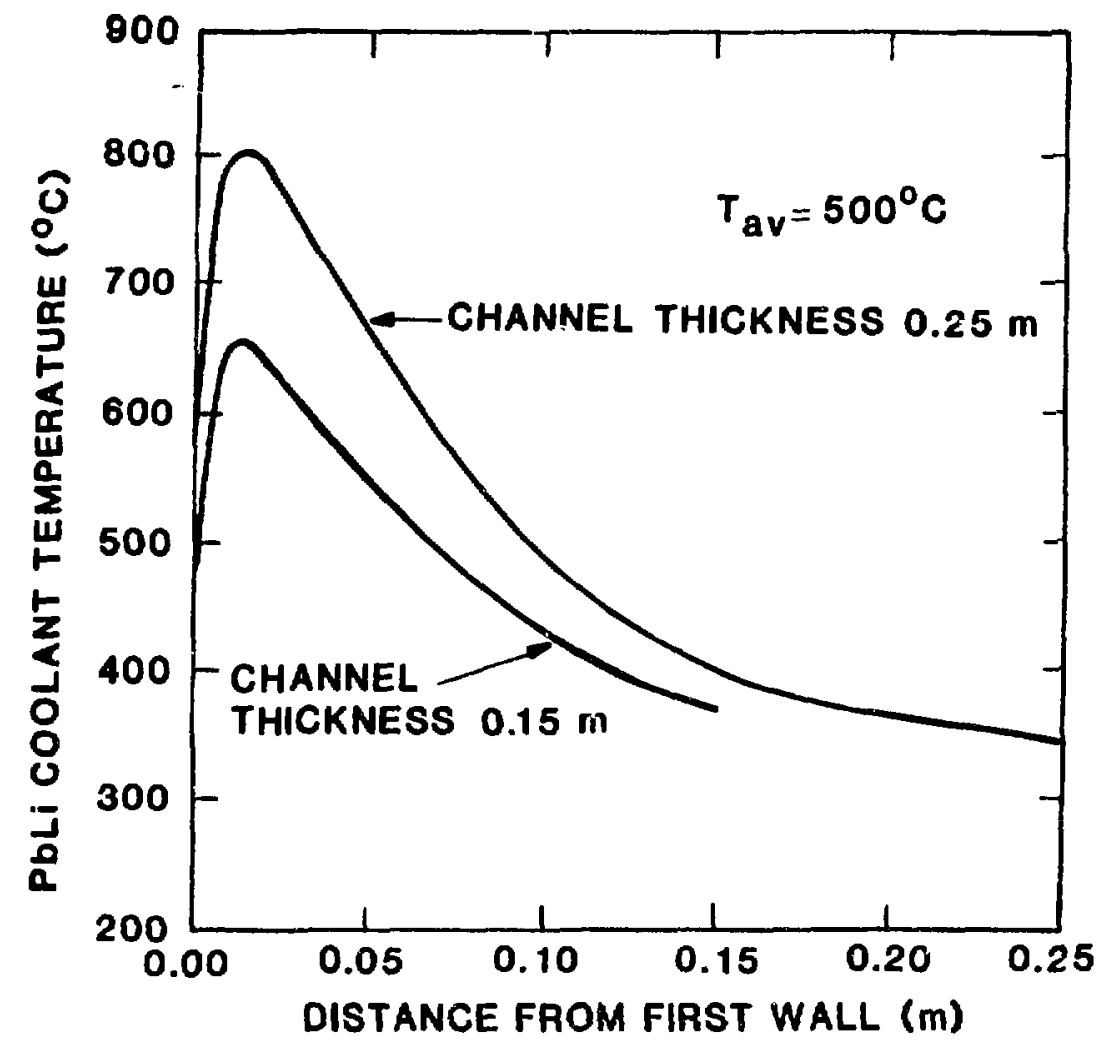

Fig. III.E-5. Transverse temperature distribution at the channel exit for the high-performance Inboard coolant channel.

Interference by the toroldal-field colls (Secs. III.F. and III.L.) require that the channel width be 1 imited to about $56 \%$ of the circumferential distance, resulting in the channel height being increased by $\sim 30 \%$. A value of $a=0.3 \mathrm{~m}$ was chosen as a satisfactory tradeoff because of the relatively low pumping power without an excessively large manifold. The thicknesses of the inside channels for configurations $\# 2$ and $\# 3$ are 0.274 and $0.167 \mathrm{~m}$, respectively. Based on the limitation caused by the transverse temperature difference and structural temperature 1imits, confj.guration \#3 was selected. Additional adjustments of design parameters were made to satisfy the restriction that the PbLi temperature in contact with stainless steel be less than $773 \mathrm{~K}$. A sumary of the resulting blanket design is given in Sec. III.L.2. (Table VI.L-V).

Stress analyses have not been performed for the blanket structure. It is anticipated that steel struts will be used to maintain proper spacing between channel walls. In anticipation of total force limics on a number of flat surfaces associated with this blanket structure, an upper limit of $\sim 1.4 \mathrm{MPa}$ 
(200 psi) was adapted for the PbLi coolant pressure in the parametric study reported in Sec. III.L.2, and used for the Einal design-point selection. The FW/B mechanical design will be governed primarlly by the desire to use "block" or single-plece maintenance schemes, wherein the FW/B along with the TFCs and coolant ducting would be removed as a single unit; the feasibility of such an operation rests with an ability to limit the total mass of the FPC.

3. Thermal Analysis of Shield. Initial FW/B neutronics parametrics studies selected a 0.50 -m-thick PbLi blanket as adequate from the viewpoint of tritium breeding. Subsequent analyses (Sec. III.B, Fig.III.B-4) indicated the benefit of adding an additional $\sim 0.10-m$-thick shield outside the breeding blanket in order to enhance the efficiency of thermal-energy recovery and to reduce the thermal loading on the colls. This $\mathrm{B}_{4} \mathrm{C} / \mathrm{W}$ shield region could in principle be cooled by the flowing PbLI blanket coolant or by a separate pressurized-water coolant, with a somewhat reduced thermal cycle efficiency resulting from the later approach. This section considers the thermal-hydraulic aspects of both a PbLi-cooled and a water-cooled shield options. Based on configurational design choices made as part of the FPC integration task (Sec. III.L.), a pressurized-water shield coolant is selected, the shleld coolant stream being combined within the blanket envelope with the FW/limiter exit-coolant stream to produce a single exit manifold of pressurized water for use in the dual-media stream cycle.

Approximately $94.5 \%$ of the energy generated by the plasma and multiplied in the FPC is absorbed in the first wall, limiters, and blanket. The remainder penetrates the blanket with $4.20 \%$ deposited into the shielding provided between the blanket and the toroidal- and poloidal-field colls to maxinize the recovery of thermal energy; the remaining $1.3 \%$ is deposited into the room-temperature colls. Energy at a rate of $143 \mathrm{MW}$ is absorbed in this shleld. The temperature. distribution within the material for a PbLi-cooled shield was computed from a one-dimensional steady-state conduction analysis using the energy-generation distribution generated by the neutronics analysis; results are summarized in Table II.E-II.

A study of the manffolding required to deliver coolant to the first wall, limiters, blanket, and shield, as well as design of the vacuum ports required for the pumped limiter, indicates that the shielding is best separated from the blanket to form both a vacuum plenum and a primary structural support. This separation makes less practical cooling the shield with PbLi blanket coolant. 
In this case, pressurized water would be used as the coolant. The shield configuration would be identical to the PbLi-cooled case (Table III.E-II), except for coolant-channel widths and the addition of a third channel in contact with the inside surface. Design parameters for a water-cooled shield are given

\section{TABLE III.E-II}

SHIELD THERMAL-HYDRAULIC DESIGN PARAMETERS (PbLL COOLANT)

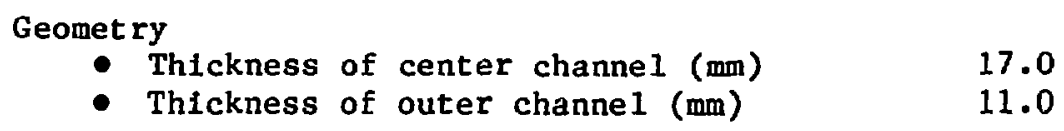

- Segment $1\left(\mathrm{~B}_{4} \mathrm{C}\right) \quad 75$

- Segment 2 (W) 40

- Segment $3\left(\mathrm{~B}_{4} \mathrm{C}\right) \quad 38$

- Segment $4(W)^{4}$

$\begin{array}{ll}\text { Total } & 143\end{array}$

TABLE III.E-III

SHIELD THERMAL-HYDRAULIC DESIGN PARAMETERS (WATER COOLANT)

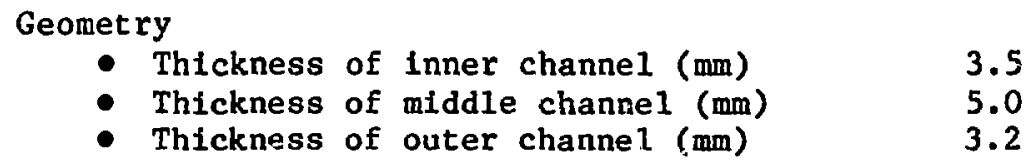

- $\mathrm{H}_{2} \mathrm{O}$ inlet 423

- $\mathrm{H}_{2} \mathrm{O}$ exit 503

- Maximum temperature In third wall 728 
In Table IIL.E-III. The water-cooled shield is adapted as the design basis for the FPC integration.

4. First-Wa11/Blanket/Shield Integration. Although the full integration of key FPC subsystems is addressed in Sec. III.L., some statement of the coolant-circuit integrations seems appropriate at this point. Figure III.E-6 gives a schematic diagam of the FPC coolant flows. As indicated in F1g. III.D-3, the water-cooled first-wall D-tubes would be brazed to a segment of a toroldal surface fabricated from a 18-mm-thick MZC copper alloy. Depending on the (passive) electrical function of the first wall as well as structural and neutronic consideration, this thickness cain be reduced. These $D$-shaped coolant tubes are arranged to provide flow in the toroidal direction. A manifold connection would join the first-wall coolant tubes to the flow channels fabricated within the second wall, thereby providing a coolant return path. Radial headers near the outboard equitorial plane would take the water coolant to and from each of $N=24$ toroldal sectors, these headers supplying pololdal manifolds in each sector for the limiter, first wa11/second wall, and ahield. The poloidal manffolds for the FW/SW system within each of 24 toroldal sectors could be located near the first-wall region and integral with the FW/SW system, or these manifolds could be located at the shield region with radial tube sheets supplying and returning coolant for each FW/SW system. To reduce the number of tubes within the B/S region, the first option was selected to demonstrate the FPC integration in Sec. III.L. ThIs option seriously reduces the tritium-breeding ratio, requiring a thinner copper first wall, $90 \% \sigma_{L 1}$ enrlchment, and a higher-albedo shield (Appendix $C$ ).

The PbLi coolant circulates through the blanket in the poloidal direction. The PbLI manifolding would also enter and exit the torus along the equatorial plane in the outboard direction. The torus may be constructed in four to elght modules if this approach is found to simplify the installation and removal of the FW/B/S/TFC assembly, compared to totally "block" or single-piece maintenance. In any case, the number of basic building blocks for off-site factory assembly would be $N=24$. Ultimately, this choice rests with the FPC mass, which will be determined through economics by the FPC radiation Iffetime. 


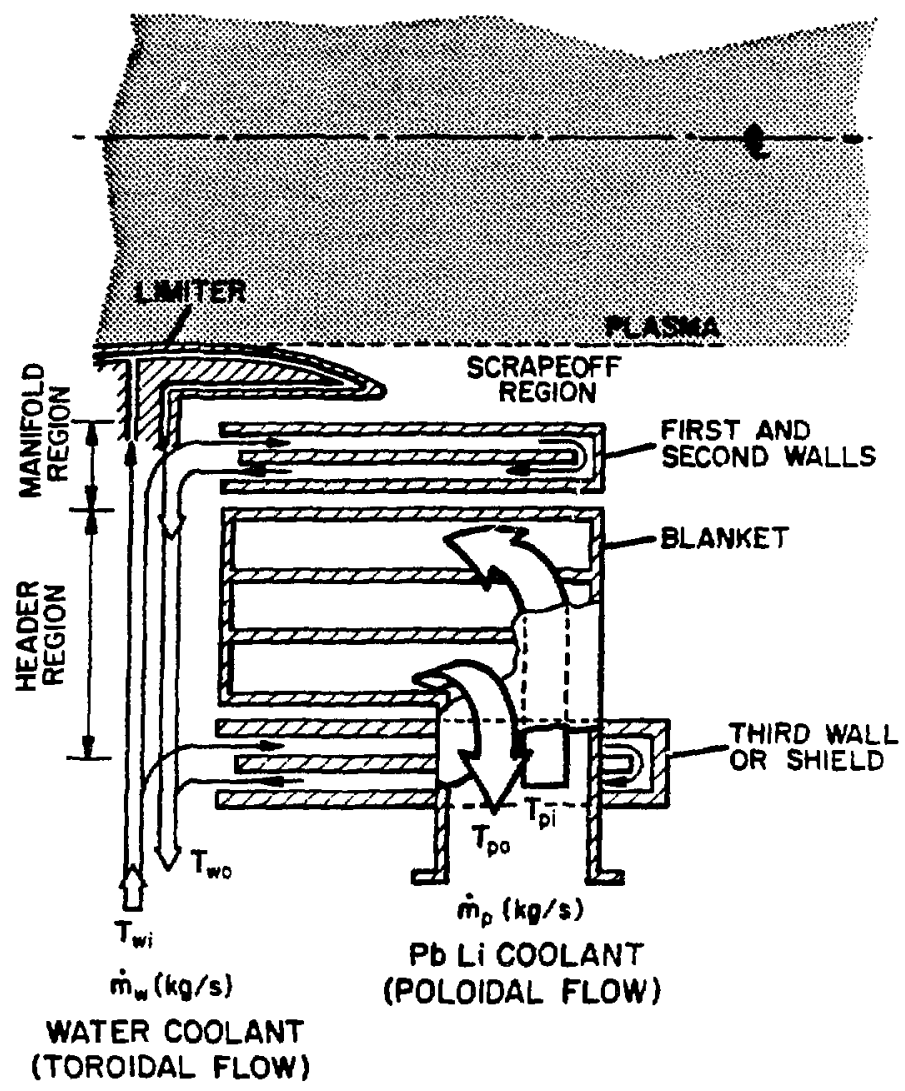

Fig. III.E 6. Schematic diagram of pressurized-water and PbLI coolant flow in one of $N \approx 24$ toroldal sectors forming the FPC. 
The CRFPR design point described in Table II.D-III requires the nominal toroidal-field-coil (TFC) and pololdal-field-coll (PFC) parameters listed on Table III.F-I. The simplified magnet model used in the parametric systems model, 15 from which Table III.F-I was generated, lumps the TFCs and PFCs into composite conductors with a representative conductor filing fraction $\left(\lambda_{c} \simeq 0.7\right)$ being specified to estimate ohmic losses (recirculating power), coll masses (and cost), and system power density (independent parameter). Figure III.E-3 illustrates this geometry and defines key notation. To advance the reactor design from the level of the Ref. 15 parametric study, both the TFC and PFC designs must be considered in more detail. The TFCs would be affixed to the FW/B/S system, forming a single toroidal unit that would be amenable to block maintenance. The PFCs on the other hand, would in part be moved to allow a

TABLE III.F-I

NOMINAL COIL PARAMETERS FOR CRFPR DESIGN

ESTIMATED FROM PARAMETRIC SYSTEMS CODE(a)

\begin{tabular}{|c|c|c|}
\hline PARAMETER & $\underline{\mathrm{TFC}}$ & $\underline{\text { PFC }}$ \\
\hline Coil thickness, $\delta_{c \phi, \theta}(\mathrm{m})$ & 0.09 & 0.36 \\
\hline Current-center minor radius, $r_{c \phi, \theta}(\mathrm{m})$ & 1.39 & 1.62 \\
\hline Total coll-set mass (tonne) & 149 & 703. \\
\hline Magnetic field at conductor, $B_{c \phi, \theta}(T)$ & $4.5 \rightarrow-0.92^{(b)}$ & 2.3 \\
\hline Stored energy, $\mathrm{W}_{\mathrm{BC} \phi, \theta^{(M J)}}$ & 542. & 1111. \\
\hline \multicolumn{3}{|c|}{ Other parameters used in magnetics calculations (from Table II.D-III) } \\
\hline \multicolumn{3}{|l|}{ - Major radius, $\mathrm{R}_{\mathrm{T}}(\mathrm{m})$} \\
\hline \multicolumn{3}{|c|}{ - Reversed toroldal field at plasma edge, ${ }^{B}{ }_{R \phi}(T)$} \\
\hline \multicolumn{3}{|l|}{ - Average plasma radius, $r_{p}(m)$} \\
\hline \multicolumn{3}{|l|}{ - First-wall radius, $r_{w}(m)$} \\
\hline \multicolumn{3}{|l|}{ - Blanket thickness, $\Delta b(\mathrm{~m})$} \\
\hline \multicolumn{3}{|c|}{ - TFC and PFC current densities, $j_{c \phi, \theta}\left(\mathrm{MA} / \mathrm{m}^{2}\right)$} \\
\hline - Pololdal field at plasma edge, ${ }^{B}{ }_{\theta}($ & 5.22 & \\
\hline
\end{tabular}

(a) Dimensions given do not reflect the added radial build that may be required for a pololdal vacuum plenum (Sec. III.L.3.).

(b) Initial (preplasma) bias field on axis could be as high as $B_{\phi_{0}}=3.24 \mathrm{~T}$, although the startup procedure described in Sec. III.G.2. Could limit $B_{\phi o}$ (setup) to $B_{R \phi}$ (ignition/burn), the low-level reversed field at ignition. 
vertical, en masse movement of the FW/B/S/TFC unit during the block maintenance operation (Sec. III.L.3.). This section gives the results of magnetics computations used to estimate the position, size, and support of TFCs and PFCs, which along with other key constraints (e.g., field ripple, magnetic islands, inductances, peak startup power, maximum voltages, etc.) needed to perform the FPC integration study.

1. Toroidal-Field-Coil System. The TFCs provide the initial bias field, $B_{\phi o}$, on which the RFP is first formed, and subsequently maintains the external reversed field, $B_{R \phi}$, with a minimum ripple in toroidal field at the plasma edge. A ripple condition is used to obtain a preliminary estimate of the discretized TFC set. As summarized in Table III,F-I and illustrated in Fig. III.F-1, the parametric systems code predicts a homogeneous (i.e., toroidally cnntinuous) TFC set of radial thickness $\delta_{c \phi} \simeq 0.09 \mathrm{~m}$ with a current center at $\mathbf{r}_{\mathbf{c} \phi} \simeq 1.39 \mathrm{~m}$ relative to a major coll radius of $R_{T}=3.79 \mathrm{~m}$. The dimensions of the discretized TFC set are obtained by specifying $N=24$ coils, each fitting snugly at the inboard equatorial plane around a $\Delta b=0.6-m-t h i c k$ blanket and shield that has a first wall positioned at $r_{w}=0.75 \mathrm{~m}$. The resulting toroidal extent of each TFC, $\ell_{c \phi}$, is used in conjunction with the TFC current density, $j_{\phi c}=6.06 \mathrm{MA} / \mathrm{m}^{2}$, obtained from the Ref. 15 paranetric systems code, to give the radial extent, $\delta_{c \phi}=0.15 \mathrm{~m}$, of the $N=24$ TFCs. The current-center radius is moved from $r_{c \phi}=1.39 \mathrm{~m}$ to $1.43 \mathrm{~m}$ to make allowance for the discretization process. These conditions apply to the steady-state operating conditions, $\mathrm{B}_{\mathrm{R} \phi}=-0.68 \mathrm{~T}$. Compared to the initial on-axis bias field, $\mathrm{B}_{\phi 0}=3.34 \mathrm{~T}$, required to inject all toroidal flux, this field level corresponds to a TFC overrating during startup by an increase by a factor of $\sim 4.9$ in TFC current density $\left(6.06\right.$ to $\left.30.0 \mathrm{MA} / \mathrm{m}^{2}\right)$ for a brief startup period should a dynamo-assisted startup prove impractical (Sec. III.F.2.). The dynamo-assisted startup, however, allows the initial toroidal bias field to equal approximately the final reversed field; the dynamo wculd increase the toroldal field internal to the plasma as the plasma current is increased, ignition is approached, and the minimum-energy RFP state is maintained for the appropriate $F-\theta$ values.

The discrete TFC set introduces a toroidal-field ripple with a periodicity and magnitude determined by the number of colls and their dimensions. The magnitude of this ripple is largest on the outboard side of the plasma and in the equatorial plane for a circular plasma cross section. Figure III.F-2 


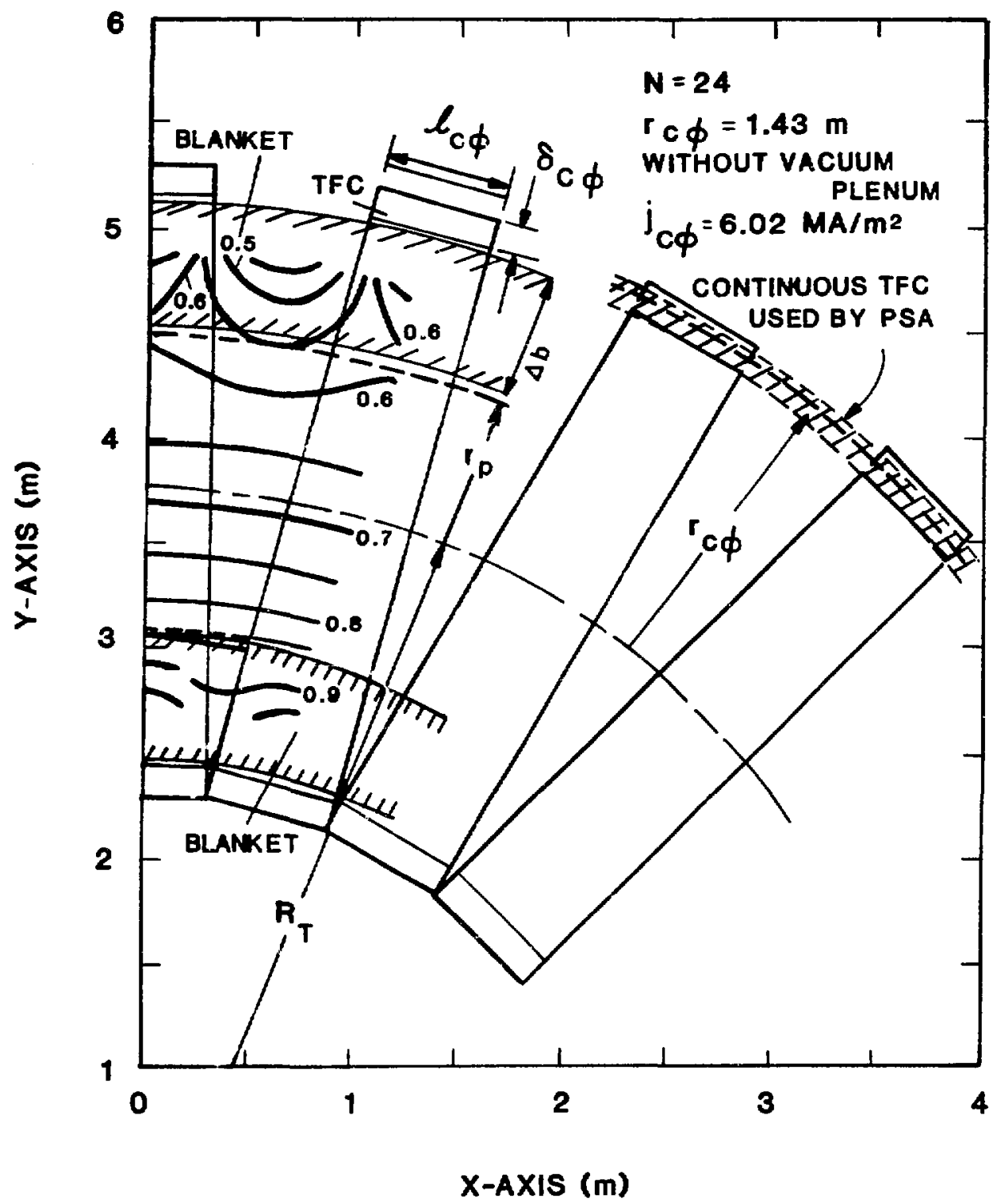

F1g. III.F-1. Toroidal-field-coll layout showing mod-B contours and plasma and FW/B locations, all for $N=24$ colls and in the equatorial plane.

illustrates the toroidal-field ripple and the nomenclature used to quantify the field ripple in order to select the TFC number and dimensions.

A11 RFP ripple criteria are based upon dimensional limitations established by magnetic island widths. Magnetic islands result from the introduction of a spatially oscillating radial field component, ${ }_{R}$, which is present in any discrete TFC set, as shown in Fig. III.F-2. It is noted that magnetic islands are not peculiar to RFPs, but also represent a concern for stellarators and 


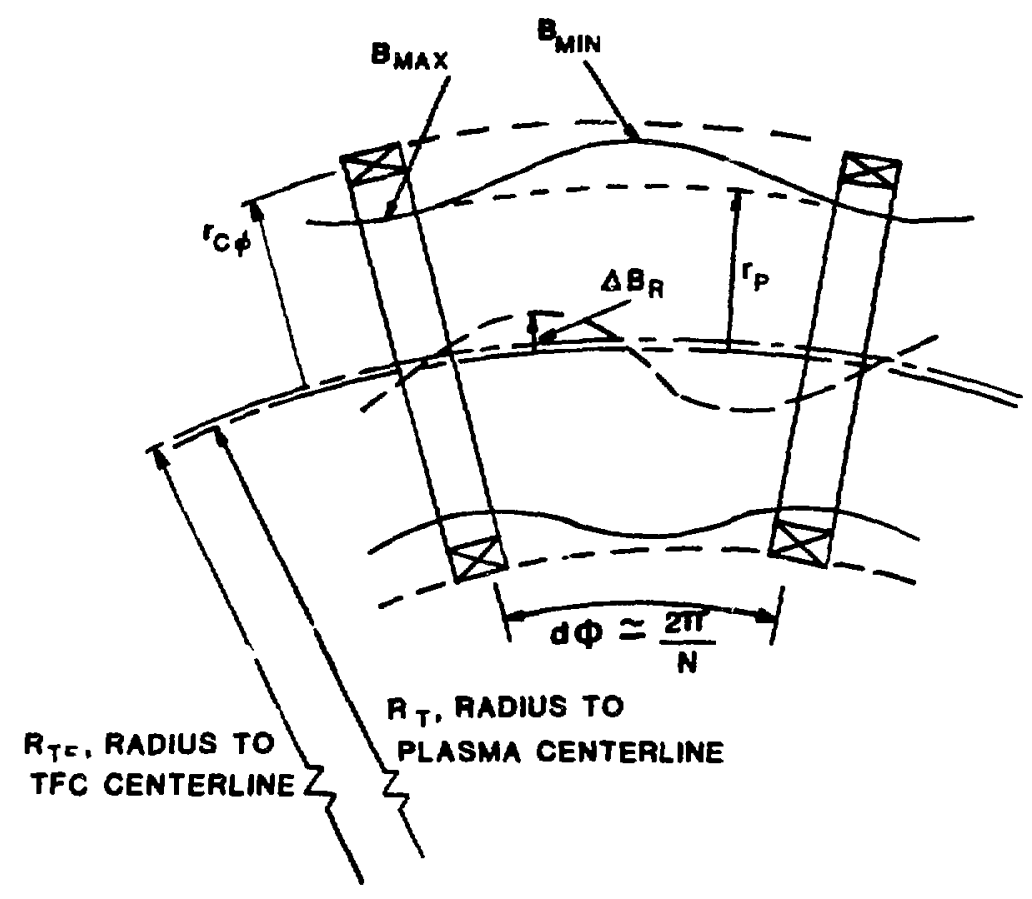

Fig. III.F-2. Schematic diagram of discrete TFC set used to specify ripple constraints.

tokamaks. Magnetic islands are thought to play a role in the tokamak disruption. The island radius, $\Delta r$, is given by 128

$$
\Delta r=4\left[\frac{r \Delta B_{R}}{n B_{\theta}(d q / d r)}\right]^{1 / 2},
$$

where $r$ is the minor radius of the resonant surface, $\Delta B_{R}$ is the amplitude of the radial helical magnetic-field perturbation, $n$ is the toroidal mode number, $B_{\theta}$ is the poloidal field, and $q=\left(B_{\phi} / B_{\theta}\right) /\left(R_{T} / r\right)$ is the safety factor. The correlation between the 1sland width and the Impact on the plasma equilibrium and transport remains to be quantified. Consequent1y, a universally accepted criteria for the maximum $\Delta B_{R}$ is not avallable for $R F P_{s}$, tokamaks, or stellarators even though the importance of minimizing the island size is 
acknowledged. One school of thought asserts that the 1sland radius for RFPs should be smaller than the distance between the plasma radius and the reversal radius to assure field reversal occurs. Another appproach argues that present-day RFP experiments obtain reversal with large field errors (1.e., $\Delta B_{R} / B_{\theta} \geq 0.01$, where $\Delta B_{R}$ is the peak in the radial field component along a field Iine, Fig. III.F-2). A reactor design criterion, therefore, should be no more stringent than an experimental design criterion. The design criteron 129 for the RFP upgrade experiment, $\mathrm{ZT}-\mathrm{H}$, is $\Delta B_{R} / \mathrm{B}_{\theta} \leq 0.003$. Both criteria are examined for the CRFPR with the $\triangle B_{R} / B_{\theta} \leqslant 0.003$ constraint being used here as the design criterion.

The evaluation of Eq. (1) and the application of the first criterion requires an estimate of $\mathrm{dq} / \mathrm{dr}$. The $q$-profile for an $R F P$ is typically taken to be parabollc:

$$
\frac{\mathrm{dq}}{\mathrm{dr}} \simeq \frac{\mathrm{q}}{\mathrm{r} / 2} \simeq \frac{2 \mathrm{~B}_{\phi}}{\mathrm{RB}_{\theta}}
$$

where $\mathrm{B}_{\phi}$ is the reversed toroidal field at the plasma edge. The island radius in the vicinity of the reversal radius is then given by

$$
\Delta r=4\left[r_{p} \Delta B_{R} R_{T} / 2 N B_{\phi}\right]^{1 / 2}
$$

Inherent in Eq. (3) are the assumptions that the toroidal node number, $n$, is given by the number of TFCE, $N$, and that the amplitude of the radial helical magnetic-field perturbation is given by the peak in the radial field component along a field line. The island width citterion for the CRFPR then becomes

$$
\begin{aligned}
\frac{\Delta r}{r_{p}} & \simeq 4\left[\left(\Delta B_{R} / B_{\phi}\right)\left(R_{T} / r_{p}\right) / 2 N\right]^{1 / 2} \\
& <1-r * / r_{w} \simeq 0.04 .
\end{aligned}
$$

where $r *$ is the radius of the reversal surface. An estimate of $\Delta B_{R}$ is obtained 
by tracing two field lines near the plasma surface, $r_{p}$, with the threedimensional vacuum-field magnetics code, TORSIDO, 51 simulating only the TFC set. The field-line traces begin at the point $\left[R=R_{j} \pm r_{p}, z=0\right\}$ and a toroidal angle $\phi_{\mathbf{n}}$ defining a given TFC plane for a given field period. The TORSIDO code monitors the field components along these field lines to determine the maximum of the radial field, $\Delta B_{R}$.

For the ideally positioned TFC sets considered by TORSIDO, ${ }_{R}$ produces no net radial displacement of field lines (i.e., $\left\langle B_{R}\right\rangle=\sqrt{ } \mathrm{Bd} / / \int \mathrm{d} \ell=0$ ). Modeling of the field errors inherent in coil construction and alignment that produce a net $\left\langle B_{R}\right\rangle$ is beyond the present level of this design; only the peak radial displacement (Fig. III.F-2) is used as a measure of $\Delta B_{R}$.

A11 cases examined by TORSIDO require the TFC to fit snugly to the blanket/shield only on the inboard side at the equatorial plane $\left(R=R_{T}-r_{p}-\Delta b, z=0\right)$. This condition is expressed as follows:

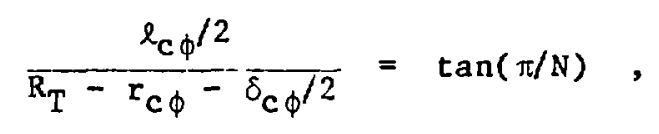

where $r_{c \phi}, l_{c \phi}$, and $\delta_{c \phi}$ are defined in Fig. III.F-l. The TFC conductor cross-sectional dimensions are related through the current density:

$$
j_{c \phi} \ell_{c \phi} \delta_{c \phi}=I_{c \phi}
$$

where $j_{C \phi}$ is the TFC current density and $I_{C \phi}$ is the TFC current per coil. Furthermore, the product $\mathrm{NI}_{c \phi}$ is held constant in order to preserve the on-axis average toroidal field at $B_{R \phi}=-0.68 \mathrm{~T}$. Equations (5) and (6) are solved simultaneously for the cofl dimensions. Parameters held fixed are listed in Table III.F-II. The results of simultaneousiy varying the number of TFCs, $N$, and the center of the TFC set, $R_{T F}$, are presented in Figs. III.F-3 and III.F-4 for the criteria established by $\Delta B_{R} / B_{\theta} \leqslant 0.003$ and $E q .(3)$. The parameterization of $\mathrm{P}_{\mathrm{LF}}$ achieves a reduction in rippie by only moving the outboard leg of the TFC outward from the blanket, thereby sitifing the peak ripple from the outboard field line to the inboard field line. An accompanying increase in the coil minor radius is given by 


$$
r_{c \phi}=1.43+2\left(R_{T F}-R_{T}\right)
$$

The CRFPR design point (Table II.D-III) with $R_{T E}=R_{T}=3.8 \mathrm{~m}$ and $N=24$ marginally complies with the constraint of $\Delta B_{R} / B_{\theta} \leqslant 0.003$, as seen in F1g. III.F-3. This design point requires an increase in $N$ and/or $R_{T F}$ to comply with the constraint of Eq. (4) shown in Fig. III.F-4. The assumptions regarding mode number $(n=N), d q / d r[E q \cdot(2)]$, and the location of the resonant surface (at $r_{p}$ ) should be noted when considering the results of $\mathrm{FIg}$. III.F-4. In additior, the numerical results of varying only the number of TFCs are presented In Table III.F-II for both the adjusted design point, $r_{c \phi}=1.43 \mathrm{~m}$, and the case where a vacuum plenum of nominal radial extent $(\sim 0.10 \mathrm{~m})$ displaces the TFCs radially outward; the need for a vacuum plenum can increase $r_{c \phi}$ to $1.53 \mathrm{~m}$. The $N=24$ case $\left(r_{c \phi}=1.43 \mathrm{~m}\right)$ has been shown in Fig. III.F-1. Figure III.F-5 gIves

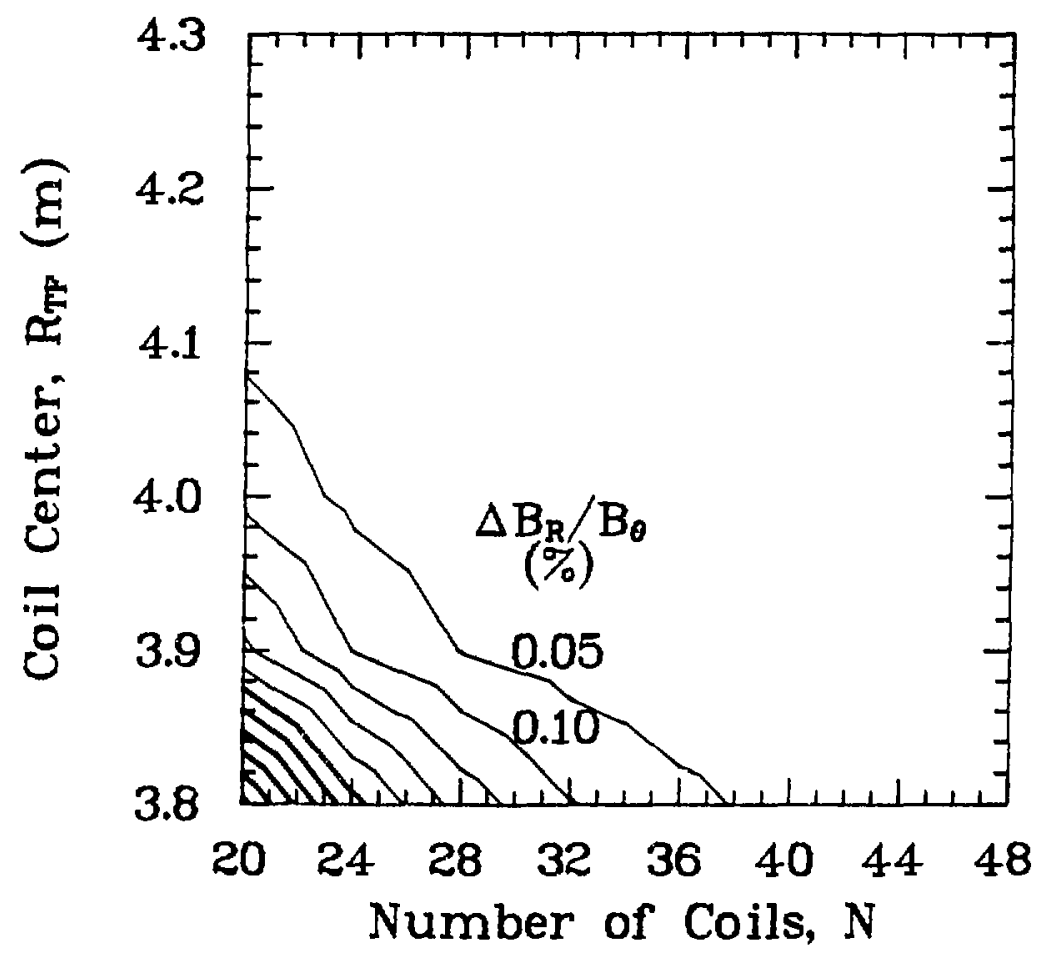

Fig. III.F-3. Equal-increment contours of $\Delta B_{R} / B_{\theta}$ plotted versus the number of TF colls and the location of the TFC center. Compliance with $\Delta B_{R} / B_{\theta}<0.003$ occurs in region of thinner contour lines. 


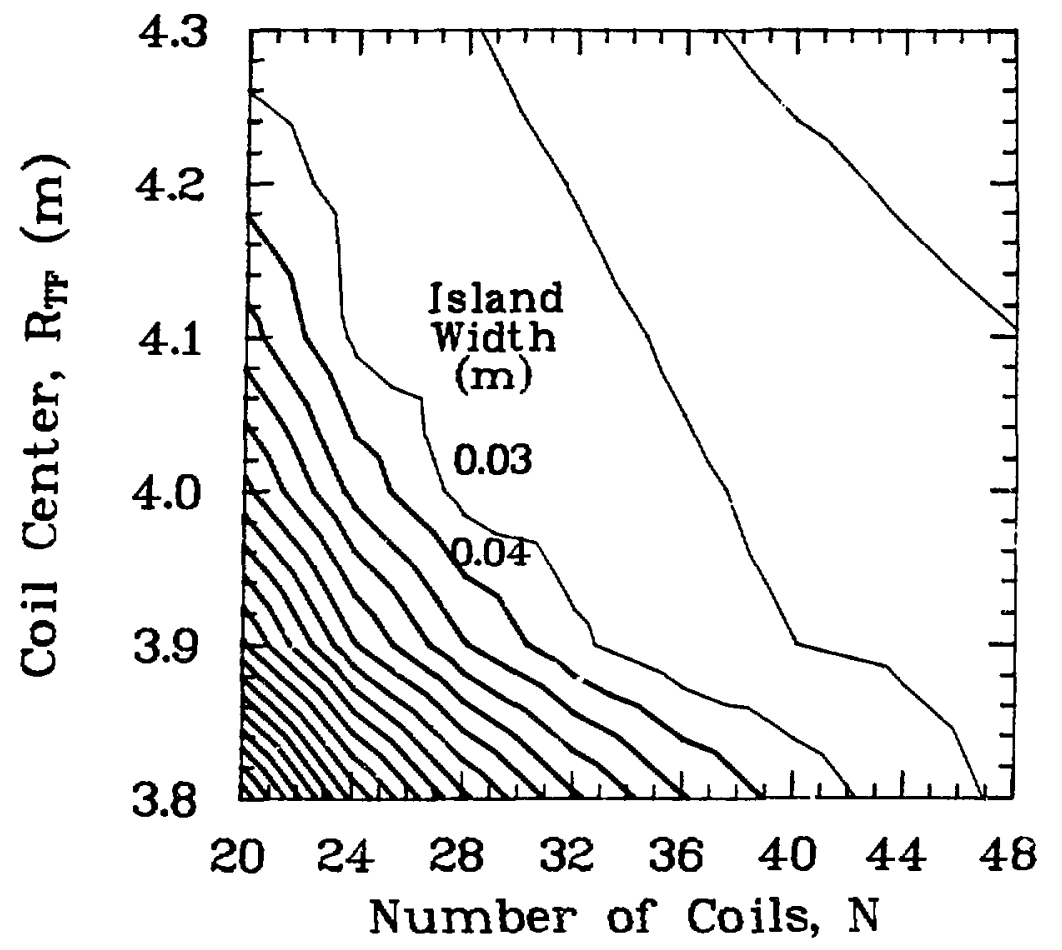

FIg. III.F-4. Equal-increment contours of $\Delta \mathrm{r}$ plotted versus the number of TFCs and the location of the TFC center. Compliance with Eq. (4) occurs in region of thinner contour lines.

the dependence of $\Delta B_{R}$ on toroldal and radial position for $N=24$ and $r_{c \phi}=1.43 \mathrm{~m}$ or $1.53 \mathrm{~m}$. The $N=24$ cases serve as the basis for formulating a design to be used in the FPC integration (Sec. III.L.). A vacuum plenum of 75-mm radial thickness can be accommodated in the radial build by doubling the current density to $12.02 \mathrm{MA} / \mathrm{m}^{2}$ and halving the radial thickness of the $r_{c \phi}=1.43 \mathrm{~m}$ case while maintaining the outer-bore diameter constant. The coll dimensions are then $r_{c \phi}=1.4625 \mathrm{~m}, \delta_{c \phi}=75 \mathrm{~mm}$, and $l_{c \phi}=0.60 \mathrm{~m}$. Use of the modified Bessel-function model in the burn simulation reduces the reversed toroldal field at the plasma edge from $0.68 \mathrm{~T}$ to $0.403 \mathrm{~T}$. Consequently, both $I_{c \phi}$ and $j_{c \phi}$ can be reduced from $0.54 \mathrm{MA}$ and $12.02 \mathrm{MA} / \mathrm{m}^{2}$ to $0.32 \mathrm{MA}$ and $7.124 \mathrm{MA} / \mathrm{m}^{2}$, respectively. The ohmic power dissipated in the TFC set, assuming copper coils with a $70 \%$ packing fraction, is given by $\mathrm{P}_{\mathrm{TFC}}^{\Omega}=1.6(10)^{-7} r_{\mathrm{c} \phi}{ }_{\mathrm{j}} \phi^{\mathrm{I}} \mathrm{c} \phi^{\mathrm{N}}$, which yields $\mathrm{P}_{\mathrm{TFC}}^{\Omega}=12.6 \mathrm{MW}$. The $\mathrm{TFC}$ design parameters are summarized in Table III.F-III. 
SUMMARY OF RIPPLE AND RADIAL FIELD CALCULATIONS MADE WITH THE TORSIDO 51 CODE FOR TFC CURREN'I RADII $r_{c}=1.43 \mathrm{~m}$ (without yacuum plenum) AND $r_{c \phi}=1.53 \mathrm{~m}(0.10-\mathrm{m}$ vacuum $p l e n u m)(a)$ FOR

IFCs CLCSELY FITTING THE B/S

\section{PARAMETER}

Number of TFCs, N

Radial fiuctuation of field Iine at outboard plasma surface $\delta_{R}(\mathrm{~mm})$

Average magnetic field along outboard field line at plasma surface $(\mathrm{T})$

Average magnetic field along inboard field line at rlasma surface (T)

TFC current, $I_{\phi}(M A)$

TFC radial thickness, $\delta_{\mathbf{c} \phi}(\omega)$ TFC toroidal length, $\ell_{c \phi}(m)$ Distance from plasma to TFC, $r_{c \phi}-\left(\delta_{\phi c} / 2+r_{p}+\delta_{R}\right)$

Ripple constraint, $\Delta B_{R} / B_{\theta}\left[B_{\theta}=5.22 T\right]$
20

$32.3(22.2) 14.6(9.3)$

0.57

0.84

0.64

0.15

$0.72(0.69) 0.60(0.57)$

$0.61(0.72) 0.63(0.73)$

$0.64(0.74)$

$0.64(0.74)$
32

(a) Values in parentheses pertain to an increase in the radial bulld caused by including a $0.10-m$ vacuum plenum; otherwise conditions remain unchanged.

2. The Pololdal-Field-Coil System. The RFP plasma is heated and confined primarily by the self-magnetic flelds generated by a strong plasma current, $I_{\phi}$, which combine with the internal toroidal fleld to produce the stabilizing highshear fleld/pressure profiles. The PFC systems, therefore, play a major role in the reactor design. The fundamental processes involved with an RFP startup are illustrated in Fig. III.F-6. An initial toroidal blas fleld, $B_{\phi_{0}}$ is produced by the TFC system. The plasma is initiated, and a changing PFC current, $I_{c \phi}$, Induces the plasma current by transformer action. The trapped initial bias 


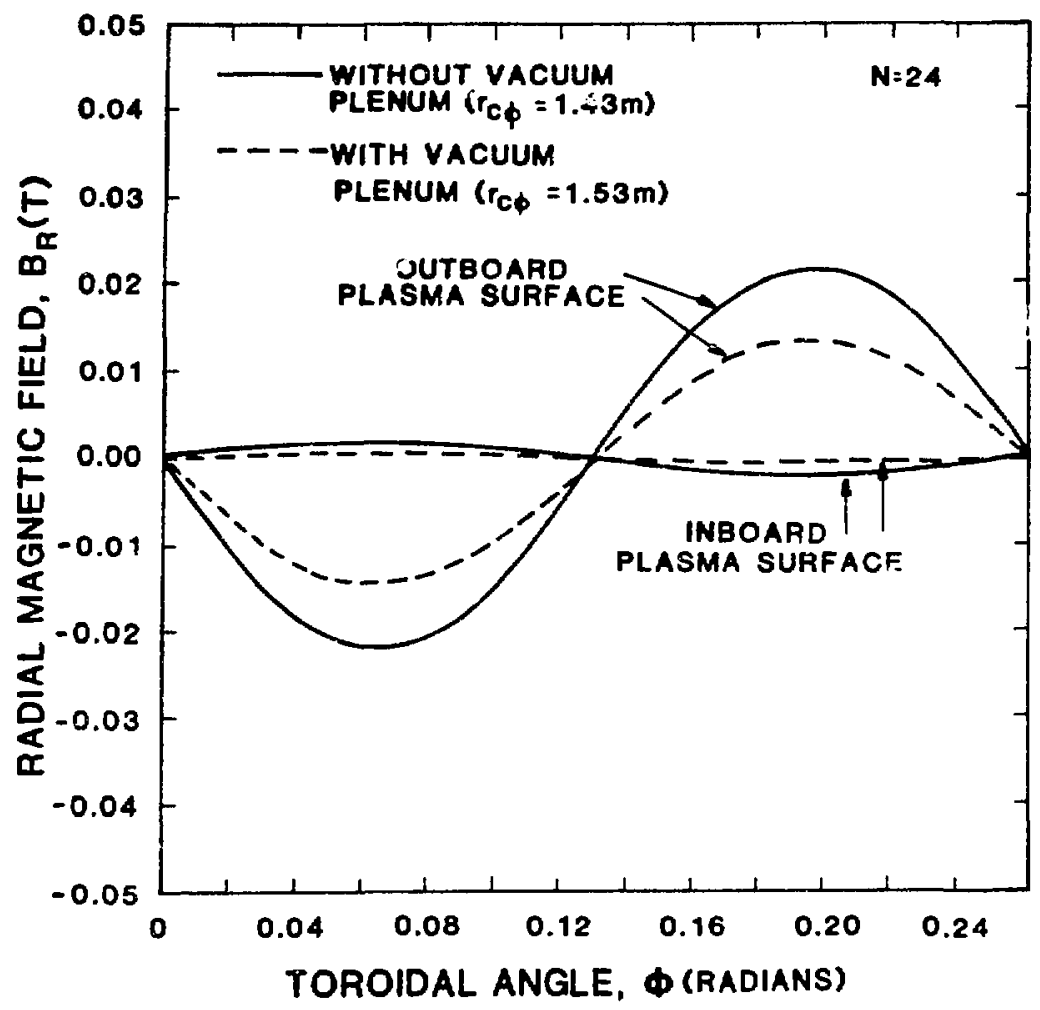

Fig. III.F-5. Radial magnetic flelds for cases with and without $0.10-m-t h i c k$ vacuum plenum for $R_{T}=3.79 \mathrm{~m}, B_{\phi}$ (axis) $=0.68 \mathrm{~T}, \mathrm{r}_{\mathrm{p}}=0.71 \mathrm{~m}$, and $j_{\phi c}=6.02 \mathrm{MA} / \mathrm{m}^{2}$.

field is compressed by the plasma current, $\mathrm{I}_{\phi}$. Simultaneously, the TFC current, $I_{c} \theta$ is reduced and ultimately reversed, achieving the familiar RFP configuration depicted in the last frame in Fig. III.F-6. The bulk of the torotdal-field energy is then provided through the dominant PFC system. Once an initial, low-energy RFP state is achieved, the plasma dynamo would be used to increase further the toroidal field trapped in the plasma. The near-minimumenergy RFP state is maintaine ${ }^{2}$ as $I_{\phi}$ is increased, turbulent plasma processes converting $I_{\phi}$ into pololdal current, $I_{\theta}$, which in turn increases the toroidal field trapped within the plasma column as $F$ and $\theta$ remain consiant near the minimum-energy state (Fig. II.D-2). The toroidal fleld required initially within the plasma, therefore, can be made small, with a major part of the required $B_{\phi}$ being added through the $F F C$ circuit and the dynamo effect. If the TFC system is sized to produce an initial field, $B_{\phi O}$, that is equal and opposite to the required reversed toroldal fleld, $B_{R} \phi$, the $R F P$ configuration would be 
TABLE III.F-III

TFC DESIGN PARAMETERS

PARAMETER

Major radius, $R_{T}(m)$

Minor radius, $r_{c \phi}(m)$

Radial thickness, $\delta_{c \phi}(\mathrm{mm})$

Toroldal length, $l_{c \phi}(\mathrm{mm})$

Current, $I_{c \phi}(M A)$

Current density, $J_{c \phi}\left(\mathrm{MA} / \mathrm{m}^{2}\right)$

Toroldal field at plasma, ${ }_{\phi}(T)$

Peak toroidal field, $B_{C \phi}(T)$

Ripple constralnt, $\Delta \mathrm{B}_{T} / \mathrm{B}_{\theta}$

Number of TFCs, $N$

Ohmic power, $\mathrm{P}_{\mathrm{TFC}}^{\Omega}(\mathrm{MW})$
VALUE

3.79

1.4625

75

600

0.32

7.124

$-0.403$

0.72

$\leqslant 0.003$

24

12.6

established at approximately 15-20\% of the ultimate plasma current for a reversal parameter, $F=B_{\phi}\left(r_{\omega}\right) /\left\langle B B_{\phi}\right\rangle$, in the range $0.1 \cdots 0.2$. The TFC system under these conditions requires two orders of magnitude less energy than the PFC circuit. Furthermore, the TFC set need not be designed for the startup overload, thereby allowing colls that are even less dominant of the FPC and leading to an easier maintenance scheme.

Before the PFC system can be designed to provide the steady-state (ignited) plasma requirements, as was done for the TFC system in Sec. III.F.1., both the plasma startup operations and a toroldal equilibrium must be defined. The following two sections address these important lssues, with the following third section (Sec. III.G.2.c.) describing the PFC circuit analysis. This more-detalled resolution of the PFC configuration predicts a system that does not differ substantially from the more-approximate predictions of the parametric systems analysis (Table III.F-I and Ref. 15).

a. Startup Operations. The plasma current in a toroidal machine is Induced by transformer action. In addition to producing the required flux change in the center of torus, the PFC system must also genezate a fleld distribution that maintains the plasms equilibrium. Both of the currentinduction and equilibrium-field functions can be provtded by a single coil 


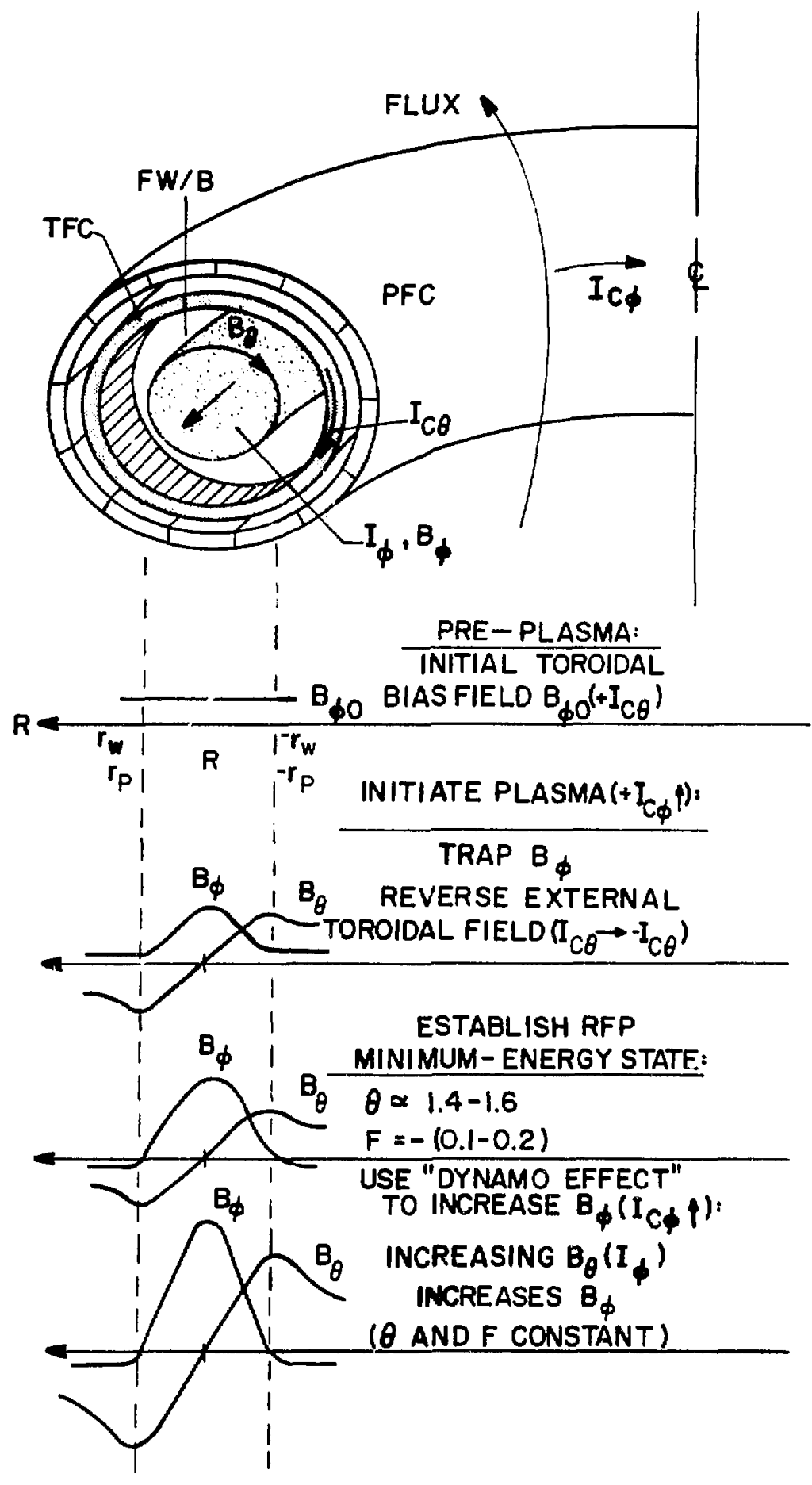

Fig. III.F-6. Fundamental principle of the RFP startup sequence. 
having the nearly uniform current distribution 1llustrated in Fig. III.F-6. This configuration greatly reduces the PFC design options, with separation of the PFC set into flux-drive (Ohmic-Heating Coll, $O H C$ ) and equilibrium (Equilibrium-Field coils, EFC) functions befing more desirable.

The OHC system provides flux change through the torus center while producing no magnetic field within the plasma minor cross section. This coil set, therefore, can be operated independently of the magnitude of the plasma current, which responds only to the change in OHC current and not the absolute current level. The stored energy and absolute fleld level in the OHC system is minimized through bipolar operation, wherein the oHC current is changed from $-I_{c \phi}$ to $+I_{c \phi}$

Plasma equilibrium, on the other hand, depends on the magnitude of $I_{\phi}$ and Is provided by the EFC set, which may or may not induce added flux change and plasma current. The EFC current must produce a nearly uniform vertical-field in the vicinity of the plasma (Sec. III.F.2.b.). The EFC current, $I_{E F}$, must closely track the plasma current, $I_{\phi^{*}}$ Although the EFC may also contribute to the flux swing and drive the plasma current, in the extreme case when all of the ohmic-heating function is integrated into the EFC set, the entire PFC current must be operated with a less-efficlent unipolar drive.

Energy can be transferred to the PFC system directly from the electrical power grid or from an intermediate energy store that initially is slowly charged from the power grid and subsequently discharged into the PFC system on a shorter time scale. The simplified circult depicted in Fig. III.F-7, where the PFCs (i.e., OHCs + EFCs) are approximately represented by a single coil, illustrates the power transfer options. The inductances designated by $\mathrm{L}_{I N}$ and $\mathrm{L}_{\mathrm{EX}}$ are assoclated with the regions or fluxes internal and external, respectively, to the PFC. An Iron core $\left(L_{E X} \rightarrow \infty\right)$ ideally couples the PFC current to the plasma current, giving $I_{c \phi} \simeq I_{\phi}$ and thereby requiring a total stored energy of $\mathrm{E}_{\mathrm{IN}}=\frac{1}{2} \mathrm{~L}_{\mathrm{IN}} \mathrm{I}_{\phi}$. The amount of iron required in a reactor would be prohibitive, considerably Increasing the system mass, cost, and maintenance complexity. The PFC current swing for an air-core system is given by flux conservation, $\mathrm{L}_{\mathrm{EX}} \Delta \mathrm{I}_{\mathrm{c} \phi}=\left(\mathrm{L}_{\mathrm{EX}}+\mathrm{L}_{\mathrm{IN}}\right) \mathrm{I}_{\phi}$; taking $\mathrm{L}_{\mathrm{EX}} \sim \mathrm{L}_{\mathrm{IN}}$, the pololdal-current swing becomes $\Delta I_{c \phi} \approx 2 I_{\phi}$

Three basic operating modes for the circuit depicted in Fig. III.F-7 are illustrated in Fig. IIL.F-8, and the decision options are listed in Fig. III.F-9. These three options are defined by whether an external energy 


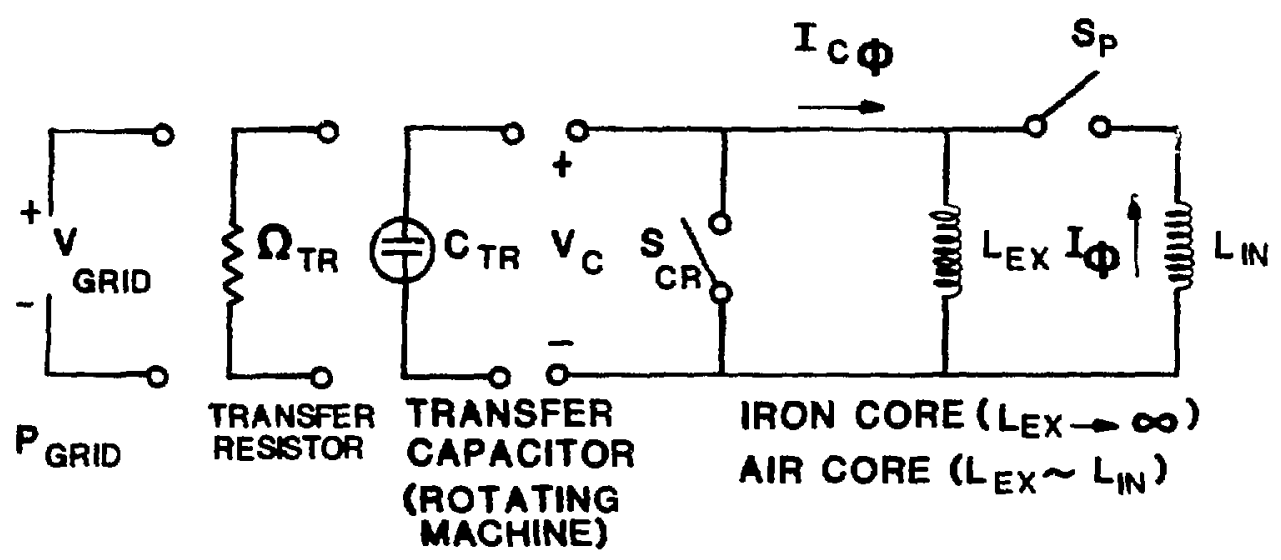

Fig. III.F-7. Simp1ifled PFC charging/discharging circuit.

store (i.e., a capacitive rotating machine) charges the PFC system or if the PFC serves as the primary (inductive) energy store. The option that uses an inductive store can undergo a bipolar swing efther through a resistive or a capacitive transfer element, thereby giving the three possible options. Using an external energy store capable of driving the PFC current from 0 to $2 I_{\phi}$ requires a total stored energy of $\frac{1}{2} L_{E X}\left(I_{c \phi}-I_{\phi}\right)^{2}+\frac{1}{2} L_{I N} I_{\phi}^{2} \simeq 2 E_{I N}$ for $L_{I N} \simeq L_{E X}$; the stored energy required is twice that for the iron-core case because of the parasitic flux stored externally to the PFC coil. The operational mode based on an external energy store minimizes external power requirements in that the rotating machine used as the main energy store can be energized over a long period of time, and the electrical grid power, $P_{G R I D}$, required is small.

Using the $\mathrm{PFC}$ as an energy-storage system significantly reduces the external energy-storage requirements. The PFC is separated into an OHC set, which produces no field within the plasma region, and an EFC set, which provides toroidal plasma equilibrium. The OHC serves as the primary energy store, this coil set being driven slowly to a current $I_{c \phi}=-I_{\phi}$ and storing the energy $E_{O H}$ $=E_{\text {IN }}$ before the plasma is initiated. The energy transfer from the coil system to the plasma can be made through either resistlve or capacitive elements, as is illustrated in Figs. III.F-8 and III.F-9. A capacitive transfer is highly efficient, requiring a rotating-machine energy store having a capacity 


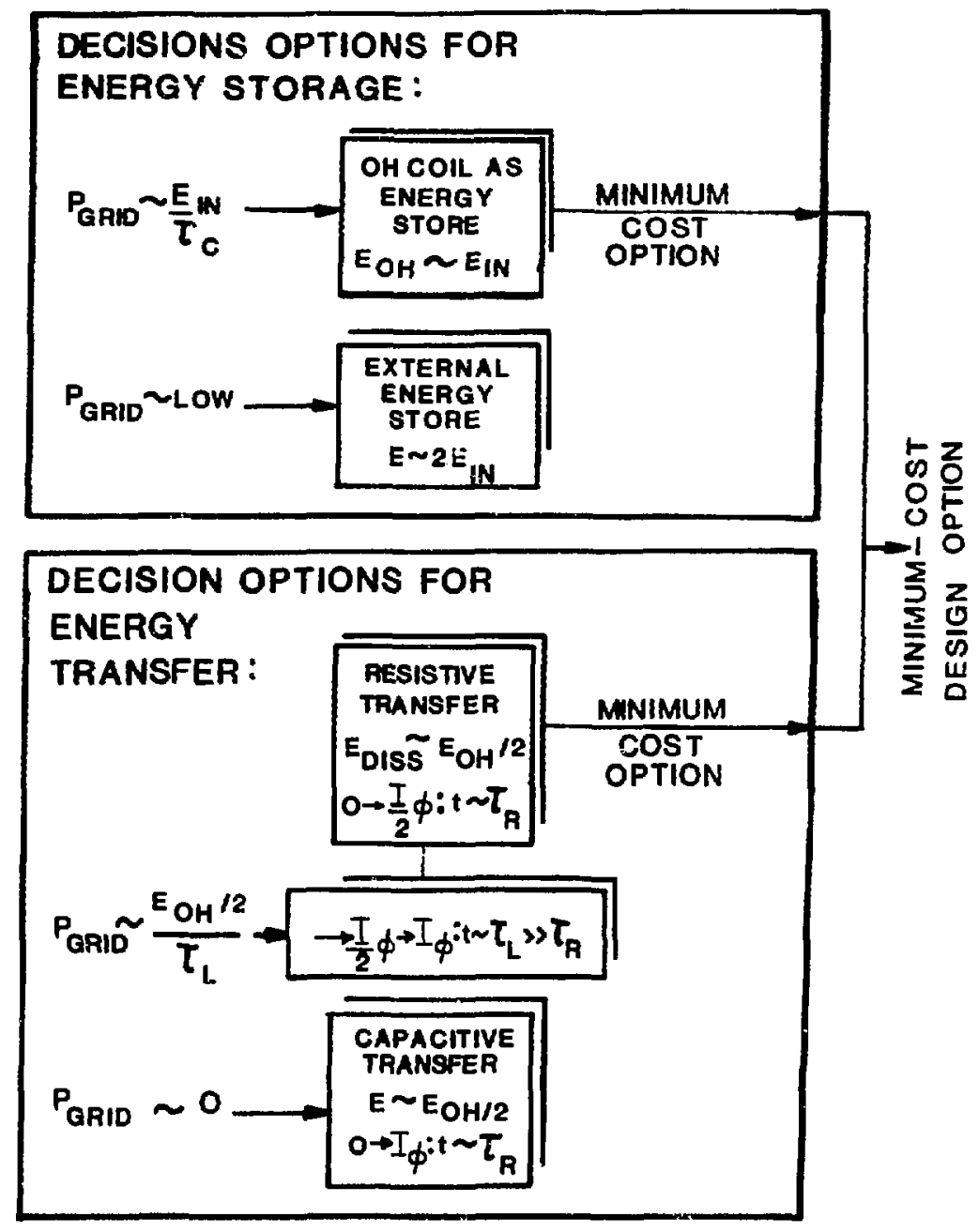

F1g. III.F-8, Decision options available for operating the PFC system, with minimum-cost choices indicated.

approximately equal to $\mathrm{E}_{\mathrm{OH}} / 2$ and providing a complete transfer of the $\mathrm{OHC}$ energy to the plasma. Power from the electrical grid is needed only during the initial charging of the OHC. A capacitive transfer is required for a short-pulsed fustion burn. This efficlent transfer allows the plasma current startup and rundown to occur in a short period of time, minimizing thermal cycling in the steam-to-electric thermal conversion system, and providing a means for efficient recovery of electromagnetic energy if large quantities of stored energy are 
required, as in the case of the large, low-power-density superconducting approaches. 53 For long-pulsed or steady-state burns envisaged for the compact systems, however, these considerations are no longer important, and the resistive transfer option becomes preferred from the viewpoints of cost, operational simplicity, and use of present-day technology.

The resistive transfer option is also depicted on Fig. III.F-8. Resistively crowbarring the OHC while simultaneously initiating the plasma and connecing the EFC across the $O H$ circuit transfers to the plasma an energy approximately equal to $\mathrm{E}_{\mathrm{IN}} / 2$; an equivalent energy is dissipated in the transfer resistor. After the resistive transfer of the PFC current, the plasma current achieves approximately 50-70\% of the design (ignition) value; electrical grid power is then used to complete the trajectory toward full plasma current and ignition. Rapid Initial current transfents, limited only by the voltage allowed on the PFC system, can be achleved by the resistive transfer scheme. Since the transfer resistor has no inherent power-handling limitations, large plasma Initiation powers can be generated without requiring either expensive on-site fower-handling or energy-storage equipment or without demanding large transients on the electrical grid.

The CRFPR design considers long-pulsed or steady-state burns wherein energy loss, duration of the startup, and degree of reversible energy transfer would not significantly impact the overall plant efficiency. The resistive startup, therefore, is adopted as the reference design. Irrespective of the energytransfer option, however, use of the OHC as an energy store as a minimum allows a fourfold reduction in the size of the rotating-machine energy storage if capacitive transfer is used; this element is completely eliminated if resistive transfer is used. Furthermore, substantial cost savings result from bipolar operation of the $\mathrm{OHC}$ system. In one limit, minimum energy requirements are achieved if an EFC is not required, while in the other limit the size of the external energy store is maximized if all the poloidal flux is driven from the EFC (1.e., no $O H C$ is used), in which case the system must be driven in a unipolar mode; these two options delimit the OHC/EFC functional split. The overall PFC design, therefore, must seek a configuration which maximizes the role of the CHC in relation to the EFC set while satisfying the need for toroldal plasma equilibrium. This consideration is of prime importance in the placement of the EFC set and is discussed in the following section. 


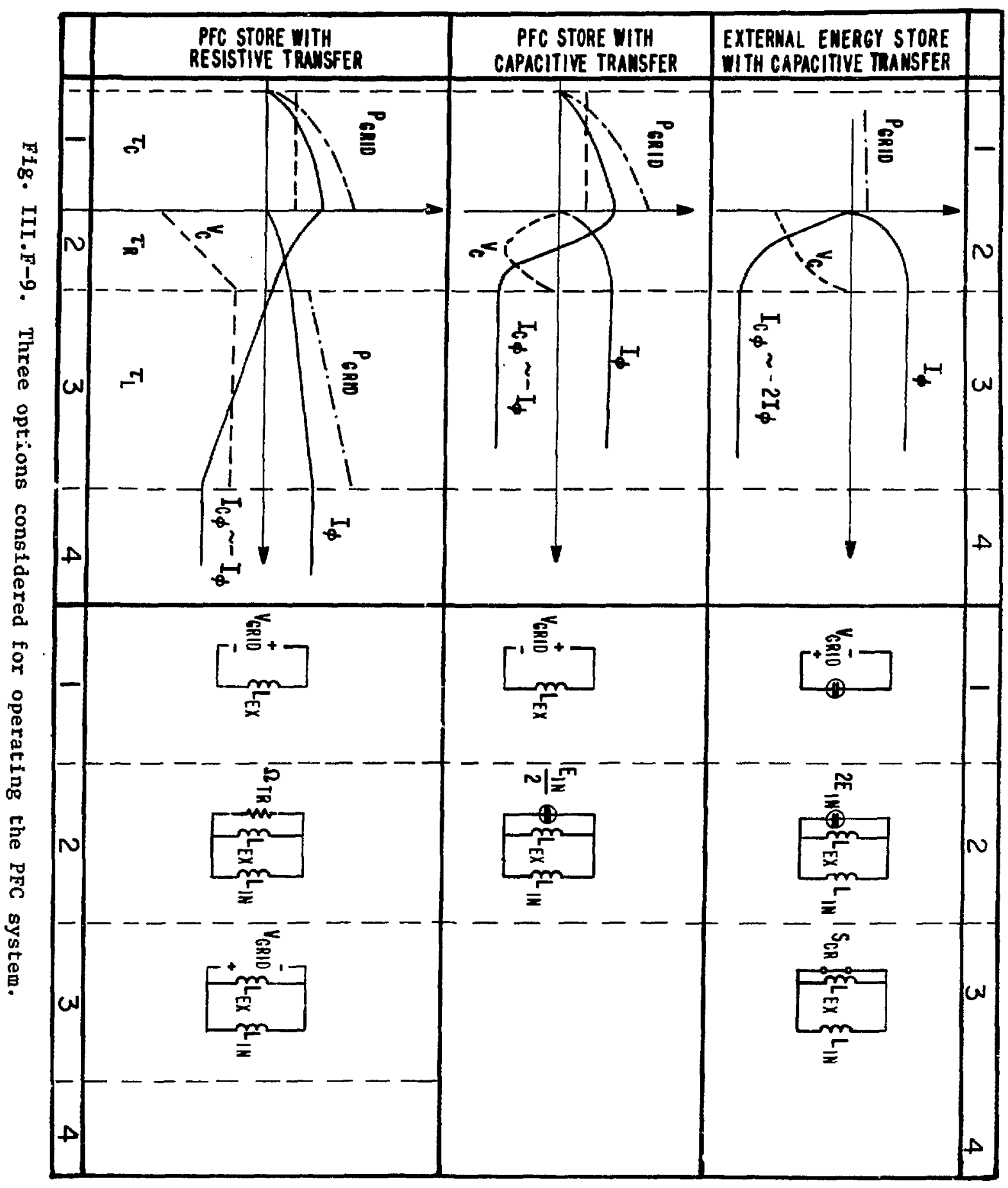


b. PFC (OHC + EFC) Conductor Locations. Considerations that impact the location and current levels in the OHCs and EFCs were discussed in Sec. III.F.2.a. and are summarized in FIg. III.F-10. Determining the proper location of the EFC and OHC conductors allows the use of single-turn selfinductances and mutual inductances to describe the interaction between the OHC, EFC, and plasma. Using these parameters and enforcing plasma equilibrium allows the calculation of all necessary coil currents. The self-inductance of the plasma is calculated analytically from the following expression:

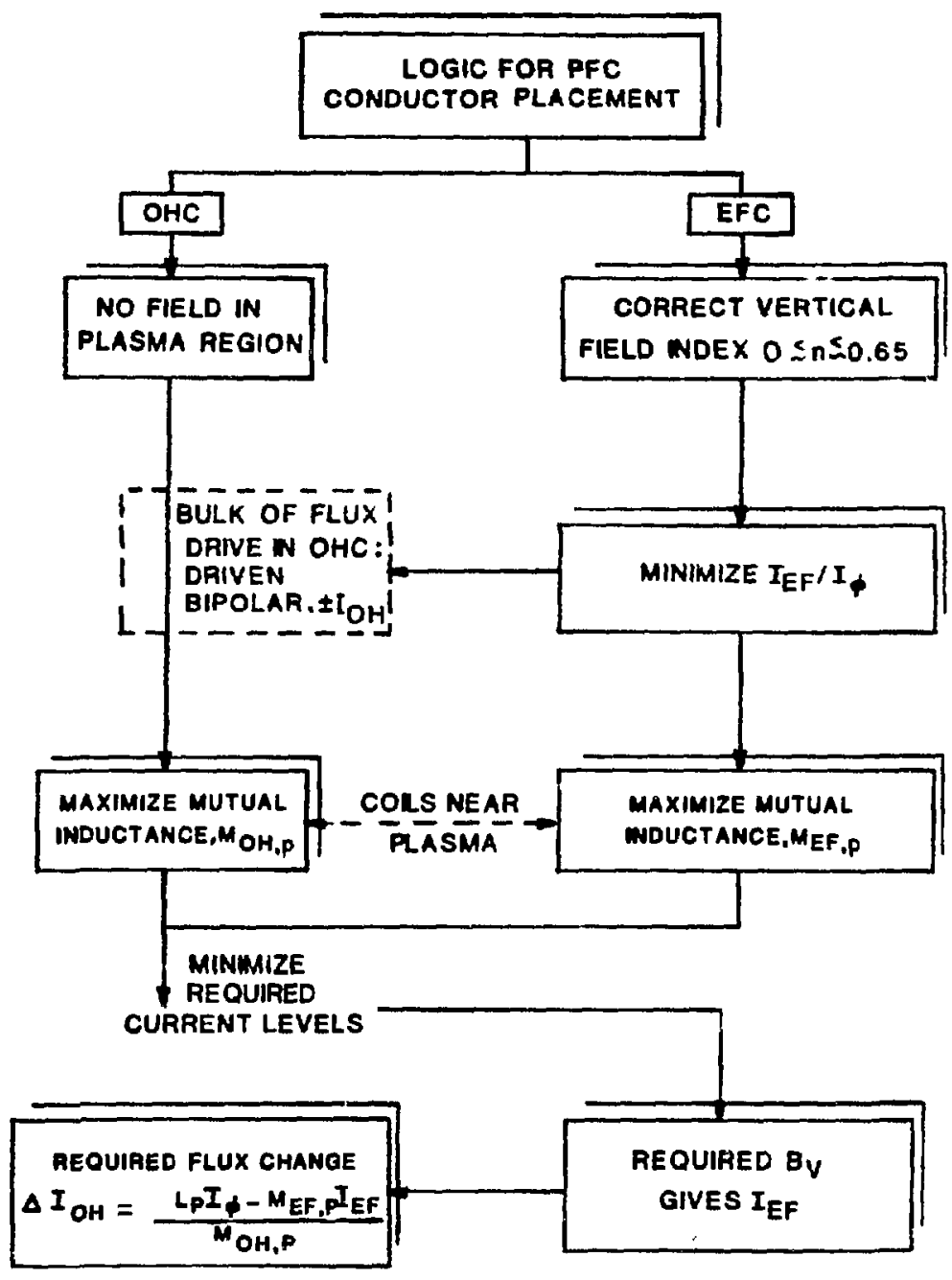

Fig. III.F-10. Major considerations that impact on the location and current levels in the OHC and EFC systems. 


$$
L_{p}=\mu_{o} R_{T}\left[\ln \left(8 R_{T} / r_{p}\right)-1.25\right] \text {, }
$$

which includes the internal energy stored by the magnetic flelds assumed to characterize the RFP. This inductance includes the internal toroidal-field energy, which is provided by the pololdal circuit through the RFP dynamo effect. Since the final plasma configuration will be established at a low current level ( $\sim 15 \%$ of the final plasma current), the plasma inductance remains virtually constant over the bulk of the plasma current rise. Only varlations in beta affect the profiles, and less than a $10 \%$ change in the plasma inductance is typically exhibited by equilibrium calculations. The actual response of the plasma inductance to changes in beta has yet to be quantified experimentally, and the use of an approximate, constant inductance is deemed appropriate. For a constant inductance, both energy and magnetic hellcity (Sec. III.K.) are automatically conserved. For all magnetics calculations performed for the PFC set, the plasma is simulated by imposing a surface current at the plasma radius, $r_{p}$, equal to the toroidal plasma current, $I_{\phi^{*}}$ Calculation of the plasma selfinductance and equilibrium are based on analytic expressions, which are expected to be adequate given the uncertainty in the plasma magnetic-field profiles. The means used to position the EFC and OHC conductors are described below.

Ohmic-ileating Col1 (OHC). As noted In FIg. III.F-10, use of the OHC as an energy storage requires large currents to flow in these windings before the plasna is inftiated. The OHC must produce a fleld distribution that excludes magnetic field from the plasma region. To determine the required OHC position, the coll current distribution is represented by a Fourier series 130 and evaluated along the coli gallery with an overall location being spectfied as 1nput. The Fourler coefficlents are then determined by a least squares minimization of the field produced inside the plasma by the OHC. The plasma is not incorporated into this calculation. The resultant continuous-current distribution is used to determine the positions of conductors carrying equal current. The correct $\mathrm{OHC}$ conductor positions for the reference design are showr. In Fig. III.F-11, where 20 equal-current windings are energized with each carrying $0.9 \mathrm{MA}$. The continuous-current distribution represented by a Fourier serles is integrated along the conductor surface and discretized into conductors carrylng equal current in order to meet the $6.1-\mathrm{MA} / \mathrm{m}^{2}$ requirement projected by 


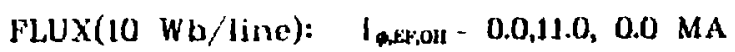

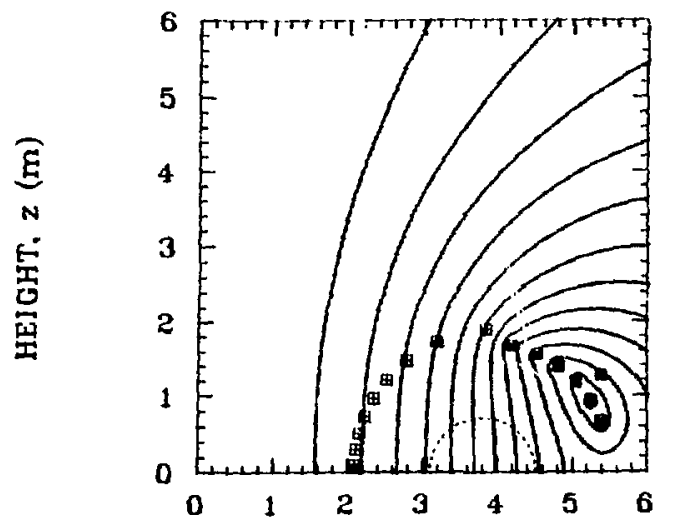

MAJOR TOROIDAL RADIUS, $\mathrm{R}_{\mathrm{T}}(\mathrm{m})$
F'luX(10 Wb;line): $I_{\text {aty;on: }}:=0.0,0.0,17.9 \mathrm{MA}$

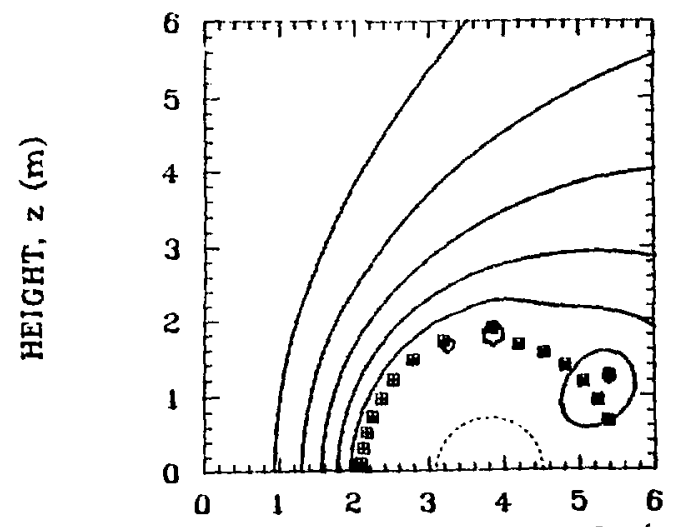

MAJOR TOROIDAL RADIUS, $R_{T}(m)$

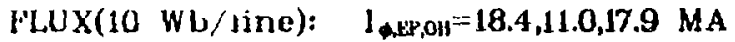

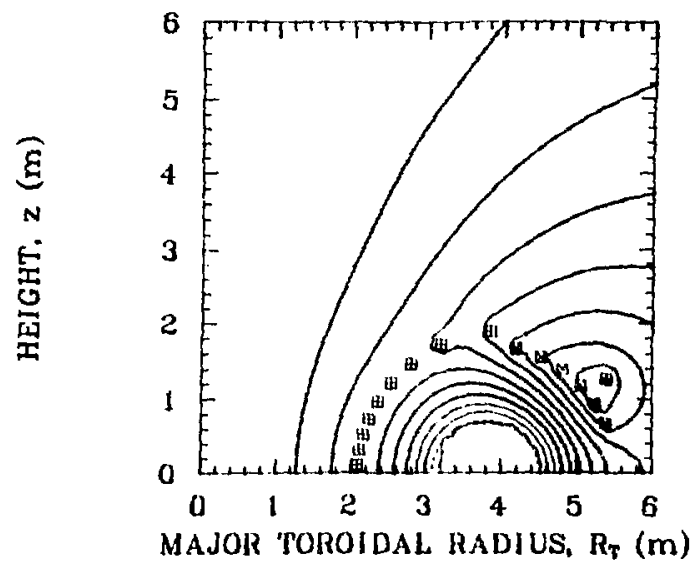

FIg. III.F-11. OHC and EFC conductor locations showing plasma position and lines of constant poloidal flux (volts).

the parametric systems code (Table III. $\bar{F}-I$ ). Equally spaced flux surfaces produced by this coil are also shown in Fig. III.F-II, where the exclusion of magnetic field from the plasma region gives essentially a constant flux in and near the plasma region.

To provide sufficient space at the outboard equitorial plane for vacuumpumping ducts and FW/limiter/B/S coolant manifolding, solutions for the OHC set exclude any conductor from the outer $10 \%$ of the PFC minor circumference. This $\sim$ 1-m gap is apparent in Fig. III.F-11. This gap only slightly perturbs the PFC solution because of the low denstey of OHC current in this outboard region. 
Maximizing the mutual inductance between the $\mathrm{OHC}$ and plasma is also accomplished by locating the inboard OHC conductors as close to the plasma as allowed by the B/S/TFC radial build.

Equilibrium-Fie1d Coil (EFC). The EFC system provides plasma equilibrium and a portion of the flux change needed to drive the plasma current. Toroidal plasma equilibrium requises the addition of a nearly uniform vertical field, $B_{v}$. The required quasi-uniform vertical field Is 131,132 approximately given below:

$$
B_{v}=\left[\mu_{0} I_{\phi} / 4 \pi R_{T}\right]\left[\ln \left(8 R_{T} / r_{p}\right)+2 \pi l_{1} / \mu_{0}+\beta_{\theta}-1.5\right],
$$

where $l_{i}$ is the inductance per unit length of the plasma; for the RFP with Bessel-function field profiles, $2 \pi l_{1} / \mu_{0}=0.5$. As seen from Eq. (9), the vertical field must vary approximately in direct proportion to the plasma current, $I_{\phi^{*}}$ This approximation is modified by a correction term that varies only $\leq 10 \%$ over the entire startup cycle. Using plasma parameters dascribing the burn $\left(I_{\phi}=18.4 \mathrm{MA}, \beta_{\theta}=0.2, r_{p}=0.71 \mathrm{~m}\right.$, and $\left.R_{T}=3.8 \mathrm{~m}\right)$, a vertical field of $B_{v}=1.44 \mathrm{~T}$ is predicted. In principle, the magnitude and distribution of the plasma current along with $\beta_{\theta}$ must be known at each point in time, with a corresponding vertical fleld consistently belng calculated. A feedback system w111 ultimately be required, although the design of such a system remains as future work. The necessary magnetic-field energies along with a first-order determination of the time-dependent currents and voltages are calculated in Sec. III.F.2.c. for a system which achieves the desired $B_{v}=1.44 \mathrm{~T}$ during the plasma burn.

To provide plasma equilibrium in both th/ horizontal and vertical directions requires a vertical field curvature that is characterized by the following decay index: 133

$$
n=\frac{\partial \ln B v}{\partial \ln r}=\frac{R}{r_{p}} \frac{B\left(R-r_{p}\right)-B\left(R+r_{p}\right)}{B\left(R-r_{p}\right)+B\left(R+r_{p}\right)},
$$

with $0<\mathrm{n}<1.5$. Constraining the plasma to a circular cross section further specifies $1320<n \leq 0.65$, which is the criterion used in this design. 
An infinice number of soiutions exist for the EFC positions needed to produce the correct vertical-field direction, magnitude, and decay index. The possible solution set is substantially reduced when fulfilling the conditions outlined in Fig. III.F-10. Minimizing the EFC current is accomplished by placing conductors outboard of the major plasma radius, $R_{T}$. Conductors located both above and below the midplane represent a short solenoidal coil in which a quasi-uniform vertical field exists. Currents in these coils also add to the current flowing in the $\mathrm{OHC}$, thereby contributing to the flux drive required to induce the plasma current. Again, to determine the necessary coil positions, the coll current distribution is represented by a Fourler series 130 and evaluated along the specified continuous EFC location. The plasma current is represented as a surface current, $I_{\phi}$, at the plasma radius, $r_{p}$. This current produces an inherent vertical field inside the radius, $r_{p}$. The Fourier coefficients are then determined by a least square minimization of the field produced by the EFC and the plasma inside the radius, $r_{p}$. The resuitant continuous-curzent distribution represented by a Fourier series is integrated along the conductor surface and discretized into equal-currerit-carrying conductors. For the reference design, the proper EFC conductor positions are also shown in Fig. III.F-11, where 12 equal-current windings are each energized with $0.9 \mathrm{MA}$. Equally spaced flux surfaces also exhtbit the required concavity of the vertical field in the plasma toward the inside of the corus. A decay index of $n=0.53$ results, assuring a circular plasma in equilibrium accordirg to the criterion outlined in Ref. 132. An EFC current of $11.0 \mathrm{MA}$ produces the required vertical field $\left(B_{v}=1.44 \mathrm{~T}\right)$ during the long-pulsed or steady state burn.

The EFCs are also excluded from the outer $10 \%$ of the coll circumference to provide space for vacuum-pumping ducts and FW/limiter/B/S coolant manif.sids. Since the EFC must produce a concave field, little EFC current would normally be present in this region. The EFC currents are specified only along the outer half-plane of che torus. Allowing EFC currents in the same direction along the inner half-plane of the torus actually subtracts from the vertical field, requiring the EFC current to increase. Additional OHC function is simply being integrated into the EFC and is a condition which is to be avolded. Localizing the EFC in the outer half-plane provides sufficient area to produce a uniform current along the minor circumference. Forcing this current to be more localized at a somewhat larger radius may lower the EFC current slightly, 
although the localization of current sightly causes construction difficulties and Eorces the conductor center away from the plasma, thereby increasing the energy-storage requirements.

Combined OHC/EFC Magnetic Properties. Several important properties of the OHC/FFC may be discerned without knowledge of the means by which the system is to be electrically driven. Single-turn, self-, and mutual inductances are

TABLE III.F-IV

OHC/EFC SINGLE-TURN PROPERTIES

PARAMETER

VALUE

Self-Inductances $(\mu \mathrm{H})$

- $\mathrm{L}_{\mathrm{p}}$

12.0

- $\mathrm{L}_{\mathrm{OH}}$

3.45

- LEF

10.6

Mutual Inductances $(\mu \mathrm{H})$

- $\mathrm{M}_{\mathrm{OH}} \mathrm{P}$

- $\mathrm{M}_{\mathrm{OH}, \mathrm{EF}}$

- $\mathrm{M}_{\mathrm{EF}, \mathrm{P}}$

5.91

Current Levels (MA)

- $I_{\phi}$

18.4

- $I_{\mathrm{EF}}$

11.0

- $\Delta \mathrm{I}_{\mathrm{OH}}$

46.8

Magnetic Fluxes (Wb)

- Plasma

220.

- EFC

65.0

- OHC 155.

EFC Parameters

- Flux change (Wb/MA turns)

- Effective coll radius, $\mathrm{R}_{E F}(m)$

- $B_{v}$ (T) at $R_{T}$

- Field Index, $n$

0.53

OHC Parameters

- F1ux change (Wb/MA turns)

- Effective coll radius, $\mathrm{R}_{\mathrm{OH}}$ (m)

2.86

- Maximum fleld at startup $\left(T, I_{\mathrm{OH}}=28.9 \mathrm{MA}\right.$ )

7.9

- Stray field at $r_{p}\left(T, I_{0 H}^{-}=28.9 \mathrm{MA}\right)$

0.01 
listed in Table III.F-IV for the PFC and EFC sets shown in Fig. III.F-11. The required plasma flux of $L_{p} I_{\phi}=220 \mathrm{~Wb}$ is partially provided by the EFC, $\mathrm{M}_{E F, p} \mathrm{I}_{E F}=65.0 \mathrm{~Wb}$, and dictates the flux change associated with the oHC current swing, ${ }_{\mathrm{OH}}, \mathrm{p}_{\mathrm{OH}}=155 \mathrm{~Wb}$. The flux surfaces for the final energized state with $I_{\phi}=18.4 \mathrm{MA}, \mathrm{I}_{\mathrm{E}_{\vec{r}}}=+11.0 \mathrm{MA}$, and $\mathrm{I}_{\mathrm{OH}}=+17.9$ are also shown in Fig. III. $\vec{r}-11$. In this particular case the total PFC current swings symmetrically from $\mathrm{I}_{\overline{\mathrm{OH}}}^{-}=28.9 \mathrm{MA}$ to $\mathrm{I}_{\mathrm{EF}}+\mathrm{I}_{\mathrm{OH}}=+28.9 \mathrm{MA}$ for purposes of mintmizing the requircd electrical grid power, as discussed in Sec. III.F.2.c.

Also given in Table III.F-IV are the effective coil radil for both OHC and EFC sets. These radil are average values to be used in subsequent calculations of nductor volume (mass) and resist:ve losses. As will be shown, this improved estimate of PFC requirements is close to the coarser predictions of the parametric systems code ${ }^{15}$ (Table III.F-I) upon which this particular costoptimized CRFPR design point is based.

c. PFC (OHC + EFC) Circuit Analysis. After the OHC and EFC conductor locations and the required total currents are defined, the operational sequence and required external driving elements remain to be determined. The PFC parameters remaining to be resolved include the time-dependent coil voltages, the conductor turns ratios, and the required conductor masses.

A simplified PFC circuit is shown in Fig. III.F-12, where single-turn parameter notation is used for illustrative purposes. The operational sequence is illustrated in Fig. III.F-13. The OHC is initially charged during the period $\tau_{c}$ to a total current $I_{0 H}^{-}$, requiring a maximum grid powar $p_{G R I D}=$

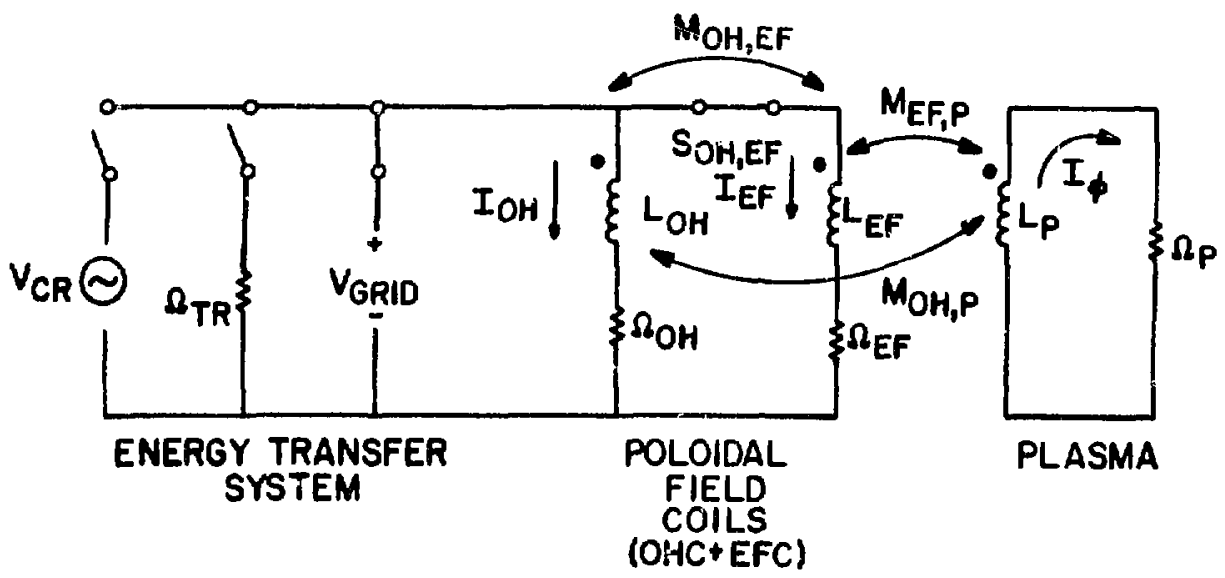

Fig. III.F-12. PFC (OHC + EFC) circuit diagram showing mutual inductions. 


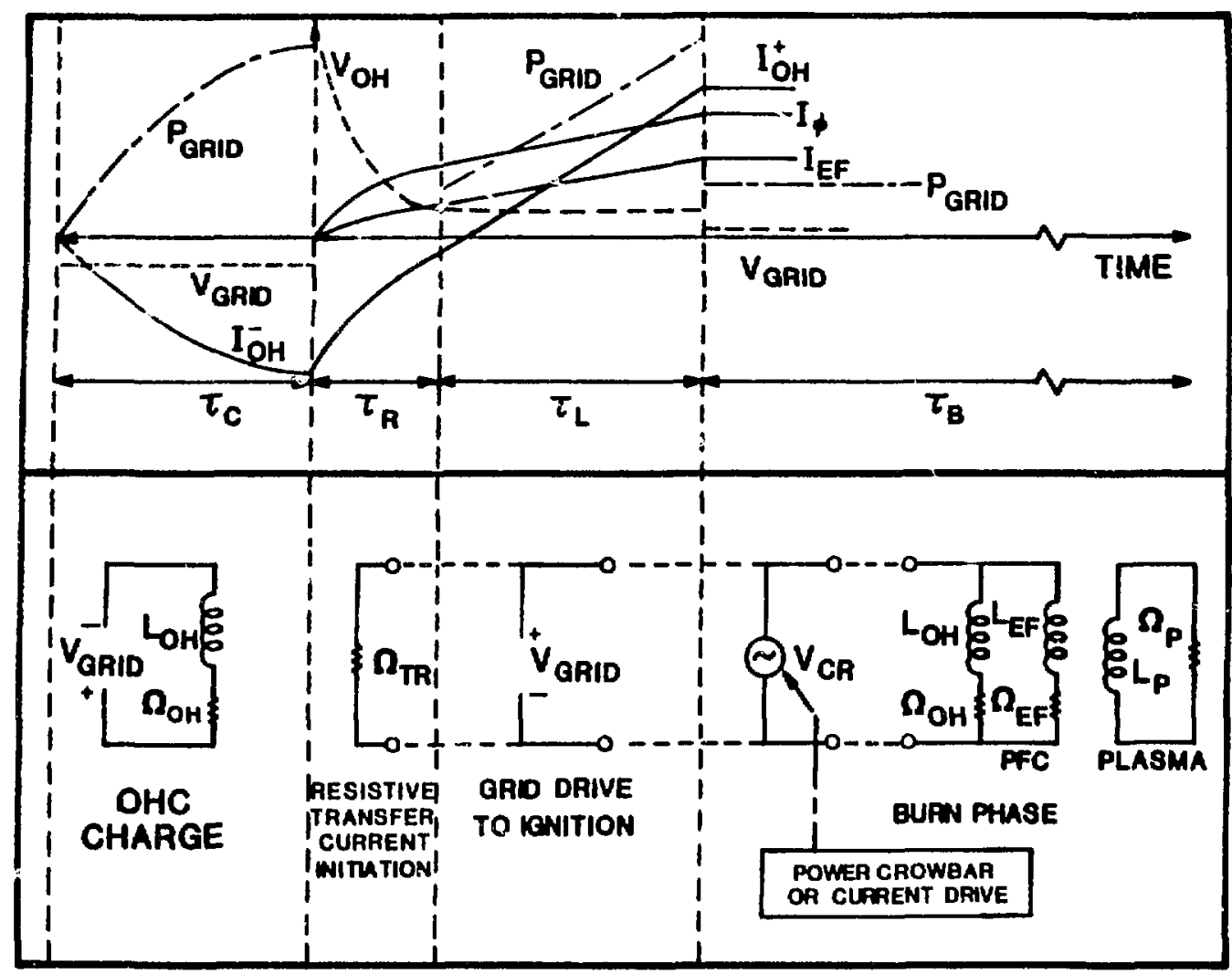

Fig. III.F-13. PFC(OHC + EFC) operational sequence.

$\mathrm{V}_{\text {GRID }} \overline{\mathrm{OH}}^{-}$- The $\mathrm{OHC}$ is then connected across the EFC set, and the plasma is simultaneously initlated while a transfer resistor, $\Omega_{T R}$, is connected across the combined OHC/EFC sets. The plasma current exponentially increases according to the following expression:

$$
I_{\phi}(t)=\frac{j H}{\Delta \bar{I}_{O H}} I_{\phi}\left(1-e^{-t / \tau_{E X P}}\right)
$$

with an initial current risettme being given by 


$$
\left(\frac{d I_{\phi}}{d t}\right)_{t=0}=\frac{I_{\overline{O H}}^{-}}{\Delta \bar{I}_{O H}} \frac{I_{\phi}}{\tau_{E X P}} .
$$

The time constant $\tau_{E X P}$ is determined from experiment to give a desired initial current rise, $\mathrm{dI}_{\phi} / \mathrm{dt}$. The resistive decay continues until $\mathrm{V}_{\mathrm{OH}}=\mathrm{V}_{\mathrm{GRID}}$, when $I_{\phi}(t)=I_{\phi}\left(\tau_{R}\right)$, and the grid voltage is again applied to drive the PFC system. Because the system is highly inductive, the plasma current at this point varies linearly according to

$$
I_{\phi}(t)=\left[I_{\phi}-I_{\phi}\left(\tau_{R}\right)\right] \frac{\left(t-\tau_{R}\right)}{\tau_{L}}+I_{\phi}\left(\tau_{R}\right),
$$

as $P_{G R I D}$ linearly increases to $P_{G R I D}=V_{G R I D}\left(I_{O H}^{+}+I_{E F}\right)$. Minimizing $P_{G R I D}$ then requires $\mathrm{I}_{\mathrm{OH}}^{-}=\mathrm{I}_{\mathrm{OH}}^{+}+\mathrm{I}_{\mathrm{EF}}$, which defines $\mathrm{I}_{\mathrm{OH}}^{-}=28.9 \mathrm{MA}$ and $\mathrm{I}_{\mathrm{OH}}^{+}=17.9 \mathrm{MA}$. The quantity of conductor present in the OHC and EFC circuit is determined by a balance that minimizes the recirculating power fraction during the plasma burn with the conductor mass and cost.

The results from the parametric systems code 15 (Table II.F-I.) predicts an optimal conductor current density of $6.06 \mathrm{MA} / \mathrm{m}^{2}$, which is applicable to $\mathrm{I}_{E F}$ and $\mathrm{I}_{\mathrm{OH}}^{+}$during the plasma burn. The final conductor sets 1 isted in Table III.F-V and described in Sec. III.F.2.d. have current densities, of 6.06 and $6.40 \mathrm{MA} / \mathrm{m}^{2}$ for the EFC and $\mathrm{GHC}$ systems, respectively. The total conductor volume is $13 \%$ more than predicted by the parametric systems code, 15 and the recirculated power is approximately $24 \%$ greater. The PFC system througn the RFP dynamo is used to provide virtually all the toroidal-field energy internal to the plasma, requiring approximately a $10 \%$ increase in the required flux change and accounting for the additional recirculated power.

The tradeoff between $\mathrm{P}_{\text {GRID }}$, conductor splits, and oHC current swings involves an economic optimization, as is illustrated in Fig. III.F-14. The general approach that minimizes $P_{G R I D}$ while using the optimal conductor current density during the burn as predicted by the systems economics model presents a logical path for parameter selection, although increasing $P_{G R I D}\left(I_{O H}^{+}\right.$) in favor of a lower recirculating power may be economically attractive depending on the 


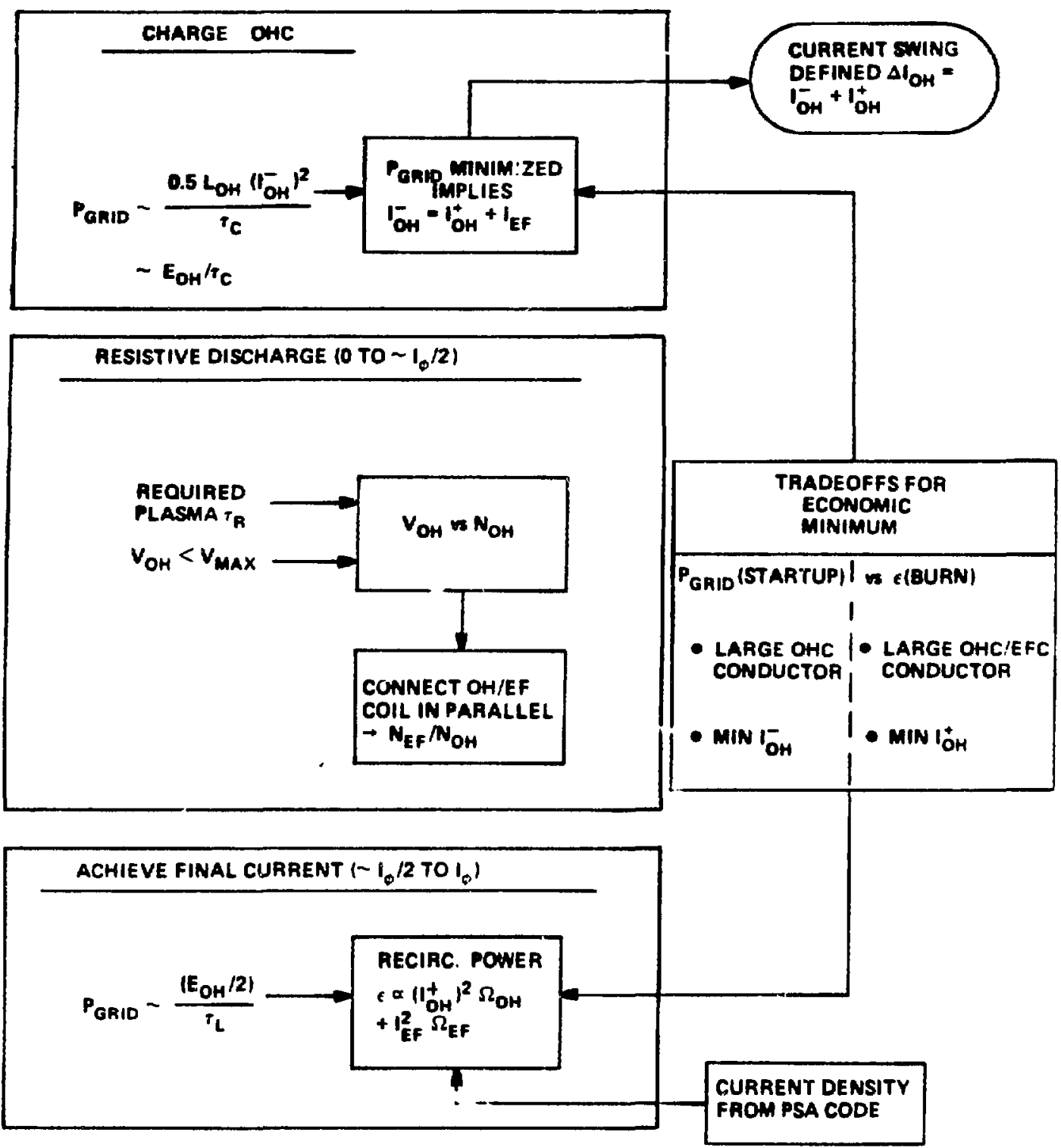

F1g. III.F-14. Schematic representation of economic tradeoffs involved in the definition of PFC drive and conductor parameters.

magnitude and avallability of electrical grid power; this tradeoff cannot be specified quantitatively at Lhis stage of the study.

Determination of the system voltages and coll turns ratio requires a complete circuit analysis. A matrix representation of the circuit, as 11lustrated in Fig: III.F-12, is given below: 


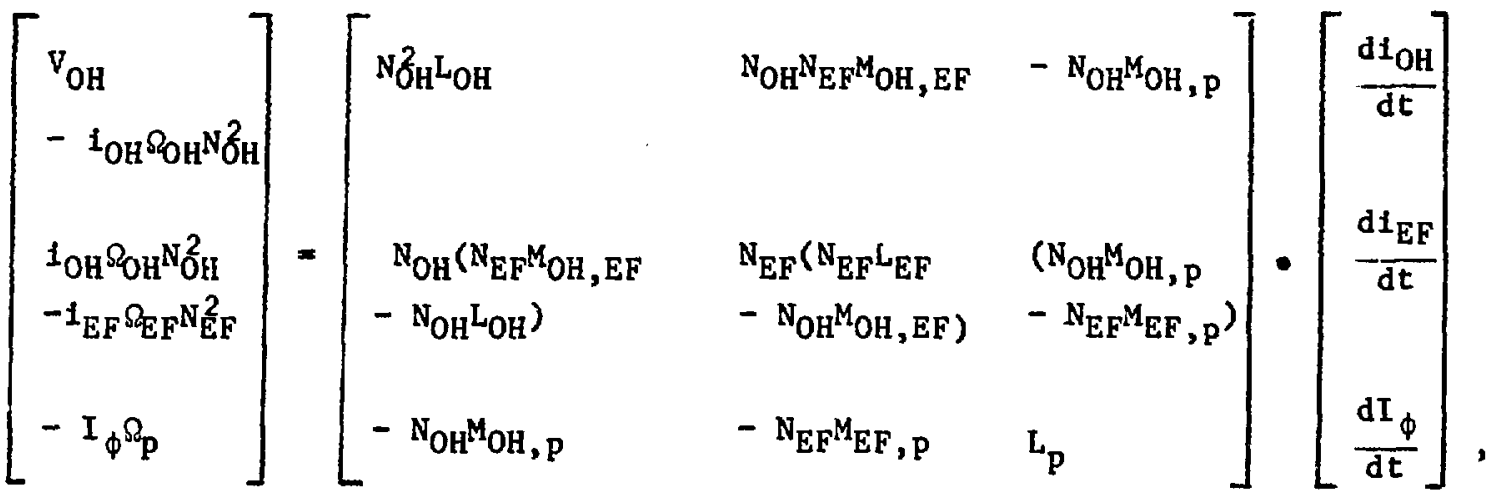

where the turis ratios $\mathrm{N}_{1}(\mathrm{i}=\mathrm{EF}, \mathrm{OH})$ are included explicitly. Lead currents are represented by lower-case letters, $i_{\mathrm{OH}}$ and $\mathrm{i}_{\mathrm{EF}}$. The above equations, including a time-dependent of the plasma resistance, $\Omega_{p}$, can be solved numerically as described in the following Sec. III.F.2.d. Since an efficient reactor requires large values of $L_{p} / \Omega_{p}$, an accurate analytic representation of the startup tranglent can be derived by taking $\Omega_{\mathrm{p}}, \Omega_{\mathrm{OH}}$, and $\Omega_{\mathrm{EF}}$ to be small. The system voltage, $\mathrm{V}_{\mathrm{OH}}$, under these conditions is given by

$$
V_{\mathrm{OH}}=N_{\mathrm{OH}}\left\{\frac{\mathrm{L}_{\mathrm{OH}}}{\mathrm{M}_{\mathrm{OH}, \mathrm{p}}}\left[\mathrm{L}_{\mathrm{p}}-\left(\frac{\mathrm{I}_{\mathrm{EF}}}{\mathrm{I}_{\phi}}\right) \mathrm{M}_{\mathrm{EF}, \mathrm{p}}\right]+\mathrm{M}_{\mathrm{OH}, \mathrm{EF}}\left(\frac{\mathrm{I}_{\mathrm{EF}}}{\mathrm{I}_{\phi}}\right)-\mathrm{M}_{\mathrm{OH}, \mathrm{p}}\right\} \frac{\mathrm{dI} \phi}{\mathrm{dt}} .
$$

Enforcing the equilibrium condition defined by Eq. (9) requires the ratio $I_{E F} / I_{\phi}$ to be approximately constant. Using this condition directly couples $\mathrm{V}_{\mathrm{OH}}$ with $\mathrm{N}_{\mathrm{OH}}$ and $\mathrm{dI}_{\phi} / \mathrm{dt}$. As seen in F1g. III.F-13, the maximum voltage, $\mathrm{V}_{\mathrm{MAX}}$, occurs at the beginning of the resistive decay. Using Eq. (11) for the plasma current transient and taking $\tau_{\text {EXP }}=0.34 \mathrm{~s}$ ylelds an experimentally characteristic initial plasma current rise of $d I_{\phi} / d t=33 \mathrm{MA} / \mathrm{s}$ for this current pulse; Eq. (15) gives $\mathrm{N}_{\mathrm{OH}}=100$ if the coil voltage is to remain below $25 \mathrm{kV}$, which is an engineering constraint imposed by this study.

T.5 uiring the EFC to be connected across the OHC during the startup transient eliminates the need for a separate power supply and forces a specific relat Sonship between $\mathrm{N}_{\mathrm{EF}}$ and $\mathrm{N}_{\mathrm{OH}}$. Again, with all resistances sma1l, Eq. (13) gives the following relationship between $\mathrm{N}_{\mathrm{EF}}$ and $\mathrm{N}_{\mathrm{OH}}$. 


$$
\frac{N_{E F}}{\bar{N}_{O H}}=\frac{\frac{L_{O H}}{M_{O H, p}}\left[L_{p}-\left(\frac{I_{E F}}{I_{\phi}}\right) M_{E F, p}\right]+M_{O H, E F}\left(\frac{I_{E F}}{I_{\phi}}\right)-M_{O H, p}}{\frac{L_{O H}, E F}{M_{O H, p}}\left[L_{p}-\left(\frac{I_{E F}}{I_{\phi}}\right) M_{E F, p}\right]+L_{E F}\left(\frac{I_{E F}}{I_{\phi}}\right)-M_{E F, p}},
$$

which for a constant ratio $\mathrm{I}_{\mathrm{EF}} / \mathrm{I}_{\phi}$ defines the EFC turns ratio. The maximum conductor lead current for both coll sets are then listed in Table III.F-V.

With the turns ratio $\mathrm{N}_{\mathrm{OH}}$ and $\mathrm{N}_{E F}$ specified from Eq.(16) to be $\mathrm{N}_{\mathrm{EF}} / \mathrm{N}_{\mathrm{OH}}=0.83$, the charging electrica1-grid voltage, $\mathrm{V}_{\mathrm{GRID}}$, is determined from the maximum imposed electrical power, $\mathrm{P}_{\mathrm{GRID}}=\mathrm{v}_{\mathrm{GRID}}{ }^{-} \mathrm{OH}_{\mathrm{H}}$, with the charge transient being described by the following expression:

$$
1_{\mathrm{OH}}=\frac{\mathrm{P}_{\mathrm{GRID}}}{1_{\mathrm{OH}}^{-} \mathrm{N}_{\mathrm{OH}}^{2} \Omega_{\mathrm{OH}}}\left(1-e^{-\Omega_{\mathrm{OH}} t / L_{\mathrm{OH}}}\right) .
$$

Because of the long charging transient, this latter expression must properly include the effect of the OHC resistance, $\Omega_{\mathrm{CAl}}$. For $\mathrm{P}_{\text {GRID }}=350 \mathrm{MWe}$ and $\mathrm{V}_{\mathrm{GRID}}=$ $1,200 \mathrm{v}$, the $\mathrm{OHC}$ current achieves $1_{\mathrm{OH}}$ in $\tau_{\mathrm{c}}=0.49 \mathrm{~L}_{\mathrm{OH}} / \Omega_{\mathrm{OH}}=10.5 \mathrm{~s}$. From Eq. (15), the resistive transient occurs from $v_{O H}=25,000$ to $1,200 \mathrm{~V}$ in $\tau_{R}=$ $3.04 \tau_{E X P}=1.03 \mathrm{~s}$ as the plasma current increases to $I_{\phi}\left(\tau_{R}\right)=10.8 \mathrm{MA}$. Finally, using Eq. (13), $d I_{\phi} / d t=\left[I_{\phi}-I_{\phi}\left(\tau_{R}\right)\right] / \tau_{L}$, and $V_{G R I D}$ in Eq. (15), the final rise of the current occurs during $\tau_{\mathrm{L}}=4.69 \mathrm{~s}$. All coll parameters are summarlzed in Table III.F-V and are used in Sec. III.L. in support of the FPC integration. Figure III.F-15 depicts the CRFPR coil cross sections and locations that have emerged from this equilibrlum and startup analyses; a comparison with the TFTR cross section is also given. 


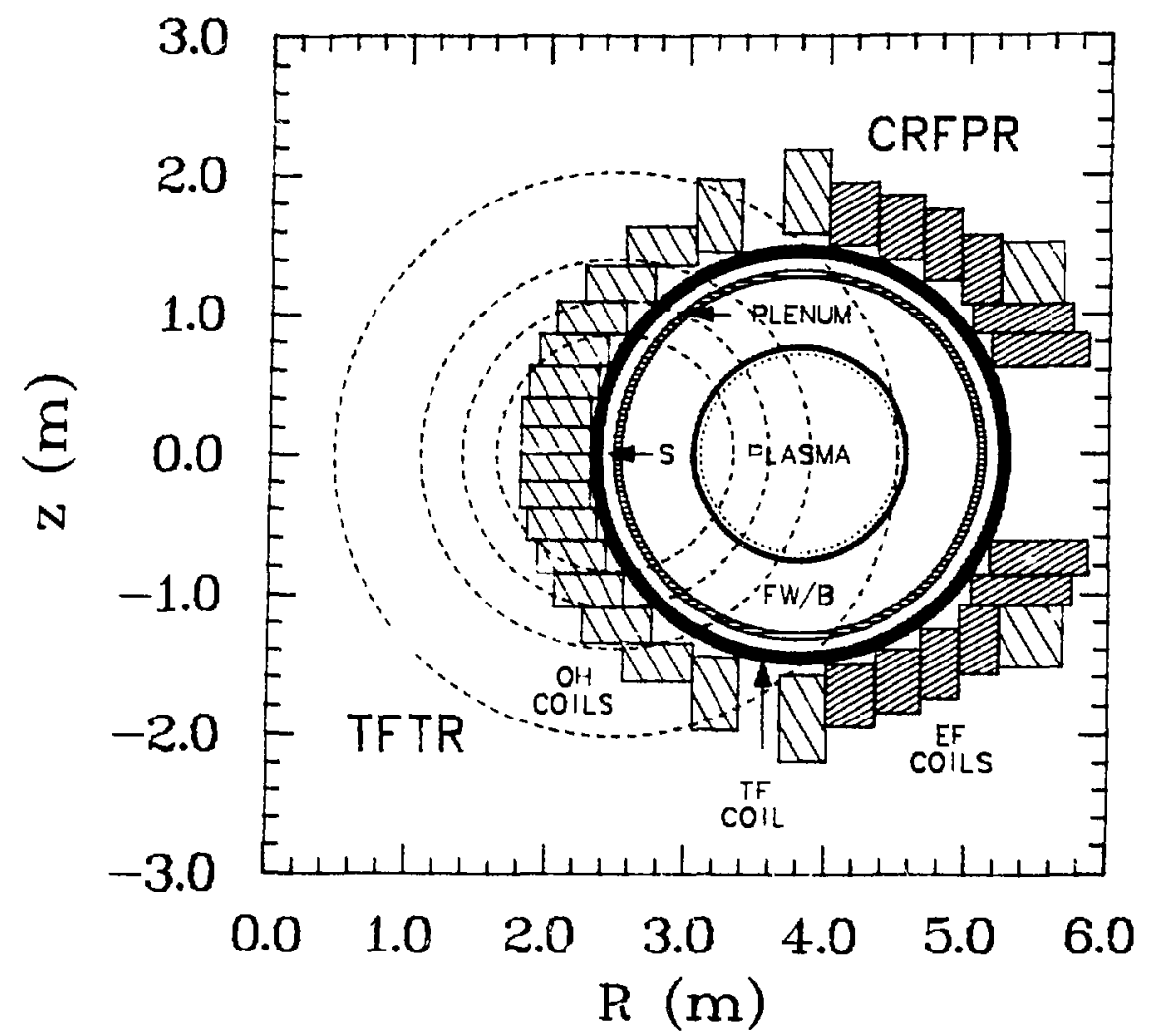

Fig. III.F-15. CRFPR coll locations and sizes predicted by startup and equilibrium analyses.

d. Resistive PFC Numerical Circuit Analysis. Inclusion of plasma and coil resistances in the PFC circuit considerably complicates the analytic circuit modeling, requiring a numerical analysis. This analysis is rigorously accomplished by coupling the PFC circuit shown in Fig. III.F-12 to the plasma simulation model, as described in Sec. III.G. and Appendix A. Using the PFC Inductance parameters listed in Table III.F-IV and the coil resistances ard turns ratios given in Table III.F-V, the complete system of circuit equations listed tin Eq. (14) can be evaluated. The plasma resistance, $\Omega_{p}$, is computed as a function of time as the plasma temperature, density, and assoclated profiles vary during the startup phase (Appendix $A$ ). Because the cotal plasma resistive heating resulting from the poloidal, $I_{\theta}$, and toroidal, $I_{\phi}$, current is supplied by the PFC circuit through the RFP dynamo effect, tne total ohmic power input defines $\Omega_{p}$ from the relationship $P_{\Omega}=I \frac{2}{\phi} \Omega_{p}$. 
TABLE III.F-V

OHC/EFC CIRCUIT AND CONDUCTOR CHARACTERISTICS $\left(\Omega_{p} \rightarrow 0\right)(a)$

PARAMETER

Electrical Grid Requirement

- Maximum power, $\mathrm{P}_{\mathrm{GRID}}$ (MWe)*

- Voltage, $\mathrm{V}_{\mathrm{GRID}}(\mathrm{V})$

EFC Characteristics

- Turns, $\mathrm{N}_{\mathrm{EF}}$

- Maximum lead current, $\mathrm{I}_{\mathrm{EF}}$ (MA)

- Maximum vo1tage, $\mathrm{V}_{\mathrm{MAX}}(\mathrm{V})$ *

- Average coll current density $\left(\mathrm{MA} / \mathrm{m}^{2}\right)$ *

- Conductor filling fraction, $\lambda_{c} *$

- Single-turn resistance, (b) ohm

- Dissipated power, $\mathrm{P}_{\mathrm{EFC}}^{\Omega}$ at $1_{\mathrm{EF}}$ (MWe)

OHC Characteristics

- Turns, $\mathrm{N}_{\mathrm{OH}}$

- Maximun lead current, $i_{O H}^{+}(M A)$

- Maximum voltage, $\mathrm{V}_{\text {MAX }}(\mathrm{V}) *$

- Average coll current density $\left(\mathrm{MA} / \mathrm{m}^{2}\right)$ *

- Conductor filling fraction, $\lambda_{c} *$

- Single-turn resistance, (b) ohm

- Dissipated power, $\mathrm{P}_{\mathrm{OHC}}^{\Omega}(\mathrm{MWe})$ at $1_{\mathrm{OH}}^{+}$

\section{Circuit Timing}

- Charge time, $\tau_{c}(s)$

- Experimental risetime, $\tau_{\mathrm{EXP}}(\mathrm{s})$ *

- Initial plasma rise, $\tau_{R}(s)$

- Final plasma rise, $\tau_{L}(s)$

\section{VALUE}

350.

1,200 .

83.

0.131

$2.5(10)^{4}$

6.1

0.7

$4 . \therefore(10)^{-7}$

53.5

100

0.025

$2.5(10)^{4}$

6.4

0.7

$1.61(10)^{-7}$

51.6

10.5

0.34

1.03

4.69

*Input parameters.

(a) As shown in Sec. III.F.2.d., Including a fini_e plasma resistance requires a $17 \%$ increase in $\mathrm{OHC}$ current swing and a $42 \%$ increase in $\mathrm{OHC}$ power consumption (73.0 MWe).

(b) Copper resistivity taken as $1.8(10)^{-8}$ ohm $\mathrm{m}$. 
After the electrical proper ties of the PFC circuit are defined, the reactor startup proceeds as described in Sec. III.G. To facilitate comparisons between nonresistive and resistive solutions to this simulation problem, a maximum grid power of 350 MWe and a coil voltage of $25 \mathrm{kV}$ are specified, as used for the analytical solution given in Sec. III.F.2.c. Inclusion of plasma resistance requires additional poloidal flux change to achleve the desired plasma surrent $\left(I_{\phi}=18.4 \mathrm{MA}\right)$. Since the EFC current is constrained to 11.0 MA-turns for equilibrium purposes, the additional resistive volt-seconds must be supplied from the OHC current swing.

The necessary external power supply requirements are best met if $I_{\mathrm{OH}^{-}}^{-}=I_{\mathrm{OH}}^{+}+I_{\mathrm{EF}}$; that is, the back-biased OHC current, $\mathrm{I}_{\mathrm{OH}}^{-}$, equals the total PFC current during the burn. For the ideal case of no plasma resistance, these currents are determined to be $\mathrm{I}_{\mathrm{OH}}^{-}, \mathrm{I}_{\mathrm{OH}}^{+}, \mathrm{I}_{\mathrm{EF}}=28.9,17.9,11.0 \mathrm{MA}$-turns, respectively. Since the resistive volt-seconds are not known prior to startup, the desired symmetric current swing must be found by iteration. A representative reactor startup, including all resistances, is shown in Fig. IIL.F-16. The plasma, EFC, and OHC current swings are shown along with the voltage applied to the PFC circuit. A summary of key circuit parameters is given in Table IrI.F-VI.

The OHC is initially charged to $-33.5 \mathrm{MA}$-turns in $16.3 \mathrm{~s}$ by the $350-\mathrm{MWe}$, 1.03-kV supply. Simultaneously paralleling the OHC and EFC while 1nitiating the plasma, a 0.0735 -ohm transfer resistor crowbars the PFC system. The peak initial coll voltage of $25 \mathrm{kV}$ induces an initial plasma current rise of approximately $32 \mathrm{MA} / \mathrm{s}$. This resistive transfer continues for a time $\tau_{R}=1.2 \mathrm{~s}$ until the coll voltage equals the power supply voltage $(1.03 \mathrm{kV})$. Under constant voltage the external power, $\mathrm{P}_{\mathrm{PFC}}$, supplied to the PFC increases approximately linearly to a maximum of $350 \mathrm{MWe}$, as is shown in Fig. III.F-17. The slow Increase in plasma current occurs over a period of $\tau_{L}=\sim 8 s$ until the ful1 18.4-MA plasma current is initiated (9.2 s). Finally, a power srowbar supply of $\sim 150$ MWe at $436 \mathrm{~V}$ is used to maintain the plasma current. This power supplies the OHC and EFC resistive losses, 73.0 and $53.5 \mathrm{MW}$, respectively, and the ohmic plasma loss of $24.5 \mathrm{MW}$ during the steady-state burn. The oHC current must be slowly increased to compensate for the resistive flux loss of $1.3 \mathrm{~Wb} / \mathrm{s}$ unless a plasma current-drive mechanism is imposed, in which case the OHC current (and power consumption) is allowed to decay to zero. 


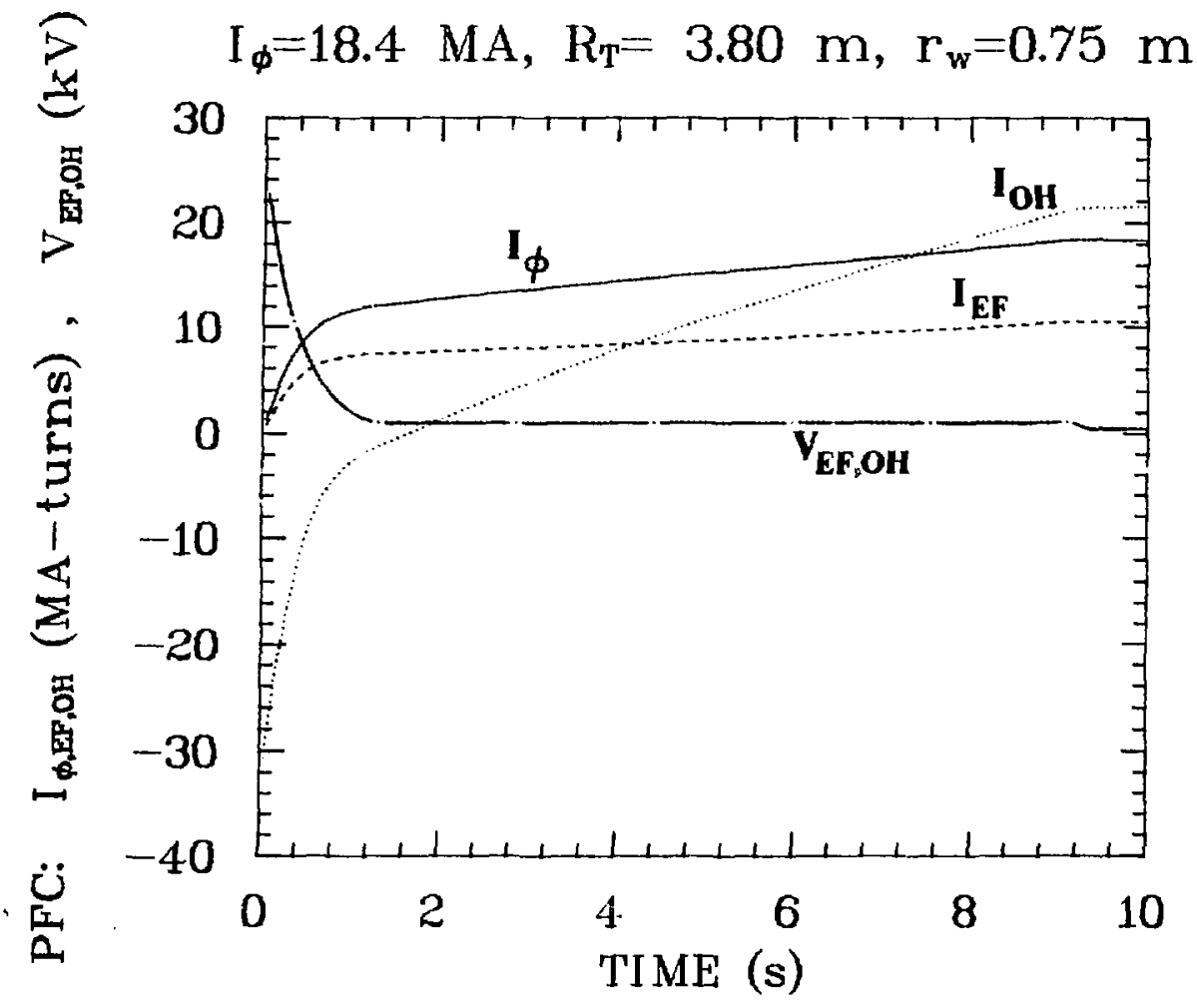

Fig. III.F-16. Representative plasma startup transient, showing plasma, EFC and OHC currents and voltages for the baseline case simulation describec in Sec. III.G.

Comparing the analytic result given in Table III.F-V to the resistive-plasma startup listed in Table III.F-VI, the additional reststive $26 \mathrm{~Wb}$ requires a $17 \%$ increase in the OHC current swing. Using the same coil set in both cases, this increased current swing inflates the recirculating power requirement from 51.6 to 73.0 MWe during the burn. An external current-drive mechanism (i.e., F- $\theta$ pumping, Sec. III.K.) may allow the oHC current to be reduced slowly to zero as the burn proceeds. The OHC is used only for inducing the plasma current and serves no equilibrium function. This coll could also be swung wore negatively when initially charged to minimize recirculacing power during the burn, although the magnetic fleld at the conductor would exceed the pregent value of $9.15 \mathrm{~T}$ at 33.5 MA-turns.

The plasia surwent trajectory during the reslstive decay phase is similar far both resfative and nonresistive solutions. The titial plasma current rise to $\sim 12 \mathrm{kA}$ requtres only $1.2 \mathrm{~s}$, which compares to the $1.03 \mathrm{~s}$ calcuiated 
TABLE III.F-VI

OHC/EFC CIRCUIT CHARACTERISTICS WITH FINITE PLASMA RESISTANCE, $\Omega_{p}$ PARAMETER

VALUE

Flux requirements (Wb)

- Total inductive

220 .

- Total resistive

26.

- EFC

65.

- $\mathrm{OHC}$

181 .

Current levels (MA-turns)

- Plasma current, $I_{\phi} \quad 18.4$

- Equilibrium-field coll, $\mathrm{I}_{\mathrm{EF}} \quad 11.0$

- Ohmic-heating-coil. swing, $\Delta_{\mathrm{OH}}$

- Initial dI $\mathrm{d}_{\phi} / \mathrm{dt}(\mathrm{MA} / \mathrm{s}) \quad 32.0$

Electrical-grid requirements

- Maximum power, $\mathrm{P}_{\text {GRID }}$ (MWe) 350.

- Voltage, $\mathrm{V}_{\mathrm{GR} I D}(\mathrm{kV}) \quad 1.03$

- Sustainment voltage at start of burn (kV) 0.436

- Recirculating power at start of burn, $\mathrm{P}_{\mathrm{PFC}}$ (MWe) 151 .

- $\mathrm{OHC}$

73.0

- EFC

53.5

- Plasma

24.5

Transfer resistor

- Resistance (ohms)

0.0735

- Maximum voltage (kV)

25.

Circuit timing (s)

- Charge time, $\tau_{C}$

- Initial plasua risetime, $\tau_{R}$

- Fina1 plasma risetime, $\tau_{\mathrm{L}}$

8.0

analytically. The continued rise of the plasma current to the design value requires $\sim 8 \mathrm{~s}$, which is significantly longer from a volt-seconds viewpoint, than the analytic value of $4.7 \mathrm{~s}$ resulting from the resistive drag of the $\mathrm{OHC}$, $E F C$, and plasma on the 350-MWe power supply.

The PFC locations, sizes, and masses, as depieted on Fig. IIJ.F-15, are summarized on Table III.F-VII according to EFC or OHC functions. Physical detalls of the $\mathrm{OHC}$ and EFC used in the analytic and numerical startup 


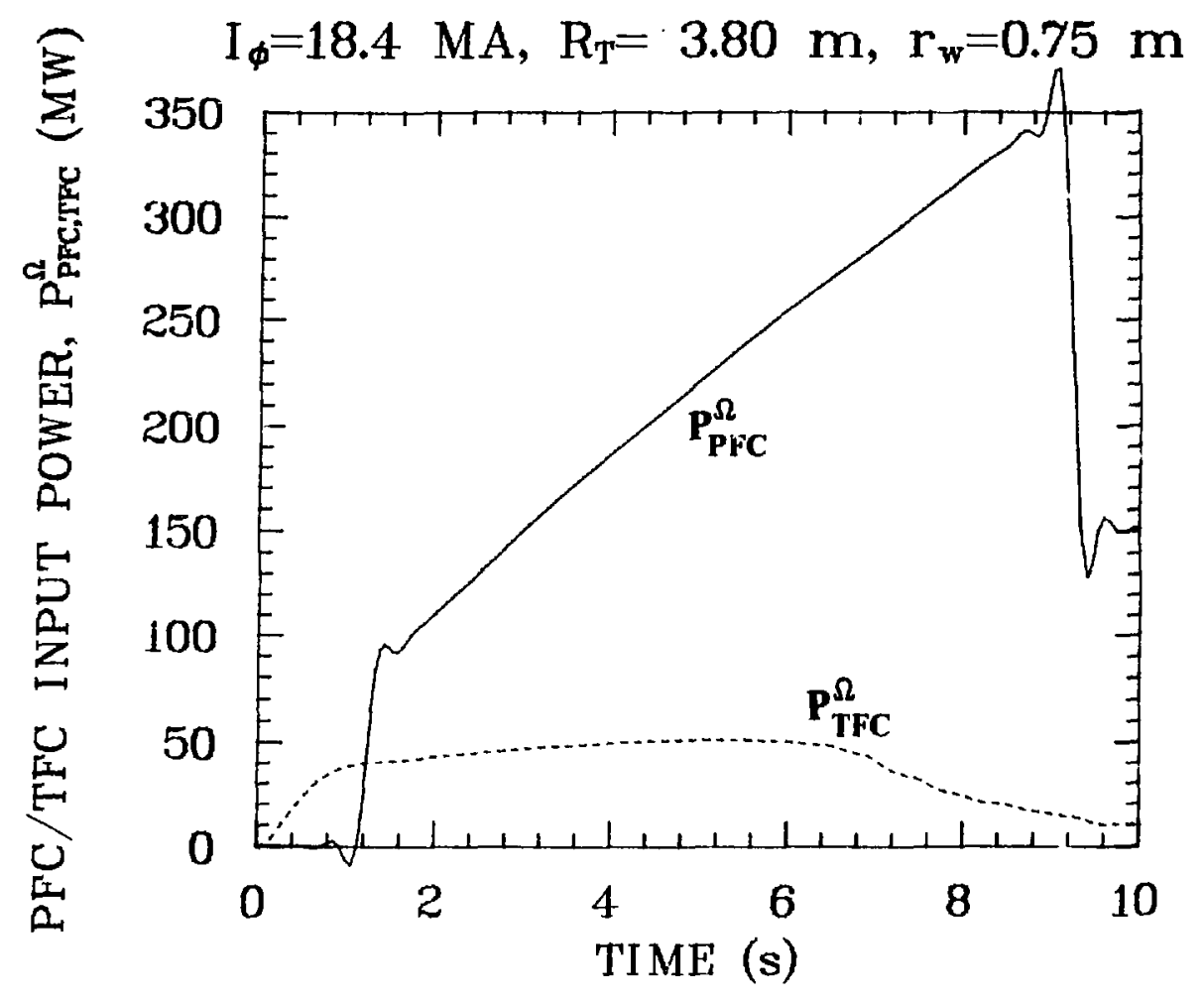

FIg. III.F-17. Power transient on PFC and TFC during the ohmic startup transient toward ignition.

calculations are given in Table III.F-VII. The total coil cross sections are glven, although individual coll closs sections vary according to avallable space. This difference is particularly true for the OHC, where limited space at the inside of the torus forces the current density to peak near the interinr and to be reduced a factor of $\sim 2$ in the outer colls, which in turn are correspondingly more lmportant from the viewpoint of power consumption because of the factor-of-three greater cfrcumference. The OHCs are connected electrically as five-turn coils in series to give a total of 100 turns.

The EFC is listed in Table III.F-VIIB as having 80 turns, which compares to 83 turns calculated from the analytic solution. Enforcing plasma equilibrium, the ratio of EFC current to plasma current must be $I_{E F} / I_{\phi} \simeq 0.60$ throughout the startup transient. For a purely inductive discharge (analytic), this requirement is enforced by $\mathrm{N}_{\mathrm{EF}}=83$. The tracking accuracy in the numerical simulation, however, depends upon both the plasma and EFC resistance. For $N_{E Q}=83$, the equilibrium coil current accurately tracks the plasma current 
TABLE TII.F-VII

PFC COIL PARAMETERS FOR REFERENCE DESIGN

\begin{tabular}{|c|c|c|c|c|c|c|c|c|}
\hline $\begin{array}{l}\text { OHC } \\
\text { SET }\end{array}$ & $\begin{array}{l}\mathrm{R} \\
(\mathrm{m})\end{array}$ & $\stackrel{z}{(m)}$ & $\delta_{(m)}$ & $\begin{array}{l}\Delta z \\
(\mathrm{~m})\end{array}$ & $\begin{array}{l}\text { Area } \\
\left(\mathrm{m}^{2}\right)\end{array}$ & $\begin{array}{l}\text { Volume } \\
\left(\mathrm{m}^{3}\right)\end{array}$ & $\begin{array}{l}\text { Mass } \\
\text { (tonne) }\end{array}$ & $\begin{array}{l}\text { Resistance } \\
\left.\text { ( } \mathrm{ohm}_{\mathrm{m}}\right)\end{array}$ \\
\hline $\begin{array}{c}1 \\
2 \\
3 \\
4 \\
5 \\
6 \\
7 \\
8 \\
9 \\
10 \\
(11-20\end{array}$ & $\begin{array}{l}2.050 \\
2.050 \\
2.100 \\
2.175 \\
2.300 \\
2.500 \\
2.800 \\
3.212 \\
3.837 \\
5.450\end{array}$ & $\begin{array}{l}0.100 \\
0.300 \\
0.512 \\
0.740 \\
0.975 \\
1.225 \\
1.495 \\
1.712 \\
1.887 \\
1.300 \\
(-z)\end{array}$ & $\begin{array}{l}0.500 \\
0.500 \\
0.500 \\
0.500 \\
0.500 \\
0.500 \\
0.500 \\
0.325 \\
0.325 \\
0.450\end{array}$ & $\begin{array}{l}0.200 \\
0.200 \\
0.225 \\
0.230 \\
0.237 \\
0.262 \\
0.275 \\
0.512 \\
0.600 \\
0.450\end{array}$ & $\begin{array}{l}0.100 \\
0.100 \\
0.112 \\
0.115 \\
0.119 \\
0.131 \\
0.138 \\
0.166 \\
0.195 \\
0.202\end{array}$ & $\begin{array}{l}1.29 \\
1.29 \\
1.48 \\
1.57 \\
1.71 \\
2.06 \\
2.42 \\
3.36 \\
4.70 \\
6.93\end{array}$ & $\begin{array}{r}9.5 \\
9.5 \\
10.9 \\
11.5 \\
12.6 \\
15.1 \\
17.8 \\
24.6 \\
34.5 \\
50.9\end{array}$ & $\begin{array}{l}3.31 \\
3.31 \\
3.02 \\
3.06 \\
3.14 \\
3.08 \\
3.29 \\
3.12 \\
3.28 \\
4.35\end{array}$ \\
\hline $\begin{array}{r}\text { EFC } \\
\text { SET } \\
1 \\
2 \\
3 \\
4 \\
5 \\
6 \\
(7-14)\end{array}$ & $\begin{array}{l}4.175 \\
4.512 \\
4.812 \\
5.087 \\
5.385 \\
5.511\end{array}$ & $\begin{array}{l}1.725 \\
1.625 \\
1.500 \\
1.325 \\
0.969 \\
0.744 \\
(-z)\end{array}$ & $\begin{array}{l}0.350 \\
0.325 \\
0.275 \\
0.275 \\
0.713 \\
0.695\end{array}$ & $\begin{array}{l}0.437 \\
0.450 \\
0.500 \\
0.500 \\
0.212 \\
0.237\end{array}$ & $\begin{array}{l}0.153 \\
0.146 \\
0.138 \\
0.138 \\
0.151 \\
0.165\end{array}$ & $\begin{array}{l}4.01 \\
4.15 \\
4.16 \\
4.39 \\
5.11 \\
5.70\end{array}$ & $\begin{array}{l}29.4 \\
30.4 \\
30.5 \\
32.3 \\
37.5 \\
41.9\end{array}$ & $\begin{array}{l}4.41 \\
4.98 \\
5.65 \\
5.98 \\
5.76 \\
5.41\end{array}$ \\
\hline $\begin{array}{l}\text { Int } \\
\text { To } \\
\text { To } \\
\text { Co } \\
\text { To } \\
\text { To }\end{array}$ & $\begin{array}{l}\text { egrate } \\
\text { tal cu } \\
\text { tal cul } \\
\text { il den } \\
\text { tal mas } \\
\text { ta! mas }\end{array}$ & $\begin{array}{l}\text { aramet } \\
\text { nt in } \\
\text { nt in } \\
y, P_{C}( \\
\text { of OHC } \\
\text { of } E F C\end{array}$ & $\begin{array}{l}\text { HCs (MA) } \\
\text { FCs (MA) } \\
e / \mathrm{m}^{3} \text { ) } \\
\text { onne) } \\
\text { onne) }\end{array}$ & $\begin{array}{l}\text { Value } \\
21.1 \\
11.0 \\
7.34 \\
394 \\
404\end{array}$ & & & & \\
\hline
\end{tabular}


TABLE III.F-VIIA

\section{PHYSICAL PARAMETERS OF POLOIDAL-FIELD COILS (OHC SET)}

\section{PARAMETER}

Number

VALUE

Coil cross section $\left(\mathrm{m}^{2}\right)$

20

Total coil volume $\left(\mathrm{m}^{3}\right)$

2.8

Total coll mass (tonne)

53.9

Conductor filling fraction, $\lambda_{c}$ 394.

Total number of turns

0.7

Resistance (ohms)

100

Maximum applied voltage $(\mathrm{kV})$

0.0016

Peak back-bias values $\left(I_{\phi}=0.0 \mathrm{MA}\right)$

- Lead current (MA)

0.335

- Average coil current density (MA/m ${ }^{2}$ )

12.0

- Dissipated power (MWe)

180.

- Maximum stored energy (MJ)

2000.

- Maximum magnet coil field (T)

9.12

- Maximum stray field at plasma (T)

0.013

Start-of-burn values $\left(I_{\phi}=18.4 \mathrm{MA}\right)$

- Lead current (MA)

0.213

- Average coll current density $\left(\mathrm{MA} / \mathrm{m}^{2}\right)$

7.7

- Dissipated power (MWe)

73.0

- Maximum magnet coll field (T)

4.5

during the resistive tartup phase $\left(<1.2 \mathrm{~s}\right.$ ) and drifts to $\mathrm{I}_{\mathrm{EF}} / \mathrm{I}_{\phi} \simeq 0.545$ at the end of startup $(\sim 10 \mathrm{~s})$. Adjusting $N_{E F}$ to 80 turns gives $I_{E F} / I_{\phi}$ ranging from 0.62 .7 to 0.57 at the beginning and end of the startup, respectively, with the equilibrium being tracked to within $\pm 5 \%$. In any case, a separate equilibrium feedback system would ultimately be required to match closely the necessary equilibrium current. 


\section{TABLE III.F-VIIB}

PHYSICAL PARAMETERS OF THE POLOIDAL-FIELD COILS (EFC SET)

\section{PARAMETER}

Number

VALUE

Total coll cross section $\left(\mathrm{m}^{2}\right)$

12

Total coll volume $\left(\mathrm{m}^{3}\right)$

1.8

Total coll mass (tonne)

55.5

Conductor filling fraction, $\lambda_{c}$

405.

Total number of curns

0.7

Resistance (ohms)

80.0

Maximum applied voltage $(\mathrm{kV})$

0.0028

During-the-burn values $\left(I_{\phi}=18.4 \mathrm{MA}\right)$

- Lead current (MA)

0.135

- Average coil current density (MA/m²)

6.05

- Dissipated power (MWe)

53.5

- Maximum magnet co:l fjeid (T)

4.5 
The parametric systems evaluation given in Ref. 15, as updated and summarized in Sec. II.D.2., has provided the guidance and basis for this design study. An accurate modeling and assessment of reactor ctartup and operation, however, remalns to be performed. Using the economically optimal design point listed in Table II.D-III as a reference, a burn simulation was performed using the CRFPR plasma simulation and systems code described in Appendix A. This time-dependent model couples the external electrical driving circult to the plasma. A complete analysis of the PFC circuit described in Sec. III.F. Is performed by the simulation code, which includes the plasmo as a nonlinear circuit element.

The plasma simulation code is based on a multparticle, time-dependent burn computation that accounts for magnetic-fleld and plasma profiles by performing Integral averages over the plasma cross section at selected time intervals. Experimentally derived plasma profiles and tranaport properties are used to generate self-consistent plasma equilibrlum conflgurations. These profiles, transport properties, and equilibrium conflgurations are adjusted as the DT/DD-fueled burn proceeds. The suprathermal (non-Maxwellian) plasma species (H, D, T, ${ }^{3} \mathrm{He}$, and ${ }^{4} \mathrm{He}$ ) are described by a Fokker-Planck formalism (Appendix A), with the Maxwellian component being subtracted from each slowing-down distribution and merged into a single beckground ion population. A complete time history of all profile-averaged plasma quantities for both DD- and DT-fueled discharges is generated. The resultant power output impinges on a two-region first-wall surface, and one-dimenalonal (cylindrical) temperatures and stresses are calculated as $a$ function of time. An overall power-plant energy balance is evaluated as the reactor achleves steady-state operation.

The magnetic-field profiles are calculated froth a one-dimensional plasma equilibrium that is consistent with plasma temperature, density and current-density profiles. The magnetic-field structure may by approximated analytically using a force-free model (1.e., no plasma pressure), giving $\vec{\nabla} \times \vec{B}=\mu_{0} \vec{j} \equiv \mu(r) \vec{B}$. If the scalar $\mu(r) \equiv \mu_{0} \vec{j} \cdot \vec{B} / B^{2}$ is 1ndependent of plasma minor radius, the Bessel-function model (BFM) results, where $B_{\phi}=A_{\phi} J_{0}(\mu r)$, $B_{\theta}=A_{\theta} J_{1}(\mu r)$, and $A_{\phi}$ and $A_{\theta}$ are constants to be determined. As derived in Appendix $A$, the plnch parameter, $\theta \equiv B_{\theta}\left(r_{p}\right) /\left\langle B_{\phi}\right\rangle$, relates $\mu, A_{\theta}$, and $A_{\phi}$ according to $\theta=\left(\mu r_{p} / 2\right)\left(A_{\theta} / A_{\phi}\right)$, where $A_{\theta}=A_{\phi}$ for the force-free, $\beta_{\theta}=0 \mathrm{BFM}$. It is noted that the plasma radius, $r_{p}$, is taken at the zero-temperature 
boundary, and hence, $r_{p} \simeq r_{w}$. The pinch parameter and reversal parameter, $F=B_{\phi}\left(r_{p}\right) /\left\langle B_{\phi}\right\rangle$, in this case, are then related as follows:

$$
F=\theta \frac{J_{0}(2 \theta)}{J_{1}(2 \theta)}
$$

Equation (1) is plotted in Fig. III.G-1 and represents the near-minimum-energy, $\beta=0$, Taylor state described in Sec. II.D.1. Presuming the magnetic fields can be approximately represented by Bessel functions when pressure is added, the relationship $\overrightarrow{\nabla p}=\vec{\nabla} \times \vec{B} \times \vec{B}$ gives $\beta_{\theta}=1.0-\left(A_{\theta} / A_{\phi}\right)^{2}$, and the pressure profile can be shown to be proportional to $\mathrm{J}_{0}^{2}(\mu \mathrm{r})$. The minimum-energy relationship between $F$ and $\theta$ is now modified by replacing $\theta$ with $\theta\left(1-\beta_{\theta}\right)^{1 / 2}$ in Eq. (1). This " $\beta$-modified BFM" is plotted for $\beta_{\theta}=0.25$ in Fig. III.G-1 but is only approximate because the perpendicular components of plasma current have been Ignored for the assumed BFM field profiles.

The self-consistent calculation described in Appendix A integrates across the field profiles and estimates the equilibrium profiles using the Maxwell equations. The experimentally derived and extrapolated temperature and density profiles are taken as $n(r) \propto T(r) \propto J_{0}(\mu r)$. This parabolic-like profile yields $\mathrm{T}_{0} / \mathrm{T}=1.6$ and $\mathrm{n}_{0} / \mathrm{n}=2.3$, where the subscrlpts denote centerline values, and $\mathrm{n}$ and $\mathrm{T}$ are averaged over the plasma cross section. The spatial dependence of $\mu(r)$ is chosen to fit experimental toroidal magnetic flux and magnetic fields measured $^{74}$ at the discharge chamber wall of ZT-40M. Th1s "Best-Fit Model" uses a constant- $\mu$ profile up to a breakpoint at $\mathbf{r}=\alpha \mathbf{r}_{\mathbf{p}}(\alpha<1)$, beyond which $\mu(\mathbf{r})$ is Iinearly extrapolated to zero as $r$ approaches $r_{p}$; the large currents at the plasma edge required by the constant- $\mu$ BFM are thereby avoided. The expressions describing this model are given in Appendix A [Eq.(A-56)]. The F- $\theta$ trajectory for $\beta_{\theta}=0$ and 0.25 is also plotted in Fig. III.G-1 for the Best-Fit Model along with the numerically computed burn trajectory, the latter being bounded by the two curves for the Best-Fit Model.

Use of the aforementioned profile assumptions results in the current-density profiles plotted in Fig. III.G-2 at the operating current of $I_{\phi}=18.4 \mathrm{M} . \mathrm{A}$. The linear extrapolation of the current density to zero is apparent for the burn case shown, which operates at $\theta=1.55, F=-0.12$, and $\beta_{\theta}=0.23$. These plasma current densities produce the toroldal and pololdal 


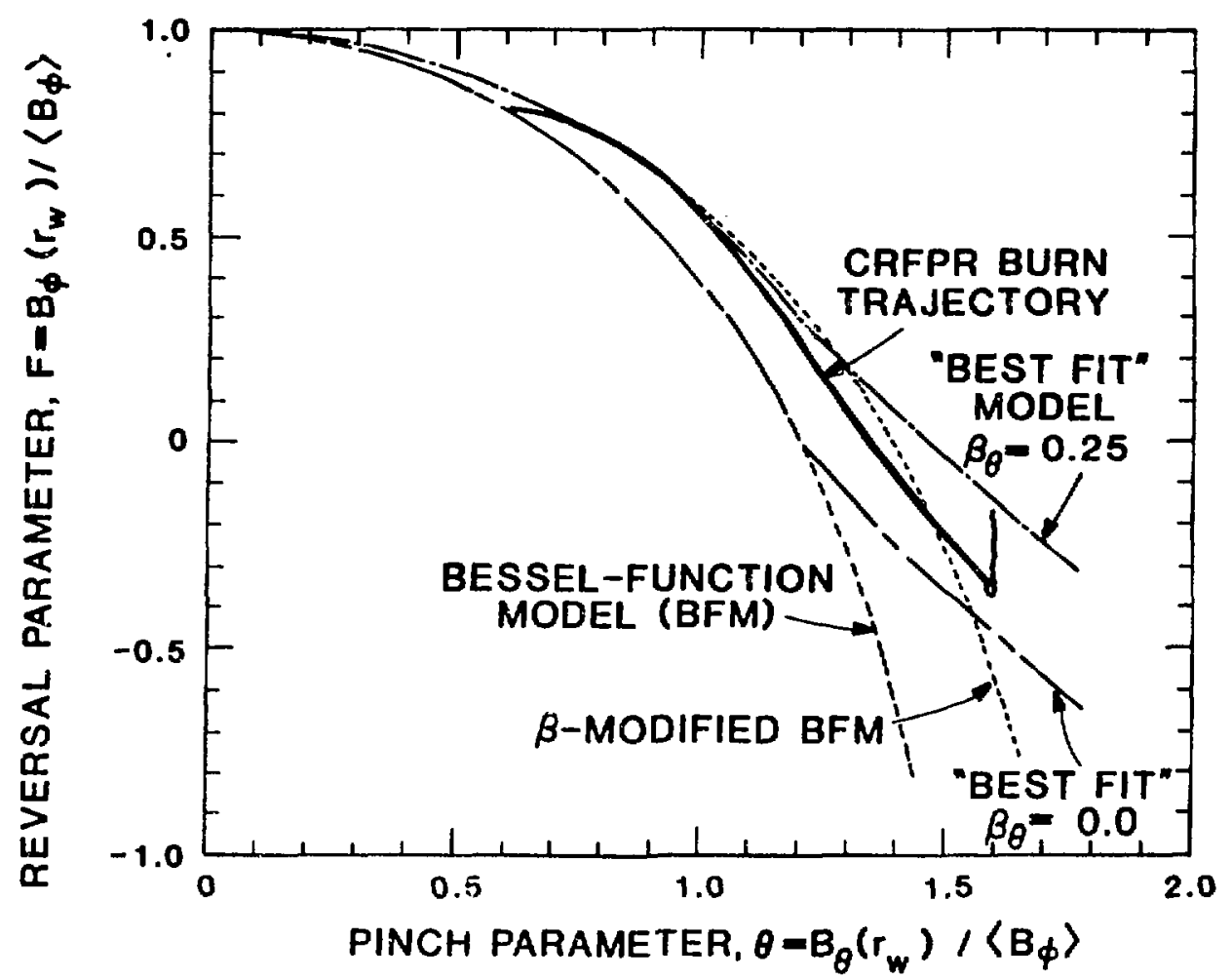

Fig. III.G-1. F- $\theta$ or Taylor diagram showing the relationship between $F=B_{\phi}\left(r_{p}\right) /\left\langle B_{\phi}\right\rangle$ and $\theta=B_{\theta}\left(r_{p}\right) /\left\langle B_{\phi}\right\rangle$, the reversal and pinch parametefs, respectively, for the BFM as well as for the "Best-Fit Model." The effects of finite beta are also shown.

magnetic fields plotted in Fig. III.G-3, which are similar to Bessel functions $B_{\phi} \propto J_{0}(\mu r)$ and $B_{\theta} \propto J_{1}(\mu r)$. Definition of the plasma profile characteristics allows equilibrium configurations to be generated and updated as the startup proceeds and the plasma approaches an ohmic ignition.

The reactor-operating scenario is illustrated in Fig. III.F-6, where an Initially uniform, vacuum toroldal fleld of $0.6 \mathrm{~T}$ is trapped inside the plasma upon initial formation. This initial toroidal field is chosen to be similar to the final value upon reversal in order to minimize engineering demands on the TFC system. The initial, tokamak-1ike condition corresponds to $F=1$ and $\theta=0$ on the F- $\theta$ diagram (Fig. III.G-1). As the plasma current increases to approximately $20 \%$ of the final value at $0.13 \mathrm{~s}$, the magnetic-field structure within the plasma is assumed to achieve the final reversed-field state with $\theta=1.55$ and $F=-0.12$ (FIg. III.G-1). Additional internal toroldal flux is assumed to be provided by the dynamo effect as the toroidal plasma current is increaseci to $I_{\phi}=18.4 \mathrm{MA}$. 


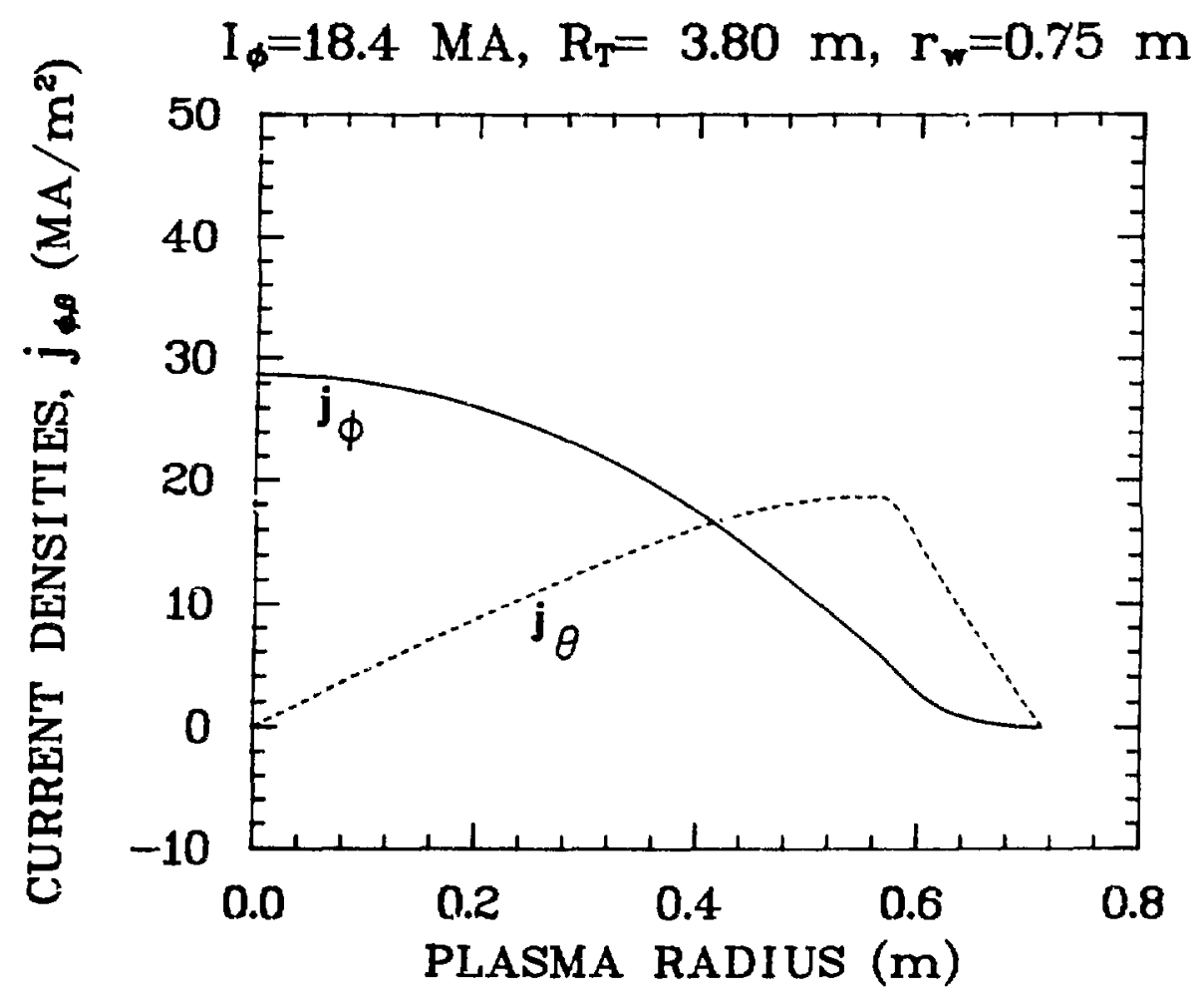

Fig. III.G-2. Current-density profiles for the $I_{\phi}=18.4-M A$ design-point simulation once steady-state is (Fig. III.G-I) achieved.

The reactor-operating scenario was descrlbed in Sec. III.F., where an analytic PFC circuit analysis was performed without including coll or plasma resistances. The plasma simulation and systems model incorporates the magnet-coll and plasma resistances, and the resultant voltage and current transients are given In Fig. III.F-16. An extended plot of the plasma current is given in Fig. III.G-4. The precharged OHC is reststively crowbarred while simultaneously initiating the plasma discharge and connecting the EFC across the OHC circuit. Approximately $2 / 3$ of the tnitially stored energy is dissipated in the transfer resistor while the plasma current rises from zero to $\sim 12$ MA in $1.2 \mathrm{~s}$. The rapid initial current transient of $32 \mathrm{MA} / \mathrm{s} 1 \mathrm{~s} 11 \mathrm{mited}$ only by the voltage allowed on the PFC system $(\leqslant 25 \mathrm{kV})$. The initlal transient is sufficient to overcome the rapid transpoit losses expected for a low-temperature plasma $\left(\tau_{E} \propto I_{\phi}^{V_{p}} r_{p}^{2} T^{v_{p}^{2}}{ }_{p}^{2} \quad\right.$ As the plasma temperature is increased, the plasma current rise can occur more slowly because of the decreased resistance and the highly inductive nature of the circuit configuration. This operational sequence m nimizes the power supply requirements for startup. 


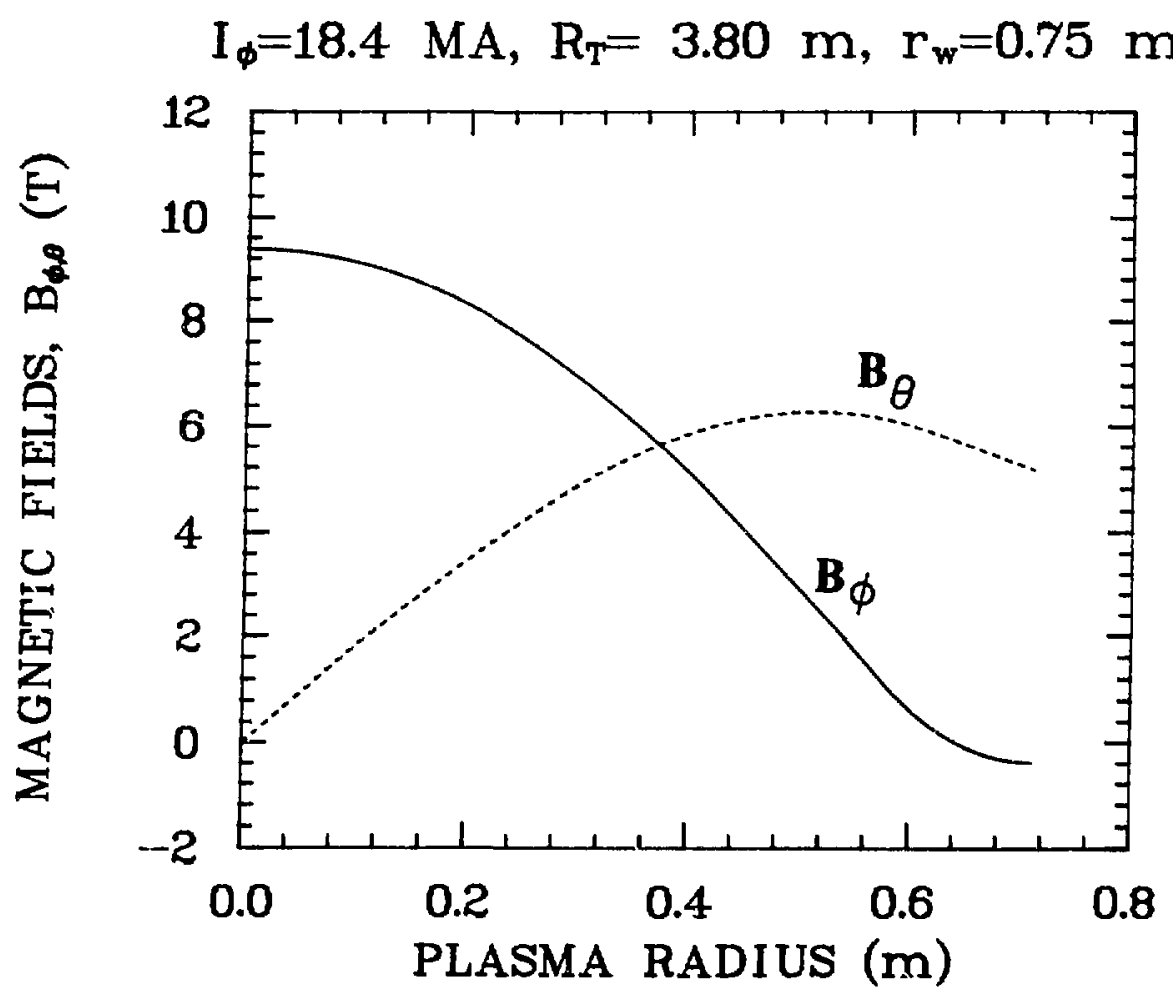

Fig. III.G-3. Magnetic-field profiles for the $I_{\phi}=18.4-\mathrm{MA}$ design-point simulation, corresponding to the current densities depicted on Fig. III.G-2.

In response to the plasma current rise plotted in Fig. III.G-4, the plasma ion and electron temperatures are expected to increase to a final operating temperature near $10 \mathrm{keV}$ through ohmic dissipation as the startup phase is completed. The density refueling rate and particle/energy transport are important in determining this temperature transient. Attributing the predominant energy loss to anomalous electron conduction, $\tau_{\text {ce }}$, a fit to ZT-40M data $\left(\tau_{c e} \sim 0.2 \mathrm{~ms}, I_{\phi}=0.12 \mathrm{MA}, r_{p}=0.2 \mathrm{~m}, \beta_{\theta}=0.1-0.15\right)$ gives a $\tau_{c e} \propto I_{\phi}^{\nu} r_{p}^{2}$ scaling (Fig. II-13), where $\nu$ is $<1.0$, but theoretically can approach 1.5 . The following scaling is adopted for this study:

$$
\tau_{c e} \simeq 5(10)^{-8} I_{\phi} r_{p}^{2} f\left(\beta_{\theta}\right)
$$

where the function $f\left(\beta_{\theta}\right)$ is given by 


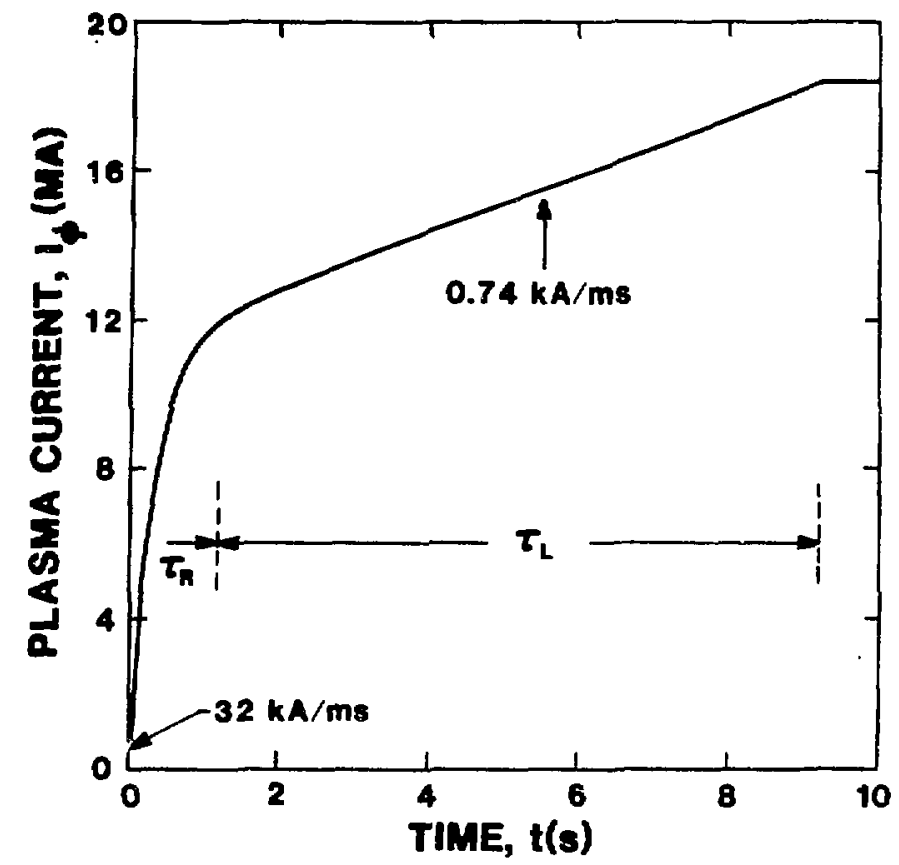

Fig. III.G-4. Expanded plot of plasma current transient.

$$
f\left(\beta_{\theta}\right)= \begin{cases}1.0 & , \beta_{\theta}<\beta_{\theta c} \\ \left(0.13 / \beta_{\theta}\right)^{2} & , \beta_{\theta}>\beta_{\theta c} .\end{cases}
$$

The function $f\left(\beta_{\theta}\right)$ is chosen to decrease $\tau_{c e}$ as a critical experimental value of $\beta_{\theta c}=0.13$ is exceeded. As discussed in Appendix $A$, the beta saturation effects on $\tau_{c e}$ exhibited by tokamak neutral-beam-heated experiments ${ }^{39}$ are assumed to apply also for the RFP. Also indicated by $2 \mathrm{~T}-40 \mathrm{M}$ experiments is the particle confinement time, which is taken as $\tau_{\mathrm{pi}} \simeq 4 \tau_{\mathrm{ce}}$; the ion-conduction time is taken as $\tau_{c i} \simeq \tau_{p i}$. Plotted in Fig. III.G-5 are the electron-conduction, $1 / \tau_{c e}$, and ion-particle, $1 / \tau_{p 1}$, loss rates as well as the inverse of the total plasma energy confinement time, $1 / \tau_{E}$, which includes both conduction and radiative losses. Also shown for comparison purposes are the classical ton-conduction time, $\tau_{\text {CLASS, }}$, and the characteristic ohmic-scailing time, toHM, explicit expressions for both being given in Appendix A. The plasma energy and particle times increase in direct proportion to the plasma current until increases in the 
poloidal beta exceeds the critical value, $\beta_{\theta c}=0.13$, which as shown in Figs. III.G-5 and III.G-6, reduces the confinement times. The total energy confinement time during the burn is only $\tau_{E} \simeq 0.24 \mathrm{~s}$ with the electron-conduction time being $\tau_{c e} \simeq 0.15 \mathrm{~s}$ at $\beta_{\theta}=0.23$.

The electron and ion density trajectories are given In Fig. III.G-7, where a starting density of $0.708(10)^{20} \mathrm{~m}^{-3}$ (1-mtorr filling pressure) is increased to $6.56(10)^{20} \mathrm{~m}^{-3}$ during the startup transient. The fueling algorithm used in this simulation attempts to maintain a constant value of $j_{\phi} / \mathrm{n}$ or $I_{\phi} / \mathrm{N}$, where $N=\pi r_{p}^{2} n$ is the plasma line density. The operating value of $I_{\phi} / N$ during the steady-state burn is $1.73(10)^{-14} \mathrm{Am}$, which corresponds to an electron-streaming parameter of $\xi=v / v_{T H} \simeq 0.08$. The, deuterium, $s_{D}$, and tritium, $s_{T}$, fueling rates are constrained to never exceed the instantaneous particle loss rate by at least $30 \%$ according to the following function:

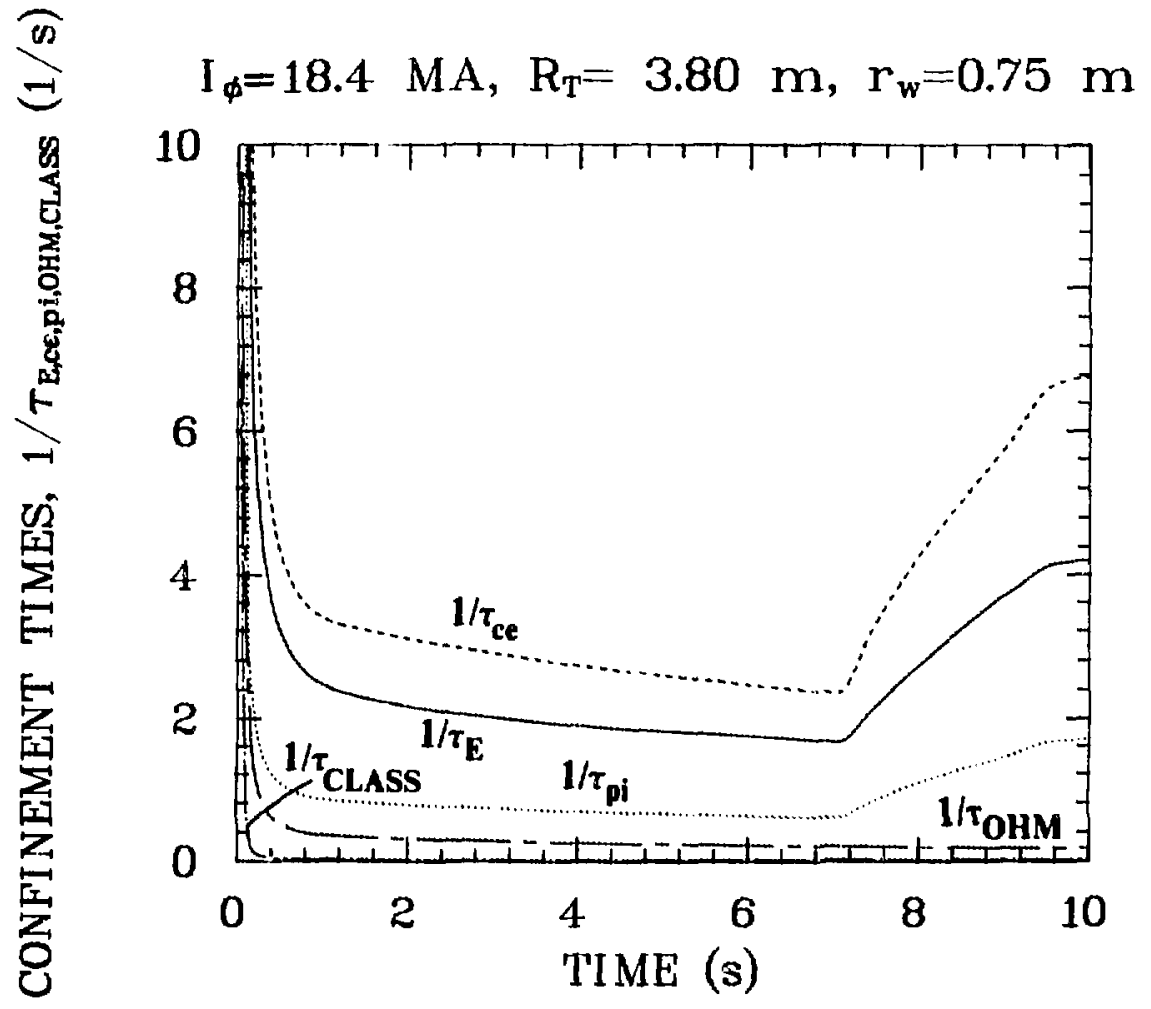

Fig. III.G-5. Dependence of electron-, ion-, and particle-conduction, times during the reactor startup using the scalings indicated by Eq. (III.G-2). The total confinement time includes both conduction and radiative $\left(Z_{\text {eff }}=1\right)$ losses. 


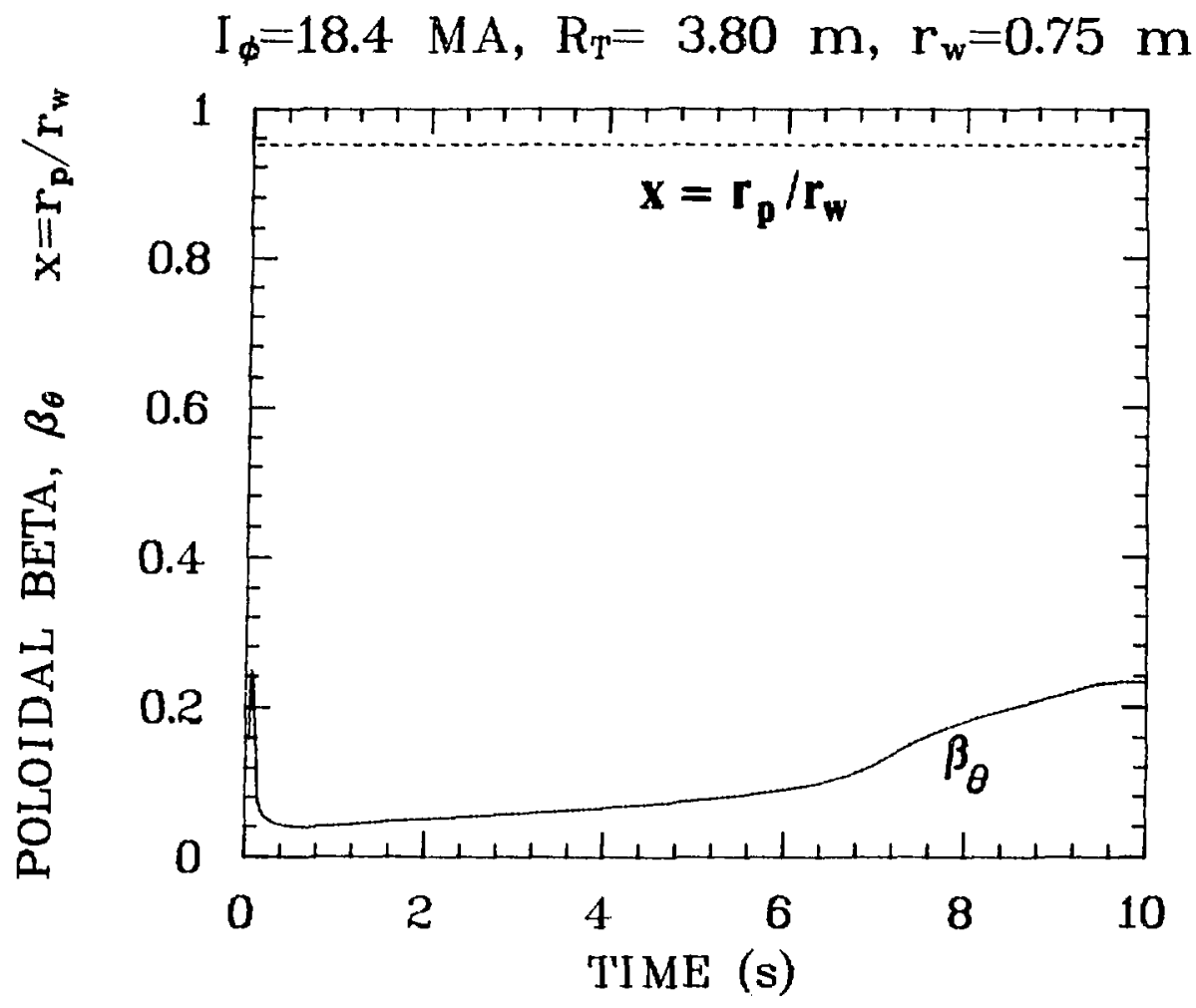

Fig. III.G-6. Time dependence of pololdal beta during the plasma startup transient.

$$
\begin{array}{ll}
\mathrm{S}_{\mathrm{D}}=\mathrm{S}_{\mathrm{T}}=1.3 \frac{\mathrm{N}_{1}}{2 \tau_{\mathrm{p} 1}}, & \mathrm{~N}_{1}<0.9737 \mathrm{~N}_{\mathrm{V}} \\
\mathrm{S}_{\mathrm{D}}=\mathrm{S}_{\mathrm{T}}=\frac{\mathrm{N}_{1}}{2 \tau_{\mathrm{P} 1}} \exp \left[10\left(1-\mathrm{N}_{1} / \mathrm{N}_{\mathrm{v}}\right)\right] & \mathrm{N}_{1}>0.9737 \mathrm{~N}_{\mathrm{V}},
\end{array}
$$

where $N_{v} \equiv I_{\phi} /\left(I_{\phi} / N\right)_{B}$ and $\left(I_{\phi} / N\right)_{B}$ is the target value during the steady-state burn. The exponential factor of 10 assumes the final line density will closely adhere to the desired value, $\mathrm{N}_{\mathrm{v}}$, and the factor 0.9737 simply assures a 


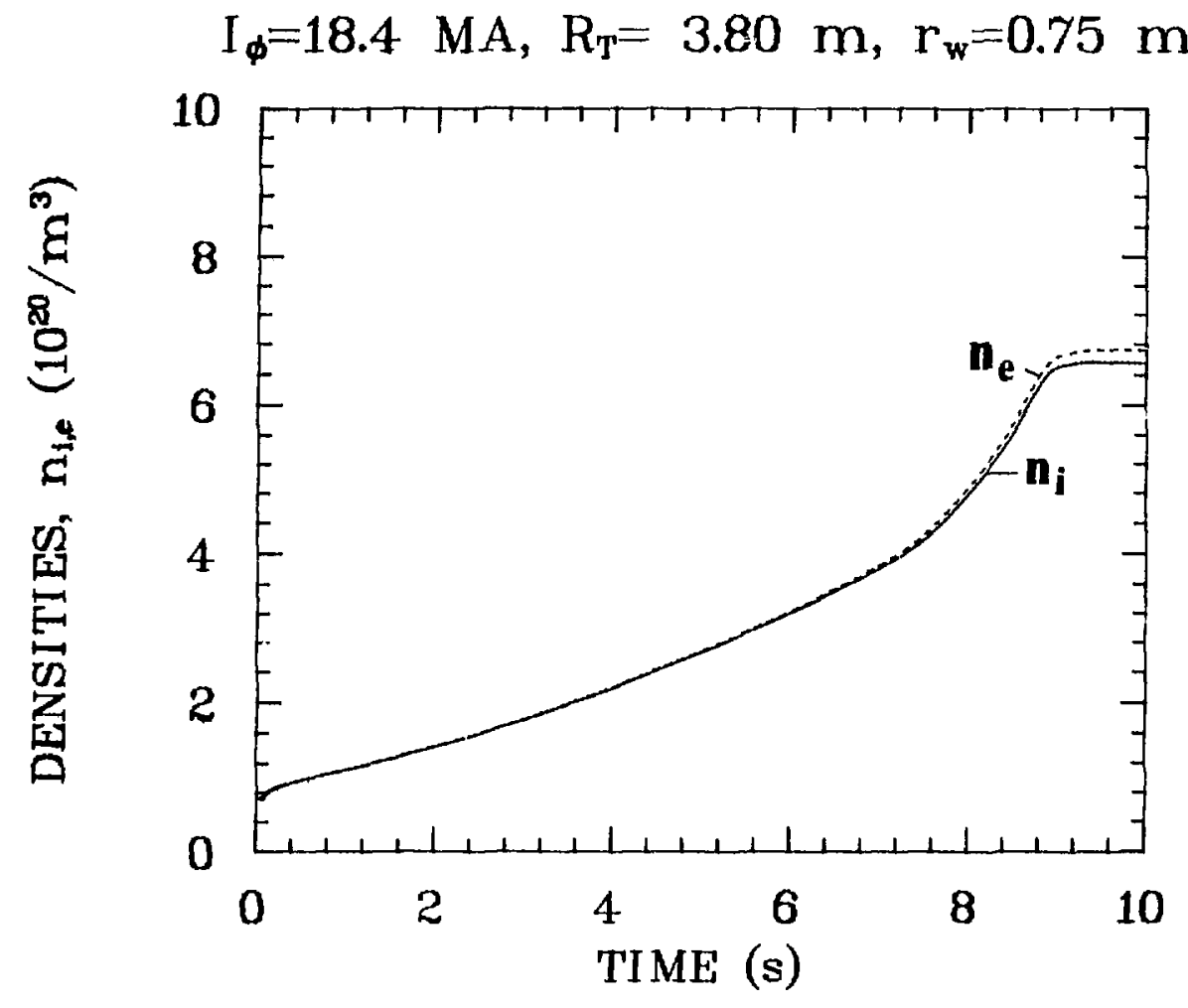

F1g. III.G-7. Time dependence of the electron and Ion density during the plasma startup transient fueling algorithm given by Eqs. (III.G-3).

continuous fueling function. This fueling algorithm avolds large Instantaneous and possibly unphysical fueling rates which may result when enforcing a strictly constant value of $I_{\phi} / \mathrm{N}$. For a major part of the startup trajectories reported here, the fueling rate is usually operating at this constraint rather than holding $I_{\phi} / \mathrm{N}$ coustant at the steady-state burn conditions. The resultant values of $I_{\phi} / \mathbb{N}$ and streaming parameter, $\xi$, are plotted in Fig. III.G-8 as a function of time, with the peak occurring as the initial, rapid current rise nears $I_{\phi}=12 \mathrm{MA}$ (Fig. III.G-4). Experimental results on $2 \mathrm{~T}-40 \mathrm{M}$ are optimal if $I_{\phi} / \mathrm{N}<(1-2) 10^{-13} \mathrm{~A} \mathrm{~m}$, above which a runaway condition may result for $\mathrm{T}_{e} \simeq$ $250-\mathrm{eV}$ ZT-40M conditions, or the streaming parameter $\xi$ may become too large, causing excessive resistance through streaming instabilities. The operating range of $I_{\phi} / \mathrm{N}=1.5-6(10)^{-14} \mathrm{Am}$ during startup is typlcal of $2 \mathrm{~T}-40 \mathrm{M}$ experimental discharges. 


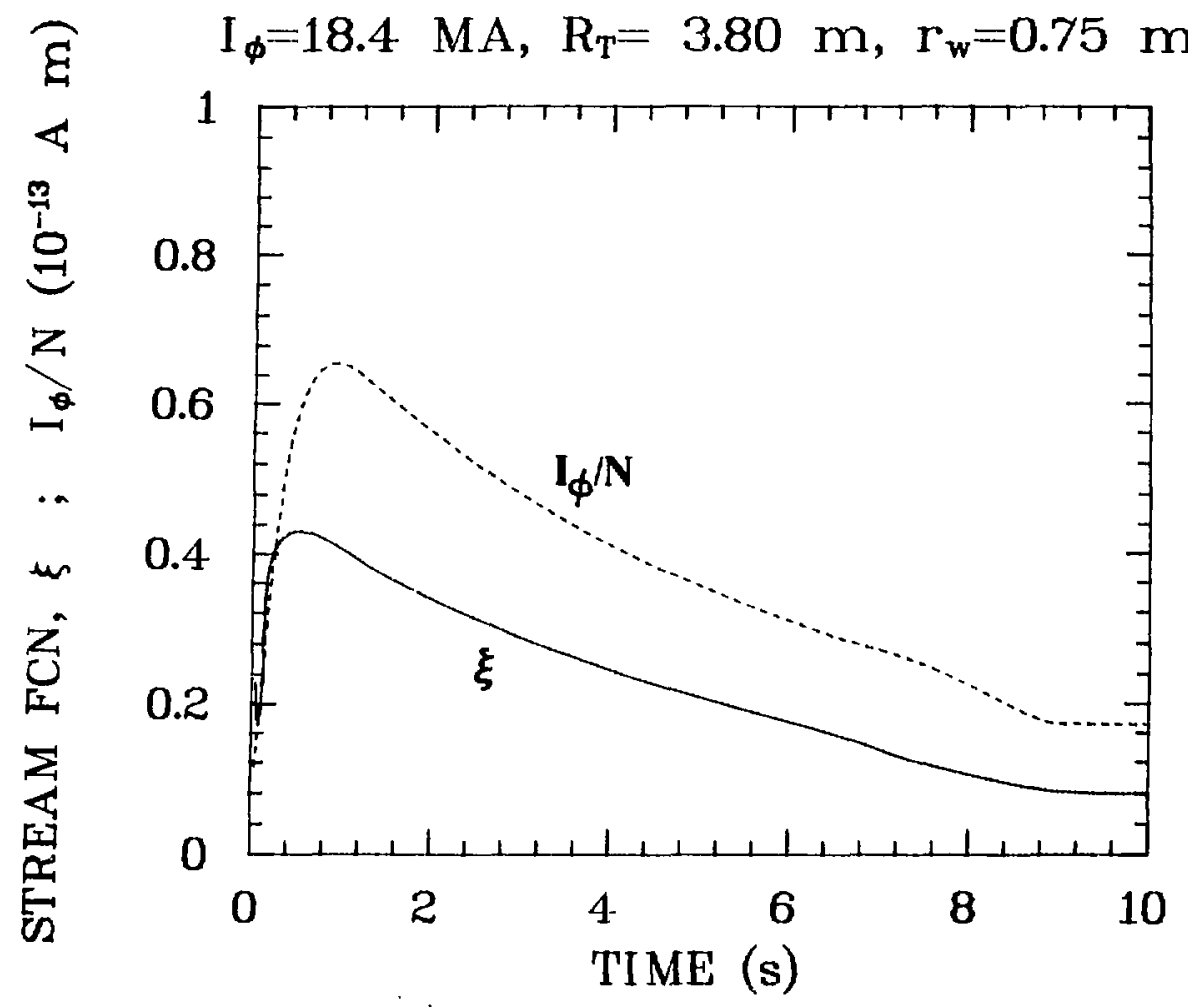

Fig. III.G-8. Time dependence of $I_{\phi} / \mathrm{N}$ and streaming parameter $\xi$ during the plasma startup transient for the fueling algorithm given by Eqs. (3).

The product of particle inventory and plasma energy confinement yields a single confinement parameter, $n \tau_{E}$, which is plotted in Fig. III.G-9. A confinement parameter of $\sim 2(10)^{20} \mathrm{~s} / \mathrm{m}^{3}$ is required to initiate the discharge ohmically using the refueling algorithm described by Eqs. (III.G-3) for $\mathrm{Z}_{\text {eff }}=1.0$. The maximum value of $n \tau_{E}$ required during ohmic startup is affected by the fueling scheme and the associated density increase and, therefore, is considered representative of a range of startup options. The value of $n \tau_{E} \simeq 1.6(10)^{20} \mathrm{~s} / \mathrm{m}^{3}$ at ignition/burn is also considered only as representat Ive in that $n \tau_{E}$ depends on density and temperature profiles.15 It is noted that the global $n \tau_{E}$ for the STARFIRE tokamak reactor design $1 \mathrm{~s} 0.64(10)^{20} \mathrm{~s} / \mathrm{m}^{2}$, where $\tau_{E}$ is the total plasma energy divided by total plasma heating power (alpha-particle plus rf power). 


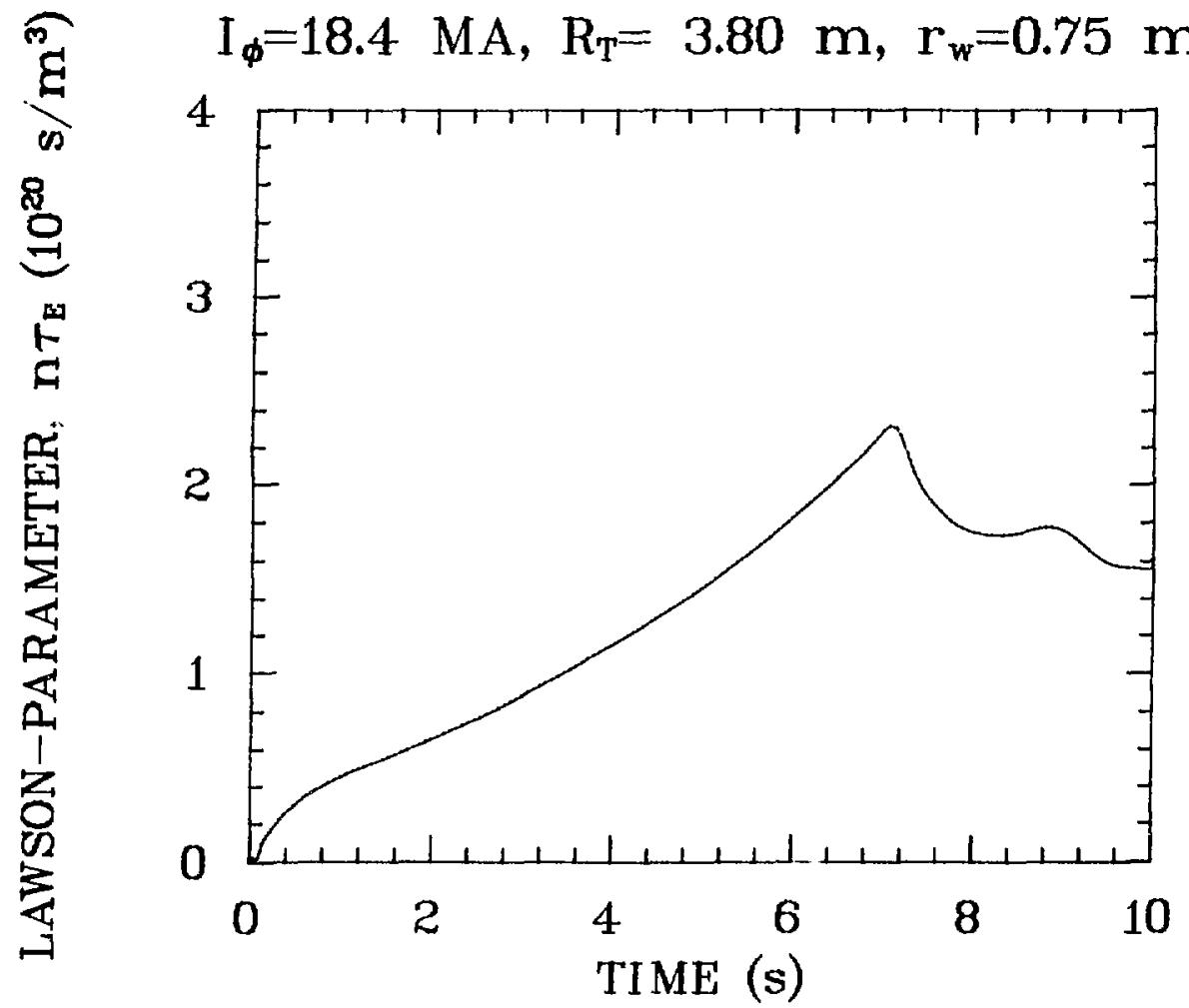

FIg. III.G-9. Time dependence of the confinement parameter, $n \tau_{E}$, during the plasma startup transient for the fueling algorithm given by Eq. (III.G-3).

The plasma temperature transients shown in Fig. III.G-10 exhibit an initially rapid rise in electron temperature in response to the Increase in plasma current and ohmic-heating power. The damped osctilation in $n \tau_{E}$ and $T_{e, 1}$ are a result of the primitive fueling algorithm used as well as the physics assoclated with alpha-particle thermalization and transport (beta) dynamics. The variation in plasma temperature is driven by the plasma powers plotted in Fig. III.G-11, which shows the total charged-particle fusion power, $P_{Q}$, as well as the conduction, $\mathrm{P}_{\mathrm{COND}}$, ohmic, $\mathrm{P}_{\mathrm{OHM}}$, bremsstrahlung, $\mathrm{P}_{\mathrm{BR}}$, and (insignificant) cyclotron, $P_{C Y C}$, powers. The initial separation between fon and electron temperature results from the low rate of equipartition when the plasma density is low. As the density increases, this temperature difference diminishes, although domisant electron heating from both ohmic and alpha-particle deposition always maintain $T_{e}>T_{1}$. As the current and plasma density increase, the alpha-parificle heating power steadily increases unt $i l P_{Q}>P_{O H M}$ at $6 \mathrm{~s}$ into the startup transient and Ignition is achieved. This initial transient toward 


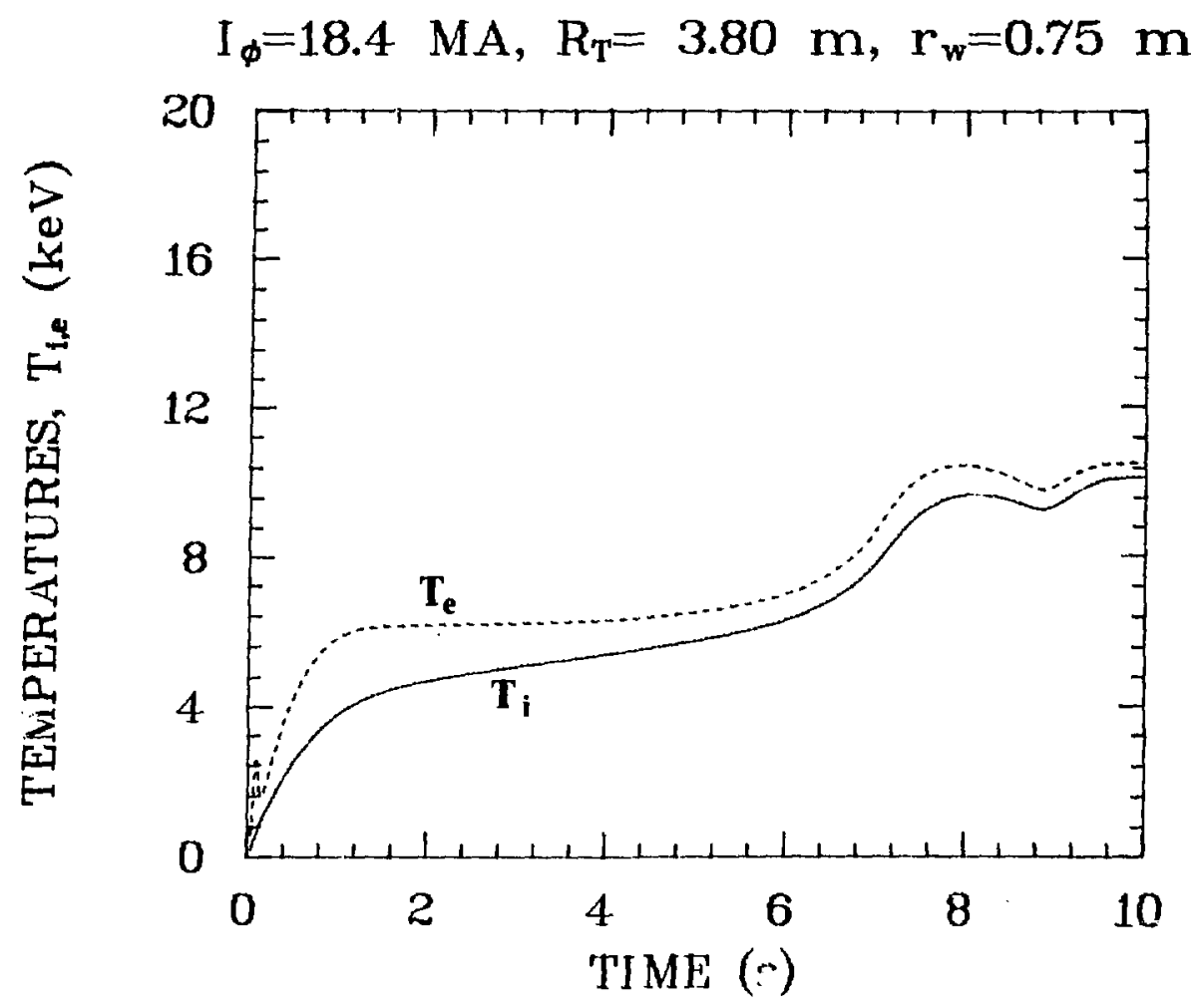

FIg. III.G-10. Time dependence of the average fon and electron temperatures during the plasma startup transient.

Ignition occurs with a nearly constant betr, as is seen from Fig. III.G-6. The rapidly increasing alpha-particle heating power forces the beta to increase, which in turn increases the transport loss [Eq. (2)]. A highly damped plasma temperature oscillation occurs as the rapid temperature increase is overcome by the enhanced transport. A steady-state burn is achieved at $9.5 \mathrm{~s}$ after plasma current intitiation.

The Fokker-Planck slowing-down model Includes the effects of the energetic (non-Maxwelifan) particles in the burn simulation. In addition to heating, the plasma energetic particles contribute to the plasma beta without producing significantly useful fusion power. The temperatures of the energetic species $\left(T, D,{ }^{3} \mathrm{He}, \alpha, H\right)$ are plotted in Fig. III.G-12, with essentially no energetic deuterium assumed to be present (Appendix A). Only the energetic alpha particles are important for this DT burn, as is shown in Fig. III.G-13. Durirg the steady-state burn, these $850-\mathrm{keV}$ alpha particles are on $1 \mathrm{y} 3(10)^{-3}$ of the total plasma ion population but contribute to $12.7 \%$ of the total plasma 


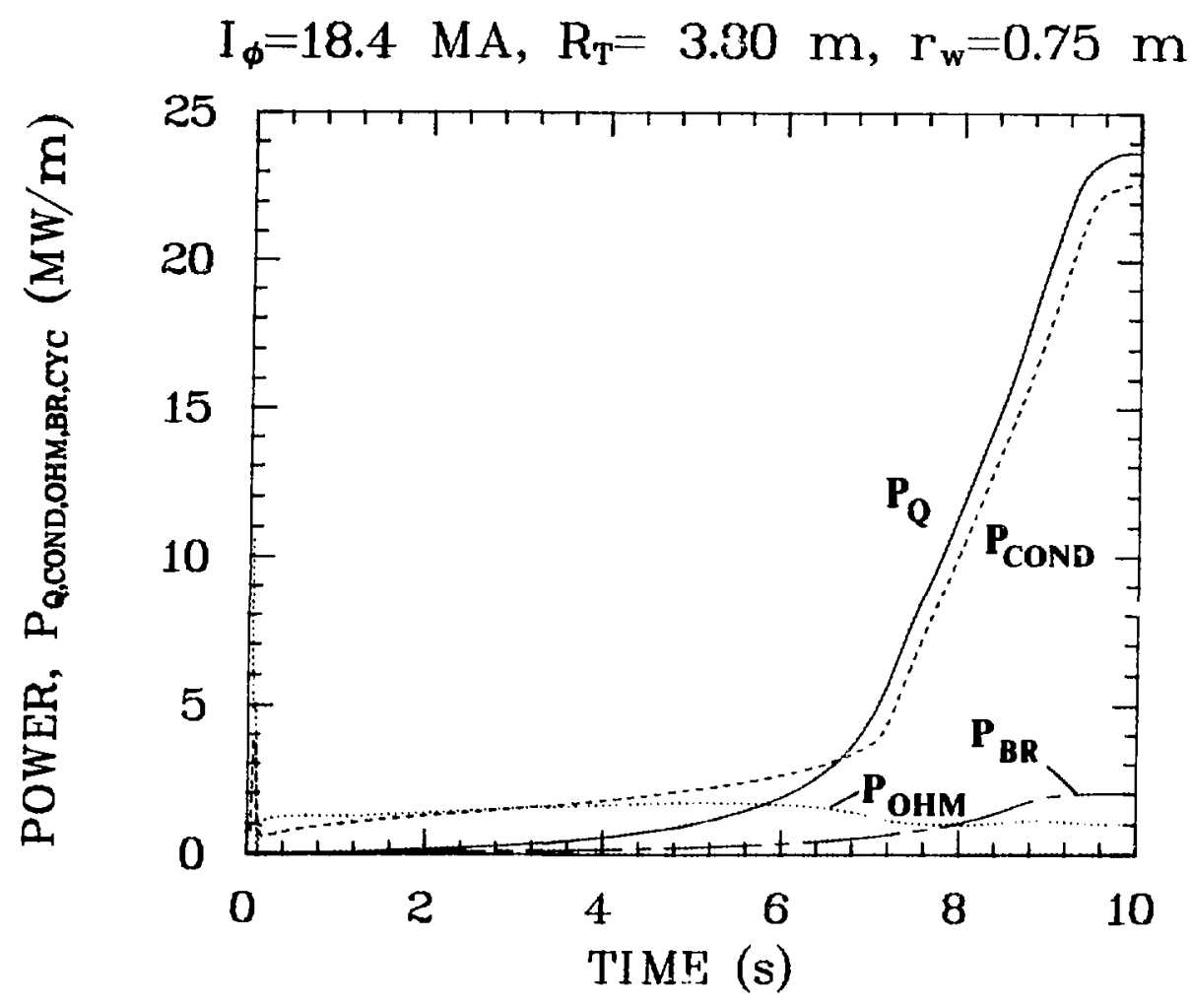

Fig. III.G-11. Time dependence of plasma powers during the plasma startup transient for the fueling algorithm given by Eqs. (3).

pressure. Maxwellian alpha particles are also present in the plasma at a concentration of $2.5 \%$ (Fig. III.G-13) and contribute $3.7 \%$ of the plasma pressure, including the added electrons. The percentage of the total pressure that is parasitic is $16.5 \%$ and requires the target plasma beta to be inflated from the 0.20 design value to the plasma burn value of 0.233 . An apparent advantage of operating at high plasma density is the relatively low resultant concentration of suprathermal particles. Low-density plasma burns ${ }^{2}$ often have parasitic energetic-particle pressure contributions in excess of $30 \%$.

The primary goal of the plasma-simulation study is the verification and/or adjustment of the CRFPR operating parameters suggested by the ear1ier studies. 15 Table III.G-I repeats Table II.D-III and includes the results from the more-detalled simulations. The parameters 1isted In Table III.G-I under "Burn Simulation Reference" are adopted hereafter for the FPC integration study given in Sec. III.L. 


\section{KEY PHYSICS AND ENGINEERING PARAMETERS FOR THE $\mathrm{P}_{\mathrm{E}}=1000-\mathrm{MWe}$ MINIMUM-COE CASES GIVEN IN TABLE II.D-III UPDATED USING THE TIME-DEPENDENT PLASMA/FPC SIMULATION CODE}

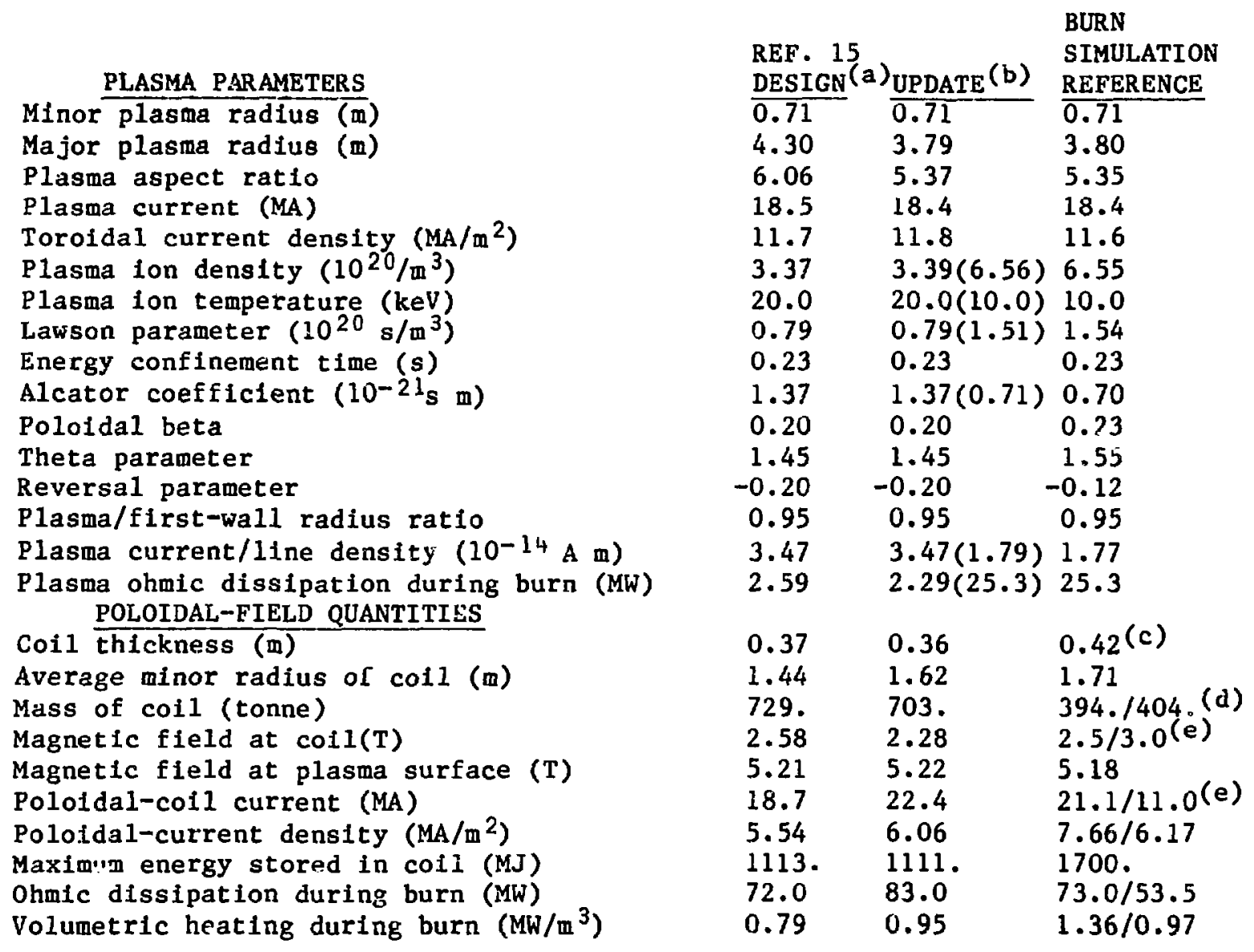

(a) Based on flat plasma temperature profile.

(b) Values in () correspond to changes resulting from the assumption of a $T(r) \propto J_{0}(\mu r)$ temperature profile.

(c) For comparison, discrete colls are lumped into a uniform annulus.

(d) Ohmic-heating col1 (OHC)/Equilibrium-field coll (EFC) parameters given.

(e) Start-of-burn values are listed. EFC current would remain unchanged, although the OHC current would be reduced to zero as current drive is provided by other sources.

(f) The starting fleld is taken to be only $0.6 \mathrm{~T}$ with the bulk of the internal plasma flux and energy provided from the PFC curcult via the dynamo effect. 
Coil thickness (m)

Average minor radius of coil (m)

Mass of coll (tonne)

Inttial toroidal bias field ( $T$ )

Reversed toroidal fleld during burn (T)

Maximum energy stored in the coil 1 (MJ)

Toroldal current density (MA/m ${ }^{2}$ )

Ohmic dissipation during burn ( $M$ )

Volumetric heating during burn ( $\mathrm{MW} / \mathrm{m}^{3}$ )

\section{ENGINEER ING SUMMARY}

Ohmic Q-value

Total thermal power (MWt)

$14.1-\mathrm{MeV}$ neutron loading (MW/m $\left.{ }^{2}\right)$

14.1-MeV blanket multiplication

First-wall radius (m)

Minor radius of system (m)

Fusion-power-core mass (tonne)

System power density ( $\left(\mathrm{Wt} / \mathrm{m}^{3}\right.$ )

Mass utilization (tonne/MWt)

Blanket/shield thickness (m)

iass of first wall/blanket (tonne)

Average blanket power density $\left(\mathrm{MWt} / \mathrm{m}^{3}\right)$

REF. 15
DESIGN (a) UPDATE (b)

BURN

SIMULATION

REFERENCE

$\begin{array}{lll}0.10 & 0.09 & 0.075 \\ 1.20 & 1.39 & 1.4625 \\ 159 . & 149 . & 72.8 \\ 3.33 & 3.34 & 0.60(\mathrm{f}) \\ 0.68 & 0.68 & 0.40 \\ 539 . & 542 . & 22.7(\mathrm{~d}) \\ 5.54 & 6.06 & 7.04 \\ 15.71 & 17.61 & 12.6 \\ 0.79 & 0.95 & 1.27\end{array}$

$37.11 \quad 32.92(27.5) 20.5$

$3350 . \quad 3389 .(3460) 3365$.

$\begin{array}{lll}19.5 & 19.6(20.0) & 19.5\end{array}$

$\begin{array}{lll}1.10 & 1.30 & 1.277\end{array}$

$\begin{array}{lll}0.75 & 0.75 & 0.75\end{array}$

$1.62 \quad 1.80 \quad 1.92$

1243. 1368 . 1105 .

$\begin{array}{lll}15.00 & 14.00 & 11.8\end{array}$

$\begin{array}{lll}0.37 & 0.40 & 0.33\end{array}$

$0.40 \quad 0.60 \quad 0.60$

355. $516 . \quad 234.1$

$\begin{array}{lll}51.92 & 36.1 & 33.8\end{array}$

(a) Based on flat plasma temperature profile.

(b) Values in () correspond to changes resulting from the assumption of a $T(r) \propto J_{0}(\mu r)$ temperature profile.

(c) For comparison, discrete colls are lumped into a uniform annulus.

(d) Ohmic-heating coll (OHC)/Equilibrium-field coil (EFC) parameters given.

(e) Start-of-burn values are listed. EFC current would remain unchanged, although the OHC current would be reduced to zero as current drive is provided by other sources.

(f) The starting field is taken to be only $0.6 \mathrm{~T}$ with the bulk of the internal plasma flux and energy provided from the PFC circuit via the dynamo effect. 


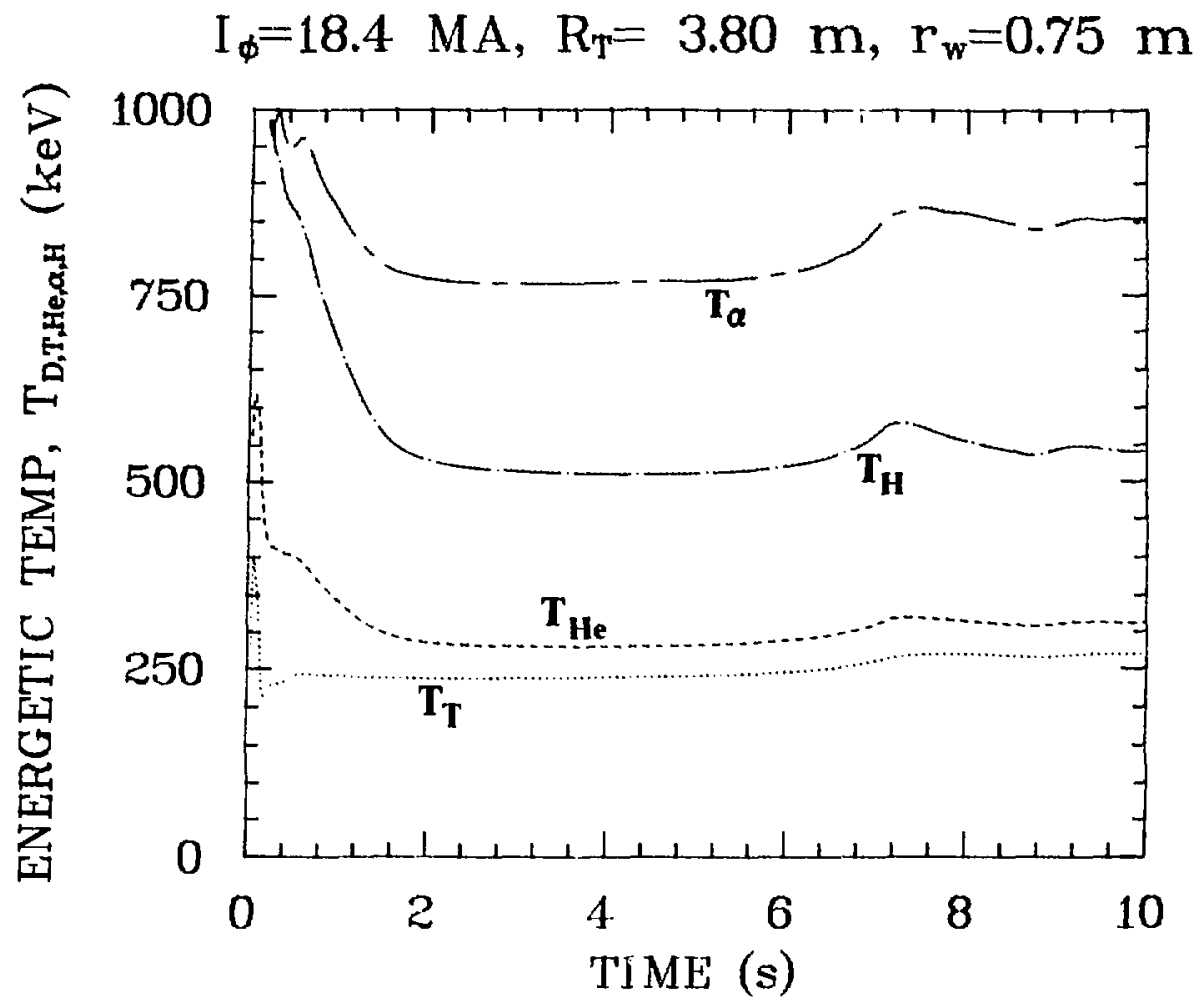

Fig. III.G-12. Time dependence of suprathermal particle temperatures during the plasma startup transient.

of primary interest when analyzing reactor startup scenarios is the first-wall thermal-mechanical transient. The plasma burn code also monitors the thermal-mechanical response of the first wall, although more-detailed engineering computations are made when completing the conceptual design. The average surface heat flux, $I_{Q w}$, and total DT neutron current, $I_{w}$, at the first wall are plotted on Fig. III.G-14. The neutron and first-wall heat fluxes are not separated by the factor of four that is expected simply because of the DT alpha-particle/neutron energy split. The average surface-neutron flux is plotted while the surface-heat flux is adjusted by $(2 / \pi) R_{T} /\left(R_{T}-r_{w}\right)$, where $2 / \pi$ accounts for the added surface area of a set of first-wall coolant tubes (Sec. III.D.), and the factor $R_{T} /\left(R_{T}-r_{w}\right)$ is a first-order approximation to consider geometric peaking in a torus. Coolant tubes near the inboard toroldal radius, $R_{T}-r_{w}$, are analyzed using the surface flux plotted in Fig. III.G-14. A volumetric neutron heating of $11.33 \mathrm{I}_{\mathrm{w}} \mathrm{R}_{\mathrm{T}}{ }^{\prime}\left(\mathrm{R}_{\mathrm{T}}-\mathrm{r}_{\mathrm{w}}\right)$, as derived from the one-dimensional neutronic calculations (Sec. III.B.), is also included in the 

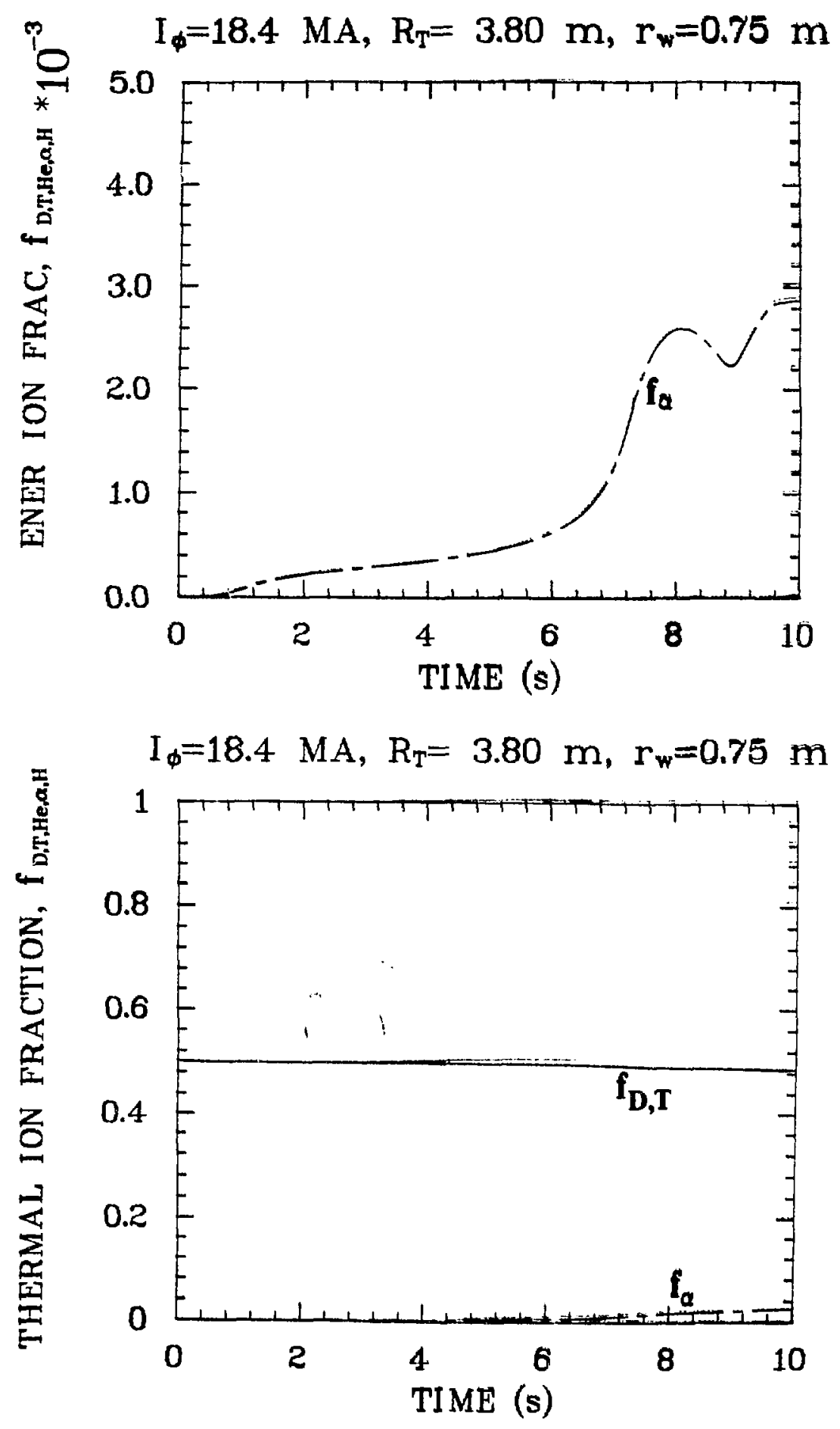

Fig. III.G-13. Time dependence of alpha-particle fraction during the plasma startup transient. 
one-dimensional heat-transfer calculation performed as an integral part of the plasma burn simulation.

The first-wall structure suggested in Sec. III.D. is used to estimate the thermal/mechanical response during the startup transient. The water-cooled copper-alloy tubes near the Inboard first-wall region have a d/2 $=24 \cdot 3-\mathrm{mm}$ Inside radius and have a $\delta=3-\mathrm{mm}$ tube-wall thickness. Using physical parameters characteristic of copper described in Appendix $A$ and cooling the tubes with pressurized water at $463 \mathrm{~K}$ having a heat-transfer coefficient of $1.14(10)^{5} \mathrm{w} / \mathrm{m}^{2} \mathrm{~K}$, the thermal response of the inside and outside coolant tube surfaces is shown in Fig. III.G-15. The resulting thermal stresses are given in Fig. III.G-15B; a maximum of $\pm 82 \mathrm{MPa}(123 \mathrm{kpsi}$ ) is indicated. For a nominal Young's modulus of $100 \mathrm{GPa}$, this stress corresponds to a thermal strain of $60.1 \%$, which is expected to be far below values considered life limiting from the viewpoint of cyclic fatigue. Inclusion of more-realistic effects [1.e., peaking factors, wall thinning because of sputtering, reduced heat transfer coefficient, radiation-degraded thermal and mechanical properties, and primary-gtress (coolant pressure) creep] will certainly reduce these margins and

$$
\mathrm{I}_{\phi}=18.4 \mathrm{MA}, \mathrm{R}_{\mathrm{T}}=3.80 \mathrm{~m}, \mathrm{r}_{\mathrm{w}}=0.75 \mathrm{~m}
$$

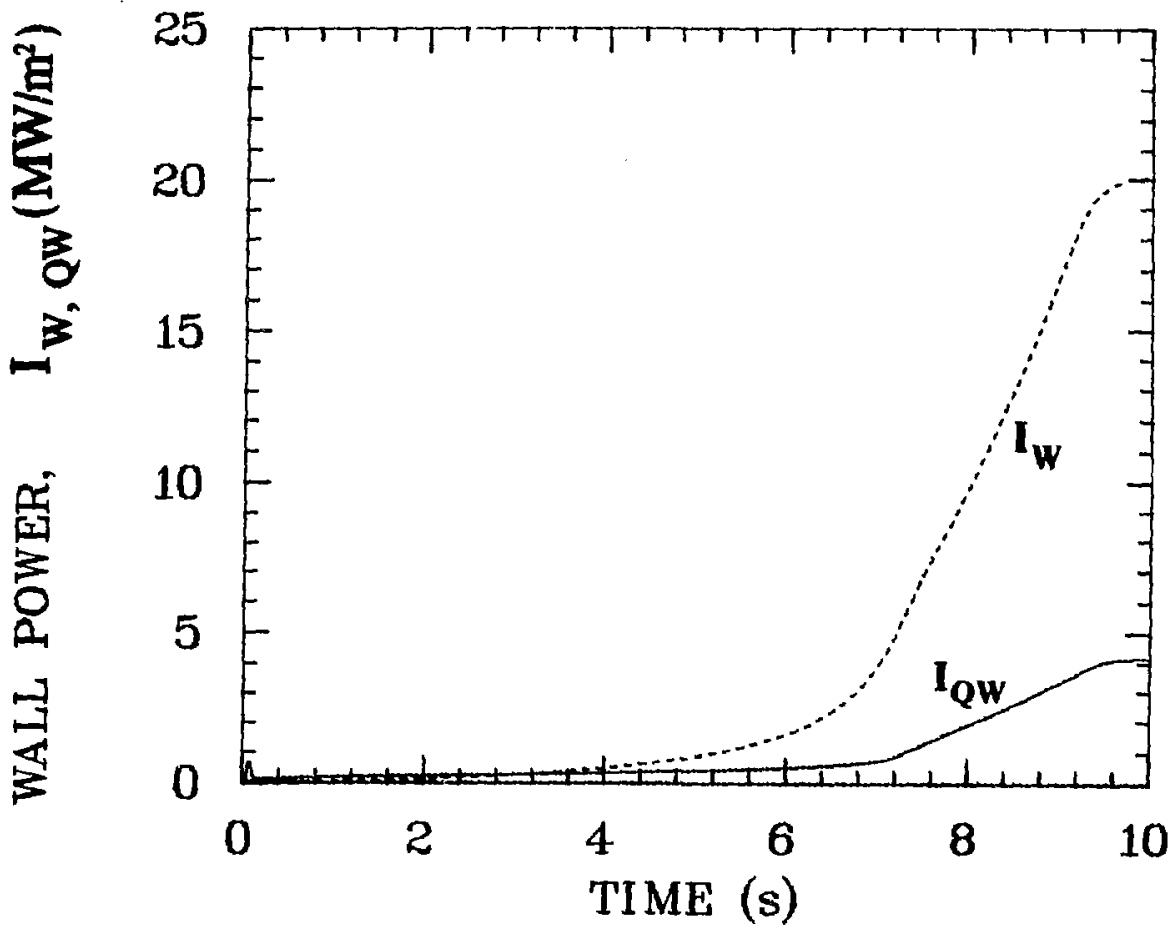

F1g. III.G-14. Time dependence of average heat and neutron power fluxes at the first wall during the plasma startup transient. 


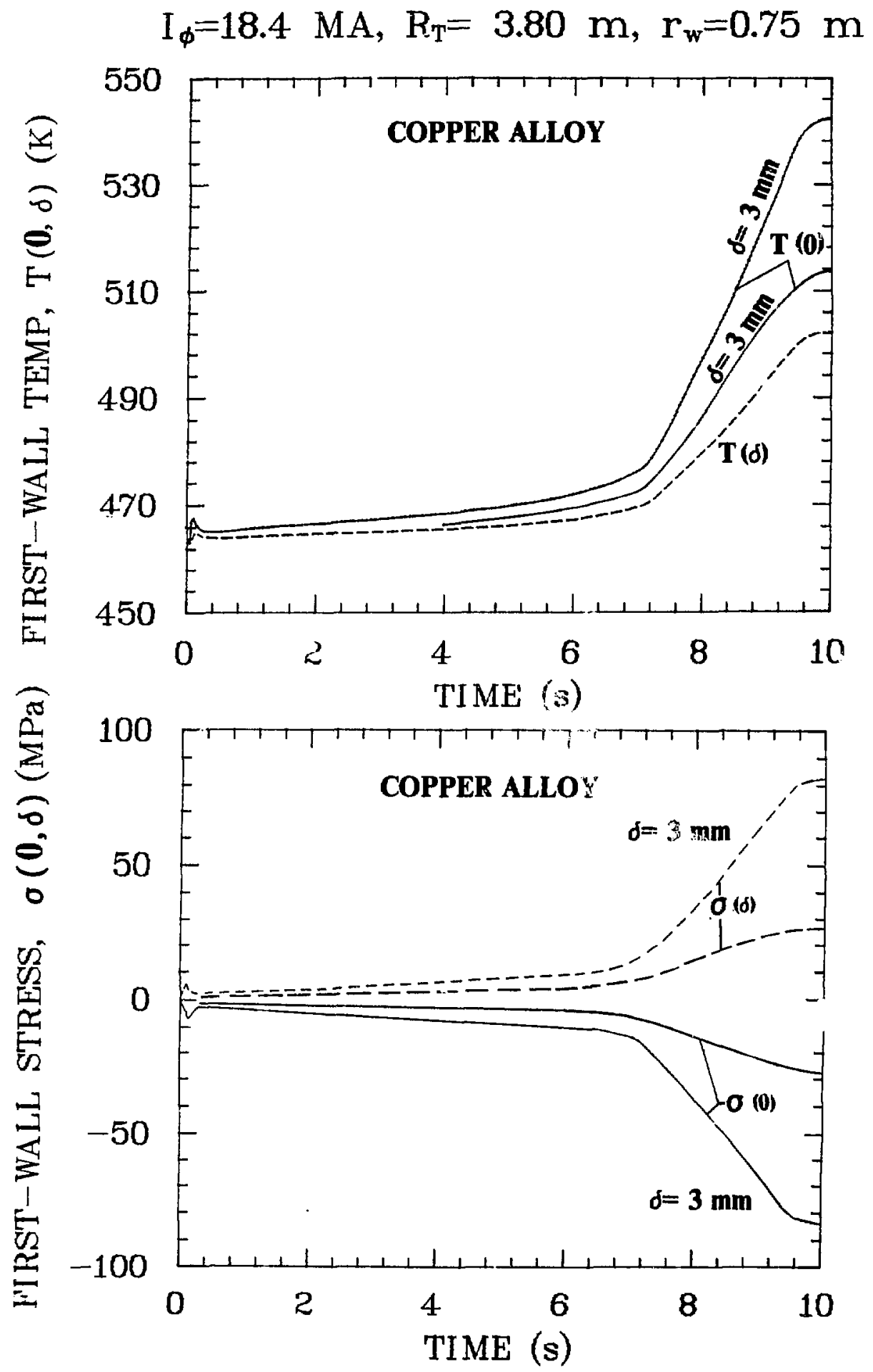

Aar transient as estimated by the plasma-simulation mode. Gestion III.D. gives a more-detailed steady-state tiverwal/mechanical analysis of the first wall. 
make stronger the case for uninimizing the number of startup transients of the kind modeled in this section. Other factors related to external power transients (Fig. III.F-17), cyclic stresses in other FPC components (i.e., blanket, shield, manifolds/headers, structure coils), and overall energy balance combine to make a strong case for extended if not steady-state burns. Thi following sections on particle control, divertors, vacuum, and current driv. emphasize a steady-state operation. 


\section{H. Particle Control By Pumped Limiters}

The mechanisms by which heat and particles emanating from the plasma interact with the reactor structure represent the premier problem for controlled fusion; a better understanding and proper control of this interaction is essential for the Iife of both plasma and flrst-wa11/1imiter systems. Uniform deposition of conductive/convective/radiative plasma power to in-vacuum component (IVC, first-wall, limiter, divertor-plate) surfaces becomes even more cructal for the compact, high-power-density systems because of the higher energy and particle fluxes expected $\left(\geq 5 \mathrm{MW} / \mathrm{m}^{2}, \geq 10^{21}\right.$ particles $\left./ \mathrm{m}^{2} \mathrm{~s}\right)$. The shorter acceptable design (chronological) life for a given fluence of neutrons (1.e., dpa or $M W y r / m^{2}$ ) or energetic particles (1.e., mm sputtering margin) must be weighted against a considerably smaller IVC system in order to evaluate the potential advantages of the compact approach.

To develop a better understanding of the plasma/first-wall interaction for the RFP configuration, tokamak models 134-137 for the edge-plasma region, or reglons where magnetic-field lines intersect physical structure (1.6., the scrapeoff region or simply "scrapeoff") have been appropriately modifled. Specifically, the simplified scrapeoff model developed and used for the tokamak FED design 137 has been 1mplemented with neutral-particle transport and is used to model the parameter range where pumped limiters may be applied to the CRFPR. The basis of this scrapeoff model is the one-dimensional, steady-state heat and particle equations. This set of equations is solved in the scrapeoff for an RFP magnetic topology. An algorithm that adjusts the shape and size of a limiter surface to assure a uniform design heat load is also implemented. Parametric results include variations of pumped-limiter performance with the number of poloidal limiters, the value of the design heat flux, the scrapeoff thickness, the plasma/scrapeoff interface conditions, the plasma/scrapeoff/first-wall recycle rate, and the degree of anomalous transport in the scrapeoff. The primary issue being addressed at this early development of the RFP plasma-edge model is heat transfer and limiter pumping efficlency rather than first-wall/1imiter sputter erosion. An improved model for the erosion must. Include a better (self-consistent) model for edge-plasma impurity/radiation transport as well as the bulk plasme transport; these are topics for future work. 
1. Scrapeoff and Limiter Mode1. The essential elements of the CRFPR gerapeoff and IImiter models are depicted in F1g. III.H-1. Because of the dominance of the pololdal fleld at the plasma-edge radius, $r_{p}$, a toroldal array of $N_{L}$ pololdal limiters is proposed. In such a configuration, the field-line connection length, $L$, between limiters can be made sufficiently long to allow an adequate amount of radial diffusion into the scrapeoff region ( $x$-axis in F1g. III.H-1) as edge-piasma particles follow the high-pitch field lines between adjacent Iimiter surfaces. The radial diffusion is assumed to be Bohm like and must be sufficient a) to counteract the tendency to concentrate heat flux onto the leading edge of the limiter, and $b$ ) to give for the purposes of pumping an acceptably large particle flux into the channel formed by the limiter and the first wall. The pololdal limiters of tor dal extent $l_{L}$ are separated by a toraldal (z-axis in F1g. III.H-1) distance, $\ell_{\downarrow}$ stth the total number being

$$
N_{L}=2 \pi R_{T} /\left(\ell_{L}+\ell_{W}\right)
$$

The average scrapeoff thickness, $\delta=r_{w}-r_{p}$, is determined by allowing space for vacuum pumpout.

BULK PLASMA BULK PLASMA

PARTICLE LOSS ENERGY OOSS

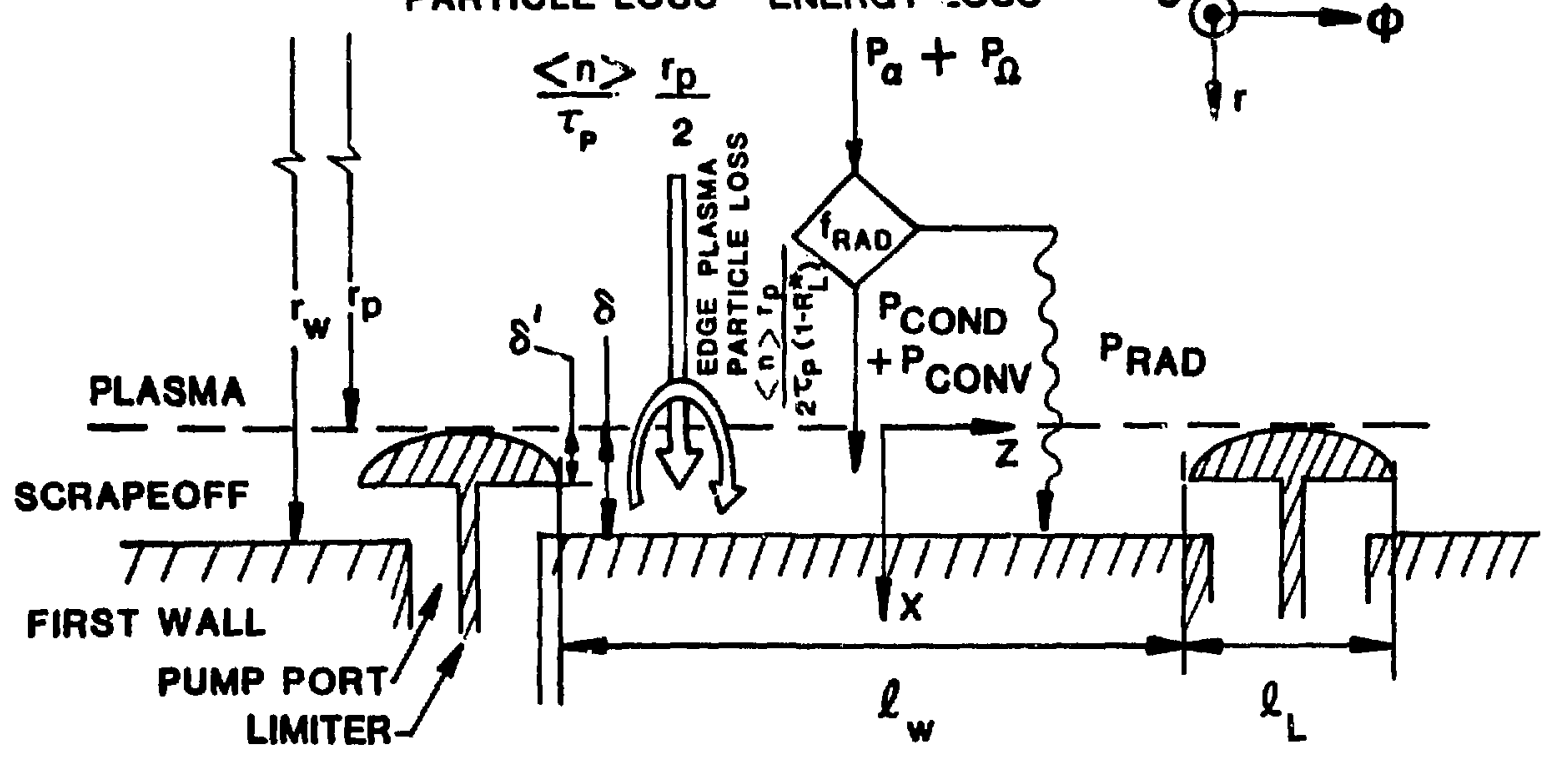

F1g. III.H-1. Schematic (distorted) diagram of the RFP pumped-11miter model. 
Although the leading edge of the limiter is assumed to be positioned at the plasma radius, $r_{p}$, image currents in fact may be established in the limiter that may cause the plasma minor radius to vary along the toroidal direction. The two-dimenstonal nature of the reversal layer, perturbations of the toroldally asymmetric conducting shell, and the effects of the radial field components on plasma confinement are not known and remain an important area for future work.

The total power being shed by the ignited plasma is $P_{\alpha}+P_{\Omega}$, where $P_{\alpha}$ is the alpha-particle power generated in the plasma, and $P_{\Omega} \ll P_{\alpha}$ is the ohmic dissipation in the bulk (ignited) plasma. The fraction $f_{R A D}$ of the cotal energy lost from the plasma is assumed to be radiated uniformly to the limiter and first-wall surfaces. The remaining power, $\left(1-f_{R A D}\right)\left(P_{\alpha}+P_{\Omega}\right)$, enters the scrapeoff region as a conductive/convective heat flux. The fraction $f_{R A D}$ is treated parametrically, although in actuality $f_{R A D}$ will be determined by the bulk- and edge-plasma impurity concentration and species, which in turn must be coupled with the dynamics of plasma transport and structural sputtering. The development and/or implementation of such a model is beyond the scope of the present study.

The bulk-plasma particle loss is measured by an average particle confinement time, $\tau_{p}$, which is equated to the bulk-plasma energy confinement time, $\tau_{\mathrm{E}} \simeq 0.21 \mathrm{~s}$, used for the cost-optimized CRFPR design point (Table II.D-III). The actual particle confinement time near the scrapeoff region, however, is expected to be decreased appreciably by edge-plasma recycling, as measured by the edge-plasma recycling coeffictent, $R_{L e}^{*}$, in order to account for the rapid rate of neutral/plasma particle exchange in the edge-plasma region (1.e., the outer part of the plasma, next to the scrapeoff). Hence, the particle flux into the scrapeoff is enhanced by a factor $1 /\left(1-R_{L e}^{*}\right)$ above that predicted from the bulk-plasma particle loss. The recycling coefficient, $R_{L e}^{*}$, must be better defined through the appropriate multi-dimensional/multi-species transport models; for the purposes of this study, however, $R_{L e}^{*}$ is treated parametrically.

The RFP scrapeoff model includes neutral-particle transport explicitly, using a modified version of the SPUDNUT ${ }^{138}$ neutral-particle transport code. All particles colliding with the first wall are returned to the scrapeoff region as an isotropic flux of neutral particles at a specific energy. The particles colliding with the limiter are reintroduced in the scrapeoff region as isotropic volumetric sources of neutral particles at an energy level to be specified in 
the code (few eV). The SPUDNUT code was modified to include volumetric sources In order to accomplish this task.

In setting up the particle and heat equations used to describe transport in the scrapeoff, an ensemble of simplifying assumptions 137 is invoked. These assumptions are

- one-dimensional (cylindrical) equations applied to the radial direction ( $1 . e .$, rapid spiral flow along field lines and along the toroidal direction).

- steady state.

- equal ion and electron densities and temperatures.

- Bohm-like values for the mass diffusivity $D$, and $x=x_{1}+x_{e}=3 D$ for the heat dirfusivity.

- $\gamma=\gamma_{e}+1$, where $\gamma_{e} \simeq \frac{1}{4} \ln \left(m_{1} T_{e} / m_{e} T_{1}\right)$ is used to represent the enhancement of the electron energy flux resulting from sheath potentials established between the bulk plasma and first-wall/1imiter surfaces.

- nonconsistent treatment of radiation.

Under these assumptions, the scrapeoff equations become

$-\frac{1}{r} \frac{d}{d r}\left(r D \frac{d u}{d r}\right)=-\frac{n}{\tau_{\|}}+s_{n}$

$-\frac{1}{r} \frac{d}{d r}\left(n \times r \frac{d T}{d r}\right)-\frac{1}{r} \frac{d}{d r}\left(3 T D r \frac{d n}{d r}\right)=-\frac{2 \gamma n T}{\tau_{\|}}-Q_{C X}-Q_{e}^{1}-Q_{1}^{1}$,

where $S_{n}$ represents the source of lons generated by fonization of neutral particles, $Q_{e}^{1}$ and $Q_{1}^{1}$ are the rates at which energy is lost because of electron and ion impact lonization, and $Q_{C X}$ is the rate at which energy is lost by Ion charge exchange with neutral particles. The time $\tau_{\|}$required for a plasma particle moving along a field line to impact a limiter surface $1 \mathrm{~s} \mathrm{~L} / \mathrm{v}_{B}$, where $\mathrm{L}$ is the $f\left\{: 1 d-11\right.$ ne connection length $\left(L \simeq \pi R_{T} q\right.$ for a two-sided toroldal limiter 
In tokamak) and $v_{\text {a }}$ is the thexmal fon velocity, $\left(k_{B} F / m_{1}\right)^{1 / 2}$. For the RFP with $\mathrm{N}_{\mathrm{L}}$ equaliy spaced pololdal limiters operating with double edges, the field-line connection length is given by

$$
L=\frac{\pi}{\frac{T}{L q}}
$$

where for the RFP $g \simeq(F / \theta) r^{2} /\left(r_{w} R_{T}\right) \cong(x F / A \theta), A=R_{T} / r_{p}$, and $x=r_{p} / r_{w}$.

Both particle and energy fluxes are spectfled as boundary conditions at the plasma/scrapeoff interface, with $R_{L e}^{*}$ being used to modify the bulk-plasma partiele $f$ lux and $f_{R A D}$ being used to adjust the partition between radiative and convection/conduction energy fluxes emanating from the bulk plasma into the edge-plasma/serapeoff region. The plasma density and temperature are indirectly opectifled at the scrapeoff/first-wall interface by requiring the respective papticle and energy fluxes onto the first wall to equal $\Gamma_{w} \equiv \alpha_{1} n v_{s}$ and $q_{w}=$ $\alpha_{2} T \Gamma_{w}$, where $\alpha_{1}$ and $\alpha_{2}$ are parameters. These latter conditions approximate an effective first-wall electrostatic sheath. Hence, both $n$ and $T$ are specifled at the first wall to match the computed gradients at that location; an iterative procedure must be used to find the approprlate value of $n$ and $T$ at the first wa11.

The 11miter radial thickness, $\delta^{-}$(FIg. 11I.H-1), is determined from a constant heat-flux condition, which provides a means to determine the limfter shape.137 The 1imiter surface facing the plasma must be shaped in order to accommodate the concentrated particle/heat flux that spirals along the highmpttoh field lines and impacts on the polofdal limiters at a glancing angle. A constant (design) heat flux, $q_{D}$, is specifled for this limiter surface, from which a limfter shape and size is iteratively determined. Figure III.H-2 gives the $x-z$ coordinate system used to determine the constant heat-flux shape for the pololdal Iimfter. Solving the scrapeoff equations gives the diffusive, $q_{\perp}$, and streaming, $q_{z}$, particle heat fluxes incident onto the fimiter, where $q_{z}$ sums all contributions to $2 \mathrm{nT} \gamma / \tau_{\|}$along the $z$-direction in the scrapeoff at constant radial position $x$. Specifying $q_{D}$ along with the equation for the Ilmfter shape, as given below, 


$$
\begin{aligned}
& q_{D}=q_{z} \sin \theta+q_{\perp} \cos \theta+q_{R A D} \cos \theta \\
& \frac{d x}{d z}=\tan \theta,
\end{aligned}
$$

gives the desired 1 imiter shape. The radiative component in Eq. $(5), q_{R A D}=\left(P_{\alpha}\right.$ $\left.+P_{\Omega}\right) f_{R A D}$, is assumed to be distributed unfformly over the first-wall/ilmiter area and is added to $q_{D^{*}}$ The radial or $x$ extent of the $11 m i t e r, \delta^{\circ}, 1 \varepsilon$ determined from the point along $x$ where $q_{z}=q_{D}$; the toroldal or $z$ extent and cross-sectional shape then follow frcm solving Eqs. (5) and (6) once $q_{z} 18$ obtalned from the solution of the scrapeoff density and temperature equations. The heat flux directed toroldally onto the limiter is taken as

$$
q_{z}=2 \gamma \mathrm{T} \Gamma_{z}
$$

where the toroldally directed particle $f 1 u x, \Gamma_{z}$, is related as follcws to the particle flux streaming along field lines, $\Gamma_{\|}$:

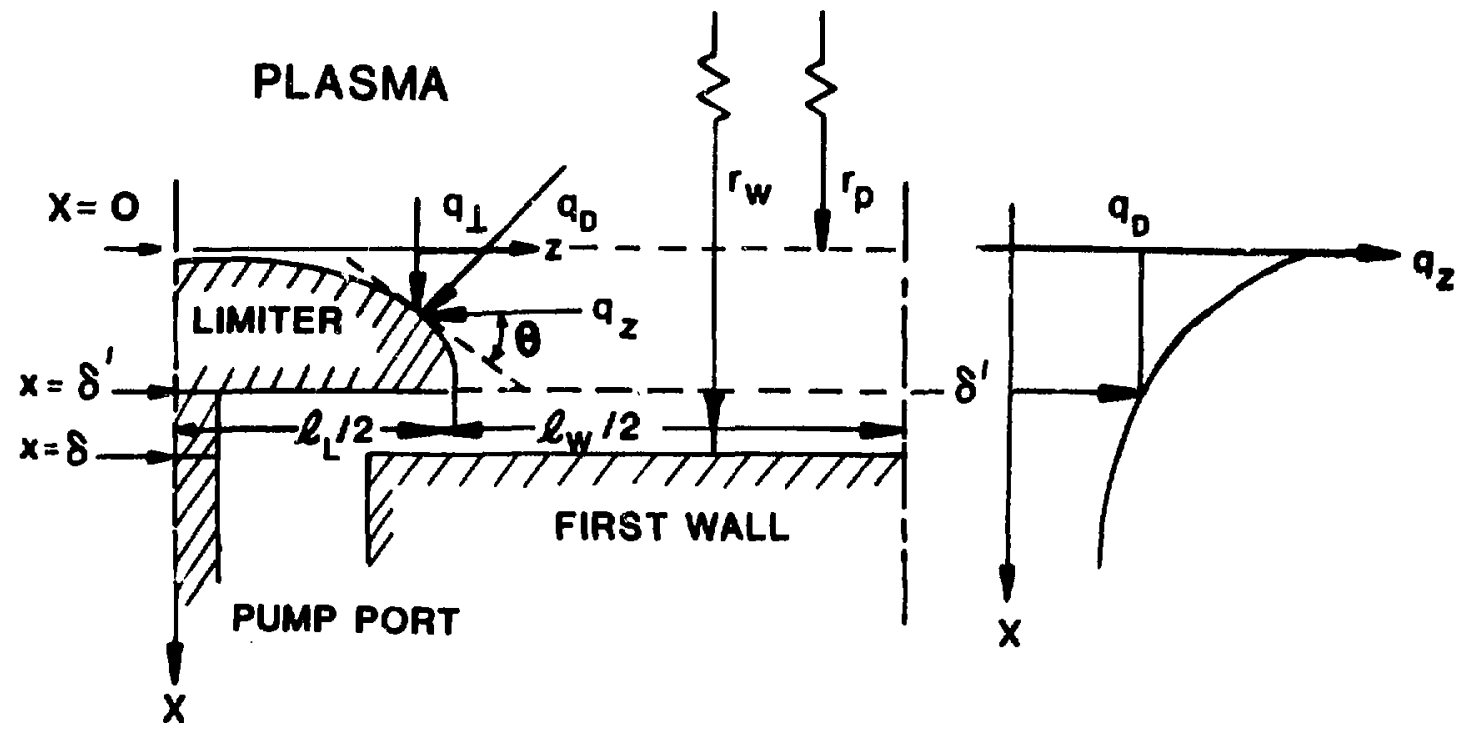

Fig. III.H-2. Limiter geometry used to compute limiter shape required for uniform heat flux. 


$$
\begin{aligned}
\Gamma_{z} & =\Gamma_{\|} \frac{B_{\phi}}{B_{\theta}\left[1+\left(B_{\phi} / B_{\theta}\right)^{2}\right]^{1 / 2}} \\
& \simeq \Gamma_{\|}\left(B_{\phi} / B_{\theta}\right)=\Gamma_{\|}\left(r / r_{W}\right)(F / \theta) .
\end{aligned}
$$

The local temperature, $T(r)$, in the scrapeoff is used in Eq. (7). Requiring that the number of particles incident upon the limiter in the differential volume $\mathrm{dx}, 2 \pi\left(\mathrm{r}_{\mathrm{p}}+\mathrm{x}\right) \mathrm{dx} \Gamma_{\mathrm{z}}$, equals the sum of the total volumetric streaming loss, $2 \pi\left(r_{p}+x\right) \mathrm{dx}\left(l_{w} / 2+l_{L} / 2-z\right) n(x) / \tau_{\|}$, along with Eq. (7) gives the following expression for $\tau_{\|}$:

$$
\tau_{\|} \simeq\left(l_{w} / 2+l_{L} / 2-z\right) \frac{n}{\Gamma_{\|}}\left(\frac{r_{w}}{r}\right) \frac{\theta}{F},
$$

where $v_{s} \simeq \Gamma_{H} / n$. This expression for $\tau_{\|}$is used in Eq. (3) together with the $q_{D}$ constraint to solve iteratively for the scrapeoff temperature and density profiles that in turn are compatible with the given limiter design (i.e., $\mathrm{N}_{\mathrm{L}}$, $q_{D}, \delta, \delta^{\prime}$, and leading-edge shape). It is noted that the value of $\delta^{-}$is a minimum in that incorporation of coolant channels into the resulting limiter structure may require the 1 imiter to be thicker than $\delta^{-}$(Sec. III.D.1.b.).

2. Parametric Results. As emphasized by Howe, 137 the sensitivity of peak and design heat fluxes at limiter surfaces to variations in a simplified edgeplasma transport mode1 renders an exact limiter design at best risky without strong direction from experiments. Hence, an emphasis is placed at this stage of the analysis on parametric sensitivity studies of limiter performance for the CRFPR design (Table II.D-III). For the $\sim 20 \mathrm{MW} / \mathrm{m}^{2}$ surface-averaged first-wall neutron loading, an average heat flux of $\sim 5 \mathrm{MW} / \mathrm{m}^{2}$ at the first wall is implied for divertorless plasma operation. The major goal of this limiter study, therefore, is to quantify the extent to which the design heat flux is increased above this irreducible minimum while maintaining a reasonable limiter number, shape, and performance (i.e., particles diffusing/streaming to the limiter channe1). 
Parameters which have been held fixed, parametrically varied, or computationally derived as part of this limiter parametrics study are summarized In Table III.H-I. A canonical base case has been defined for $\mathrm{N}_{\mathrm{L}}=24$ (nominally one limiter per $\mathrm{TFC}$ ), $\mathrm{R}_{L e}^{*}=0.85, \mathrm{D} \simeq 1 \mathrm{~m}^{2} / \mathrm{s}$ (approximately the Bohm diffusion rate), and $q_{D}=6 \mathrm{MW}^{\prime} \mathrm{m}^{2}$; sensitivity studies are performed about this base case. Density, temperature, particle flux, and energy flux profiles through the scrapeoff are shown in Fig. III.H-3 for a range of specified radiation fractions, $f_{R A D}$, for the base-case parameters summarized in Table III.H-I. Decreasing $f_{R A D}$ requires a higher particle convection/conduction heat flux at the scrapeoff. Generally, increasing $f_{R A D}$ decreases $q_{1}$ at the edgeplasma/scrapeoff boundary, which in turn causes the observed decrease in plasma temperature in this region, since the particle flux into the scrapeoff remains unchanged and the energy per particle, therefore, must decrease. Decreasing the edge-plasma/scrapeoff temperature causes $\tau_{\|} \propto 1 / T^{1 / 2}$ to increase, which in turn decreases the streaming loss, $n / \tau_{\|}$, which then increases the density in this region if $\Gamma_{1}$ remains fixed. Figure III.H-3 displays this general behavior.

The magnitude of the energy fluxes, $q_{2} \simeq 20-100 \mathrm{MW} / \mathrm{m}^{2}$, indicates that the limiter shape will be nearly parallel to the first wall if the design heat $f(u x$, $\mathrm{q}_{\mathrm{D}} \simeq 6 \mathrm{MW} / \mathrm{m}^{2}$, at the limiter surface is to be maintained. Figure III.H-4 gives the toroidal energy flux incident upon the limiter as well as the limiter shapes required to meet the $q_{D}=6 \mathrm{MW} / \mathrm{m}^{2}$ constraint. As $f_{R A D}$ decreases, the limiter coverage fraction, $\ell_{L} /\left(l_{L}+l_{W}\right)$, increases to maintain $q_{D}=6 \mathrm{MW} / \mathrm{m}^{2}$ at all points on the limiter leading-edge surface. The high toroidal energy fluxes in the first few millimeters of the scrapeoff are a consequence of the relatively slow cross-field diffusion velocity (somewhat less than Bohm-like rates) compared to the parallel field streaming velocity, even for the high-pitch (i.e., long connection length, $L$ ) edge-plasma region of the RFP. Increasing the number of limiters combines two competing effects (1.e., less total energy per limiter versus shortened reconnection length and decreased $\tau_{\|}$) to relieve this problem somewhat; either $D$ must be increased much above the Bohm-like values used here, or $f_{\text {RAD }}$ must be increased. On the bases of the results given in Fig. III.H-4, $\mathrm{F}_{\mathrm{RAD}}$ much below 0.90 will give unwieldly limiter designs for the $\mathrm{q}_{\mathrm{D}} \simeq 6 \mathrm{MW} / \mathrm{m}^{2}$ 1imit imposed.

The dependence of limiter performance [i.e., $l_{L} /\left(l_{L}+l_{w}\right), f_{p}, f_{E}, l_{L}$, $\left.\delta^{\prime} / \delta\right]$ on $N_{L}, q_{D}$, and $f_{R A D}$ is illustrated in Figs. III.H-5 and III.H-6. Typically, for $N_{L} \geq 20-30$, the opposing effects of less energy and particles 
FIXED, VARIED, AND DERIVED PARAMETERS USED IN PUMPED-LIMITER PARAMETRIC STUDY

FIXED RARAMETERS

Major/first-wall/plasma radil, $R_{T} / r_{w} / r_{p}(m)$

Plasma volume/first-wa11 area, $v_{p}\left(m^{3}\right) / A_{w}\left(m^{2}\right)$

Alpha-particle power, $\mathrm{P}_{\alpha}(\mathrm{MW})$

Ohmic-heating power $\mathrm{P}_{\Omega}(\mathrm{MW})$

Bulk-plasma particle loss, $\langle n\rangle v_{p} / \tau_{p}\left(10^{23} / s\right)$

Average plasma density, $\langle\mathrm{n}\rangle\left(10^{20} / \mathrm{m}^{3}\right)$

Bulk-plasma energy confinement time, $\tau_{E}(s)$

Reversal parameter, $F$

Pinch parameter, $\theta$

Rotational transform at first wall, $t\left(r_{w}\right)=1 / q$

Limiter radial extent, $\delta(m)=r_{w}-r_{p}$

Particle flux first-wall boundary coefficient, $\alpha_{1}$

Energy flux first-wall boundary coefficlent, $\alpha_{2}$

VAR IED PARAMETERS

Fractional plasma energy lost by radiation, $f_{\mathrm{RAD}}$

Edge-plasma recycling coefficient, $R_{\mathrm{Le}}^{*}$

Particle diffusivity in scrapeoff, $D\left(\mathrm{~m}^{2} / \mathrm{s}\right)$

Thermal diffusivity in scrapeoff, $\chi\left(\mathrm{m}^{2} / \mathrm{s}\right)$

Number of 11miters, $\mathrm{N}_{\mathrm{L}}=2 \pi R_{\mathrm{T}} /\left(\mathrm{L}_{\mathrm{L}}+f_{\mathrm{W}}\right)$

Design heat flux on first-wall/1imiter, $q_{D}\left(\mathrm{MW} / \mathrm{m}^{2}\right)$

Particle heat flux at scrapeoff, $q_{1}\left(M W / \mathrm{m}^{2}\right)$

Particle flux at scrapeoff, $\Gamma_{1}\left(10^{21} / \mathrm{m}^{2} \mathrm{~s}\right)$

DERIVED PARAMETERS

Edge-plasma density, $n\left(r_{p}\right)\left(10^{20} / \mathrm{m}^{3}\right)$

Edge-plasma temperature, $T\left(r_{p}\right)(e V)$

Limiter length, $\ell_{1}(\mathrm{~m})$

Limiter coverage fraction, $\ell_{L} /\left(l_{L}+l_{W}\right)$

Limiter thickness, $\delta^{*}(\mathrm{~m})$

Fraction scrapeoff particles under limiter, $f_{p}$

Fraction scrapeoff particle energy under limiter, $f_{E}$
VALUE 15

$4.30 / 0.75 / 0.71$

$42.8 / 127.3$

615.6

28.6

0.69

3.40

0.21

$-0.2$

1.45

41.6

0.04

0.25

6.0

$0.85+0.95$

$0.0 \rightarrow 0.85$

$0.5 \rightarrow 10.0$

3D

$10+100$

$6 \rightarrow 20$

$5.35\left(1-f_{\mathrm{RAD}}\right)$

$4.56 /\left(1-R_{L e}^{*}\right)$

1.38

45.9

0.38

0.38

0.0115

0.34

0.12 

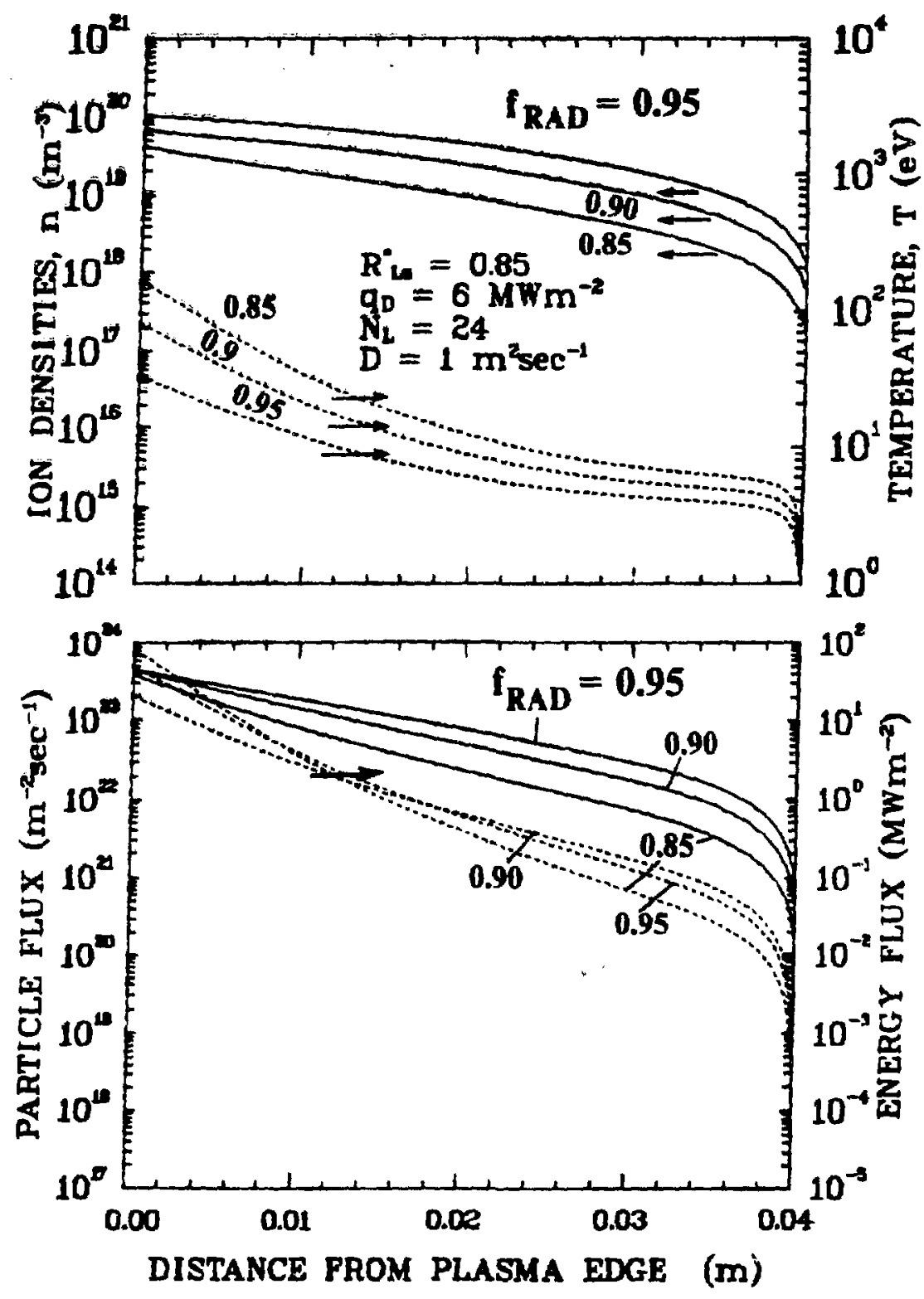

Fig. III.H-3. Density, temperature, particle flux, and energy flux profiles in the scrapeoff for variations in the fraction, $f_{R A D}$, of the total plasma energy loss appearing as (edge) radiation for the basecase parameters indicated. 

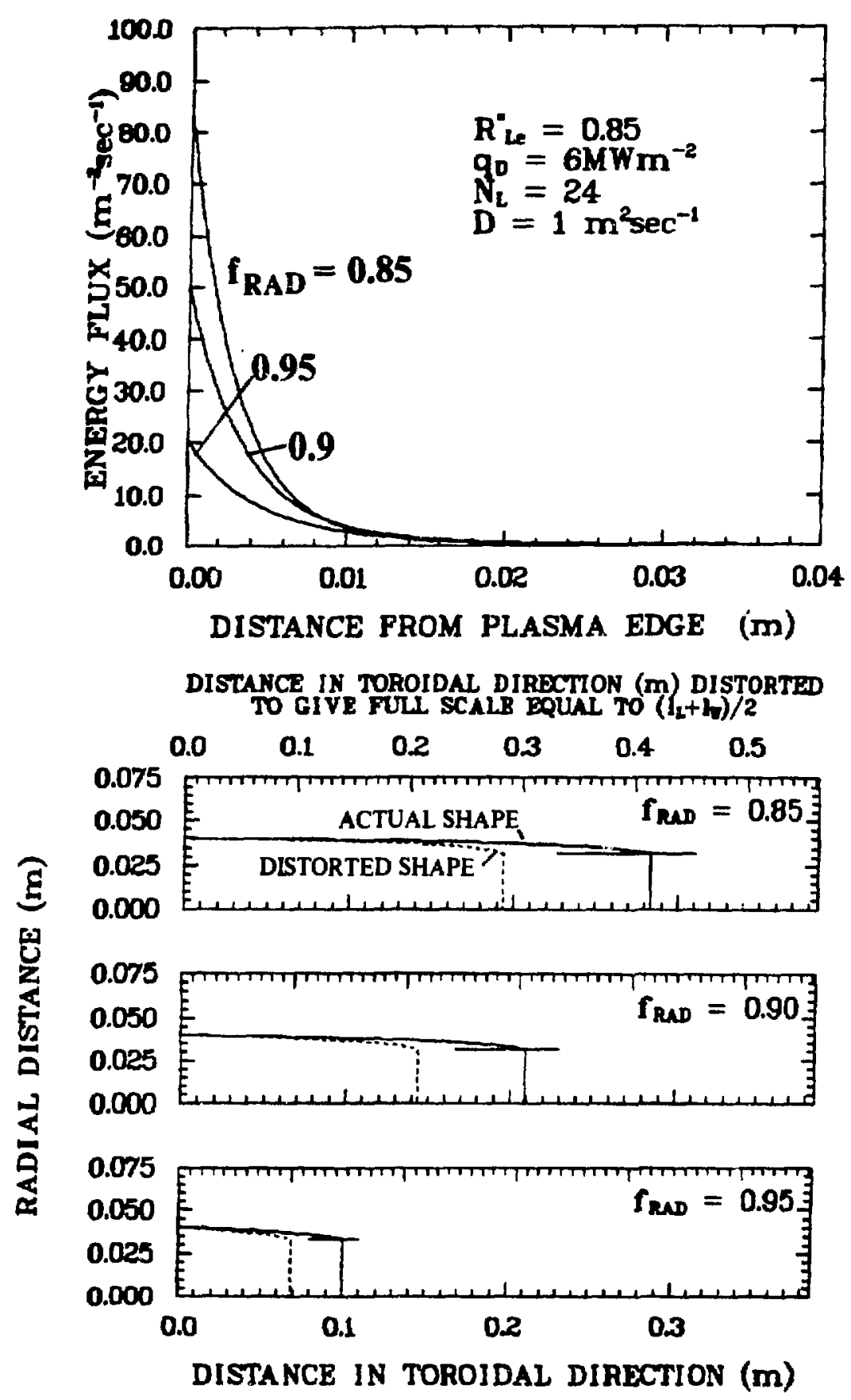

Fig. III.H-4. Dependence of limiter shape on $f_{R A D}$ for the base-case parameters indicated. The 1 imiter cross sections are indicated both on an actual scale and a scale that gives the proportion of $\left(l_{L}+l_{w}\right) / 2$. The toroldal heat flux, $q_{Z}$, is also shown as a function of distance into the scrapeoff. 


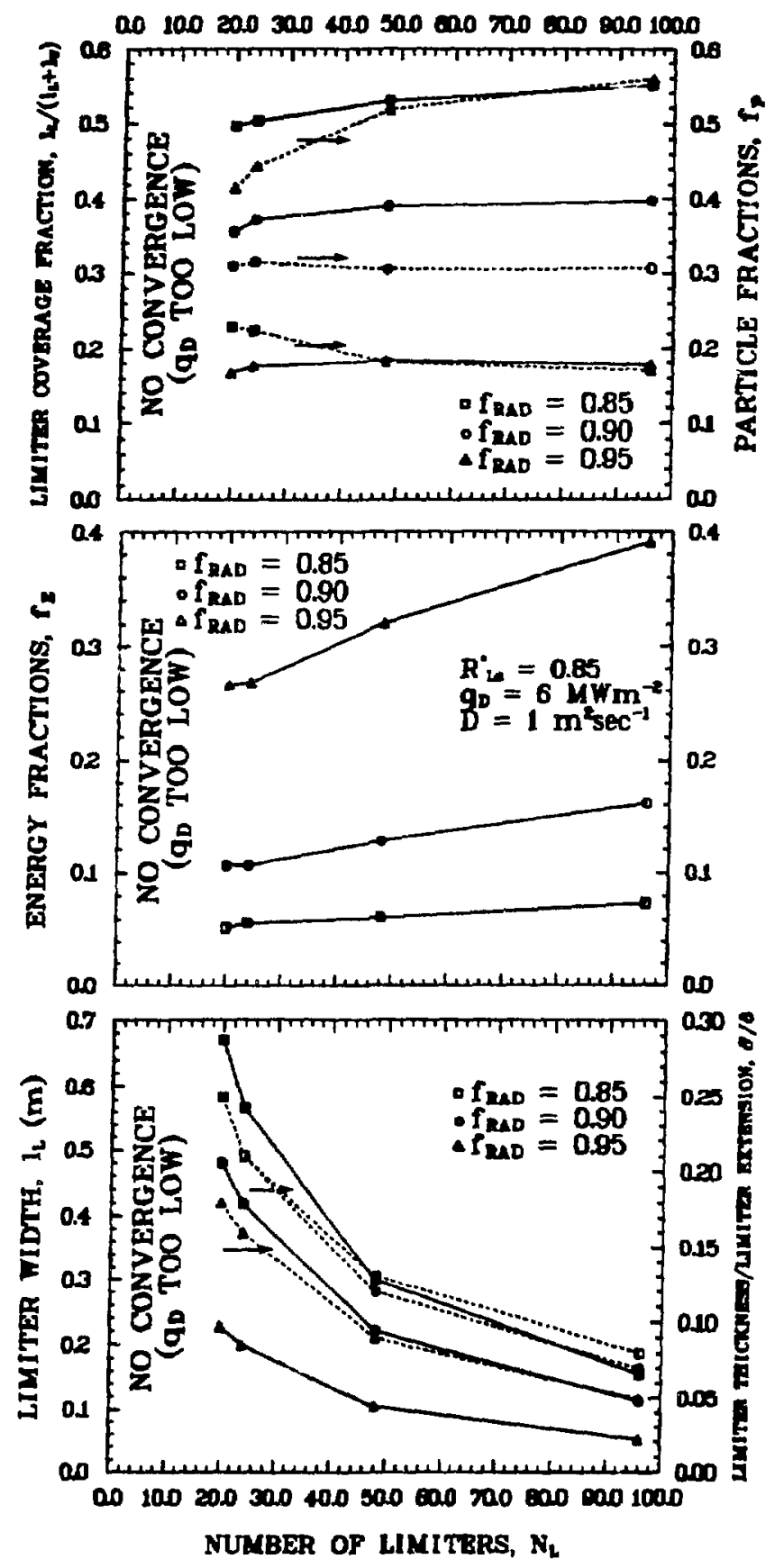

Fig. III.H-5. Dependence of 1 imiter performance on number of limiters, $\mathbb{N}_{L}$, and radiation Eraction, $f_{R A D}$, for $q_{D}=6 \mathrm{MW} / \mathrm{m}^{2}$. 


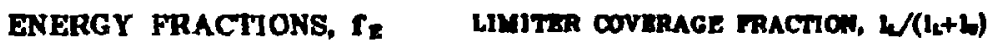
م⿱⺈

$\pm 0$

眥总

突架

吕
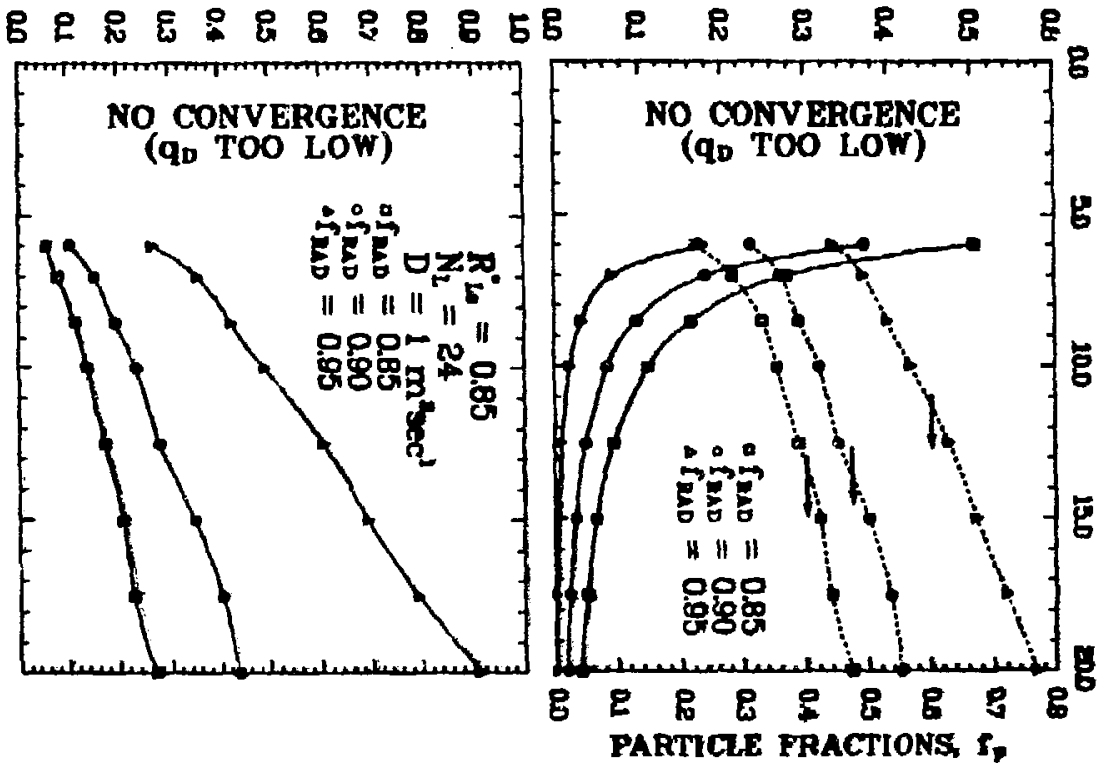

$\stackrel{t}{\underline{x}}$ 
versus reduced connection length as $N_{L}$ increases is 1llustrated. Increasing the design heat flux beyond $q_{D} \simeq 6 \mathrm{MW} / \mathrm{m}^{2}$ improves the limiter performance, although the base-case value of $q_{D}$ is already large.

For the base case, which now includes $f_{R A D}=0.90$, the dependence of 1imiter performance on the edge recycling coefficient, $R_{L e}^{*}$ is shown on Figs. III.H-7, III.H-8, and III.H-9. The dependence of the profiles on $R_{L e}^{*}$ (FIg. III.H-7) is explained as follows. Increasing $R_{L e}^{*}$ leads to a decrease in the effective, edge-plasma particle confinement time, $\tau_{p}\left(1-R_{L e}^{*}\right)$, so that both the particle $f$ lux and density at the scrapeoff/edge-plasma interface increase. Since the energy flux through this interface remains unchanged, the energy per particle decreases at this interface. The decrease in plasma temperature then leads to an increased $\tau_{\|}$. Since $\Gamma_{\perp} \simeq \mathrm{nv} \propto n \mathrm{~T}^{1 / 2}$, with $\Gamma_{\perp}$ Increasing with increased $R_{L e}^{*}$, the scrapeoff/edge-plasma density increases faster than $T^{1 / 2}$ decreases, indicating that the streaming loss $n / \tau_{\|} \propto n T^{1 / 2}$ has a net increase; the dependence of the fluxes on $R_{L e}^{*}$ depicted in Fig.III.H-7 results.

The ability to achieve an acceptable limiter performance depends primarily on a) the particle energy flux incident upon the scrapeoff (i.e., $f_{R A D}$, and b) the capability for rapid radial transport to spread this energy flux radially outward, away from, and under the 1imiter. Figures III.H-10, III.H-11, and III.H-12 show the effect on profiles and limiter performance as the particle diffusivity, $D$, is parametrically varied above and below the base-case value of $D=1 \mathrm{~m}^{2} / \mathrm{s}$, with the relationship $\chi=3 \mathrm{D}$ bef ng maintained. The general behavior observed is explained by noting that $D(d n / d x)_{x=0} \simeq \int\left(n / \tau_{\|}\right) d x$ is nearly a constant. Hence, increases in $\mathrm{D}$ result in decreased density gradients. The constraint that $\chi=3 D$ makes this tendency even stronger for flat temperature gradients with $q_{\perp}$ held fixed. The dependence of limiter performance on $D$ is summarized in Fig. III.H-12. The maximum in the fraction of particles transported into the limiter channel, $f_{p}$, as $D$ is changed indicates that for $D \leqslant 1 \mathrm{~m}^{2} / \mathrm{s}$ the majority of the particles impinge on the limiter leading edge, whereas for $D>1 \mathrm{~m}^{2} / \mathrm{s}$ the majority of the particles intersect the first wall and, hence, are assumed lost at the wall or ionlzed in the bulk plasma. The previous selection of Bohm-like $D=1 \mathrm{~m}^{2} / \mathrm{s}$ for the base case has indeed been fortuitous from the viewpoint of optimizing the limiter pumping effectiveness.

On the basis of this scrapeoff parameter study, the varied and derived limiter parameters listed on Table III.H-II are adopted for a specific limiter point design. The limiter design parameters for the Ref. 15 case and the update 


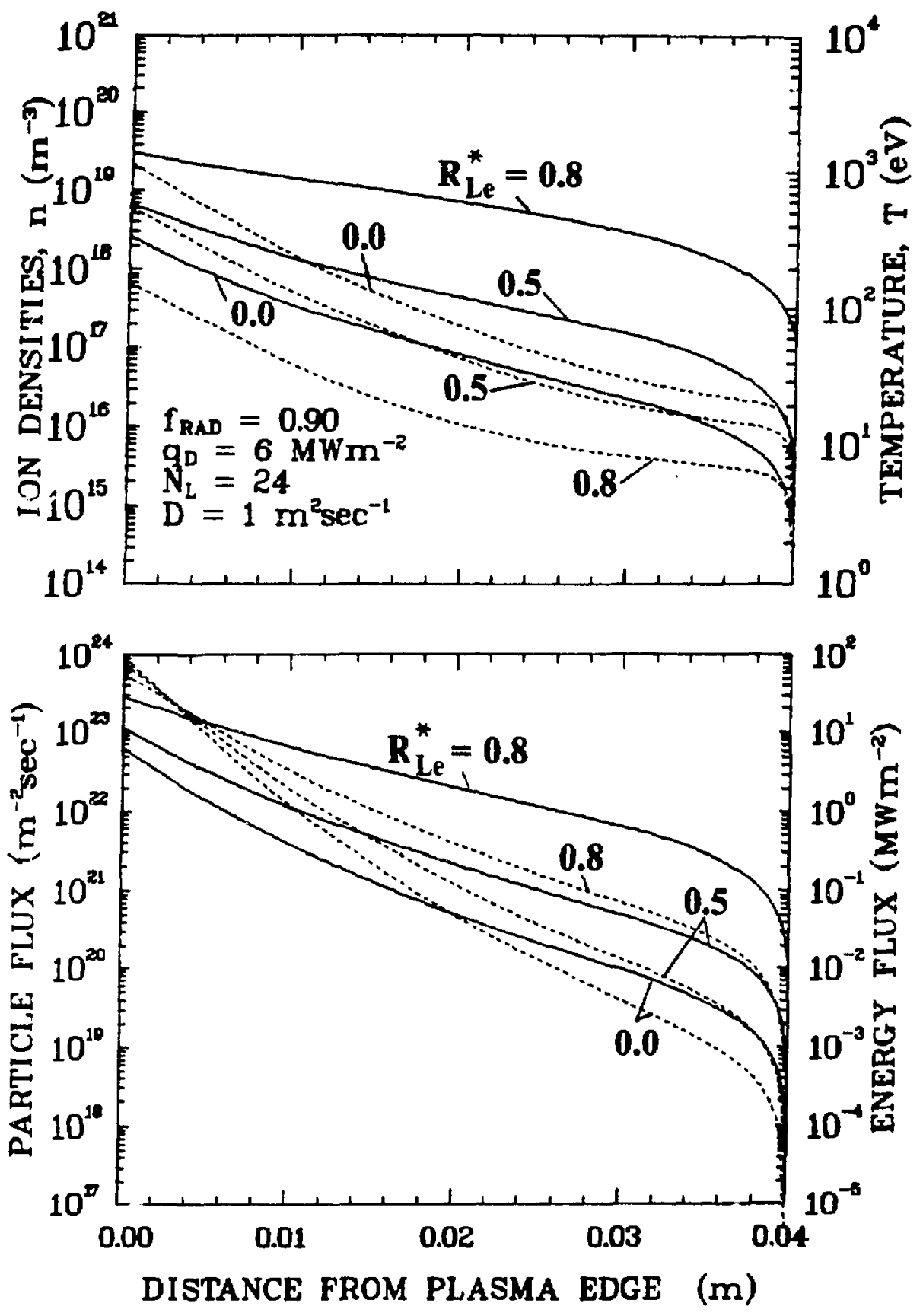

Fig. III.H-7. Density, temperature, particle flux, and energy flux profiles in the scrapeoff as a function of the edge recycling coefficlent, $R_{L e}^{*}$, for the base-case parameter indicated. 

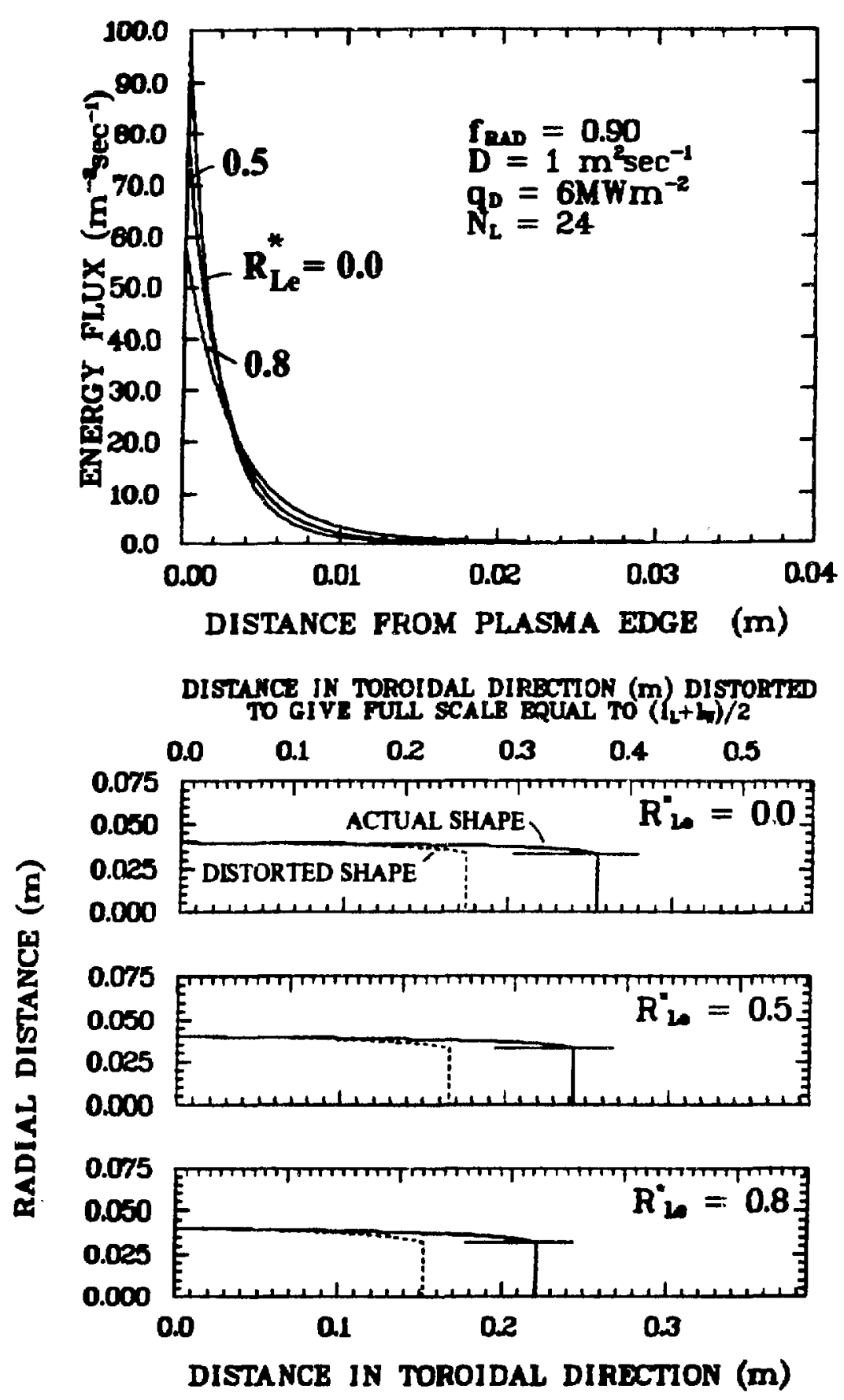

Fig. III.H-8. Dependence of limiter shape on $\mathrm{R}_{\mathrm{Le}}^{*}$ for the base-case parameters indicated. The limiter cross sections are indicated both on an actual scale and a scale that gives the proportion of $\left(l_{L}+l_{W}\right) / 2$. The toroidal heat flux, $q_{z}$, is also shown as a function of distance into the scrapeoff. 


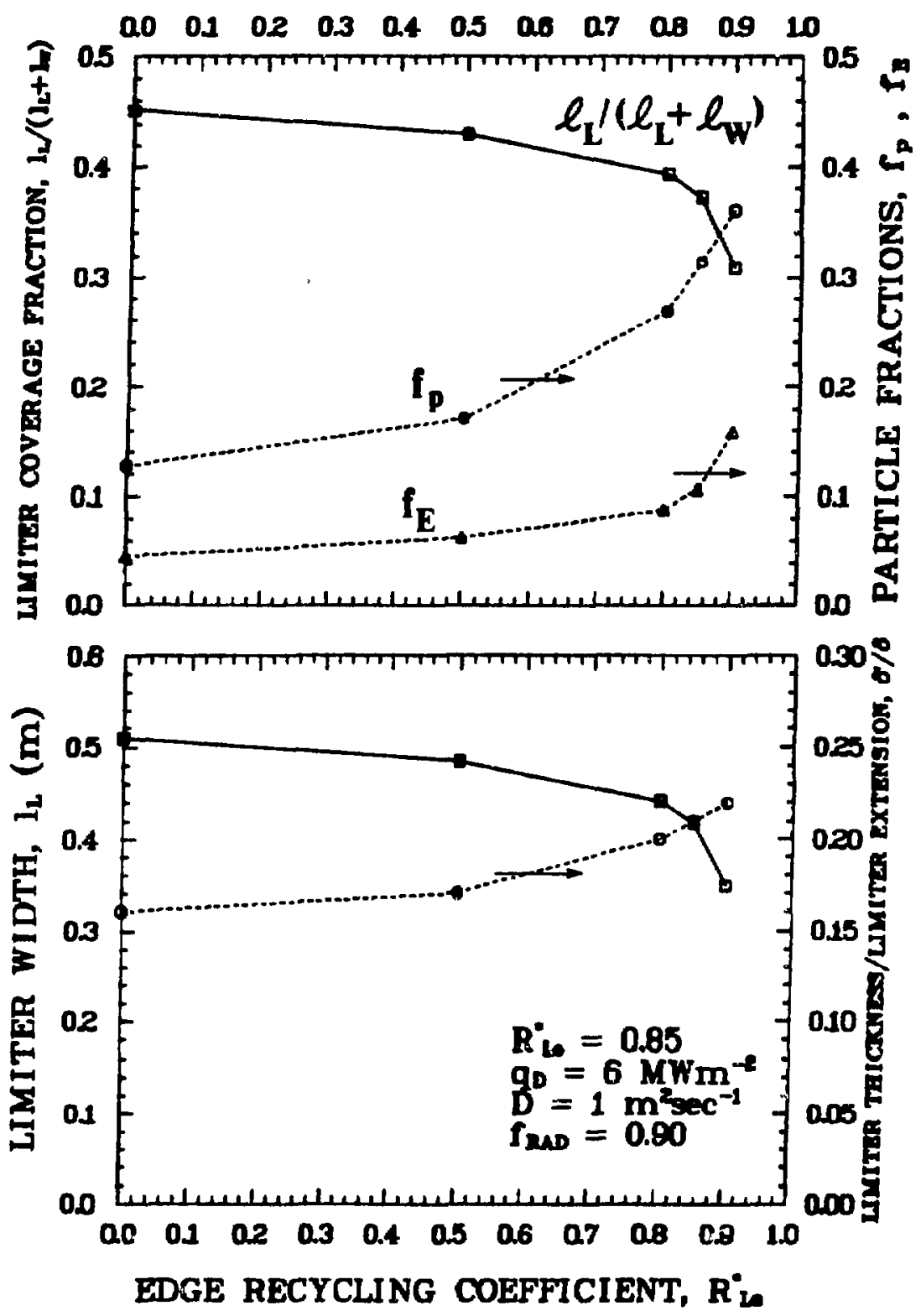

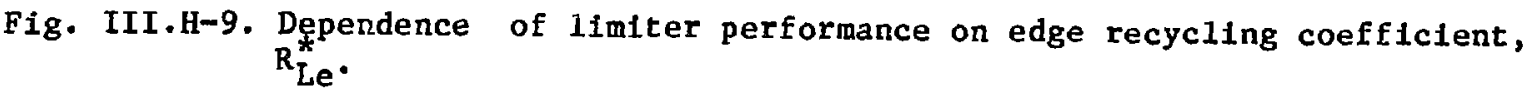




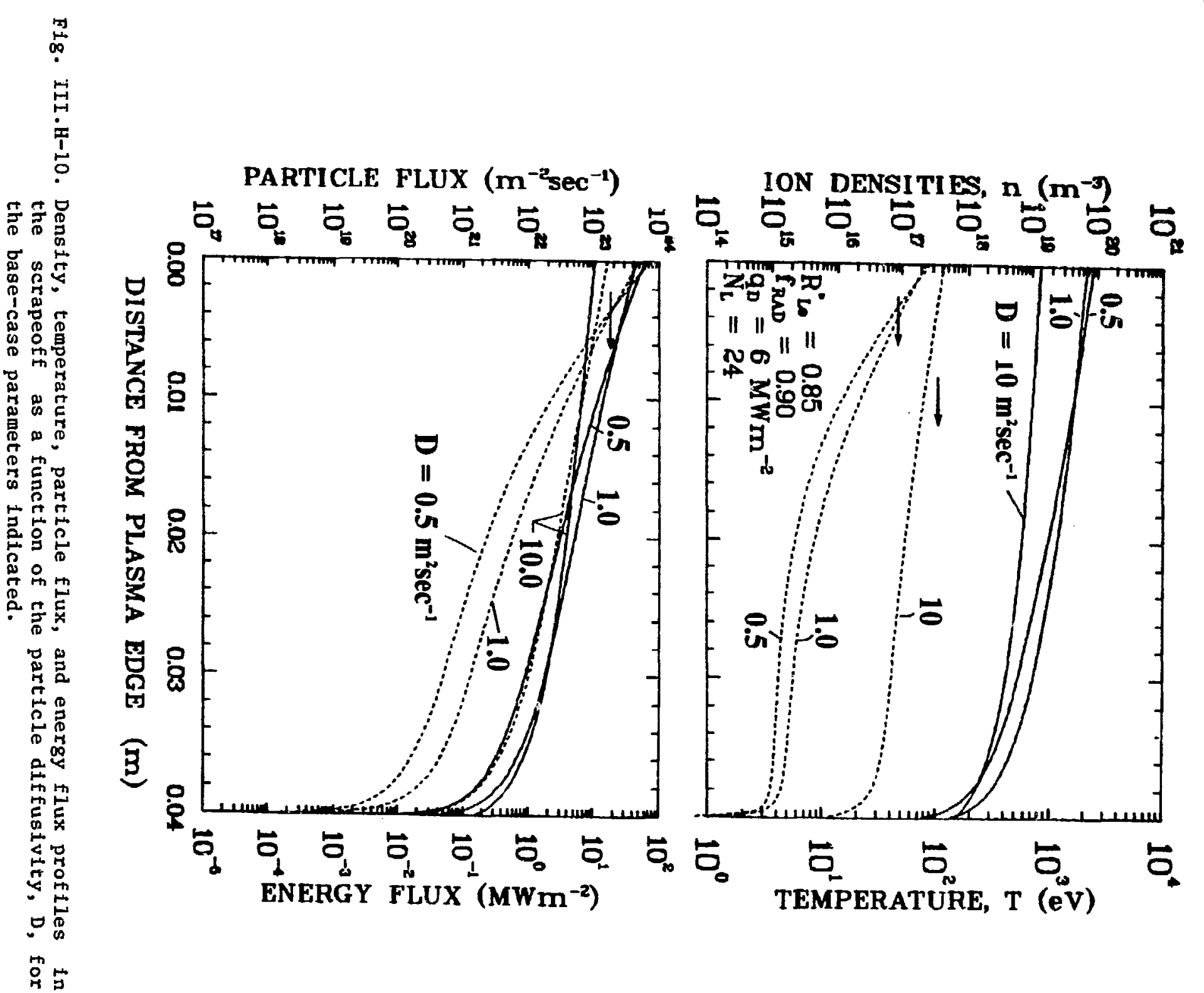




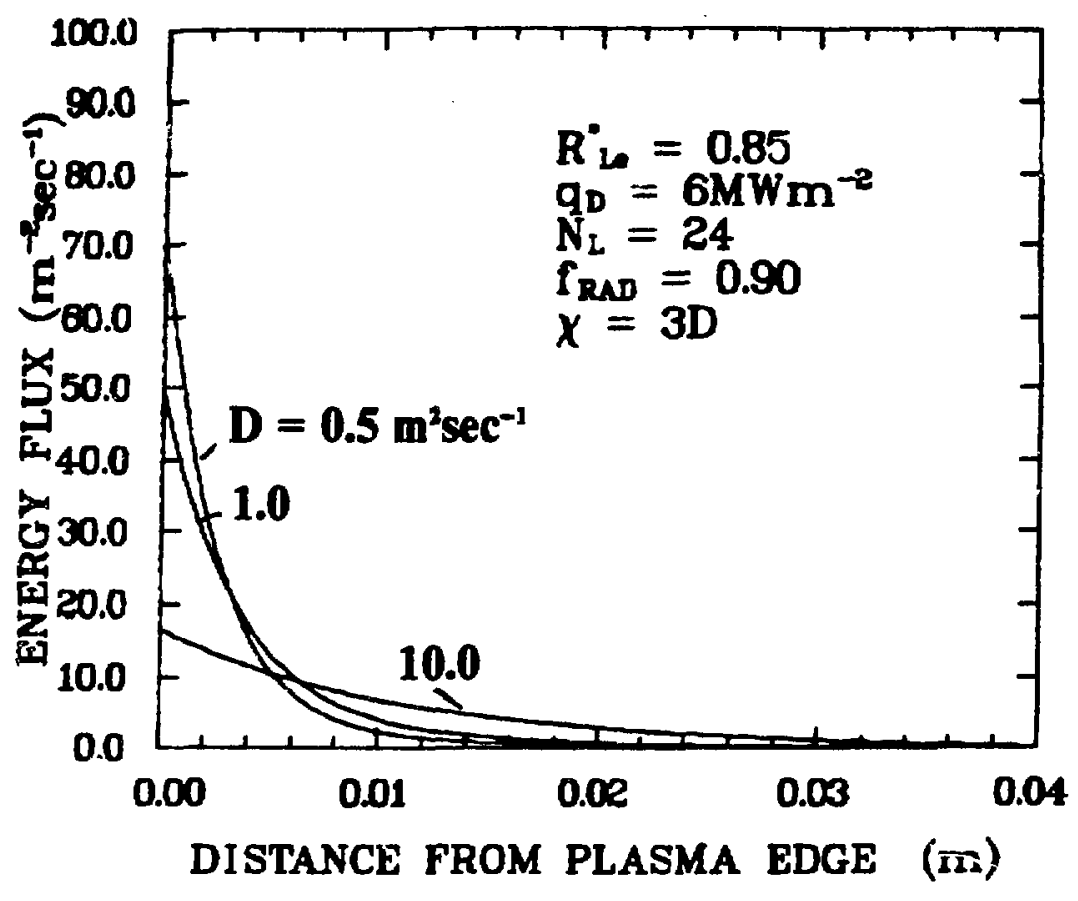

DISTANCE IN TOROIDAL DIRECTION (m) DISTORTED TO GIVE FULL SCALE EQUAL TO $\left(\mathrm{l}_{2}+\mathrm{H}_{\mathrm{W}}\right) / 2$

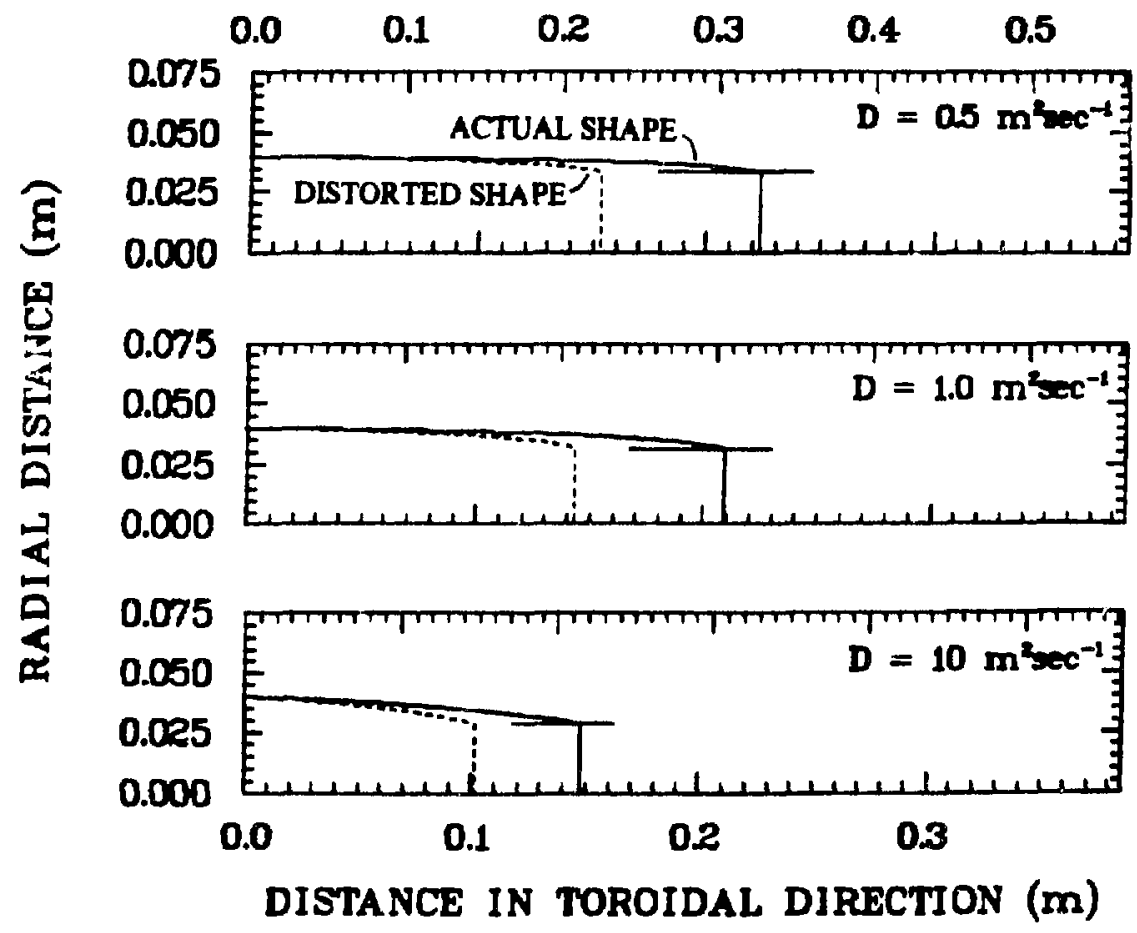

Fig. III.H-11. Dependence of limiter shape on particle diffustvity, $D$, for the base-case parameters indicated. The 1 imiter cross sections are Indicated both on an actual scale and a scale that gives the proportion of $\left(l_{L}+l_{w}\right) / 2$. The toroldal heat flux, $q_{z}$, is also shown 2.3 a function of distance into the scrapeoff. 

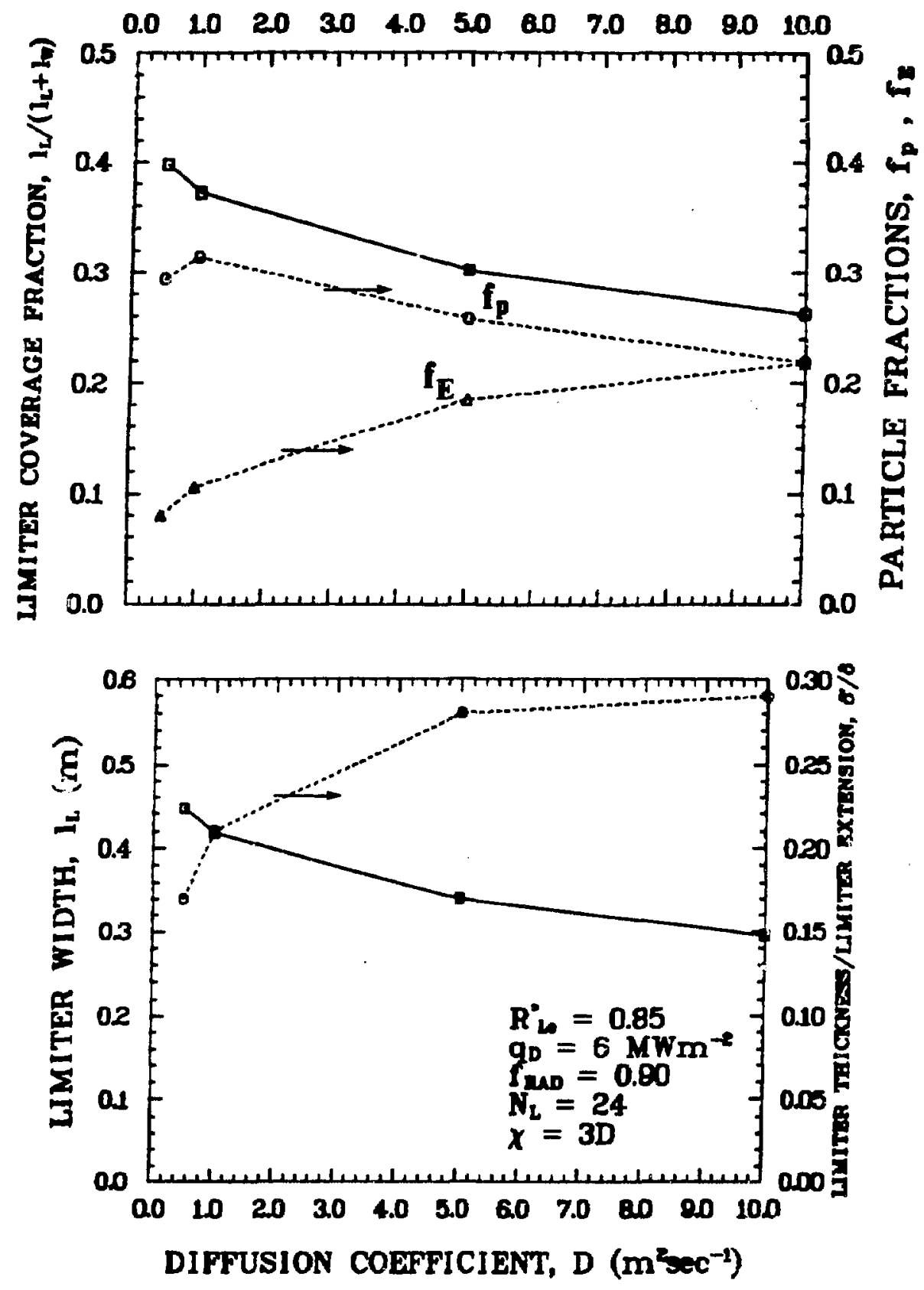

Fig. III.H-12. Dependence of limiter performance on particle diffusivity, D, for the base-case parameters indicated. 
POINT-DESIGN PARAMETERS FOR A PUMPED-LIMITER SYSTEM

PARAMETER
REF. 15

0.21

4.30

Major toroldal radius, $\mathrm{R}_{\mathrm{T}}(\mathrm{m})$

Average particle density, $\langle\mathrm{n}\rangle\left(10^{20} \mathrm{~m}^{-3}\right)$

Alpha-particle power, $\mathrm{P}_{\alpha}(\mathrm{MW})$

Ohmic-heating power, ${ }_{\Omega}(\mathrm{MW})$

Plasma energy radiation fraction, $f_{R A D}$

Edge-plasma recycling coefficient, $R_{\text {Le }}^{*}$

Number of 1 imiters, $\mathrm{N}_{\mathrm{L}}=2 \pi \mathrm{R}_{\mathrm{T}} /\left(l_{\mathrm{L}}+l_{\mathrm{w}}\right)$

Design heat flux, $q_{D}\left(M W / m^{3}\right)$

Particle heat flux at scrapeoff, $q_{\perp}\left(\mathrm{MW} / \mathrm{m}^{2}\right)$

Particle flux at scrapeoff, $\Gamma_{1}\left(10^{21} / \mathrm{m}^{2} \mathrm{~s}\right)$

Temperature at edge, $\mathrm{T}_{\mathbf{e}}(\mathrm{eV})$

Neutra1-atom pressure at 11miter slot, mtorr 12.6

Ion edge density, $n_{e}\left(10^{19 / m^{3}}\right)$
Neutra1 edge density, $n_{n e}\left(10^{17} / \mathrm{m}^{3}\right)$

Particles entering scrapeoff $\left(10^{23} / \mathrm{s}\right)$

Particles golng to limiter $\left(10^{24} / \mathrm{s}\right)$

Particles golng to wall $\left(10^{23} / \mathrm{s}\right)$

Limfter coverage fraction, $l_{L} /\left(l_{L}+l_{w}\right)$

Limiter toroidal extent, $l_{L}(m)$

Limiter radial extent, $\delta^{-}(\mathrm{mm})$

Limiter thickness-to-extent ratio, $\delta^{\prime} / \delta$

Fraction particles under limiter, $f_{p}$

Fraction particle energy under limiter, $f_{E} 0.11$

Fraction total plasma energy loss to limiter, $\sim f_{R A D} l_{L} /\left(l_{L}+l_{w}\right)+\left(1-f_{R A D}\right)$

Scrapeoff recycling coefficlent, $R_{L s}^{*}$

Inversion probability, $k$
6.85

1.88

4.62

1.00

0.66

0.37

0.42

8.4

0.21

0.31

0.43

0.57

0.66

(a) for internal structure and coolant ducts (Sec. III.D.2.b.).
UPDATE

$T(r)=\operatorname{CONST} \quad T(r) \propto J_{0}(\mu r)$

0.23

3.79

6.74

546.5

25.3

0.90

0.80

24

6.0

0.54

5.21

45.9

18.6

13.76

2.32

5.53

1.62

1.29

0.38

0.38

$8.4(11.4)(\mathrm{a})$

$0.21(0.29)(a)$

$0.34(0.24)^{(a)}$

$0.12(0.068)(a)$

0.096

0.41

0.44

0.34

0.68

0.49

$0.73(0.69)^{(a)}$ 
case (Table II.D-III), where the major radius is $R_{T}=3.79 \mathrm{~m}$, average plasma density is $n=6.74(10)^{20} \mathrm{~m}^{-3}$, and particle confinement time, $\tau_{E}=023 \mathrm{~s}$, are listed as are the scrapeoff recycling coefficient, $R_{L s}^{*}$, and the inversion probability, $k$. The scrapeoff recycling coefficient is computed by the limiter code and refers to the recycling in the scrapeoff and is similar to $R_{\mathrm{Le}}^{*}$ defined for the edge-plasma region. The inversion probability gives the probability that a particle neutralized at the first wall and back of the limiter slot in the absence of pumping returns to the limiter slot (i.e., is lonized in the scrapeoff rather than in the edge or bulk-plasma region). The results from Table III.H-II Indicate poloidal limiters of realistic number, size, and shape are possible for the CRFPR design point and a limiter design heat flux of $\mathrm{q}_{\mathrm{D}}<6 \mathrm{MW} / \mathrm{m}^{2}$. The assumptions made in developing this limiter/scrapeoff model, however, require further examination before a detailed mechanical design is warranted. In addition to the assumptions listed previously, the following 1imitations should be noted:

- one-dimensional neutral-atom transport model (SPUDNUT)

- approximate interface with the bulk plasma

- absence of a self-consistent treatment of impurity transport, radiation $\left(f_{\mathrm{RAD}}\right)$, and first-wall/limiter sputtering

- simplified (parametric) treatment of radial electrostatic sheaths at the first wall and no treatment of electrostatic effect along the toroidal direction

- sputtering, impurity transport, and redeposition, although estimated, require more accurate modeling.

The use of the SPUDNUT model to estimate the Inversion probability, $k$, and the inability to model properly neutral-particle interactions with the underside of the limiter generally underestimates $k$ and, therefore, overestimates the limiter pumping speed. A more realistic model (e.g., Monte Carlo) is needed to estimate this crucial parameter, particularly as the engineering design requires the limiter thickness, $\delta^{\circ}$, to increase in nrder to accommodate more support and cooling structure beyond the minimum value, $\delta_{i N}$, required to assure $q_{D}=6 \mathrm{MW} / \mathrm{m}^{2}$. Figure III.H-13 gives the dependence of $f_{p}, f_{E}, k$, and the average neutral-atom pressure as the limiter-slot thickness is varfed for the design point suggested on Table III.H-II, these results again limited by the 
assumptions listed above. The neutral-atom pressure given in Fig. III.H-13 is a radial average using the neutral-atom density profile returned by SPUDNUT and the local plasma temperature profile. This average pressure decreases nearly linearly with increasing $\delta^{-}$( $1 . e$. , for fixed $\delta$, the limiter slot is diminished), nominally giving a value that is close to that used in the estimate of the vacuum requirements (Sec. III.J.).

The behavior of the particle and energy fractions, the neutral-atom pressure, and inversion factor plotted in Fig. III.H-13 as a function of the limiter thickness for the base-case designs must be explained in terms of both the model limitations as well as the physics that this model is attempting to describe. The curves of the $f_{p}$ and $f_{E}$ versus $\delta^{-}$can be explained by noting that increasing the limiter thickness will increase the number of particles striking the leading edge of the limiter, thereby decreasing $f_{p}$ and $f_{E}$. As noted previously, $k$ is estimated using an adapted version of the SPUDNUT code (a volumetric neutral-particle source has been added at each mesh point) to model neutral-atom emissions from the limiter back surface. A transparent limiter is assumed while following trajectories and ionization events for calculating the Inversion probability, $k$. By assuming a transparent limiter the reflection of neutral atoms on the backside of the limiter is ignored and, hence, $k$ will be underestimated. The larger $\delta^{\circ}$, the thinner the limiter slot, and, therefore, $k$ can be expected to decrease with increasing $\delta^{-}$. A more realistic (two-dimensional) model must be implemented to evaluate the behavior of $k$ in the presence of limiter reflections. The decrease in $k$ as $\delta^{-}$is increased can be explained by simple geometric arguments and the modelistic limitations associated with the absence of limiter/neutral-atom interactions (1.e., reflections). In this case $k$ is a parameter indicating the ease of neutral-atom pressure build-up behind the limiter, and, therefore, a drop in neutral-atom pressure with increasing $\delta^{\prime}$ is consistent with the computed $k$ behavior.

The Table III.H-II parameters are used to Incorporate the pumped limiter into the FPC integration and to size vacuum-pumping ducts, but final design choices must awalt answers to the above-listed questions. It is in this spirit that the potential for magnetic divertors in RFPs has been examined as an alternative impurity-control scheme. 


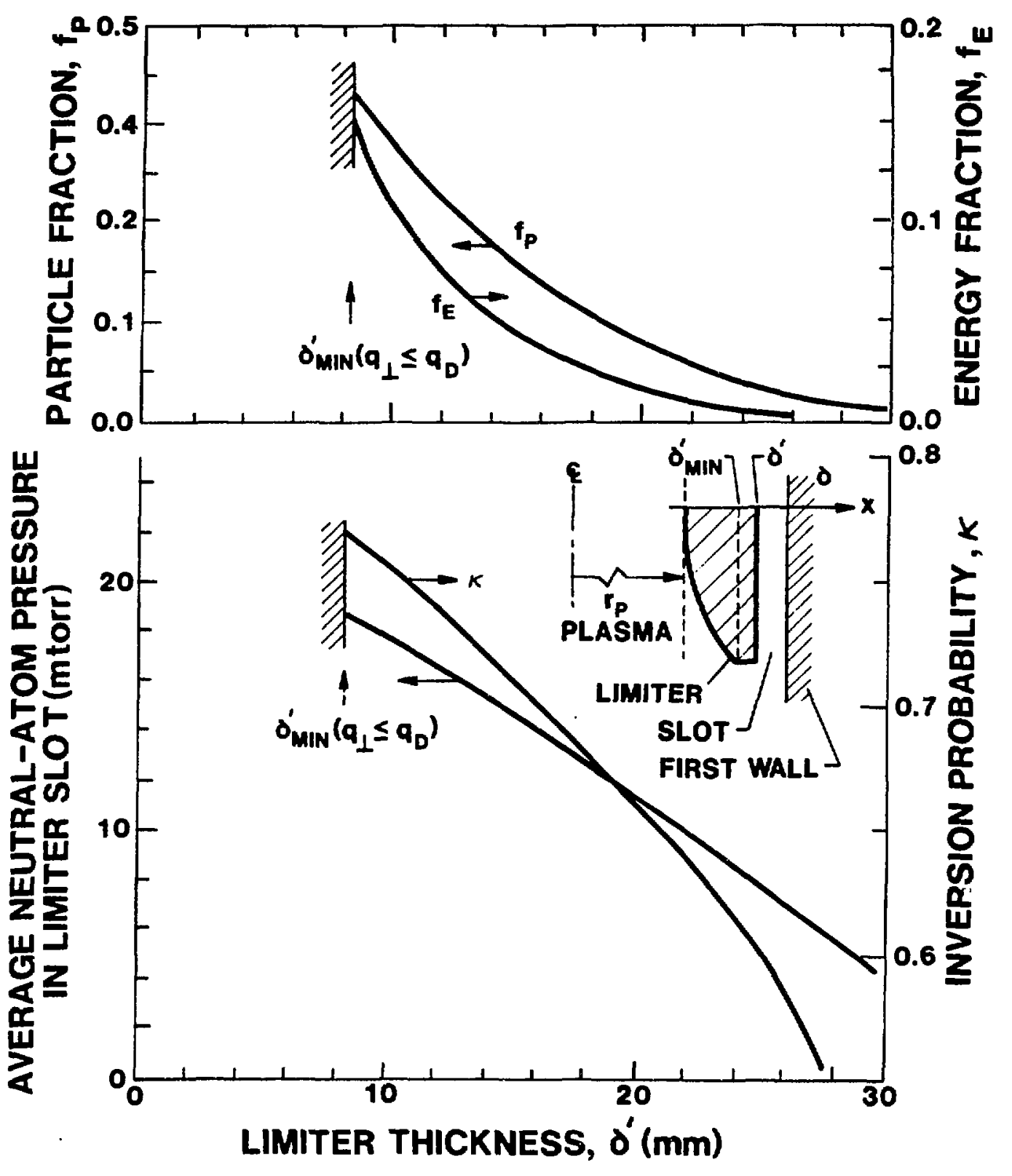

Fig. III.H-13. Dependence of particle and energy fractions, $f_{p}$ and $f_{E}$, the inversion probability, $K$, and the average neutral-atom pressure in the limiter slot as the limiter thickness (and slot width) changes for the base-case parameters listed in Table III.H-II. 


\section{Magnetic Divertor}

Although the pumped limiter has served as the focus of the impurity control for this study (Sec. III.H.), as well as providing a means to determine the essential elements of the vacuum-system design (Sec. III.J.), preliminary consideration has been given to the use of magnetic divertors in an effort to reduce heat/particle fluxes onto surfaces having a direct view of the plasma. As for the pumped limiter, magnetic divertors in an RFP configuration have recelved little attention, and new development was required. The material presented herein should be considered exploratory and is the beginning of a continuing effort in an important area for RFPs.

The FPC volume required by a pumped limiter generally is substantially less in toroidal devices than the volume needed for a magnetic divertor. Because of the large premium placed on compactness and a reduced total FPC mass for the CRFPR, the pumped limiter is the natural first choice for the impurity-control scheme if both schemes are equally viable. Present experimental data on Impurity-control in tokamaks, ${ }^{139}$ however, indicate better plasma performance in devices with a magnetic divertor than in devices using a limiter because of the isolation from the plasma chamber. Although these results are not sufficlently definitive to eliminate the pumped limiter as an impurity-control scheme, the preliminary magnetic divertor design presented below, nevertheless, was performed to examine the feasibility of diverting field lines in an RFP and to gauge the 1mpact of a magnetic divertor on the overall FPC size.

A magnetic divertor design study must begin with a selection of the field component to be nulled and ultimately diverted to an external structural surface. Most magnetic divertor designs pertain to tokamaks. These designs predominately null the minority poloidal field, and are a choice that can be supported for the following reasons. The plasma equilibrium equation, $\vec{j} \times \vec{B}=\vec{\nabla}$, indicates that nulling a component of the magnetic field will result in a reduction in the gradient of the plasma pressure In the vicinity of the null for comparable plasma current densities. The reduction in the volume-averaged beta that inevitably results can be minimized if only the minority field is nulled. A second consideration is the effect a field null will have on particle and energy confinement. Particle confinement times generally scale in proportion to $B$ raised to a positive exponent, for example, $\tau \propto B$ for Bohm diffusion and $B^{1.8} \rightarrow B^{2.0}$ for empirical scalings 140 using $\left\langle n_{e}\right\rangle \propto \beta B^{2} / T$. A reduction in confinement in the vicinity of a fleld null would reduce the volume- 
average confinemert time. Thirdly, the currents required in the divertor coils scale linearly with the magnitude of the field component to be nulled; the energy stored by and forces on the divertor coils are minimized by nulling the minority-field component. Hence, minimization of the effects on beta, confinement time, and divertor coll all indicate that the minority field is the preferred field to null. Diverting the minority poloidal field in a tokamak requires that the divertor colls be interlocked with the TFC set. A few tokamak experiments do null the majority fleld in an attempt to avoid the problems assoclated with interlocked coils. 141 The minority fleld in an RFP, however, is toroldal, and the preferred minority toroidal-field component can be nulled without the engineering com: lexity of interlocking colls.

The simplest design of a toroldal-field divertor for an RFP merely adds TFCs of the approprlate size and current to the main TFC set. The divertor should not only lsolate the region of fleld-line contact with structural surfaces from the plasma but also should provide a path along which the plasma exhaust can deposit energy. This goal is accomplished with divertor coils of radius sufficiently large to encase the main TFC set. The current in the coil that nulls the toroldal fleld must be sufficlently large to cancel the field from the TFC set. Viewing the nulling coils in each of $N_{D}$ toroidal divertors as a solenold which must cancel the fleld from the $N$ toroldal-field colls, then the current in each divertor coll, $I_{D}$, is approximately related to the current in each TFC, $I_{T F}$, by the relationship

$$
\mathrm{N}_{\mathrm{D}} \mathrm{I}_{\mathrm{D}} \simeq-\mathrm{NI} \text { TF }
$$

A set of such divertor colls will null the toroldal field indiscriminately within the bore of TFC set. A pair of colls that flank the divertor colls are Introduced with a combined current equal in magnitude but opposite in sign to that in the divertor coll in order to localize the nulling effect of the divertor coils approximately to within the distance between the divertor coil and its flanking colls. The flanking colls only afford gross control of the location of the toroidal-field null and the size of the penetration in the FPC needed to accommodate the divertor channel. A more precise method of controlling the characteristics of the fleld null, and in particular its toroidal extent, is necessary to reduce the degree to which the tritium-breeding 
blanket is displaced by the divertor channe1, the size of tha gap required in the conducting first wall (1.e., may effect plasma equilibrium and stability), and the amplitude of the toroidal ripple induced, the last resulting in the formation of magnetic islands that affects both transport and field reversal (Sec. III.F.1.). Quantitative estimates of the latter two effects have not yet been made. Demonstrating a method of controlling the toroidal extent of the field null and field ripple, however, was deemed necessary for this study.

The method chosen for controlling the toroldal extent of the field perturbation introduced another divertor-flanking-coil (DFC) set with less current than the main divertor coil set but located closer to the plasma edge. Hence, the DFCs are encased by the TFC set, and a smaller gap between the divertor coil and the DFCs result. A divertor design with four toroidal divertors for the CRFPR magnetics parameters (Sec. III.F.) is shown in Fig. III.I-l, where only the TFC set, the main divertor coil set, and the DFC set have been simulated by the three-dimensional vacuum-field magnetics code, TORSIDO. 51 The main divertor coll encasing the TEC set has a current of $1.6 \mathrm{MA}$, while the secondary DFCs encased within the TFC set have a current of $0.4 \mathrm{MA}$. The effect of the secondary DFC set is evident in Fig. III.I-1 from the pinching of the diverted field lines near the plasma edge. The property of localizing the divertor action to the outboard side of the plasma is characteristic of divertor designs similar to that depicted in Fig. III.I-1. A more compact divertor design is possible that yields nearly the same diversion of field lines by making the current of the diverting colls return throigh the DFCs rather than through the inboard side of the torus, as shown in Fig. III.I-1. Such a design remains for future work. In addition, fleld-line tracings are needed to include the simulation of the PFC set and the plasma currents to yield a full threedimensional picture of the divertor as well as to reveal adequate formation of separatrix surfaces and the control of magnetic islands. This calculation also must be coupled with a transport and radiation mode? to determine the length of the divertor channel necessary to provide cooling of the plasma exhaust, as well as plasma stability and tritium-breeding calculations to quantify the permissible size of the toroidal gap. A rode similar to ZEPHYR 142 will be used for the latter purpose. Generally, work in both pumped-limiter and magnetic-divertor schemes for RFP impurity control represents an important, ongoing effort, both promising advantages relative to confinement schemes with a dominant toroldal-field component. 


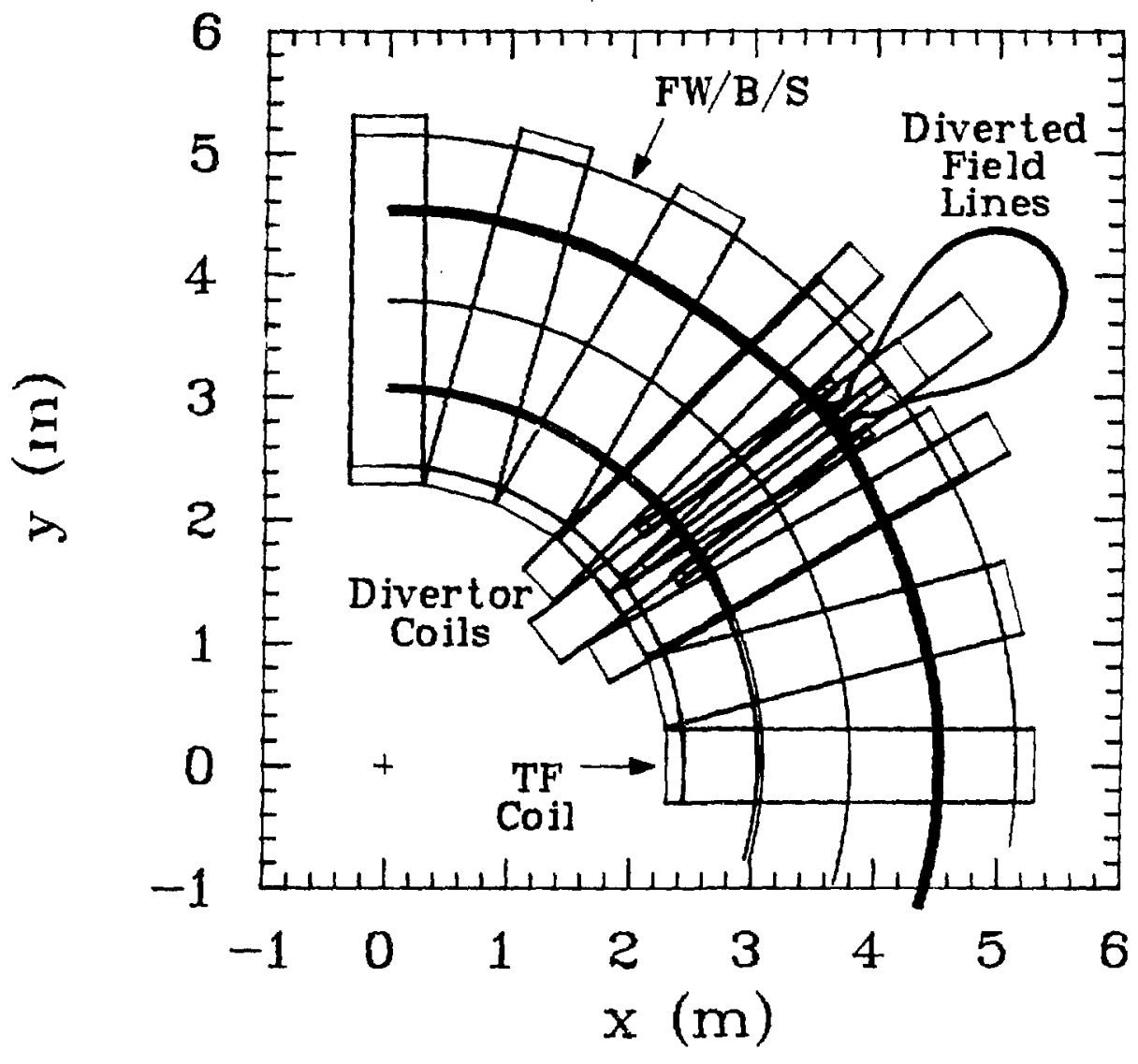

Fig. III.I-1. Field-line tracings in the equatorial plane for a field period of the CRFPR with four toroldal-field divertors. 
J. Vacuum and Fueling

The primary function of the CRFPR vacuum system is the removal of excess impurities and helium ash generated by fusion reactions. Purging of the hellum ash is accompanied by the removal of large quantities of unburned DT fuel for subsequent reprocessing and reinjection tnto the plasma chamber as fresh fuel (pellets). Design of the vacuum system requires a calculation that is self-consistent with the plasma-edge,limiter model (Sec. III.H.) and subject to constraints imposed to minimize neutron and gamma-ray streaming through vacuum channels, thereby maximizing blanket coverage and efficlency. The external fueling rate must balance the combined DT exhaust and burnup in steady state. The vacuum system is considered in Sec. III.J.1, and the pellet-fueling requirements are discussed in Sec. III.J.2.

1. Vacuum Syster. The rate of generation and, hence, removal of helium ash by the fusion reaction is given by

$$
\phi_{\mathrm{He}}(\mathrm{He} / \mathrm{s})=2 \pi \mathrm{r}_{\mathrm{w}} 2 \pi \mathrm{R}_{\mathrm{T}} \frac{\mathrm{I}_{\mathrm{w}}}{14.1 \mathrm{k}_{\mathrm{B}}},
$$

which for $\mathbf{r}_{w}=0.75 \mathrm{~m}, R_{T}=3.8 \mathrm{~m}, I_{w}=19.5 \mathrm{MW} / \mathrm{m}^{2}$, and $k_{B}=1.602(10)^{-16} \mathrm{~J} / \mathrm{eV}$ equals $9.74(10)^{20} \mathrm{He} / \mathrm{s}$ and compares with $1.34(10)^{21} \mathrm{He} / \mathrm{s}$ for the STARFIRE tokamak design; ${ }^{3}$ the helium generation rate, of course, scales directly with fusion power. The corresponding helium gas load under standard conditions $\left[273 \mathrm{~K}, 1.013(10)^{5} \mathrm{~Pa}\right]$ is

$$
\phi_{\mathrm{He}}^{\mathrm{STP}}(\mathrm{Pa} \mathrm{m} / \mathrm{s})=6.58(10)^{-2} \mathrm{r}_{w} \mathrm{R}_{\mathrm{T}} \mathrm{I}_{\mathrm{w}}
$$

corresponding to $3.66 \mathrm{~Pa} \mathrm{~m} / \mathrm{s}(27.8$ \& torr/s). At the elevated operating temperature of the limiter slot/first wall ( $1 . e ., \sim 600 \mathrm{~K}$ ), the hellum gas load becomes $8.05 \mathrm{~Pa} \mathrm{~m} / \mathrm{s}$ for the CREPR design point.

The DT (molecular) gas load being pumped must be calculated consistently with the pumped-1imiter model (Sec. III.H.) as follows:

$$
{ }_{\phi_{\mathrm{DT}}}^{\mathrm{STP}}\left(\mathrm{Pa} \mathrm{m}{ }^{3} / \mathrm{s}\right)=0.84(10)^{-22} \frac{\langle\mathrm{n}\rangle \mathrm{V}_{\mathrm{p}}\left(1-\mathrm{R}_{\mathrm{DT}}\right)}{\tau_{\mathrm{p}}\left(1-\mathrm{R}_{\mathrm{Le}}\right)\left(1-\mathrm{R}_{\mathrm{L}_{s}}\right)},
$$

where the average plasma density $1 \mathrm{~s}\langle\mathrm{n}\rangle=6.55(10)^{20} \mathrm{~m}^{-3}$, the plasma volume is $v_{p}=37.8 \mathrm{~m}^{3}, \quad \tau_{p}=0.59 \mathrm{~s}$ is the core-plasma particle confinement $t$ ime, $R_{D T}$ is the 1 imiter-slot reflection coefficient, $R_{L e}^{*}=0.80$ is the edge-plasma recycling 
coefficient, and $R_{\mathrm{Ls}}^{*}=0.68$ is tne scrapeoff recycling coefficient. For these conditions, $\phi_{\mathrm{DT}}(\mathrm{DT} / \mathrm{s})=3.27\left(10 ; 22\left(1-\mathrm{R}_{\mathrm{DT}}\right)\right.$ becomes

$$
\phi_{\mathrm{DT}}^{\mathrm{STP}}\left(\mathrm{Pa} \mathrm{m} \mathrm{m}^{3} / \mathrm{s}\right)=55\left(1-\mathrm{R}_{\mathrm{DT}}\right)
$$

The limiter-slot reflection coefficlent, $\mathrm{R}_{\mathrm{DT}}$, can be expressed by

$$
\mathrm{R}_{\mathrm{DT}}=1-\mathrm{f}_{\mathrm{DT}} \varepsilon_{\mathrm{DT}}
$$

where $\mathrm{E}_{\mathrm{DT}}=0.24$ (Table III.H-II) is the fraction of D'r particles entering the limiter slot and $\varepsilon_{\mathrm{DT}}$ is the DT particle transmission probability, as defined in Ref. 3 (p. 8-111). The value of $\varepsilon_{D T}$ depends on the detalls of the vacuumsystem model, as will be shown below. Following the discussion gi en in Ref. 3, a mass flow balance in the limiter-slot for species $i$ (i.e., either He or DT) requires that

$$
V_{01} \frac{d\left(P_{01}+P_{1 i}\right) / 2}{d t}=\phi_{1}-F_{R 1} P_{1 i}-F_{011}\left(P_{1 i}-P_{0 i}\right)\left(1-\kappa_{i}\right),
$$

where $P_{O_{i}}$ is the gas pressure at the limiter-slot entrance, $P_{1 i}$ is the pressure of species 1 in the back of the slot region, $V_{01}$ is the volume of the slot region, $F_{\mathrm{RI}}$ is the effective conductance of the vacuum system beyond the slot for species $1, F_{011}$ is the conductance of the slot region for species 1 , and $\kappa_{1}$ is the inversion probability for spectes 1 [1.e., $K_{\mathrm{He}}=0.99$ (assumed) and $K_{\mathrm{DT}}=0.69$ (Table III.H-II) ). The three terms on the right-hand side of Eq. (4) describe the gas load entering the limfter slot, the outflow through the vacuum system, and the backflow to the plasma chamber, respectively. Steady-state operation allows the simplification to the following expression:

$$
\left.\phi_{1}=F_{R 1} P_{11}[]+\gamma_{1}\left(1-P_{01} / P_{11}\right)\left(1-\kappa_{1}\right)\right]
$$

where $\gamma_{1} \equiv F_{011} / F_{\mathrm{RI}}$ is the ratio of the 11miter-slot conductince to that of the downstream vacuum channel for spectes $i$.

The transmission probability $[E q \cdot(3)]$ is given by $\varepsilon_{1}=F_{R 1} P_{11} / \phi_{1}$, which upon substitution in Eq. (5) gives 


$$
\varepsilon_{1}=\frac{1}{1+\frac{\gamma_{1}\left(1-P_{01} / P_{11}\right)\left(1-\kappa_{1}\right)}{}},
$$

and is shoin on Fig. III.J-1 in the limit $P_{0 i} / P_{11} \ll 1$.

The schematic "unit cell" of the CRFPR vacuum system being modeled is illustrated in Fig. III.J-2, which also introduces the dimensional notation used in the vacuum conductance models. Fixed parameters are summarized on Table III.J-I and have been chosen on the basis of preliminary estimates of the overall size of the torus (Table II.D-III), blanket/shield dimensions (Fig. III.B-5.), limiter geometry (Table III.H-II), and coll sizes (Tables III.F-III and III.F-V). FIgure III.J-2 represents a first level of engineering integration of the FPC and is needed to size the vacuum system. This process of FPC integration is continued in more detail in Sec. III.L.

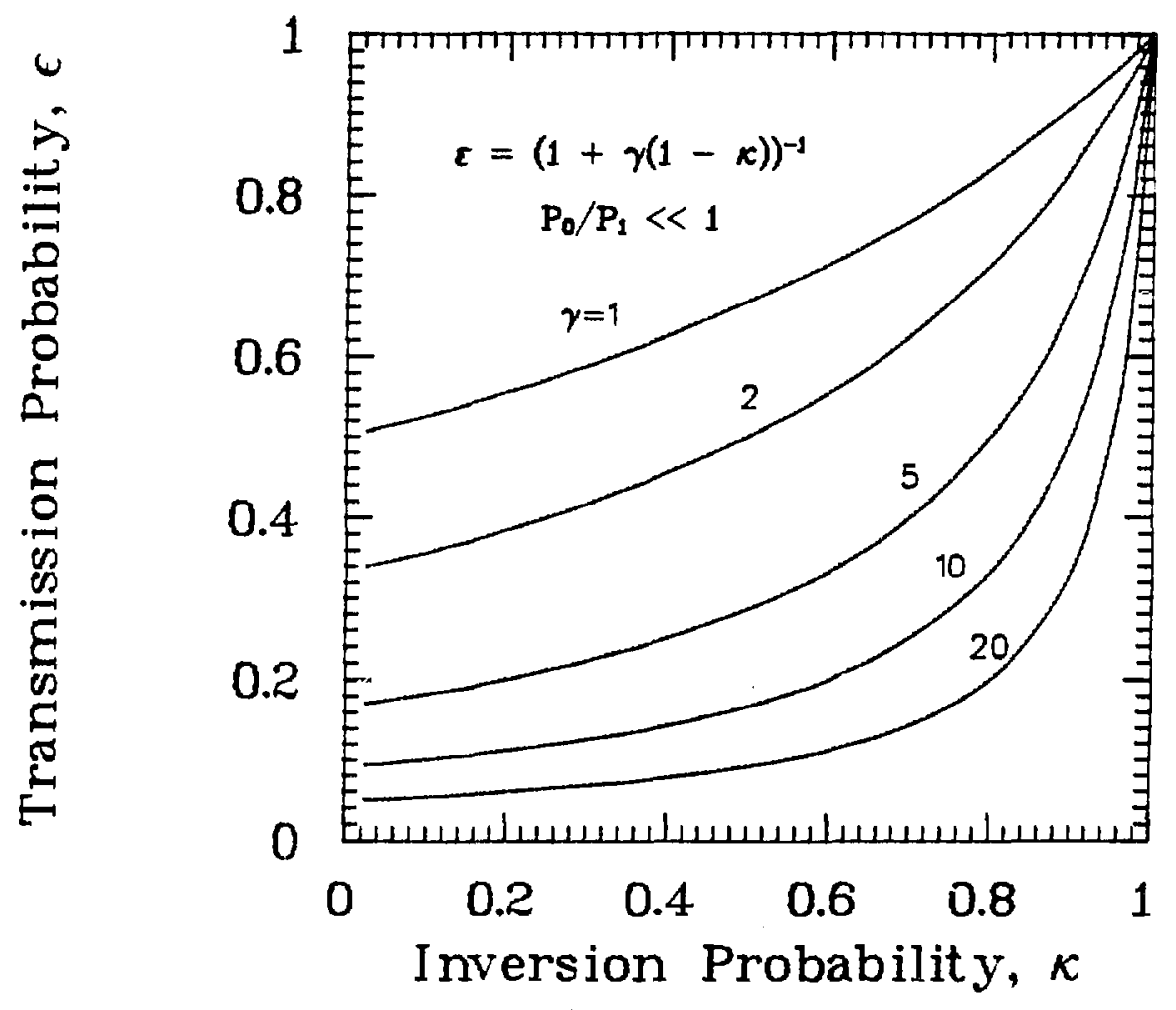

F1g. III.J-1, Dependence of the required vacuum-system transmission probability, $\varepsilon$, on the Inversion probability, $K$, for various conductance ratios, $\gamma$ (Ref. 3 ). 


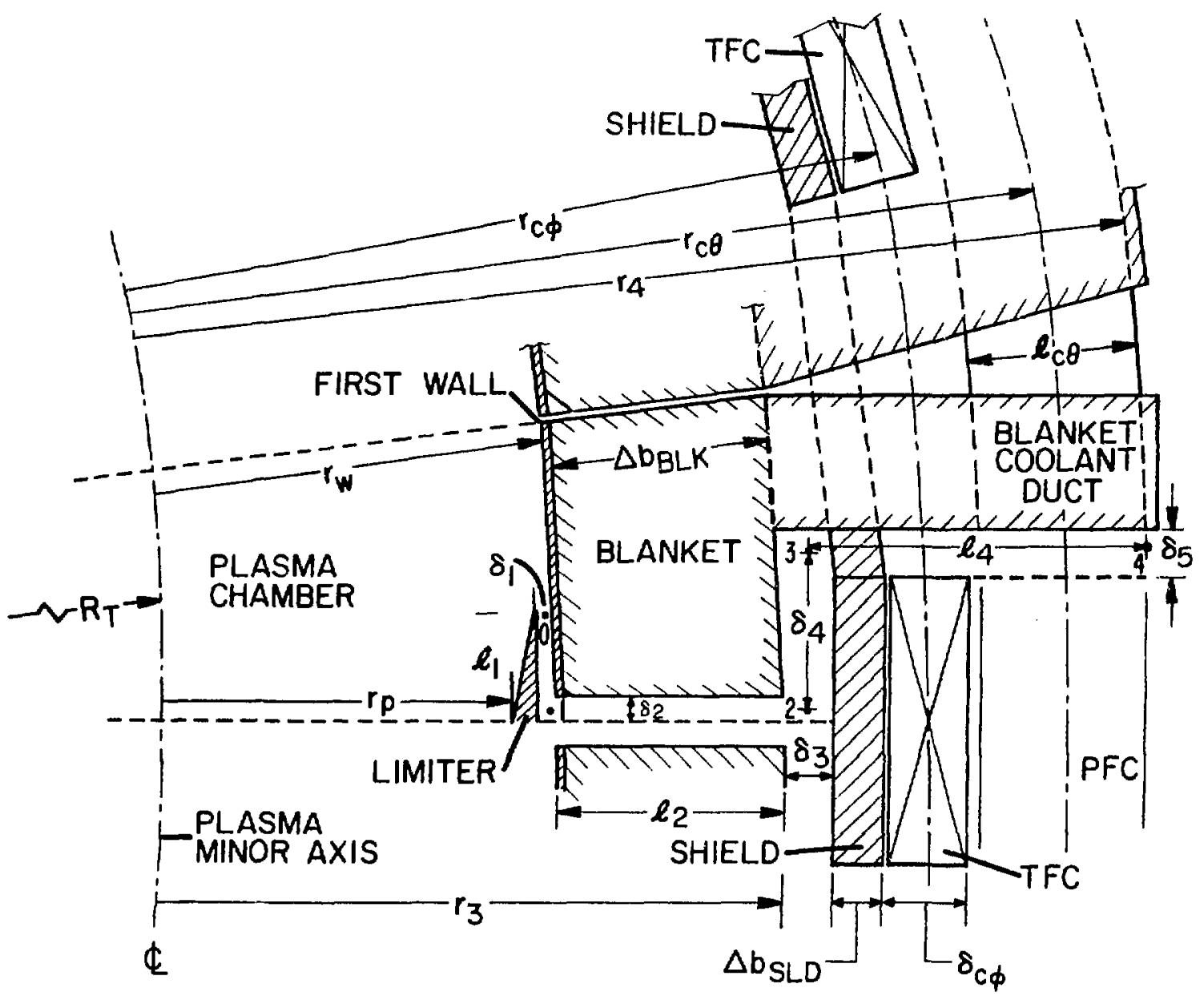

Fig. III.J-2. Schematic CRFPR "unit cel1" vacium-system model showing geometry and key notation.

The vacuum channel outboard of the limiter slot is sequentially composed of a) a radially divergent slot between adjacent blanket modules, b) an annular plenum extending around the outboard poloidal clrcumference of the torus which separates the blanket module from the shield module and provides an interruption in the neutron-streaming path from the plasma chamber to the external environment, and c) a rectangular exhaust channel on the outboard side of the torus which is constrained on either side to thickness $\delta_{5}$ by a TFC and the blanket coolant ducts and above and below to helght $\delta_{6}$ by the bianket coolant ducts for the outboard blanket annulus. In the integrated FPC design, placement of limiter and first-wall coolant manifolds and headers may be anticipated to interface somewhat with the idealized unit-cell geometry described and used here. The channel opens into the vacuum enclosure surrounding the FPC beyond the system radius, $r_{8} \simeq r_{4}$, which 1 s taken for the purposes of these vacuum 
TABLE III.J-I

FIXED PARAMETERS FOR VACUUM CALCULATION

\begin{tabular}{|c|c|}
\hline Dimensions $(m)$ : & Value \\
\hline Major toroidal radius, $R_{T}$ & 3.8 \\
\hline Minor first-wal1 radius, $r_{w}$ & 0.75 \\
\hline System radius, $r_{4} \simeq r_{s}$ & 1.69 \\
\hline Limiter-slot thickness, $\delta_{i}$ & 0.0285 \\
\hline Radius of 1imtter underside, $r_{1}=r_{w}-\delta_{1}$ & 0.7215 \\
\hline Exhaust--channel thickness, $\delta_{5}(a) \quad:$ & 0.06 \\
\hline Exhaust-channel height, $\delta_{6}$ & 0.72 \\
\hline Radial-gap length, $l_{2} \simeq \Delta_{\mathrm{FW}} / \mathrm{SW}+\Delta \mathrm{b}_{\mathrm{BLK}}$ & 0.525 \\
\hline Exhaust-channe1 length, $\ell_{4}$ & 0.75 \\
\hline Shield thickness, $\Delta \mathrm{b}_{\text {SLD }}$ & 0.10 \\
\hline TFC thickness, $\delta_{c \phi}$ & 0.075 \\
\hline TFC length, $\ell_{c \phi}$ & 0.60 \\
\hline $\begin{array}{l}\text { TFC current-center radius, } r_{c \phi} \\
\text { Temperatures }(K):\end{array}$ & 1.4625 \\
\hline$\langle\mathrm{T}\rangle_{01}$ & 600 \\
\hline$\langle\mathrm{T}\rangle_{23}$ & 500 \\
\hline$\langle\mathrm{T}\rangle_{4}$ & 350 \\
\hline Pressures (mtorr): & \\
\hline Helium, $P_{1}$ & 5 \\
\hline DT, $\quad \begin{array}{l}\mathrm{P}_{1}=2 \mathrm{P}_{2}=10 \mathrm{P}_{3} \\
\mathrm{P}_{4}\end{array}$ & $\begin{array}{l}10 \\
0.04\end{array}$ \\
\hline Parameters From Pumped-Limiter Model (Table & III. $H-I I):$ \\
\hline Limiter-slot thickness, $\delta_{1}(\mathrm{~m})$ & 0.0285 \\
\hline Limiter-slot length, $\ell_{L}(m)=l_{L} / 2$ & 0.19 \\
\hline Reflection coefficient, $\mathrm{R}_{\mathrm{DT}}$ & 0.90 \\
\hline Inversion probability, $\kappa_{D T}$ & 0.69 \\
\hline $\begin{array}{l}\text { Fraction of particles into limiter slot, } f_{\mathrm{DT}} \\
\text { Transmission probability, } \varepsilon\end{array}$ & $\begin{array}{l}0.24 \\
0.40(b)\end{array}$ \\
\hline
\end{tabular}

(a) In the actual design, two such thicknesses are combined on one side of the TFC to pump each sector $(N=24)$.

(b) A goal [Eq. (6) and Ref. 3]. 
calculations to have a pressure $P_{4}=0.04$ mtorr. The vacuum enclosure for the purpose of these estimates is taken to be a cylindrical chamber with 9-m radius and 5-m helght glving a nominal volume (less the volume of the FPC) of $\sim 1000 \mathrm{~m}^{3}$.

Vacuum conductances for these channels are calculated using a composite expression that is valid for both viscous and free-molecular flow as well as for the transition regime. Combining a viscous-flow conductance, $F_{V}\left(\mathrm{~m}^{3} / \mathrm{s}\right)$, and free-molecular-flow conductance, $F_{M}\left(m^{3} / s\right)$, gives the following general relationship:

$$
F=F_{V}+Z F_{M}
$$

where $Z$ is given by the Knudsen expression, 143

$$
Z=\frac{1+(8 / \pi)^{1 / 2} \frac{2 \delta}{R_{1}\left\langle v_{1}\right\rangle}\left\langle P_{1}\right\rangle}{1+1.235(8 / \pi)^{1 / 2} \frac{2 \delta}{\eta_{1}\left\langle v_{1}\right\rangle}\left\langle P_{i}\right\rangle} \text {. }
$$

The symbol $\delta$ is a characteristic duct dimension, where $\left\langle D_{i}\right\rangle$ $=\left(\mathrm{P}_{\mathrm{IN}}+\mathrm{P}_{\mathrm{OUT}}\right) / 2$ is the average pressure of the $1^{\text {th }}$ species in the segment, $\left\langle v_{1}\right\rangle=\left(8 \mathrm{k}_{B}\langle T\rangle / \pi \mathrm{m}_{1}\right)^{1 / 2}$ is the average molecular speed of the gas in the segment, and $\eta_{1}$ is the gas viscosity given by

$$
\eta_{1}(P a s)=0.499 m_{i}\left\langle v_{1}\right\rangle /\left(\sqrt{2} \pi d_{1}^{2}\right)
$$

where $m_{1}$ is the molecular mass (amu) and $d_{i}$ is the effective molecular diameter. Values of $d_{1}$ for $H e$ and DT are used to return the empirical values of $\eta_{\mathrm{He}}(298 \mathrm{~K})=1.99(10)^{-5} \mathrm{~Pa} \mathrm{~s}$ and $\eta_{\mathrm{DT}}(298 \mathrm{~K})=1.41(10)^{-5} \mathrm{~Pa} \mathrm{~s}$. For purposes of the present calculation, properties of the He/D $/ T_{2} / D T$ exhaugt are computed using the average properties of the dominant (DT) effective molecular species (1.e., $\mathrm{m}_{1}=5 \mathrm{amu}$ ).

The viscous-flow conductances, $F_{V}\left(m^{3} / s\right)$, for the channels illustrated on F1g. III.J-2 are given by the following expressions: 14 - 245

$$
\left.F_{V O 1}=\frac{\pi}{8 \eta_{1} l_{1}} r r_{w}^{4}-r_{1}^{4}-\frac{\left(r_{w}^{2}-r_{1}^{2}\right)^{2}}{\ln \left(r_{w} / r_{1}\right)}\right]\left\langle P_{01}\right\rangle
$$




$$
\begin{aligned}
& F_{\mathrm{V} 12}=\frac{3.287 \delta_{2}^{3}}{\eta_{2} \ln \left(r_{3} / r_{\mathrm{W}}\right)}\left\langle\mathrm{P}_{12}\right\rangle \\
& \mathrm{F}_{\mathrm{V} 23}=\frac{4.797(10)^{-3} \mathrm{Y}_{3} \delta_{3}^{2} \delta_{4}^{2}}{\eta_{3} l_{3}}\left\langle\mathrm{P}_{23}\right\rangle \\
& F_{\mathrm{V} 34}=\frac{4.797(10)^{-3} \mathrm{Y}_{4} \delta_{5}^{2} \delta_{6}^{2}}{\eta_{4} l_{4}}\left\langle\mathrm{P}_{34}\right\rangle,
\end{aligned}
$$

where the gas viscosity, $\eta_{j}$, is evaluated for the average local conditions, and $\left\langle P_{j k}\right\rangle \equiv\left(P_{j}+F_{k}\right) / 2$. The functions $Y_{1}$ are geotetry-dependent corrections.143-145 For convenfence and consistency with Eq. (4), $P_{4} \simeq 0.04$ mtorr. The analogous free-molecular-flow conductances, $F_{M}\left(m^{3} / s\right)$, are given by the following expressions: $143-145$

$$
\begin{aligned}
& F_{M 01}=\frac{\pi}{6} \frac{\left(r_{w}^{2}-r_{3}^{2}\right)^{2}}{\left(r_{w}+r_{3}\right) l_{1}}\left\langle v_{1}\right\rangle \\
& F_{M 12}=\frac{4 \pi \delta_{2}^{2}}{3 \ln \left(r_{3} / r_{w}\right)}\left\langle v_{2}\right\rangle \\
& F_{M 23}=\frac{2}{3} \frac{\delta_{3}^{2} \delta_{4}^{2}}{\left(\delta_{3}+\delta_{4}\right) l_{3}}\left\langle v_{3}\right\rangle \\
& F_{M 34}=\frac{2}{3} \frac{\delta_{5}^{2} \delta_{6}^{2}}{\left(\delta_{5}+\delta_{6}\right) l_{4}}\left\langle v_{4}\right\rangle,
\end{aligned}
$$

where the average molecular speeds, $\left\langle v_{1}\right\rangle$, are evaluated locally. In the above expressions, the Clausing factors are taken ${ }^{145}$ to be $Y_{3} \approx 1.2$ and $Y_{4} \simeq 1.5$, and orifice/aperture corrections have been neglected. The most serious approximation employed in Eqs. (10C) and (11C) is the treatment ${ }^{52}$ of the vacuum plenum as a rectangular duct with effective length $i_{3}=\left(2 \pi r_{3}\right) / 3$. A design allowance for these effects is included in the selection of the reference vacuum-system configuration. For purposes of $\mathrm{Eq}$. (4), the combined conductance beyond the limiter slot is given by

$$
\frac{1}{F_{R 1}}=\frac{1}{F_{121}}+\frac{1}{F_{231}}+\frac{1}{F_{341}}
$$


for the $i^{\text {th }}$ gas species. The approprtate viscous [Eqs.(10)] and free-molecular-flow [Eqs. (11)] expressions are combined by means of Eq. (7) to obtain the local vacuum conductances in the transition-flow regime.

The vacuum system is examined parametrically in the dimensions $\delta_{2}$ and $\delta_{3}$ to establish self-consistent combinations with the desired transmission probability, $\varepsilon[E q \cdot(3)]$. The blanket coverage factor, $f_{b c} \equiv 1-\left(N \delta_{2} / \pi \mathbb{R}_{T}\right)$, depends only on $\delta_{2}$ for fixed $N$ and $R_{T}$. It is desirable that $f_{b c}$ exceed 0.95 from the viewpoints of high blanket efficiency and adequate tritium breeding. For the present, reference design $\delta_{2} \leqslant 0.025 \mathrm{~m} \mathrm{yields} \mathrm{f}_{\mathrm{bc}}>0.95$. The present configuration with $r_{w}=0.75 \mathrm{~m}, \quad \Delta_{\mathrm{FW}} / \mathrm{SW}=0.02 \mathrm{~m}, \quad \Delta \mathrm{b}_{\mathrm{BLK}}=0.505 \mathrm{~m}$, $\Delta b_{S L D}=0.10 \mathrm{~m}$, and $r_{c_{\varphi}}=1.4625 \mathrm{~m}$ is consistent with $\delta_{3}=0.055 \mathrm{~m}$. These constraints are Imposed on the results displayed in Fig. III.J-3, showing $\varepsilon$ for the He/DT exhaust gas mixture as a function of $\delta_{2}$ and $\delta_{3}$. Since $\varepsilon_{1}>\left(1-R_{1}\right) / F_{1} \simeq 0.4$ is necessary to satisfy plasma impurity requirements, the region of heavier-lined contours of $\varepsilon>0.4$ on Fig. III.J-3 are of present interest. A more-sophisticated model, including the flow impedance introduced by orifices and bends or the insertion of limiter/first-wall coolant headers in the vacuum channel (Sec. III.L.3.), can be expected to degrade this initlal estimate of system performance. Th1s degradation is anticlpated here by selection of a design point near the $\varepsilon=0.5$ contour rather than the $\varepsilon=0.4$ contour. Table III.J-II gives parameters for this interim vacuum-system design. Pending a more-detalled analysis consistent with a "design to construct," geometric parameters are chosen conservatively in anticipation of unaccounted orifice and elbow impedances while simultaneously meeting the vacuum-system performance goals without seriously reducing blanket coverage. Because the vacuum calculation is coupled to the results of the edge-plasma/limiter model (Sec. III.H.), a significant change in the results of that analysis should prompt a reassessment of the vacuum system as well. Possible changes in the vacuum-system design resulting from modest changes in the limiter design can be estimated qualitatively. For example, a decrease in the 1imfter-slot length, $l_{1}$, results in an increase in the $11 \mathrm{miter-s}$ lot conductance, $F_{01}$, and gas backilow. To maintain a given value of the transmission probability, $\varepsilon$, the downstream conductance, $F_{R}$, must increase to preserve $\gamma=F_{01} / F_{R}$ for a fixed value of the Inversion probability, $K$. The larger value of $F_{R}$ is obtained by increasing $\delta_{2}$ and $\delta_{3}$, all other parameters being fixed. By similar logic, an increase in the limiter-slot length results in a decrease in the required value 


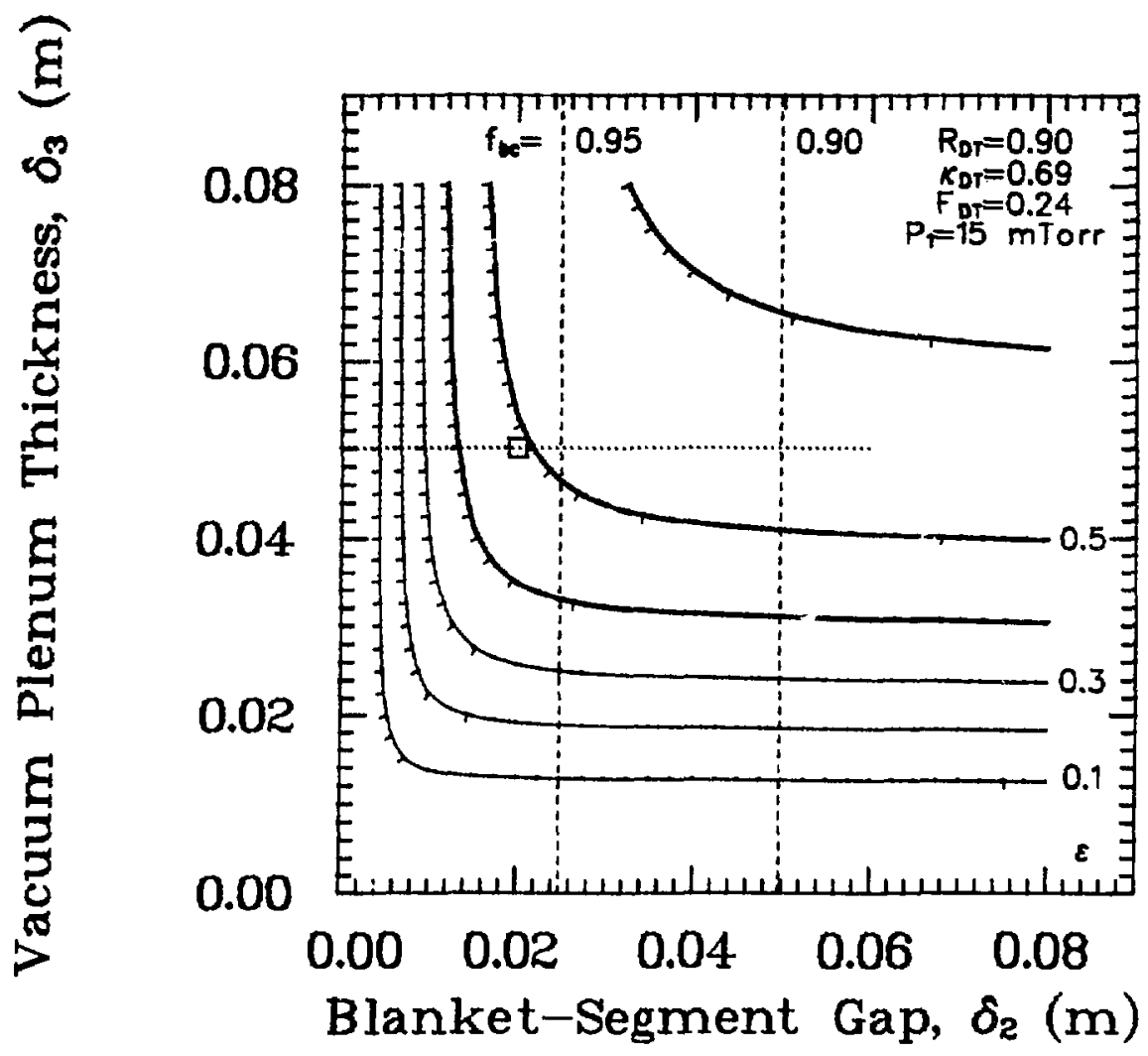

F1g. III.J-3. Dependence of He/DT exhaust gas transmission probability, $\varepsilon$, on blanket-segment gap thickness, $2 \delta_{2}$, and plenum annular thickness, $\delta_{3}$, for the flxed parameters of Table III.J-I. A conservative design point at $\delta_{2} \simeq 0.02 \mathrm{~m}$ and $\delta_{3} \simeq 0.05 \mathrm{~m}$ is shown with blanket coverage factor $f_{b c} \approx 0.96$. Operation above (below) the dashed line at $\delta_{3}=0.05 \mathrm{~m}$ requires increases (decreases) in the reference $\mathrm{FPC}$ radial build.

of $F_{R}$. The smaller value of $F_{R}$ is obtained by decreasing $\delta_{2}$ and $\delta_{3}$. If the driving pressure, $P_{1}$, at the back of the limiter slot changes, the assumed downstream channel pressures are also changed proportionally. To maintain the reference gas load, for a given geometry (1.e., fixed $\delta_{2}$ and $\delta_{2}$ ), the transmission probability, $\varepsilon$, must increase (decrease) as $P_{1}$ decreases (increases). Finally, if the value of $k$ changes, the required target value of $\varepsilon$ ananges in the same direction. The magnitude of the change can be estimated Erow Elg. III.J-1. If the reference value of $\mathrm{F}_{01}$ changes $[\mathrm{Eq} \cdot(3)]$, the target 
CRFPR INTERIM VACUUM-SYSTEM DESIGN PARAMETERS

\section{Parameter}

Unit-ce11 plasma chamber volume $\left(\mathrm{m}^{3}\right)$

Gap between adjacent blanket segments, $2 \delta_{2}$ (m) (a)

Blanket coverage parameter, $f_{b c}$

Limiter-slot thickness, $\delta_{1}(\mathrm{~m})$

Limiter-slot length, $\ell_{1},(\mathrm{~m})$

Radial-gap length, $\ell_{2}$ (m) $=\Delta \mathrm{b}_{\mathrm{BLK}}+\Delta_{\mathrm{FW} / \mathrm{SW}}$

Plenum annulus thickness, $\delta_{3}(\mathrm{~m})$

Effective plenum length, $\delta_{4}(m)=\ell_{c \phi} / 2$

Shield thickness, $\Delta b_{S L D}(m)$

Exhaust-channel thickness, $2 \delta_{5}(\mathrm{~m})(\mathrm{a})$

Exhaust-channel height, $\delta_{6}(\mathrm{~m})$

Exhaust-channe1 length, $\ell_{4}(m)$

Reflection coefficient, $\mathrm{R}_{D T}$

Edge-plasma recycling coefficient, $R_{\text {Le }}^{*}$

Scrapeoff recycling coefficient, $R_{L s}^{*}$

Inversion probability, $K_{D T}$

Fraction of particles into limiter slot, $f_{D T}$

Transmission probability, $\varepsilon$

Conductance ratio, $\gamma=F_{01} / F_{R}$

Limiter-slot conductance, $F_{01}\left(\mathrm{~m}^{3} / \mathrm{s}\right)$

Vacuum-channe 1 conductance, $F_{R}\left(m^{3} / s\right)$

Total He gas load, $\phi_{\mathrm{He}}^{\mathrm{STP}}(\mathrm{Pa} \mathrm{m} / \mathrm{s})$

Tota1 DT gas load, $\phi_{\mathrm{DT}}^{\mathrm{STP}}(\mathrm{Pa} \mathrm{m} / \mathrm{s})$

Total He mass flow rate $(\mathrm{kg} / \mathrm{s})$

Total DT mass flow rate $(\mathrm{kg} / \mathrm{s})$
Value

0.88

0.04

0.96

0.0285

$0.19^{(b)}$

0.525

0.05

0.30

0.10

0.12

0.72

0.75

0.90

0.80 (b)

$0.68^{(b)}$

0.69 (b)

$0.24^{(b)}$

$0.49^{(c)}\left[0.40^{(d)}\right]$

8.35

16.7

2.0

3.66

5.5

$6.5(10)^{-6}$

$2.7(10)^{-4}$

(a) The unit-cell value is doubled to give the $(\mathbb{N}=24)$ sector value.

(b) From Table III.H-II.

(c) Value provided to allow for orifice and elbow effects.

(d) Optimistic minimum requirement resulting from idealized model. 
value of $E$ changes in the opposite direction to preserve the value of $R_{D T}$. Generally, a moderate amount of flexibility exists in the FPC vacuum and gas load design to accommodate changes in other subsystems with boundaries that define vacuum ducts and associated conductances.

2. Pellet Fueling. One approach for refueling a fusion reactor is the periodic injection of cryogenic $\left(p_{0} \simeq 250 \mathrm{~kg} / \mathrm{m}^{3}\right)$ DT pellets. 146 As the pellets penetrate the plasma, ablation or evaporation contributes to the particle inventory. Larger initial pellet radius or higher injection speed allows deeper penetration into the plasma. It is desirable to limit the individual pellet particle inventory to a small fraction, $g$, of the nominal plasma particle inventory to reduce fluctuations in the density and fusion power during refueling. The time interval between pellet injection is $\tau_{I}(s)$.

The pellet ablation process has been considered by several authors. $147-150$ The basic model applied here is the so-called "neutral ablation cloud" model. 148 Corrections for the perturbation of the local magnetic field by the pellet lead to somewhat (up to $\sim 50 \%$ ) reduced pellet ablation rates and longer lifetimes. 149 The surface ablation of a spherical pellet with initial radius $a(m)$ is represented by the following expression for the time-rate-of-change of the pellet radius: 148

$$
\dot{a}(\mathrm{~m} / \mathrm{s})=\frac{-1.0(10)^{-8} n e^{1 / 3} \mathrm{e}^{5 / 3}}{\mathrm{a}^{2 / 3} \mathrm{~m}^{1 / 3}},
$$

where $m(a m u)$ is the molecular weight of the fuel $\left(e . g ., 5\right.$ amu for DT), $n_{e}\left(m^{-3}\right)$ is the target plasma electron density, and $T_{e}(k e V)$ is the target plasma electron temperature. Integration of $\mathrm{Eq}$. (13) in the form

$$
\int_{0}^{a} a^{2 / 3} d a=\int_{0}^{t p} \frac{-1.0(10)^{-8} n^{1 / 3} \mathrm{~T}^{5 / 3}}{m^{1 / 3}} d t,
$$

gives the pellet lifetime, $t_{p}(s)$, or the time after initial injection $(t=0)$ at wich the pellet completely evaporates $(a \rightarrow 0)$. Assuming a constant pellet Injection speed, $u(m / s)$, the maximum pellet penetration distance, $d(m)$, is given By $J=u t_{p}$. For radial plasma density and temperature profiles of the form 
$\left[1-\left(r / r_{p}\right)^{\nu}\right]$, where $r_{p}(m)$ is the plasma radius and $\nu$ is a parameter, Eq (14) can be rewritten in the following form

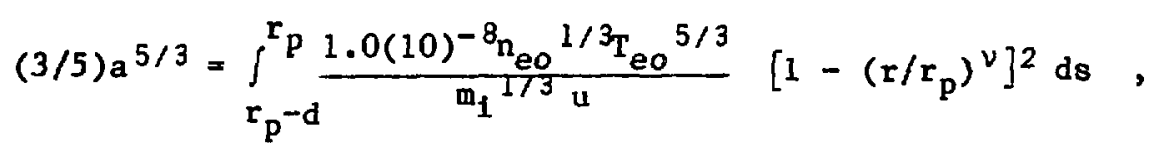

where $s=r_{p}-d$ is the distance along a perpendicular injection trajectory and $n_{e o}$ and $T_{e o}$ are the central $(r=0)$ values of plasma density and temperature, respectively. For the profile approximation used here, $n_{\text {eo }}=\langle n\rangle(v+2) / v$ and $T_{\text {eo }}=\langle T\rangle(v+2) / v$. Injection beyond the center of the plasma column is not of interest, so that solutions of $\mathrm{Eq}$. (15) can be limited to the range $0<\mathrm{d}<\mathrm{r}_{\mathrm{p}}$. The solution of $\mathrm{Eq}$. (15) is

$$
\begin{aligned}
u(m) & =1.67(10)^{-8} \frac{\mathrm{n}_{e o}{ }^{1 / 3} \mathrm{~T}_{e 0}{ }^{5 / 3} \mathrm{r}_{\mathrm{p}}}{\mathrm{m}^{1 / 3} \mathrm{a}^{5 / 3}}\left[\frac{\mathrm{d}}{\mathrm{r}_{\mathrm{p}}}-\frac{2}{(v+1)}\left(\frac{\mathrm{d}}{\mathrm{r}_{\mathrm{p}}}\right)^{(v+1)}\right. \\
& +\frac{1}{(2 v+1)}\left(\frac{\mathrm{d}}{\mathrm{r}_{\mathrm{p}}}\right)^{(2 v+1)],}
\end{aligned}
$$

which gives the required injection speed as a function of normalized penetration distance, $d / r_{p}$, for fixed plasma and pellet properties. Parabolic profiles $(\nu=2)$ for both plasma temperature and density give profiles that are close to the Bessel-function profiles assumed for the CRFPR reference design.

The time interval between pellet injections is given by $\tau_{I}(s)=M / M$, where $M$ is the pellet mass $\left(\rho_{0}=250 \mathrm{~kg} / \mathrm{m}^{3}, n_{0}=3.18(10)^{28} \mathrm{~m}^{-3}\right)$ and $\dot{M}=2.78(10)^{-4}$ $\mathrm{kg} / \mathrm{s}$ is the required mass flow rate (Table III.J-II). Pellet-fueling parameters for several combinations of assumed ratios of pellet to plasma inventory, $g$, and normalized penetration distance, $d / r_{p}$, are summarized in Table III.J-III. Assuming advanced laser-ablation technology, the pellet injection speed is Iimited to $\mathrm{u}<100 \mathrm{~km} / \mathrm{s}$, requiring $\mathrm{g}>0.1$ and $\mathrm{d} / \mathrm{r}_{\mathrm{p}}<0.33$. Near-term injector technology based on pneumatic or centifugal acceleration can attain $u=2 \mathrm{~km} / \mathrm{s}$, which is inadequate for this application. This result is more pessimistic than earlier projections. ${ }^{37}$ A model including magnetic-shielding effects would give somewhat lower estimates of the required pellet speed. Direct penetration of the pellet to the central plasma may not be 
necessary if the fuel can diffuse inward. This diffusion process is assumed for the STARFIRE design, ${ }^{3}$ which is based only on edge-plasa refueling. Calculation of pellet refueling of the central cell of the MARS design ${ }^{5}$ using a similar model have projected $u \simeq 43 \mathrm{~km} / \mathrm{s}$ for $\mathrm{d} / \mathrm{r}_{\mathrm{p}}=1.0$ and $\mathrm{u} \simeq 1.2 \mathrm{~km} / \mathrm{s}$ for $\mathrm{d} / \mathrm{r}_{\mathrm{p}}=0.2$. In both cases, $8=0.1$ and $a \simeq 4 \mathrm{~mm}$. A similar calculation for the WITAMIR design ${ }^{4}$ yields $u \simeq 114 \mathrm{~km} / \mathrm{s}$ for $g=0.1$ and $a \simeq 3 \mathrm{~mm}$. Clearly, deep refueling by pellet injection represents a development area for magnetic fusion in general.

\section{PELLET-FUELING PARAMETED VARIATIONS $(a)$}

\begin{tabular}{|c|c|c|c|}
\hline Pellet Inventory/P lasma Inventory, $g$ : & $\underline{0.01}$ & $\underline{0.05}$ & $\underline{0.10}$ \\
\hline Pellet radius, $a(\mathrm{~mm})$ & 0.98 & 1.67 & 2.11 \\
\hline Pellet injection time, $\tau_{I}(s)$ & $3.56(10)^{-3}$ & $1.78(10)^{-2}$ & $3.56(10)^{-2}$ \\
\hline Pellet mass, $M(\mathrm{~kg} /$ pe1let $)$ & $9.89(10)^{-7}$ & $4.95(10)^{-6}$ & $9.89(10)^{-6}$ \\
\hline Pellet velocity, $u(\mathrm{~km} / \mathrm{s})$ & $t$ & + & $\downarrow$ \\
\hline \multicolumn{4}{|l|}{ Penetration depth, $d / r_{p}$ : } \\
\hline 0.25 & 276 & 113 & 77 \\
\hline 0.333 & 356 & 146 & $99(b)$ \\
\hline 0.50 & 487 & 199 & 136 \\
\hline 0.75 & 595 & 243 & 165 \\
\hline 1.0 & 614 & 251 & 171 \\
\hline
\end{tabular}

(a) Fixed parameters: $\quad v=2,\langle\mathrm{n}\rangle=6.55(10)^{20 \mathrm{~m}^{-3}}\langle\mathrm{~T}\rangle=10 \mathrm{keV}, \quad \mathrm{r}_{\mathrm{p}}=0.71 \mathrm{~m}$, (b) ${ }^{\tau} \mathrm{p}=0.59 \mathrm{~s}$, DT injection rate, $\dot{\mathrm{M}}=2.78(10)^{-4} \mathrm{~kg} / \mathrm{s}$. 


\section{K. Current Drive}

Noninductive means of steady-state current drive have been proposed fur the RFP. 78-81 Unlike the tokanak, the toroldal and pololdal currents in the RFP are closely coupled, and it is possible in principle to create a time-average toroidal voltage within the plasma without requiring a time-varying magnetic flux to link the torus. As described in Appendix $A$, the minimum-energy RFP state is defined 151-152 primarily by holding the toroidal flux, $\phi$, and the magnetic helicity, $K=\int \vec{A} \cdot \vec{B} d \tau$, invariant within a conducting shell of radius $r_{w}$ surrounding the plasma, where $\vec{A}(\vec{V} \times \vec{A}=\vec{B})$ is the magnetic vector potential and the integration is perfurmed over the volume enclosed by the conducting shell. The magnetic helicity is simply a measure of the degree to which flux tubes within the conducting shell are interlinked. The helicity remains invariant and a global minimum energy is maintained while an external circuit parameter (e.g., voltage, $V_{\theta}$, applied to a TFC) is varied to change the toroidal flux external to the plasma. Intrinsic plasma processes related to turbulence and instabilities, therefore, generate toroidal voltages and currents within the plasma in order to increase (or reduce) poloidal flux to maintain the helicity constant and the plasma in a minimum-energy state. This nonlinear coupling between plasma and magnetic flelds through the F- $\theta$ diagram (Fig. II.D-2) can be used to "rectify" current oscillations created at external colls into a net steady-state current within the plasma. This "F- $\theta$ pumping" is envisaged to transform toroidal magnetic flux (poloidal currents) into toroidal currents (poloidal magnetic flux) through the plasma "dynamo" responsible for maintaining the minimum-energy RFP configuration.

Little analysis and design beyond that described in Refs. 78-81 is avallable to describe current-drive systems based on $F-\theta$ pumping for elther experiment or the reactor. This section describes the process and develops a methodology by which future engineering evaluations of $\mathrm{F}-\theta$ pumping current drive can proceed. In developing and evaluating these expressions along the lines described in Refs. 78 and 81 , the circuit parameters, definitions, and approach summarized in Table III.K-I are used. The plasma is described in terms of $K, \phi$, and $W_{M}$, and the relationshlp between these parameters and the circuit varlables (i.e., resistances, inductances, currents, voltages) constitutes the ovelall current-drive model. The time dependence of $\mathrm{K}, \phi$, and $W_{M}$ result ${ }^{81}$ directly from the Maxwe11 equations: 
SUMMARY OF DEFINITIONS AND NOTATION USED TO MODEL "F- $\theta$ PUMPING" CURRENT DRIVE

\section{Definition}

Toroidal voltage on plasma

Toroldal current in plasma

Poloidal voltage applied to toroldal-field coll

Pololdal current flowing in external conductor

Plasma resistance

Magnetic hellcity, where $\vec{B}=\vec{\nabla} \times \vec{A}$

Toroidal flux

Total field energy within conducting shell

Vacuum toroldal Inductance

Inverse aspect ratio

Average toroldal fleld within shell

Reversal parameter (Fig. II.D-2)

Pinch parameter (Fig.II.D-2)

\section{Parameter}

$\mathrm{V}_{\phi}$

I. $\phi$

$v_{\theta}=\dot{\phi}$

$I_{\theta}$

$\mathbf{R}_{\text {p }}$

$\mathrm{K}=\frac{1}{\mu_{\mathrm{o}}} \overrightarrow{\mathrm{A}} \cdot \overrightarrow{\mathrm{B}} \mathrm{dV} \mathrm{p}$

$\phi=2 \pi \int_{0}^{x_{w_{B}}}(r) r d r$

$W_{M}=\int\left(B^{2} / 2 \mu_{0}\right) d \tau$

$L_{0}=\mu_{0} r^{2} / 2 R_{T}$

$\varepsilon=r_{\mathrm{W}} / \mathrm{R}_{\mathrm{T}}$

$\left\langle B_{\phi}\right\rangle=\phi / \pi r_{w}{ }^{2}$

$F=B_{\phi}\left(r_{w}\right) /\left\langle B_{\phi}\right\rangle=L_{o} I_{\theta} / \phi$

$\theta=B_{G}\left(r_{w}\right) /\left\langle B_{\phi}\right\rangle=L_{0} I_{\phi} / \varepsilon \phi$

$$
\begin{aligned}
& \frac{d K}{d t}=2 \phi V_{\phi}-2 \int \eta \vec{j} \cdot \vec{B}_{d} V_{p} \\
& \frac{d \phi}{d t}=+v_{\theta}
\end{aligned}
$$

$$
\begin{aligned}
& \frac{d W_{M}}{d t}=I_{\phi} V_{\phi}-I_{\theta} V_{\theta}-\int \eta \vec{j} \cdot \vec{j} d V_{p} \\
& \vec{E}=\overrightarrow{n j}-\vec{v} \times \vec{B}\left(E_{\phi}=\eta j_{\phi}-\alpha B B_{\phi}\right),
\end{aligned}
$$


where the last expression gives an Ohm's law corrected for the plasma dynamo effect. With the plasma resistance defined by

$$
I^{2} \phi_{p}=\int \eta \vec{j} \cdot \vec{j} d v_{p}
$$

the plasma helicity, flux, and energy equations become

$$
\begin{aligned}
& \frac{d K}{d t}=2 \phi\left(V_{\phi}-I_{\phi} R_{p}\right) \\
& \frac{d \phi}{d t}=+V_{\theta} \\
& \frac{d W_{M}}{d t}=I_{\phi} V_{\phi}-I_{\theta} V_{\theta}-I_{\phi}^{2} R,
\end{aligned}
$$

where in Eq. (1) the helicity dissipative term, $2 \int \vec{\eta} \vec{j} \cdot \vec{B} d \tau$, is given exactly by $2 \phi I \phi_{p}$ for the Bessel-function model (BFM). It is readily seen from either $\mathrm{Eq}$. (1) or (6) that if the time-average $\langle\mathrm{dK} / \mathrm{dt}\rangle$ is to equal zero, then $2\left\langle\phi V_{\dot{\phi}}\right\rangle$ must equal the dissipation term, which means $\phi$ and $v_{\phi}$ " should be nominally in phase; hence, $v_{\phi}$ and $v_{\theta} \simeq \dot{\phi}$ should be out of phase by $90^{\circ}$.

A statement of $W_{M}$ and $\dot{W}_{M} \equiv \mathrm{dW}_{M} / \mathrm{dt}$ in terms of plasma and circult parameters is given below:

$$
\begin{aligned}
& W_{M}=\frac{1}{2} L_{p} I_{\phi}^{2}+\frac{\phi^{2}}{2 L_{\theta}} \\
& \dot{W}_{M}=V_{\phi} I_{\phi}+V_{\theta} I_{\theta}-I_{\phi}^{2} R_{p} .
\end{aligned}
$$

The plasma inductance, $L_{p}$, is a function of the field profiles and, for example, can be derived using the BFM or variants thereof described in Appendix $A$. Combining Eqs. (7), (8), and (9) using Faraday's Law $\left(V_{\theta}=+\dot{\phi}\right)$ leads to the following expression for the toroldal voltage around the plasma, $v_{\phi}$ : 


$$
V_{\phi}=I_{\phi} R_{p}+\left[L_{p}+\frac{\theta}{2} \frac{d L_{p}}{d \theta}\right] \dot{I}_{\phi}+\left[\frac{1-F}{\varepsilon \theta}-\frac{\varepsilon \theta^{2}}{2 L_{o}} \frac{d L_{p}}{d \theta}\right] V_{\theta}
$$

Adoption of a positive Faraday's Law for the toroidal circuit is based upon a convention that orients $\phi$ in the same direction as $I_{\phi}$. When the toroidal and poloidal circuits are coupled through the $F-\theta$ diagram a) toroidal current can be driven by an applied poloidal (TFC) voltage, $V_{\theta}$, and $b$ ) the plasma responds as a nonlinear circuit element through the $F-\theta$ diagram. Sinusoidal variations in $V_{\theta}$ and $\mathrm{V}_{\phi}$ applied to the THC and PFC sets thereby couple nonlinearly to drive a net toroidal dc current. Equation (10) has been solved for the complete ZT-40M circuit, 78 showing the theoretical feasibility of $F-\theta$ current drive. In order to solve Eq. (10), the plasma Inductance, $L_{p}$, must be expressed as a function of the pinch parameter, $\AA$. Using the BFM field profiles (Appendix $A$ ) and equating the total magnetic energy stored within the plasma to $\frac{1}{\Delta} L_{p} I_{\phi}^{2}$ gives the following expression for $\mathrm{L}_{\mathrm{p}}$ :

$$
\mathrm{L}_{\mathrm{p}}=\frac{2 \mathrm{~L}_{\mathrm{o}}}{\varepsilon^{2}}\left[1+(2 F+1)(F-1) / 2 \theta^{2}\right] \text {, }
$$

where $F=\theta \mathrm{J}_{0}(2 \theta) / \mathrm{J}_{1}(2 \theta)$ for the $\mathrm{BFM}$, and included in $\mathrm{Eq} \cdot(11)$ is the energy needed to compress an initially uniform toroidal flux into the $B_{\phi} \propto J_{0}(\mu r)$ distribution. The purely poloidal inductance for the $B F M$ is $L_{p \theta}=2 W_{B} \theta / I=$ $\left(L_{o} / \varepsilon^{2}\right)\left[1+\left(F / \theta^{2}\right)(F-1)\right]$. The plasma resistance, $R_{p}$, used in Eq. (10) is also dependent upon profiles through the factor $8_{0 H M}$, with $R_{p}$ giver by

$$
R_{\underline{p}}=2 g_{O H M}\langle\eta\rangle / \varepsilon^{2} R_{T},
$$

where $\langle\eta\rangle$ is the classical resistivity evaluated at the average plasma temperature. For the BFM and a flat temperature distribution, $g_{O H M}$ is given by

$$
g_{\mathrm{OHM}}=2\left(\theta^{2}+F^{2}\right)-F \text {. }
$$

The profile factor, $g_{O H M}$, is often referred to as the resistance "anomaly" 
factor, a notation that can be misleading in that as given above $8_{0 \mathrm{HM}}$ is a measure of the extended current path resulting from the "screwing-up" of the high-pitch field Iines. For the BFM model without deep reversal $(\theta<1,6)$, $8_{\text {OHM }}$ typically is $\sim 3$.

The $F-\theta$ coupled poloidal and toroidal circuit equations can be solved for $I_{\phi}$ once driver functions are selected for $\phi$ (i.e., $\phi=\phi_{0}+\delta \phi \cos \omega t, v_{\theta}=\dot{\phi}=$ $\delta \phi \omega \sin \omega t)$ and $v_{\phi}\left(i . e ., v_{\phi}=\delta v_{\phi} \cos \omega t\right)$. This procedure must be performed with some assurance that the time-averaged magnetic helicity is constant. The constraint that the time average of $\mathrm{dK} / \mathrm{dt}$ should be zero can be used to establish limits on the magnitude of the fleld oscillations required to sustain a given toroldal plasma current. If the ohmic dissipation for both the induced and driven cases are similar, and if the induced case operates with $\phi_{0}$ and $v_{\phi_{0}}$ for $d K / d t=0$, then $d K / d t$ for the driven case is given by Eq. (6) with $V_{\phi o} \equiv I_{\phi} R_{p} \cdot$ Hence, 80

$$
\frac{d K}{d t} \simeq 2 \phi\left(v_{\phi}-v_{\phi 0}\right)
$$

Using the driver functions given below,

$$
\begin{aligned}
& \phi=\phi_{0}+\delta \phi \cos \omega t \\
& v_{\phi}=\delta v_{\phi} \cos \omega t,
\end{aligned}
$$

the following expression for a time average of $\mathrm{dK} / \mathrm{dt}$ for the driven case results:

$$
-\langle\mathrm{dK} / \mathrm{dt}\rangle=\delta \phi \delta \mathrm{v}_{\phi}+2 \phi_{0} \mathrm{~V}_{\phi \circ}
$$

For $\langle\mathrm{dK} / \mathrm{dt}\rangle \simeq 0$, the following conditions on the amplitudes of toroidal flux and voltage oscillation result: 


$$
\left(\frac{\delta \phi}{\phi_{0}}\right)\left(\frac{\delta V_{\phi}}{V_{\phi}}\right)=-2
$$

Given that toroidal flux oscillations much above $\delta \phi / \phi_{0} \approx 0.2-0.3$ are expected to impact seriously the RFP configuration, the ac toroidal voltage needed to drive a de toroidal current with $\langle\mathrm{dK} / \mathrm{dt}\rangle \approx 0$ can be 6-10 times greater than the voltage needed to sustain an inductively driven RFP current drive. The ac toroidal voltage probably will have to be applied at a frequency that is above $1 /$ (diffusion time) but lower than $1 /$ (relaxation time).

Many physics and engineering questions remain to be address before the viability of F- $\theta$ pumping for RFP current drive can be assessed. of particular concern and importance are the following issues:

- Effects of $\delta I_{\phi} / I_{\phi}$ and $\delta \phi / \phi$ variations on

- bulk-plasma transport

- plasma-edge effects (Iimiter and divertor performance)

- constancy of first-wall thermal loading, fusion yield, and blanket power density.

- Overall plant efficiency

- magnitude of reactive power and fraction of total stored energy transferred

- efficiency or Q-value of F- $\theta$ pumping current-drive.

- Overa11 EPC layout

- use of separate versus main coils to oscillate $\mathrm{B}_{\phi}$ and $\mathrm{B}_{\theta}$ at the plasma surface, and position of driver coils if separate from main coils

- interaction of driver coils with other FPC subsystems, particularly those related to coolant lines, divertors, magnets, and blanket neutronics performance (e.g., tritium breeding).

To assess the tradeoff between $\delta \phi / \phi_{O}, \delta V_{\phi} /\left\langle I_{\phi} R_{p}\right\rangle, \omega, \delta I_{\phi} /\left\langle I_{\phi}\right\rangle$, reactive power, $P_{R}$, and the energy stored in the current-drive circuits, $E_{S}$. Eq. (10) has been solved numerically' for the $B F M$ under the constraint that $\langle\dot{I}\rangle_{\phi}$ and $\langle\dot{K}\rangle$ are zero. The voltage drive functions given by Eq. (15) are used, and 
Table III.K-II lists the $F$ ana $\theta$ dependences of $R_{p}$ and $L_{p}$, as dictated by the constant- $\mu$ BFM, as well as key plasma parameters. The dependencies of $\delta \mathrm{I}_{\phi} /\left\langle\mathrm{I}_{\phi}\right\rangle$, $\delta V_{\phi^{\prime}}\left\langle I_{\phi^{R}} R_{p}, P_{R}\right.$, and $E_{s}$ on $\delta \phi / \phi_{\rho}$ and $w$ are shown on Fig. III.K-1 for the CRFPR operating conditions and the BFM. Selecting $\delta \phi / \phi_{0}=0.01 \mathrm{ylelds}$ a value of $\delta I_{\phi} /\left\langle I_{\phi}\right\rangle$ near the minimum of 0.003 ; reduced $\delta V_{\phi} /\left\langle I_{\phi} R_{p}\right\rangle$ relative to the value at the $\delta I_{\phi} /\left\langle I_{\phi}\right\rangle$ minimum are possible if higher $I_{\phi}$ oscillations are tolerable. Table III.K-II gives the associated parameters for this current-drive design point. For this closely coupled system the dissipative power, $\mathrm{P}^{*} \simeq 5.74 \mathrm{MW}$, entirely supplies the plasma ohmic losses and gives a current-drive efficlency of $\left\langle I_{\phi}\right\rangle / P^{*} \approx 3.2 \mathrm{~A} / \mathrm{W}$, which is expected co decrease by $\sim 2$ for an engineered current-drive system. The energy stored in the poloidal and toroidal current-drive circuits is $0.26 \mathrm{MJ}$. For the Modified Bessel-Function model (MBFM) and $T(r) \propto J_{0}(\mu r)$ the expected plasma resistive dissipation (Sec. III.G.) is $25.3 \mathrm{MW}$. If the $\mathrm{OHC}$ current were driven to zero ( $\mathrm{P}_{\mathrm{OHC}}^{\Omega}=72 \mathrm{MW}$ ), once achieving steady state, a maximum of $36 \mathrm{MW}$ current drive at $50 \%$ efficlency would be avaliable without degradation of the overall reactor power balance.

To assess the effects of using more-realistic $\mu$ profiles, the calcuiations depicted in Fig. III.K-1 were repeated using the MBFM (Appendix A). The magnetic fields for the MBFM are obtained by numerically integrating Eq. A-45 (Appendix A) with a $\mu$ profile that is constant in the range $0 \leqslant r \leqslant \alpha_{p}$ and ramps linearly to zero for $\alpha r_{p} \leqslant r \leqslant r_{p}$, with $\alpha$ given by Eq. A-56. The pressure profile is held proportional to $\mathrm{J}_{0}^{2}(\mu \mathrm{r})$ and normalized to correspond to the $\beta_{\theta}=0.23$ design point (Table III.G-I). The resulting magnetic-field profiles are used to generate the reversal parameter, F, and the total magnetic energy, $w_{M}$, from which $L_{p}$ can be derived as a function of $\theta$. A cubic spline fit to $L_{p}(\theta)$ is used to generate $d L_{p} / d \theta$. A comparison of $F, L_{p}$, and $d L_{p} / d \theta$ derived from the BFM and MBFM are presented in Pig. III.K-2. The MBFM results in a reversal that is less shallow for the same $\theta$ than obtained for the $B F M$; $a$ smaller inductance and a substantlally smaller value of $d_{\mathrm{p}} / \mathrm{d} \theta$ for $\theta>1.3$ also result. A minor anomaly occurs in $\mathrm{dL}_{\mathrm{p}} / \mathrm{d} \theta$ at $\theta \sim 1.2$ because of a discontinuity in the derivatives of $L_{p}$ of all orders; this discontinuity does not affect the calculations since the region of interest is $1.4<\theta<1.7$.

Another consequence of the MBFM is that Eq. (6) becomes an approximation to Eq. (1) because of the varying $\mu$ profile. The conservation of helicity and energy denoted by Eqs. (6) and (8), therefore, cannot be satisfied simultaneously. A conserving set of equations can be recovered by introducing a 
CRFPR PLASMA PARAMETERS FOR A 1000-MWe(net) MINIMUM-COE DESIGN POINT USED TO SIMULATE F- $\theta$ PUMPING CURRENT DRIVE

\section{PLASMA PARAMETER}

Minor plasma radius, $r_{p}(m)$

Major toroldal radius, $R_{T}(m)$

Inverse plasma aspect ratio, $\varepsilon=r_{p} / R_{T}$

Average plasma temperature, $T(\mathrm{keV})$

Average plasma density, $n\left(10^{20} / \mathrm{m}^{3}\right)$

Toroldal plasma current, $I_{\phi o}$ (MA)

Energy confinement time, $\tau_{\mathrm{E}}(s)$

$F-\theta \operatorname{mode} i\left[F=\theta J_{0}(2 \theta) / J_{I}(2 \theta)\right]$

Reversal parameter, $F=B_{\phi}\left(r_{w}\right) /\left\langle B_{\phi}\right\rangle$

Pinch parameter, $\theta=B_{\theta}\left(r_{w}\right) /\left\langle B_{\phi}\right\rangle$

Pololdal beta, $\beta_{\theta}$

Streaming parameter, $I_{\phi} / \mathrm{N}\left(10^{-14} \mathrm{Am}\right)$

classfcal plasma resistivity, $\langle\eta\rangle\left(10^{-10} \Omega \mathrm{m}\right)$

CIRCUIT PARAMETERS

Toroldal plasma inductance,

$$
L_{\mathrm{O}}(\mathrm{H})=\mu_{\mathrm{o}} R_{\mathrm{T}} \varepsilon^{2 / 2}
$$

Pololdal plasma inductance, $\mathrm{L}_{\mathrm{p}}(\mathrm{H})=$

$$
\left(L_{o} / \varepsilon^{2}\right)\left[1+\left(F / \theta^{2}\right)(F-1)\right]
$$

Toroldal flux, $\phi_{0}(\mathrm{~Wb})=\mathrm{L}_{0} \mathrm{I}_{\phi} / \varepsilon \theta$

"Screw-up" factor, $8_{0 H M}=2\left(\theta^{2}+F^{2}\right)-F$

Plasma resistance, $R_{p}(o h m)=2 g_{0 H M}\langle\eta\rangle / \varepsilon^{2} R_{T}$

Plasma poloidal flux, $L_{p} I_{\phi}(W b)$

Plasma ohmic voltage, $V_{\phi}=I_{\phi} R_{p}(V)$

Plasma decay time, $\mathrm{L}_{\mathrm{p}} / \mathrm{R}_{\mathrm{p}}(\mathrm{s})$
VALUE

0.71

3.8

0.19

20. [flat]

$5 \cdot\left[\mathrm{J}_{0}^{2}(\mu \mathrm{r})\right]$

18.5

0.23

BFM

$0.0-(-0.6)$

$1.2-(-1.4)$

0.2

5.8

3.4

\section{VALUE}

$$
8.3(10)^{-8}
$$

$$
(2.4-3.6)(10)^{-16}
$$

5.9-6.9

2.9-3.0

$$
1.5(10)^{-8}
$$

44. -66 .

0.3

164. -234 . 
TYPICAL $F-\theta$ PUMPING CURRENT-DRIVE PARAMETERS

PARAMETER

Fractional Flux Swing, $\delta \Phi /\langle\Phi\rangle$

Voltage Swing, $\delta V_{\phi} /\left\langle I_{\phi^{2}}\right\rangle$

Toroldal Voltage, $\left\langle I_{\phi} R_{p}\right\rangle(V)$

Current Swing, $\delta \mathrm{I}_{\phi} /\left\langle\mathrm{I}_{\phi}\right\rangle$

Current Swing, $\delta I_{\phi}(M A)$

Frequency, $\omega(\mathrm{Hz})$

Dissipative Power, P*(MW)

- Poloida1, $\mathrm{P}_{\theta}^{*}(\mathrm{MW})$

- Torolda1, $\mathrm{P}_{\phi}^{\star}(\mathrm{MW})$

Efficlency Factor, $\left\langle I_{\phi}\right\rangle / P^{*}(A / W)$

Stored Energy, $E_{\mathbf{g}}$ (MJ)

\section{VALUE}

0.01

200.

0.31

0.0042

$0.078^{(a)}$

50 .

5.74

3.44

2.30

$3.2 *$ (b)

0.26

(a) If the $\mathrm{P}_{\mathrm{OHC}}^{\Omega} \simeq 72 \mathrm{MW}$ were made avallable entirely to support current-drive inefficiencies once steady state was achieved and the OHC set was run down to zero current, a maximum level of current drive of $36 \mathrm{MW}$ at $50 \%$ efficiency $(>0.26 \mathrm{~A} / \mathrm{W}$ ) would be allowed without changing the overall, base-case reactor power balance. This value compares to the plasma resistive dissipation of $25.3 \mathrm{MW}$ for the MBFM and $T(r) \propto J_{0}(\mu r)$.

(b) This amounts to $\delta \mathrm{I}_{\phi} /\left\langle\mathrm{I}_{\phi}\right\rangle=0.0042$, which translates to a $1.70 \%$ power fluctuation for constant beta.

multiplicative factor into the $2 \phi I \phi_{p}$ term of Eq. (6), which contains the appropriate profile weighting. This approach, however, is not warranted since the resistive dissipation described by Eq. (5) and appearing in Eq. (3) is not classical in nature but results from current fluctuations. The procedure adopted here maintains Eq. (6) intact and replaces $R_{p}$ in Eqs. (8) and (10) with the product $f_{A} R_{p}$, where $f_{A}$ is an effective anomaly factor. By requiring $\langle\dot{K}\rangle=\left\langle\dot{I}_{\phi}\right\rangle=0$, the factor $f_{A}$ is determined numerically to be $\sim 0.52$ for the CRFPR operating conditions (Table III.K-I) using the MBFM. The only difference in the assumed operating conditions for the two models occurs in the pinch parameter, $\theta$, which is 1.25 for the BFM and 1.55 for the MBFM; F $=0.122$ for both models. In addition, the value of $R_{p}$ listed in Table III.K-I is used 

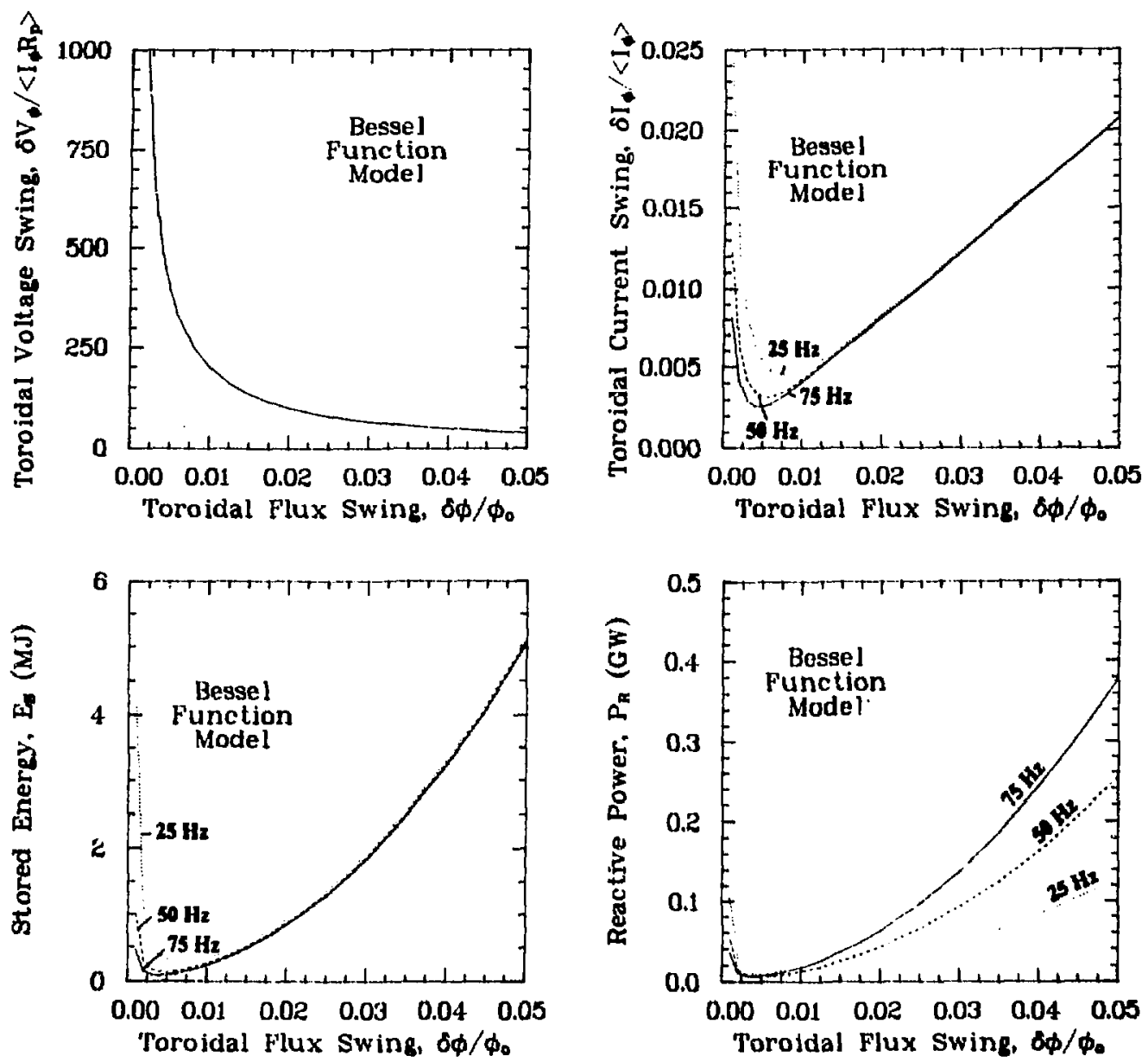

F1g. III.K-1. Parametric evaluation of Eq. (10) for F- $\theta$ pumping current drive using Eqs. (15) as drivers and the plasma parameters listed in Table III.K-I.

Independent1y of the value of $\theta$, which to some extent represents an approximation.

The dependencles of $\delta I_{\phi} /\left\langle I_{\phi}\right\rangle, \delta V_{\phi} /\left\langle I_{\phi} f_{A} R_{p}\right\rangle, P_{R}$, and $E_{g}$ on $\delta \phi / \phi_{0}$ and $w$ are shown on F18. III.K-3 for the MBFM. The minlmum in $\delta I_{\phi} /\left\langle I_{\phi}\right\rangle, P_{R}$, and $E_{B}$ for the MBFM are slightly shallower and occur at higher values of $\delta \phi / \phi_{0}$ compared to the BFM. Substantlally larger differences in these three parameters between the two models occur at values of $\delta \phi / \phi_{0}>0.02$, where the larger-amplicude osclllations In $\theta$ can sample the corresponding greater differences in $L_{p}$ and $\mathrm{dL}_{\mathrm{p}} / \mathrm{d} \theta$ predicted by the two models (Fig. III.K-2). The MBFM results for $\delta V_{\phi} /\left\langle I_{\phi} f_{A} R_{p}\right\rangle$ have been 

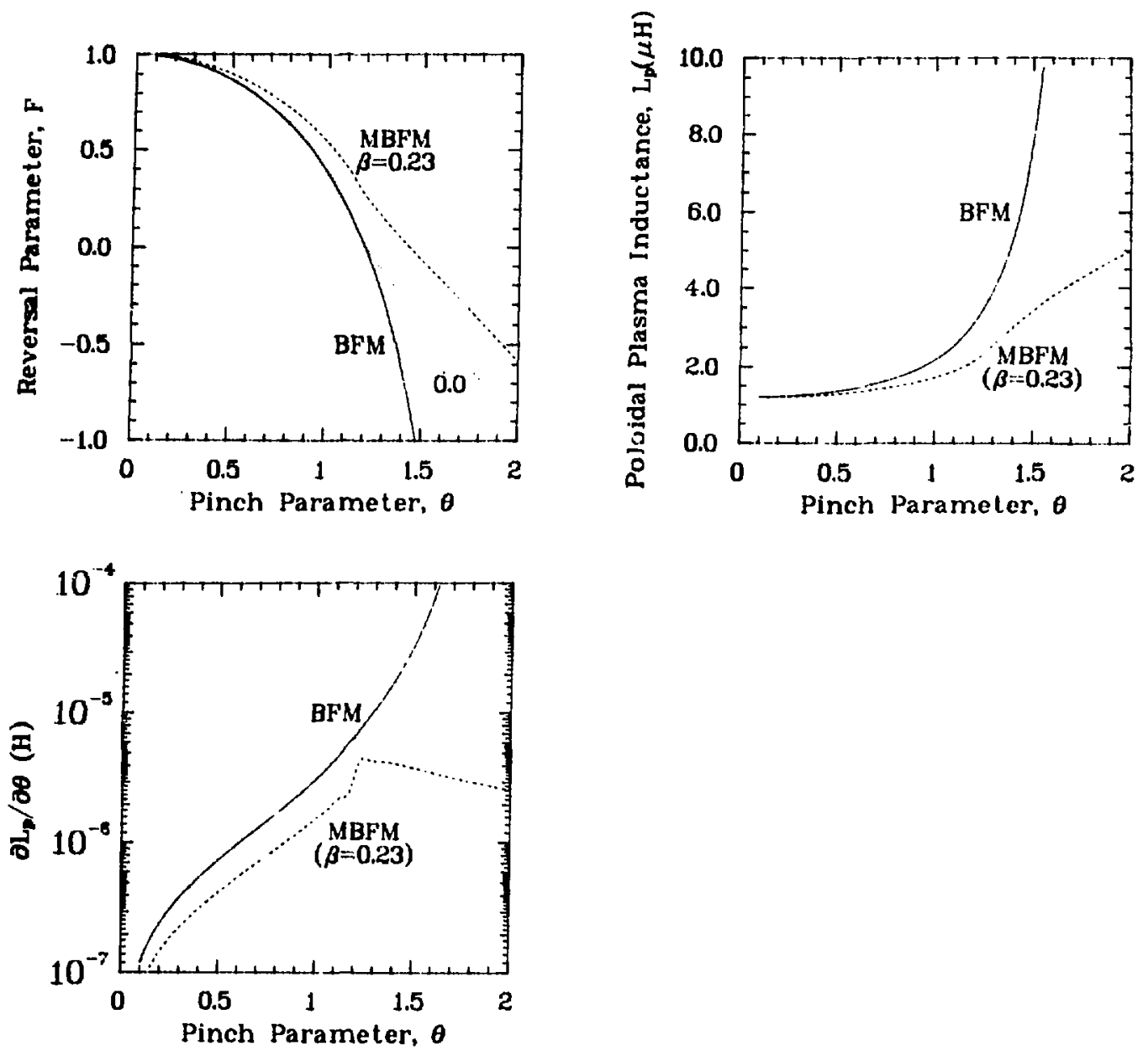

FIg.III.K-2. A comparison of the BFM and the MBFM calculations of $F, L_{p}$, and $d L_{p} / d \theta$ for $F-\theta$ pumping current drive.

Increased by $1 / f_{A}$ over the $B F M$, which is directly attributable to using $R_{p}$ for a resistance In Eq. (6) and $f_{A} R_{p}$ for a resistance in Eq. (10).

It remains for future work to model $F-\theta$ pumping current drive using even more realistic $\mu$ profiles in confunction with a detalled time-dependent simulation of both plasma and circuit response (Sec. III.G.). The results of such a simulation would be needed to perform a complete circuit design and to integrase the resulting current-drive circuitry realistically into both the FPC mechanical/electrical/thermal design as well as into the CRFPR energy balance. Based on the present scoping analysis of a simplified RFP plasma, however, the 

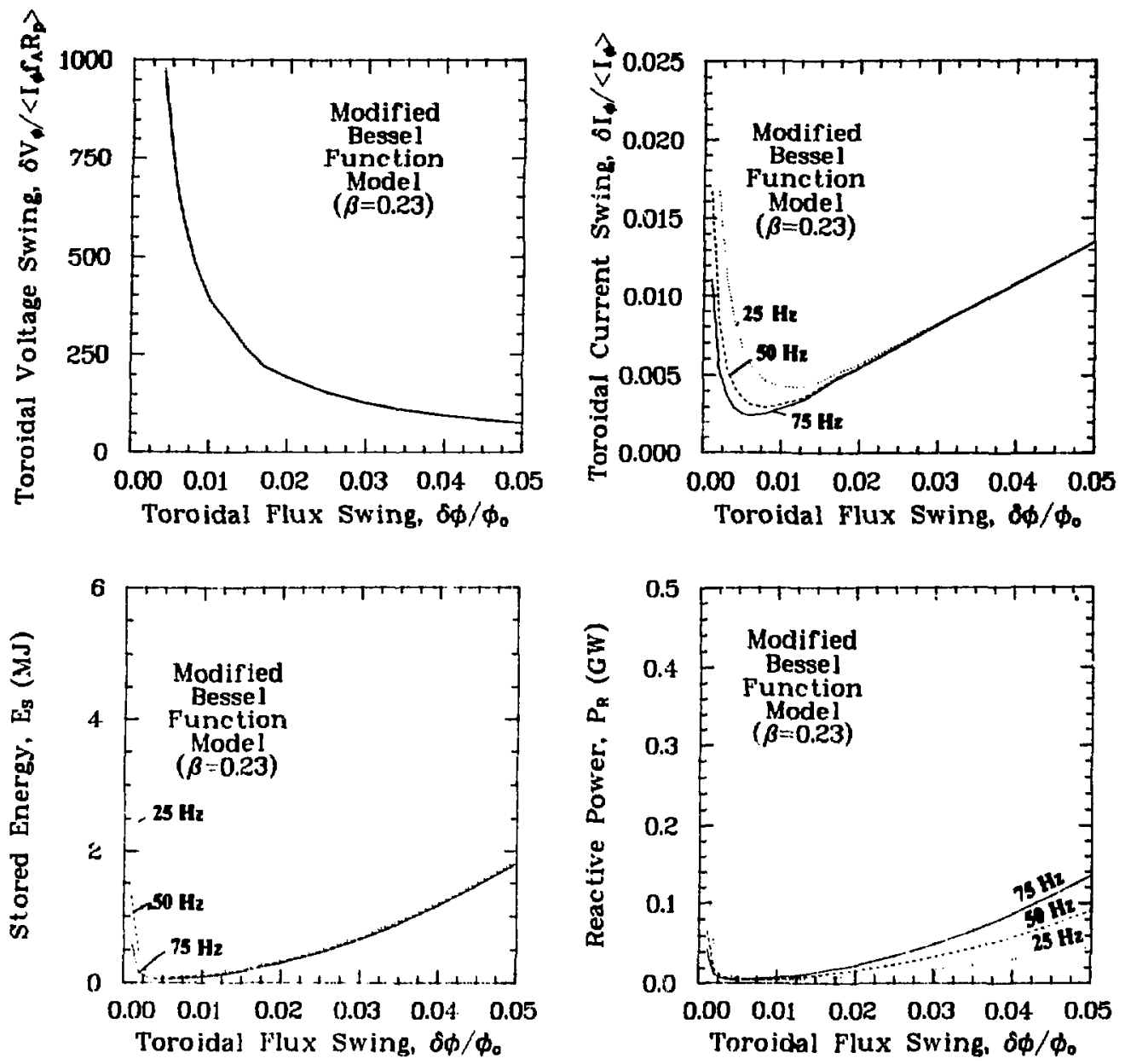

Fig. III.K-3. Parametric evaluation of Eq. (10) with a resistance of $f_{A} R_{p}$ for F- $\theta$ pumping current drive using Eqs. (15) as drivers and the plasma parameters listed on Table III.K-I with $g_{\text {OHM Independent }}$
of $\theta$.

application of F- $\theta$ pumping to drive steady-state currents in reactor-relevant RFPs using relatively low-technology power supplles appears to be promising. The key FPC 1ssues remaining to be resolved revolve around the position and design of these low-voltage/low-current driver colls and the Impact of these colls on the blanket neutronic performance if located near the first wall. If the F- $\theta$ pumping can be operated at sufficlently low frequency, oscillation of the main TFCs and PFCs become possible, thereby ellminating the impact on blanket performance. 


\section{Fusion-Power-Core Integration}

This section integrates key FPC subsystems described individually throughout Sec. III. This FPC integration is performed at three levels: power-plant energy balance, thermal-hydraulic design, and mechanical design. Specification of a given set of design parameters at a given level of integration requires parameters to be fixed at the previous level. Each are discussed in the order given above.

1. Power-Plant Energy Balance Integration. The power distribution throughout the FPC as suggested by the neutronics analysis (Sec. III.B.) and modifled by preliminary destgns of first-wall (Sec. III.D.), blanket (Sec. III.E.), and pumped-limiter (Sec. III.H.) subsystems lead to the global power-plant energy balance depicted on Fig. III.L-1. Coupling these results with the more-realistic plasma and circuit simulations described in Sec. III.G. gives the baseline power balance summarized in Table III.L-I.

The power balance used to complete this FPC integration is based on

- water-cooled pumped limiter

- water-cooled first wall and second wall

- water-cooled shield (to reduce manifolding coiplexity)

- ohmic and nuclear heating in TFC and PFC not used by thermal-conversion cycle

- use of a steam cycle with an intermediate heat exchanger between PbL1 loop and steam generator

- aside from ohmic power required by the plasma and coils, all pumplng power and other auxlliary needs are held to within $\mathrm{f}_{\mathrm{AU}}:<0.07$ of the gross electrical power, ${ }_{\mathrm{ET}}$.

Under these conditions $1403 \mathrm{MW}$ or $41.7 \%$ of the total recoverable thermal energy, $\mathrm{P}_{\mathrm{TH}}=3365 \mathrm{MW}$, appears in the pressurized-water coolant loop. The total unrecoverable energy deposited into the TFC/PFC set (ohmic plus nuclear heating) amounts to $5.4 \%$ of $\mathrm{P}_{\mathrm{TH}}$, with the ratio of total ohmic dissipation, $\mathrm{P}_{\Omega}+\mathrm{P}_{\mathrm{TFC}}^{\Omega}+\mathrm{P}_{\mathrm{PFC}}^{\Omega}=164.4 \mathrm{MW}$, to the fusion power, $\mathrm{P}_{\mathrm{N}}+\mathrm{P}_{\alpha}=2732.5 \mathrm{MW}$, being 16.6; the plasma $Q$-value, $Q_{p} \equiv\left(P_{N}+P_{\alpha}\right) / P_{\Omega}$, is 108. It is roted that although F- $\theta$ pumping current drive has been assumed, other than the ohmic dissipation in the plasma, the baseline power balance does not include a separate current-drive power requirement. The current-drive subsystem is not sufficiently resolved for such an assessment, and a closely coupled system has been assumed. If F- $\theta$ pumping current drive proves feasible, however, the ohmic power required by the 


\section{CRFPR BASELINE POWER-BALANCE SUMMARY}

PARAMETER

VALUE

Major toroldal radius, $R_{T}(m)$

3.79

First-wall radius, $r_{w}(m)$

0.75

First-wall area, $A_{W}\left(m^{2}\right)=(2 \pi)^{2} r_{W} R_{T}$

112.1

Major circumference, $2 \pi R_{T}(m)$

Fusion neutron first-wa11 loading, $I_{w}\left(M W / m^{2}\right)$

Fusion neutron power, $\mathrm{P}_{\mathrm{N}}(\mathrm{MH})$

Alpha-particle power, $P_{\alpha}(M W)$

546.5

Plasma ohaic power, ${ }^{(a)} P_{\Omega}(\mathrm{MW})$

First-wall (and 1 imiter) tuclear heating, $(b) P_{F W}(M W)$

Second-wa11 nuclear heating, (b) ${ }_{\mathrm{P}_{\mathrm{SW}}}(\mathrm{MW})$

Blanket nuclear heating, $(\mathrm{b}) \mathrm{P}_{\mathrm{BLK}}(\mathrm{MW})$

2057.6

Shield nuclear heating, $(b) P_{S L D}(M W)$

Recoverable nuclear heating, $\mathrm{M}_{\mathrm{N}} \mathrm{P}_{\mathrm{N}}(\mathrm{MW})=$

$$
\mathrm{P}_{\mathrm{FW}}+\mathrm{P}_{\text {S. }}+\mathrm{P}_{\mathrm{BLK}}+\mathrm{P}_{\mathrm{SLD}}
$$

Energy multiplication, $\mathrm{M}_{\mathrm{N}}$

Total recoverable thermal power,

$$
\mathrm{P}_{\mathrm{TH}}(\mathrm{MW})=\mathrm{M}_{N} \mathrm{P}_{\mathrm{N}}+\mathrm{P}_{\alpha}+\mathrm{P}_{\Omega}
$$

Toroidal-field coll heating, $\mathrm{P}_{\mathrm{TFC}}(\mathrm{MW})$

- nuclear heating ${ }^{(b)} \mathrm{P}_{\mathrm{TFC}}^{\mathrm{N}}(\mathrm{MW})$

30.0

- ohmic heating, (c) $\mathrm{P}_{\mathrm{TFC}}^{\Omega}$ (MW)

Pololdal-field coil heating, $\mathrm{P}_{\mathrm{PFC}}(\mathrm{MH})$

- nuclear heating(b) P PFC (MW)

- ohmic heating, (d) $\mathrm{P}_{\mathrm{PFC}}^{\Omega}$ (MW)

Fraction plasma power radiated, $f_{\mathrm{RAD}}$

Fraction transport loss to limiter, $\mathrm{f}_{\mathrm{L}}$

Fractional 11miter coverage, $l_{L} /\left(l_{L}+l_{w}\right)$

Limiter length $\left(N_{L}=24\right), l_{L}(m)$

0.38

Radiated power, $P_{\text {RAD }}(M W)=f_{R A D}\left(P_{\alpha}+P_{\Omega}\right)$

Transport power, $P_{T R}(M W)+\left(1-f_{R A D}\right)\left(P_{\alpha}+P_{\Omega}\right)$

Power to limiter, $P_{L}(M W)=f_{L} P_{T R}+\left(P_{R A D}+P_{F W}\right) l_{L} /\left(l_{L}+l_{W}\right)$

440.8

Total power to first wal1, $P_{\mathrm{FW}}(M W)=\left(1-f_{\mathrm{L}}\right) \mathrm{P}_{\mathrm{TR}}+$

$$
\left[l_{W} /\left(l_{L}+l_{W}\right)\right]\left(P_{\mathrm{RAD}}+P_{\mathrm{FW}}\right)
$$


Power back-leakage from blanket to $\mathrm{SW}$, (e) $\mathrm{P}_{\mathrm{BL}}(\mathrm{MW}) \quad 96.0$

Total power to $\mathrm{FW} / \mathrm{SW}$ system, $\mathrm{P}_{\mathrm{FW} / \mathrm{SW}}^{+}(\mathrm{MW})=\mathrm{P}_{\mathrm{FW}}+\mathrm{P}_{\mathrm{SW}}+\mathrm{P}_{\mathrm{BL}}$

Total power to water-cooled first-wall systems $(f), P_{F W / S W}+P_{L} 1259.9$

Total power to magnet coolant, $\mathrm{P}_{\mathrm{TFC}}(\mathrm{MW})+\mathrm{P}_{\mathrm{PFC}}(\mathrm{MW})$

Estimated thermal-conversion efficiency, $\eta_{\mathrm{TH}}$

Gross electric power, $\mathrm{P}_{\mathrm{ET}}(\mathrm{MW})=\eta_{\mathrm{TH}} \mathrm{P}_{\mathrm{TH}}$

Auxiliary power needs, $P_{A U X}(M W)=f_{A U X} P_{E T}\left(f_{A U X}=0.05\right)$

183.6

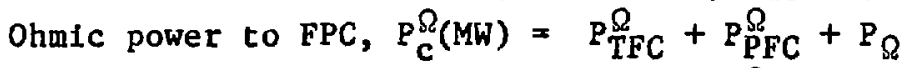

Recirculating power, $P_{c}(\mathrm{MW})=\mathrm{P}_{\mathrm{AUX}}+\mathrm{P}_{\mathrm{C}}^{\Omega}$

Recirculating power fraction, $\varepsilon=\mathrm{P}_{\mathrm{c}} / \mathrm{P}_{\mathrm{ET}}$

Plant efficiency, $\eta_{\mathrm{p}}=\eta_{\mathrm{TH}}(1-\varepsilon)$

Net electrtic power, $\mathrm{P}_{\mathrm{E}}(\mathrm{MW})=\mathrm{P}_{\mathrm{ET}}(1-\varepsilon)$

$$
\begin{gathered}
0.365^{(g)} \\
1226.7^{(g)} \\
62.6 \\
164.4 \\
227.0(g) \\
0.185^{(g)} \\
0.297^{(g)} \\
1000 .(g)
\end{gathered}
$$

(a) Based on peaked temperature proftle, $T(r) \propto J_{0}(\mu r)$.

(b) Refer to Table III.B-V.

(c) For the $\theta$ and $F$ values used in the systems code $\left(B_{R} \phi=-0.68 \mathrm{~T}\right)$, a continuous TFC would generate $17.6 \mathrm{MW}$ ohmic dissipation. ${ }^{\phi}$ The more-detalled plasma simulations (modified $B F M, \theta=1.55, F=0.12, \beta_{\theta}=0.23, B_{R}=0.4 T$ ) and the use of discretized coils gives the value of $P_{T F C}^{S}$ used in this power balance.

(d) A continuous PFC set used in the paranetric systems code predists 83.0 MW for $\mathrm{P}_{\mathrm{PFC}}^{\Omega}$. The results used here are based on the magnetics computations of a descretized $P F C$ set for $P_{E F C}^{\Omega}=53.5 \mathrm{MW}$ and $P_{O H C}^{\Omega}=73.0$, as described in Sec. III.F.2.d.

(e) Estimated in Sec. III.E. as penalty incurred when a structural temperature limit of $\sim 500^{\circ} \mathrm{C}$ set by PbLi corrosion limit and amounts to $4.66 \%$ of $\mathrm{P}_{\mathrm{BLK}}$.

(f) Amounts to $37.4 \%$ of $\mathrm{P}_{\mathrm{TH}}$, which increases to $1403.2 \mathrm{MW}$ or $41.7 \%$ of $\mathrm{P}_{\mathrm{TH}}$ if the shield, $P_{\text {SLD }}$, is also cooled with pressurized water.

(g) Values based on thermal-hydraulics optimization and integration study described in Sec. III.L. 2 . 


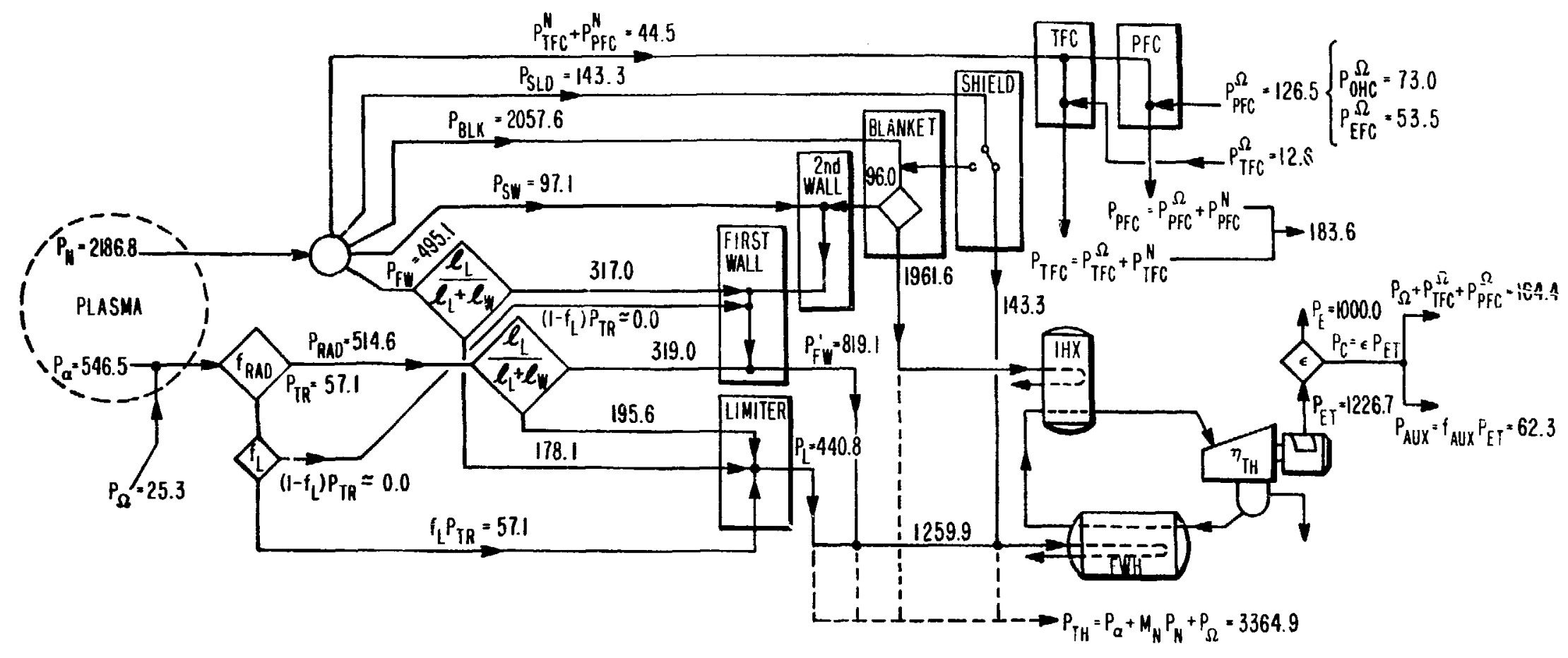

GLCOAAL POWER-PLANT BALANCE FOR CRFPR

$$
\begin{aligned}
\eta_{T H} & =0.365 \\
\epsilon & =0.185 \\
\eta_{P} & =0.297
\end{aligned}
$$

Fig. III.L-1. Global power-plant energy balance. Refer to Table III.L-I for definition of notation. Base-case values are shown. 
OHC set ( $73 \mathrm{MWe)}$ could be reduced to zero, with the corresponding increase in the overall plant efficlency, $\eta_{p}$, of approximately two percentage points. This increase amounts to 73 MWe avallable a) to sell to the grid and decrease the recirculating power fraction, $\varepsilon$, or b) to be applied to the current-drive system. For the latter option, current-drive inefficiencies as low as $0.26 \mathrm{~A} / \mathrm{W}$ could be supplied without altering the power balance given on Table III.L-I.

The task of the thermal-hydraulic design integration given in the following subsection is to resolve the fluid and manifolding parameters for this dual-media (1.e., pressurized-water and PbLi) power conversion system. These thermal-hydraulic parameters must be defined within the constraints imposed by the need for adequate thermal-conversion and overall plant efficiency, $\eta_{T H}$ and $\eta_{\mathrm{p}}=(1-\varepsilon) \eta_{\mathrm{TH}}$, respectively, and engineering limit: associated with system temperature, pressure, manifolding topology, and pumping power. Once $\eta_{\mathrm{TH}}$ is specified, the baseline values listed on Table III.L-I can be applied to determine the recirculating power fraction, $\varepsilon$, and hence $\eta_{p}$. Figure III.L-2 depicts this tradeoff, which indicates the desirability for $\eta_{\mathrm{TH}}>0.36$ if $\eta_{\mathrm{p}}$ is to be maintained above 0.30 . Since over $40 \%$ of the thermal power appears in the pressurized-water loop, this power must be used for steam generation rather than being used solely for feedwater heating. A similar power split has been reported for the MARS design, 6 pointing to a general need for more-careful design consideration of dual-media power cycles as applied to unique fusion needs.

2. Therma1-Hydraulic Design Integration. Sections III.D. and III.E. address general thermal-hydraulic aspects of the first wall/1imiter and blanket/shield, respectively, without directly considering the means by which the pressurized-water and PbLi power streams would be comblied to yleld an optimal thermal-conversion efficiency, $\eta_{\mathrm{TH}}$. In addition, the quesilons of coolant(s) manifolding, headering, and pumping power must be assessed from both viewpoints of FPC assembly and maintenance and gross power balance. Furthermore, these 1ssues must be resolved at this level of study on the basis of the one-dimensional neutronic studies given in Sec. III.B.; preliminary sensitivity studies of obvious multidimensional effects resulting from more-detalled design can be found in Appendix $C$, although these results are not directly incorporated into the present study. On the basis of the power flow given in Sec. III.L.1., the first-wall/1imiter/shield system delivers a total of 1403 MW to a pressurized-water coolant circuit, and the PbLi-cooled blanket 


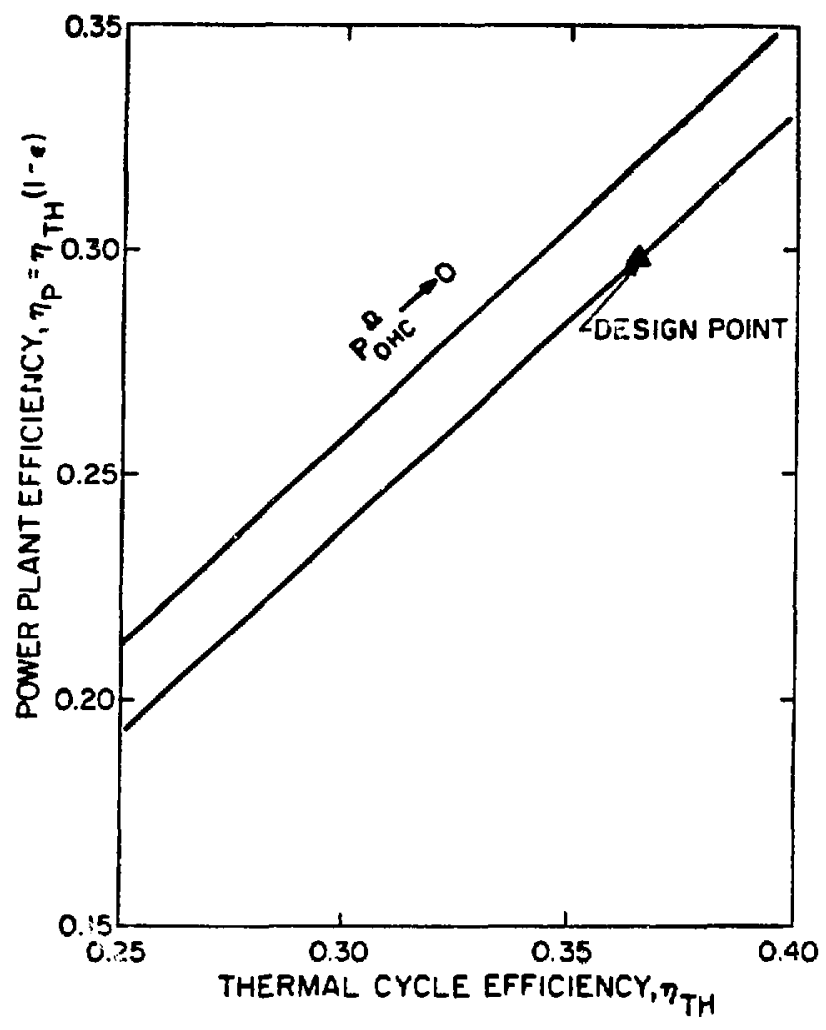

F1g. III.L-2. Dependence of plant efficiency, $\eta_{p}$, on therma1-conversion: efficiency, $\eta_{T H}$, for the dual-media system depicted on Fig. III.L-1 and Table III.L-I. The maximum impact of current drive, which allows the current in the OHC to be reduced to zero, is also shown.

recelves $1962 \mathrm{MW}$ for a total of $3365 \mathrm{MWt}$. This section addresses the topology questions of manifold/headers and the questions of total pumping power and overall thermal-conversion efficiency from the viewpoint of a fully integrated FPC as constralned by the aforementioned neutronic (tritium-breeding) considerations.

a. Thermal-Conversion Efficlency. The high-heat-fllix characteristics of the power flow depicted in Fig. III.L-1 gives the dual-media cycle with a ratio of power to $\mathrm{PbLi} / \mathrm{H}_{2} \mathrm{O}$ of 1.4 . This ratio is not unlike a similar cycle suggested for the MARS design, 5,6 which has a $\mathrm{PbLI} / \mathrm{H}_{2} \mathrm{O}$ power ratio of $\sim 2.0$. Determination of an optimum cycle efficlency for such dual-media systems is an Important area of future work for advanced fusion systems in general but could not be considered by this study. The approach adopted here computes an Ideal cycle efficiency and then reduces this tdeal efficiency by a factor shown 
achievable by more-detalled stean-power-cycle studies. Typically, conversion efficlencies in excess of $75 \%$ of the thermodynamic ideal value can be achieved ${ }^{3}$ for a :ingle-media power conversion system. It is assumed that the dual-media cycle can also achleve $75 \%$ of the ideal efficiency by using several stages of reheat for a cycle driven by both high-temperature PbLi and lower-temperature pressurized-water coolants. In addition, extraction from the turbine at various pressures will probably be desirable for feedwater heating. Extensive steam-power-cycle analysis and cost tradeoff studies will be needed before the compromise between increased efficiency and system complexity (i.e., reliability and cost) can be resolved.

Figure III.L-3 depicts an Ideal thermodynamic conversion cycle in the form of a temperature-entropy diagram for both a single-media and a dual-media system. In computing the Ideal cycle efficiency, the following assumptions are made:

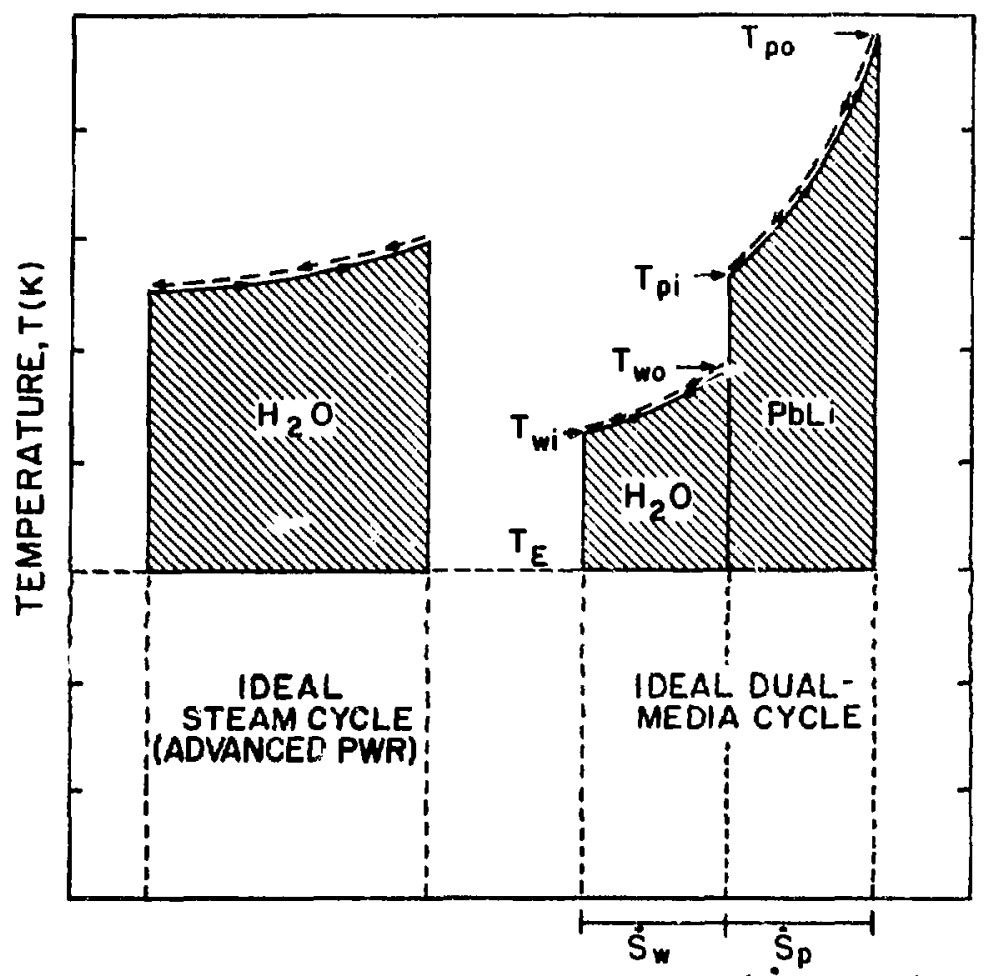

RATE OF ENTROPY GENERATION, $\triangle \dot{S}$ (MW/K)

FIg. III.L-3. Temperature-entropy diagrams for ideal single-media and dual-media thermal-conversion cycles, where in both cases heat is rejected to a coimmon temperature $\mathrm{T}_{E}$. 
- the heat sources are fluids having specifled supply and return temperatures, $T_{0}$ and $T_{i}$, respectively.

- heat is transferred from the heat source fluid to an ideal heat engine in a reversibie, constant-pressure steady-flow process; temperature differences and frictional losses are assumed zero for this ideal cycle.

- the heat engine rejects heat to a $300-K$ environment through a reversible, isothermal process.

If $P_{\mathrm{TH}}$ is the total thermal heat input and the fraction $f_{w}$ of $P_{T H}$ appears in the pressurlzed-water loop, then energy and entropy balances on the water (w) and PuLI (p) streams give the following relationships:

$$
\begin{array}{ll}
\mathbf{f}_{w} P_{T H} & =\dot{m}_{w} c_{p, w}\left(T_{w o}-T_{w 1}\right) \\
\left(1-f_{w}\right) P_{T H} & =\dot{m}_{p} c_{p, p}\left(T_{p o}-T_{p 1}\right) \\
\dot{S}_{w} & =\dot{m}_{w} c_{p, w} \ln \left(T_{w 0} / T_{w 1}\right) \\
\dot{S}_{p} & =\dot{m}_{p} c_{p, p} \ln \left(T_{p o} / T_{w 1}\right),
\end{array}
$$

where the inlet and outlet temperatures for both streams are depicted on Fig. III.L-3, as are the respective entropy production terms, $\dot{S}_{j}$. The mass flow rates are $\dot{m}_{j}$, and the specific heats at constant pressure for the $j^{\text {th }}$ fluid are ${ }_{{ }_{p}} \mathrm{p}, j^{*}$ Since the useful work (electrical power) is $P_{E T}^{I}=P_{T H}-\dot{Q}$, where $\dot{Q}=T_{E}\left(\dot{S}_{w}+\dot{S}_{p}\right)$ is the heat rejection rate, then the ideal effictency for the conversion of ${ }^{P_{T H}}$ ro $\mathrm{P}_{\mathrm{ET}}^{\mathrm{T}}$ is

$$
\begin{aligned}
\eta_{\mathrm{TH}}^{\mathrm{T}} & =1-\frac{\dot{Q}}{\mathrm{P}_{\mathrm{TH}}} \\
& =1-\mathrm{T}_{\mathrm{E}}\left[\mathrm{f}_{\mathrm{w}} \frac{\ln \left(\mathrm{T}_{\mathrm{wo}} / \mathrm{T}_{\mathrm{w} 1}\right)}{\mathrm{T}_{\mathrm{wO}}-\mathrm{T}_{\mathrm{w}}}+\left(1-\mathrm{f}_{\mathrm{w}}\right) \frac{\ln \left(\mathrm{T}_{\mathrm{po}} / \mathrm{T}_{\mathrm{p} 1}\right)}{\mathrm{T}_{\mathrm{pO}}-\mathrm{T}_{\mathrm{p} 1}}\right] .
\end{aligned}
$$

The real conversion efficiency $1 \mathrm{~s}$ taken as $\eta_{\mathrm{TH}}=0.75 \eta_{\mathrm{TH}}^{\mathrm{T}}$. As seen from Table III.L-I, $\mathrm{f}_{\mathrm{w}}=0.417$. Since $\mathrm{T}_{\mathrm{E}} \approx 300 \mathrm{~K}$, the relationships between the varlous temperatures, flow rates, coolant duct/manifold/header parameters, and pumping powers remain to be deterinined before $\eta_{T H}$ can be estimated. The factor 0.75 is assumed achievable at a reasonable cost in a real engineering system. 
It is noted that the ideal Carnot efficiency is approached as $\mathrm{T}_{w 1} \rightarrow \mathrm{T}_{\text {wo }} \rightarrow \mathrm{T}_{\mathrm{pi}} \rightarrow \mathrm{T}_{\text {po }}$, but of course the flow rates, pressure drops, and pumping power become large if a finite amount of power (1.e., $\mathrm{P}_{\mathrm{TH}}$ ) is to be transported through a diminishing temperature difference. The following section examines the major tradeoffs between $T_{1 j}$, system pressures, and pumping powers.

b. Therma1-Hydraulics Design Parametrics Study. Figure III.L-4 deplcts the PC coolant flow pattern for both the pressurized-water and PbLi loops. The simplified merhodology described in SE.S. III.D. and III.E. has been combined to examine the sensitivity of TH and pusp powex to changes in prinary design

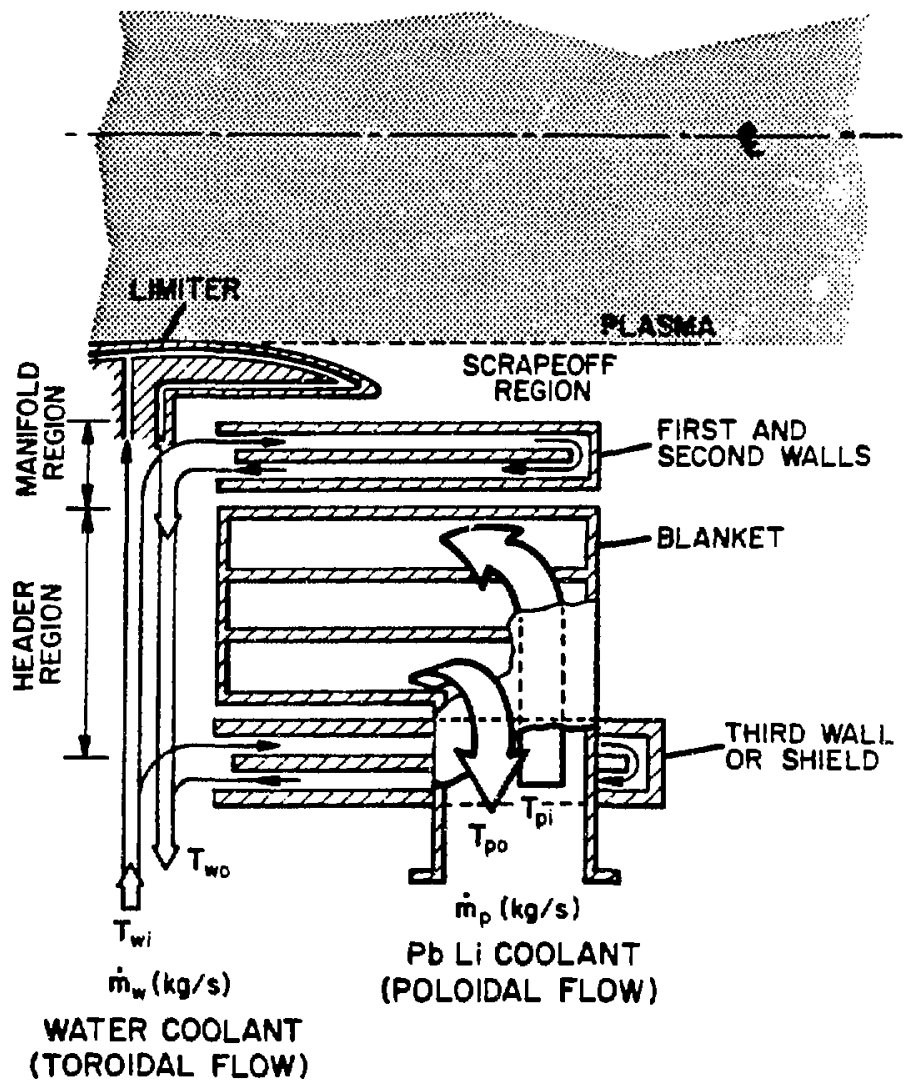

F1g. III.L-4. Schematic layout of FPC coolant loops showing the pressurized-water and PbLi loops. The relative sizes of the water-cooled limiter, first and second wall, PbL1-cooled blanket, and water-cooled shield have been distorted to illustrate the respective flow paths. 
variables subject to the following fixed design parameters and specific constraints:

- Major radius, $R_{T}=3.79 \mathrm{~m}$

- First-wall radius, $r_{\mathrm{w}}=0.75 \mathrm{~m}$

- Number of toroidal segments, $N=24$

- Pump efficiencies, TPUMp $=0.75$

- Generator efficiency, $\eta_{\text {GEN }}=0.98$

- Thermal-conversion efficiency, $\eta_{\mathrm{TH}}=0.75 \eta_{\mathrm{TH}}^{\mathrm{I}}$

- First-wa11, second-wa11, limiter, and blanket/shield dimensions

- Limiter coolant-channel geometry (Table III.D-II)

- Cooling loads and heat fluxes of all components (Table III.L-I)

With these parameters fixed, the following limits and constraints are imposed:

- Pbli outlet temperature, $T_{\text {po }}=773 \mathrm{~K}$

- Minimum water subcooling in first/second wall and in limiter coolant channels, $\Delta \mathrm{T}_{\mathrm{SAT}}=10 \mathrm{~K}$

- Maximum water flow velocity in all channels and manifolds, $v=10 \mathrm{~m} / \mathrm{s}$

- Total pump power must remain less than $f_{A U X}=0.07$ of the total electric power, $P_{E T}$

Upon establishing these fixed or constrained variables, the sensitivity of $\eta_{\mathrm{TH}}$ and pump power was examined as a function of the following design variables:

- cooling-water pressure

- inlet cooling-water temperature, $T_{w i}$

- inlet Pbli temperature, Tpi

- number of first- and second-wall coolant tubes allowed to operace i aries

- insude diameter of first-wall coolant tubes, $d_{i}$

Using this hierarchy of fixed, constrained, and variable parameters, an optimizing procedure was used to compute individually design parameters for the limiter, the first/second wall, the shield, and the blanket. A design estimat: for the pressurized-water manifold/header system, pumping power, and cycle efficiency was then obtained. Eact computation is described separately below.

Limiter Analysis. An initial inlet coolant velocity is assumed for the Iimiter, and an energy balance is used to determine the mass flow for a fixed coolent-channel geometry (Sec. III.D.2.b.). A thermal analysis determines the 
bulk water temperature and corresponding inner-wall temperature at the leading edge of the limiter; this location is viewed as the critical design point for the limiter. If the coolant temperature at this critical point is less than the saturation temperature by more than $\Delta \mathrm{T}_{\mathrm{SAT}}$, the flow velocity is adjusted until this condition is satisfied. Upon convergence, the pressure drop and associated pumping power for the limiter are determined.

First-Wal1/Second-Wal1 Analysis. By means of a thermal stress analysis and a given inside tube diameter, the tube-wall thickness and outside diameter are determined (Sec. III.D.2.a.). These dimensions combine with other fixed reactor dimensions to determine the number of first-wall tubes for each of $N=24$ toroidal sectors. For a given value of $\mathrm{T}_{\text {wo }}$, energy balance determines the mass flow rate through the tube. The minimum coolant-water subcooling is determined through a thermal analysis, and $T_{\text {wo }}$ is iteratively adjusted until the design coolant subcooling, $\Delta \mathrm{T}_{\mathrm{SAT}}$, is achieved. Upon convergence, the pressure drup and pumping power are computed along with the mass flow rate through the first-wall/second-wall system, which for the purposes of this analysis is assumed simply joined in series.

Shield Analysis. The heat fluxes and power densities for the shield are low compared to the limiter and first/second-wall systems. Generally, the shield coolant temperature is operated at the allowed subcooling, $\Delta T_{S A T}$ and energy balance determines the required mass flow rate. Typically, the pumping power and prassure drop for this system are small.

Pressurized-Water Manifold/Header Design. These systems consist of manifold, inlet headers, and outlet headers for both the limiter and first-wall/second-wall systems. Sizing of these systems to a minimum that is consistent with pressure-drop and pumping-power constraints is important from the viewpoint of assembly and the amount of blanket and vacuum-systems "real estate" required for mutual accommodation. The previously computed mass flow rates are used along with a specified allowable flow velocity to determine Inside diameters of the various manifolds and headers. Separate manifolds and headers are specified for each inlet and outlet circuit for each of $N=24$ toroidal segments. A stres: analysis is used to compute the tube-wall thicknesses and, hence, outside diameter and FPC space allocation. Pressure drops, including local losses in bends, and pumplng-power requirenents are estimated for each manifold/header and added to that of the system being served. 
Blanket Analysis. With the PbLi inlet temperature, $\mathrm{T}_{\mathrm{p}}$, specifled, energy balance gives the mass flow rate, which in turn yields the pressure drop and pumping power using the MHD equations, field profiles, and poloidal coolant-duct configuration described in Sec. III.E.

Cycle Efficiency. The mass flow rates and water outlet temperatures from the limiter, first/second-wall, and shield systems are used to compute the total coolant-water flow rate, $\dot{\mathrm{m}}_{\mathrm{w}}$, and mixed-mean temperature, $T_{w 0^{\circ}}$ Using the mass flow rate, pressure drops, pumping power, and $\mathrm{PbLi}$ temperatures, the ideal thermal-conversion efficiency, $\eta_{\mathrm{TH}}^{\mathrm{I}}$, is estimated and reduced by $75 \%$ to give $\eta_{\mathrm{TH}}$. The turbine output, $\mathbf{P}_{\mathrm{ET}}$, is the product of $\eta_{\mathrm{TH}}$, $\eta_{\mathrm{GEN}}=0.98$, and $\mathrm{P}_{\mathrm{TH}}$ *

c. Thermal-Hydraulics Design Paiametrics Study Results. This section gives the results of sensitivity studies as the key desigr parameters were varied. The sensitivity of $\eta_{\mathrm{TH}}$ and $\eta_{\mathrm{TH}}\left(1-\varepsilon_{\text {pump }}\right)$ to changes in coolant-water pressure and PbLi inlet temperature, $T_{p i}$, is shown on Fig. III.L-5, where Epump is the ratio of total pumping power ( $\eta_{\text {pump }}=0.75$; to $P_{E T}$. The corresponding PbLi pressure drop and a direct expression of $\varepsilon_{\text {pump }}$ as a function of $T_{p i}$

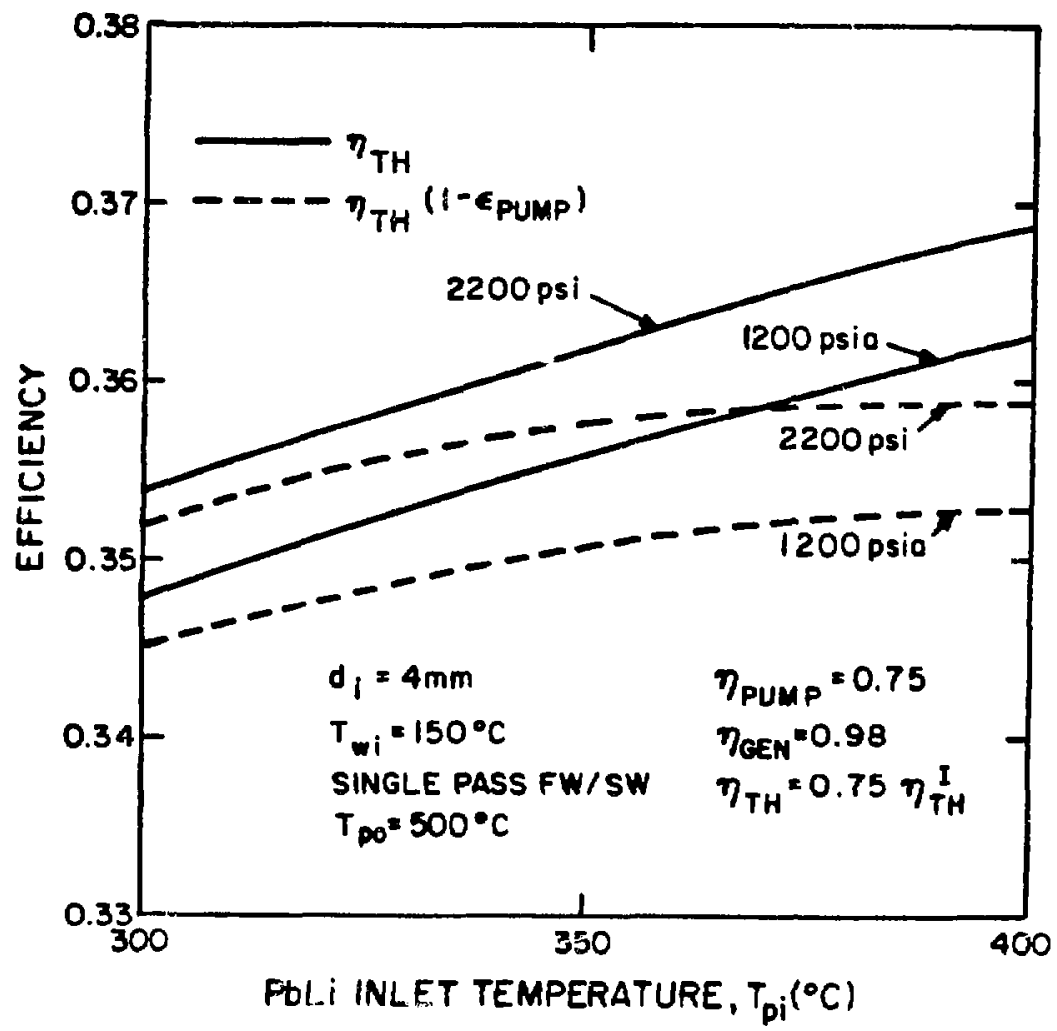

Fig. III.L-5. Effect of PbLi inlet temperature, $T_{p i}$, and cooling-water pressure on overal? cycle efficiency, $\eta_{\mathrm{TH}}$. 
$\left(\mathrm{T}_{\mathrm{po}}=500^{\circ} \mathrm{C}\right)$ is shown on Fig. III.L-6. As for the pressurized-water coolant circuit, the PbLi system appears to be 11 mited by pressure and nembrane stresses rather than by pumping-power limits. The increase in cycle efficiency is small in the range $\mathrm{T}_{\mathrm{p}^{i}}=350-400^{\circ} \mathrm{C}$, while the blanket pressure increases by $50 \%$. Although a detalled structural analysis of the PbLi blanket cannot be made until a more-complete design is avallable, it is anticipated that PbLi pressures above $\sim 200 \mathrm{psi}$ w 11 cause difficult structural and fabrication problems that counteract the advantage of low pressure generally attributed to 1iquid-metal coolants. For these reasons, a PbLI inlet temperature of ${ }^{\mathrm{i}} \mathrm{pI}=350^{\circ} \mathrm{C}$ (Fig. III.L-6) is selected for the canonical design.

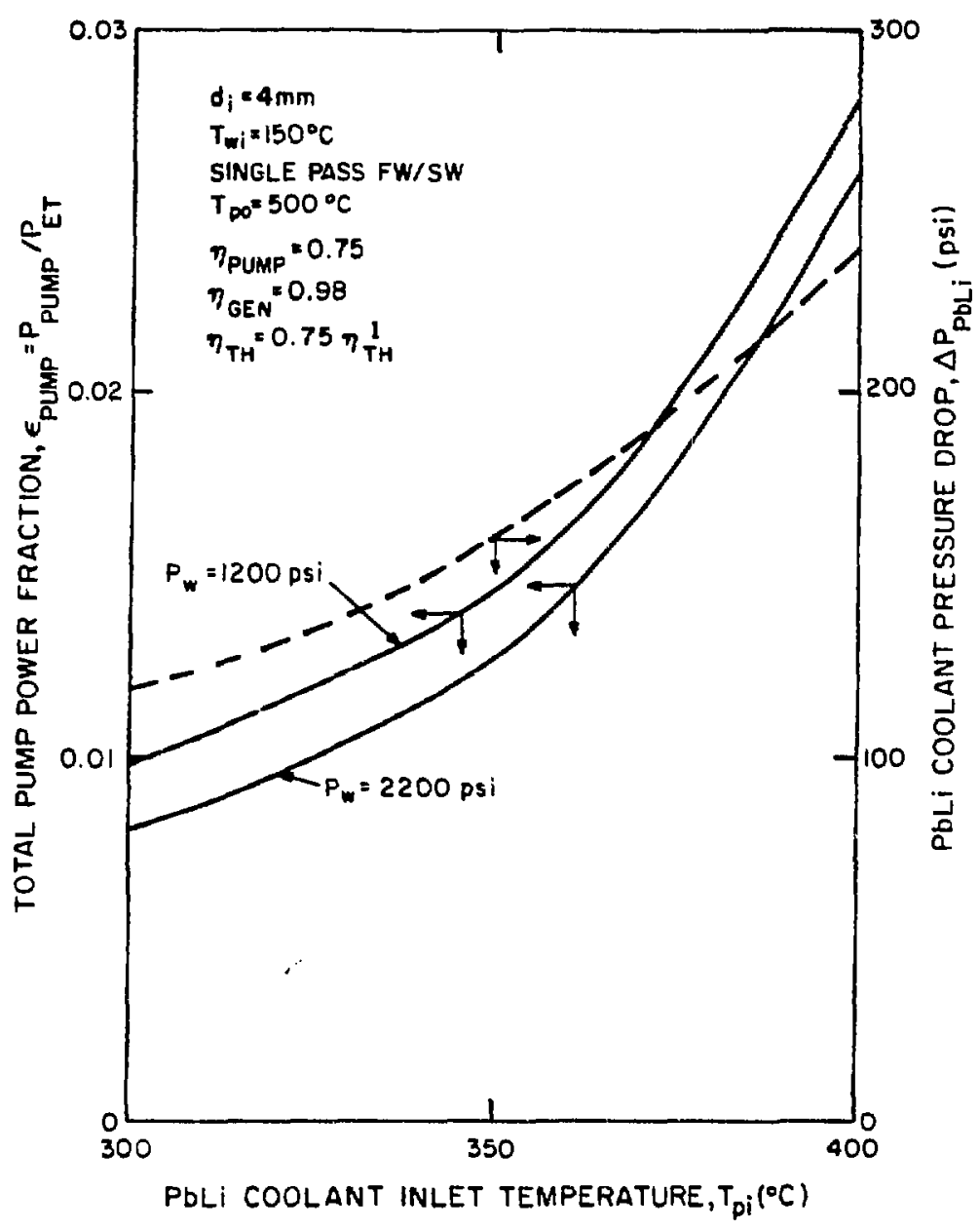
Fig. III.L-6. Effect of PbLi inlet temperature, ${ }^{T}{ }_{p i}$, on pumping power and $P b L i$
pressure drop. 
The overall efficiency, $\eta_{T H}$, increases with increasing cooling-water pressure but with diminishing return as the critical pressure (3206 psi) is approached. Increasing the water coolant pressure above $15.2 \mathrm{MPa}$ (2200 psi) yields little additional increase in thermal-conversion efficiency. The $15.2 \mathrm{MPa}$ value is close to PWR conditions and is selected for this canonical design point. Figure III.L-7 illustrates the effects of inlet coolant-water temperature, $T_{w i}$, and first-wall coolant-tube inside diameter, $d_{i}$, on the thermal-conversion exficlency, whereas FIg. IIL.L-8 gives the corresponding effects of $d_{1}$ and $T_{w i}$ on the flow velocity and pressure drop. For inlet coolant-water temperatures above $\sim 190^{\circ} \mathrm{C}$, the $10-\mathrm{m} / \mathrm{s}$ flow velocity 1 imit is exceeded; hence, $\mathrm{T}_{\mathrm{wi}} \simeq 190^{\circ} \mathrm{C}$ wis selected for the canonical design. The minimum allowable coolant-tube diameter needed to assure flow velocities below $\sim 10 \mathrm{~m} / \mathrm{s}$ is approximately $\dot{0} \mathrm{~mm}$.

The parametric results given in Figs. III.L-5 through III.L-8 are based on a single-pass FW/SW coolant circuit. No significant increase in efficiency was observed for multiply connected FW/SW tube arrays. Table III.L-II suinmarizes the key reactor thernal parameters for the canonical design evolved in this section. Tables III.L-III, III.L-IV, III.L-V, and III.L-VI give the key

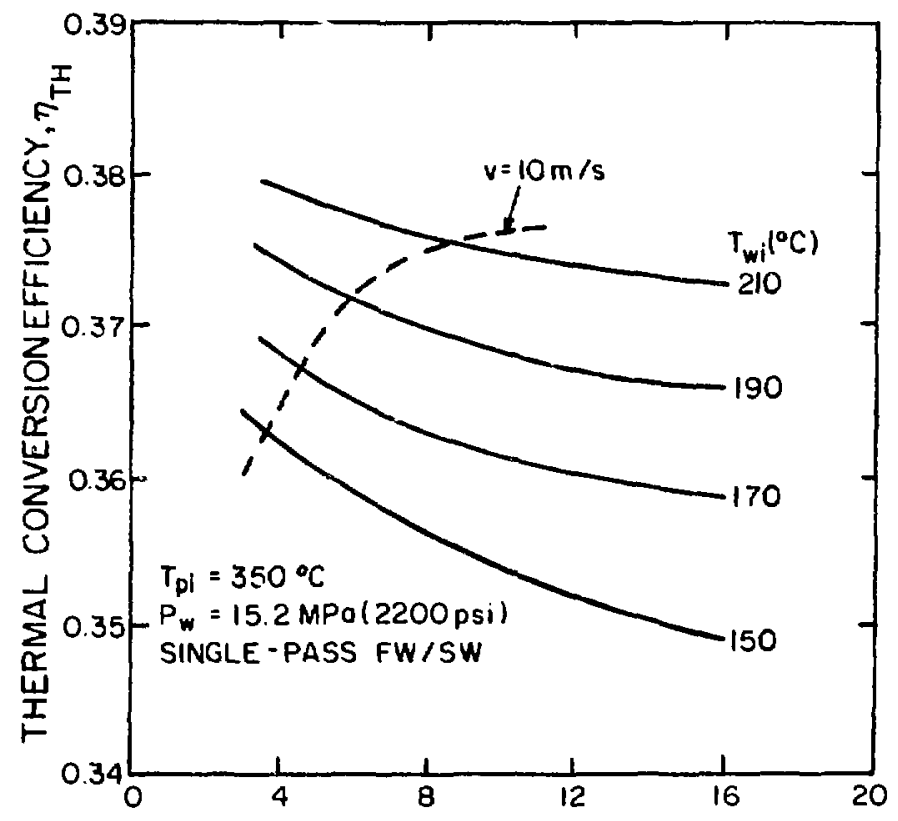

FIRST-WALL TUBE INSIDE DIAMETER, $d_{i}(\mathrm{~mm})$

Fig. III.L-7. Dependence of thermal-conversion efficiency on FW tube diameter and inlet water coolant temperature. The locus of $10-\mathrm{m} / \mathrm{s}$ flow velocities is also indicated. 


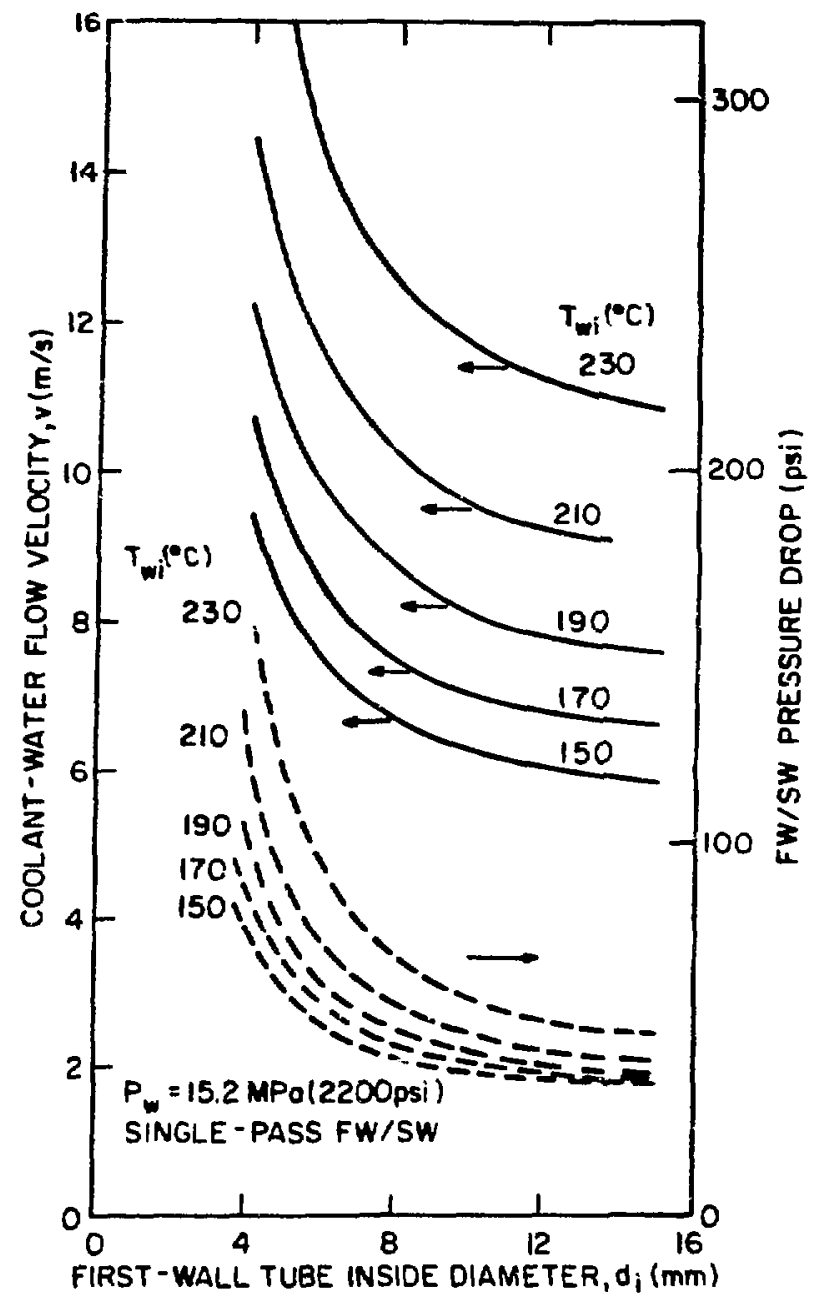

Fig. III.L-8. Dependenze of FW tube flow velocity and pressure drop on FW tube diameter and inlet water coolant temperature.

thermal-hydraulic design parameters for the key FPC subsystems as well as manifold/header sizes for use in the FPC mechanical integration.

The sensitivity of the theraal-hydraulics design point being suggested by these parametric results is besc illustrated by a plot of $T_{p o}-T_{p i}$ versus $T_{p o}$ that is constrained by a number of physical limitaticns. Figure iII.L-9 shows the pinch-point limits for a $10-K$ minimum temperature difference between $T_{\text {po }}$ and the steam generator outlet; two steam-generator pressures are shown. With Eq. (3) along with $\eta_{\mathrm{TH}}^{*}=0.75 \eta_{\mathrm{GEN}} \eta_{T H}\left(1-\varepsilon_{\text {pump }}\right), \eta_{\mathrm{GEN}}=0.98$, and the reference design values of $\mathrm{f}_{\mathrm{w}}=0.417, \mathrm{~T}_{\mathrm{E}}=300 \mathrm{~K}$, and $\mathrm{T}_{\mathrm{wO}} / \mathrm{T}_{\mathrm{wi}}=526.6 \mathrm{~K} / 463.0 \mathrm{~K}$, 11nes of 
TABLE III.L-II

KEY THERMAL PROPERTIES FOR THE CRFPR CANONICAL DESIGN

\section{PARAMETER}

Total recoverable thermal power, $\mathrm{P}_{\mathrm{TH}}(\mathrm{MW})$

Pressurized-water coolant

- power, $\mathrm{P}_{\mathrm{THW}}$ (MW)

- flow rate, $\dot{\mathrm{m}}_{\mathrm{w}}(\mathrm{kg} / \mathrm{s})$

- Inlet temperature, $T_{w i}(K)$

- outlet temperature, $\mathrm{T}_{\text {wo }}(\mathrm{K})$

- pressure (MPa/psi)

- pumping power (MW)
VALUE

3364.9

1403.2

4898.8

463.

536.6

$15.2 / 2200$

$2.8^{(a)}$

1961.7

72,840 .

623

773

$1.1 / 161$.

$13.2^{\text {(a) }}$

0.372

0.365

$1226.7^{(b)}$

$0.013^{(a)}$

$164.4^{(\mathrm{c})}$

0.134

0.038

0.185

0.297

1000 .
(a) Based on $\eta_{\text {pump }}=0.75$.
(b) Based on $\eta_{T H}=0.372$ and $\eta_{G E N}=0.98$.
(c) Table III.L-I, $P_{\Omega}+P_{\text {TFC }}^{\Omega}+P_{\text {PFC }}^{\Omega}$. 
Overall dimensions

- toroidal extent, $l_{\mathrm{L}}(\mathrm{m})$

0.38

- maximum radial thickness, $\delta^{\prime}$ (mm)

11.5

- stand-off distance from first wa11, $\delta(\mathrm{mm})$

40.

Coolant configuration (toroidal flow)

- channel height (mm)

0.8

- channel width (mm)

4.0

- wall thickness to outside surface (mm)

0.8

- wall thickness between channels (mm)

1.00

- number of channels per limiter

942.

Limiter thermal rating

- total cooling rate for all limiters, $\mathrm{P}_{\mathrm{L}}(\mathrm{MW})$

440.8

- design heat $\mathrm{flux}, \mathrm{q}_{D}\left(\mathrm{MW} / \mathrm{m}^{2}\right)$

6.0

- maximum limiter temperature (K)

645 .

Coolant properties (pressurized water)

- Inlet temperature, $\mathrm{T}_{\mathrm{wI}}(\mathrm{K})$

463.

- outlet temperature, $\mathrm{T}_{\text {wo }}(\mathrm{K})$

545.

- nominal coolant velocity, $v(\mathrm{~m} / \mathrm{s})$

10.0

- total mass flow rate, $\dot{\mathrm{m}}_{\mathrm{w}}(\mathrm{kg} / \mathrm{s})$

1311.2

- pressure drop in channel (MPa)

0.27

Manifold/header properties

- inlet manifold (behind limiter), $\operatorname{ID} / O D(\mathrm{~mm})$

$63.0 / 74.3$

- outlet manifold (behind limiter), $\operatorname{ID} / O D(\mathrm{~mm})$

$66.7 / 78.6$

- inlet header (single radial run), ID/OD(mm)

$89.1 / 105.0$

- outlet header (single radial run), ID/OD(mm)

$94.3 / 111.0$

- total limiter circuit pressure drop (MPa)

0.55

- limiter pumping power(a) (MW)

0.54

- total limiter circuit pumping power(a) (MW)

0.94

(a) Pump efficiency is $\eta_{\text {Pump }}=0.75$. 
TABLE-III.L-IV

FIRST-WALL/SECOND-WALL THERMAL-MECHANICAL DESIGN PARAMETERS

PARAMETER

Number of segments, $N$

Material

Overall dimensions of segments

- toroldal extent, $l_{\mathrm{W}}(\mathrm{m})=2 \pi \mathrm{R}_{\mathrm{T}} / \mathrm{N}$

- minor radius, $r_{w}(m)$

- major toroidal radius, $\mathrm{R}_{\mathrm{T}}(\mathrm{m})$

Coolant configuration (toroldal flow)

\section{VALUE}

24

MZC Copper/sS

- "D-tube" ID/OD(m)

3.78

- number of D-tubes per half-segment (inlet or outlet) 589

- tube-wall thickness, $\delta(\mathrm{mm})$

1.0

- FW/SW flow configuration single-pass, series

FW/SW thermal racing

- $\mathrm{FW}$ heat $\mathrm{f} I \mathrm{ux}, \mathrm{I}_{\mathrm{QW}}\left(\mathrm{MW} / \mathrm{m}^{2}\right)$

- FW heat-flux power (MW)

319.1

- FW volumetric nuclear heating (peak/average, $M W / \mathrm{m}^{3}$ )

$239.0 / 218.0$

- FW nuclear-heating power (MW)

307.0

- FW total power, $\mathrm{P}_{\mathrm{FW}}^{\prime}(\mathrm{MW})$

626.1

- SW nuclear-heating power, $\mathrm{P}_{\mathrm{SW}}(\mathrm{MW})$

97.1

- SW back leakage frcm blanket, $\mathrm{P}_{\mathrm{BL}}(\mathrm{MW})$

96.0

- total power to $\mathrm{FW} / \mathrm{SW}(\mathrm{MW})$

819.1

- coolant inlet temperature, $\mathrm{T}_{\mathrm{WI}}(\mathrm{K})$

463.

- coolant outlet temperature, $\mathrm{T}_{\text {wo }}(\mathrm{K})$

518.

- coolant mass flow rate, $\dot{\mathrm{m}}_{\mathrm{w}}(\mathrm{kg} / \mathrm{s})$ 3315.4

Manifold/header Properties

- inlet manifold, ID/OD(mm)

$100 . / 118$.

- outlet mantfold, ID/OD(mm)

$105 . / 123$.

- inlet header, ID/OD(mm)

142./167.

- outlet header, ID/OD(mm)

$148 . / 174$.

- tota1 FW/SW circuit pressure drop (MPa)

- FW/SW pumping power (MW)

0.91

- total FW/SW circult pumping power (MW) 
TABLE-III.L-V

BLANKET THERMAL-MECHANICAL DESIGN PARAMETERS

PARAMETER

Number of segments

Material

Overall dimensions

- toroidal extent, $l_{W} / 2(m)$

- radial extent, $\Delta b(\mathrm{~m})$

- inside radius (m)

Coolant configuration (poloidal flow, Fig. III.E-2)

- channel thicknesses with increasing radius, $\alpha_{i}(m)$

$0.148 / 0.136 / 0.206$

- channel thicknesses entering manifold (m)

- channel wall th:ckriess (mm)

Blanket thermal rating

- total cooling rate, $\mathrm{P}_{\mathrm{BLK}}(\mathrm{MW})$

- peak power density $\left(\mathrm{MW} / \mathrm{m}^{3}\right)$

- Average power density (MN/m $\left.{ }^{3}\right)$

- Maximum structural temperature (K)

Coolant properties

- inlet temperature, $\mathrm{T}_{\mathrm{pi}}(\mathrm{K})$

- outlet temperature, $\mathrm{T}_{\mathrm{po}}(\mathrm{K})$

- nominal coolant velocity in channels, $v_{1}(\mathrm{~m} / \mathrm{s})$

- nominal coolant velocity in mantfold $(\mathrm{m} / \mathrm{s})$

- total mass flow rate, $\dot{m}_{\mathrm{p}}(\mathrm{kg} / \mathrm{s})$

- pressure drop in blanket/manifold (MPa)

- pumping power (MW)(a)
VALUE

48.

$\mathrm{HT}-9 / \mathrm{PbLi}$
0.48

0.50

0.775
$0.201 / 0.053 / 0.036$

5.0

1961.7

255

27

773

623

773

$1.25 / 0.50 / 0.23$

1.29

72,840 .

1.11

13.2

Manifold/header properties

- radial extent beyond blanket (m)

- height(n)

0.30

(a) Pump effictency is $r_{\text {Pump }}=0.75$. 
TABLE III.L-VI

SHIELD THERMAL-MECHANICAL DESIGN PARAMETERS

\section{PARAMETER}

Number of segments, $N$

Material

Total power rating, $\mathrm{P}_{\mathrm{SLD}}(\mathrm{MW})$

Coolant mass flow rate, $\dot{m}_{\text {SLD }}(\mathrm{kg} / \mathrm{s})$

Coolant inlet temperature, $\mathrm{T}_{\mathrm{SLD}, \mathrm{I}}$ (K)

Coolant outlet temperature, $\mathrm{T}_{\mathrm{SLD}, \mathrm{o}}(\mathrm{K})$

\section{VALUE}

24

$\mathrm{B}_{4} \mathrm{C} / \mathrm{W} / \mathrm{SS} / \mathrm{H}_{2} \mathrm{O}$

143.3

272.2

463.

597.

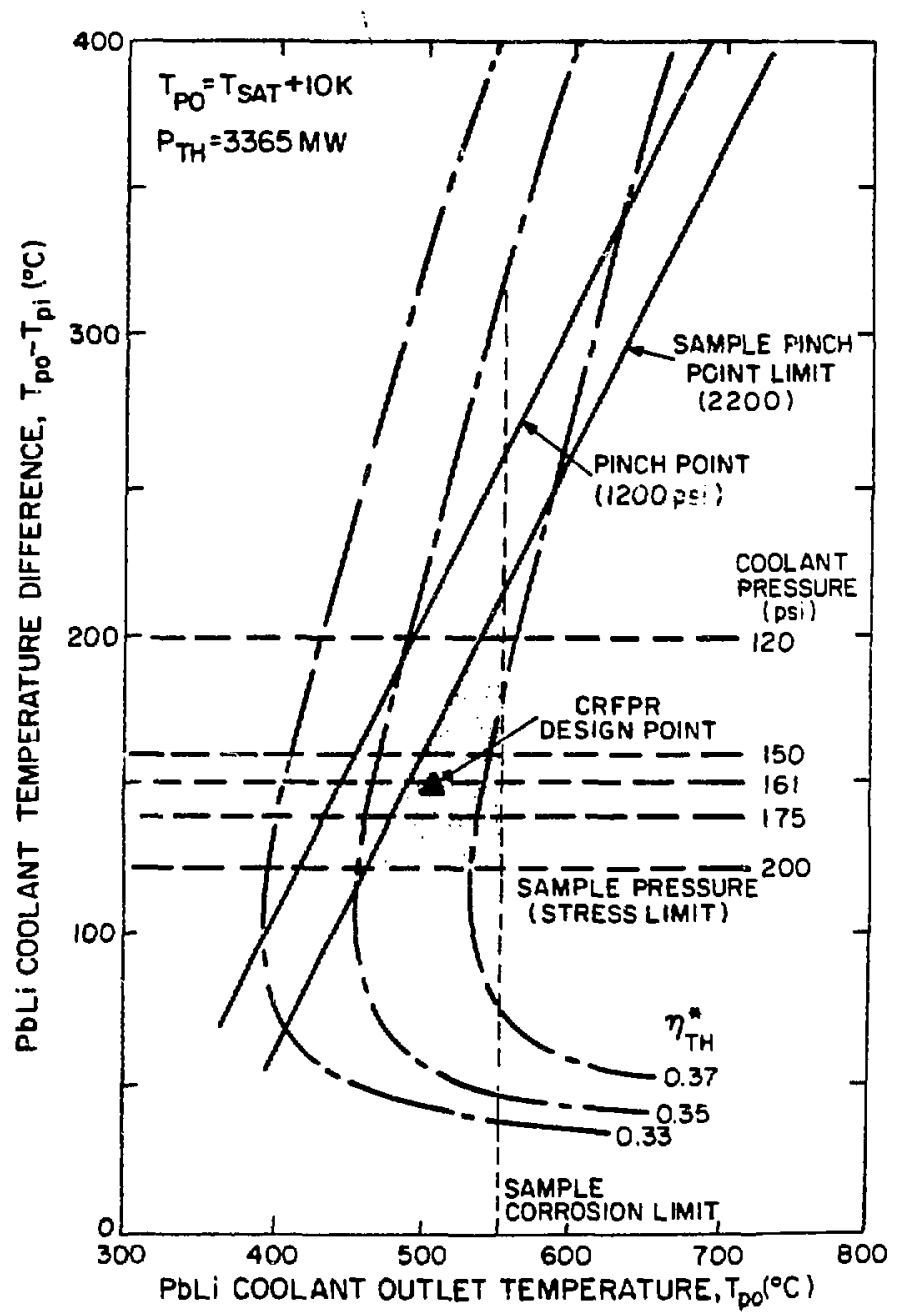

Fig. III.L-9. Thermal-hydraulics operatir:g window for the PbLi-cooled blanket showing pinch-point, pressure, efficiency, and (suggestive) corrosion limits. 
constant $\eta_{\mathrm{TH}}^{*}$ can be generated on Fig. III.L-9. Combined, these IImits (e.g., $\leqslant 200 \mathrm{psi}$ pressure stress, $6550^{\circ} \mathrm{C}$ corrosion, $\eta_{\mathrm{TH}}^{*}$ thermal-conversion/pumping power, 2200 psi pinch-poin. 1imits) define a suggested operating window and related design-point sensitivities. Both more-detalled analyses and data bases are required before the engineering and materials sansitivity of the thermal-hydraulic design window illustrated on Fig. III.L-9 can be fully resolved.

3. Mechanical Design Integration. Preliminary consideration has been given to the integration of several FPC subsystems of the CRFPR at a conceptual level to define options in anticipation of more-detailed engineering designs. Constraints imposed include maintenance of the basic reference parameters ${ }^{15}$ as modified by the subsystem conceptual designs developed in this study. Summartzed "below are specific parameter lists used to guide this FPC mechanical design incegration.

- Key plasma and FPC characteristics

- Overall power balance

- Impurity-control (poloidal pumped limiters) -physics and mechanical characteristics -thermal-mechanical design

- Copper-alloy first wall

-nuclear

-thermal-mechanical

- Self-cooled PbLi blanket

- nuclear

-thermal-mechanical

- Hot shield

-nuclear

-thermal-mechanical

- Magnet coils

-nuclear

-TFC

electrical

locations

$-\mathrm{PFC}$

electrical

locations

- Fueling and vacuum
Table III.G-I

Table TII.L-I

Table II:-H-IT

Table III.L-III

Tables III.B-V,VI,VII

Table III.L-IV

Tables III.B-V,VI

Table III.L-V

Table III.B-V,VI

Table III.L-VI

Tables III. B-V, VI, VII

Table III.F-III

Table III.F-II

Table III.F-VIIA, B

Table III.F-VII

Table III.J-III 
Appendix B gives a standard reactor design table based on the data summarized in the above-1isted tables. Only the FPC mechanical integration is considered here, with the interface between the FPC and support eystems (i.e., primary containment building, maintenance support equipment, primary heat-transfer circuits, etc.) being treated by future studies.

The choice of the impurity-control system has a strong influence on the FPC layout (Sec. III.H.). The goal of a block maintenance scheme and the minimum FPC weight constraint also influences the FPC layout. The number, size. location, and kind of penetrations through the $\mathrm{FW} / \mathrm{B} / \mathrm{S}$ as well as the positions of the EFCs are determined by these choices. All fluid lines are positioned at or near the outboard equatorial plane to facilitate pumping, power distribution, FPC disconnects, and vertical, single-plece FPC removal and replacement. Figure III.L-10 gives a FPC cross-sectional view, showing the space allotted in

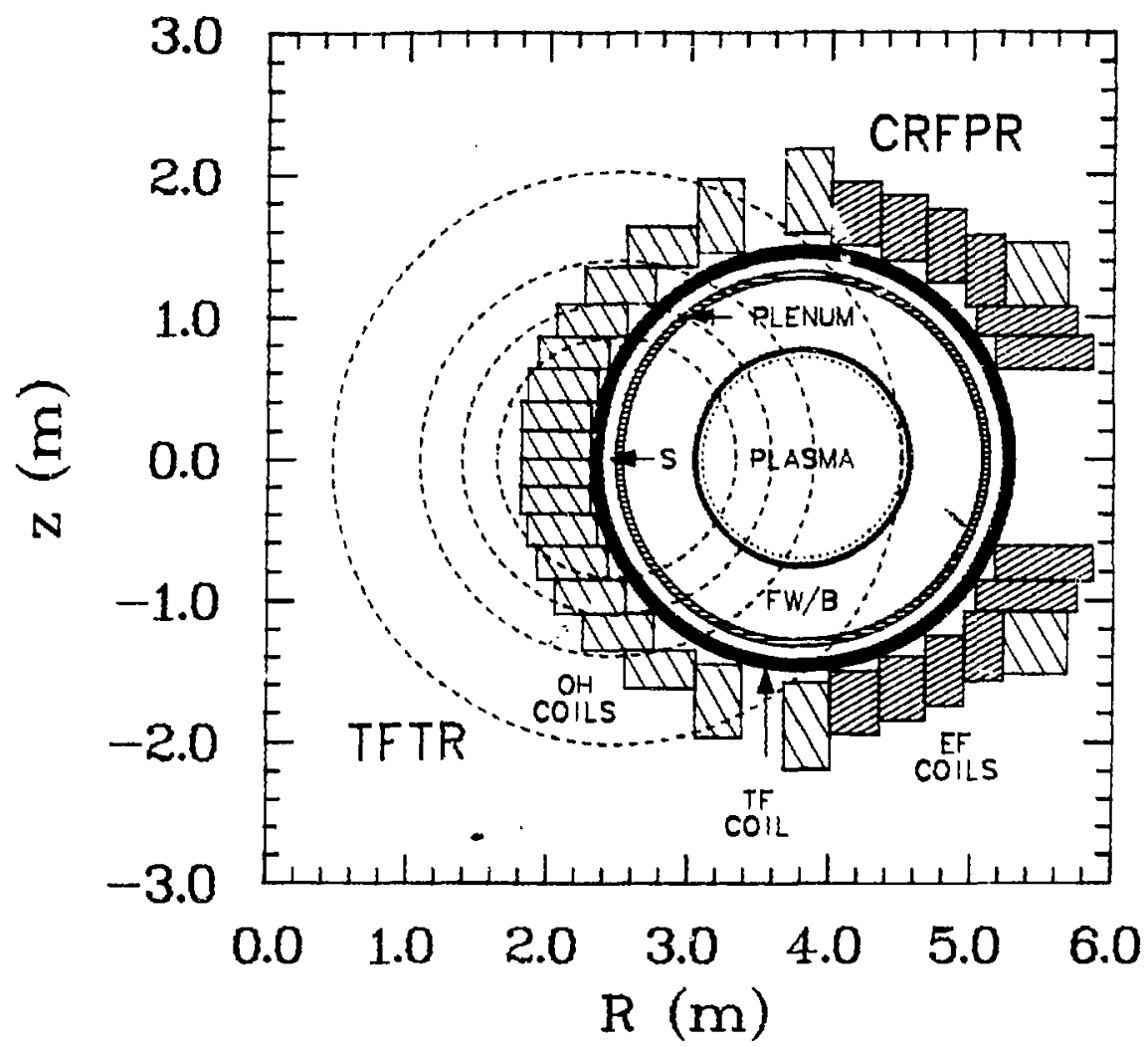

Fig. III.L-10. Cross-sectional view of FPC showing space allottec in the EFC set to accommodate service penetrations. A cross section of the TFTR tokamak experiment ${ }^{36}$ is also shown for comparison. The TFTR poloidal-field coils have been omitted. 
FPC MASSES BASED ON NEUTRONICS CANONICAL BLANKET (FIg. III.B-5)

\begin{tabular}{|c|c|c|c|c|}
\hline Region & $\frac{\text { Thickness }}{(\mathrm{m})}$ & $\frac{\text { Volume }}{\left(m^{3}\right)}$ & Composition & Mass \\
\hline First wall & 0.02 & 2.28 & $60 \% \mathrm{Cu}, 40 \% \mathrm{H}_{n} \mathrm{O}$ & 12.3 \\
\hline Second wall & 0.005 & 0.579 & $60 \%$ PCASS , $40 \% \mathrm{H}_{2} \mathrm{O}$ & $2.7,(a)$ \\
\hline $\begin{array}{l}\text { Blanket } \\
\text { Shield }\end{array}$ & $\begin{array}{l}0.500 \\
0.10\end{array}$ & $\begin{array}{l}16.9 \\
19.88\end{array}$ & $\begin{array}{l}5 \% \text { PCASS }, 95 \% \text { PbLI } \\
40 \% \text { B } 4 \text { C, } 40 \% \text { W } \\
10 \% \text { PCASS } 10 \% \text { H, }\end{array}$ & $\begin{array}{l}30.2 \times 0 \\
188.9\end{array}$ \\
\hline TFCs & 0.075 & 9.92 & & 72.8 \\
\hline PFCs & $0.42(b)$ & 108.6 & $70 \% \mathrm{Cu}, 10 \%$ PCASS & $($ Subtota1 $=307)$ \\
\hline - $\mathrm{OHC}$ & var. & 53.6 & $10 \% \mathrm{H}_{2} \mathrm{O}, 10 \% \mathrm{MgO}$ & \\
\hline - EFC & var. & 55.0 & at $80 \%$ density & $\begin{array}{l}404 . \\
(\text { Tota } 1=1105)\end{array}$ \\
\hline
\end{tabular}

\footnotetext{
(a) Contains $\sim 720$ tonnes of PbLi in blanket and $\sim 80$ tonnes of PbLi in manifolds to outside of PFC.

(c) Average or smeared thickness.
}

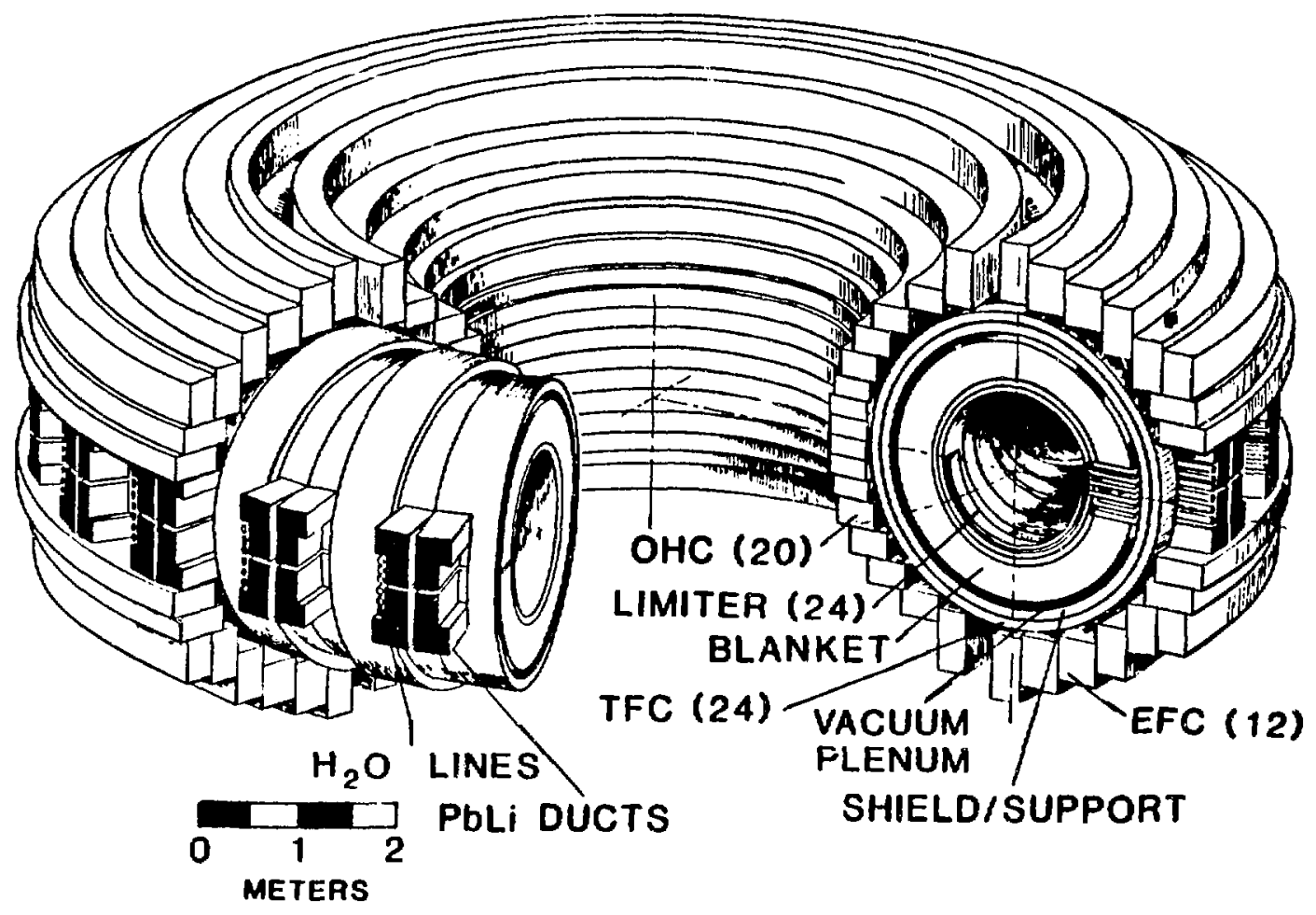

Fig. III.L-11. Overview of arrangement of PFC components. 

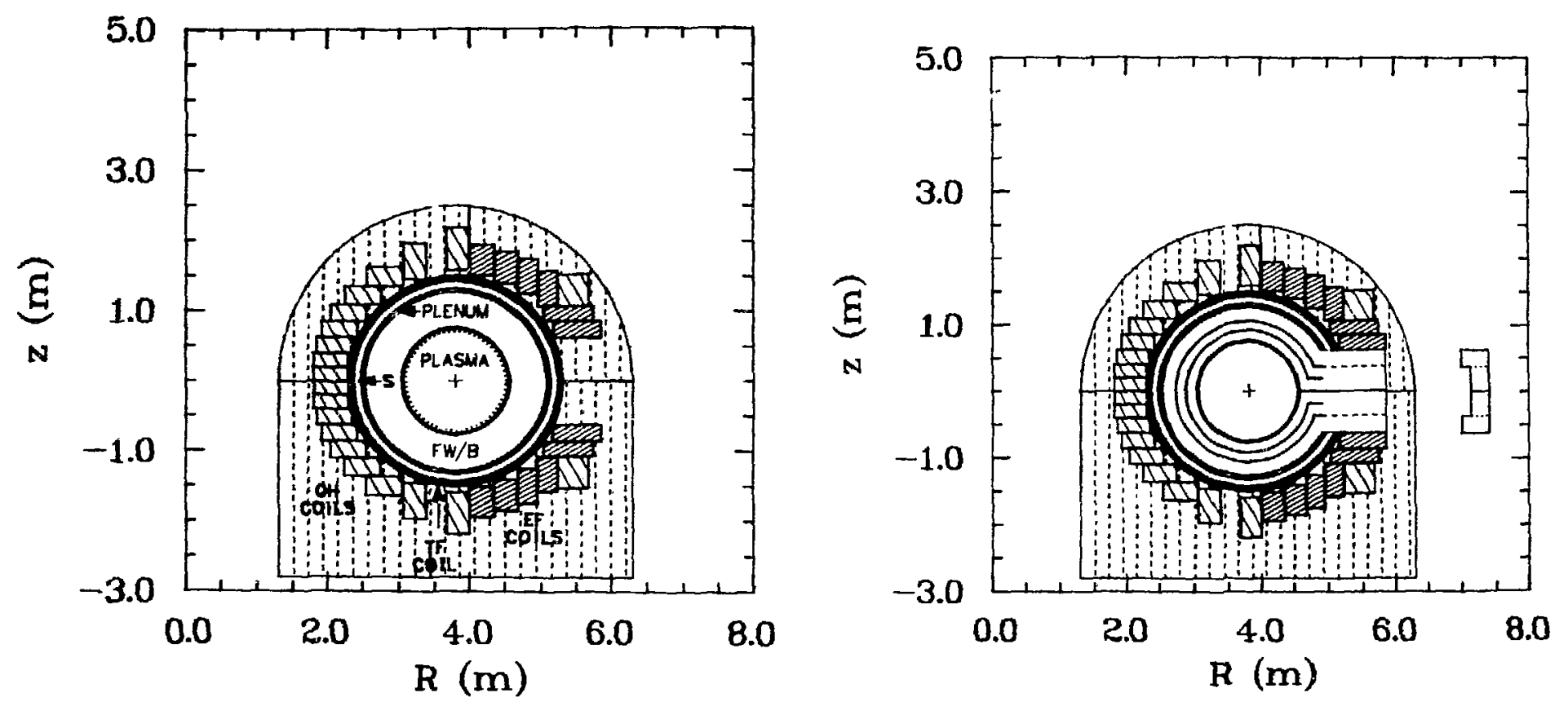

Fig. III.L-12. FPC cross section showing PEC support structure, PbLi blanket, and outboard iocation of coolant ducts (right-hand illustration). 


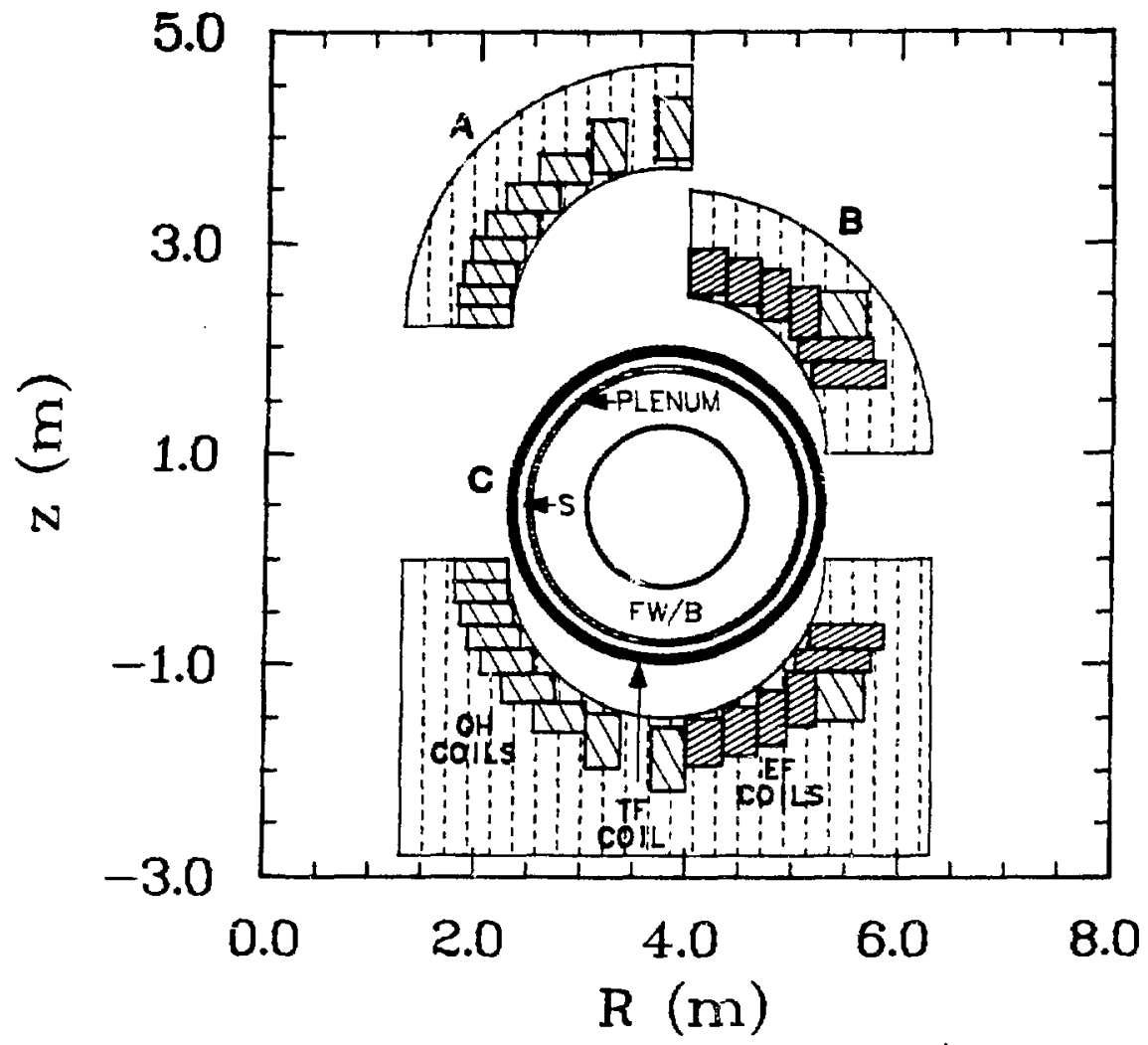

Fig. III.L-13. Illustration of batch maintenance removal/replacement scheme for FW/B/S/TFC torus (C), requiring removal or upper PFC shell assemblies ( $A$ and $B, 146$ and 253 tonne, respectively) prior to the vertical lift of the drained and decoupled FW/B/S/TFC unit (307 tonne).

the EFC set to accommodate these service penetrations. A cross section of the TFTR tokakmak experiment ${ }^{36}$ is also shown for comparison; the TFTR poloidal-field coils have been omitted.

Table III.L-VII summarizes the FPC component masses as modified by discretized the coil sets. The basic components of the 1105-tonne FPC are illustrated in Fig. III.L-11. The relatively tight array of PFCs allows close electrical coupling to the plasma and a minimization of coil mass and stored energy while simultaneously limiting access to the underiying TFCs (73 tonne) and $\mathrm{FW} / \mathrm{B} / \mathrm{S}$ (234 tonne, excluding the $\sim 800$ tonne of PbLi coolant) to only the outboard equatorial plane. A 1.25-m gap is provided between the two outermost EFCs for all FPC service penetrations. A cross-sectional view of the FPC is shown on Fig. III.L-12, illustrating the PFC support structure and the ducting for the self-cooled PbLi blanket. The FW/B/S/TFC torus (307 tonne, excluding coolants) underlying the 798-tonne PFC set would be installed and removed as a 


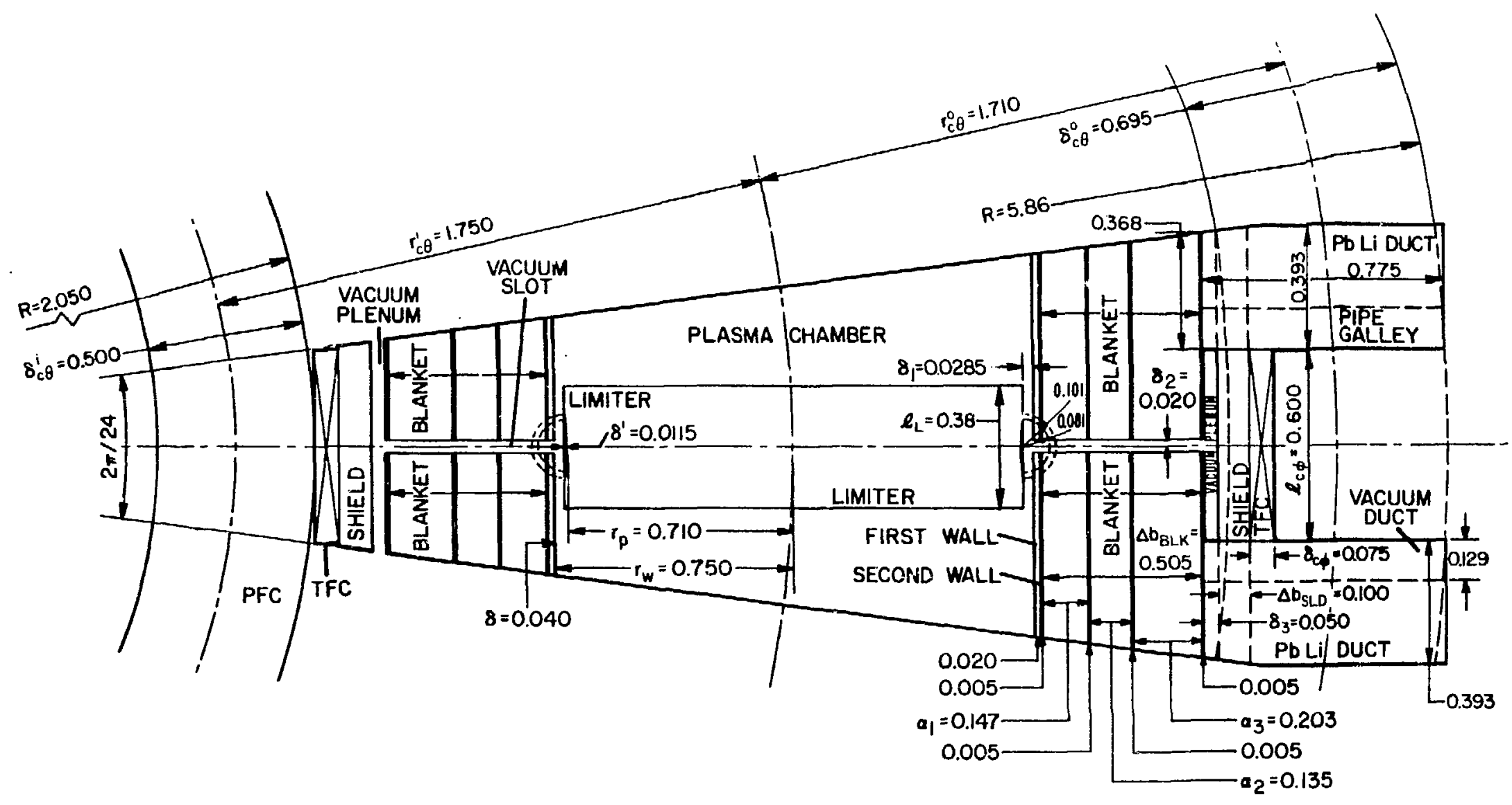

F1g. III.L 14. Plan view of one of 24 toroidal sectors that are fabricated and assembled off site to form the FPC. 
single unt after removal of the top two $P F C$ quadrants, as is illustrated in Fig. III.L-13. The FW/B/S/TFC torus is segmented into 24 toroldal sectors, each centered on a TFC, for purposes of off-site fabrication and assembly. The $\sim$ 1100-tonne PbLi-filled (720 tonne in blanket and 80 tonne in manifolds to outside of PFC) FW/B/S/TFC torus would be supported from below by a saddle or strongback arrangement extending through the lower gap in the PFC set (Fig. III.L-12).

Figure III.L-14 gives a cross-sectional cut through the equatorial plane of one of $N=24$ toroldal sectors, illustrating typical components and nominal dimensions. The 24 poloidal limiter blades (one per sector) are arrayed in the TFC midplane. The limiter blade stand-off is $30 \mathrm{~mm}$ from the first-wall surface, presenting a total limiter-slot area of $\sim 6.8 \mathrm{~m}^{2}$, which compares to $\sim 15 \mathrm{~m}^{2}$ for the toroidal limiter suggested for the STARFIRE tokamak. ${ }^{3}$ Each of the $N=24$ toroldal sectors contains two poloidal blanket sections separated by a radically diverging vacuum-pumping slot that connects the back of the limiter to the vacuum plenum formed by the outboard side of the blanket structure and the water-cooled shield and primary structural support. Two right-angle bends are introduced into this vacuum channel to reduce neutron streaming. The shield annulus is also segmented toroidally for purposes of initial fabrication and assembly. This shield defines the outer radial vacuum boundary as well as providing a basic structural member to each toroidal segment. The TFCs would be received by and secured to this structure in order to react overturning and centering forces, small as they may be for the RFP ( $2.7 \mathrm{MN})$. The low-field TFCs are self-supporting ( 3 tonne each) against outward radial forces. Square apertures are provided in each structure/shield between TFCs at the outboard equatorial plane for vacuum ducts (to cryopumps or a vacuum tunnel), pressurized-water coolant, and PbLi coolant. Each of the 24 sectors would be welded into a single-piece torus, again with the water-cooled shield providing primary structural support both within and to the FW/B/S/TFC sector.

A cruclal issue for the FPC design approach adapted by this study is the need to remove all fusion power and fuel/ash/impurity through the area defined in the vicintty of the outboard equatrial plane by the EFCs and the TFCs. As shown in Sec. III.L.2. (FIg. III.L-4), fusion power would be carried from the FPC by the coolant streams summarized in Table III.L-VIII. A manifolding/headering scheme is suggested in Fig. III.L-15 that meets the thermal-hydraulic criteria established in Sec. III.L.2. The pressurized-water 


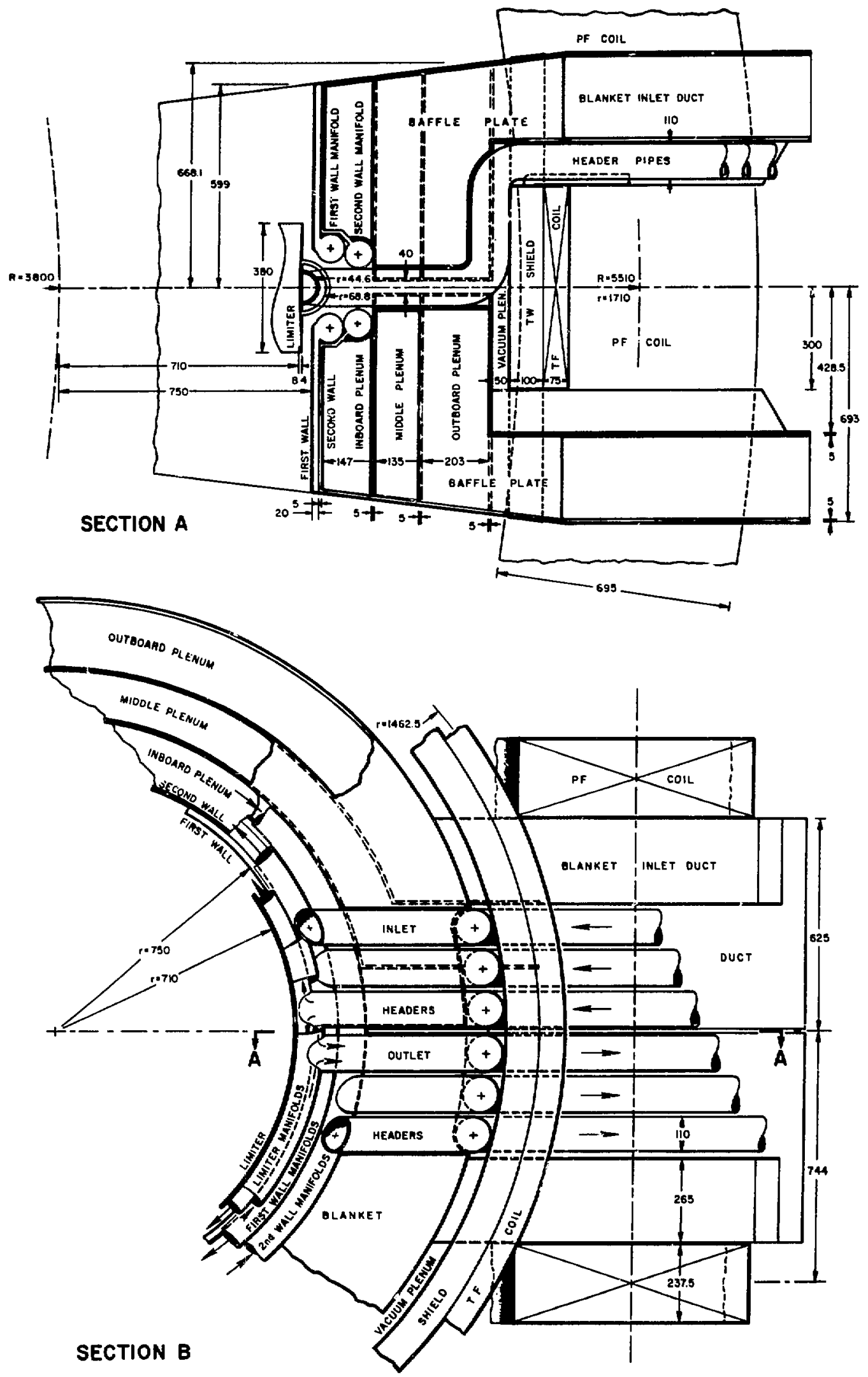



Fig. III.L-15. Mantfolding and headering scheme suggested for each of $N=24$
toroidal segments to remove heat at the levels given in
Table III.L-VIII.

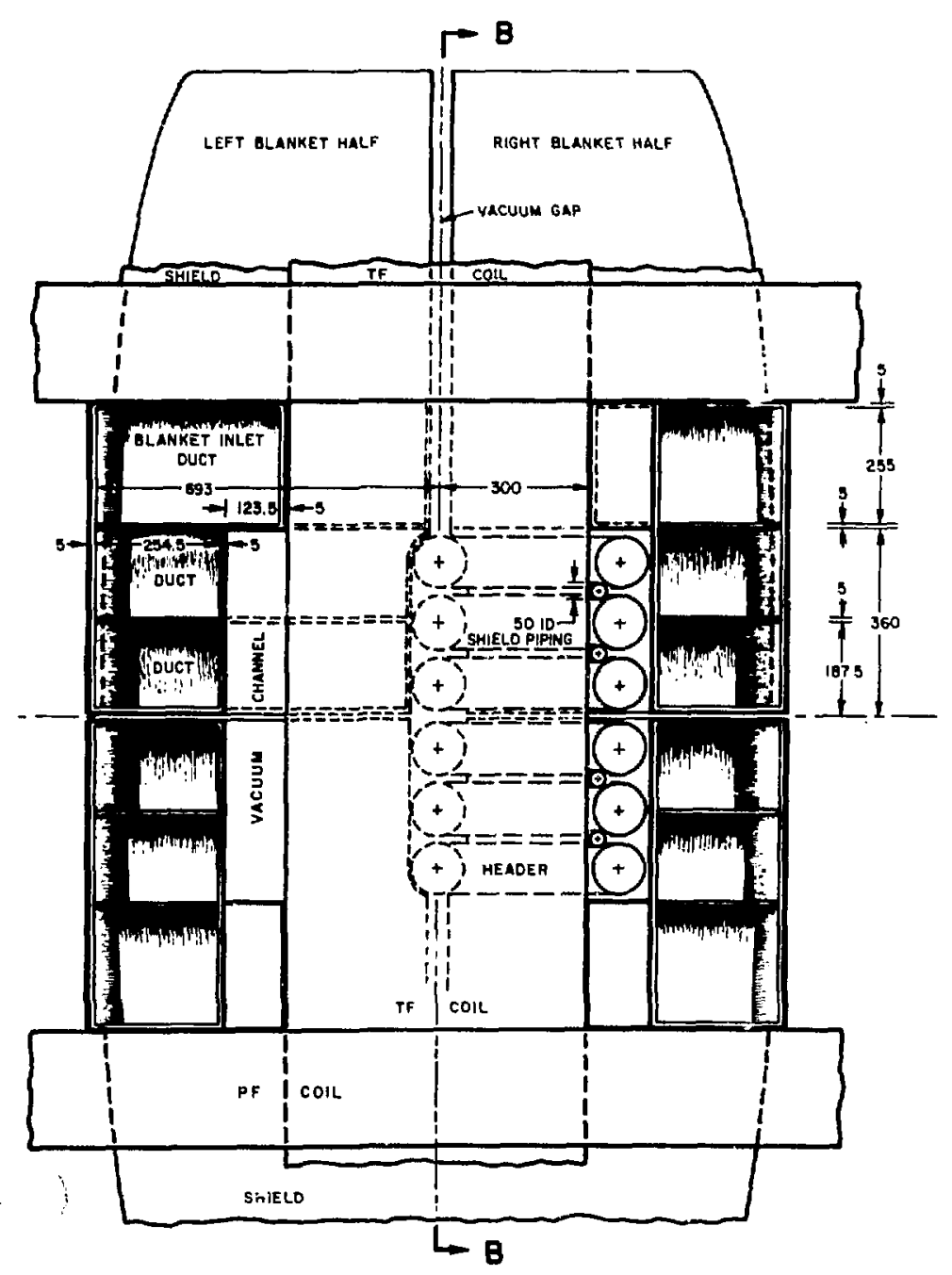


TABLE III.L-VIII

SUMMARY OF POWER AND MASS FLOW FROM FPC PER $N=24$ SECTORS

\begin{tabular}{llcc} 
Source & Fluid & Mass Flov $(\mathrm{kg} / \mathrm{s})$ & Power (MW) \\
\cline { 2 - 3 } & & & \\
Limiter & $\mathrm{H}_{2} \mathrm{O}$ & 54.6 & 18.4 \\
First-wall/second-wall & $\mathrm{H}_{2} \mathrm{O}$ & 138.1 & 34.1 \\
Shield & $\mathrm{H}_{2} \mathrm{O}$ & 11.3 & 6.0 \\
$\quad$ & & 204.0 & 58.5 \\
Blanket & $\mathrm{PbLi}$ & 3035.0 & 81.7 \\
Vacuum & $\mathrm{DT} / \mathrm{He}(10)^{-6}$ & $0.27 / 0.51$ & --
\end{tabular}

manifold for each pumped limiter would be positioned directly behind the poloidal limiter blade as concentric hemicylinders, with a single pair of inlet/outlet headers radially traversing the blanket/shield near the outboard equatorial plane. The limiter detail shown in Figs. III.L-15 and III.L-16 gives the coolant manifolding and flow paths. A number of options for the pressurized-water manifolding/headering scheme for the FW/SW system can be suggested. First, from the viewpoint of minimizing the number of tubes delivezing coolant water to the $\mathrm{FW} / \mathrm{SW}$, it is desirable to integrate the inlet and outlet manifolds into the respective first-wa11 and second-wall systems, using only single pairs of inlet/outlet headers at or near the outboard equatorial plane to supply and return coolant. This approach is also illustrated on the orthographic layout given in Fig. III.L-15. Figure III.L-17 gives a detailed view of this FW/SW manifolding scheme in which the separated first-wall (copper-alloy) and second-wall (HT-9) coolant structures would be mated and joined by a return-coolant ring manifold as part of the sector assembly sequence. The adverse effects on the FW/B/S nuclear performance (tritium breeding) by displacing PbLi with presurized-water manifolds near the critical first-wall region and the means by which tritium breeding can be enhanced is dis:ussed in Appendix $C$. An alternative or backup option for pressurized-water coolant manffolding/headering to correct the deleterious effects on the tritium-breeding ratio expected by the scheme depicted in Figs. III.L-15 and III.L-17 would place the manifolds at or behind the shield 290 

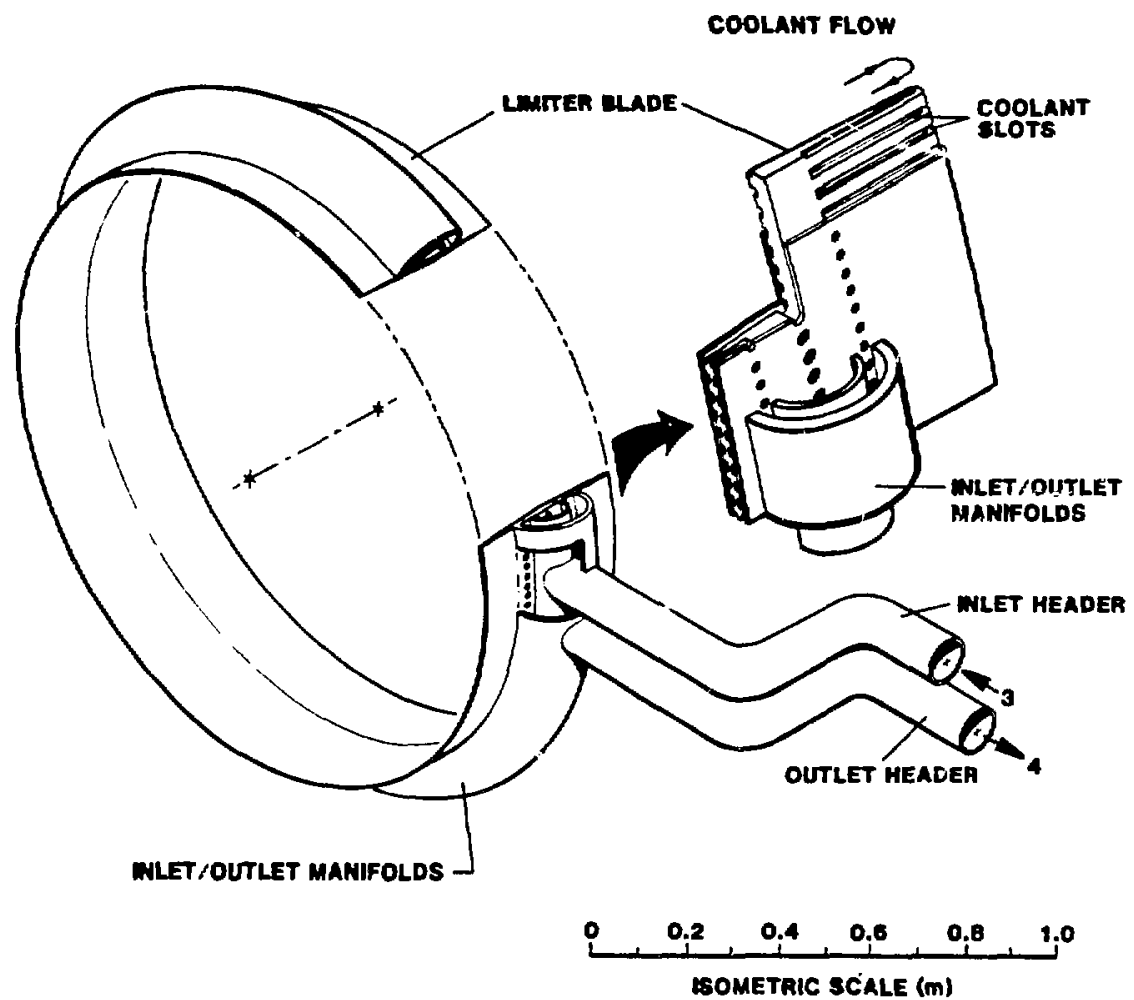

Fig. III.L-16. Detafled view of pumped poloidal ifiter coolant circuit.

region. In this case, which is also examined in Appendix $C$, radial tube sheets located at the intersector positions would deliver and return pressurized-water coolant to and from the FW/SW system. These tube oheets and associated poloidal manifolds would be located at the outer radial surfaces of each toroidul sector rather than within the sector, as was adapted for the approach depicted in Fig. III.L-15. For the purposes of completing this design integration study, however, the manifolding scheme depicted in Fig. III.1-15 is used along with the minor design changes suggested by the sensitivity studies of tritium breeding given in Appendix $C$. The first-wall manifolding scheme for the arrangement depicted in Fig. III.L-15 is now addressed in more detall.

As seen by the orthonormal sketch given in Fig. III.L-15 and the isometric sketch of a toroidal sector given in FIg. III.L-18, the split-flow limiter coolant would be provided by a concentric, hemicylindrical manifold serviced by $\sim 110-\mathrm{mm}-0.0$. headers extending radially just above and below the equatorial plane. Each side of the FW/SW within a given sector would also be serviced by independent poloidal manifolds and radial headers. A total of six 110-mm-0.D. pressurized-water headers would be brought out and around the TFCs in the 


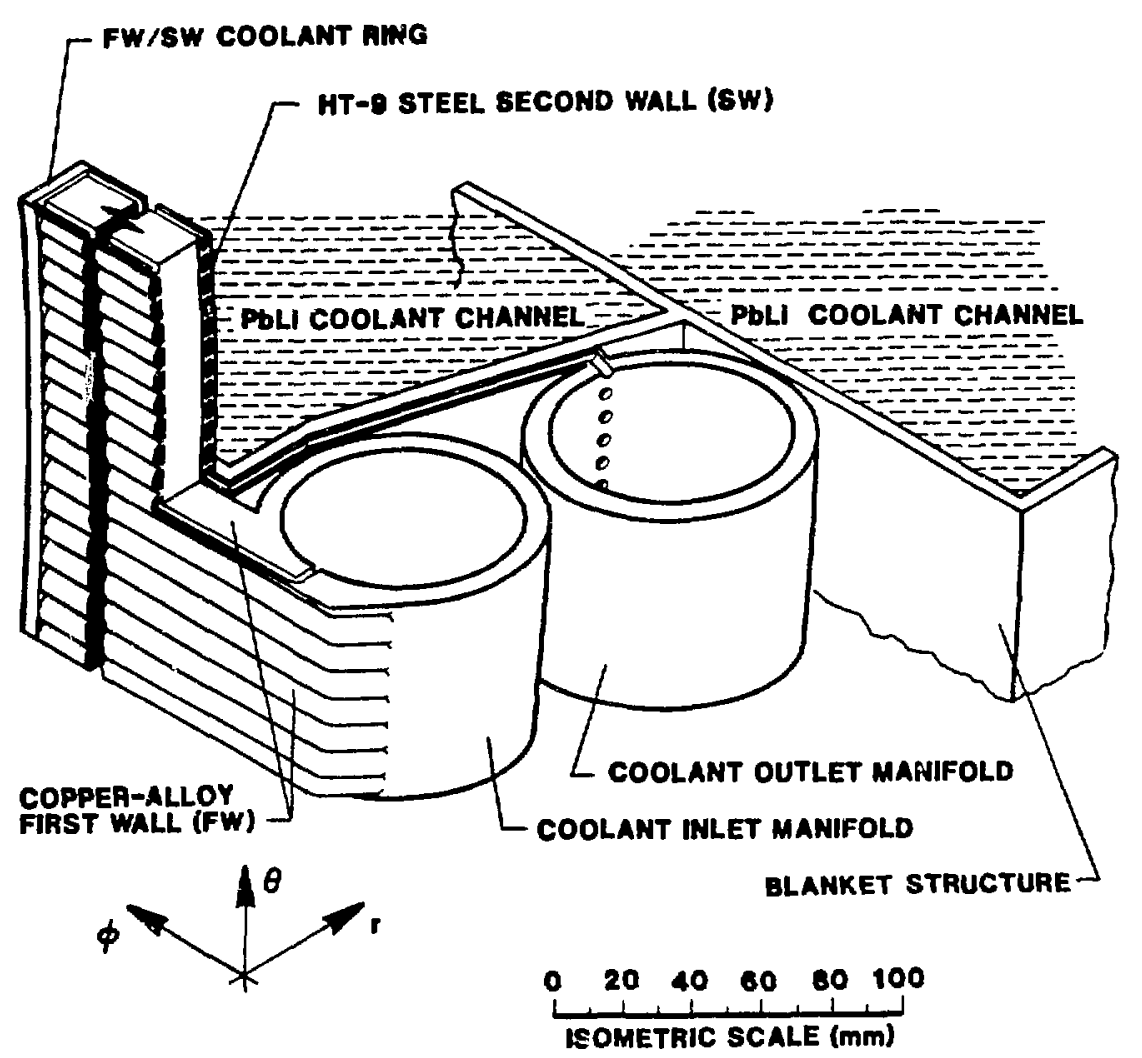

Eig. III.L-17. Detailed view of the first-wa1l/second-wall manifolding scheme for the minimum-tubulation option that places inlet/outlet manifolds near the first-wall region.

equatorial plane, as is shown in Fig. III.L-15. Four 40-mm-0.D. pressurized-water pipes would also service the FPC shield in the same vicinity as for the limiter and FW/SW service entries. The 1.25-m vertical gap between the outboard EFCs is acceptable from a magnetics viewpoint (Sec. III.F.2.), giving a vertical-field index of $n=0.53$. An area of $0.095 \mathrm{~m}^{2}$ at the $T F C$ current center is ailowed for the pressurized-water header galley, the comparable area on the other side of the TFC being dedicated to vacuum pumping. The remaining $\sim 0.39 \mathrm{~m}^{2}$ (total) is used for PbLi flow to and from each blanket half within the toroidal segment; this flow area 1 s $>95 \%$ of the design goal established in Table III.L-V. Similarly, the pressurized-water headers suggested in Fig. III.L-15 meet the design criteria established in Tables III.L-III and III.L-IV (maximum flow velocity is actually $12 \mathrm{~m} / \mathrm{s}$ in the exit FW/SW headers). 


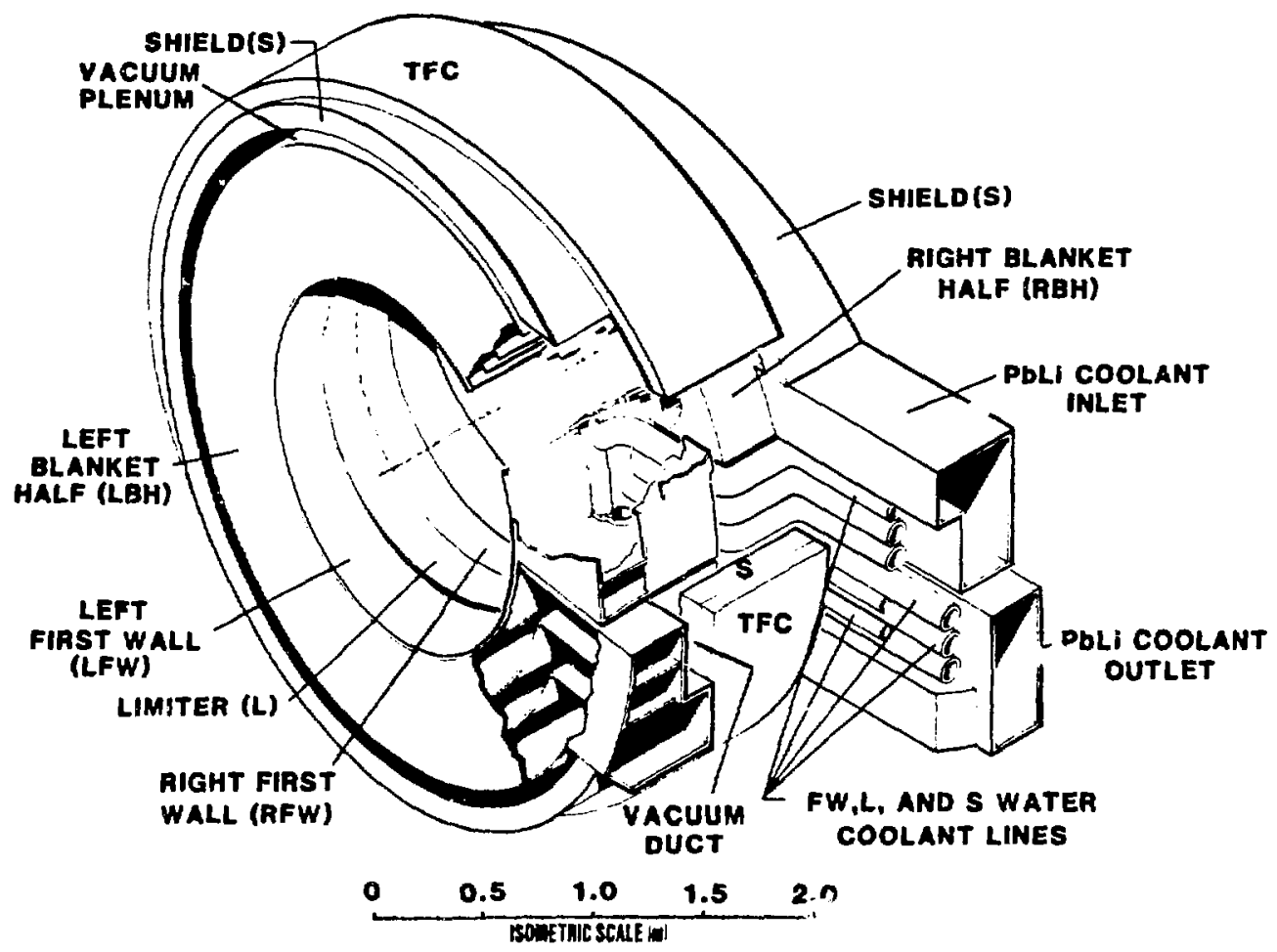

Fig. III.L-18. Detalled isometric view of toroldal FPC sector showing limiter and FW/SW manifolding and headering scheme and the PbLi coolant ducting.

Approximately $10 \%$ of the $\mathrm{FW} / \mathrm{B} / \mathrm{S}$ volume 1 s taken by the manifold/headering scheme suggested in Fig. III.L-15, which reduces the tritium-breeding ratio to below unity since the blanket volume removed is in the high-importance first-wall region. Means to recover the tritlum-breeding ratio are a) decrease in first-wall thickness (Fig. III.B-7), b) modestly increase the blanket thickness, c) Increase the ${ }^{6} \mathrm{~L} I$ enrichment (now is 60\%), d) Increase the IIthium content (i.e., stagnant, canned lithlum), e) incorporate more neutron multiplication, f) adopt exoblanket manifolds and the problem of tube sheets aaking radial connections with the $\mathrm{FW} / \mathrm{SW}$ system $_{\Downarrow}$ or g) Improve the neutron reflectivity or albedo of the thin, heat-recovering shield. The results of preliminary two-dimensional neutronics studies that explore the above-listed options are given in Appendix C. Emerging from these studies is the desire to a) thin the copper-alloy first wall from $20 \mathrm{~mm}$ to $5 \mathrm{~mm}$, b) enrich the lithium from $60 \%$ to $90 \% 6_{\mathrm{L} 1}$, and c) 1mprove the shield albedo by replacing the 
$\mathrm{H}_{2} \mathrm{O} / \mathrm{B}_{4} \mathrm{C} / \mathrm{W}$ shield with $\mathrm{H}_{2} \mathrm{O} / \mathrm{HT}-9$. These modifications have little practical impact on the design presented herein but should be incorporated into ongoing reactor design efforts.

Figure III.L-18 gives an isometric sketch of a CRFPR sector, showing the multiturned TFCs. Not shown are the dominant PFCs (Fig. III.L-11) or the current-drive coils. Electrical leads to the multiturn TFCs and PFCs are considerably reduced in size from the coils. In order to maximize the coupling (1.e., minimize stray inductances), the low-current poloidal current-drive colls ideally would be integral with the first-wall/limiter, along with similarily placed toroidal-field current-drive coils. As is shown in Fig. III.B-7 and Appendix C, however, placement of current-drive colls near the first-wall region will adversely effect tritiun breeding, and a crucial tradeoff between current-drive and neutronic efficiency remains for future study. The main TFC and PFC sets can be used to drive current, if the drive frequency can be lower than the $\sim 50 \mathrm{~Hz}$ assumed in this study. If $\mathrm{F}-\theta$ pumping can be lised to drive dc current in the CRFPR, the OHC set shown in FIg. III.L-10 is needed only for startup. Reduction in the size of the OHCs may result, which in turn would also allow a reduced EFC set for the same cost and recirculating power fraction.

Each of the $\mathrm{N}=24$ toroidal sectors would be fabricated, assembled, and tested at an off-site factory where the environment for quality assurance can be better controlled. Figure III.L-19 concludes this FPC mechanical integration study by depicting a sequence of assembly steps anticipated for a sector assembly. After assembling (Step \#1) the TFC and structural shield (S), the left blanket half (LBH) (includes the return SW coolant manifold) is inserted (Step 非) and made captive of the assembly by subsequent welding of the return pressurized-water coolant header (\#6) for the left first-wall assembly (LFW). The LFW and the assoctated inlet FW/SW coolant mantfold/header (\#1) is inserted (Scep \#3) and made captive of the assembly upon welding the FW/SW return coolant ring. Step 玤 then inserts the limiter (L), along with the associated inlet/outlet headers ( $\$ 3$ and $\# 4$ ). The limiter is made captive of the assembly upon executing step 非 and inserting the right first-wall (RFW) assembly and its associated inlet coolant header (\$2). Step \#6 makes the RFW captive of the assembly upon inserting the right blanket half (RBH), the associated SW coolant return header ( $\$ 5)$, and the $\mathrm{FW} / \mathrm{SW}$ return coolant ring. The resulting FPC sector (Fig. III.L-18), upon electrical, fluid, and vacuum testing, would be assembled into a torus (Step $\# 7$ ), again ysing the shield as the primary structure and 294 
vacuum boundary. The asserably of the full FW/B/S/TFC torus co'sld also be made at the factory site, with fully integrated testing being performed at either the factory, on site, or both.

The scheduled (annual) CRFPR maintenance approach envisages removal/replacement of the inner FW/B/S/TFC torus. A second FW/B/S/TFC torus would be assembled and subjected to pressure, flow, and electrical checkout prior to the substitution. As illustrated in Fig. III.L-13, access to the inner $\mathrm{FW} / \mathrm{B} / \mathrm{S} / \mathrm{TFC}$ torus is obtained by decoupling and lifting the upper inner oHC shell (A, 146 tonne) and the upper outer EFC shell (B, 253 tonne). The FW/B/S/TFC torus, (C, 307 tonne) drained of PbLi coolant and remotely decoupled from all electrical and primary coolant lines, is then lifted as a unt vertically out of the saddle formed by the lower PFC shell/base, which remains in place. The FW/B/S/TFC torus is transferred to a hot cell for refurbishment. Attachment of vacuum pumps direcily to the FPC and locating the vacuum bo:undary under the coll annulus, as was done for the STARFIRE tokamak ${ }^{3}$ and other fusion systems, is possible for the CRFPR. Such an approach is consistent with a stationary FPC and a "patch" maintenance approach. The FPC size reduction for a compact high-power-density device, however, allows serious consideration of batch maintenance, whereby an integrated FW/B/S/TFC torus would be removed anc replaced as a single unit. To reduce the encumbrances in handling this unit, it may be preferable to locate the entire PFC in an evacuated chamber. Such a vacuum approach has been proposed for several of the conventional fusion reactors but appears even more practical for compact systems such as the CRFPR, where the total evacuated volume based on a pill-box chamber with $R \simeq 9 \mathrm{~m}$ and $\mathrm{h}=5 \mathrm{~m}$ is $\sim 1000 \mathrm{~m}^{3}$. Design detail of systems beyond the FPC systems is needed, however, before these options can be adequately assessed. 


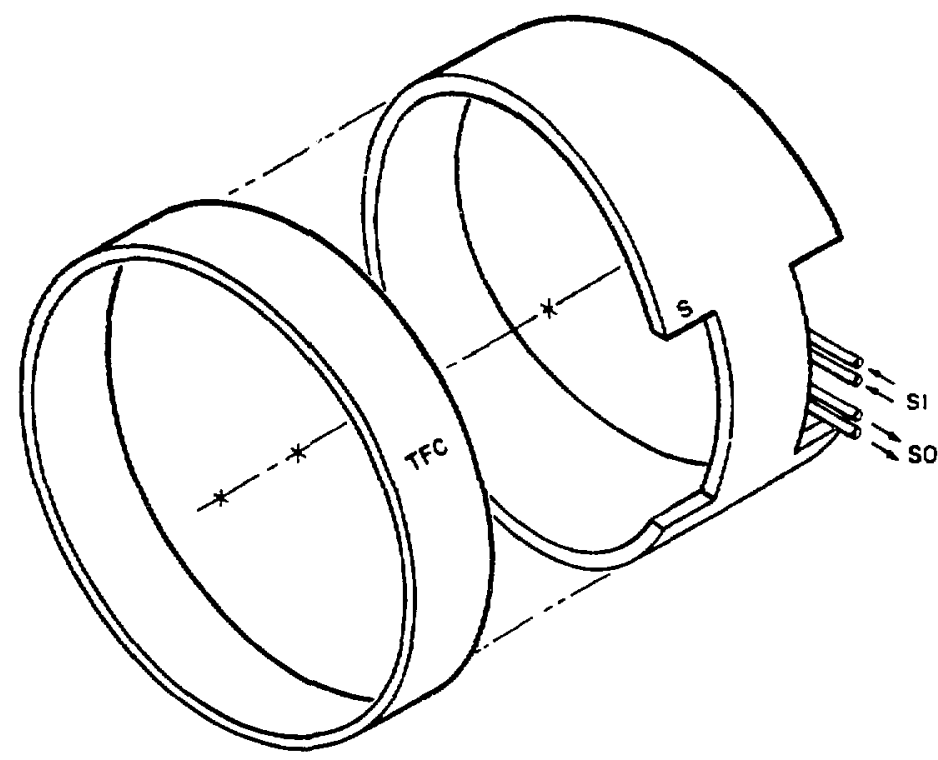

STEP 1 ASSEMBLE TOROIDAL-FIELD COIL (TFC) ONTO STRUCTURAL SHIELD(S)

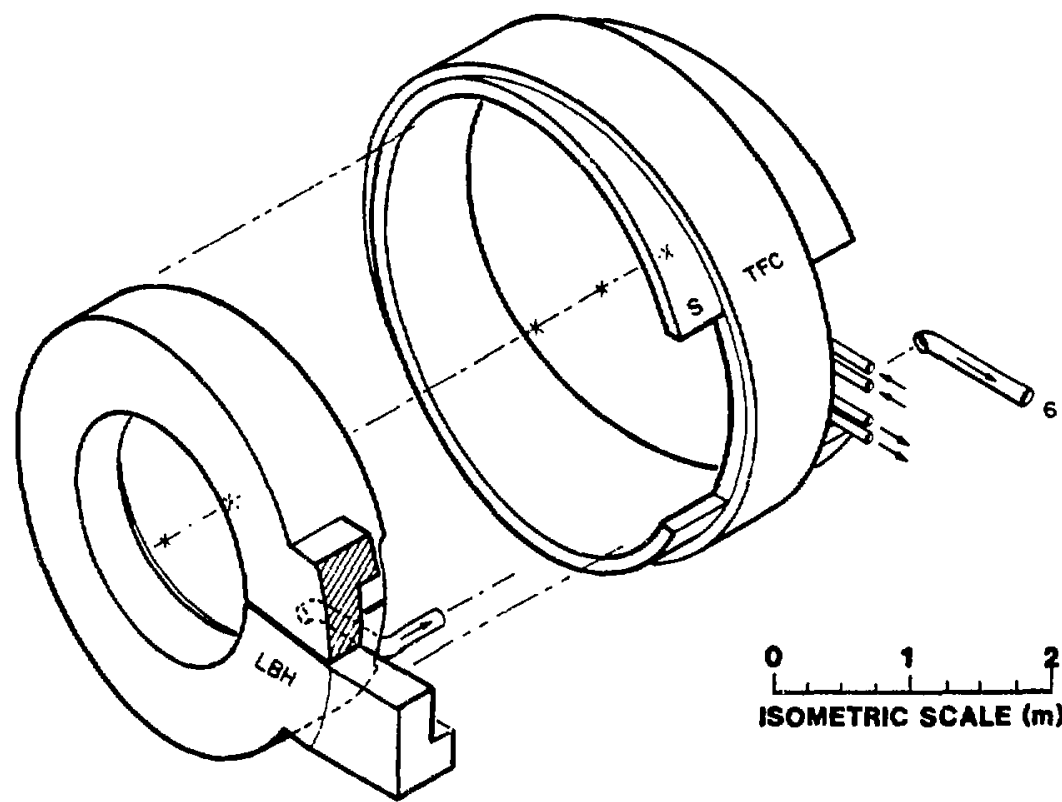

STEP 2 INSERT LEFT BLANKET HALF (LBH) AND COOLANT OUTLET FRUM SECOND WALL $(* 6)$

Fig. III.L-19. Assembly sequence envisioned for each of $N=24$ FW/B/S/TFC sectors based on a near-first-wa11 manifold/headering scheme. 


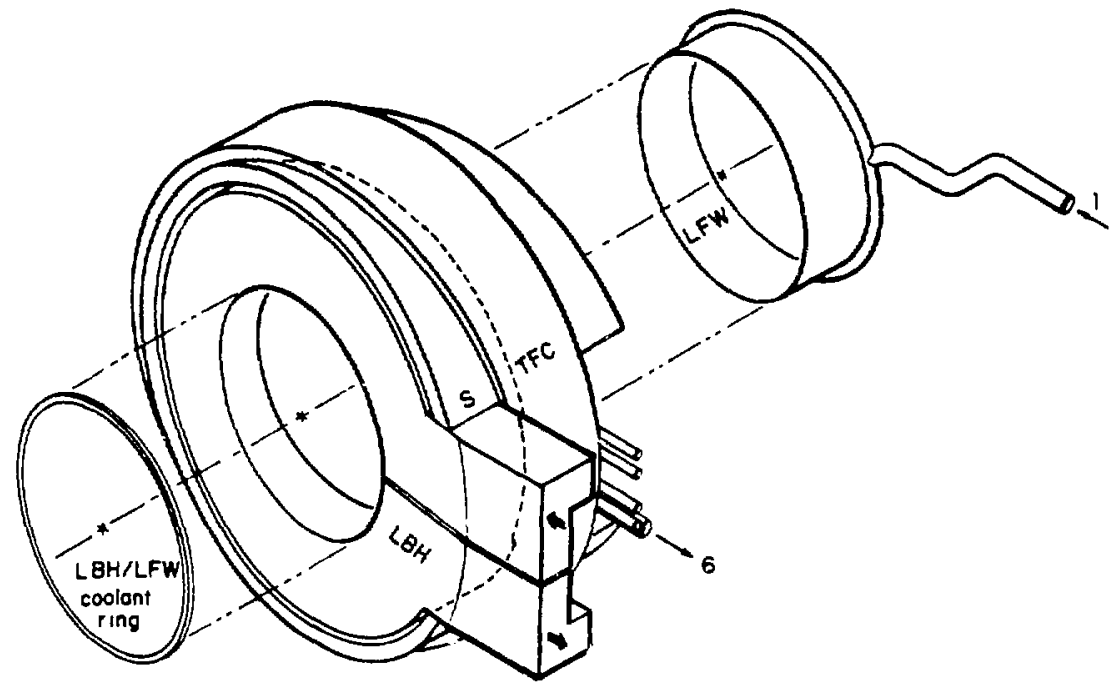

STEP 3 INSERT LEFT FIRST WALL (LFW), ASSOCIATED SECOND WALL COOLANT INLET (*1),AND LFW/LBH CUOLANT RING (*1 INLET, *6 OUTLET)

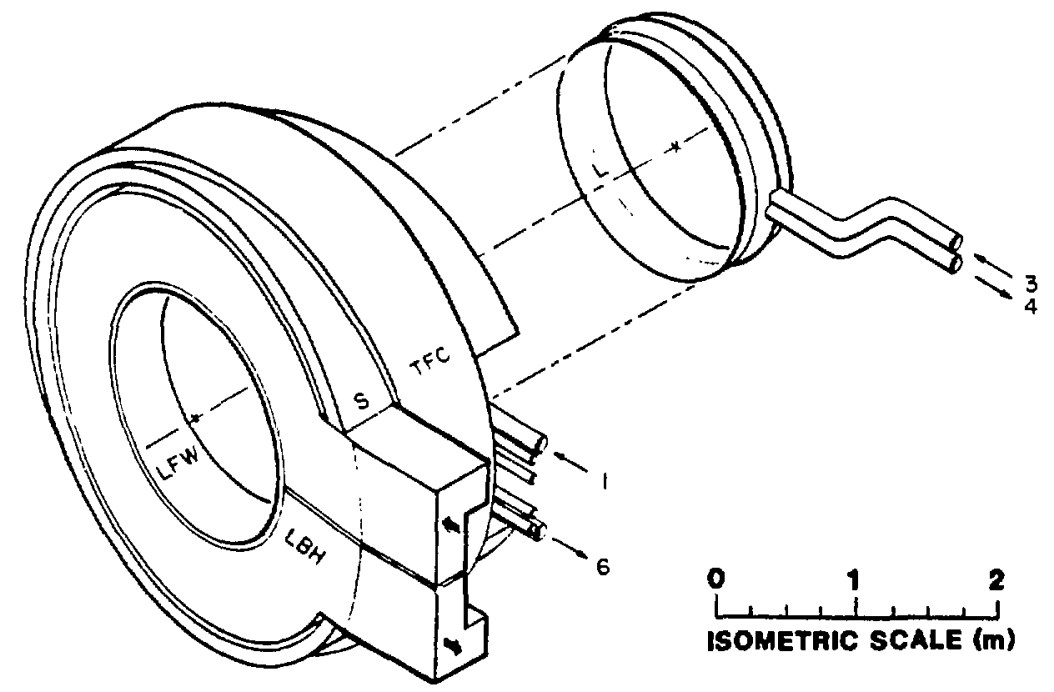

STEP 4 INSERT POLOIDAL LIMITER AND ASSOCIATED COOLANT INLET (*3) AND OUTLET (*4)

Fig. III.L-19, continued. 


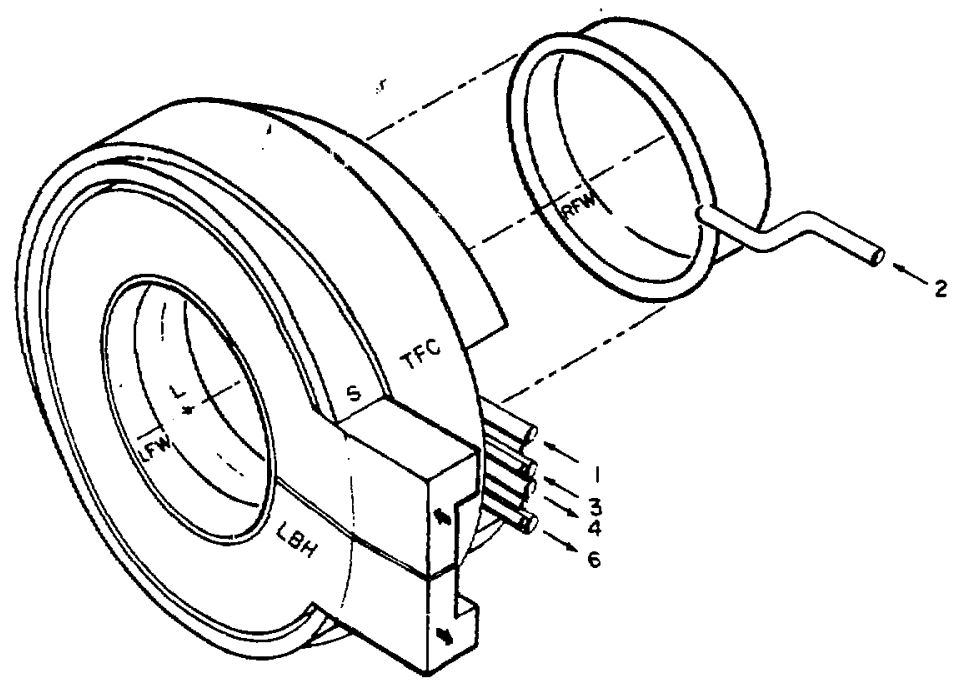

STEP 5 INSERT RIGHT FIRST WALL (RFW) AND ASSOCIATED COOLANT INLET $(* 2)$

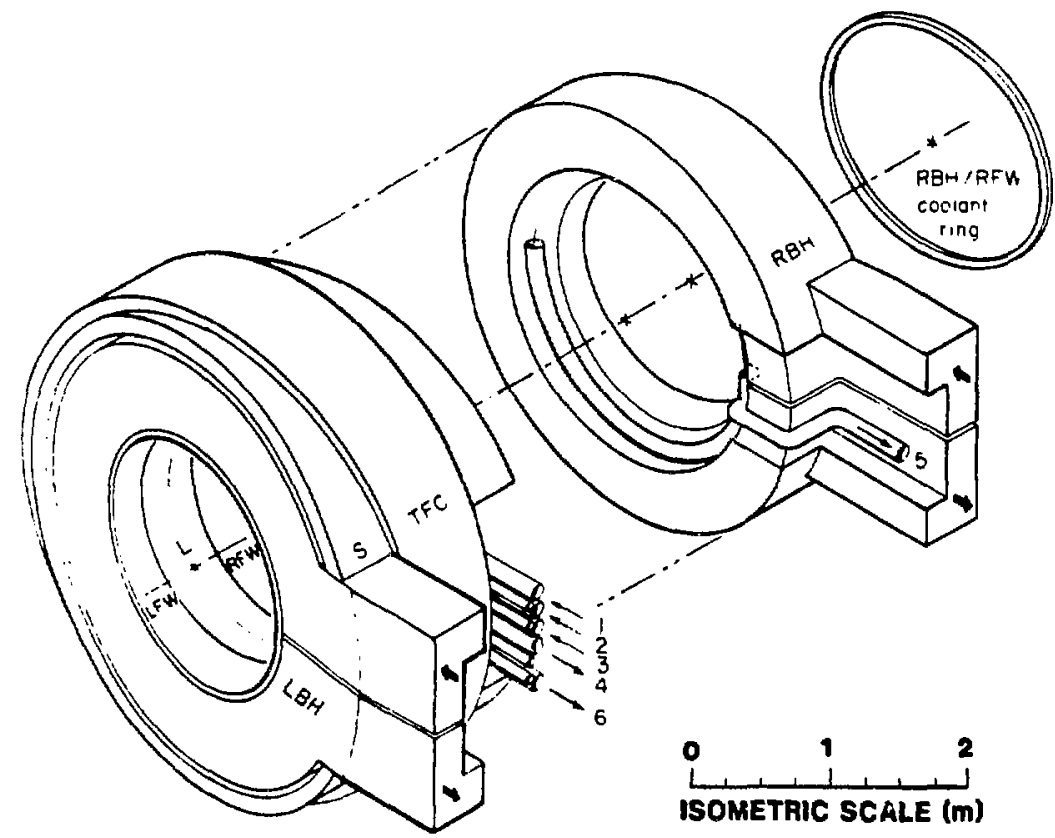

STEP 6 INSERT RIGHT BLANKET HALF (RBH), ASSOCIATED SECOND-WALL COOLANT OUTLET (*5), AND RFW/RBH COOLANT RING ( $* 2$ INLET, *5 OUTLET)

Fig. III.L-19, continued. 


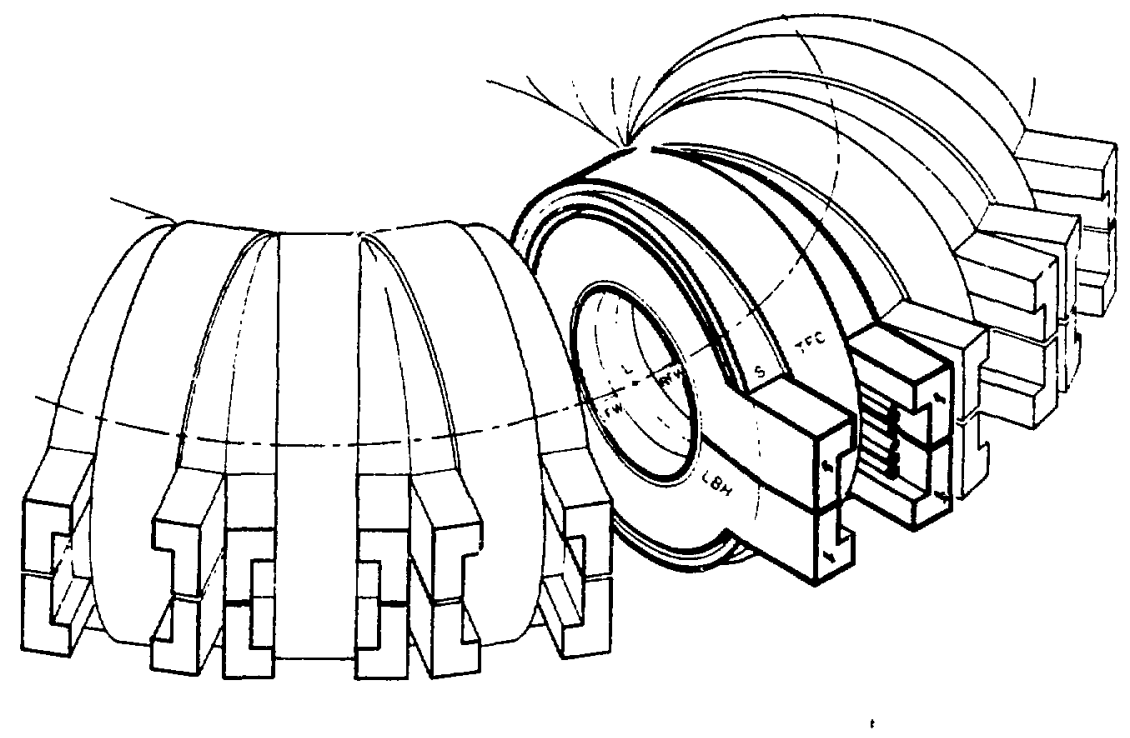

STEP 7 ASSEMBLY OF FW/B/S/TFC TORUS FROM N=25 SECTORS

Fig. III.L-19, continued. 


\section{G. Conclusions}

The physics and engineering characteristics of key plasma engineering systems have been broadly described, quantifled, and integrated for a high-wall-loading, compact RFP reactor. The economic and operational benefits of the compact high-power-density approach can be attained by the RFP since it is one of a class of approaches that can confine high-beta plasma without excessive toroldal flelds at external conductors. Hence, efficient, resistive-coil systems are possible with a FPC mass and volume reduced by factors in excess of 20 when compared with superconducting systems of similar power rating; both reduced cost and single-plece FPC maintenance of a Factory-produced system become possible. Furthermore, unique and highly-effictent plasma-heating and steady-state current-drive systems that are inherent to the RFP become a possibility. Although this study stressed impurity control by high-wall-coverage (poloidal) pumped limiters, the ability to use closely-coupled resistive colls allows serious considerations of (toroidal) magnetic divertors and represints a high-priority area for future study; the practicality of magnetic divertors is greatly enhanced by the better coupling allowed in a resistive-coil system. Lastiy, although this study sicressed the minimum-cost, $1000-\mathrm{MWe}$ (net), $\sim 20-\mathrm{MW} / \mathrm{m}^{2}$ (neutrons) design, detail parametric studies show acceptable cost peraltles for lower-wall-loading systems [5-10 $\mathrm{MW} / \mathrm{m}^{2}$ (neutrons)] of nominally the same physical size but operating with reduced power density and delivering reduced total power. The RFP, therefore, presents a "robust" plasma confinement system capable of providing a range of reactor systems that are compact in both physical size and/or net power output while assuring acceptable cost and levels of engineering feasibility for a range of assumed levels of physics (beta, transport) performance. Maintenance of the regenerative RFP dynamo at higher plasma pressures while retalning the already reactor-relevant beta values with increasing current is central to achieving this ideal end product and, therefore, represents a major experimental objectlve of the RFP physics program. 


\section{ASSESSMRNT}

This study concludes with an assessment of the physics (Sec. IV.A.) and technology (Sec. IV.B.) needed to achieve the reactor parameters summarized in Appendix B. Section IV.C. gives an economic assessment that indicates directions for future study and design innovation based on results showing quantit itively the tradeoffs between cost, first-wall neutron loading, net plant power, FPC size (mass, complexity, batch versus patch maintenance), and piasma confinement.

\section{A. Physics Assessmeni}

The cost-optimized design suggested in Sec. III.L and summarized in Appendix $B$ depends on the confinement of a high-pressure plasma by self-generated magnetic fields that are large within the plasma $\left[B_{\phi}(0) \approx 9.5 \mathrm{~T}\right.$, $\left.B_{\theta}\left(r_{p}\right)=5.2 \mathrm{~T}\right]$ and diminish considerably at the colls $\left(B_{c \phi}=0.72 \mathrm{~T}\right.$, $B_{c \theta}=4.5 \mathrm{~T}$ ). The possibility of confining efficiently steady-state plasmas of moderate beta in a minimum-energy state using water-cooled copper-alloy coils located outside a thin high-power-density blanket offers a fusion option with considerible developmental, economic, and operational promise. The RFP is one of a class of confinement schemes with such promise. Rapid experimental and theoretical advances have occurred for the RFP in the 1980s, showing a reactor-relevant scaling $\left[\tau_{\mathrm{E}} \propto I_{\phi}^{\gamma_{\mathrm{p}}}{ }_{\mathrm{p}} \mathrm{f}\left(\beta_{\theta}\right)\right.$, with $\left.\nu>1\right]$ at reactor-relevant betas $\left(\beta_{\theta} \simeq 0.1-0.2\right)$ but for densities $\left[2-5(10)^{19} \mathrm{~m}^{-3}\right]$ and temperatures $(300-500 \mathrm{eV})$ that remain to be increased by factors of 10-20. These relatively small $\left(V_{p}=0.8-1.0 \mathrm{~m}^{3}\right)$, low-current $\left(I_{\phi}=0.3-0.5 \mathrm{MA}\right)$, short-pulsed (2-30 ms) experiments must demonstrate increased confinement times by increasing primarily $I_{\phi}$ along with more moderate increases in $r_{p}$. Simultaneously, both $\beta_{\theta} \geqslant 0.1$ and an efficient dynamo (i.e., nearly classical resistivities) must be maintained. Progress toward these first-priority physics goals must occur in high-power-density ohmic discharges while slmultaneously dealing with high heat and particle fluxes on critical components and maintaining acceptably low values of $\mathrm{Z}$ eff and the streaming parameter, $I_{\phi} / \mathrm{N}$. Control of fleld errors, wall conditioning, and the need for active impurity-control and fueling schemes $i_{r}$ these Inherently smaller, high-current-density devices are also important physics goals. This approach towards ignition, burn, and ultimately the reactor through large Increases in plasma current and low-to-moderate increases in minor plasma radius strongly couples engineering technology with plasma physics at an early stage of development. Although presenting problems from a physics 
viewpoint, the early coupling of physics and technology in a small, reactorrelevant device may offer significant development advantages related to reduced cost and schedules. The central physics issues summarized above combine with the need to understand better plasma-edge effects and divertor physics in the RFP magnetics geometry. Key issues for the RFP in these latter areas include scrapeoff physics (charge exchange, radiation, transport), the magnitude and direction of electrical sheath potentials, optimal separatrix geometry, control of magnetic islands, divertor channel magnetics, and transport. Lastly and most central to the RFP is the need to resolve better the role played by the conducting first-wall shell in providing plasma stability, particularly with respect to the effects of pololdal breaks and shell penetrations (vacuum, limiter, divertor, etc.). The ability to provide plasma services while minimizing the local field errors is crucial to the coitrol of transport and reducing local peaking of the already high first-wall energy deposition projected for the compact RFP. 
B. Technology Assessment

Technology development needs for fusion can generally be assessed according to the following systems:

- Plasma-Heating Systems

- startup

- sustenance (ignition)

- Particle-Control Systems

- fueling

- particle removal

- vacuum

- Plasma Current-Drive Systems

- Nuclear Systems

- In-vacuum components (IVC)

- blanket (B)

- shield (S)

- Magnetic Systems

- TFC

- PFC (OHC + EFC)

- divertor coils

- current-drive coils

- feedback-control coils

- Electrical and Mechanical Systems

- energy transfer and storage (ETS)

- FPC structural support

- assembly and maintenance

- Tritlum Systems

- fueling and particle control

- breeding

- containment

- Balance-of-Plarit Systems

The material developed in Sec. III. for each of these reactor systems represents the state of the knowledge for RFP reactor projections, wuch of this information being extracted and extended from counterpart systems being proposed for the more thoroughly examined tokamak and tandem-mirror reactors. This section summarizes the technology needs, performance requirements, design options, and related key issues that have emerged from this study for each major FPC subsystem.

1. Plasma-Heating System. Plasma heating during startup to ignition is provided by ohmic dissipation of plasma currents induced by a water-coolcd copper PFC set operating at low $(4-5 \mathrm{~T})$ to moderate $(8-9 \mathrm{~T})$ flelds. This $\sim 25-\mathrm{kV}, 2-\mathrm{GJ}$ bipolar system should be easily developed. The means by which the PFC is powered depends on the plasma energy and magnetic flux loss incurred during startup; the RFP scaling used in this study allows the startup power to be supplied from the electrical grid at power levels of 200-300 MWe for times on the order of 8-i0 s (Fig. III.G-4) and a total flux of $246 \mathrm{~Wb}$. A varsened 
transport, as reflected by the $\tau_{E} \propto I_{\phi}^{\nu} r_{p}^{2}$ scaling with the current exponent $\nu$ falling below $\sim 0.8$, increases flux and power requirements, eventually necessitating an oit-site energy-transfer element (1.e., a homopolar motor/generator). Even with the added cost of on-site energy storage, the relatively small amounts of stored and transferred energy ( 2 GJ compared to $\geqslant 10 \mathrm{GJ}$ for the OHC/EFC set and $\sim 50 \mathrm{GJ}$ for the TFC set in STARFIRE ${ }^{3}$ ) characterize this system as near state of the art.

Once the DT plasma is Ignited, the plasma heating system (1.e., PFC system) must supply $P_{\Omega}=25.3-\mathrm{MW}$ ohmic losses to the plasma and $\mathrm{P}_{\mathrm{PFC}}^{\Omega}=126.5-\mathrm{MW}$ ohmic losses in the PFCs, of which $\mathrm{P}_{\mathrm{OHC}}^{\Omega}=73.0 \mathrm{MW}$ is dissipated in the $\mathrm{OHC}$ set. If F- $\theta$ pumping current drive (Sec. III.K.) proves feasible, the OHC current can be reduced to zero, this power then being avallable to supply the current-drive power needs or for sale to the electrical grid. Generally, in terms of coll current density $\left(s_{c \phi} \simeq 6-7 \mathrm{MA} / \mathrm{m}^{2}\right)$, power density $\left(1-2 \mathrm{MW} / \mathrm{m}^{3}\right.$, including nuclear heating), and conductor/structural configuration, these low-voltage coils ( $<250 \mathrm{~V} /$ turn) require a technology that differs little from that used for TFTR. ${ }^{36}$ Inorganic (e.g., powdered-MgO) low-voltage Insulation that most probably would be co-swaged with the conductor and water-coclant channels will be required to harden these colls for operation in a more intense radiation fleld, however. In principle, these colls are similar to magnets used in paiticle accelerators. 107,108

2. Particle Control Systems.

a. Fueling. The CRFPR plasma would be refueled by advanced laser-ablation pellet-acceleration techniques rather than presently avallable pneumatic or centrifugal pellet infectors. The pellet injectors would operate to support or augment plasma-edge refueling at a level that remains to be determinad. The CRFPR plasma is 6-7 times more dense than piasmas proposed for fusion approaches like STARFIRE, ${ }^{3}$ but the factor of 3-4 reduction in plasma radius leads tu pellet injector requirements that differ little from the requirements generally envisaged for fusion. Injection of a DT pellet containing $\sim 10 \%$ of the plasma inventory every $\sim 0.4 \mathrm{~s}$, penetrating one-third of the plasma radius ( $\sim 50 \%$ of plasma volume), requires an injection speed of $\sim 100 \mathrm{~km} / \mathrm{s}$ and advanced laser-ablation technology. Under steady-state conditions, for the same $n \tau_{p}$, and for simflar total fusion powers, the pellet size and injection rate are independent of concept; these needs are generic to magnetic fusion. 
b. Particle Removal. As for other fusion systems, considerable uncertainty surrounds the pumped-limiter particle removal scheme adopted for the CRFPR design. A purely batch burn for these more-dense plasmas would give an unacceptably short burn pulse from the viewpoint of pulse power, energy transfer/storage, and thermal-mechanical fatigue; a means of helium-ash rewoval and impurity control, therefore, must be postulated. The higher average neutron and thermal load at the first wall ( $I_{w}=19.5 \mathrm{MW} / \mathrm{m}^{2}$ and $I_{Q w}=5-6 \mathrm{MW} / \mathrm{m}^{2}$, respectively) requires a larger fraction of the avallable first-wall area to serve a limiter function for a given split between radiative and convective energy loss from the plasma. Thirty-eight percent of the $112-\mathrm{m}^{2} \mathrm{first}$ wall is devoted to 24 poloidal limiter, compared to $\sim 7 \%$ of the $780-\mathrm{m}^{2}$ STARFIRE first wall being devoted to a single toroidal limiter. Both systems require a large fraction, $f_{R A D}>0.9$, of the total plasma energy loss to be radiated uniformly over the first-wa1' ...iter area $\left(4.6 \mathrm{MW} / \mathrm{m}^{2}\right.$ for CRFPR, $1.0 \mathrm{MW} / \mathrm{m}^{2}$ for STARFIRE) in order that manageable loc heat fluxes impinge onto the limiter as particle transport. The average heat flux at the limiter assciated only with particle transport is equal $\left(1.3 \mathrm{MW} / \mathrm{m}^{2}\right)$ for both the CRFPR and STARFIRE. The pumped limiter for both the high-power-density and low-power-density systems represents an advanced heat-transfer system, complicated by potentially high particle fluxes, sputter-erosion/redeposition rates, and neutron damage rates. In terms of heat and particle fluxes, however, the limiter represents a generic issue for fusion and should not be more difficult for the compact approach. The close coupling of the pumped limiter with the first-wall/blanket structure in the CRFPR, however, requires that the limiter survive for the life of the FPC, whereas more frequent changeouts can be postulated for the larger, low-power-density systems.

The plasma for the higher-power-density systems must coexist with a potentially higher instantaneous flux of inward-moving, high-atomic-number impurities, although the total impurity influx and critical impurity fractions for a self-heated plasma are not dependent on power density. Maintenance of a given impurity fraction, however, should be easier for the higher density plasmas. For either high- or low-power-density systems, it may be necessary, therefore, a) to operate with replenishable low-atomic-number coatings (probably more feasible in a system with an order-of-magnitude less first-wall/limiter area), or b) establish a high-atomic-number radiating gas mantle surrounding a plasma that is capable of preventing high-Z impurities from entering the plasma 
core. Generally, hot plasma edges are preferred in these circumstances. These ideas remaln to be substantiated, and the impurity-transport and gas-recycled models advanced so far for the RFP are primitive, particulazly with respect to a self-consistent treatment of edge-plasma radiation, sputtering, and impurity transport. This area deserves considerably more work to guide both existing and planned experiments as well as more-futuristic reactors.

Only preliminary estimates of impurity/ash-control schemes based on magnetic divertors have been maie in this study. The ability to divert the minority toroidal field presents an option for the RFP not generally avallable to the tokamak, which either diverts the minority (poloidal) field and must deal with the problem of Interlocked colls, or diverts the majority (toroidal) field and must address the problems of high forces, currents, and degraded (plasma pressure) confinement. Furthermore, the ability to use resistive coils located closer to the plasma leads to a magnetic divertor scheme of considerably enhanced efficiency as measured in terms of energy and space requirements. Resistive divertor colls may in fact be required even in otherwise superconducting low-power-density systems. Examination of the toroidal-field divertor for RFPs is being vigorously pursued for both the reactor and for the near-term, high-current RF? experiments. Key issues for the latter revolve around the increased energy (and cost) sto Id in the coil set and the effects of local perturbations in the conducting shell that surrounds the plasma.

c. Vacuum Pumping. All steady-state systems of similar total fusion power, governed by essentially the same edge-plasma physics, must deal with stmilar quantities of exhaust gas (1.09 MPa m$/ \mathrm{s}$ GWt for the CREPR compared to $0.79 \mathrm{MPa} \mathrm{m} / \mathrm{s}^{3}$ GWt for STARFIRE ${ }^{3}$ ). Expressed per unit first-wali area, the required pump speed for the smaller, high-power-density systems must be greater by approximately the increase in neutron first-wall loading. The increased fraction of the first-wall area that may be devoted to vacuum pumping can be reduced somewhat by a) operating with higher recycle rates, and/or b) taking advantage of the shorter radial flow path (1.e., thinner blanket/shield) to the pumps affordtd by the generally smaller FPC. Once outside the FPC, the vacuum-pumping requirement becomes almost independent of concept. The smallness of the high-power-density FPC, however, may make the "vacuum building" more feasible which for the size of the FPC being considered is more aptly termed a "vacuum room" (18-m diameter $\times 5-m$ high). 
Generally, the key issues for the vacuum-pumpling system remain similar for all fusion concepts: maintenance of acceptable impurity levels; neutron streaming through the vacuum ducts; reduced blanket efficiency (i.e., tritium breeding, energy recovery, shielding); and choices between vacuum building versus vacuum chamber versus vacuum trenches versus individuai pumps. Specific to the RFP, however, is the inadequate understanding of the role of the electrically conducting first wall, its function with respect to short-term plasma stability/equilibrium, and the degree to which this conducting first-wall shell can be divided or otherwise segmented for vacuum, impurity control, and assembly purposes. These vacuum slots can also cause fleld errors that in turn exacerbate the problems of high heat-flux and local peaking. The complexity of each problem and uncertainty in this area taken separately has prevented a clear analytical resolution; coupling these with the problems of locally enhanced transport, equilibrium/stability at local shell gaps, and the vacuum exhaust, therefore, presents an uncertainty that will remain unresolved for some time to come, in spite of its crucial impact on the operating space avallable for the CRFPR or other similar systems. Progress in this area will more likely occur on the experimental rather than the theoretical front.

3. Plasma Current-Drive System. Only preliminary analyses have been made to estimate the "F- $\theta$ pumping" current-drive parameters (Table III.K-II) for the RFP. The model used assumes ideal coupling between the current-drive colls and the plasma. Positioning of these low-frequency ( $50 \mathrm{~Hz})$, low-voltage (20-40 times the piasma loop voltage or $\sim 5-15 \mathrm{~V}$ ) colls near the first wall is inferred by these analyses in order to maintain both good coupling and low reactive energy $\left(E_{s} \simeq 260 \mathrm{~kJ}\right.$ ). Since $1 \mathrm{t}$ is desirable to minimize the associated reactive power $\left(\omega E_{S} \sim 13 \mathrm{MW}\right)$ and to maximize the circuit Q-value, future work in this area should focus on developing means of actively driving oscillating currents in or near each of the 24 first-wall sectors. Furthermore, if lower-frequency current drive (few $\mathrm{Hz}$ ) is possible, the existing PFC/TFC systems can be oscillated directly. Given that the base-case OHC losses ( $P_{O H C}^{\Omega} \simeq 72 \mathrm{MW}$ ) could be applied to drive a less-ideal current-drive circuit, considerable margin exists for supplying large current-drive inefficiencies $(>0.26 \mathrm{~A} / \mathrm{W})$ without seriously affecting the overall CRFPR power balance and recirculating power fraction. The tradeoff between current-drive efficiency and blanket neutronics performance as affected by increased near-first-wall structure and propensity 
for reduced tritium breeding remains a crucial issue for future design studies of the $F-\theta$ pumping current-drive circuit.

It is noted that the $0.42 \%$ plasma current fluctuation associated with F- $\theta$ pumping current drive/translates into a $1.70 \%$ power oscillation under constant-beta plasma operation. This level of fluctuation appears tolerable from the viewpoint of ofcillations in the thermal power delivered to all coolant circuits. For the copper-alloy first-wall/limiter system, this power oscillation corresponds to a secondary (thermal) strain fluctuation of $\leqslant 10^{-5}$ applied at $50 \mathrm{~Hz}$, and should present no serious fatigue problem. The effects of a $\sim 0.42 \%$ fluctuation imposed on the majority (pololdal) edge-plasma field or any minority-field (toroldal) divertor operation must be quantiiled by future magnetics calculations, but no serious problems are envisaged for magnetic divertor opericion in this connection at this time.

Preliminary/experimental evidence from $\mathrm{ZT}-40 \mathrm{M}$ indicates that separate oscillation of poloidal and toroid : circuits, respectively, is accompanied by a plasma response that maintained an oscillating trajectory along the minimum-energy $-\theta$ diagram. Given the continued operation of an efficient RFP dynamo to malntain the constant-helicity plasma, F- $\theta$ pumping of steady-state plasma currents should be possible. Demonstration of current drive in small, low-temperature, resistive plasmas, however, is considerably more difficult than for reactor-gride plasmas. The plasma resistance and dynamo-related anomalies represent a key issue for $F-\theta$ pumping current drive. Unlike high-frequency current drives that attempt to modify electron distribution functions to drive a net plasma current, the strong coupling of poloidal and toroidal circuits that allows the possibility for low-frequency $F-\theta$ pumping for RFPs actually becomes easter as reactor conditions are approached.

4. Nuclear Systems.

a. In-Vacuum Components. The in-vacuum components (IVCs) include the first-wall, the limiter, divertor channel walls, divertor plates, and other diagnostic surfaces in direct view of cold or hot plasma. This study and the following discussion focuses entirely on the first wall (Tabie III.L-IV) and 1imiter (Table III.L-III) issues.

The combination of high average design heat fluxes $\left(\sim 5 \mathrm{MW} / \mathrm{m}^{2}\right)$, potentially large fluxes of energetic $(\leqslant 1 \mathrm{keV})$ particles and high neutron fluxes, power densities, and fluences ( $\leqslant 20 \mathrm{MN} / \mathrm{n}^{2}, \sim 250 \mathrm{MW} / \mathrm{m}^{3}, 10-15 \mathrm{MWyr} / \mathrm{m}^{2}$, respectively) couples with the major role played by these IVCs on plasma performance, 
operational viability (1.e., refueling, impurity/ash control), and overall plant efficiency ( $>30 \%$ thermal power recelved by these systems) to make this system one of the most important and difficult to resolve. The $112-\mathrm{m}^{2}$ first-wall area for the CRFPR is comparable to the $62-\mathrm{m}^{2}$ limiter area for STARFIRE, ${ }^{3}$ which has a first-wall arca of $780 \mathrm{~m}^{2}$; the limiter area for STARFIRE is $7 \%$ of the total first-wall area, compared to $\sim 38 \%$ for the CRFPR. In terms of physical heat flux, the entire first wall for the CRFPR operates under limiter-like heat fluxes, with the actual limiter surface receiving a majority of the particle flux. Both the STARFIRE and CRFPR designs assume $f_{\text {RAD }}>0.9$ of the alpha-particle and externally applied heatirg power radiates uniformly over the first-wall/limiter surfaces; both systems have comparable limiter areas which recelve nearly the same heat flux in the form of plasma particles $\left(1.3 \mathrm{MW} / \mathrm{m}^{2}\right)$. Unlike the lower-power-density systems, however, the sputter fluence lifetime (mm erosion) for the CRFPR must occur when the neutron fluence lifetime (MWyr $/ \mathrm{m}^{2}$ ) is reached, since more-frequent changeout of the limiter alone is probably not feasible for the compact systems. As noted in Sec. IV.B.2., the impact of the erosion rates on the performance of a smaller but more-dense plasma is also an area where more theoretical insight is needed; regenerative, low- $\mathrm{Z}$ coatings may also be required, an approach that may be more feastble for the smaller, uniformly high-heat-flux surfaces suggested for the CRFPR.

The key issues for the IVCs envisaged for the CRFPR remain similar to those for fusion in general; the erosion at unknown but potentially serious rates of high-heat-flux surfaces and the possible need for low-Z, erodable coatings. Furthermore, the high-power-density systems are less -olerant of local heat-particle-flux peaking. Although the design of the first-wall/limiter coolant system would be eased by economically allowed reductions in neutron first-wa11 loading by factors of 2-3 (Sec. IV.C.), the uncertainties in erosion rates and the associated impact on both plasma and IVC design will remain large and crucial. Lastly, the combination of high-heat-flux IVCs and high-power-density blankets leads to the choice of pressurized-water-cooled IVCs and self-cooled liquid-metal breeding blankets, both driving a dual-media thermal cycle; a more complicated and potentially more costly thermal conversion system results if high overall thermal efficiencies are to be maintained (Sec. IV.B.8.). 
A long list of nuclear material issues can be identified for the high-strength copper alloys being suggested for the IVCs. Alloy stability in the intensely damaging and transmuting neutron flux under simultaneously imposed thermal and coolant-pressure stresses requires considerable study. A short menu for future work in this area is given below.

- Radiation creep of a stressed, high-performance coolant system.

- Radiation swelling of both disperston-hardened and solution-hardened copper-allny systems.

- Effects of large transmutation rates of $\mathrm{Cu}$ (Ni and $\mathrm{Zn}$ production), causing increased hardness/strength, reduced thermal conductivity, and increased electrical resistivity, depending on final location of the transmutation products ( $1 . e_{.}$: matrix versus grain boundaries or separate inclusions).

- Fabrication, assembly, and joining methods that allow use of dissimilar metals that may share the same $\left(\mathrm{H}_{2} \mathrm{O}\right)$ coolant system operating under high pressures $(1000-2000$ psi).

- Effects of helium and gas pruduction within the alloy structure by nuclear reactions and the impact of these gases on mechanical properties.

- Corrosion of copper alloy and ferritic steel by an oxidizing coolant that is subjected to radiolytic decomposition.

- Development of other high-heat-flux alloys that can deal with the above-listed problems.

b. Blanket. The high nuclear power density $\left(255 \mathrm{MW} / \mathrm{m}^{3}\right.$ peak, $27 \mathrm{MW} / \mathrm{m}^{3}$ average) within the PbLi blanket for this $I_{w}=19.5 \mathrm{~mW} / \mathrm{m}^{2}$ design point eliminates as an option solid breeders that are separately cooled by pressurized water, molten salts, or pressurized helium gas. The potential for either self-cooled or separately cooled FLIBE was not examined because of the wider thermal and breeding margins afforded by self-cooled liquid-metal blankets. Furthermore, because of reduced chemical reactivity with the pressurized-water coolants used to perform substantial cooling functions for the IVCs, and because of the neutron- and energy-multiplying advantages of lead, the $60 \%{ }^{6}$ Li-enriched $\mathrm{Pb}_{83} \mathrm{Li}_{17}$ compound was selected initially for the CRFPR blanket. A comprehensive optimization of this high-power-density blanket from materials, thermal, neutronic, and cost viewpoints was not possible; however, the results emerging from other larger studies instead were used, and more optimal blanket designs based on self-cooled PbLI are likely. The CRFPR blanket design (Table III.L-V), however, seems to provide a reasonable cholce, would operate efficlently in the 
low-field RFP environment, and offers a considerably simplified overall system compared to gas- or water-cooled solid-breeder systems.

The use of a thick $(\sim 20-\mathrm{mm})$ copper-alloy first wall in conjunction with a simpler first-wall/second-wall coolant manffolding scheme degrades the tritium-breeding ratio for the canonical blanket design used in this design study when examined by more realistic two-dimensional models. As shown in Appendix $C$, the tritium-breeding ratio can be recovered by a) reduced first-wall thickness, b) added enrichment of $\left.\sigma_{L}, c\right)$ improved albedo of the thin exoblanket shield, d) modest increases in blanket thickness, e) replacement of the $\mathrm{H}_{2} \mathrm{O}$ coolant with $\mathrm{D}_{2} \mathrm{O}$, and $\left.f\right)$ shifting the water-coolant manifolds from the firstwall region to the mid-blanket region or shield region. The first three design changes (decrease first-wall thickness from 20 to $5 \mathrm{~mm}$, increase $6_{\mathrm{L} i}$ enrichment from $60 \%$ to $90 \%$, replace $\mathrm{H}_{2} \mathrm{O} / \mathrm{B}_{4} \mathrm{C} / \mathrm{W}$ shield with $\mathrm{H}_{2} \mathrm{O} / \mathrm{HT}-9$ ) represent minor design changes and would be adopted by future studies. Slight increases in blanket thickness $(\sim 0.1 \mathrm{~m})$ are also acceptable from the viewpoint of maintaining an optimal design. The use of $a D_{2} 0$ coolant loop is viewed as a costly complication, and the placement of the water-coolant manifolds outside the blanket requires the use of radial, intersegment flow through tube sheets, although no added pumping power would be required; these latter two options are held at lower priority as a means to improve tritium-breeding margins in these thin, high-power-density blankets.

The need to satisfy percelved corrosion limits at the inboard structural (second) wall requires water cooling at that point, the first-wall return coolant being used for that purpose. This design complication causes a somewhat reduced overall thermal efficiency and can possibly be eliminated by a more exacting design of the blanket coolant-duct structure, possibly leading to increased pump power. These kinds of trade studies should be considered in future, more-elaborate studies. A better resolution of structural corrosion limits which are based on coolant-duct plugging problems rather than structural thinning limits could also reduce the need for a separately cooled second wall and the attendant reductions in thermal efficiency, increased design complexity, and a lessened separation of water and liquid-metal coolant circuits. The key issues for this blanket as applied to the CRFPR revolve around the following issues: 
- Maximum allowable structural temperature established by plugging-related corrosion limits. This issue strongly effects thermal efficiency, design complexity, safety, rellability, pumping power, and structural alloy choice (austenitics versus ferritics versus refractories).

- Tritium recovery from and inventory in the blanket and primary coolant loops.

- Structural design of blanket to support both the pumping pressures and inertial loads under high-power-density conditions for both transient (Including selsmic events) and steady-state conditions.

- Removal of heat from a highly nonuniform source while controlling local heating within both the coolant and the surrounding structure.

- Better resolution of the complex tradeoffs between overall nuclear performance, thermal efficiency, pumping powers, operational safety, design complexity, and cost associated with these higher-power-density blankets.

c. Shield. The CRFPR design proposes a thin $(\sim 0.10-\mathrm{m}) \mathrm{H}_{2} \mathrm{O} / \mathrm{Fe} / \mathrm{B}_{4} \mathrm{C} / \mathrm{W}$ shield positioned outside the blanket to allow $>99 \%$ recovery of the fusion energy and to minimize the nuclear heating in the water-cooled copper colls. This shell also defines the primary vacuum plenum connecting 24 radial intersector vacuum ducts to an outboard, equatorial-plane exit vacuum duct. The primary structural support and fluid-line chroughputs for the first wall, limiter, blanket, and TFCs are also provided by this system. The thermal (Table III.L-VI), mechanical, and nuclear demands on this shielding shell are minimal, although this shield forms a keystone to each of the $N=24$ sectors and provides the assembly for the full FPC torus and the single-piece (batch) maintenance scheme. The means by which these off-site subassembly, assembly, and testing operations would be conducted are based on a highly conceptual design, more detailed studies being required to illuminate problems in a system that at present: appears to be straightforward. The key issues for the shleld system revolve around the transient and steady-state loads exerted on a system that must react a large and complex array of electromagnetic, high-pressure-fluid, and gravitational forces. Replacement of the tungsten in this shield by other less costly material must also be examined, since the shield comprises $62 \%$ of the FW/B/S/TFC mass. Such redesign is advisable from the neutronics viewpoint also, In that Improved albedo would enhance tritlum breeding in the thin, high-power-density blanket. The reflector function rather than the shield function should be stressed by future work in this area with the use of 
pressurized-water-cooled steel rather than the $\mathrm{H}_{2} \mathrm{O} / \mathrm{Fe} / \mathrm{B}_{4} \mathrm{C} / \mathrm{W}$ composite adopted in this study as the canonical design.

d. Afterheat and Radwaste. Only preliminary estimates of the safety and environmental issues celated to the higher-power-density FPCs have been made, and the increased concern for nuclear afterheat and radwaste disposal (1.e., near-surface versus deep-geological burial) presents an important area for future study. Loss of coolant to the pumped limiter would result in its temperature increasing to $\sim 67 \%$ of the melting point if the limiter radiated to an actively-cooled first wall with an emissivity of 0.4 and without any direct conduction to a heat sink through structural supports. Roughened copper at room temperature can achieve an emissivity of 0.2 , and another increase of 0.2 can occur at the operating temperature. Loss of pressurized-water coolant to both limiter and first-wall/second-wall circuits would force these systems to within $79 \%$ and $58 \%$ of the (copper-alloy) melting point if the blanket remains actively cooled. The additional loss of PbLI coolant flow in the blanket will lead to a limiter and first-wall temperature rise that depends on the amount of natural convection allowed by the PbLi coolant circuit. Actual melting of any structural surface is unlikely for emissivities above 0.4 , but the resulting thermal excursion will cause alloy changes to an extent that will probably leave overheated components unusable. For emissivities below 0.2 under conditions where all coolant flow ceases and only natural convection in the PbLi is operative, local melting of the limiter may occur. Considerably more modeling, however, is required before the extent of active emergency cooling can be assessed for these high-power-density systems and the dependence of these needs on neutron first-wall loading is resolved. Genera11y, the afterheat problem is viewed primarily as a problem for the plant investment rather than one of public safety per se; emergency core cooling is expected to be a plant (investment) safety system, unlike the broader role played by similar systems in fission plants.

Neutron activation of the various FPC components has been estimated only at the level described in Table III.B-IX, with an emphasis being placed on the long-term problems of ${ }^{60} \mathrm{Co}$ and $63 \mathrm{Ni}$ gaiterated in the copper-alloy first wall, the TFC, and the PFC. The 20-min-thick $\left(\sim 2.2 \mathrm{~m}^{3}\right)$ canonical first-wall model will be reduced to 5-mm thickness in future designs for the neutronics reasons described in Appendix $\mathrm{C}$. The estimates of ${ }^{60} \mathrm{Co}$ and $63 \mathrm{NI}$ activity for the 20-mmthick first wall are based on a one-dimensional beginning-of-life model and give 
$1.03(10)^{7}$ and $2.05(10)^{6} \mathrm{Ci} / \mathrm{m}^{3}$, respectively, after one year of full-power operation at $I_{\mathrm{w}}=19.5 \mathrm{MW} / \mathrm{m}^{2}$. These concentrations are reduced by factors of $1.8(10)^{-4 / 2.9(10)^{-4}}$ and $1.1(10)^{-5} / 1.7(10)^{-5}$ for ${ }^{60} \mathrm{Co} / 63 \mathrm{NI}$ at the TFC and PFC, respectively. The Class $A$ and class $C$ near-surface burial limits ${ }^{153}$ for ${ }^{60} \mathrm{Co} / 63 \mathrm{NI}$, respectively; are $700 / 35$ and $-17000 \mathrm{Ci} / \mathrm{m}^{3}$; no entry for class $\mathrm{C}$ burial of ${ }^{60} \mathrm{Co}$ is avallable. ${ }^{153}$ Hence, in order for the first wall to qualify alone for Class $C$ near-surface burlal based on $63_{\mathrm{N} 1}$, dilution by a factor of $\sim 290$ would be required. If the first wall remains attached to the (drained) blanket and shield, the intrinsic system dilution factor for the copper-alloy first wall alone amounts to 70 , and further dilution by a factor of $\sim 4$ would be required. The cholces between dilution followed by class $c$ near-surface burial, disposal of a more-concentrated mass in deep-geological burial, or recycle of an important but active material ultimately must be made on the basis of economics and sound environmental/safety practices. Lastly, the radwaste issue for the TFCs and PFCs after one full-power year differ by factors of $2.9(10)^{-4}$ and $1.7(10)^{-5}$, respectively, for the $63_{\mathrm{N} 1}$ isotope; many years of full-power operation should be possible before the Class $C$ near-surface burial limits are exceeded in these FPC components.

The short- and long-term activation of other isotopes in the FPC (e.g., Pb, Fe, minor constitutents in the HT-9 and copper alloys) can present problems for near-surface disposal, Ref. 153 listing 27 key elements of concern. More detafled radioactivity analyses remain to be performed for the design reported here or variations thereof. The concerns related to short-term maintenance and long-term radwaste disposal remain to be quantifled in terms of related cost penalties and percelved licensing constraints. The tradeoffs between system economics and safety remain to be resolved; the inherently safe, "walk away" FPCs must be operated with such lower power density and high mass utilization as to raise serious questions of economic viability. Similar concerns have been cited even for counterpart approaches suggested for "second-nuclear-era" fission systems. 154 More detailed study is required to resolve whether the associated price is acceptable particularly when a) the afterheat issue for fusion may not be so much a public safety issue as one of protecting the plant (FPC) Investment, and b) whether radwaste dilution for near-surface burial should occur by operational design (i.e., lower power density and related alloy cholces), be a post-operations decision, or simply be abandoned in favor of more concentrated deep-geological burlal. 
5. Magnet Systems. The main confining fields are provided by induced and ultimately driven currents that in turn are generated by currents flowing in external TFCs and PFCs. In addition to the TFC and PFC sets, separate current-drive coils may be required, although a crucial tradeoff with blanket neutronics performance remains to be resolved. If very-low-frequency $F-\theta$ pumping current drive proves feasible, the primary PFC/TFC system can also be used for current drive. Lastly, feedbaci control of local instabilities may have to be provided by conductors dispersed outside the blanket/shield area, which like the F- $\theta$ pumping current-drive colls would operate with low current and low voltage. The current-drive and feedback circuits and associated performance goals have not been sufficiently resolved to permit an assessment other than the need to resolve better the controlling physics. In addition, little work beyond concept definition has been done for the toroidal divertor coil and associated magnetics. This section, therefore, deals only with the TFC and PFC sets required for plasma confinement.

a. Toroidal-Field Coils. As seen from Table III.F-III, in terms of size (1.46-m current radius, 75-mm radial thickness, 0.6-m toroldal extent, 3.0-tonne mass), peak field $\left(B_{c \phi}=0.7 \mathrm{~T}\right)$, engineering current density $\left(j_{c \phi}=7.1 \mathrm{MA} / \mathrm{m}^{2}\right)$, and ripple constraint ( $N=24,0.3 \%$ at the plasma surface), the TFC should not represent a major R\&D issue. The strong radiation environment, even outside the thin blanket/shield system, however, necessitates the use of powdered inorganic electrical insulation (e.g., MgO) co-swaged with coolant channels ( $0.5 \mathrm{MW}$ ohmic, $1.25 \mathrm{MW}$ nuclear per $\left.\mathrm{TFC}, \sim 2(10)^{4} \mathrm{r} / \mathrm{s}\right)$ and conductor. The reaction of internal conductor loads, the resolution of body forces (2.7 MN), and the means by which power and coolant are delivered to these multiturn coils have been estimated and should not present a serious design problem.

A key issue for the TFC design is the degree to which current overloading is required during the inftial injection of toroldal blas flux just prior to the plasma initiation. The extent to which the RFP dynamo can generate internal toroldal flux from the conversion of toroidal current will dictate the startup demands placed on the TFC system. The present design takes maximum credit for the RFP dynamo, wherein the initial bias field is equal to the final reversed field, $B_{R \phi}=-0.4 \mathrm{~T}$, and the dynamo ultimately creates the steady-state on-axis toroldal field, $B_{\phi}(0) \simeq 9.5 \mathrm{~T}$, during the $\sim 8-10 \mathrm{~s}$ plasma current rise towards Ignition. The extreme condition for TFC operation would require the plasma chamber to be filled with a toroldal flux equal to that present in the steady- 
state, ignited plasma, $\phi=\left\langle\mathrm{B}_{\phi}\right\rangle \pi_{\mathrm{p}}^{2} \cong\left|\mathrm{B}_{\mathrm{R} \phi}\right| \pi \mathrm{r}_{\mathrm{p}}^{2} / F \cong 5-6 \mathrm{~Wb}$. In this case the initial toroldal bias fleld would be increased to $3-4 \mathrm{~T}$, requiring a transient current density in tive TFC of $\sim 10$ times the steady-atate value; the advantages of increasing the toroldal fleld within the plasma through the RFP dynamo are apparent.

b. Pololdal-Field Colls. The PFC set dominates the drained FPC mass: 394 tonne for the OHCs, 404 tonne for the EFCs, 73 tonine for the TFCs, and 234 for the $\mathrm{FW} / \mathrm{B} / \mathrm{S}$, with an additional $\sim 800$ tonne of 'bL1. The maximum field for the back-biased ( $I_{\phi}=0.0 \mathrm{MA}$ ) condition is $9.0 \mathrm{~T}$, which decreases to $4.5 \mathrm{~T}$ upon achievement of the full plasma current $\left(I_{\phi}=18.4 \mathrm{MA}\right)$. Because of the good electrical coupling between the OHC and plasma, the external PFC current amounts to $I_{c \phi}=22 \mathrm{MA}$, and the total stored energy is $1.7 \mathrm{GJ}$, which is a factor of $\sim 6$ less than for the $\operatorname{STARFIRE}^{3}\left(\mathrm{I}_{\phi}=10.1 \mathrm{MA}\right)$.

Like the TFC set, the PFCs require only state-of-the-art technology, with the exception of the need for radiation hardening and the use of inorganic (e.g., powdered-Mg0) electrical insulation. The tightly integrated FPC requires the lower PFC elements to form a receiving support bed or strong back for the FW/B/S/TFC, whereas the upper PFC elements must be vertically mobile for purposes of assembly and malntenance of the FW/B/S/TFC unit. The conductor gap in the EFC set positioned about the outboard equatorial plane is needed for flutd and electrical services to the FPC and presents no serious magnetics (equilibrium) problem. Considerable mechanical and electrical design is required, however, to assure an optimal electrical lead configuration for these multiple-turn series/parallel conductors; it is expected that PFC conductor locations, sizes, and current densities w111 change somewhat under closer design scrutiny. The structures and force analyses also have been limited and deserve additional consideration, particularly in view of the role played by the PFCs in the overall support and maintenance scheme. Lastly, the PFC se: (i.e., the OHCs, specifically) represents the primary energy storage for a grid-powered startup; the preliminary assessment of this aspect of the CRFPR is given in the following section.

6. Electrical and Mechanical Systems. This section briefly reviews and assesses what little deslgn hes been carried out on electrical and mechanical systems that directly support the FPC. 
a. Energy Transfer And Storage (ETS). The primary ETS systems would deliver startup power to the PFC, and little information is available beyond the definition of the OHC/EFC circuit given in Table III.F-V. The F- $\theta$ pumping current drive and stability/equilibrium feedback control will also require an ETS system, but considerably more work is required before these systems can be defined and, therefore, subjected to a preliminary assessment.

The primary energy storage for the startup power is the OHC/EFC set, which is charged on a 15- to 20-s time scale directly from the electrical grid. With the electrical grid removed from the FPC circuit, the RFP is established and the plasma current is raised to $\sim 12 \mathrm{MA}$ in $\tau_{\mathrm{R}}=1.2 \mathrm{~s}$, the initial current ramp being $32 \mathrm{MA} / \mathrm{s}$. The electrical grid is then reconnected to the FPC circuit once the required drive power drops to acceptable levels; the plasma current then ramps to the final value of $I_{\phi}=18.4 \mathrm{MA}$ in $\tau_{L} \simeq 8 \mathrm{~s}$. The peak grid power approaches $350 \mathrm{MWe}$ at $1 \mathrm{kV}$. Upon ignition and achievement of a steady-state burn, $\sim 246 \mathrm{~Wb}$ are required (26 Wb resistively consumed). The OHC, EFC, and plasma require $73.0,53.5$, and 24.5 MWe, respectively, to supply ohmic dissipation; it is also proposed to supply this 150.0-MWe load from the electrical grid. As noted in Sec. IV.B.3., the OHC currents would be driven to zero upon initiation of the plasma current drive, with the 73.0-MWe grid power requirement being used in part by the $F-\theta$ pumping circuit.

If the startup power requirement is too large, then a rotating machine (e.g., homopolar motor/generator) capable of receiving, storing, and transferring $\sim 1$ GJ will be required. The use of the power grid versus the use of an infrequentiy operated on-site ETS system represents a modest economics rather than technical feasibility issue and is influenced most strongly by unresolved physics (transport) issues.

b. FPC Structural Support, Assembly, and Maintenance. The FPC mechanical integration described in Sec. III.L.3. proposes an off-site FPC assembly from 24 Identical toroidal segments that have been separately subjected to electrical, thermal-hydraulic, and vacuum testing. After similar tests of the fully assembled FW/B/S/TFC assembly, that unit would be shipped to the powerplant site and loaded into the PFC set and associated support structure. The problem of making and breaking a large number of pressurized-water and PbLi coolant-header connections can be reduced somewhat by partially including these fluid mains into the $\sim 300-t o n n e$ FW/B/S/TFC prior to lowering into the cradle formed by the PFC set, pillar supports, and associated strong back. As for any 
remote maintenance operation of this kind, the joining of high-pressure coolant lines, high-current electrical leads, and vacuum ducts (if a vacuum pillbox is not used) represents a key development area.

The key engineering issue for the tightly integrated FPC depicted in Fig. III.L-16 is that of system reliabllity. An assessment of reliability is not possible without a more fully evolved design and (ideally) related operating experience and data base. An Interesting trade exists between the mean-time-to-failure and the mean-time-to-repair and should be given high priority in assessing the relative merits of the high-power-density approach, as reflected in the annual plant factor, $p_{f}$, and ultimately in the COE. A key Issue deserving further study is the relative ease and restart rellabflity associated with full-FPC maintenance compared to replacement of a small fraction of a considerably larger torus.

7. Tritium systems. No work was done on tritium system requirements other than the vacuum design presented in Table III.J-I. The technical requirements of fueling and particle control, tritium breeding, and tritium containment for the CRFPR will be similar to other systems based on a self-cooled PbLi blanket. Tritium solubility 1 and extraction from the PbLi breeder remaln as crucial unknowns, with a 1-ppm concentration giving a $0.8-\mathrm{kg}$ blanket inventory; at least as much tritium is expected in the PbLi coolant external to the FPC. Tritium transport into the primary water coolant through the water-cooled second wall represents an area of concern; other water/PbLi interfaces within the FPC are at least doubly contained. For the recycle coefficients listed in Table III.J-I, the flow of tritium through the pellet-injector/plasma/1imiter/vacuum loop amounts to $1.7(10)^{-4} \mathrm{~kg} / \mathrm{s}$, compared to $0.073 \mathrm{~kg} / \mathrm{s}$ flowing in the PbLi coolant if tritium could be malntained at a l-ppm level. The rate of tritium leakage from the primary coolant streams into the intermediate heat-exchanger loops and eventually into the environment through the steam generator and turbine cannot be assessed until a steam cycle design is avallable, but tritium containment in this area is viewed as a crucial issue.

8. Balance-of-Plant Systems. A Balance-of-Plant. (BOP) design was not conducted, nor was a specific steam cycie suggested. The power and flow split between the pressurized-water $(4900 \mathrm{~kg} / \mathrm{s}, 1403 \mathrm{MW})$ and $\mathrm{PbLi}(72,840 \mathrm{~kg} / \mathrm{s}$, $1962 \mathrm{MW}$ ) coolant loops is such that considerable steam raising will be required by the water loop; the power delivered to the primary coolant water is too large for use only in feedwater heating while simultaneously expecting a good overall 
plant efficiency. Hence, the design of a high-efficiency dual-media power cycle represents an important area for future work. A similar area of concern can be Identified for the MARS tandem-mirror reactor. 5,6 The efficient use of dual-media cycies may become a necessity for higher-power-density tokamak reactor designs.

A major goal of the compact, high-power-density approaches is to separate and isolate better the FPC and other nuclear support systems from an ideally nonnuclear BOP. Licensing procedures, construction costs and schedules, quality assurance, and overall plant operations would be considerably improved by a better separation and definition of nonnuclear versus nuclear systems. The level of FPC integration with the BOP provided by this study has not been sufficient to assess the advantages offered by the compact, high-power-density systems in this respect, although strong advantages are perceived on a qualitative basis. Future study should stress this important area, while simultaneously selecting BOr systems and layouts that minimize the pathways between the nuclear FPC and the nonnuclear BOP. 


\section{Economic Assessment}

The CRFPR design originally selected for study from the Ref. 15 parametrics study (Table II.D-III) is a minimum-COE, $P_{E}=1000-\mathrm{MWe}(\mathrm{net}), I_{\mathrm{w}}=19.5 \mathrm{MW} / \mathrm{m}^{2}$ system. Although Figs. II.D-6 and II.D-7 show the effects on COE of decreasing $P_{E}$ and $I_{w}$, respectively, the primary goal of the present study was to examine through a conceptual but fully integrated FPC design the problems and promise of the 1000-MWe, high-power-density reactor. Minimum-COE, 100n-MWe power plants, however, may not present an optimal system with which to demonstrate technical and economic risk for fuston. Furthermore, the choice of the high-power-density, 1000-MWe system was infiuenced by perceived needs based on recent utility history in the U.S., with unpredictable future markets perhaps requiring a broader spectrum of capacities. Part of that future spectrum possibly may include systems with higher $\operatorname{COE}$ if the total capital investment could be reduced. In addition, the technology required of the $I_{W}=19.5 \mathrm{MW} / \mathrm{m}^{2}$ base case being considered here may be too advanced for risk demonstration, and both lower first-wall neutron loading and lower net power systems may be more optimal for purposes of market penetration and confidence building. Lastly, the tradeoff betwen system performance, cost, and the degree of passive safety is yet to be resolved for fission power, 154 and relevant options should also be considered for fusion. For these reasons, the parametric systems model used originally in Ref. 15 to Identify the minimum-COE system of the 1000-MWe(net) class has been used to examine the economics and operational characteristics of lower-wa11-loading, lower-power-density CRFPR designs. These systems, nevertheless, remain compact and high-power-density when compared to most superconducting fusion approaches proposed to date, recognizing that the factors of 10-30 in engineering power density that presently separate these two approaches to fusion power give a broad range of options.

1. The Parametric Systems Mode1. The parametrics model originally reported in Ref. 15 has been generalized by allowing consideration of arbitraxy confinement times in search of minimum-COE, rasistive-coll RFP reactors. Figure IV.C-1 gives a logic diagram of this mode1, and Fig. IV.C-2 depicts both the simplifled geometry and engineering energy balance used by this model. The overall physics and englneering models, systems approach, and data base are reported in Ref. 15. Table IV.C-I lists both fixed and varied parameters used to assess the cost sensitivity, as well as defining key notation. The fixed physics and engineering parameters list on Table IV.C-I are similar to the 
base-case values suggested in Table II.D-III and modified by the analyses given in Sec. III. As Indicated on Fig. IV.C-1, the parametrics model identifies optimal system parameters in a set of nested search loops centered on a convergence operation for the engineering $Q$-value, $Q_{E}=1 / \varepsilon$, and the specified net electric power, $P_{E}$, where $\varepsilon$ is the recirculating power fraction. For a given total coil thickness, $\delta_{c}$, this inner iteration searches for the value of $Q_{E}$ that yields the specified $P_{E}$ as the split between the TFC and PFC geometry varies, subject to the constraints of equal (but unspecified) coil current densities and the matching of fixed engineering and physics parameters indicated in Fig. IV.C-1. The total coil thickness $\delta_{c}$ that produces a minimum-COE design for an otherwise fixed geometry, including plasma minor radius, is first determined after convergence of the set $\left(Q_{E}, P_{T H}\right)$ for a given $P_{E}$. The outerloop optimum is then determined as a function of plasma radius, $r_{p}$, which shows a higher-order (lower) COE minimum. Generally, $r_{p}$ is used as a display variable, with the respective minimum-COE design corresponding to a particular value of $P_{E}$ and aspect ratio. The outermost loop then varies the plasma aspect ratio, $A=R_{T} / r_{p}$, in search of an even lower minimum-COE system, although within realistic bounds, the optimum in $A$ is fairly flat for the RFP. These fully-cost-optimized CRFPR design points are then examined as a function of $P_{E}$ and the physics, engineering, and economic variailes listed both on Fig. IV.C-I and Table IV.C-I.

2. CRFPR Cost Tradeoffs. This parameter study examines primarily the effects of decreased neutron first-wall loading under conditions of fixed net electric power and under conditions of decreased net electric power. As seen from Fig. IV.C-1, $I_{w}$ emerges as an output and is expressed as a function of $r_{p}$ for all minimum-COE designs of constant $P_{E}$. and $A$.

The first set of calculations varies the aspect ratio for the $P_{E}=1000-M W e(n e t)$ design point in order to demonstrate the relative insensitivity of the minimum-COE point to changes in this variable. Figure IV.C-3 shows a shallow COE minimum in $A$, but for all intents and purposes the $C O E$ for these cost-optimized designs is independent of A. Subsequent sensitivity studies, therefore, $f i x$ at the base-case value of 5.35 . Shown also on Fig. IV.C-3 is the dependence of the Unit Direct Cost [UDC( $\$ / k W e)], Q_{E}$, and $I_{w}$ on $r_{p}$, each curve giving the locus of minimum-COE designs for the indicated value of $r_{p}$. 


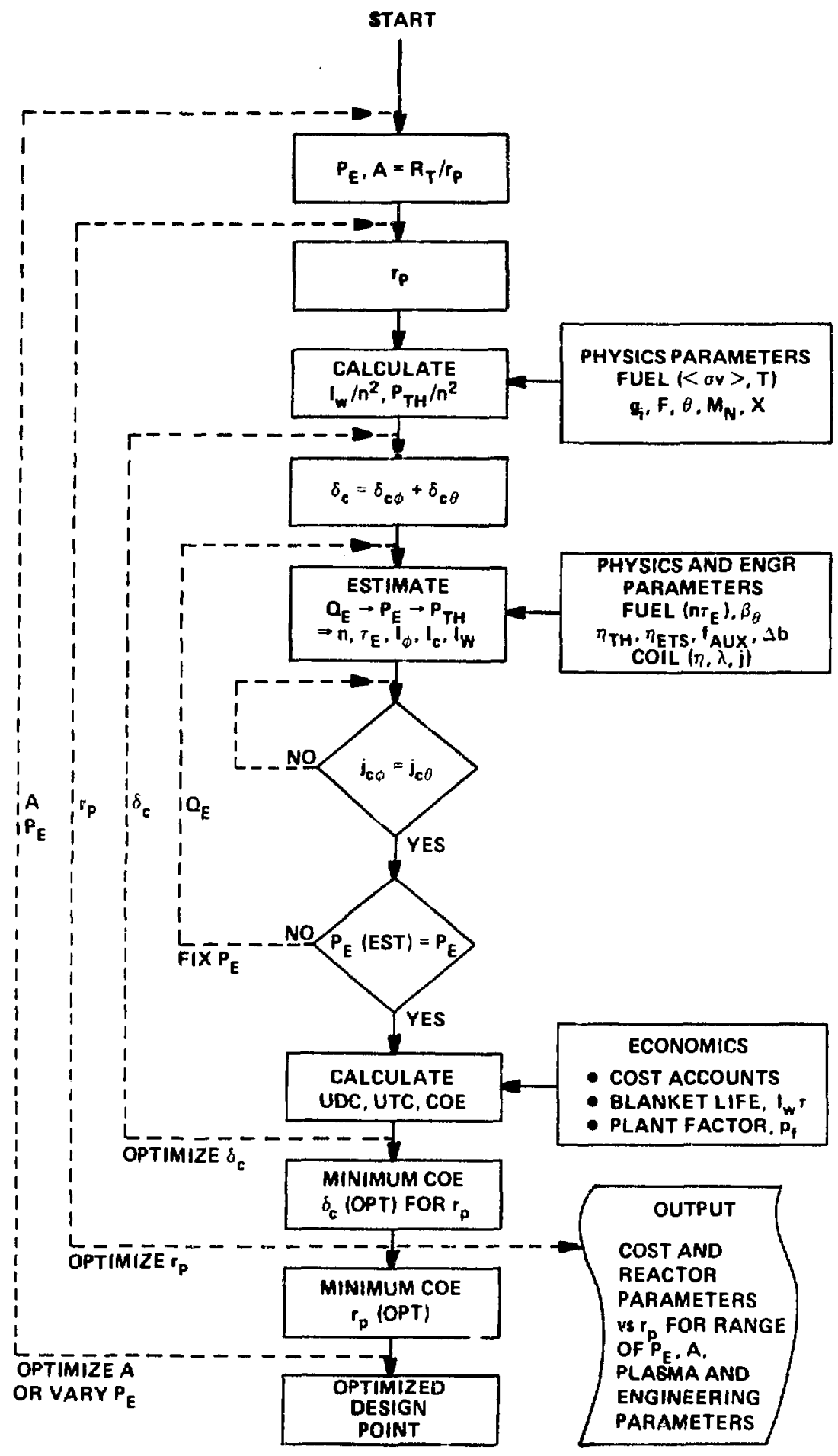

Fig. IV.C-1. Logic diagram for the simplified parametrics systems model used to assess economic sensitivity of the CRFPR. 

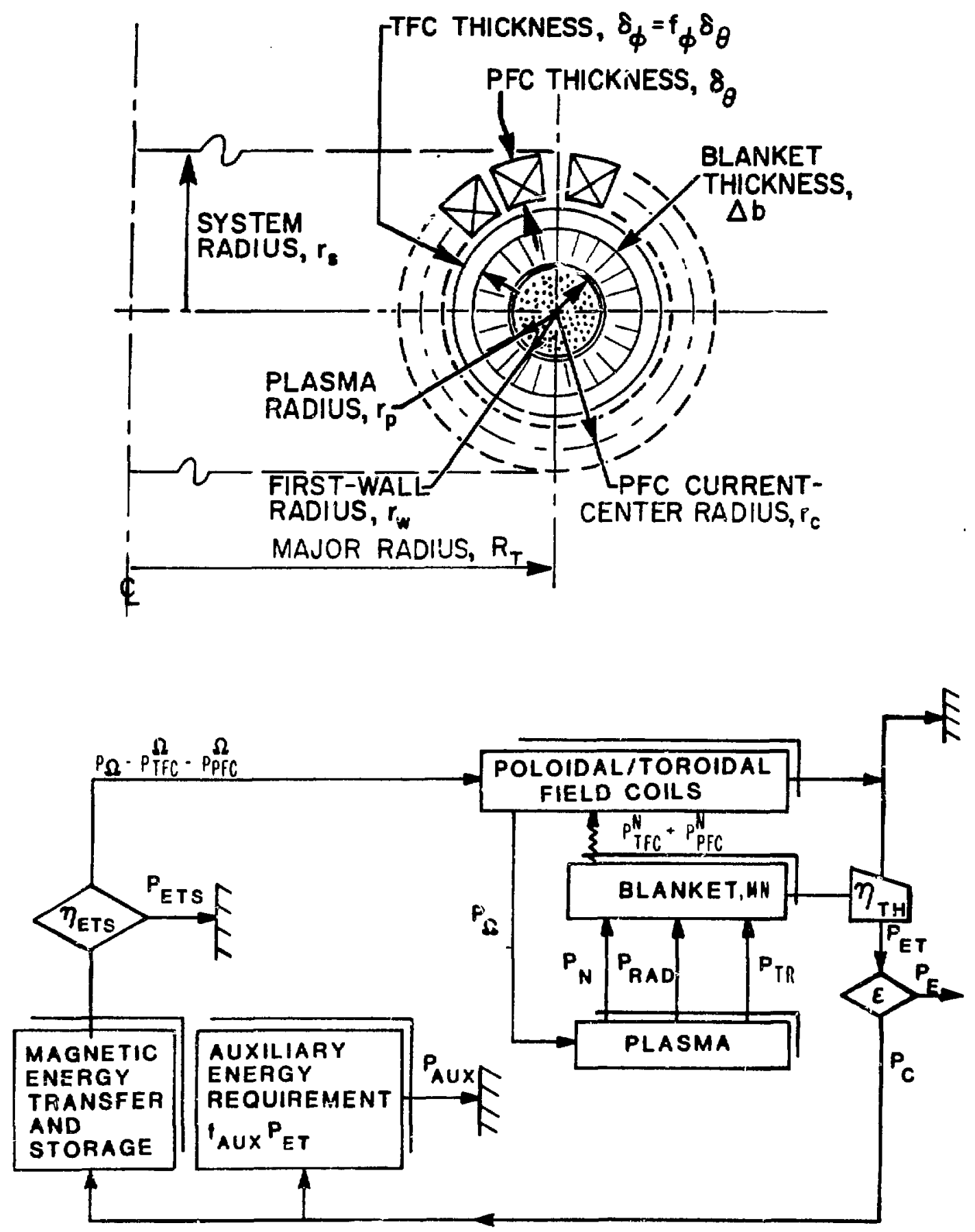

Fig. IV.C-2. Geometry and engineering encrgy balance used in the simplified parametrics systems model of CRFPR. 
LIST OF FIXED AND VARIED PARAMETER USED IN COST SENSITIVITY STUDY

\section{PLASMA PARAMETERS}

Minor plasma radius, $r_{p}(m)$

Major plasma radius, $\mathrm{R}_{\mathrm{T}}^{\mathrm{P}}(\mathrm{m})$

Plasma aspect ratio, $A=R_{T} / r_{p}$
Plasma density, $n\left(10^{20} / \mathrm{m}^{3}\right)$

Plasma temperature, $T(\mathrm{keV})$

Frofile factors, $g_{i}$

Ignition parameter, $n \tau_{\mathrm{E}}\left(10^{20} \mathrm{~s} / \mathrm{m} ; 2\right)$

Alcator coefficient, $\tau_{E} / \mathrm{nr}_{\mathrm{p}}^{2}\left(10^{-2} \mathrm{l}_{\mathrm{s}} \mathrm{m}\right)$

$P$ inch parameter, $\theta$

Reversal parameter, $F$

Plasma/wall radius ratio, $x=r_{p} / r_{w}$

Poloidal beta, $\beta_{\theta}$

ENGINEERING PARAMETERS

Thermal-conversion efficlency, $\eta_{\mathrm{TH}}$

Blanket/shield thickness, $\Delta b(m)$

Energy transfer and storage efficlency, 证TS

Auxiliary power fraction, $\mathrm{f}_{\mathrm{AUX}}$

Coil parameters

- electrical resistivity, $\eta\left(10^{-8}\right.$ ohm m)

- conductor filling fraction, $\lambda_{c}$

Net electrical power, $\mathrm{P}_{E}$ (MWe)

ECONOMIC PARAMETERS (c)

Return on Investment (\%/yr)

Operating cost (\%/yr)

First-wal1/blanket life, $I_{w} \tau\left(\right.$ MWyr $\left./ \mathrm{m}^{2}\right)$

Plant factor, $p_{f}=\left(365-\tau_{u}-\tau_{s}\right) / 365$

- unscheduled, $\tau_{u}$ (days/yr)

- scheduled, $\tau_{s}$ (days/yr)

$>1 \mathrm{replacement} / \mathrm{yr}$

$<1$ replacement/yr

- unit costs $\left(\$ / \mathrm{kg}, \$ / \mathrm{m}^{3}\right)$
VALUE (BASE CASE)

Varied $(0.71)$
Varied $(3.80)$
Varied $(5.35)$
Varied $(6.55)$
$10\left[\mathrm{~T}(\mathrm{r}) \propto \mathrm{J}_{\mathrm{o}}(\mu \mathrm{r})\right]$
$\mathrm{BFM}(\mathrm{MBFM})$
$1.60\{1.54)$
$5.0(\mathrm{a})$
$1.45(1.55)$
$-0.2(-0.12)$
0.95
$0.20(0.23)$

0.35

1.30

0.60

1.0 (b)

0.07

1.8

0.7

Varied (1000.)

15

2

15

$\leqslant 0.76$

60

${ }_{28}^{28} p_{f} I_{W} /\left(I_{W} \tau\right)$

Ref. 15 (d)

(a) Used for comparison purposes only.

(b) Inferred long-pulsed or steady-state operation.

(c) Based primarily on the guidelines given in Ref. 68 and modified by subsequent large-reactor studies. ${ }^{3}$ All costs are referenced to 1980 dollars.

(d) Generally conservative relative to the STARFIRE cost data base ${ }^{3}$ for the FPC costing. 


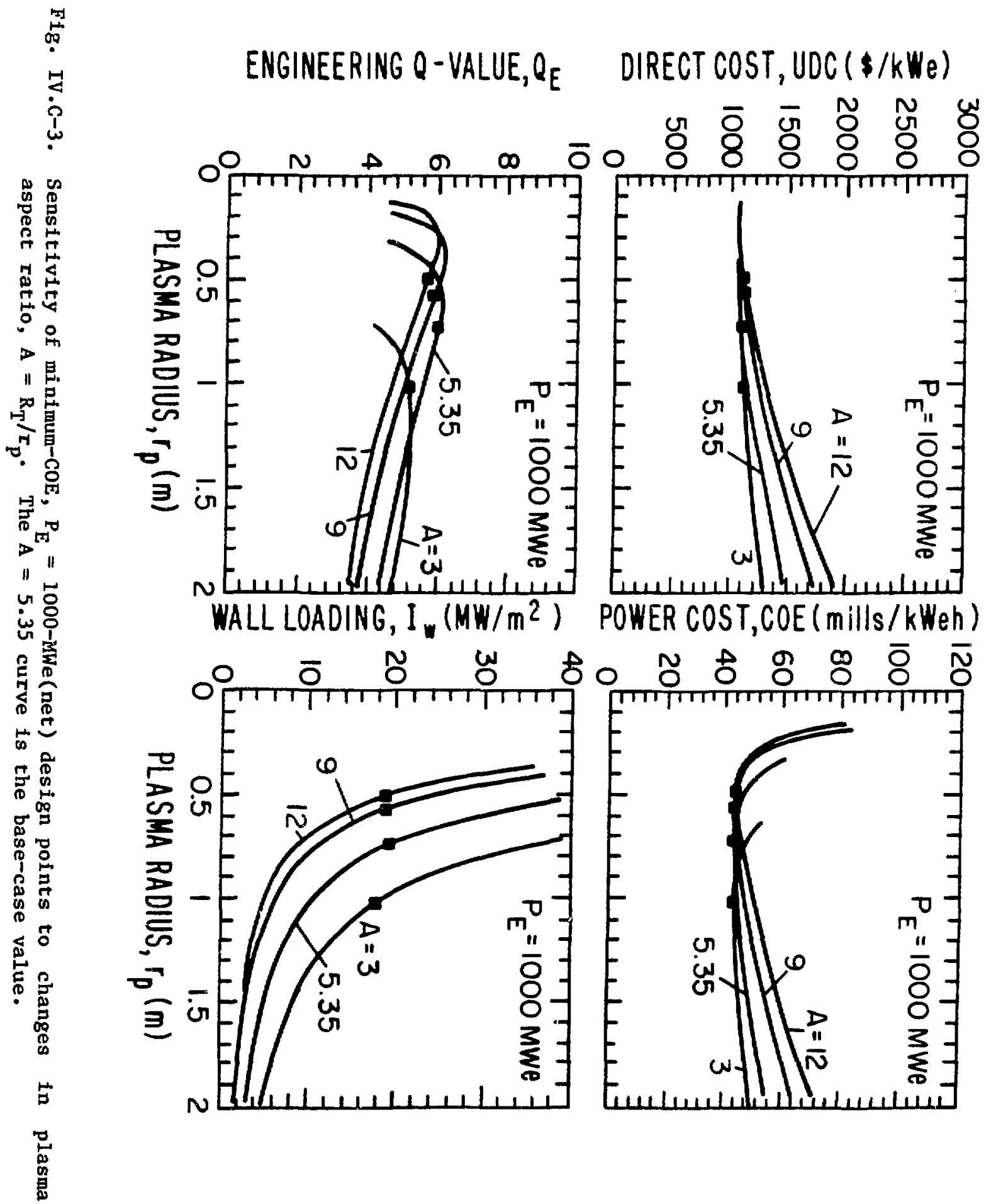


The cost and efficiency changes resulting in the dependence of $\operatorname{COE}$ on $r_{p}$ depicted in Fig. IV.C-3 are ca sed by a number of competing effects. The following observations are listed to aid the interpretation of these results.

- Increased $r_{p}$ for a fixed $P_{E}$ requires that $I_{w}$ decrease. Since $P_{E}$ is approximately proportional to $I_{w} r_{p^{2}}$, increased A decreases $I_{w}$ for fixed $r_{p}$, as is illustrated on Fig. IV.C- 3 . This argument is based simply on geometry. As $I_{w}$ decreases because of increased $r_{p}$ : the FPC power density decreases, resulting in an increased UDC. The resuiting increase in UDC drives $C O E$ upward as $r_{p}$ is increased at fixed $P_{E}$ and $A$.

- The increase in COE with increased $r_{p}$ is also caused by an increase in recirculating power delivered to the resistive colls (i.e., $Q_{E}$ decreases with increased $r_{p}$, as seen from Fig. III.C-3). The $Q_{E}$ decreases because of increased coll resistive losses. As $r_{p}$ increases, pressure balance demands slightly increased plasma currents, which in turn will effect the coll currents. Since pressure balance dictates $I_{W} \propto I_{\phi}^{4} / r_{p}^{3}$, the total power is proportional to $I \frac{4}{A} / r_{p}$, which for fixed $\mathrm{P}_{E}$ and $A$ approximately required $I_{\phi}$ o $r_{p}^{l / 4}$. The coll currents, however, may actually decrease because of better electrical coupling, depending on the value of $A$ when $r_{p}$ is increased. Increasing $r_{p}$ for a fixed $A$ increases $R_{T}$ and hence the coil resistance, which tends to override any decrease in coil current, giving a net increase in resistive coll losses and decreases in $Q_{E}$ as $r_{p}$ increases.

- As $r_{p}$ decreases for a fixed $A$, the electrical coupling between plasma and coll rapidly decreases, increased coll currents are then required to drive a given plasma current, and $Q_{E}$ rapid1y decreases. The COE rapidly increases as $r_{p}$ is decreased.

- As $r_{p}$ decreases for fixed $A$ and $P_{E}$, the rapidly increasing $I_{w} \propto 1 / r_{p}^{2}$ causes the plant factor, $p_{f}$, to diminish, in accordance with the algorithm given in Table IV.C-F. This decrease in $p_{f}$ also contributes to the rapid increase in $C O E$ as $r_{p}$ is decreased.

In summary, decreasing $Q_{E}$ and $p_{f}$ contributes to Increasing CoE with decreasing $r_{p}$, and decreasing (more slowly) $Q_{E}$ along with increasing UDC causes the $\operatorname{COE}$ to increase as $r_{p}$ increased. The COE optimum depicted in Fig. IV.C-3 results, this optimum being relatively insensitive to $A$, with a shallow $C O E$ minimum occurring near the base-case value of $A=5.35$.

From the sensitivity analysis given in Fig. IV.C-3 it is clear that systems with lower values of $I_{w}$ for a given $A$ are possible for increased $r_{p}$ and moderate increases in COE. Systems with lower $I_{w}$ are also possible for FPCs of the same physical size as the base case by simply generating less power. The cost tradeoff associated both with off-optimum systems $\left[r_{p}>r_{p}(O P T)\right]$ and lower- $P_{E}$ systems is displayed in Fig. IV.C-4, where $r_{p}(\mathrm{OPT})$ is the plasma radius at which the COE is minimized. The general behavior of these curves follows that 
described for Fig. IV.C-3. The rapid increase in the minimum-COE as $P_{E}$ is decreased res (from the strong (nuclear) economy of scale built into the costing procedure and data base used, 68 as depicted graphically on Fig. II.D-7. Again, the increase in COE for plasma radii below $r_{p}$ (OPT) results from rapidly decreasing $Q_{E}$ (1.e., poor electrical coupling to plasma) and $p_{F}$ (1.e., increased $I_{w}$ and decreased FPC chronological life), whereas the Increasing COE for $r_{p}>r_{p}(O P T)$ results from decreasing $Q_{E}$ (1.e., increased coil resistance as $R_{T}$ increases) and increasing UDC (i.e., increased FPC mass and poorer mass utilization). The values of plasma and coll currents for the parameter study described by FIg. IV.C-4 are shown on Fig. IV.C-5 as $r_{p}$ varies away from $r_{p}(O P T)$. As previously noted, operation to the left of the minimum-COE point results in poor electrical coupling, increased coll currents and ohmic losses, decreased $Q_{E}$, and rapidly increased COE. In addition, the rapid increase in $I_{w}$ as $r_{p}$ decreases also decreases the plant availability, again causing the $\operatorname{COE}$ to increase rapidly. The good coupling to the right of $r_{p}$ (OPT) is not sufficient to overcome the increased coll resistance or the $Q_{E}$ decreases, and $C O E$ Increases again.

The changing plasma density and FPC mass utilization that occurs in the parametric variations are illustrated on Fig. IV.C-6. The Alcator parameter, $n r_{p}^{2}$, varies by only a factor of 2.3 over the range of minimum-COE points depicted. The FPC mass utilization lies in the 0.4- to 0.6-tonne/MWt range for the minimum-COE design point and rapidly increases for lower neutron first-wall loadings, although only moderate increases in COE occur (Fig. IV.C-4) with increased $r_{p}$. These FPC mass utilizations, although increasing, remain far below those predicted for superconducting fusion approaches (a value of 5.7 tonne/MWt is predicted for STARFIRE ${ }^{3}$, Table II.C-IB). The possibility for batch, single-piece FPC maintenance is compromised, however, for the higher-COE, lower $-I_{w}$ designs.

The comparison of the economic confinement time, $\tau_{E}$ (OPT), with RFP scaling is shown on Fig. IV.C-7, which also indicates the minimum-COE design point for the range of $P_{E}$ considered. Following the algorithm described on Fig. IV.C-1, $\tau_{E}(O P T)$ is the energy confinement time required to achieve the global minimum or local minimum-COE values; no transport scaling restricts this optimization. Figure IV.C-7 demonstrates how the goal of minimum $C O E$ must be tempered with the realities of physics. The $\tau_{E} \propto I_{\phi}{ }_{\phi}^{r}{ }_{p}^{2} f\left(\beta_{\theta}\right)$ RFP scaling remains to be documented fully, particularly for the current exponent and the beta function. 

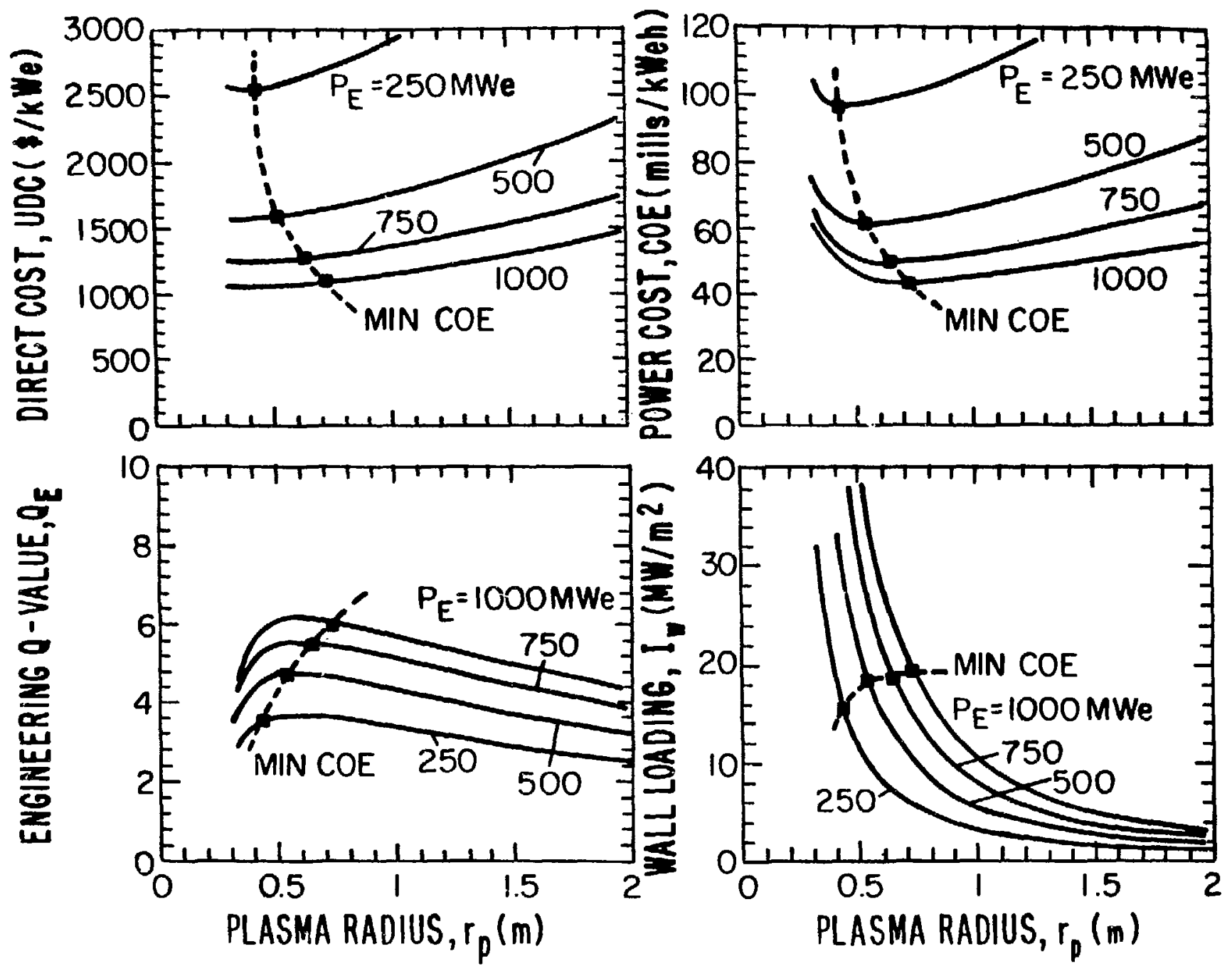

Fig. IV.C-4. Sensitivity of minimum-COE design points to changes in net electric power, $P_{E}$, for the $A=5.35$ base case, showing $U D C, C O E, I_{w}$, and $Q_{E}$ as a function of $r_{p}$ and $P_{E}$. 

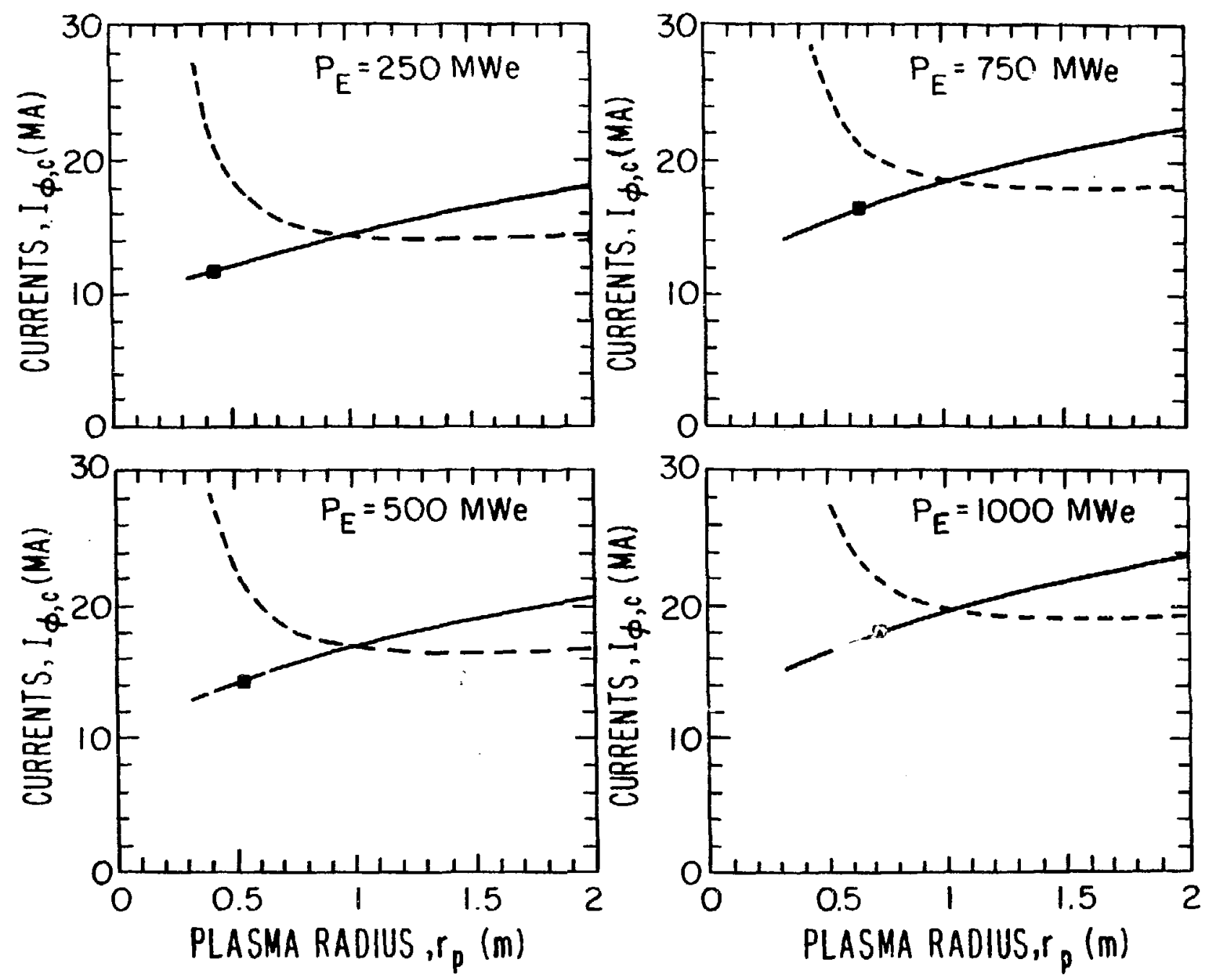

Fig. IV.C-5. Sensitivity of plasma (solid line) and coil (dashed line) currents as a function of $r_{p}$ and $P_{E}$ for the conditions depicted on Fig. IV.C-4. The solid curves give $I_{\phi}$, whereas the dashed curves give $I_{\phi c}$ • 

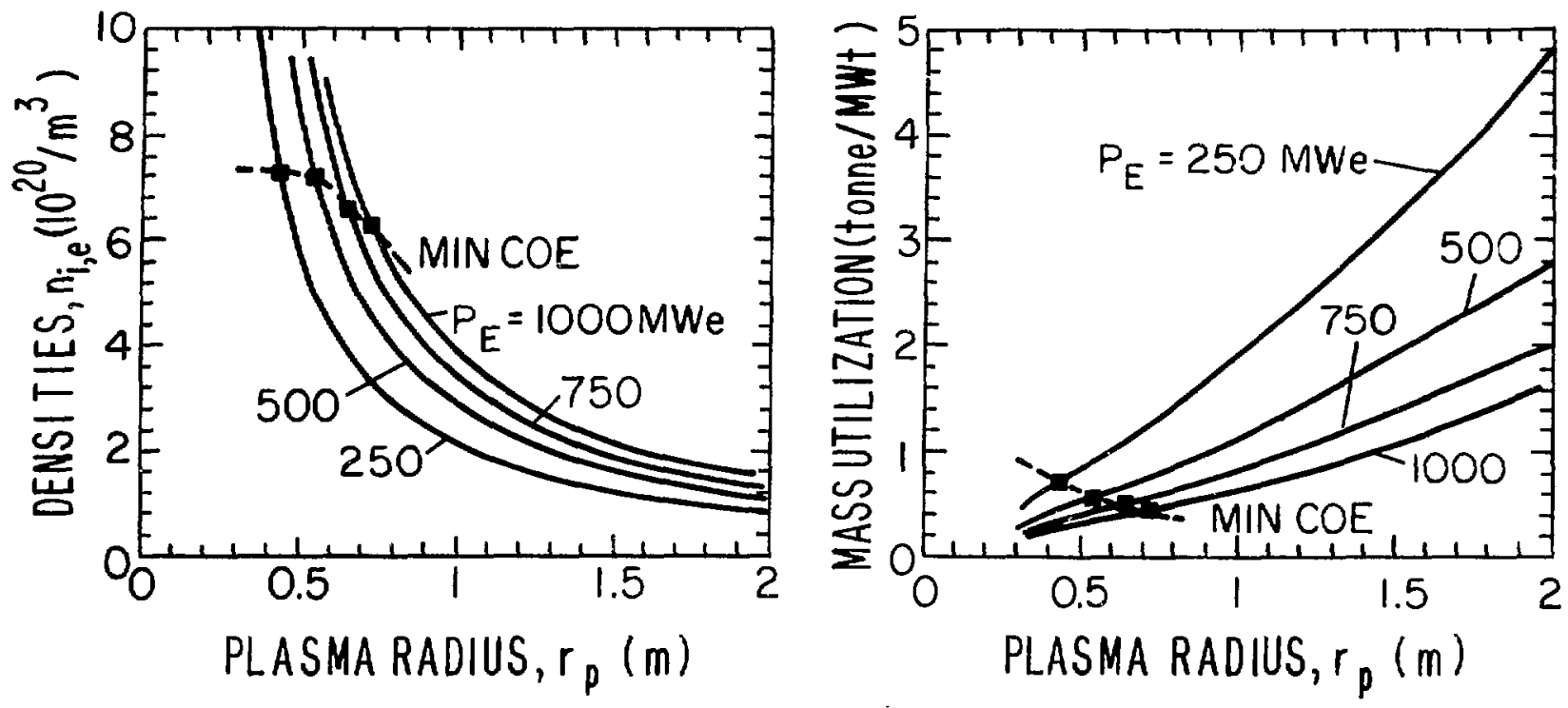

F1g. IV.C-6. Sensitivity of plasma density and FPC mass utilization as a function of $r_{p}$ and $P_{E}$ for cost-optimized designs.

Nevertheless, the margins between physics and econoulcs can be charted on Fig. IV.C-7, and a comfortable operating reglme is indicated for $P_{E}>750$ MWe and $v>1$. For lower values of $\mathrm{P}_{\mathrm{E}}$, Fig. IV.C-7 indicates that the minimum-COE design is not attainable for the RFP scaling used (1.e., $v>1$ ).

The $I_{w} / P_{E} / C O E$ tradeoff deplcted on Fig. IV.C.-4 defines the margins between technology (1.e., high heat/particle-flux surfaces, high-power-density blankets, batch versus patch maintenance, off-site versus on-site monolithic FPC construction, e:c.) and economics. The margins among $I_{w} / P_{E} / C O E$ and physics (transport) is best seen by combining the features of the COE and $I_{w}$ plots on Fig. IV.C-4 with the $\tau_{E}(O P T)=\tau_{E}$ (RFP) intersection on Fig. IV.C-B. Figure IV.C-8 graphically demonstrates this window for $\tau_{E}(R F P) \propto I_{\phi}^{{ }_{r}}{ }_{p}^{2} f\left(\beta_{\theta}\right)$ over a range of current exponents, that window opening or closing depending on whether the current exponent increases or decreases. The uniqueness of Fig. IV.C-8 rests with the comparison on a single plot of key technological 

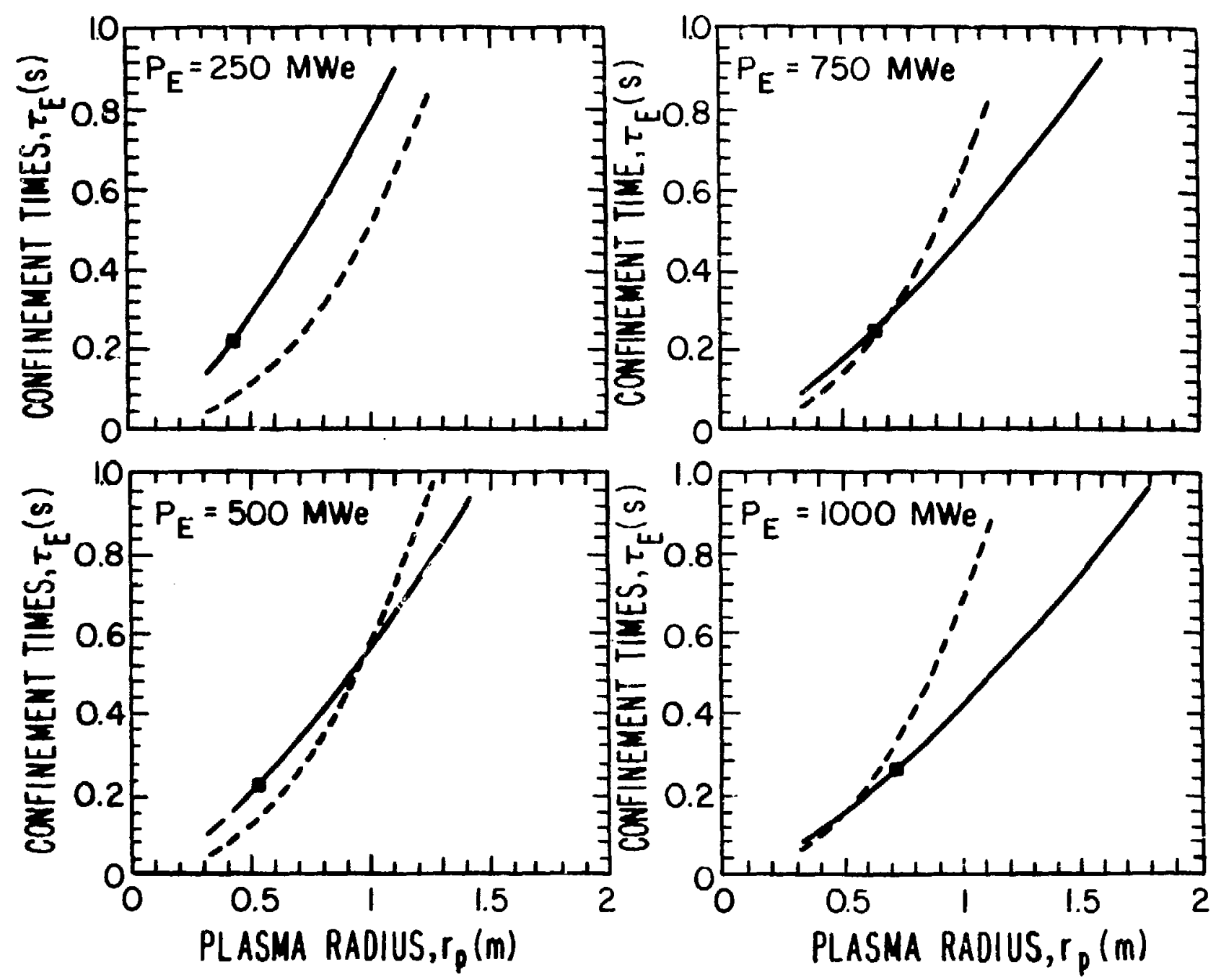

Fig. IV.C-7. Comparison of "economic" confinement time (solid line) $\tau_{E}$ (OPT), with RFP scaling [Eq. (A-35) and Eq. (A-36), dashed curve] for the parametric sensitivity results depicted on Fig. IV.C-4. 


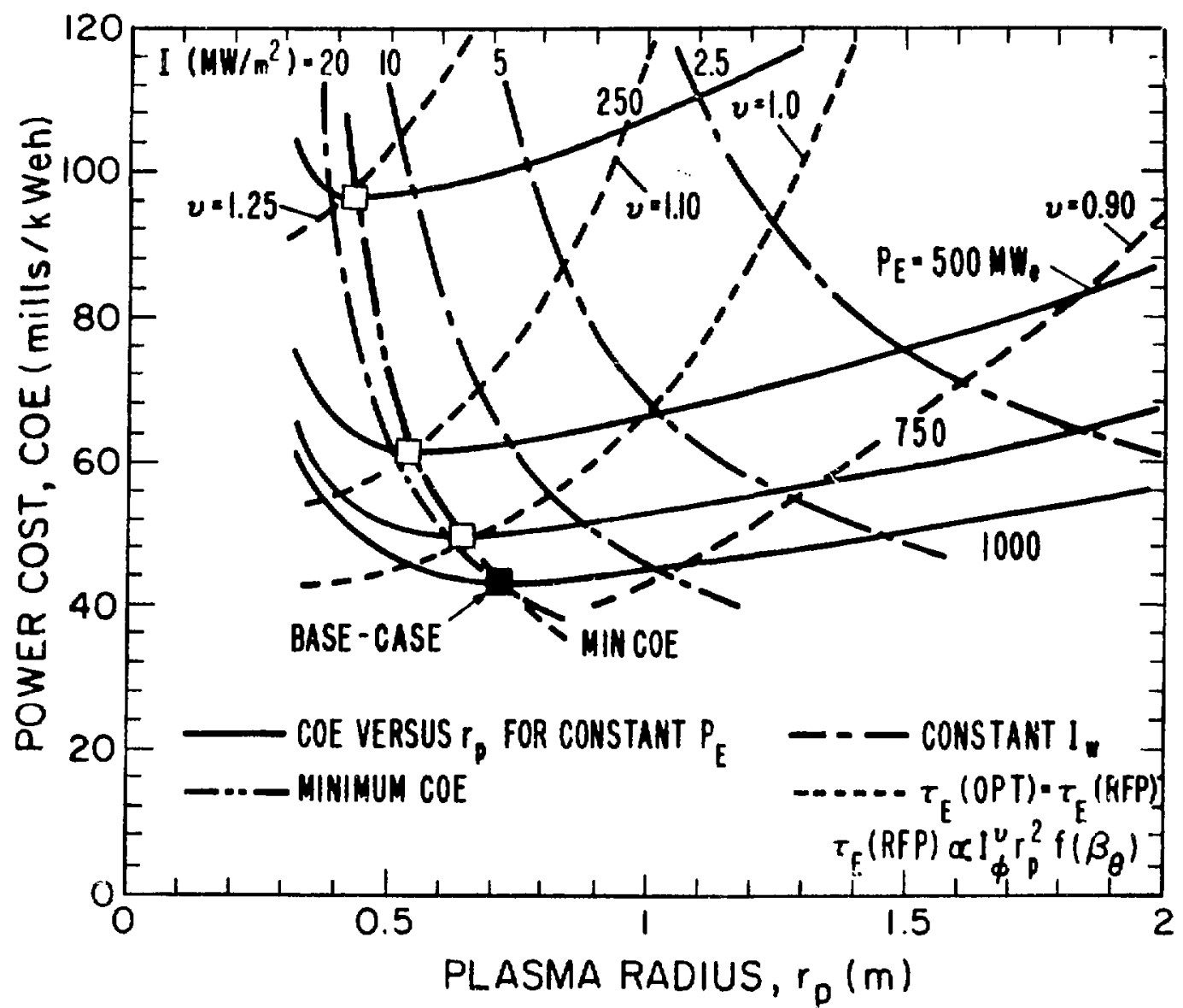

Fig. IV.C-8. Dependence of $C O E$ on $r_{p}$ for a range of $P_{E}$ values. Also shown are lines of constant $I_{w}$. The condition where $\tau_{E}$ (OPT) $=\tau_{E}$ (RFP) is also shown for $\tau_{E}(R F P) \propto I_{\phi} Y_{p}^{2} f\left(\beta_{\theta}\right)$ scaling for a range of $v$ values.

$\left(I_{w}\right)$, economic $\left(\operatorname{COE}, P_{E}\right)$, physics $\left(v, r_{p}\right)$; and institutional $\left(P_{E}\right)$ variables for the RFP.

\section{Conclusions}

This study and the concept assessment that has emerged has dealt primarily with the definition of both the physics and technology margins that allow access to the design windows described on Fig. IV.C-8. On the basis of this model development, systems study, and conceptual design, it is generally concluded that the RFP approach can operate within broad physical, technical, and economic margins while promising a unique, realistic, and robust approach to competitive magnetic fusion energy. Many physics technology and economics issues remain to 332 
be resolved, however, particularly with respect to the tradeoff between power density, development cost, $\mathrm{COE}$, and safety and environment issues. The rosuits of this framework study provides a basis and direction for a more comprehensive power plant study that could contribute to a better understanding of these important tradeoffs and issues for the RFP and related systems. 


\section{REFERENCES}

1. C. A. Flanagan, D. Steiner, and G. E. Smith, "Fusion Engineering Device Design Description," Oak Ridge National Laboratory report ORNL/TM-7948 (December 1981).

2. "INTOR, International Tokamak Reactor, Phase I," IAEA report STI/PUB/619, Vienna, (1982).

3. C. C. Baker, M. A. Abdou, R. M. Arons, A. E. Bolon, C. D. Boley, J. N. Brooks, et al., "STARFIRE - A Commerica1 Tokamak Fusion Power Plant Study," Argonne National Laboratory report ANL/FPP-80-1 (September 1980).

4. B. Badger, K. Audenaerde, J. B. Beyer, D. Braun, J. D. Callen, G. A. Emmert, et al., "WITAMIR - I: A University of Wisconsin Tandem Mirror Reactor Design," University of Wisconsin report UWFDM-400 (September 1980).

5. B. G. Logan, "Mirror Advanced Reactor Study (MARS)," Nuc1. Technol./Fusion 1, 563 (1983).

6. J. D. Gordon and B. G. Logan, "Magnetic Mirror Fusion Power Systems," Proc. Ilth Energy Technol. Conf., Washington, D.C. (March 1984) (also TRW report TRW-FRE-009, (1984)).

7. B. Badger, F. Avendt, H. M. Attaya, K. Audenaerde, H. Ave1, J. B. Beyer, et al., "TASKA: A Tandem Mirror Fusion Engineering Facility," University of Wisconsin report UWFDM-500 (March 1982).

8. T. K. Fowler and B. G. Logan, "Tandem Mirror Technology Demonstration Eacility," Lawrence Livermore National Laboratory report UCID-19193 (September 1981).

9. C. C. Baker, G. A. Carlson, and R. A. Krakowsk1, "Irends and Developments in Magnetic Confinement Fusion Reactor Concepts," Nucl. Technol./Fusion, 1, 5-78 (January 1981).

10. Report on the Third IAEA Technical Committee Meeting and Workshc. on Fusion Reactor Design-III, Tokyo, Japan, October 5-16, 1981, Nuc1. Fusion 22, 671-719 (1982).

11. W. M. Stacey, Jr., C. C. Baker, R. W. Conn, R. A. Krakowski, D. Steiner, and K. I. Thomassen, "Future Technology Requirements for Magnetic Fusion An Evaluation Based on Conceptual Design Studies," Nuc1. 'Iechno1./Fusion S, $266(1984)$.

12. R. A. Krakowski and R. L. Hagenson, "Compact Fusion Reactors," Proc. 5th ANS Topical Meeting on the Technology of Fusion Energy, Knoxville, TN (April 26-28, 1983), Nucl. Technol./Fusion 4, 1265 (September 1983).

13. M. Mitchell Waldrop, "Compact Fusion: Small is Beautiful," Science 219, 154 (1983). 
14. R. L. Hagenson, R. A. Krakowski, and H. Dreicer, "High-Power-Density Approaches to Magnetic Fusion Energy: Problems and Promise of Compact Reversed-Field Pinch Reactors (CRFPR), "Stockholm Symp. on New Trends in Unconventional Approaches to Magnetic Fusion (June 16-18, 1982) J. Nucl. Instr. and Methods 207, 241 (1983).

15. R. L. Hagenson and R. A. Krakowski, "Compact Reversed-Field Pinch Reactors (CRFPR): Sensitivity Study and Design-Point Determination," Los Alamos National Laboratory report LA-9389-MS (July 1982).

16. R. L. Hagenson and R. A. Krakowski, "Engineering Design of a Compact RFP Reactor (CRFPR)," Pruc. 5th ANS Topical Meeting on the Technology of Fusion Energy, Knoxville, TN (Apri1 26-28, 1983); Nucl. Technol./Fusion 4, 1284 (September 1983).

17. R. L. Hagenson, C. G. Bathke, M. E. Battat, D. J. Blevins, G. E. Cort, M. J. Embrechts, et al., "Design of A Compact Reversed-Fleld Pinch Reactor (CRFPR)," Proc. 10th IEEE Symposium on Fusion Engineering, Philadelphia, PA (December 5-9, 1983) IEEE Cat. No. 83CH1916-6 NPS I, 853 (1983).

18. R. F. Bourque, "OHTE Reactor Concepts," Proc. 9th Symp. on Eng. Prob. of Fusion Research II, 1851, Chicago, IL, (October 26-29, 1981).

19. R. A. Jacobsen, C. E. Wagner, R. E. Covert, "Systems Studies of High-Field Tokamak Ignition Experiments," J. Nucl. Fus. 3, (4), 217 (1983).

20. S. N. Rosenwasser, R. D. Stevenson, G. Listvinsky, D. L. Vrable, J. E. McGregor, and N. Nir, "Materials and Design Aspects of the Riggatron TM Tokamak," Proc. 3rd Topical Meeting on Fusion Reactor Materials, Albuquerque, NM (September 19-22, 1983) to be published in J. Nuc1. Mater.(also, Internationa1 Fusion Energy Systems Company, Inc. report II-100-83 (September 1983)).

21. W. Kopendorfer, "The ZEPHYR Experiment," Fus. Technol., 1980, p. 77, Pergamon Press, NY (1S81).

22. D. R. Cohn, L. Brombery, J. E. C. Willlams, H. Becker, R. LeCla1re, and T. Yang, "Near-Term Tokamak Reactor Designs with High-Performance Resistive Colls," Proc. Third Tech. Comm. Meeting and Workshop on Fusion Reactor Design and Technol., Tokoyo, Japan (October 15-16, 1981), IAEA-TC-392/11 I, 173 (1983).

23. H. J. Willenberg, "Definition and Conceptual Design of a Small Fusion Reactor," Mathematical Sciences Northwest report MSNW-1159 (February 1981).

24. A. E. Robson, "A Conceptual Design for an Imploding-Liner Fusion Reactor," Megagauss Physics and Technol., p. 425, Plenum Publishing Corp., NY $(1980)$.

25. R. L. Miller and R. A. Krakowsk1, "Reassessment of the Slowly-Imploding Liner (LINUS) Fusion Reactor Concept," Proc. 4th Top. Mtg. Technol. of Controlled Nuclear Fusion, CONF-801011 II, 825, King of Prussia, PA (October 14-16, 1980). 
26. R, E. Olson, J. G. Gilligan, E. Greenspan, and G. H. Miley, "The Spheromak Approach to High Power Density Reactors," Proc. 9th Symp. on Eng. Prob. Fusion Research II, 1898, Chicago, IL (October 26-29, 1981).

27. R. L. Hagenson and R. A. Krakowski, "A Compact-Torus Fusion Reactor Based upon the Field-Reversed Theta Pinch," Proc. 4th Top. Mtg. Technol. of Controlled Nuclear Fusion, CONF-801011 III, 1120, King of Prussia, PA (October 14-16, 1980).

28. R. L. Hagenson and R. A. Krakowsk1, "A Compact-Toroid Fusion Reactor Based on the Field-Reversed Theta Pinch," Los Alamos National Laboratory report LA-8758-MS (March 1981).

29. A. C. Smith, C. P. Ashworth, D. G. Abreu, D. M. Woodall, G. A.Carlson, W. S. Neef, et al., "The Prototype Moving-Ring Reactor," Proc. 9th Symp. on Eng. Prob. of Fusion Research II, 1867, Chicago, IL (October 26-29, 1981).

30. J. G. Gilligan, G. H. Miley and D. Driemeyer, "Conceptual Design of a Compact Field Reversed Mirror Reactor - SAFFIRE," Proc. 4th Top. Mtg. Technol. of Controlled Nuclear Fusion, CONF-801011 II, 840, King of Prussia, PA (October 14-17, 1980).

31. R. Hancox, R. A. Krakowski, and W. R. Spears, "The Reversed-Field Pinch Reactor (RFPR) Concept, "Nuc1. Eng. and Design 63, 2, 251 (1981).

32. R. L. Miller, "General Reactor Considerations Eor Stellarators," Los Alamos National Laboratory document LA-UR-82-2667, presented to 4 th International Stellarator Workshop, Cape May, NJ (September 13-15, 1982).

33. H. A. B. Bodin and A. A. Newton, "Review Paper: Reversed-Field Pinch Research," Nuc1. Fus. 20, 1255 (1980).

34. D. A. Baker and W. E. Quinn, "The Reversed-Field Pinch, "Edward Teller, ed., Fusion I, Part A, 438 (Academic Press, NY) (1981).

35. "Mirror-Based and Field-Reversed Approaches to Magnetic Fusion," Course and Workshop, International School of Plasma Physics, Varenna, Italy (September 7-17, 1983).

36. Tokamak Fusion Reactor Final Design Report," Princeton Plasma Physics Laboratory report PPPL 1475 (August 1978).

37. R. A. Krakowski, J.E. Glancy, and A. E. Dabiri, "The Technology of Compact Fusion Reactor Concepts," presented at the Workshop in Compact Fusion Reactors, Washington, DC (September 31 -October 1, 1982) Nucl. Techno1./Fusion 4 , 342 (1983).

38. "MIT Tokamak Aicator C Exceeds Lawson Criterion," Physics Today 20 (February 1984). 
39. M. Nagami and the JAERI Team, D. Overskel and the GA Team, "High- $\beta$ Injection Experiments with Shaped Plasmas in Doublet-III," Proc.9th Intern. Conf. on Plasma Physics and Controlled Nuclear Fusion Research, Baltimore, USA, paper IAEA-CN-41/A-2, I, 27 (September 1982).

40. D. Q. Hwang, M. Bitter, R. Bundy, A. Cesallo, R. Chrien, S. Cohen, et al., "High-Power ICRF and ICRF Plus Neutrai-Beam Heating on PLT," ibid, IAEA-CN-41/11, II, 3 .

41. P. C. Efthimion, M. Bell, W. R. Blanchard, N. Bretz, J. L. Cecchi, J. Coonrad, et al., "Initial Confinement Studies of Ohmically Heated Plasmas in the Tokamak Fusion Test Reactor," Phys. Rev. Lett. 52, 1492 (1984).

42. K. Uo, A. Iiyoshi. T. Obiki, o. Motojima, and S. Morimoto, A Sasaki, et al., "Heliotron Studies," Proc 9th Intern. Conf. on Plasma Fhysics and Controlled Nuclear Research, Baltimore, USA. IAEA-CN-41/L-3, II, 209 (September 1982).

43. G. Cattanei, D. Dorst, A. Elsner, G. Grieger, H. Hacker, J. Hartfuss, et al., "Neutral-Irjection Heating In the Wendelsteln VII-A Stellarator," ibid, IAEA-CN-41/L-5, II, 241 .

44. D. A. Baker, M. D. Bausman, C. J. Buchenauer, L. C. Burkhardt, G. Chandler, J. N. DiMarco, et al., "Performance of the 2T-40M Reversed-Field Pinch with an Inconel Liner," ibid, IAEA-CN-41/H-2-1, I, 587 (1982).

45. V. Antoni, M. Bogatin, A. Buffa, C. Bunting, S. Costa, I. Giudicott1, et al., "Studies on High Density RFP Plasmas in the Eta-Beta II Experiment," ibid, IAEA-CN-41/H-4, I, 619 (1982).

46. K. Ogawa, Y. Maejima, T. Shimada, Y. Hirano, P.G. Carolan, C. W. Gowers, et al., "Experimental and Computational Studies of Reversed Field Pinch on $\overline{T P E} \overline{-I R}(M), "$ Ibid, IAEA-CN-41/H-1, I, 575 (1982).

47. M. Fujiwara, T. Kamimura, M. Hosokawa, T. Shoji, H. Iguchi, et al., "Experimental and Numerical Studies on Plasma Confinement in Nagoya Bumpy Torus (NBT)," ibid, IAEA-CN-41/L-2, II, 197.

48. D. J. Rej and M. G. Tuszewski, "A Zero-Dimensional Model for Fleld-Reversed Configurations," Proc. 5th Symp. on Physics and Tech. of Compact Torolds in the Magnetic Fusion Program, Bellevue, WA (November $16-18,1982$ ).

49. C. W. Barnes, T. R. Jarboe, I. Henins, A. R. Sherwood, S. O. Knox, R. Gribble, et al., "Spheromak Formation and Operation With Background Filling Gas and A Solid Flux Conserver in CTX," Nucl. Fus. 24, 267 (1984).

50. T. C. Simonen, D. E. Baldwin, S. L. Allen, W. L. Barr, T. A. Casper, Y. J. Chen, et al., "TMX Tandem Mirror Experiments and Thermal Barrier Theoretical studies," Proc 9th Intern. Conf. on Plasma Physics and Controlled Nuclear Fusion Research, Baltimore, USA. IAEA-CN-41/G-1, I, 519 (September 1982). 
51. R. L. Miller, C. G. Bathke, R. A. Krakowsk1, F. M. Heck, L. Green, J. S. Karbowski, et al., "The Modular Stellarator Reactor; A Fusion Power Plant," Los Alamos National Laboratory report LA-9737-MS (July 1983).

52. C. G. Bathke, D. J. Dudziak, R. A. Krakowsk1, W. B. Ard, P. A. Bowers, J. W. Davis, et a1., "ELMD Bumpy Torus Reactor and Power Plant Conceptual Design Study," Los Alamos National Laboratory report LA 8882-MS (August 1981).

53. R. L. Hagenson, R. A. Krakowsk1, and G. E. Cort, "The Reversed-Field Pinch Reactor (RFPR) Concept," Los Alamos Scientific Laboratory report LA-7973-MS (August 1979).

54. United Engineers and Constructors, Inc., "1000-MWe Central Station Power Plants Investment Cost Study," U.S. AEC report WASH-1230 (June 1972).

55. J. Ha11, The Story of the Construction of Berieley Nuclear Power Station, Leonard Hill Books, London (1963).

56. A. E. Waltar and A. B. Reynolds, Fast Breeder Reactors, Pergamon Press, NY (1982).

57. G. C. Dale (ed.) and J. M. Bowerman, "The Safety of the AGR," UK Central Electricfty Generating Board and South of Scotland Electricity Board (1982).

58. UK Central Electricity Generating Board, "A Technical Outline of Sizewell "B': The British Pressurized Water Reactor " (1983).

59. E. W. Merrow, S. W. Chapel, and C. Worthing, "A Review of Cost Estimation in New Technologies: Implications for Energy Process Plants," Rand Corporation report $R-2481-D O E$ (July 1979).

60. I.. S. Caldwell, "Utility Financing - Risk and Return," Nuclear News 27, 46 (April 1984).

61. J. Payne, "Looking for a Way Back Home," Nuclear News 26, 31 (May 1984).

62. "Nuclear Plant Involvement Affects Rates, Ratings," Nuclear News 27, 39 (May 1984).

63. C. F. McDonald and D. L. Sonn, "A New Small HTGR Power Plant Concept with Inherently Safe Features - An Engineering and Economic Challenge," Proc. American Power Conference, Chicago, IL, 45, 818 (1983).

64. "Small Reactors: Is the Nuclear Industry on the Verge of a Large Step Backward?," The Energy Daily (December 22, 1983).

65. C. E. Behrens, "Economic Potential of Smaller-Sized Nuclear Plants in Today's Economy," The Library of Congress Congressional Research Service report 83-621 ENR (January 1984).

66. R. A. George and C. K. Paulson, "A Nuclear Plant Design for the 1990 s - Meeting Tomorrow's Needs," Proc. American Power Conference, Chicago, IL, 45, 799 (1983). 
67. R. Carruthers, "Criteria for the Assessment of Reactor Potential," Unconventional Approaches to Fuslon, B. Brunelli and G. G. Leatton (eds.), p. 39-45, Plenum Press, NY (1982).

68. S. C. Schulte, W. E. Bickford, C. E. Willingham, S. K. Ghose, M. G. Walker, "Fusion Reactor Design Studies--Standard Unit Costs and Cost Scaling Rules," Pacific Northwest Laboratory report PNL-2987 (September 1979).

69. A. A. Hollis, "An Analysis of the Estimated Capital Cost of a Fusion Reactor," UKAEA Harwe11 report AERE-R 9933 (June 1981).

70. J. B. Taylor, "Relaxation of Toroldal Discharges," 3rd Topical Conf. on Pulsed High-Beta Plasma, Culham, UK, p. 59 (1975).

71. D. A. Baker, C. J. Buchenauer, L. C. Burkhardt, J. N. DiMarco, J. N. Downing, and A. Haberstich, "Initlal Reversed-Field Pinch Experiments on ZT-40M with a Metallic Vacuum Liner," 10th European Conf. on Controlled Fus, and Plasma Physics, Moscow, USSR (September 14-19, 1981) also Los Alamos National Laboratory document LA-UR-81-1413.

72. P. G. Carolan, L. Firth, C. W. Gowers, G. C. H. Heywood, I. H. Hutchinson, A. A. Newton, V. A. Piotrowicz, M. R. C. Watts, P. D. Wilcox, and H. A. B. Bodin, "Initial Results from the HBTXIA Reversed Field Pinch Experiment," 10th European Conf. on Cont. Fus. and Plasma Physics, Moscow, USSR (September 14-19, 1981).

73. A. A. Newton, LI Yin-An, J.W. Long, and B. C. Yeung, "Numerical Investigation of Reversed Field Pinches," 3rd Topical Conf. on Pulsed High, Beta Plasmas, Culham, UK, p. 323 (1975).

74. K. F. Schoenberg, R. F. Gribble, and J. A. Phillips, "Zero-Dimensional Simulations of Reversed-Field Pinch Experiments," Nucl. Fusion 22, 1433 (1982).

75. R. Gerwin and R. Kelnigs, "Dynamo Theory: Can Amplification of Magnetic Field Profiles Arise from a Cross-Field Alpha Effect," Los Alamos National Laboratory report LA-9290-MS (April 1982).

76. M. J. Schaffer, "A Plasma Model with Dynamo for Sustained Reversed Fleld Pinches," GA Technologies report GA-Al6759 (May 1982).

77. K. F. Schoenberg, R. W. Moses, Jr., and R. L. Hagenson, "Plasma Resistivity in the Progress of a Reversed-Field Dynamo," submitted to Physics of Fluid (1983), also Los Alamos National Laboratory document LA-UR-83-1739.

78. K. F. Schoenberg and R. F. Gribble, "Oscillating Field Current Drive for Reversed-Field Pinch Discharges," Proc. IEEE Mtg. on Plas. Sci., Ottawa (May 17-19, 1982).

79. K. F. Schoenberg, C. J. Buchenauer, R. J. Massey, J. G. Melton, R. W. Moses, R. A. Nebel, and J. A. Phlllips, "F- $\theta$ Pumping and Field Modulation Experiments on a Reversed Field Pinch Discharge," Phys. Fluids 27, 548 (1984). 
80. M. K. BevIr and J. W. Gray, "Relaxation, Flux Consumption and Quasi Steady State PInches," Proc. RFP Theory Workshop, Los Alamos National Laboratory Apri1 28 - May 2, 1980, Los Alamos National Laboratory report LA-8944-C, p. 176 (January 1982).

81. M. K. Bevir, C. G. Gimblett, and G. Miller, "Quast-Steady-State Current Drive by Plasma Relaxations," Proc. IAEA Technical Meeting on Non-Inductive Current Drive in Tokamaks, Culham Laboratory (April 1983).

82. R. D. O'De11, F. W. Brinkley, Jr., and D. R. Marr, "Users Manual for ONEDANT: A Code Package for One-Dimensional, Diffusion-Accelerated, Neutral-Particle Transport," Los Alamos National Laboratory report LA-9184-MS (February 1982).

83. D. Garber (ed.), "ENDF/B-IV Summary Documentation," Brookhaven National Laboratory report BNL-17541 (ENDF-201) (October 1975).

84. M. E. Bactat, R. J. LaBauve, and D. W. Muir, "The GAMMON Activation Library," Los Alamos Scientific Laboratory report LA-8040-MS (September 1979).

85. D. K. Sze, R. Clemmer, and E. T. Cheng, "LiPb, A Novel Material for Fusion Applications," Proc. of the 4th Top. Mrg. on the Tech. of Controlled Nuc1. Fus., III, 1786, King of Prussia, PA (October 14-17, 1980).

86. R. F. Bourque, "Flowing $\mathrm{Pb}_{83} \mathrm{Li}_{17}$ Liquid-Metal Coolant in Fusion Reactors with High-Power-Densities and Magnetic Fields," Nucl. Technol./Fusion $\underline{3}$, 493 (1983), also GA Technologies report GA-Al6662 (June 1982).

87. M. Abdou (project manager), "Blanket Comparison and Selection Study: Interium Report," Argonne National Laboratory report ANL/FPP/TM-177 (September 1983).

88. R. W. Conn, "Tokamak Reactors and Structural Materials," J. Nucl. Mater. 85 and 86,9 (1979).

89. D. L. Smith, "Blanket Materials for DT Fusion Reactors," ibid., p. 19.

90. R. E. Nygren, "Material Issues in the Fusion Engineering Device," Ibid., p. 31 .

91. M. A. Abdou, R. F. Mattas, D. L. Smith, and G. L. Kulcinski, "Important Material Consisderations in INTOR," ibid., p. 41.

92. R. R. Hasiguti, "Japanese Program of Materials Research for Fusion Research," 1bid., p. 51 .

93. J. Nihoul, "The European Programe of Fusion Materials," ibid., p. 57.

94. R. A. Krakowsk1, "Materials Needs for Compact Fusion Reactors," 3rd Top. Mtg. on Fusion Reactor Materials, Albuquerque, MM (September 19-22, 1983) to be published in J. Nucl. Mater. 
95. L. J. Perkins, "Material Considerations for Highly Irradiated Normal-Conducting Magnets in Fusion Reactor Applications," ibid.

96. I. N. Sviatoslavsky and W. C. Young, "Structural Design Features for Commercial Fusion Power Reactor Magnet Systems," Nucl. Eng. and Design 58, 207 (1980).

97. M. A. Hoffman, "Heat Flux Capabilities of First-Wall Tube Arrays for an Experimental Fusion Reactor," Nucl. Eng, and Design 64, 283-299 (1981).

98. G. P. Yu, "Relationship of Material Properties to the Design of a Fusion Reactor First Wa11," ScD. Thesis, Dept. of Nucl Eng., MIT (May 1981).

99. C. K. Youngdal and D. L. Smith, "Stress and Lifetime Limitations of First-wal1 Structural Materials," J. Nucl. Mater. 85 and 86, 153 (1979).

100. P. W. Taubenblat, H. R. Opic, and Y. T. Hsu, "A New Copper Alloy with High Strength and Conductivity," Metals Englneering Quarterly (November 1972).

101. G. E. Cort, A. L. Graham, and K. E. Christensen, "A High-Flux First-Wall Design for a Small Reversed-Field Pinch Reactor," AIAA/ASME Conf . on Fluid, Plasma, Thermophysics, and Heat Transfer, St. Louis, MO (June 8-11, 1982).

102. O. K. Harling, G. P. Yu, N. J. Grant, and J. E. Meyer, "Application of High-Strength Copper Alloys for a Fusion Reactor First Wall," Proc. 2nd Top. Mtg. on Fus. Reactor Materials, Seattle, WA (August 9-12, 1981).

103. F. W. Wiffen, T. C. Reuther, and R. E. Gold, "Copper and Copper Alloys for Fusion Reactor Applications: Summary Report of a DOE/OFE Workshop," Proc. 3rd Topical Mtg. on Fusion Reactor Materlals, Albuquerque, NM (September 19-22, 1983).

104. M. A. Hoffman and R. W. Werner, "Heat Flux Limitations on First-Wall Shields for Early Fusion Machines," Proc. 1st Topical Meeting on the Technology of Controlled Nuclear Fuston, CONF-740402-P1, 619-633 (Apr11 16-18, 1974) (also Lawrence Livermore National Laboratory report UCRL-75622 (Apri1 1974)).

105. L. J. Perkins and G. L. Kulcinski, "Material Considerations for Highly Irradiated Normal-Conducting Magnets in the MARS Tandem Mirror Reactor," Proc. 3rd Top. Mtg. on Fus. Reactor Materials, Albuquerque, NM (September $19-22$, 1983).

106. L. J. Perkins and G. L. Kulcinski, "Economic Design Optimization of the $\mathrm{LiPb}$ Blanket for the Mirror Advanced Reactor (MARS)," Proc. 5th ANS Topical Meeting on the Technology of Fusion Energy, Knoxville, TN (April 26-28, 1983), Nucl. Technol./Fusion 4, 1107 (1983).

107. A. Harvey, "Radiation-Hardened Magnets Using Mineral Insulated Conductors," Los Alamos Scientific Laboratory report LA-5306-MS (June 1973). 
108. R. J. Grieggs, D. J. Liska, and A. Harvey, "Radiation-Hardened Field Coils for FMIT Quadrupoles," 1983 Particle Accelerator Conf., Santa Fe, NM (March 21-23, 1983).

109. L. E1-Guebaly, L. J. Perkins, and C. Maynard, "MARS Axicell Damage and Shielding Analysis," Proc. 5th ANS Top. Mtg. on the Tech. of Fus. Energy, Knoxville, TN (April 26-28, 1983), Nucl. Technol./Fusion 4, 1171 (September 1983).

110. G. F. Hurley, J. C. Kennedy, F. W. Clinard, Jr., R. A. Vangman, and W. R. McDonnel, "Structural Properties of $\mathrm{MgO}$ and $\mathrm{MgAl}_{2} \mathrm{O}_{4}$ after Fusion Neutron Irradiation Near Room Temperature," 2nd Top. Mtg. on Fus. Reactor Materials, Seattle, WA (August 9-12, 1981).

111. L. J. Perkins, "Dose-Rate Conductivity Enhancement in Ceramic Insulators: A New Shielding Problem?," Trans. Amer. Nucl, Soc. 44, 131 (1983).

112. J. A. Horak and T. H. Blewitt, "Fast Neutror Irradiation Induced Resistivity in Metals," Phys. Stat. Sol (A) $\underline{9}, 721$ (1972).

113. W. A. Harrison, "Resistivity Due to Dislocations in Copper," J. Phys. Chem. Solids 5,44 (1958).

114. R. M. Brick, A. W. Pense, and R. B. Gordon, Structure and Properties of Engineering Materials, McGraw-Hi11, NY, p. 66 (1977).

115. N. F. Mott and H. Jones, The Theory of the Properties of Metals and Alloys, 269-287, Dover, NY (1936).

116. T. H. Blewitt and T. J. Koppena1, Radiation Effects, W. F. Sheeley, ed., Gordon and Breach, NY p. 585 (1967).

117. L. D. Glowinsk1, J. M. Lanore, C. Foche, and Y. Adda, "Etude de $1 a$ Formation des Cavites D“irradiation Dans e Cuivre IV-Etude des Mecanismes," J. Nucl. Mater. 61, 41-52 (1976).

118. W. J. Duffin and F.A. Nichols, "The Effect of Irradiation on Diffusion-Controlled Creep Processes," J. Nuc1. Macer. 45, 302-316 (1972/73).

119. W. H. Rohsenow, "Boiling," Handbook of Heat Transfer, W. H. Rohsenow and J. P. Hartnett, eds. (McGraw-Hi11, NY, 1973), Sec. 13, 13.34-13.63.

120. W. C. Reynolds, "Effect of Wall Heat Conduction on Convection in a Circular Tube with Arbitrary Circumferential Heat input," Intern. Jour. of Heat and Mass Trans. 6, 925 (1963).

121. J. A. Fillo and J. R. Powe11, "Coupled Conduction-Turbulent Convention in a Circular Tube," Procedures of the 6th Inter. Heat Transfer Conf. 2, 181, Toronto (1978).

122. A. L. Chan, J. W. Baughn, and M. A. Hoffman, "Nonuniform Circumferential Heat Flux Experiments in a Circular Tube," ASME Winter Annual Meeting, 75-WA/MT-52, Houston, TX (November 1975). 
123. W. R. Gambill and N. D. Greene, "Bolling Burnout with Water in Vortex Flow," Chem. Eng. Progress 54, 68-76 (1958).

124. R. G. Lawton, "The AYER Heat Corduction Computer Program," Los Alamos Scientific Laboratory report LA-5613-MS (May 1974).

125. D. L. Smith, M. Abdou, P. Adler, C. Baker, D. Berwald, J. Bethin, et al., "Fusion Reactor Blanket Shield Design Study," Argonne National Laboratory report ANL/FPP-83-1 (October 1983).

126. M. Abdou, C. Baker, J. Brooks, D. DeFreece, D. Ehst, R. Mattas, et al., "A Demonstration Tokamak Power Plant Study, (DEMO)," Argonne National Laboratory report ANL/FPP/82-1 (September 1982).

127. U.S. Department of Energy, "Materials Handbook for Fusion Energy Systems."U.S. DOE report DOE/TIC - 10122 (1980).

128. R. L. Spencer, "Magnetic Islands and Stochastic Field Lines in the RFP," Proc. RFP Theory Workshop, Los Alamos, MM (April 29-May 2, 1980), Los Alamos National Laboratory report LA-8944-C, p. 129 (January 1982).

129. P. Thullen (ed.), "ZT-40U: Conceptual Design of a 2-MA Reversed-Field Pinch Experiment," Los Alamos National Laboracory document LA-UR-83-1375 (1983).

130. R. W. Moses, Jr. and J. K. Ballou, "Inductive Shielding for Pulsed Energy Storage Magnets," IEEE Trans. on Magnetics, MAG-11, 493-496 (March 1975).

131. V. D. Shafranov, Reviews of Plasma Physics, 2 (Consultaris Bu:eau, p.103, NY) (1966).

132. J. M. Greene, J. L. Johnson, and K. E. Weimer, "Tokamak Equilibrium," Phys. Fluids 14, 671 (1971).

133. V. S. Mukhovatov and V. D. Shafranov, "Plasma Equilibrium in a Tokamak," Nuc1. Fusion 11, 605 (1971).

134. M. Petravic, D. Heifetz, D. Post, W. Langer, and C. Singer, "A Model for the Performance of Polodial Divertors," 9 th Inter. Conf. on Plasma Physics and Controlled Nucl. Fus. Research, Baltimore, MD, IAEA-CN-41/D-3-2, I, 323 (September 1-8, 1982).

135. A. K. Prinja, R. W. Conn, S. Talmadeg, D. Helfetz, and D. Post, "Physics of Particle Exhaust in Pumped Limiters," J. Nuc1. Mater. 111-112, 279 (1982).

136. M. Ulrickson, "Optimum Shapes for Pumped Limiters," Princeton Plasmal Physics Laboratory report PPPL-1901 (May 1982).

137. H. C. Howe, "Physlce Considerations for the FED Limiter," Oak Ridge Nationa1 Laboratory report ORNL/TM-703 (1982).

138. K. Audenaerde, G. A. Emmert, and M. Gordinier, "SPUDNUT: A Transport Code for Neutral Atoms in Plasma," J. Comp. Phys. 34, 268 (1980). 
139. Symp. on Energy Removal and Particle Control in Toroidal Fusion Devices, Princeton, NJ (July 26-29, 1983).

140. W. Pfelffer and R. E. Waltz, "Emplrical Scaling Laws for Energy Confinement in Ohmically-Heated Tokamaks," Nucl. Fusion 19, 51 (1979).

141. K. B. Axon, J. E. Bradley, J. Burt, W. H. M. Clark, M. Dunstan, et al., "Results from DITE Experiment," Plasma Phys. and Controlled Nucl. Fusion Res. III, 201, Baltimore (September 1-8, 1982).

142. P. J. Harbour and J. G. Morgan, "Models and Codes for the Plasma Edge Region," Culham Laboratory report CLM-R234 (December 1982).

143. D. G. Worden, "Flow of Gases Through Tubes and Orifices," in S. Dushman and J. M. Lafferty, eds., Scientific Foundations of Vacuum Technique, (John Wiley \& Sons, Inc., NY) (1962).

144. G. L. Weissler and R. W. Carlson, Vacuum Physics and Technology (Academic Press, NY) (1979).

145. R. Loevinger, "Fundamental Considerations in Vacuum Practice," in A. Guthrie and R. K. Wakerling, eds., Vacuum Equipment and Techniques (McGraw-Hil1 Book Company, Inc.) (1949).

146. S. L. Milora, "Review of Pellet Fueling," J. Fusion Energy 1, 15 (1',1).

147. S. L. Gralnick, "Solid Deuterium Evaporation in a Fusion Plasma," Nuc1. Fus. 13, 703-713 (1973).

148. P. B. Parks and R. J. Turnbul1, "Effect of Transonic Flow in the Ablation Cloud on the Lifetine of a Solld Hydrogen Pellet in a Plasma," Phys. Fluids 21, 1735-1741 (1978).

149. R. P. Gilliard and K. Kim, "Significance of Magnetic Shielding in the Ablation of a Solid Hydrogen Pellet in a Plasma," IEEE Transaction on Plasma Science PS-8, 477-484 (December 1980).

150. W. A. Houlberg, M. A. Iskra, H. C. Howe, and S. E. Attenberger, "Pellet-A Computer Routine for Modeling Pellet Fueling in Tokamak Plasma," Oak Ridge National Laboratory report ORNL/TM-6549 (January 1979).

151. J. B. Taylor, "Relaxation of Toroidal Plasma and Generation of Reversed Magnetic Field," Phy. Letts. 33, 1139 (1974).

152. J. B. Taylor, "Relaxation of Toroidal Discharges to Stable States and Generation of Reversed Magnetic Field," Proc. 5th Inter. Conf. on Plasma Physics and Cont. Nuc1. Fusion Research 1, 161, Tokyo, Japan (November 11-15, 1974).

153. F. M. Mann, "Reduced Activation Calculations for the Starfire First Wall," Hanford Engineering Development Laboratory report HEDL-TME-83-27 (October, 1983). 
154. A. M. Weinberg, I. Speiwak, "Inherently Safe Reactors and a Second Nuclear Era" Science 224, 1394 (June 1984). 


\begin{tabular}{|c|c|}
\hline AFC & Alternative Fusion Concept \\
\hline AGR & Advanced Gas-Cooled Reactor \\
\hline $\mathbf{B}$ & Blanket \\
\hline B/S & Blanket/Shield \\
\hline BOP & Balance of Plant \\
\hline BWR & Bolling-Water Reactor \\
\hline c & $\operatorname{Cot1}$ \\
\hline $\mathrm{COE}$ & Cost of Electricity (mills/kWeh) \\
\hline CRFPR & Compact Reversed-Field Pinch Reactor \\
\hline DBTT & Ductile to Brittle Transition Temperature \\
\hline DFC & Divertor Flanking Coil \\
\hline DT & Deuterfum-Tritium \\
\hline D\&T & Development and Technology \\
\hline EBT & Elmo Bumpy Torus \\
\hline EFC & Equilibrium-Field Coll \\
\hline EI & Escalation/Interest \\
\hline ETR & Experimental Test Reactor \\
\hline FED & Fusion Engineering Device \\
\hline FPC & Fusion Power Core (FW/B/S/C) \\
\hline FPY & Ful1-Power Year \\
\hline FW & First Wa11 \\
\hline HHF & High Heat Flux \\
\hline HMG & Homopolar Motor/Generator \\
\hline HPD & H1gh Power Density \\
\hline IHX & Intermediate Heat Exchanger \\
\hline INTOR & International Toroldal Reactor \\
\hline IVC & In-Vacuum Component \\
\hline LMFBR & Liquid-Metal Fast-Breeder Reactor \\
\hline LWR & Light-Water Reactor \\
\hline MARS & Mirror Advanced Reactor Study \\
\hline$M / G$ & Motor/Generator \\
\hline MSR & Modular Stellarator Reactor \\
\hline MTTF & Mean-Time-To-Fallure \\
\hline MTTR & Mean-Time-To-Repair \\
\hline
\end{tabular}




$\begin{array}{ll}\text { NA } & \text { Not Applicible } \\ \text { NBT } & \text { Nagoya Bumpy Torus } \\ \text { O\&M } & \text { Operation and Maintenance } \\ \text { OHC } & \text { Ohmic-Heating Coil } \\ \text { OHTE } & \text { Ohmically Heated Toroidal Experiment } \\ \text { PFC } & \text { Poloidal-Field Coll } \\ \text { PWI } & \text { Plasma-Wall Interaction } \\ \text { PWR } & \text { Pressurized-Water Reactor } \\ \text { RFP } & \text { Reversed-Field Pinch } \\ \text { ROI } & \text { Return on Investment } \\ \text { RPE } & \text { Reactor Plant Equipment } \\ \text { SG } & \text { Steam Generator } \\ \text { SOL } & \text { Scrapeoff Layer } \\ \text { SOTA } & \text { State of the Art } \\ \text { SP } & \text { Superphenix } \\ \text { TBD } & \text { To Be Determined } \\ \text { TDC } & \text { Total Direct Cost } \\ \text { TFC } & \text { Toroidal-Field Coil } \\ \text { TFTR } & \text { Tokamak Fusion Test Reactor } \\ \text { TMR } & \text { Tandem-Mirror Reactor } \\ \text { UDC } & \text { Unit Direct Cost ( } / \mathrm{kWe)} \\ \text { UTC } & \text { Unit Total Cost ( } / \mathrm{kWe)} \\ & \end{array}$




\section{ACKNOWLEDGEMENTS}

The authors wish to acknowledge the useful discussions with J. N. DiMarco, J. N. Downing, R. W. Moses, Jr., K. F. Schoenberg, and R. L. Spencer of Los Alamos on a wide range of physics and techalcal copfce dealing with the extension of present RFF physics understanding to describe reactor-1ike conditions. The consultation and advice on materials issues by F. W. Clinard, Jr. Is greatly appreciated. C. R. Mynard contributed significantly to the illustrative mechanical layout of the fusion power core; D. J. Blevins and G. E. Cort are similarly acknowledged. C. Copenhaver contributed to the limiter heat-transport assessment. During the course of this study one of the authors (RAK) made an extended visit to the Culham Laboratory and expresses his appreciation for the insights developed during that stay, particularly through conversations with R. Hancox, P. I. H. Cook, W. R. Spears, G. J. Butterworth, and D. E. T. F. Ashby. Lastly, the unflaggingly accurate and rapld typing and editing skills of Marion Clark and 01ivia Moss throughout the evolution of this report are especially acknowledged. 
Appendix A. CRFPR Burn Model and Reactor Design Code

The time-dependent simulation of the CRFPR couples the external electrical driving circuit to a profile-averaged zero-dimensional plasma mode1. A complete analysis of the polotdal-field circuit is performed, as described in Sec. III.F.2.; this analysis includes the plasma as a nonlinear circuit element. Experimentally derived plasma profiles and transport properties are used to generate self-consistent plasma equilibrium configurations that are adjusted as the DT/DD-fueled burn proceeds. The concentration and energies of spectes from the constituent reactions $\left(\mathrm{H},{ }^{3} \mathrm{He},{ }^{4} \mathrm{He}, \mathrm{T}\right)$ are followed in $t$ ime with a Fokker-Planck slowing-down formulation. Power output from the plasma impinges on a two-region first-wall surface, and one-dimensional temperature and stress distributions within the first wall are calculated as a function of time. An overall power-plant energy balance is evaluated as the reactor achieves a steady state, with an option to perform a self-consistent analysis of F- $\theta$ current drive.

The RFP systems code is based on a multiparticle, time-dependent burn computation that accounts for effects related to magnetic-field and plasma profiles by performing integral averages over the plasma cross section at selected time intervals. Magnetic fleld, $\vec{B}(r)$, and current density profiles, $\vec{j}(r)$, are calculated using the Maxwell equations, these profiles being computed self-consistently with input plasma density, temperature, and $\mu(r)$ profiles, where $\vec{J}_{\|}(r)=\left[\mu(r) / \mu_{0}\right] \vec{B}(r), \quad \mu_{0}=4 \pi(10)^{-7} \mathrm{H} / \mathrm{m}$, and $\mu(r)$ is defined by this expression for $j_{\|}(r)$. With these one-cimensional equilibrium relationships, profile form factors are derived numerically and used to simulate a one-dimensional calculation while maintaining the time efficiency of a zero-dimensional "point-model" code. This kind of calculation can not prodict profile evolution, which instead must be provided as a function of time. This limitation, however, does not seriously compromise the burn predictions when the level of avallable experimental information in this area is considered. One-dimensional computer codes require spatially resolved transport coefficients, which even for the well-studied tokamak are generally unavailable. Present experiments typically provide only approximate profile information and bulk-plasma transport losses. This level of knowledge is ideally sulted as input to the profile-averaging computer code described in this section; the use of multidimensional transport codes tend to create an unwarranted level of 
confidence in computed results in that the predictive capabilities remain based on comparison with integral Experimental results.

The CRFPR burn model provides the following specific information:

- Time history of currents, voltages, powers, and energies in the poloidal-field-coil (PFC) and toroidal-field-coll (TFC) circuits, with the PFC circuit driving the burn computation. Applying appropriate circuit switching sequences and voltages while incorporating the plasma as a nonlinear circuit element allows an accurate simulation of reactor operation.

- Self-consistent, one-dimensional (cylindrical) plasma equilibria using experimentally derived plasma density, $n(r)$, temperature, $T(r)$ and current-density [1.e., $\mu(r)]$ profiles. Profile updating is performed whenever the plasma beta or boundary conditions have significantly changed over a given period of time.

- Complete time history of plasma properties is generated, including parameters that are constrained to satisfy stability requirements (particularly $\beta_{\theta}, \theta$, and $F$ ). This calculation includes profile averaging of all plasma quantities versus time for a DT- or DD-fueled burn.

- Temperature and density fractions of all energetic ionic species are determined. These non-Maxwellian suprathermal plasma species (ik, $\mathrm{D}, \mathrm{l},{ }^{3} \mathrm{He}$, and ${ }^{4} \mathrm{He}$ ) are described by a Fokker-Planck formalism with the Maxwellian component being subtracted from each slowing-down distribution and merged into a single background ion population.

- Complete reactor energy balance and a listing of all systen energy requirements that culminate in a final expression for the engineering Q-value, $Q_{E}$, or recirculating power fraction, $\varepsilon=1 / Q_{E}$, are complited. Results are displayed for a pulsed-plasma system, as directly simulated by the computer code and assuming the reactor performance at code termination represents a steady-state burn corfiguration. Details of this energy balance calculation are given in Sec. A.7.

- The option exists to model the reversed-field pinch, spheromak, tokamak, EBT, or stellarator assuming -ppropriate experimental temperature, density, and current profiles are avallable. If the plasma minor cross section is noncircular, these profiles must be mapped into a circular surface having an effective circular cross section equivilent to the actual surface being modeled.

- A simultaneous, time-dependent thermal response of the first wall during startup, burn, and plasma quench is generated. This one-dimensional heat-transfer calculation is made for a two-region first wall (1.e., coated or tiled surfaces) that can be cooled with a variety of coolant choices. 
- A time-dependent mechanical/structural response of the first wall is computed simultaneously with the burn simulation, producing a one-dimensional stress history of the same structure used in the above-mentioned heat-transfer calculation.

- A file is created for interactive use with a standardized reactor costing code. 1

The modelistic details of the CRFPR systems and burn code are presented below. This description is specifically written for CRFPR simu? ations, although the model is easily extended to other fusion concepts. Burn similations nave been performed for tokamaks, stellarators, bumpy tori, and compact corolds by using concept-specific proflie information and appropriate transport. The CRFPR code described herein is benchinarked against the DD/STARFIRE tokamak results and has shown excellent agreement. 2

\section{A.1. Computer Model for Burn Simulation.}

The plasma behavior is described by a set of first-order differential equations. These time-dependent equations describe particle inventories, plasma temperatures, spatial varlations, and a range of integrated plasma powers. The more general form or the calculation uses deuterium as the primary fueling source. The reaction rates, $R_{i j}$, of intercst are summarized below:

$$
\begin{aligned}
& \mathrm{R}_{\mathrm{DDp}}: \mathrm{D}+\mathrm{D} \rightarrow \mathrm{T}(1.01 \mathrm{MeV})+\mathrm{p}(3.03 \mathrm{MeV}) \\
& \mathrm{R}_{\mathrm{DDn}}: \mathrm{D}+\mathrm{D} \rightarrow{ }^{3} \mathrm{He}(0.82 \mathrm{MeV})+\mathrm{n}(2.45 \mathrm{MeV}) \\
& \mathrm{R}_{\mathrm{DT}}: \mathrm{D}+\mathrm{T} \rightarrow{ }^{4} \mathrm{He}(3.52 \mathrm{MeV})+\mathrm{n}(14.06 \mathrm{MeV}) \\
& \mathrm{R}_{\mathrm{DHe}}: \mathrm{D}+{ }^{3} \mathrm{He} \rightarrow{ }^{4} \mathrm{He}(3.67 \mathrm{MeV})+\mathrm{p}(14.67 \mathrm{MeV}) .
\end{aligned}
$$

The spatially averaged density of five fon species listed above $\left(H, D, T,{ }^{3} \mathrm{He}\right.$, and $\left.{ }^{4} \mathrm{He}\right)$ are computed as a function of time. Rate equations used to calculate the time variation of the line density, $N_{j}=n_{j} \pi r_{p}^{2}$, are given be low:

$$
\begin{aligned}
& \dot{\mathrm{N}}_{\alpha}=-\mathrm{N}_{\alpha M} / \tau_{\mathrm{pI}}+\mathrm{R}_{\mathrm{DT}}+\mathrm{R}_{\mathrm{DHe}} \\
& \dot{\mathrm{N}}_{\mathrm{H}}=-\mathrm{N}_{\mathrm{HM}} / \tau_{\mathrm{pi}}+\mathrm{R}_{\mathrm{DHe}}+\mathrm{R}_{\mathrm{DDP}}
\end{aligned}
$$




$$
\begin{aligned}
& \dot{\mathrm{N}}_{\mathrm{He}}=\mathrm{S}_{\mathrm{He}}-\mathrm{N}_{\mathrm{HeM}} / \tau_{\mathrm{PI}}+\mathrm{R}_{\mathrm{DDn}}-\mathrm{R}_{\mathrm{DHe}} \\
& \dot{\mathrm{N}}_{\mathrm{T}}=\mathrm{S}_{\mathrm{T}}-\mathrm{N}_{\mathrm{TM}} / \tau_{\mathrm{PI}}+\mathrm{R}_{\mathrm{DDP}}-\mathrm{R}_{\mathrm{DT}} \\
& \dot{\mathrm{N}}_{\mathrm{D}}=\mathrm{S}_{\mathrm{D}}-\mathrm{N}_{\mathrm{DM}} / \tau_{\mathrm{pI}}-\mathrm{R}_{\mathrm{DT}}-2 \mathrm{R}_{\mathrm{DDp}}-2 \mathrm{R}_{\mathrm{DDn}}-\mathrm{R}_{\mathrm{DHe}},
\end{aligned}
$$

where He designates ${ }^{3} \mathrm{He}, \alpha$ designates $4 \mathrm{He}$, and $\dot{\mathrm{N}}_{\mathrm{j}}=\mathrm{dN}_{\mathrm{j}} / \mathrm{dt}$. Total particle Inventories, Including both Maxwellian specles, $\mathrm{N}_{j M}$, and energetic (non-Maxwellian or superthermal) particles, are taken into account by these rate equations. This formulation separates the plasma into five energetic species, each through a Fokker-Planck model thermalizing against a homogeneous-background Maxwellian plasma of electron and ion at respective temperatures. The Maxwellian component of the energetic particle-distributiou functions is continuously subtracted and merged into the thermal ion background. Particle loss is assumed to occur only from the Maxwellian plasma according to a loss time $\tau_{p 1}$. Neutral-gas fueling at a rate $s_{j}(1 / m s)$ is included for deuterium, tritium, and ${ }^{3} \mathrm{He}$. The reaction rates for each spectes are evaluated from the following equations:

$$
\begin{aligned}
& \mathrm{R}_{\mathrm{DDn}}=\frac{1}{2} \mathrm{~g}_{\mathrm{DDn}} \mathrm{n}_{\mathrm{D}}^{2}\langle\mathrm{ov}\rangle_{\mathrm{DDn}^{\mathrm{A}} \mathrm{p}} \\
& \left.\mathrm{R}_{\mathrm{DDp}}=\frac{1}{2} \mathrm{~g}_{\mathrm{DDp}} \mathrm{n}_{\mathrm{D}}^{2}\langle\sigma \mathrm{v}\rangle\right\rangle_{\mathrm{DDp}} \mathrm{A}_{\mathrm{p}} \\
& R_{D T}=g_{D T} n_{D} n_{T}\langle\sigma v\rangle_{D T} A_{P}
\end{aligned}
$$

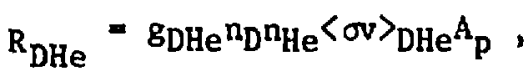

where the plasma cross-sectional area is $A_{p}=\pi r_{p}^{2}, r_{p}$ is the plasma radius defined as the cold-temperature $[T(r) \rightarrow 0\}$ plasm: boundary, and $g_{1 j}$ is a profile factor. These equations use Maxwellian-averaged cross sections ${ }^{3,4}$ and total particle densities (i.e., energet:lc plus Maxwellian densities). Because the energetic particle population ils typically very small $(<1 \%)$ for the DT 
densittes being considered here, the non-Maxwellian contribution to the reaction rate is simply approximated by using total densities in the rate equations. A discussion of the nonthermal effects of energetic particles is given in Ref. 2 . The proftle-averaged wetghting functions, $g_{i j}$, are evaluated from an expression of the following form.

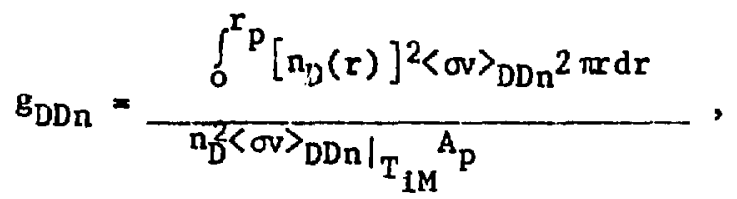

with the other profile terms analogously derived. The radial dependence of the total ion density, $n_{1}(r)$, and all consituent densities $\left(n_{D}, n_{T}, n_{H e}, n_{\alpha}, n_{H}\right)$ are taken to be identical. Integrating over the plasma radius, the average ion density is given by

$$
n_{1}=\int_{0}^{r_{p}} n_{1}(r) 2 \pi r d r / A_{p}
$$

The density-weighted average Maxwellian temperature is given by the following expression:

$$
T_{1 M}=\int_{0}^{r} p T_{1 M}(r) n_{1}(r) 2 \pi r d r / N_{1}
$$

where $N_{1}=n_{1} A_{p}$.

Changes in the plasma radius Introduced by adiabatic dimensional changes are taken into account by the following plasma energy balance:

$$
d\left(3 p \pi r_{p}^{2} / 2\right) / d t=P_{S U M}-p d\left(\pi r_{p}^{2}\right) / d t
$$

where $p$ is the total plasma pressure and $P_{S U M}$ is the sum of all plasma powers as defined below and is expressed here on the basts of a unit toroidal length. The second term in Eq. (A-7) subtracts the direct-conversion work pc:formed by a high-beta plasma expanding against confining magnetic fields and generally is negligible for the conditions being examined for the CRFPR. The components of $P_{\text {SUM }}$ are defined below: 


$$
\mathrm{P}_{\mathrm{SUM}}=\mathrm{P}_{\mathrm{Q}}-\mathrm{P}_{\mathrm{RAD}}-\mathrm{P}_{\mathrm{COND}}+\mathrm{P}_{\mathrm{OHM}}+\mathrm{P}_{\mathrm{AUX}} \cdot
$$

In the order listed, these powers (MW/m) are related to total charged-particle generation, radiation, thermal conduction, ohmic heating, and (optional) auxiliary heating; each power component is described in Sec. A.3. The plasma radius variation is negligible for the CRFPR, and $d x / d t$ is equal to zero, where $x=r_{p} / r_{w}$ is the plasma radius normalized to the radius of the first material surface.

A global accounting of the plasma energy, $W_{p}$, is evaluated from Eq. (A-7), which can be rewritten as follows:

$$
\dot{\mathrm{w}}_{\mathrm{p}}=-\frac{4}{3} \mathrm{w}_{\mathrm{p}} \frac{\dot{\mathrm{x}}}{\mathrm{x}}+\mathrm{P}_{\mathrm{SUM}} \text {, }
$$

where the first term on the right-hand side represents direct-conversion work, if applicable. The Maxwellian component of the plasma energy is defined from Eqs. $(A-5)$ and $(A-6)$ as

$$
\left(W_{p}\right)_{M}=3 k_{B}\left(N_{1 M} T_{1 M}+N_{e} T_{e}\right)
$$

and the Maxwellian electron and ion energy equations are given below:

$$
\begin{aligned}
& \frac{d\left(\frac{3}{2} N_{i M} k_{B} T_{I M}\right)}{d t}=-N_{i M} k_{B} T_{i M} \frac{2 \dot{x}}{x}-P_{C I}, \\
& \frac{d\left(\frac{3}{2} N_{e} k_{B} T_{e}\right)}{d t}=-N_{e} k_{B} T_{e} \frac{2 \dot{x}}{x}+P_{O H M}-P_{R A D}-P_{C e},
\end{aligned}
$$

where the electron/Ion conduction powers, ${ }^{P_{C j}}(j=i, e)$, have been introduced and $k_{B}=1.602(10)^{-16} \mathrm{~J} / \mathrm{kev}$.

Nine first-order differential equations have been introduced up to this point: five rate equations ( $H, D, T,{ }^{3} \mathrm{He},{ }^{4} \mathrm{He}$ ), one total energy equation, two particle energy equations (Ion and electron), and one plasma-expansion (pdV 
work) equation. In addition, all individual plasma powers are integrated with time, including the total charged-particle power, neutron power for both DD and DT reactions, direct-conversion work, Bremsstrahlung, cyclotron radiation into the first wall and first-wall penetrations, line radiation, ohmic heating, and electron and ion thermal conduction. Finally, the five rate equations [Eqs. (A-2)] are integrated, which in turn provide the population of energetic particles to be used as input to the Fokker-Planck model. A total of 24 equations are then integrated using a fourth-order Runge-Kutta method. This portion of the program follows the total species densities, plasma radius, total plasma energy, Maxwellian species temperature, and density variation resulting from plasma column radius changes or energy/particle losses. In addition, all plasma powers are Integrated, and the production of energetic particles is computed for use by the Fokker-Planck model.

\section{A.2. Fokker-Planck Slowing-Down Calculation.}

The slowing down of energetic charged particles in the plasma is described by a Fokker-Planck model. The Fokker-Planck routine receives the incremental energetic particle additions each time step. This calculation does not modify the total species densities, plasma radius, or total plasma energy determined from the main integrating routine. Only the Maxwellian electron and ion energy equations need be modifled by the Fokker-Planck equations, along with the Maxwellian number density of each Ionic species. Five species are separately followed: hydrogen, $\mathrm{p}(3.03$ and $14.67 \mathrm{MeV})$; tritium, $\mathrm{T}(1.01 \mathrm{MeV})$; ${ }^{3} \mathrm{He}$, He $(0.82 \mathrm{MeV}) ;{ }^{4} \mathrm{He}, \alpha(3.52$ and $3.67 \mathrm{MeV})$; and deuterium, $\mathrm{D}(\mathrm{NES})$. Energetic deuterlum species are produced only through nuclear-elastic-scattering (NES) events, which have been shown ${ }^{2}$ to be insignificant in the density range of interest to this study. Allowances for an energetic distribution of deuterium, therefore, are not necessary, although an account for this species is retained in event neutral-beam heating studies or the examination of lower-density systems are desired.

Incremental additions of energetic particles are passed from the integration routine to the Fokker-Planck model. Energetic particles are added to the respective aistribution functions as a Gaussian distribution that is centered about the "birth" energy with a velocity spread given by

$$
\Delta v_{S}=\left(2 k_{B} T_{i M} / M_{i M}\right)^{1 / 2}
$$


The properties of Maxwellian background species are used. A more-accurate expression would use the temperature corresponding to the peak of the cross section-velocfty product, ov, for the particular reacting species, 4 although this higher-order effect is not incorporated into this model. This spread of the Gaussian birth spectrum also includes the recoil energy of the interacting Maxwellian species. Comparing the energy content of the incremental distribution function, $\Delta f_{G}$, to that of the birth energy, $\Delta n_{s} E_{s}$, leads to adjustment of the background temperature that accounts for the loss of Maxwellian plasma energy resulting from the disappearance of the reacting species. Additions to the distribution function, $f_{s}$, for the energetic species are then given by

$$
\Delta f_{s}\left(n / v^{3}\right)=\frac{\Delta n_{s}}{4 \pi^{3 / 2}\left(\Delta v_{s}\right) v_{s}{ }^{2} e^{\left[\left(v-v_{s}\right) / \Delta v_{s}\right]^{2}}}
$$

where the velocity $v_{s}$ corresponds to the birth energy of the energetic species. The two alpha-particle and proton species are added to their respective distribution functions, leading to a double-peaked slowing-down spectrum. ${ }^{5}$

At a given time step after a pulse of energetic particles is added to the respective superthermal distribution, a superthermal slowing-down calculation is then performed for all species, thereby transferring energy to the background (Maxwellian) electron and fon species. This model is also used to calculate the Maxwellian electron/ion equilibration. The Maxwellian component of each distribution function is subtracted after each time step, and these particles are added to the background tons. The background ion veloctty is given by the following expression:

$$
v_{1}=\left(2 k_{B} T_{i M} / M_{1 M}\right)^{1 / 2}
$$

with the revised distribution function being given by

$$
f^{\prime}=f-\frac{\Delta n_{j M}}{\pi^{3 / 2} v_{1}^{3}}-\left(v / v_{1}\right)^{2} .
$$

The quantity $\Delta n_{j M}$ is defined so that $f^{\prime}\left(n / v^{3}\right)>0.0$ for each velocity mesh in the velocity range $0<v<4 v_{1}$. The quantity $\Delta n_{j M}$, therefore, represents the incremental Maxwellian ion densities added to the background population for each 
energetic species. The revised distribution function, $f^{\prime}$, then consists of purely non-Maxwellian particles.

After the slowing-down calculation is performed, the Maxwellian Ion/electron energy is reinitialized along with the total Maxwellian Ion density and the Maxwellian density of each ion component. After redistributing the energy and densities (Maxwellian versus energetic species) within the plasma, the integrating routine proceeds to another time step. It is important to limit the Fokker-Planck calculation to redistributing energy among particles within the plasma, with the profile-integrating routine governing total particle inventories and total plasma energies.

\section{A.3. Plasma Powers and Profile Averages.}

This section summarizes the analytic expressions used to describe all plasma energy gains and losses. In all cases, the powers are evaluated locally and integrated over the plasma cross section using the time-evolving, but functionally assumed, radial profiles. A typical power may be generally represented as a function of minor plasma radius according to

$$
P(r)=f[B(r), j(r), n(r), T(r)],
$$

with $P(r)$ being a function of magnetic field, $B(r)$, current density, $f(r)$, plasma density, $\mathfrak{n}(r)$, and plasma temperature, $T(r)$. The computer model reduces these powers to profile-average quarilities given by

$$
P=g f(B, j, n, T)
$$

The weighting functions, $g$, are given by

$$
g=\frac{\int_{0}^{r} p_{P(r) 2 \pi r d r}}{P A p} .
$$

The parameters in Eq. $(A-18)$ are defined as follows:

$$
\begin{aligned}
& B \equiv B_{\phi o}, \\
& j \equiv I_{\phi} / A_{p},
\end{aligned}
$$




$$
\begin{aligned}
& n \equiv \int_{0}^{r} p(r) 2 \pi r d r / A_{p}, \\
& T \equiv \int_{0}^{r} p T(r) n(r) 2 \pi r d r / n A_{p},
\end{aligned}
$$

where the peak toroldal field on axis, $B_{\phi_{0}}$, the toroldal plasma current, $I_{\phi}$, and the plasma cross-sectional area, $A_{p}=\pi x_{p}^{2}$, are used. These predefined quantities then provide the basis from which the weighting factors are derived. The above definitions are arbitrary, with specific definitions producing a specific set of weighting functions. The following list of powers are given in units of watts per meter of toroldal length and are expressed in the form of $\mathrm{Eq}$. (A-18) with the inferred calculation of weighting functions as defined by Eq. $(A-19)$.

a. Energetic-Particle Heating. Using the four reaction rates specifled by Eqs. (A-3), the energetic charged-particle (heating) powers are given by

$$
\begin{aligned}
& P_{H}=\left[R_{D D p}(3.03)+R_{D H e}(14.67)\right] 10^{3} k_{B}, \\
& P_{T}=R_{D D p}(1.01) 10^{3} k_{B}, \\
& P_{H e}=R_{D D n}(0.82) 10^{3} k_{B}, \\
& P_{\alpha}=\left[R_{D T}(3.52)+R_{D H e}(3.67)\right] 10^{3} k_{B},
\end{aligned}
$$

where the hydrogen, tritium, ${ }^{3} \mathrm{He}$, and ${ }^{4} \mathrm{He}$ rates are known. The total charged-particle power retained by the plasma is given by

$$
\begin{aligned}
\mathrm{P}_{\mathrm{Q}} & =\mathrm{P}_{\mathrm{H}}+\mathrm{P}_{\mathrm{T}}+\mathrm{P}_{\mathrm{He}}+\mathrm{P}_{\alpha} \\
& -3 \mathrm{k}_{\mathrm{B}} \mathrm{T}_{\mathrm{IM}}\left(0.8 \mathrm{R}_{\mathrm{DT}}+0.75 \mathrm{R}_{\mathrm{DDn}}\right) .
\end{aligned}
$$

Two Maxwellian Ions are lost for each fusion reaction, with the energy being contributed to the energetic fusion products. If the reaction products are charged, this energy is retalned in the plasma and no net energy loss occurs. This additional energy is added to the charged particles and is inherently taken into account by the Gaussian energy spread of the energetic particles that in turn is added to the Fokker-Planck distribution functions. That portion of $P_{Q}$ 
associated with the charged-particle energy loss corresponds to the energy carried away from the system by the fusion neutrons. These neutron powers are given by

$$
\begin{aligned}
& P_{D T n}=R_{D T}\left[(14.1) 10^{3} k_{B}+3 k_{B} T_{i M}(0.80)\right], \\
& P_{D D n}=R_{D D n}\left[(2.45) 10^{3} k_{B}+3 k_{B} T_{i M}(0.75)\right],
\end{aligned}
$$

with the quantities 0.75 and 0.80 accounting for the fractions $f$ Maxwellian-species energy given to the neutrons in each reaction.

b. Ohmic Heating. The ohmic heating power is given by

$$
P_{O H M}=g_{O H M} \eta_{\|} j^{2} A_{p}
$$

where the following expression for the clussical plasma resistivity 5,6 is used:

$$
\eta_{\|}\left(\text {ohm m) }=9.62(10)^{-10_{Z}}{ }_{\text {eff }} \ln N / \gamma_{E} \mathrm{~T}^{3 / 2}\right.
$$

The parameter $z_{e f f}$ is the sum of $n_{j} z_{j}^{2}$ divided by the sum of $n_{j} z_{j}$ taken over all ion species $j$. The Coulomb logarithm is defined as follows:

$$
\begin{aligned}
& \Lambda=4.907(10)^{17} \mathrm{~T}_{\mathrm{e}} \mathrm{e}^{3 / 2 / 2} \mathrm{eff}_{\mathrm{f}} \mathrm{e}^{1 / 2}\left(\mathrm{~T}_{\mathrm{e}}<0.0362 \mathrm{keV}\right), \\
& \Lambda=\Lambda\left(0.0362 / \mathrm{T}_{\mathrm{e}}\right)^{1 / 2}\left(\mathrm{~T}_{\mathrm{e}}>0.0362 \mathrm{keV}\right) .
\end{aligned}
$$

The value of $\gamma_{E}$ is approximated by the following expression:

$$
\gamma_{E}=0.582+0.418\left[\left(z_{\text {eff }}-1\right) / z_{\text {eff }}\right]^{2} \text {. }
$$

c. Radiation Power. The radiation power, $\mathrm{P}_{\mathrm{RAD}}$, consists of Bremsstrahlung, Iine, and cyclotron radiations. Impurity radiation is modeled by oxygen and uses fitted analytic functions. ${ }^{7}$. Any number of impuritles may be added to the plasma assuming the ion species are fully sicripped. The expression for Iine radiation, however, Includes only oxygen. The average Bremsstrahlung power is 8

$$
P_{B R}=5.35(10)^{-37} g_{B R} n_{e^{2}}^{2} e_{f f} e^{1 / 2} A_{p}
$$


The cyclotron radiation leaving a nonabsorbing plasma ${ }^{8}$ is given by

$$
{ }^{P} C Y=6.20(10)^{-17} n_{e^{B}}{ }^{2} T_{e}\left(1+T_{e} / 204\right)
$$

Accounting for absorption and assuming the walls surrounding the plasma are nonreflecting, ${ }^{B}$ the actual fraction of cyclotron radiation leaving the plasma is given by

$$
k_{L}=2.1(10)^{-3} T_{e}^{7 / 2}\left(c B \varepsilon_{0} / 2 r_{p} n_{e}\right)^{1 / 2},
$$

where $\varepsilon_{0}=10^{-9} / 36 \pi \mathrm{F} / \mathrm{m}$ is the permittivity of free space, the speed of light is $c=3(10)^{8} \mathrm{~m} / \mathrm{s}$, and the electronic charge is given by $\mathrm{e}=1.502(10)^{-19} \mathrm{C}$.

The fraction of $P_{C Y} \frac{T}{Y}$ leaving an absorbing plasma surrounded by a reflecting cylindrical wall is given as the function $k_{C}$ by Krajcik. ${ }^{9}$ For a first-wall hole fraction $\mathrm{f}_{H}$, therefore, the cyclotxon radiation power leaving the plasma and escaping through these holes is

$$
\mathrm{P}_{\mathrm{CY}}^{\mathrm{H}}=\mathrm{g}_{\mathrm{CYH}} \mathrm{P}_{\mathrm{CY}}^{\mathrm{T}} \mathrm{k}_{\mathrm{L}} \mathrm{f}_{\mathrm{H}_{\mathrm{p}}}^{\mathrm{A}_{\mathrm{p}}}
$$

The power leaving the plasma and absnrbed by the first wall is

$$
\mathrm{P}_{\mathrm{CY}}^{W}=g_{C Y W} \mathrm{P}_{\mathrm{CY}}^{\mathrm{T}} k_{C}\left(1-\mathrm{f}_{\mathrm{H}}\right) A_{\mathrm{p}}
$$

and the total power leaving the plasma is given by

$$
\mathrm{P}_{\mathrm{CY}}=\mathrm{P}_{\mathrm{CY}}^{\mathrm{H}}+\mathrm{P}_{\mathrm{CY}}^{\mathrm{W}}
$$


i. izansport, As with most approaches to magnetically cisnfined fusion energy, transport scaling represents a key uncertainty fsi any reactor projection. A transport-based confinement time scaling per se is not yet available for RFPs. By equating the ohmic heating power density, $\eta j^{2}$, to an energy loss rate, $3 \mathrm{nk}_{\mathrm{B}} \mathrm{T} / \tau_{E}$, of unspectfled mechanism, an "ohmic" scaling results, $\tau_{E}\left(\right.$ OHMIC) $\propto \beta_{\theta} T^{3 / 2} r_{p}^{2} / z_{e f}$, where the plasma resistivity is assumed to be classical and pressure balance is invoked. The coeffictent in this expression for $\tau_{\mathrm{E}}$ (OHMIC) depends on plasma density, temperature, and current-density [f.e., $\mu(r)]$ profiles. This ohmic scaling for a flat and a $J_{0}(\mu r)$ temperature profile with $p(r) \propto J_{0}^{2}(\mu r)$ is compared in Fig. A-1 for a number of key RFP experiments that have reported energy confinement times while operating at a centerline temperature, $T(0)$. The weak dependence of the coefficient $\tau_{E}($ OHMIC $) /\left(\beta_{\theta} T(0)^{3 / 2} r_{p}^{2} / z_{\text {eff }}\right)$ on $z_{\text {eff }}$ is shown, and a nominal beta of $\beta_{\theta} \simeq 0.2$ was used to show this dependence. Little physics other than the assumption of classical resistivity has been used, except to state that the beta

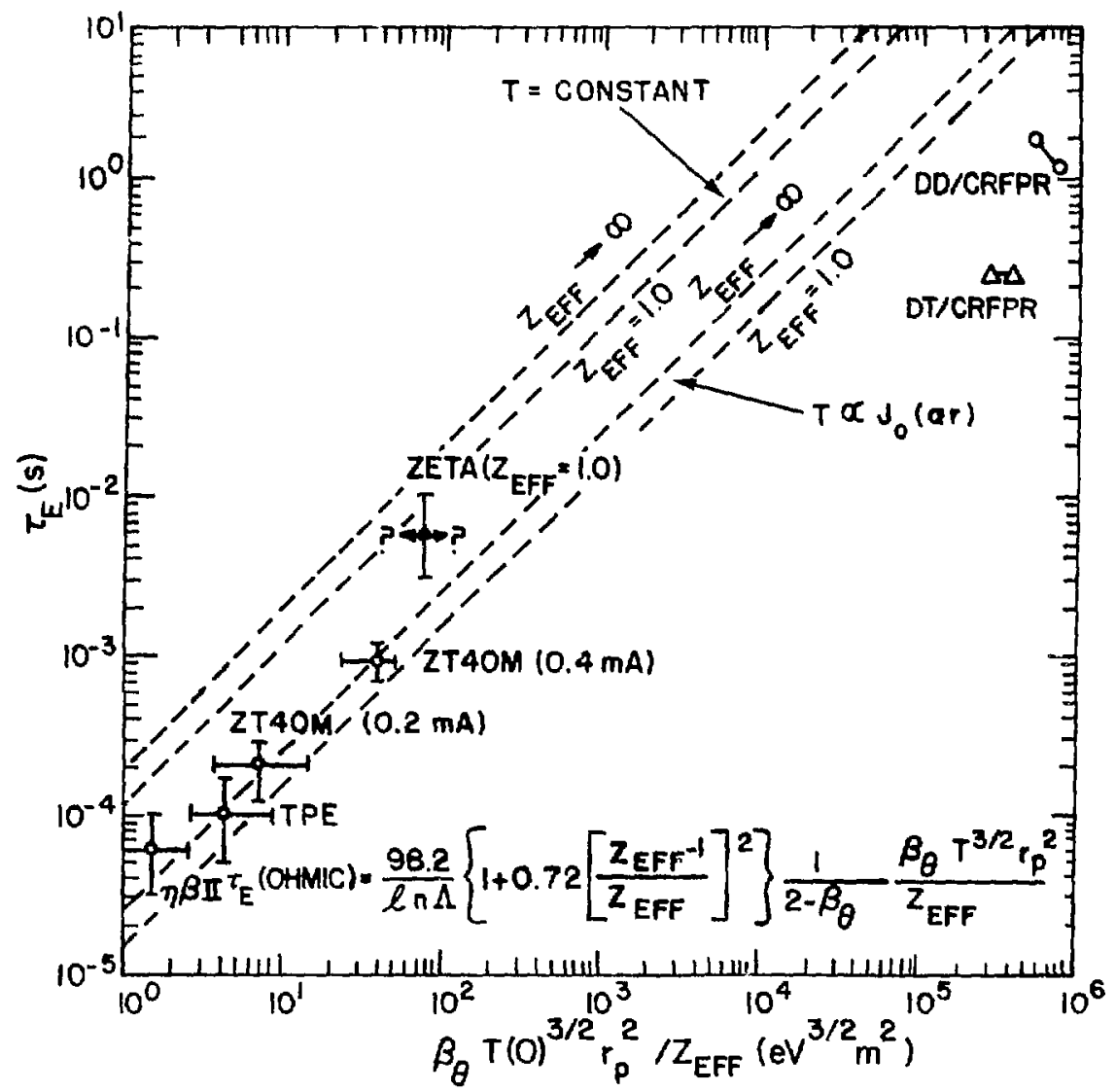

F1g. A-1. Comparison of ohmic transport scaling with a number of RFP experiments. 
Is limited to a specific value by the transport mechanism. If the parameter $I_{\phi} / \mathrm{N}$ is then presumed to be relatively constant as the toroldal plasma current, $I_{\phi}$, is increased, the relationship $I_{\phi} \propto T$ then results. This dependence is generally expected on the basis of pressure balance with both $\beta_{\theta}$ with $I_{\phi}$ nominally fixed. For present-day experiments $I_{\phi}=0.1-0.2 \mathrm{MA}$, and projected reactors have $I_{\phi}=18 \mathrm{MA}$, both operating with $I_{\phi} / \mathrm{N}$ in the range (1.5-8) $10^{-14} \mathrm{~A} \mathrm{~m}$. The ohmic scaling would then give

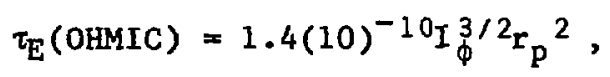

where $I_{\phi}$ has units of amperes. The coefficient has been benchmarked to the $\mathrm{ZT}-40 \mathrm{M}$ experiment 10 at $I_{\phi}=120 \mathrm{kA}$, where $\tau_{\mathrm{E}} \simeq 0.2 \mathrm{~ms}$. It is emphasized, however, that the current exponent being predicted from $\mathrm{ZT}-40 \mathrm{M}$ is near unity.

Self-consistent experimental evidence to date generally has shown $\tau_{E} \propto I_{\phi}^{\nu} f\left(\beta_{\theta}\right)$ for a range of RFPs, where $v=1.0-1.5$; the lower exponent is exhlbited by $\mathrm{ZT}-40 \mathrm{M}$, and the higher values are indicated by TPE-1RM. 11 For the present reactor simulations, the more pessimistic scaling for the electron conduction time is taken:

$$
\tau_{c e}=5(10)^{-\gamma_{1}} r_{\phi}^{2} f\left(\beta_{\theta}\right),
$$

where $I_{\phi}$ is expressed in units of amperes, the coefficient is again benchmarked to $\mathrm{ZT}-4 \mathrm{OM}$ experiment, 10 and again emphasizing that the current exponent is near unity for $2 \mathrm{~T}-40 \mathrm{M}$. Beta saturation effects in neutral-beam-heated tokamaks $12-14$ have indicated the value of $\tau_{c e}$ is reduced by $\left(1 / \beta_{\theta}\right)^{2}$ as a critical value of beta, $\beta_{\theta C}$, is exceeded. Typicaliy, $f\left(\beta_{\theta}\right)$ equals unity for $\beta_{6}>\beta_{\theta C}$ and $\left(\beta_{\theta C} / \beta_{\theta}\right)^{2}$ otherwise, where $\beta_{\theta C} \simeq 0.13$ is representative of present ohmically heated RFPs. The following functional forms emerge and are used for $f\left(\beta_{\theta}\right)$ :

$$
f\left(\beta_{\theta}\right)\left\{\begin{array}{l}
=1\left(\beta_{\theta}<0.13\right) \\
=\left(0.13 / \beta_{\theta}\right)^{2}\left(\beta_{\theta}>0.13\right) .
\end{array}\right.
$$

Having defined the electron conduction time, preliminary experimental measurements Indicate the ion-particle confinement time is given approximately by 15 


$$
\tau_{p 1} \simeq 4 \tau_{c e} .
$$

The ion-conduction time is assumed equivalent $\left(\tau_{c 1} \simeq \tau_{p 1}\right)$.

The above equations define the transport model used in the reactor plasma simulations. Other energy confinement times are also calculated for comparison purposes. Such comparisons are particularly crucial and useful for the RFP, where an intrinsic or physics-based transport scaling is not avallable, economics dictate a particular value of $\tau_{E}=\tau_{E}(O P T)$, and in the process of reconciling the two with ohmic scaling it becomes useful to examine the "benchmarks" established by classical or Alcator scaling laws. The classical Lon-conduction time is given below:

$$
\tau_{\text {CLASS }}=\frac{19.08 \mathrm{~T}_{1}^{3 / 2} r_{p}^{2}}{\left(\beta_{\theta} a_{1}^{1 / 2} \ln \Lambda\right)},
$$

where the atomic mass for DT is taken as $a_{i}=2.5$. A Bohm-related confinement time is given by

$$
\tau_{\mathrm{BOHM}} \simeq \frac{200 \mathrm{r}_{\mathrm{w}}^{2} \mathrm{~B}_{\theta}\left(\mathrm{r}_{\mathrm{p}}\right)}{62.5 \mathrm{~T}_{\mathrm{e}}},
$$

where a factor of 200 multiplies the usual Bohm time. Finally, a tokamak-related Alcator scaling of the following form is used for comparisons:

$$
\tau_{c e}(\mathrm{ALC}), \simeq 4(10)^{-2 l_{n r_{p}^{2}}} .
$$

A.4. Magnetic-Field, Density, and Temperature Profiles. This section specifies the magnetic-field profiles used to model reactor startup, burn, and rundown (plasma quench). A complete one-dimensional plasma equilibrium is performed at selected time intervals while imposing the experimentally derived plasma density, temperature, and current-density profiles and while satisfying pertinent edge-plasma boundary conditions. Following the formulation suggested 
by ortolani, 16 the magnetic-field spatial distribution 1 s described by speclfying the current density both parallel and perpendicular to the magnetic field. This procedure separates the magnetic-field variations resulting from plasma pressure gradients from the much larger force-free parallel current component; typically, field variations from pressure gradients are ustally small for $\beta_{\theta} \leqslant 0.2$.

From the Maxwe1l equations, assuming a static electric field, it follows that

$$
\begin{aligned}
& \vec{\nabla} \times \vec{B}=\mu_{0} \vec{j}=H_{0}\left(\vec{j}_{\|}+\vec{j}_{1}\right) \\
& \vec{j} \times \vec{B}=\vec{\nabla}_{p} .
\end{aligned}
$$

Performing a cross-product operation,

$$
(\vec{j} \times \vec{B}) \times \vec{B}=(\vec{j} \cdot \vec{B}) \vec{B}-B^{2} \vec{j}=\vec{\nabla} p \times \vec{B}
$$

The parallel and perpendicular current components are given by

$$
\begin{aligned}
& \vec{j}_{\|}=\frac{(\vec{j} \cdot \vec{B}) \vec{B}}{B^{2}}=\overrightarrow{\mu B} \\
& \vec{j}_{\perp}=\frac{\overrightarrow{\nabla p} \times \vec{B}}{B^{2}}
\end{aligned}
$$

Expanding Eq. (A-43) in cylindrical coordinates where $\phi$ and $\theta$ are the major and minor toroldal angles, respectively, yields the folluwing working equations used to evaluate $B_{\theta}$ and $B_{\phi}$ :

$$
\begin{aligned}
& -\frac{\partial B_{\phi}}{\partial r}=\mu_{0} j_{\theta}=\mu B_{\theta}+\mu_{0} \frac{\partial p}{\partial r} \frac{B_{\phi}}{B^{2}} \\
& \frac{1}{r} \frac{\partial}{\partial r}\left(r B_{\theta}\right)=\mu_{0} j_{\phi}=\mu B_{\phi}-\mu_{0} \frac{\partial p}{\partial r} \frac{B_{\theta}}{B^{2}} .
\end{aligned}
$$

Except for spectal cases, these equations must be numerically integrated. Taking $\mu$ as a constant, the analytic Idealized Bessel-function model (BFM) may be derived for $p(r)=0$. In this case 1 follows that 


$$
\begin{aligned}
& { }_{B_{\theta}}(r)=A_{\theta} J_{l}(\mu r) \\
& B_{\phi}(r)=A_{\phi} J_{0}(\mu r),
\end{aligned}
$$

where $J_{0}(\mu r)$ and $J_{1}(\mu r)$ are Bessel functions of the first kind with $A_{\theta}=A_{\phi^{*}}$ Using Eq. $(A-44)$, the plasma current densities are given by

$$
\begin{aligned}
& j_{\phi}(r)=\frac{\mu A_{\theta} J_{0}(\mu r)}{\mu_{0}} \\
& j_{\theta}(r)=\frac{\mu A_{\phi} J_{1}(\mu r)}{\mu_{0}} .
\end{aligned}
$$

The toroidal plasma current, $I_{\phi}$, must equal the integral of $j_{\phi}$ over the plasma cross section, which leads to

$$
A_{\theta}=B_{\theta}\left(r_{p}\right) / J_{1}\left(\mu r_{p}\right),
$$

where $r_{p}$ is defined as the first material $\left[T\left(r_{p}\right)>0\right]$. Parameters of experimental interest are defined as follows:

$$
\begin{aligned}
& \theta=B_{\theta}\left(r_{p}\right) /\left\langle B_{\phi}\right\rangle \text { (pinch parameter) } \\
& F=B_{\phi}\left(r_{p}\right) /\left\langle B_{\phi}\right\rangle \text { (reversal parameter), }
\end{aligned}
$$

where

$$
\left\langle B_{\phi}\right\rangle=\frac{2}{\mathbf{r}_{\mathbf{p}}^{2}} \int_{0}^{r_{p}} B_{\phi} \mathrm{rdr} .
$$

The following expression is for the pinch parameter in terms of $\mu(r)$ results:

$$
\theta=\frac{\mu r_{p}}{2} \frac{A_{\theta}}{A_{\phi}},
$$

where $A_{\theta}=A_{\phi}$ for the force-free $\left(\beta_{\theta}=0\right)$ BFM. The relationship between $F$ and $\theta$ then becomes

$$
F=\frac{\theta J_{0}(2 \theta)}{J_{1}(2 \theta)}
$$

A major shortcoming of the force-free BFM ts the absence of plasma pressure. 
From the Maxwell equation $\nabla p=\nabla \times \vec{B} \times \vec{B} / \mu_{0}$ and assuming the Bessel Function magnetic-field profiles are approximately maintained in the presence of plasma pressure, the following relationship between $A_{\theta}$ and $A_{\phi}$ results:

$$
\beta_{\theta}=1-\left(A_{\theta} / A_{\phi}\right)^{2}
$$

where the experimentally defined poloidal beta is given by

$$
\beta_{\theta}=\frac{\langle p\rangle}{\bar{B} z\left(r_{p}\right) / 2 \mu_{0}}
$$

For this approximate model the plasma pressure profile is described by $p(r) \propto J_{0}^{2}(\mu r)$. Observing the BFM equations, only $\theta$ and $\beta_{\theta}$ need be provided to establish the magnetic-field profile. The mugnitude of the fields ase then determined by $I_{\phi}$ and $r_{p}$. Even when numerically solving Eqs. (A-45), for the generalized field and current profiles, similar information is needed. The good agreement between the Bessel-function mode: and actual MHD stable fleld profiles is shown in $\mathrm{Fig}$. A-2, where vacuum flelds are taken beyond the radius, $r_{p}$.

Although the magnetic-field profiles for the Bessel-function model are in good agreement with the numerically computed profiles, this model has two limitations. The pressure-modified magnetic-field profiles are not described accuratily by Bessel functions, and the experimental pressure profile may substantially deviate from the $p(r) \times J_{0}^{2}(p r)$ functional dependence. Because $\beta_{\theta}=0.1-0.2$, large changes in the pressure profile have little nffect on the magnetic-field structure. Secondly, the current density profile given by Eqs. (A-47), in the BFM can be substantially different from the experimentally observed current densfty. The $B F M$ in particular requires $\mu_{0} j_{\theta}=\mu B_{\theta}$, forcing a large current density near the plasma edge where the plasma temperature is approaching zero and the plasma resistance is large. This condition is experimentally unphysical and overestimates the effective resistive heating in 'he plasma.

This shortcoming is resolved by direct numerical integration of $\mathrm{Eq} \cdot(\mathrm{A}-45)$. In this case the expertmental density, $n(r)$, and te:uperature, $T(r)$, profiles are 1 mposed along with the current density profiles using $\mu_{0} j_{\|}=\mu \mathrm{B}$, where $\mu(r)$ is a function of radius. The current density profile is forced to zero at the plasma edge by requiring $\mu(r) \rightarrow 0$ as $r+r_{p}$. The numerical solution of $E q \cdot(A-45)$ is straightforward if the central beta, $\beta_{0}=\mathrm{p}_{0} /\left(\mathrm{B}_{\phi_{0}}^{2} / 2 \mu_{0}\right)$, and the radial 


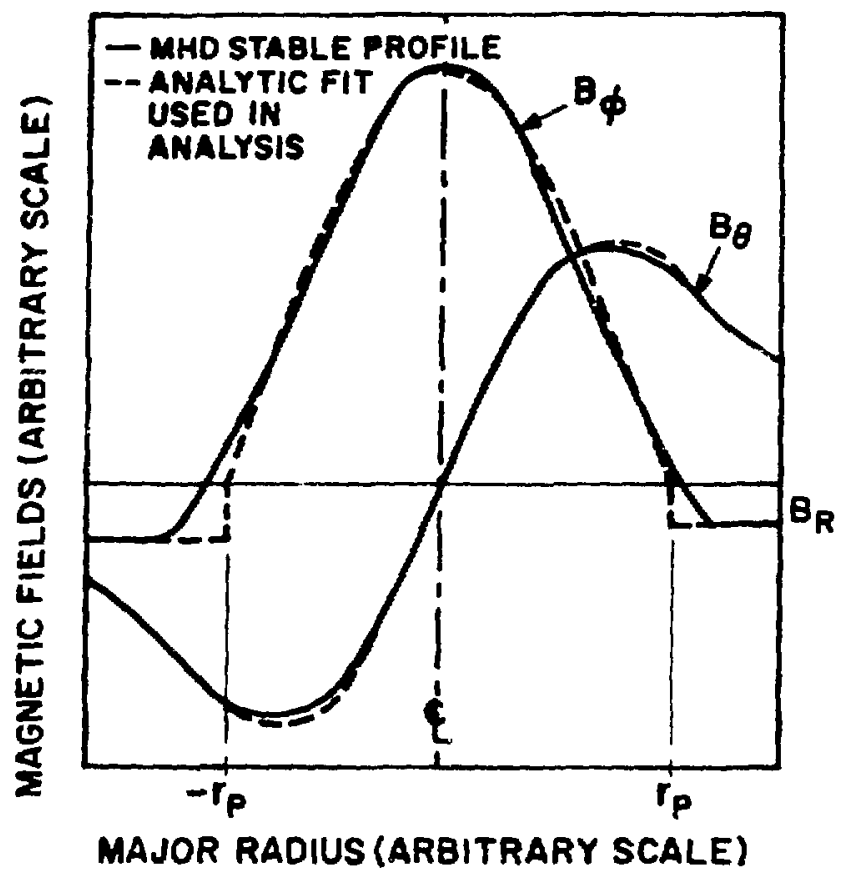

Fig. A-2. Comparison of Besse1-function model with numerically computed field profiles.

derivative of the pololdal magnetic fleld, $\left(\partial B_{\theta} / \partial r\right)_{r}=0$, are known a priori. In fact, for $B_{\phi} \gg B_{\theta}$, as for a stellarator and to Elrst order for a tokamak, this condition is indeed appropriate, assuming $n(r), T(r)$, and $\mu(r)$ are known. For a reversed-field pinch and the spheromak, where $B_{\theta} \simeq B_{\phi}$, and for an exact calculation of tokamak profiles, whers $B_{\theta} \sim 0.1 B_{\phi}$, the centerilne quantiries must be derived, requiring numerical iteration of the profiles until the boundary conditions are satisfied.

Imposing $n(r), T(r)$, and $\mu(r)$ profiles, solutions for $B(r)$ and $f(r)$ having the desired values of $\beta_{\theta}$ and $\theta$ are found. A given iteration requires numerical Integration of Eqs. (A-45) and comparison of derived results with the desired values of $\beta_{\theta}$ and $\theta$. The new 1teration then adjusts the centerline beta and pololdal magnetic-field derivative. Simultaneous iteration on two variables uses Newton's method, and the resultant iteration converges quadratically since the number of significant places doubles on each iteration. Like the trinsport $[\mathrm{Eq} \cdot(\mathrm{A}-35)]$, the input profiles are referenced to the $2 \mathrm{~T}-40 \mathrm{M}$ device. 10 operating with a toroidal plasma current of $120 \mathrm{kA}$ in a $0.2-\mathrm{m}$ minor radius 
machine, the centerline electron temperature exceeds $300 \mathrm{eV}$ at a time of 2 ms into the discharge. With powtr crowbar operation greater than 20-ms steady-state discharges with poloidal betas of $\beta_{\theta} \sim 0.1$ have been obtained. Thomson-scattering measurements of $T_{e}$ at a minor radius of 0.14 m show roughly $2 / 3$ of the central temperature, which is indicative of $T(r) \propto J_{0}^{1 / 2}(\mu r)$. Interferometric density measurements may be approximately represented by $n(r) \propto J^{3 / 2}(\mu r)$, implying a pressure profile of $p(r) \propto J_{0}^{2}(\mu r)$, which is the approximate BFM solution. At reactor-like plasma temperatures, better confinement properties are projected because of higher plasma currents, and more-gradual temperature gradients are expected. Based on these considerations, - simple Bessel-function temperature and density profiles $\left[T(r) \propto J_{0}(\mu r)\right.$, $n(r) \propto J_{0}(\mu r)$ where, $T(r)$ and $n(r) \rightarrow 0$ as $\left.r \rightarrow r_{p}\right]$ are taken for the reactor plasma. In this case the peak-to-average density is 2.3 and the density-weighted peai-to-average temperature is 1.6 . As determined by the parametric systems code 17 for a fixed pressure profile, $p(r) \propto J_{0}^{2}(\mu r)$, the specific shape of $n(r)$ and $T(r)$ has little impact on the reactor power output; as these profiles are changed, the operating temperature and density must be adjusted to maintain the same power output, and this in turn may affect other parameters such as the plasma resistive losses.

The RFP field profile is then determined when the current density profile is specified through $\mu(r)$. The spatial dependence of $\mu(r)$ is chosen to provide an accurate fit to the experimentally measured toroidal magnetic flux and to the toroldal and poloidal magretic flelds at the discharge chamber wall. A "best-fit" model uses a constant $\mu(r)$ until the breakpoint $r=\alpha r_{p}(\alpha<1)$, where $\mu(r)$ is linearly extrapolated to zero at $r=r_{p}$. This breakpoint is described by a fitted function as toilows: 18

$$
\begin{aligned}
& \alpha=1.0 \quad(\theta<1.2) \\
& \alpha=1.92-\mathrm{f}(\theta) / 2.6 \quad(1.2<\theta<2.5),
\end{aligned}
$$

where the function $f(\theta)$ is given by

$$
f(\theta)=0.04623+1.47121 \theta+1.21618 \theta^{2}-0.83678 \theta^{3}+0.13193 \theta^{4} .
$$


A.5. Equilibrium and Stability. The reversed-field force-free configuration was predicted analytically by Taylor ${ }^{19}$ as the lowest plasma energy state $\theta>1.2$ and negligible beta for a plasma confined inside a perfectly conducting shell. From scs. A.4. the pinch parameter, $\theta$, and reversal parameter, $F$, are related according to $F=\theta \mathrm{J}_{0}(2 \theta) / J_{1}(2 \theta)$, which is plotted in Fig. A-3 for the zero-beta Taylor state. Numerical calculations ${ }^{19}$ have shown the existence of high-beta stable states $\left(\beta_{\theta}=0.3-0.4\right)$ in the $F-\theta$ range, $\theta=1.5-2.0$ and $F=-0.5$ to -1.0 , which is denoted as the high-beta model. A

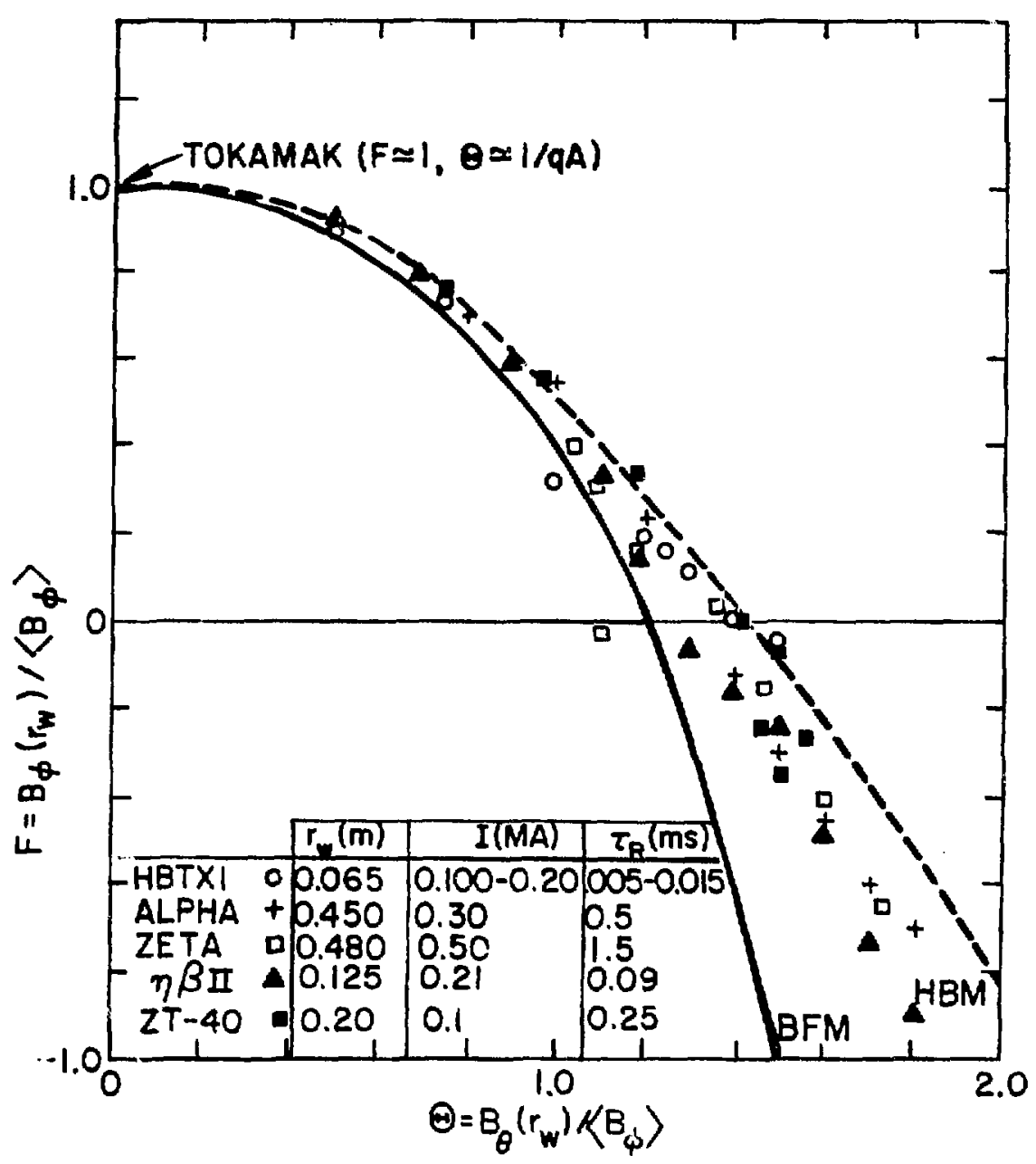

Fig. A-3. Universal $F-\theta$ curve showing data from four RFP machines, where $F=B_{\phi}\left(r_{w}\right) /\left\langle B_{\phi}\right\rangle$ and $\theta=B_{\theta}\left(r_{w}\right) /\left\langle B_{\phi}\right\rangle$. The theoretical curve for the Bessel-function mode1 18 is shown. The dotted curve is for the high-beta model. 19 
substantial body of experimental evidence has verified the Taylor theory, which is used for the reactor calculations.

The actual $F-\theta$ trajectory used in the computer analysis must be consistent with the one-dimensional plasma equilibrium which satisfies the Maxwell equations. The experimentally derived and extrapolated temperature and density profiles are taken as $n(r) \propto T(r) \propto J_{o}(\mu r)$. Forcing the current density to zero at the plasma edge according to the "best-fit" model [Eq. (A-56)] gives the $F-\theta$ trajectories depicted in Fig. A-4 for $\beta_{\theta}=0$ and 0.25 . Also given in Fig. A-4 is an approximate beta-modifled analytic Bessel-function solution, where $F=\theta^{*} J_{0}\left(2 \theta^{*}\right) / J_{1}\left(2 \theta^{*}\right)$ and $\theta^{*}=\theta\left(1-\beta_{\theta}\right)^{1 / 2}$.

Recent experimental data also indicate a minimization of plasma losses if the F- $\theta$ trajectory is followed during plasma startup and burn. The "best-fit" F- $\theta$ trajectory provides the relationship between the toroidal, $B_{\phi}$, and poloidal, $B_{\theta}$, fields during startup/burn at a given pololdal beta, $\beta_{\theta^{*}}$ Experimental

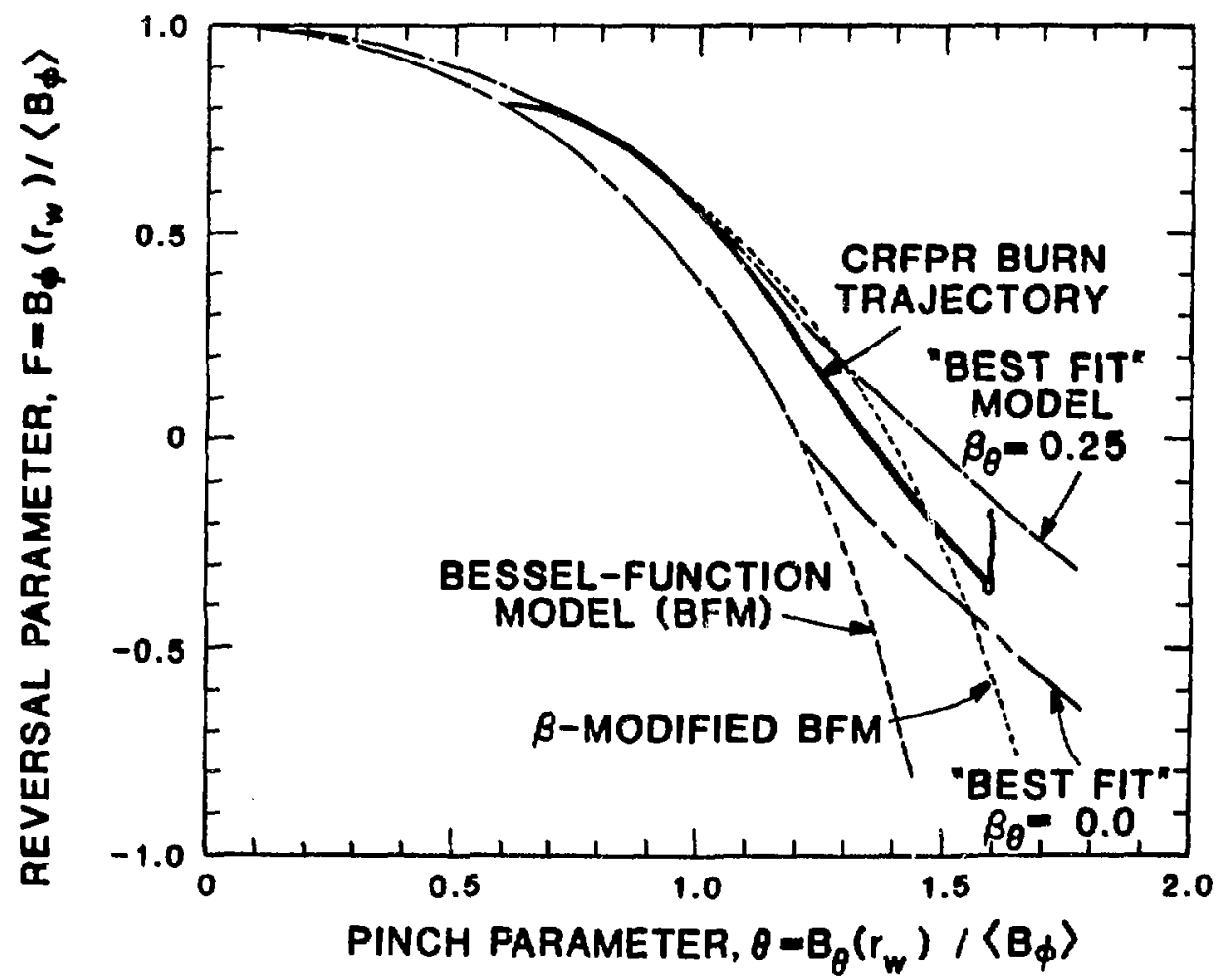

Fig. A-4. Taylor diagram showing the $\beta=0$ Taylor curve, the high-beta model, the numerical "best fit" model for $\beta_{\theta}=0.0$ and 0.25 , and the startup trajectory proposed for the CRFPR. 
values of $\theta=1.55$ and $F=-0.1$ to -0.15 at $\beta_{\theta}=0.1-0.25$ dictate the magneticfield structure during the burn simulation.

The presence of a conducting shell provides plasma stability and equilibrium for a relatively short $(\sim 0.1 \mathrm{~s})$ time scale. For longer times external feedback conductors must provide the tmage currents required to stabilize the $m=1$ mode, as predicted by theory. Gross plasma equilibrium is also provided externally by an external vertical field for times longer than 0.1 s. An expression for the required quasi-uniform vertical field is 20,21

$$
B_{v}=\left(\mu_{0} I_{\phi} / 4 \pi R_{T}\right)\left[\ln \left(8 R_{T} / r_{p}\right)+2 \pi l_{1} / \mu_{0}+\beta_{\theta}-1.5\right]
$$

where $l_{1}$ is the Inductance per unit length of plasma. For the RFP with Bessel-function profiles, $2 \pi i_{1} / \mu_{0}=0.5$. To provide flasma equilibrium in both the horizontal and vertical directions requires the vertical field to have a curvature that is characterized by the following decay index:

$$
\mathbf{n}=-\frac{\partial \ln B}{} \mathbf{v}=\left(\frac{R}{\partial \ln r}\right) \frac{B\left(R_{T}-r_{p}\right)-B\left(R_{T}+r_{p}\right)}{r_{p}\left(R_{T}-r_{p}\right)+B\left(R_{T}+r_{p}\right)},
$$

where $n$ should lie between 0.0 and 1.5. Constraining the plasma to have a circular cross section further specifies that $n$ be in the range of $0<\mathrm{n} \leq 0.65 .22$

A.6. First-Wa11 Thermal-Mechanica1 Response. A critical engineering subsystem in any fusion reactor is the first wall and the in-vacuum components (IVCs) directly exposed to the plasma. Even preliminary reactor designs must apply first-wall thermal fatigue constraints. For this reason the CRFPR computer model directly couples the thermal-mechanical analysis of the first wall and the dynamic simulation of plasma burn. This first-wall thermal-mechainical response serves only a monitoring function, the more detailed analyses of the kind described in Sec. III.D. being used for design purposes.

A one-dimensional heat-transfer calculation follows first-wall temperature variations subject to surface cooling by a specified coolant. A one-dimensional structural analysis is also directly coupled to the first-wall thermal calculation. Both thermal and structural analyses allow two material regions, 
although a single water-cooled first wall is used in the burn calculations reported here.

The average surface heat flux incident on the first wall is given by

$$
I_{Q W}\left(W / m^{2}\right)=\left(P_{B R}+P_{L I N E}+P_{C Y}^{W}+P_{C O N D}\right) /\left(2 \pi r_{W}\right)
$$

where Bremsstrahlung, 11ne, cyclotron (1nto the wa11), and conduction powers per unit toroidal length are used. Volumetric heating by the energetic neutrons may also be Included, this input being available from the neutronics analysis (Sec. III.B.). The volumetric heating in a 20-mm-thick copper-alloy first wall by $14.1-\mathrm{MeV}$ neutrons amounts to $11.2 \mathrm{MW} / \mathrm{m}^{3}$ for every $\mathrm{MW} / \mathrm{m}^{2}$ of $14.1-\mathrm{MeV}$ neutrons Incident on the first wall. Information is not available for the $2.45 \mathrm{MeV}$ neutron species, which is small for the 50/50 oT fuel being used here.

The powers used for the heat-transfer calculation are given by

$$
\begin{aligned}
& I_{Q W}\left(W / m^{2}\right)=I_{Q W}(2 / \pi) R_{T} /\left(R_{T}-r_{W}\right) \\
& q_{N}\left(W / m^{3}\right)=11.2 I_{W} R_{T} /\left(R_{T}-r_{W}\right),
\end{aligned}
$$

where the factor $2 / \pi$ accounts for the added surface area oi a first-wall tube array. The factor $R_{T} /\left(R_{T}-r_{w}\right)$, where $R_{T}$ is the major radius, is used as a first-order consideration of toroidal power peaking.

The one-dimensional two-region thermal-mechanical subroutine uses the physical properties listed in Table A-I for copper alloy and stainless steel. The stainless-steel region is taken to be diminishingly small for the CRFPR first wall. With the copper tube dimensions, the coolant temperature, and heat-transfer coefficient as input, the thermal/mechanical characteristics can be determined.

A.7. Reactor Energy Balance. A reactor energy balarice is calculated directly by the simulation code when the conditions at the time of code termination represent a steady-stace condition. Figure A-5 deplcts the general energy flow dlagram for the CRFPR, which is an approximation to the power balance given in Fig. III.L-1. The plasma produces neutron, $P_{N}$, radiation, ${ }_{\mathrm{RAD}}$, and conduction, $\mathrm{P}_{\mathrm{COND}}$, powers, which eventually appear as thermal power delivered to the first wall and blanket. All thermal energy delivered to the first wall and bianket coolant is converted with a thermal efficiency, $\eta_{T H}$, to 
TABLE A-I

FIRST-WALL PHYSICAL PARAMETERS

\begin{tabular}{lcc} 
REgION I & REgION 2 \\
PARAMETER & 316 STAINLESS STEEL & COPPER ALLOY \\
\hline
\end{tabular}

$\begin{array}{lll}\text { Density, } \rho\left(\mathrm{kg} / \mathrm{m}^{3}\right) & 7,970 & 8,880 \\ \text { Thermal conductivity, } \mathrm{k}(\mathrm{W} / \mathrm{m} \mathrm{K}) & 19.5 & 324 . \\ \text { Heat capacity, } c_{\mathrm{p}}(\mathrm{J} / \mathrm{kg}) & 560 . & 394 . \\ \text { Therma1 expansivity, } \alpha(1 / \mathrm{k}) & 17.7(10)^{-6} & 19.3(10)^{-6} \\ \text { Young's Modulus, } \mathrm{E}(\mathrm{Pa}) & 1.67(10)^{11} & 1.38(10)^{11} \\ \text { Poisson's ratio, } \nu & 0.29 & 0.36\end{array}$

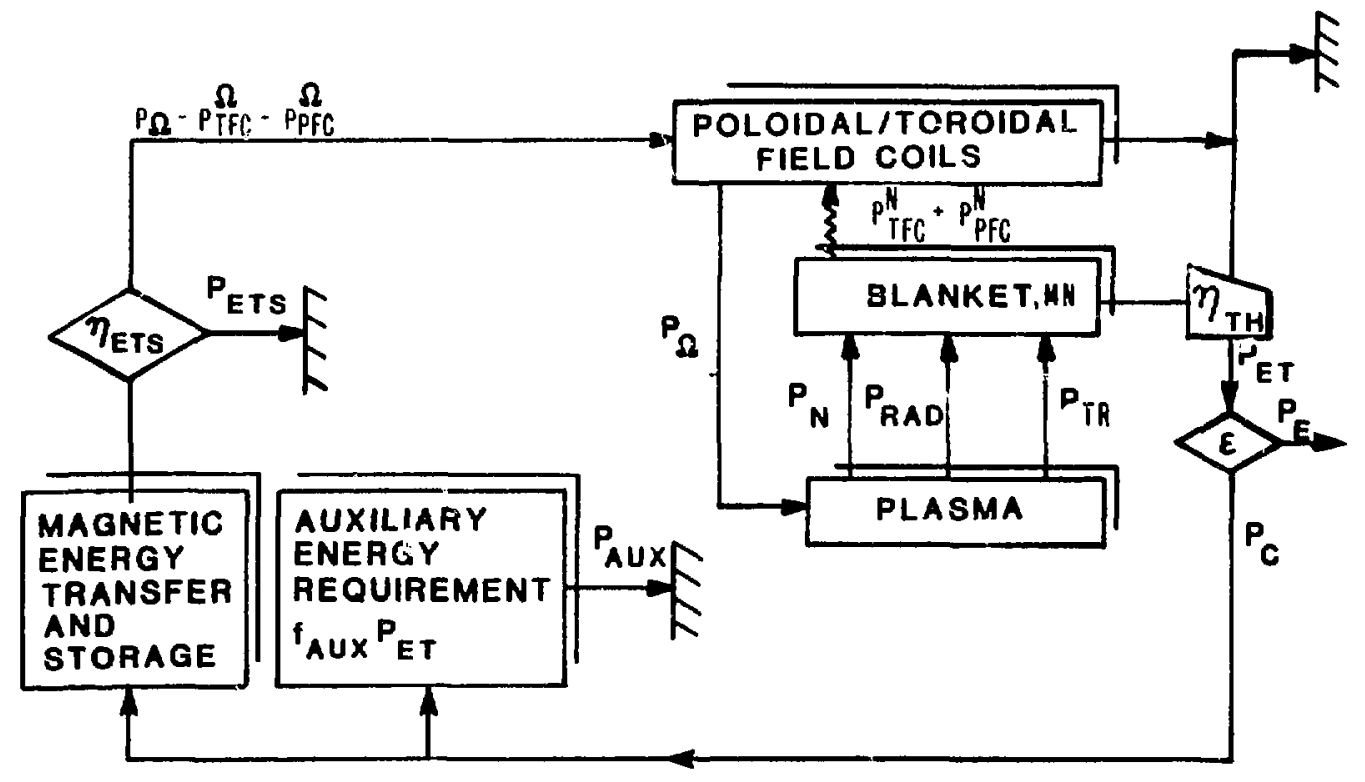

Fig. A-5. CRFPR power balance for a long-pulse or steady-state burn. 
produce a gross (total) electric power, $\mathrm{P}_{\mathrm{ET}}$. Auxiliary power requirements $\mathrm{P}_{\mathrm{AUX}}$ (pumps, plant operation, etc.) are given as a fraction $f_{A U X}$ of $P_{E T}$ along with power transferred to the magnet coils. The ohmic power losses in the poloidal, $\mathrm{P}_{\mathrm{PFC}}^{\Omega}$, and toroidal, $\mathrm{P}_{\mathrm{TFC}}^{\Omega}$, magnet coils are assumed nonrecoverable, but the plasma ohmic losses, $\mathrm{P}_{\Omega}$ appear in the thermal-conversion cycle. Inefficiencies in supplying $\mathrm{P}_{\mathrm{TFC}}^{\Omega}, \mathrm{P}_{\mathrm{PFC}}^{\Omega}$, and $\mathrm{P}_{\Omega}$ are represented by $\mathrm{P}_{\mathrm{ETS}}$, which is important primarily for pulsed systens. A fraction $\varepsilon$ of the total electrical power, $P_{E T}$, must be recirculated as makeup or recirculating power, $P_{C}=\varepsilon P_{E T}$. Tise net electric power is $P_{E}=(1-\varepsilon) P_{E T}$, and the overall plant efficiency is $\eta_{\mathrm{p}}=(1-\varepsilon) \eta_{\mathrm{TH}}$. An engineering Q-value, $\mathrm{Q}_{\mathrm{E}}$, is defined as a measure of system performance.

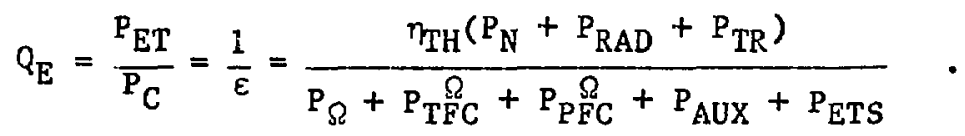

As for the thermal-mechanical analyses described in the previous subsection, the calculation of $Q_{E}$ at this level serves only a monitoring function, with more detailed and integrated calculations of the .ind summarized in Sec. III.L. being used for the final wesign-point determination.

REFERENCES

1. S. C. Schulte, W. E. Blckford, C. E. Willingham, S. K. Ghose, M. G. Walker, "Fusion Reactor Design Studies - Standard Unit Costs and Cost Scaling Rules," Pacific Northwest Laboratory report PNL-2987 (September 1979).

2. R. L. Hagenson and R. A. Krakowski, "An Advanced-Fuel Reversed-Field Pinch Fusion Reactor (DD/RFPR): Preliminary Considerations," Los Alamos National Laboratory report LA-9139-MS (February 1982).

3. I. M. Hively, "Convenient Computational Forms for Maxwellian Reactivities," Nucl. Fus. 17, 873-876 (1977).

4. G. H. Miley, H. Towner, and N. Ivich, "Fusion Crcis Sections and Reactivities," University of Illinois report CO0-2218-17 (1974).

5. ‥ L. Miller, "Alpha-Particle Considerations Relevant to the Reversed Field Pinch Reactor (RFPR)," Proc. of the Reversed-Field Pinch Theory Workshop, Los A.lamos, NM (April 29-May 2, 1980), Los Alamos National Laboratory report LA-8944-C, p. 39 (January 1982). 
6. S. I. Braginskii, "Transport Processes in a Plasma,"Reviews of Plasma Physics 1, H. A. Leontovich, ed., Consultants Bureau, NY (1965).

7. K. I. Thomassen (Ed.), "Ohmic Heating Systems Study for a Tokamak EPR," Los Alamos Scientific Laboratory report (CTR Division) submitted to the Dịvision of Fusion Energy in the Department of Energy (1977).

8. D. J. Rose and M. Clark, Jr. , Plasmas and Controlled Fusion, 2nd ed., The M.I.T. Press, Cambridge, Mass. (1965).

9. R. A. Krajcik, "The Effect of a Metallic Reflector Upon Cyclotron Radiation," Nucl. Fus, 1.2, 7 (1973).

10. D. A. Baker, M. D. Bausman, C. J. Buchenauer, L. C. Burkhardt, G. Chandler, J. N. DiMarco, "Performance of the ZT-40M Reversed-Field Pinch with an Inconel Liner," 9th International Conference on Plasma Physics and Controlled Nuclear Fusion Research, Baltimore, USA, paper IAEA CN-41/H-2-1, I, 587 (September 1982).

11. K. Ogawa, Y. Maejima, T. Shimada, Y. Hirano, P. G. Carolan, C. W. Gowers, et al., "Experimental and Computational Studies of Reversed Field Pinch on $\overline{T P E} \overline{-I R}(\mathrm{M}), "$ ibid, IAEA-CN-41/H-1, I, 575.

12. M. Nagami and the JAFRI Team, D. Overskei and the GA Team, "High- $\beta$ Injection Experiments with Shaped Plasmas in Doublet-III," ibid, IAEA-CN-41/A-2, I, 27.

13. D. Johnson, M. Bell, M. Bitter, K. Bol, K. Brau, T. Crowley, et al., "High Beta Experiments with ieutral Beam Injection on PDX," ibid, IAEA $\mathrm{CN}-41 / \mathrm{A}-1, \mathrm{I}, 9$.

14. M. Murakami, S. C. Bates, J. D. Bell, C. E. Bush, A. Carnevali, B. A. Carreras, et al., "Beta Scaling Experiments in ISX-B," 1bid, IAF.A-CN-41/A-4, $\underline{\text { I, }} 5 \overline{7}$.

15. D. A. Baker, E. J. Caramana, A. Haberstich, A. R. Jacobson, E. M. Little, K. F. Schoenberg, K. S. Thomas, P. G. Weber, "Preliminary Scaling of ZT-40M with Poloidal Limiters," RFP Workshop, Los Alamos National Laboratory, Los Alamos, NM (1983).

16. S. Ortolani, " $\mu$ and Pressure Mode1," Instituto Gas Ionizzat1, Padova, Italy (1983).

17. R. L. Hagenson and R. A. Krakowski, "Compact Reversed-Field Piniah Reactors (CRFPR): Sensitivity Study and Design-Point Determination," Los Alamos National Laboratory report LA-9389-MS (July 1982).

18. J. B. Taylor, "Relaxation of Toroldal Discharges," 3rd Topical Conf. on Pulsed High-Beta Plasma, Culham, UK, p. 59 (1975).

19. A. A. Newton, Li Yin-An, J. W. Long, and B. C. Yeung, "Numerica之 Investigation of Reversed Field Pinches," 3rd Toptcal. Conf. on Pulsed High-Beta Plasmas, Culham, UK, 323-328 (1975). 
20. V. D. Shafranov, Reviews of Plasma Physics 2, 103 (Consultants Bureau, NY, 190̈6).

21. J. M. Greene, J. L. Johnsion, and K. E. Weimer, "Tokamak Equilibrium," Phys. of Fluids 14,671 (1971).

22. V. S. Mukhovatov and V. D. Shafranov, "Plasma Equilibrium in a Tokamak," Nucl. Fus. 11, 605 (1971). 


\section{APPENDIX B: Table of CRFPR Design Parameters}

This table contains the comprehensive and uniform design data for the CRFPR. The forat of this table follows the DOE/OFE guidelines. 1 Superscripted numbers in par $n$ theses refer to notes found at the end of this table.

TABLE OF REACTOR DESIGN PARAMETERS

\section{Parameter}

Unit

Value

1. Characteristic Machine Dimensions

1.1. Reactor envelope (la)

1.1.1. Helght (coil outside diameter)

m

3.90

1.1.2. Width (coil outside diameter)

m

3.90

1.1.3. Length (major circumference)

m

23.9

1.1.4. Volume of reactor envelope

$\mathrm{m}^{3}$

285

1.2. Plasma chamber

1.2.1. Major toroldal radius, $R_{T}$

m

3.80

1.2.2. Minor radius

m

0.75

1.2.3. Plasma volume, $V_{p}$

$\mathrm{m}^{3}$

37.7

1.2.4. Plasma chamber volume

$\mathrm{m}^{3}$

42.2

1.2.5. Wall surface area

$\mathrm{m}^{2}$

112

1.2.6. Number of sectors, $\mathrm{N}^{(1 \mathrm{~b})}$

24

\section{Plasma Parameters (Steady State)}

2.1. Plasma dimensions

2.1.1. Major toroidal radius, $R_{T}$

m

3.80

2.1.2. Average minor radius, $r_{p}$

2.2. Average ion density, $n_{i}$

m

0.71

2.3. Average alpha-particle density, $\mathbf{n}_{\alpha}$

$10^{20} / \mathrm{m}^{3}$

6.55

2.4. Energy confinement time, $\tau_{E}$

$10^{20} / \mathrm{m}^{3}$

0.19

2.5. Electron corisinement time, $\tau_{\text {pe }}$

s

0.23

2.6. Ion confinement time, $\tau_{p 1}$

$\mathbf{s}$

0.15

2.7. Average Lawson parameter, $n_{1} \tau_{E}$

$s$

0.59

2.8. Average poloidal beta, $(2 a)\left\langle\beta_{\theta}\right\rangle$

2.9. Average plasma toroidal current

2.10. Average ion temperature, $\left\langle\tilde{s}_{1}\right\rangle(2 b)$

2.11. Average electron temperature, $\left\langle\mathrm{T}_{\mathrm{e}}\right\rangle(2 \mathrm{~b})$

$10^{20} \mathrm{~s} / \mathrm{m}^{3} \quad 1.54$

2.12. Average alpha-particle energy, $\left\langle\mathrm{I}_{\alpha}\right\rangle^{(2 c)}$

$\begin{array}{ll}- & 0.23 \\ \mathrm{MA} & 18.4 \\ \mathrm{keV} & 10 . \\ \mathrm{keV} & 10 . \\ \mathrm{keV} & 805 .\end{array}$


2.13. Effective plasma ion

$$
\text { sharge, }{ }^{(2 d)} Z_{\text {eff }}
$$

2.14. Reactor cycle

2.14.1. Burn pulse length

2.14.2. Total pulse length

2.15. Fuel cycle

2.16. Plasma-heating method

2.16.1. Plasma-heating power ${ }^{(2 e)}$

2.16.2. Plasma-heating frequency

2.17. Plasma energy gain, ${ }^{(2 f)} Q_{p}$

3. Power-Output

3.1. Plasma fusion power, $P_{F}=P_{N}+P_{\alpha}$

3.1.1. 14.1-MeV neutron power, $\mathrm{P}_{\mathrm{N}}$

3.1.2. 3.5-MeV alpha-particle power, $P_{\alpha}$

3.2. Reactor thermal power

3.2.1. Power to first wall

3.2.2. Power to limiter

3.2.3. Power from blanket, $\left(M_{N} \simeq 1.28\right)$

3.2.4. Power to shield

3.3. Blanket power amplification

$$
\text { factor, } \mathrm{M}_{\mathrm{N}}
$$

3.4. Plasma chamber power density

$$
\text { (total cycle time average) (3a) }
$$

3.5. Plasma power density (3b)

3.6. Engineering power density $(3 c)$

3.7. Blanket power density (3d)

3.8. Total tharmal power to

$$
\text { conversion cycle, }{ }^{(3 e)} P_{T H}
$$

3.9. Plant gross electrical output, $P_{E T}$

3,10. Plant net electrical output, $P_{E}$

3.11. Thermal cycle efficiency, $\eta_{\mathrm{TH}}$

3.12. Net plant efficiency, $\eta_{p}=\eta_{T H}(1-\varepsilon)$

3.13. Recirculating power fraction, $\varepsilon^{(3 f)}$

4. Reactor Coolant System

4.1. Blanket coolant

4.2. Blanket outlet temperature (hot leg)

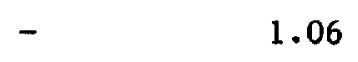

$\mathbf{s}$

s

-

-

$\mathrm{MH}$

$\mathrm{GHz}$

$$
-
$$

MWt

2732.5

MWt

2186.0

MWt

546.5

MWt

3354.9

MWt

626.0

MWt

440.8

MWt

2057.6

Mwt

143.3

$-$

1.28

$M T / m^{3}$

64.8

$M W t / m^{3}$

72.5

$M W t / m^{3}$

11.8

$M W t / m^{3}$

30.6

MWt

3364.9

MWe

1226.7

MWe

1000 .

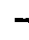

-

0.365

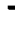

-

0.297

0.185

$\begin{array}{ll}- & \mathrm{Pb}_{83} \mathrm{Li}_{17} \\ \mathrm{~K} & 773\end{array}$


4.3. Blanket inlet temperature (cold leg)

4.4. Blanket cuilet pressure

$\mathrm{MPa}$

$\sim 0.0$

4.5. Blanket inlet pressure

$\mathrm{MPa}$

1.1

4.6. Blanket coolant flow rate (tota1)

$\mathrm{kg} / \mathrm{s}$

72,840

4.7. Blanket structural material(4a)

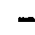

4.8. First-wall coolant type

4.9. First-wall outlet temperature

4.10. First-wall inlet temperature

4.11. First-wall outlet pressure

4.12. First-wall inlet pressure

4.12. First-wall coolant flow rate (total)

4.14. Ictal number of blanket coolant loops

4.15. Type of blanket coolant circulator

4.16. Power input to each circulator (4b)

4.17. Peak temperature in case

of 1oss-of-coolant-flow accident

4.17.1. First wall

K

TBD

4.17.2. Blanket multiplier

$\mathrm{K}$

TBD

4.17.3. Breeder

4.18. Thermal energy storage (4b)

$\mathrm{K}$

TBD

$\mathrm{J}$

not required

5. Intermediate Coolant System

TBD

6. Steam Genezation (SG) System

TBD

6.1. Steam outlet temperature

K

TBD

6.2. Steam outlet pressure

$\mathrm{MPa}$

TBD

6.3. Steam flow rate (total)

$\mathrm{kg} / \mathrm{s}$

TBD

0.4. Feedwater temperature

K

TBD

6.5. Number of steam generators (SG) per loop

TBD

6.6. Number of reactor sectors per SG

TBD

6.7. SG materials, shell/tube

T\&D

7. Shield Coolant System

7.1. Total power deposited in the shield

MWE

143.3

7.2. Shield coolant type

7.3. Shield outlet temperature

$-\mathrm{H}_{2} \mathrm{O}$

K

463

7.4. Shield inlet temperature

K

597

7.5. Coolant outlet pressure

$\mathrm{MPa}$

15.2

7.6. Coolant inlet pressure

MPa

15.2 


\section{Reactor Auxiliary System}

8.1. Vacuum pumping system

8.1.1. Plasma chamber pressure

8.1.2. Plasma chamber volume

8.1.3. Total vacuum volume(8a)

8.1 .4 . Number of vacuum pumps $(8 \mathrm{~b})$

8.1.5. Regeneration interval

8.1.6. Helfum-pumping speed (per pump)

$\begin{array}{ll}\mathrm{Pa} & 2.0 \\ \mathrm{~m}^{3} & 42.2 \\ \mathrm{~m}^{3} & 60.6 \\ - & \mathrm{TBD} \\ \mathrm{h} & \mathrm{TBD} \\ \mathrm{m}^{3 / \mathrm{s}} & \mathrm{TBD}\end{array}$

8.2. Magnet cooling system (chilled $\mathrm{H}_{2} \mathrm{O}$ )

\subsubsection{TFC cooling load (8c) \\ 8.2.2. PFC cooling load $(8 \mathrm{~d})$}

8.3. Plasma-heating-system cooling load (3f,8d)

8.4. Plasma-fueling system

\subsubsection{Type ${ }^{(8 e)}$ \\ 8.4.2. Fuel composition \\ 8.4.3. Fueling rates}

8.4.4. Pellet diameter

8.4.5. Pellet injection frequency

8.5. Tritium-processing and recovery system

8.5.1. Total tritium inventory

8.5.2. Yulnerable tritium Inventory

8.6. Impurity control system

8.6.1. Limiter coolant type

8.6.2. Limiter outlet temperature

8.6.3. Limiter Inlet temperature

8.6.4. IImiter outlet pressure

8.6.5. Limiter inlet pressure

8.6.6. Limiter coolant flow rate
MWt

MWt

MWt

42.6

141.0

73.0

$\begin{array}{ll}- & \text { DT Pe1let } \\ - & 50 \% \mathrm{D}, 50 \% \mathrm{~T} \\ \mathrm{~g} / \mathrm{s}(\mathrm{D}) & 0.11 \\ \mathrm{~g} / \mathrm{s}(\mathrm{T}) & 0.17 \\ \mathrm{~mm} & 4.22 \\ \mathrm{~s}^{-1} & \sim 28\end{array}$

kg TBD

$\mathrm{kg}$ TBD

Pumped IImfter

$\begin{array}{ll}- & \mathrm{H}_{2} \mathrm{O} \\ \mathrm{K} & 545 \\ \mathrm{~K} & 463 \\ \mathrm{MPa} & 15.2 \\ \mathrm{MPa} & 15.4 \\ \mathrm{~kg} / \mathrm{s} & 1311.2\end{array}$

9. Reactor Components

9.1. First wall/blanket

9.1.1. Structural material (first-wa11/blanket) -

9.1.2. Breeding material

9.1.3. Neutron multiplier material

9.1.4. Breeding ratio $(9 a)$
MZC-Copper/HT-9 $\left.\mathrm{Pb}_{83}{ }^{\mathrm{LI}_{17}}{ }^{(60 \%} 6_{\mathrm{LI}}\right)$ $\mathrm{Pb}$

1.11 
9.1.5. Number of modules $(9 b)$

9.1.6. Weight of modules $(9 b)$

9.1.7. Welght of largest single component

9.1.8. Dimensions of largest component (9c)

9.1.9. First-wall power loading

9.1.9.1. 14.1-MeV neutron current

9.1.9.2. Energetic alpha-particle $\mathrm{flux} \mathrm{MW} / \mathrm{m}^{2}$

9.1.9.3. Radiation, charge-exchange, and conduction power to

first wall

9.1.9.4. Radiation, charge-exchange, and conduction/convection

power to limiter

9.1.9.5. First-wall life

MHyr $/ \mathrm{m}^{2}$

$\begin{array}{ll}- & 1 \\ \text { tonnes } & 307 \\ \text { tonnes } & 307 \\ \text { m } & \text { torus, 3.8-m } \times 1.5-m\end{array}$

$M W / \mathrm{m}^{2}$

19.5

0.0

$\mathrm{MW} / \mathrm{m}^{2} \quad 5.0$

9.2. Shielding

9.2.1. Material

9.2.2. Number of modules

9.2.3. Weight of each module

tounes

15

6.0

9.2.4. Weight of largest single component

tonnes

$\mathrm{H}_{2} \mathrm{O} / \mathrm{W} / \mathrm{B}_{4} \mathrm{C} / \mathrm{Fe}$

1

188.9

(1ncluded in 9.1.6.)

188.9

(Included in 9.1.6.)

9.2.5. Dimensions of largest component

m

torus,

$3.8-\mathrm{m} \times 1.43-\mathrm{m}$

\subsection{Magnets}

9.3.1. Superconduction

9.3.2. Conductor material (insulator)

9.3.3. Structural material

9.3.4. Operating temperature

9.3.5. Coolant

9.3.6. Mean stress in col1(9d)

9.3.7. Maximum force transmitted to building $(9 \mathrm{e})$

9.3.8. Maximum fleld(9f)

9.3.9. Field on plasma axis $(9 \mathrm{~g})$

$\begin{array}{ll}- & \text { no } \\ - & \text { Cu-alloy (Hgo) } \\ - & \text { PCASS } \\ \mathrm{K} & 300-350 \\ & \mathrm{H}_{2} \mathrm{O} \\ \mathrm{MPa} & 7-90 \\ \mathrm{MN} & 2.7 \\ & \\ \mathrm{~T} & 4.5 \\ \mathrm{~T} & 9.5 \text { (toroidal) }\end{array}$


9.3.10. Field at plasma surface

9.3 .11 . Number of magnets

$$
\begin{aligned}
& 9.3 .11 .1 \text {. Toroldal-field colls } \\
& 9.3 .11 .2 \text {. Equilibrium-field colls } \\
& 9.3 .11 .3 \text {. Ohmic-heating colls }
\end{aligned}
$$

9.3.12. Mean coll radius

$$
\begin{aligned}
& \text { 9.3.12.1. Toroidal-field coll } \\
& \text { 9.3.12.2. Poloidal-field coil }
\end{aligned}
$$

9.3.13. Total stored energy (full torus)

$$
\begin{aligned}
& \text { 9.3.13.1. Toroidal-field coll } \\
& \text { 9.3.13.2. Pololdal-field coil }
\end{aligned}
$$

9.3.14. Weight of largest single

$$
\text { component }(9 h)
$$

9.3.15. Dimenston of largest single component

9.4. Energy transfer and storage( 91$)$

9.5. Plasma heating

9.5.1. Type

9.5.2. Frequency

9.5.3. Power to the plasma

9.5.4. Transmission method

9.5.5. Power reflected from plasma

9.5.6. Power loss in transmission

9.5.7. Power loss in amplifiers

9.5.8. Fower loss in power supplies

9.5.9. System input power

9.5.10. Heating time

9.5.11. Number of wavegulde grills

9.5 .12 . Number of amplifiers

\section{Electrical Power Requirements}

10.1. Cold-plasma start-up power from grid 10.2. Auxiliary power requirements (10a)

$$
\text { (normal operation) }
$$

10.2.1. Electrical energy storage

$\begin{array}{ll}- & 24 \\ - & 12 \\ - & 20 \\ \text { m } & 3.31 \\ \text { m } & 1.46 \\ \text { m } & 3.80 \\ \text { GJ } & 1.72 \\ \text { GJ } & 0.02 \\ \text { GJ } & 1.7\end{array}$

\section{tonnes}

m

200

torus, $4.8-\mathrm{m} \times 1.0-\mathrm{m}$

Not required

Ohmic

GHz

NA

$\mathrm{MW}$

25.3

-

MW

Induction

MW

0.0

MW

73.0

MW

NA

MW

TBD

s

$\sim 100$

8-10

- NA

- NA

MWe/s $\quad 300 / 8$

MWe

62.6

MHe

none required 
10.2.2. Magnet nower supply (other than ohmic losses) (10b)

10.2.3. First-wall circulators (10c)

$\begin{array}{ll}\text { MWe } & 0.0 \\ \text { MWe } & 1.85 \\ \text { MWe } & 0.94 \\ \text { MWe } & 13.2 \\ \text { MWe } & 0.0 \\ \text { MWe } & \text { NA } \\ \text { MWe } & \text { TBD } \\ \text { MWe } & \\ \text { MWe } & \text { TBD } \\ \text { MWe } & \text { TBD } \\ \text { MWe } & \text { TBD } \\ \text { MWe } & \text { TBD } \\ & \text { TBD }\end{array}$

\section{Builoings}

11.1. Reactor Building

11.1.1. Characteristic dimensions

m

TBD

11.1.2. Enclosed volume

11.1.2.1. Free volume(11a)

$\mathrm{m}^{3}$

TBD

11.1.2.2. Total volume

$\mathrm{m}^{3}$

TBD

11.1.3. Minimum wall thickness for

m

TBD shielding

11.1.4. Internal pressure

$\mathrm{MPa}$

TBD normal/accident (11b)

11.1.5. Containment atmosphere

$-\quad$ TBD

11.2. Turbine Buflding

11.2.1. Characteristic dimensions

$\begin{array}{ll}m & \text { TBD } \\ \mathrm{m}^{3} & \text { TBD }\end{array}$

11.2.2. Enclosed volume

11.3. Reactor Service Building

11.3.1. Characteristic dimensions

m

TBD

11.3.2. Special functions (1.e., hot Tritium handling, radwaste, cells, blanket processing hot cells, maintenance equipment, etc.) and storage

12. Reactor MaInt enance

12.1. First-wa:1/blanket/shield

12.1.1. Annual percentage

$\%$ area/yr 100 
12.1.2. Area

12.1.3. Weight (12a)

12.2. Radioactive material storage for life of plant ( $30 \mathrm{yr}$ )

12.2.1. Total long-term storage $(12 b)$

12.2.2. Remaining recycled blanket storage

12.2.3. Total recycled reactor equipment ${ }^{(12 c)}$ (after plant decommissioning)

12.3. Reactor avallability

12.4. Overall plant availability (12d) $\mathrm{m}^{2} / \mathrm{yr}$ 112 tonnes/yr 45.2 $\mathrm{m}^{3} /$ tonnes $(12 \mathrm{~b})$

$270 / 1356(65 \%$ compression 2400/7356(concrete f111)

$m^{3} /$ tonnes $\quad$ TBD

$\mathbf{m}^{3} /$ tonnes $\mathrm{TBD}$

TBD

0.75

\section{Footnotes for Reactor Design Table}

1. Charactertstic Machine Dimensions

(a) The reactor envelope encloses the pololdal-fleld coils and has the dimensions given with a circumference (length) of $23.9 \mathrm{~m}$ (at the major toroldal radius of $3.8 \mathrm{~m}$ ).

(b) Sectors are used only for off-site assembly of FW/B/S/C, with full torus being installed as a single unit into reactor cavity.

2. Plasma Parameters

(a) Includes average alpha-particle pressure, total beta is 0.12 .

(b) Based on $T(x) \propto J_{0}(\mu r)$ profiles and BFM pressure profiles.

(c) Thermalized Maxwellian component has been subtracted.

(d) Based only on steady-state alpha-particle density, $\mathrm{Z}_{\text {eff }}=\left(1+4 \mathrm{f}_{\alpha}\right) /\left(1+2 \mathrm{f}_{\alpha}\right) \mathrm{f}_{\alpha}=\mathrm{n}_{\alpha} / \mathrm{n}_{\mathrm{I}}=0.029$.

(e) At steady state, 200-300-MWe grid power required during bipolar startup.

(f) Based on ratio of fusion power to ohmlc dissipation in plasma, inclusion of ohmic power dissipated in TFCs and PFCs gives a Q-value of 16.62 .

3. Power Output

(a) Based on fusion power (2732.5 MWt) divided by vacuum chamber volume $\left(42.2 \mathrm{~m}^{3}\right)$.

(b) Based on fusion power (2732.5 MWt) divided by plasma volume $\left(37.7 \mathrm{~m}^{3}\right)$. 
(c) Based on total thermal power (3364.9 MWt) and net volume (285 $\mathrm{m}^{3}$ ) enclosed by and including magnets.

(d) Based on first-wall, blanket and shield volume ( $110 \mathrm{n}^{3}$ ) and thermal power actually delivered to primary coolant (3364.9 MWt). Peak value of blanket power density is $255 \mathrm{MWt} / \mathrm{m}^{3}$ and actual average for the blanket is $26.9 \mathrm{MWt} / \mathrm{m}^{3}$.

(e) Does not include primary-coolant pumping power.

(f) Includes $73 \mathrm{MW}$ delivered to OHCs, which under steady-state operation could be reduced to zero.

4. Reactor Coolant System

(a) $\mathrm{HT}-9$ is a ferritic steel $(85.0 \mathrm{Fe} / 11.5 \mathrm{Cr} / 1.0 \mathrm{Mo} / 0.55 \mathrm{Mn} / 0.5 \mathrm{Ni} /$ $0.5 \mathrm{w} / 0.4 \mathrm{~s} 1 / 0.3 \mathrm{~V} / 0.2 \mathrm{C}$ ).

(b) Energy stored in the $\sim 800$-tonne PbL1 coolant contained within the FPC amounts to 65 GJ over the temperature range from room temperature to $773 \mathrm{~K}$, when ratio to the total thermal output gives $\sim 20 \mathrm{~s}$.

7. Shield Coolant System

(a) Thin (0.10-m) shield operates at first-wall/shield conditions and contributes to overall reactor power cycle.

\section{Reactor Auxiliary System}

(3) Vacuum volumes within the FrC include $4.5 \mathrm{~m}^{3}$ for the 24 radial slots, $10.7 \mathrm{~m}^{3}$ for the vacuum plenum, =nd $1.7 \mathrm{~m}^{3}$ for 24 ducts out to outer edge of PFCs.

(b) Design choice between separate cryogenic pumps at each of 24 ducts (one per sector) or the use of a "vacuum room" remalns to be made.

(c) Of this total, $30.0 \mathrm{MW}$ is nuclear heating and 12.6 is ohmic heating.

(d) Of this total, $14.5 \mathrm{MW}$ is nuclear heating, 126.5 is ohmic heating, of which $73.0 \mathrm{MW}$ appears in the OHCs and $53.5 \mathrm{MW}$ appears in the EFCs.

(e) Pellet speed $=1.4 \mathrm{~km} / \mathrm{s}$ with ratio of pellet inventory to plasma inventory equal to 0.05 .

\section{Reactor Components}

(a) Theoretical one-dimensional breeding ratio; does not include losses, decay, first-wall area reduction.

(b) The first-wall/blanket/TFC forms a single, integral unit; weight does not include the $\sim 800$ tonne coolant.

(c) When toroidal in shape, the dimenstons indicate effective major and and minor radil, respectively. 
(d) Lower value at PFC \#12 (upper, inboard) and higher value at PFC \#6 (equatorial plane, inboard); compares to $550 \mathrm{MPa}$ (TFC) and $14 \mathrm{MPa}$ (PFC) for STARFIRE and $127 \mathrm{MPa}$ (TFC/ARE) for EBTR.

(e) Centering force for TFC.

(f) Maximum field at inboard side of PFC.

(g) Toroldal field at plasma edge is $-0.40 \mathrm{~T}$ and increases to $-0.72 \mathrm{~T}$ at the TFC.

(h) Total mass of PFC is 798 tonne, with EFC being 404 tonne and the OHC being 394 tonne. Each EFC and OHC set would in turn be divided into two segments for maintenance purposes.

(1) If a grid-assisted startup proves unfeasible, a transferred energy store of $\sim 2$ GJ will be required.

10. Electrical Power Requirements

(a) Taken nominally as $\mathrm{f}_{\mathrm{AUX}}=0.05$ of the gross electrical power, $\mathrm{P}_{\mathrm{ET}}=1226.7 \mathrm{MWe}$

(b) Refer to item 10.3.

(c) Based on $75 \%$ pump efficiency.

\section{Bulldings}

(a) Includes volume of reactor hall and primary-coolant component room; value used to calculate overpressure in event of coolant line rupture.

(b) Normal operating pressure is slightly less than atmospheric, as in the present-day nuclear power plants. The accident condition is assumed to be the loss of primary coolant into the containment building.

\section{Reactor Maintenance}

(a) Based on replacing entire first wall and blanket each year of operation, and recycling the shield (188.9 tonne) and TFCs ( 72.8 tonne).

(b) Includes first-wa11, blanket, and limiter components.

(c) Includes blanket and first wall, all shlelding, colls, primary structure and support, Limiters, and vacuum cryopumps (if used), but excludes longterm storage in Item 12.2.1.

(d) Based on 60 day/year unscheduled maintenance and 28 d/FPC replacement, this algorithm being sensitive to fusion neutron wall loading and giving an optimal wall loading based on COE.

REFERENCE

1. C. R. Head, "Standard Fusion Reactor Design Study Contents," Department of Energy, Office of Fusion Energy letter RS\&A:CRH: 非78 (March 7, 1979). 
Appendix C. Two-Dimensional Neutronics Analyses

\section{C.1. Introduction}

Estimates of the nucleonics performance of the first wall, blanket, and shleld (FW/B/S) design presented in this report were based on analyses of the results of a one-dimenstonal neutronics model and parametrics study leading to the "canonical" model described in Sec. III.B. That parameter survey varied the thickness of the blanket and shield as well as the composition of primary materials: PCASS (structure), $\mathrm{H}_{2} \mathrm{O}$ (FW/1imiter/shield coolant), $\mathrm{B}_{4} \mathrm{C} / \mathrm{W}$ (shleld), and $\mathrm{Pb}_{83} \mathrm{LI}_{17}$ (enrlched to $60 \%$ in GL, blanket breeder and coolant). The caiculations were performed using the one-dimensional, discrete-ordinates code ONEDANT $^{l}$ and a 30-neutron/12-gamma-ray group 1ibrary based on ENDF/B-IV data. ${ }^{2}$

The one-dimensional aspects of the $\mathrm{FW} / \mathrm{B} / \mathrm{S}$ design are for the most part wel1 represented by the canonical model. Inclusion of the 11miter, the vacuum ducts, and the manifolds and headers cooling the limiter, first wall, and second wall, however, give significant two-dimensional characteristics to the FW/B/S model. A two-dimensional neutronics analysis, therefore, was performed to assess the effects on the blanket performance predicted by the one-dimensional model. In addition, the effects of using ENDF/B-V cross sections ${ }^{3}$ and other changes in the FW/B/S materials (e.g., HT-9 ferritlc steel was substituted for PCASS, 1mproved shielding materials) could also be assessed.

of particular interest to this postdesign neutronfics analysis is the tritium-breeding ratio (TBR). The one-dimensional calculation for the canonical FW/B/S model predicted a value of 1.108 (Table III.B-V). Displacement of a portion of the PbLi blanket by the manifold, headers, and vacuum ducts, particularly in regions near the first wall where the greatest contribution to tritium breeding occurs, however, is expected to degrade TBR seriously for the blanket design that evolved in Sec. L. In addition, a.calculated TBR of $\sim 1.10$ is necessary to allow for calculation uncertainties in the tritium recovery efficiencies. 4

After describing the two-dimensional neutronics model used in this postdesign assessment in Sec. C.2., the neutronics codes and cross sections are described in Sec. C.3. Section C.4 gives the parametric results for the referenced $\mathrm{FW} / \mathrm{B} / \mathrm{S}$ model and a range of design changes available to obtain adequate tritium bresding in the highly two-dimension model that has evolved through the process of integrating the FPC (Sec. L.). In the spirit of this framework study, design directions are indicated to reconcile the blanket 
performence with the two-dimensional nature of this problem, only tritium breeding is computed, and more-detailed assessment of neutronic responses and design in two-dimensions must await future work.

\section{C.2. Two-Dimenstonal Mode1}

As illustrated in Fig. C-1, the CRFPR torus is composed of $N=24$ sectors each forming a portion of the first-wall/second-wall, blanket, shleld, and TFC. A half-sector, which is actually wedge shaped, was taken as the unit cell and modeled as a finite cylinder. Because this unit cell is for the most part poloidally symmetric about the plasma minor axis and toroldally symmetric about the module center plarie, the origin for the coordinate system was taken at the Intersection of this plave and the plasma centerline. The length of the cylindrical unit cell was taken as one-half the length of the plasma minor axis through the sector. The $F W / B / S$ minor radil were specified at the actual design values (FIg. III.B-5). The outer radius of the calculational unit cell was taken as $1.75 \mathrm{~m}$, this value belng arbitrarily selected to yield a $0.25-\mathrm{m}$ thickness for the region representing the PFCs. This region is approximately $40 \% \cot 1$ and $60 \%$ vold, and the $0.25-\mathrm{m}$ thickness corresponds to a few neutron mean-free-paths, giving a reasonable cutoff for the purposes of this calculation.

The two-dimenstonal model is depicted In FIg. C-2. Detalls of the geometry for the 1imiter, the first wall/second wall (FW/SW), and the associated manifold region are shown in $\mathrm{Fig} \cdot \mathrm{C}-3$. The modeling of this cructal region was simplified by homogenizing the manifold reglons for both the limiter and the FW/SW into zones composed of structure (HT-9), coolant $\left(\mathrm{H}_{2} \mathrm{O}\right)$, and vold. This homogeneous modeling is also shown in Fig. C-3. The volume fractions for the various materlals that compose the regions in Fig. C-2 are given In Table C-I.

\section{C.3. Neutronics Calculations}

a. Codes. The neutronics calculations were performed with a combination of computer codes: the two-dimenstonal, discrete-ordinates codes TRIDENT-CTR, 5 TWODANT; $^{6}$ the Monte Carlo code MCNP; ${ }^{7}$ and the one-dimensional, discrete-ordinates code ONEDANT. ${ }^{1}$ This combination of codes was needed both to benchmark the varlous calculations against each other and to utflize the particular assets of each (1.e., spatial resolution versus running time).

The calculations using TRIDENT-CTR used the triangular mesh capabilities, which allowed detalled modeling of the geometry. In addition, the disk storage system used In TRIDENT-CTR allowed a much larger problem [1.e., finer mesh, 


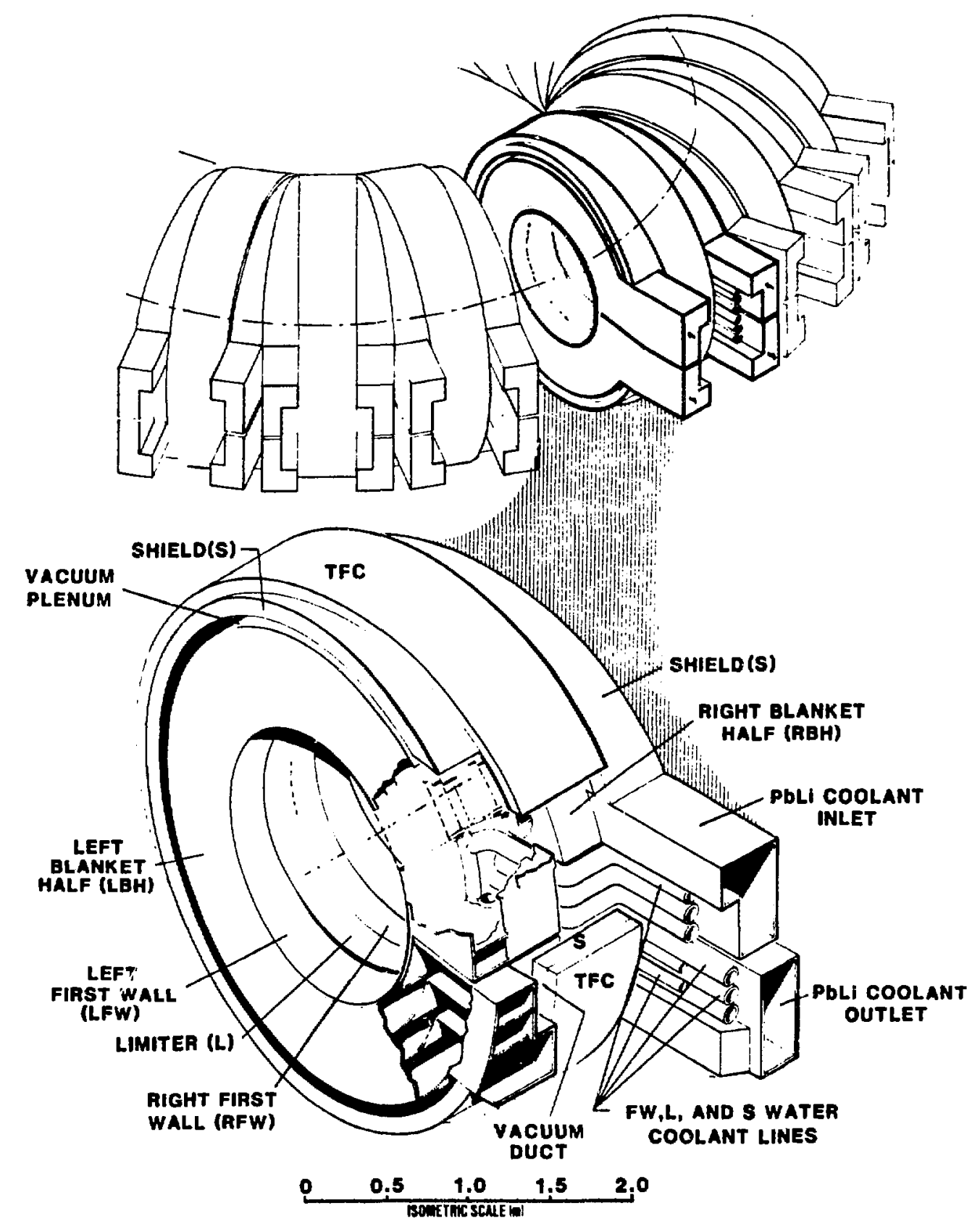

Fig. C-1. Detailed isometric view of FW/B/S/TFC torus and one of 24 sectors arranged to form the CRFPR torus. The calculation unit cell for the two-dimensional neutronics models takes one-half of this sector in cylindrical geometry. Pololdal-field colls are not shown. 


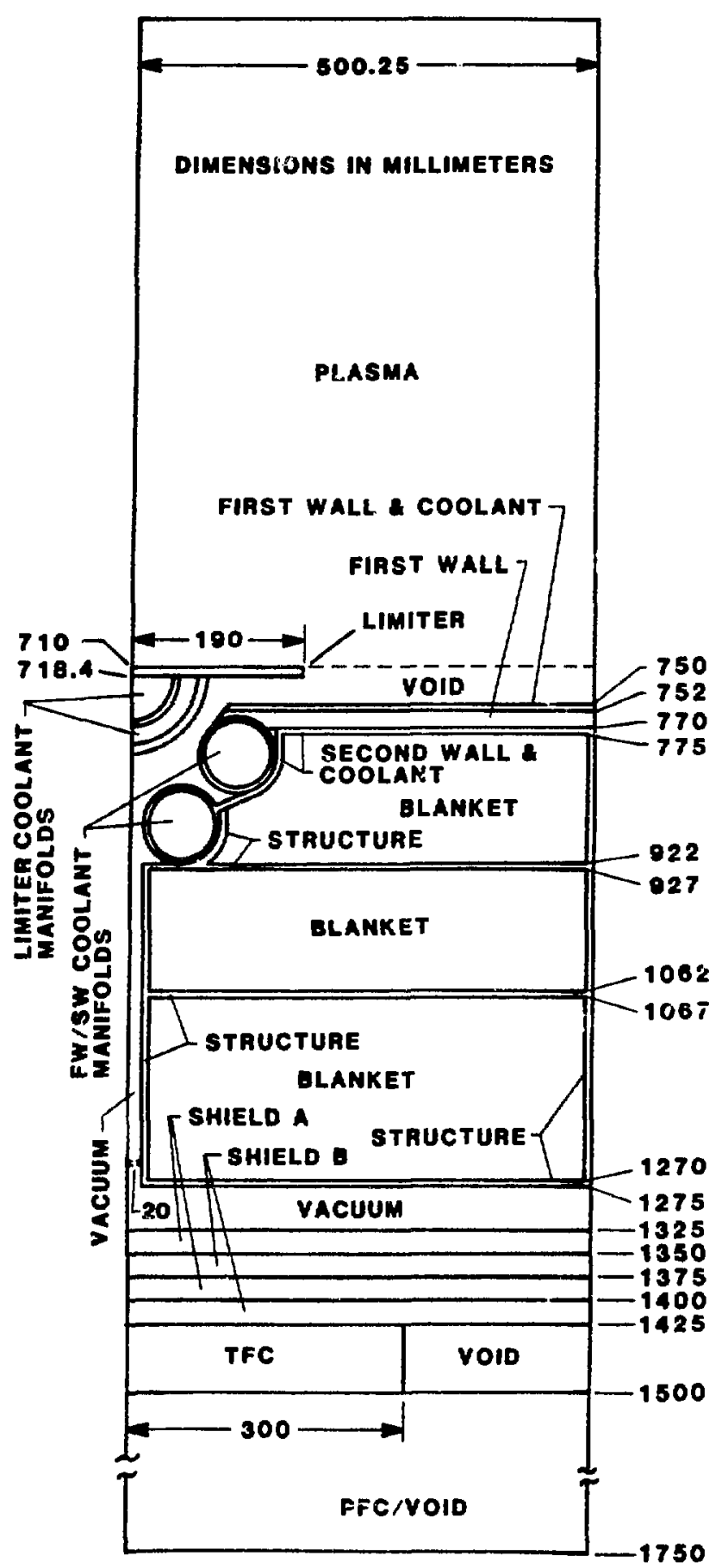

F1g. C-2. Calculation untt cell for two-dimensional CRFPR FW/B/S/C model to reflect in cylindrical geometry the effects of limiter and $\mathrm{FW} / \mathrm{SW}$ manifolding as well as vacuum ducting. 


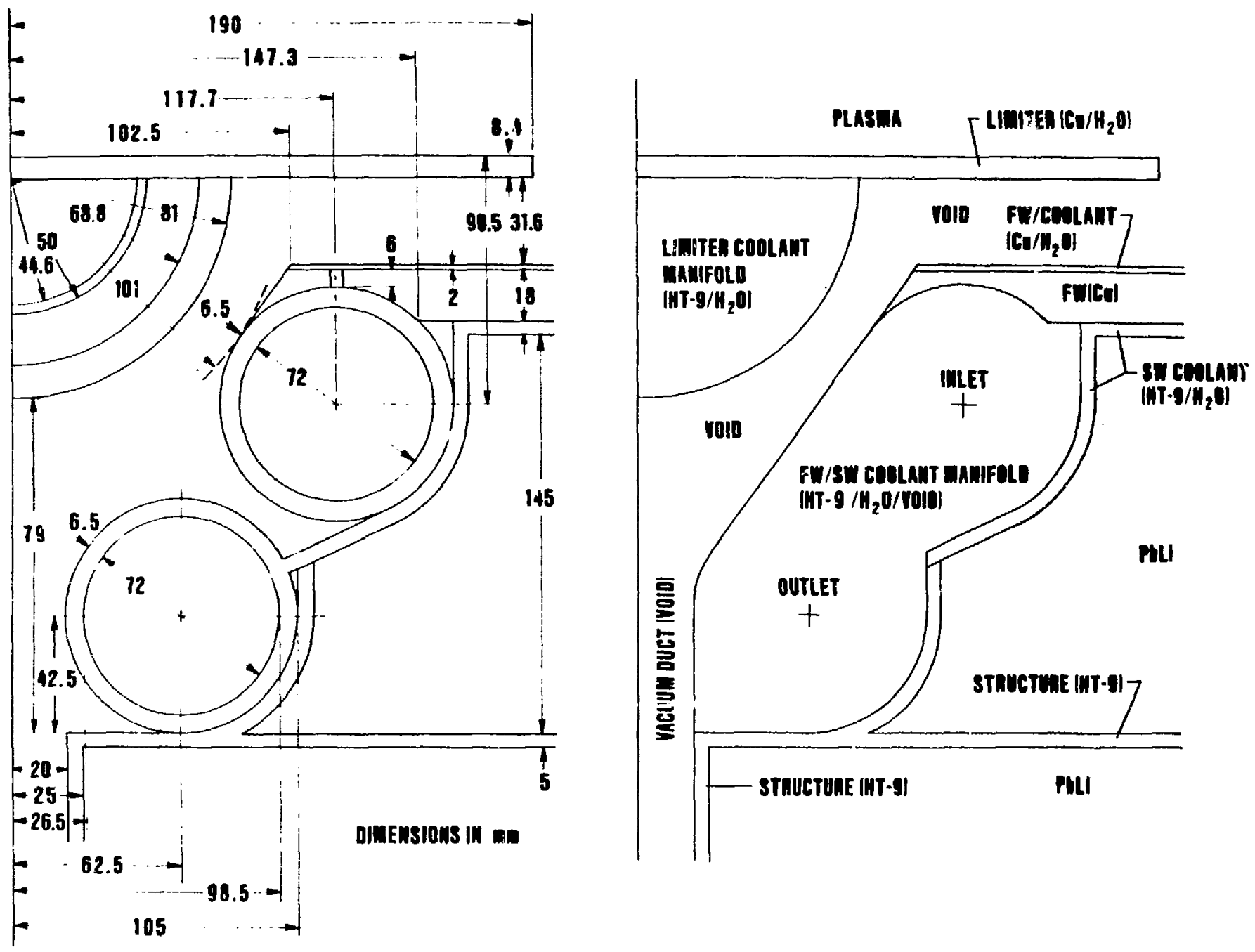

Fig. C-3. Detailed view of limiter, $\mathrm{FW} / \mathrm{SW}$, and associated $\mathrm{H}_{2} \mathrm{O}$ coolant manifolds (re: Fig. III. $\mathrm{L}_{-17}$ ) as well as homogenized version for use in two-dimensional neutronics models (TRIDENT-CTR and MNCP). 


\section{TABLE C-I \\ VOLUME FRACTIONS OF MATERIALS \\ USED IN TWO-DIMENSION NEUTRONICS MODEL}

\begin{tabular}{|c|c|c|}
\hline Region & Materials & Volume Fractions $(a)$ \\
\hline Limiter & $\mathrm{Cu}$ & 1.0 \\
\hline Limiter manifold & $\mathrm{HT}-9 / \mathrm{H}_{2} \mathrm{O}$ & $0.3564 / 0.5334$ \\
\hline inirst wall (with coolant) & $\mathrm{Cu} / \mathrm{H}_{2} \mathrm{O}$ & $0.5890 / 0.1654$ \\
\hline First wall (FW) & $\mathrm{Cu}$ & 1.0 \\
\hline Second wall (SW) & $\mathrm{HT}-9 / \mathrm{H}_{2} \mathrm{O}$ & $0.6400 / 0.3034$ \\
\hline FW/SW coolant manifold & $\mathrm{HT}-9 / \mathrm{H}_{2} \mathrm{O}$ & $0.2364 / 0.5085$ \\
\hline Blanket & PbL1 & 1.0 \\
\hline Blanket structure & HT -9 & 1.0 \\
\hline Shield Layer A & $\mathrm{W} / \mathrm{PCASS} / \mathrm{H}_{2} \mathrm{O}$ & $0.8 / 0.1 / 0.079$ \\
\hline Shield Layer B & $\mathrm{B}_{4} \mathrm{C} / \mathrm{PCASS} / \mathrm{H}_{2} \mathrm{O}$ & $0.8 / 0.1 / 0.079$ \\
\hline TFC & $\mathrm{Cu} / \mathrm{PCASS} / \mathrm{MgO} / \mathrm{H}_{2} \mathrm{O}$ & $0.7 / 0.1 / 0.1 / 0.0986$ \\
\hline PFC & $\mathrm{Cu} / \mathrm{PCASS} / \mathrm{MgO} / \mathrm{H}_{2} \mathrm{O}$ & $0.2815 / 0.0402 / 0.0402 / 0.0397$ \\
\hline
\end{tabular}

higher-order scattering $\left(\mathrm{P}_{3}\right)$, and coupled neutron and gamma rays] than was possible for TWODANT. The restart capabilities of the longer-running TRIDENT-CTR also allowed the converged tluxes from one case to be used as initial values for subsequent calibrations, thereby decreasing the computing Etme required to converge a new problem.

Although TWODANT calculations were limited to a coarse-wesh, $P_{1}$-scattering approximation and a number of neutron energy groups established by core storage, this code runs considerably faster than TRIDENT-CTR, allowing a larger number of two-dimensional parametric calculations to be made. Calculations were made only for an integral quantity (tritlum-breeding ratio) and no attempt was made to assess the accuracy of the spatial varlation in the calculated reaction rates.

The use of MCNP allowed the assessment of self-shielding effects, as well as permitting a check on the cross sections and effects of secondary energy and angular distribution. The TWODANT and TRIDENT-CTR codes used the same multigroup cross-section library, while MCNP uses a detailed pointwise library. The geometry modeled in MCNP was exactly that used in TRIDENT-CTR (i.e., the 
surface segments corresponded to the triangular mesh zones specified for TRIDENT-CTR).

b. Cross Sections. The source of the multigroup cross sections used in the TRIDENT-CTR, TWODANT, and ONEDENT calculations was the MATXS6 1 ibrary $^{8}$ that $^{-}$ was generated from ENDF/B-V data ${ }^{3}$ with the code NJOY. The MATXS6 11brary is an 80/24 group, coupled neutron/gamma-ray set. This 1ibrary was collapsed to a $30 / 12$ group structure for the calculations performed herein. This approach also allowed a comparison to be made with the results for the canonical FW/B/S model described in Sec. III.B. as well as an assessment of the effects of the weighting spectrum used to collapse the cross-section set. Multipgroup libraries were generated using the TRANSX $\operatorname{code}^{B}$ in whtch mixing of macromaterials $\left(\mathrm{e} . \mathrm{g} \cdot, \mathrm{H}_{2} \mathrm{O}\right)$, was performed. The weighting spectrum for collapsed librarles was elther input, or the MATXS6 "built-in" spectrum was used.

Calculations were performed with ONEDANT for the canonical FW/B/S model with the $80 / 24$ group library to generate varlous spatially averaged group fluxes for use in TRANSX as welghting functions. The $80 / 24$ group calculation yielded a TBR value of 1.075 , which compares with 1.108 reported in Sec. III.B. using the 30/12 group library based on ENDF/B-IV data. This difference most probably results from the more-recent $7_{L} 1$ evaluation in ENDF/B-V. Group weighting functions were generated as the flux average over the entire blanket, over the first $0.10 \mathrm{~m}$ of blanket, and over the middle $0.10 \mathrm{~m}$ of the 0.50 -m-thick canonical blanket. Collapsed 30/12 1ibraries were then created using these weights as well as the built-in spectrum. Calculations with ONEDANT were then made using each of these libraries. The results for the calculated TBR are summarized in Table C-II. Little difference exists between the TBR values obtained using the first $0.10 \mathrm{~m}$ of blanket and the entire blanket since the flux in the first $0.10 \mathrm{~m}$ of blanket dominates the blanket average. Based on this analysis, subsequent calculations used a library collapsed to $30 / 12$ neutron/gamma-ray groups using the flux spectrum averaged over the entire canonical blanket.

c. Calculational Specifications. The TRIDENT-CTR mesh used in the calculations is shown in FIg.C-4, with a more detalled view of the manifold/header region behind the limiter given in Fig. C-5. A total of 1521 triangles are included in the mesh depicted on Fig. C-4. The calculations used $\mathrm{S}_{8} \mathrm{P}_{3}$ approximations and the $30 / 12$ neutron/gamma-ray group structure described previously. An 1sotropic $14.1-\mathrm{MeV}$ neutron source was distributed throughout the 
COMPARISON OF RESULTS OF CROSS SECTION

COLLAPSING ANALYSIS USING TRITIUM-BREEDING RATIO AS A MEASURE

Source Collapsing Spectrum $\quad \frac{\text { Group Structure }}{\text { (Neutron/Gamma) }} \quad$ Calculated TBR

\begin{tabular}{|c|c|c|c|}
\hline$X S L I B A(a)$ & Built-in(CLAW-IV) & $30 / 12$ & $1.108^{(b)}$ \\
\hline MATXS6 & - & $80 / 24$ & 1.074 \\
\hline MATXS6 & Built-in & $30 / 12$ & 1.078 \\
\hline MATXS6 & Total blanket average & $30 / 12$ & 1.071 \\
\hline MATXS6 & First $0.10 \mathrm{~m}$ of blanket & $30 / 12$ & 1.071 \\
\hline MATXS6 & Middle $0.10 \mathrm{~m}$ of blanket & $30 / 12$ & 1.056 \\
\hline
\end{tabular}

$\overline{(\bar{a})}$ Generated by G. L. Moodruff from ENDF/B-IV data.

(b) Using canonical model and ONEDANT.

plasma volume. Initial TRIDENT-CTR calculations were converged within $10^{-4}$, with later calculations being relaxed to $10^{-3}$ to conserve on computer time.

The $20 \times 24$ coarse-mesh boundaries used in the TWODANT calculations coincided with the region boundaries shown in Fig. C-2 with the exception of the FW/B region behind the limiter. The coarse-mesh geometry for this region is shown in $\mathrm{Fig}$. C-6. Two fine-mesh intervals were spectified for most coarse-mesh intervals with the exception of the broadest coarse-mesh intervals. In this case, up to five fine-mesh intervals were used. The TWODANT calculations were $S_{8}{ }^{P_{1}}$ approximations using only the 30 neutron energy groups. The neutron source was specified as in TRIDENT-CTR, and all calculations were converged to within $10^{-3}$, again to conserve on computer time.

The MCNP calculations were performed in three-dimensional geometry using the identical two-dimensional model specified for TRIDENT-CTR. The tally used was a track length estimate of the flux in the reglons containing PbLi multiplied by the ${ }^{6} \mathrm{LI}$ and $7_{\mathrm{LI}}$ tritium-production cross sections. For this integral tally 10,000 source particles gave relative standard deviations iess than $1.0 \%$. The ENDF/B-V cross sections were used.

C.4. Parametric Results

a. Reference (Canonica1) FW/B/S Mode1. A base-case TRIDENT-CTR calcu1ation for the reference model yielded a TBR of 0.785 compared to the value of 1.108 reported in Table III.B-V for the ONEDANT model and the "canonical" blanket. Subbreeding of this magnitude indicated that the blanket design or 


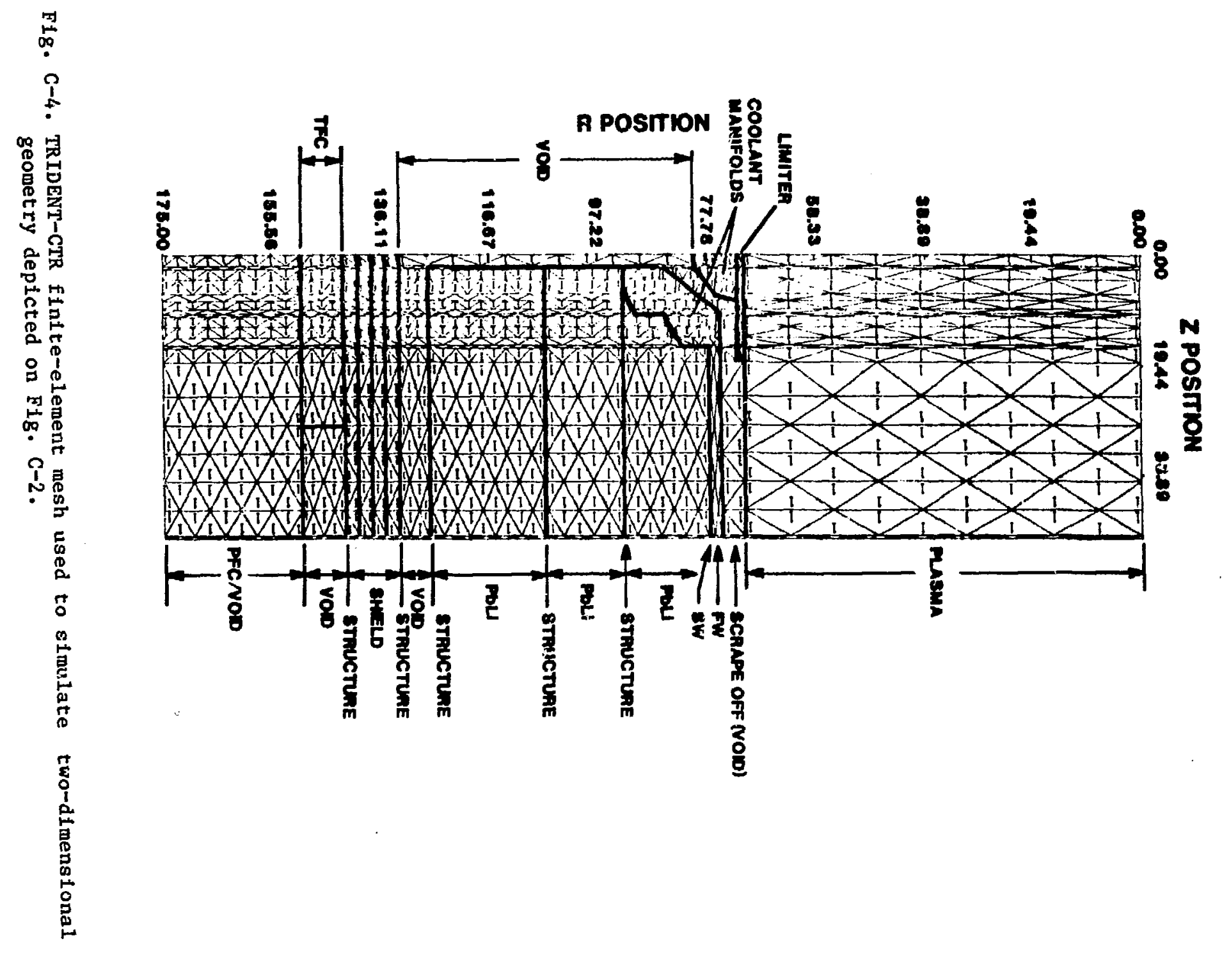




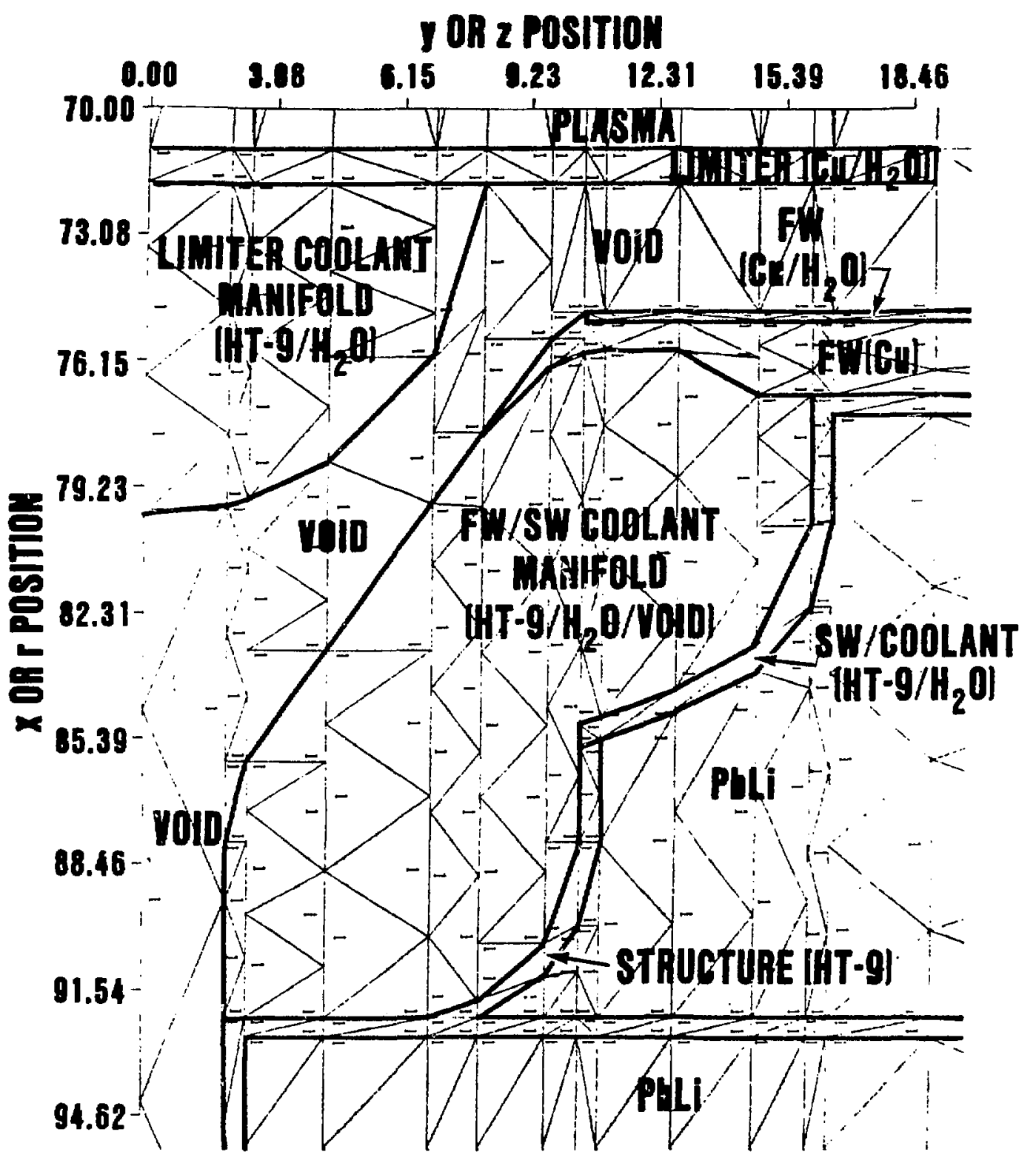

F1g. C-5. Detall of TRIDENT-CTR finite-element mesh used to simulate the two-dimenstonal limtter, $\mathrm{FW} / \mathrm{SW}$, and manifold geometry depicted on Fig. C-4. 

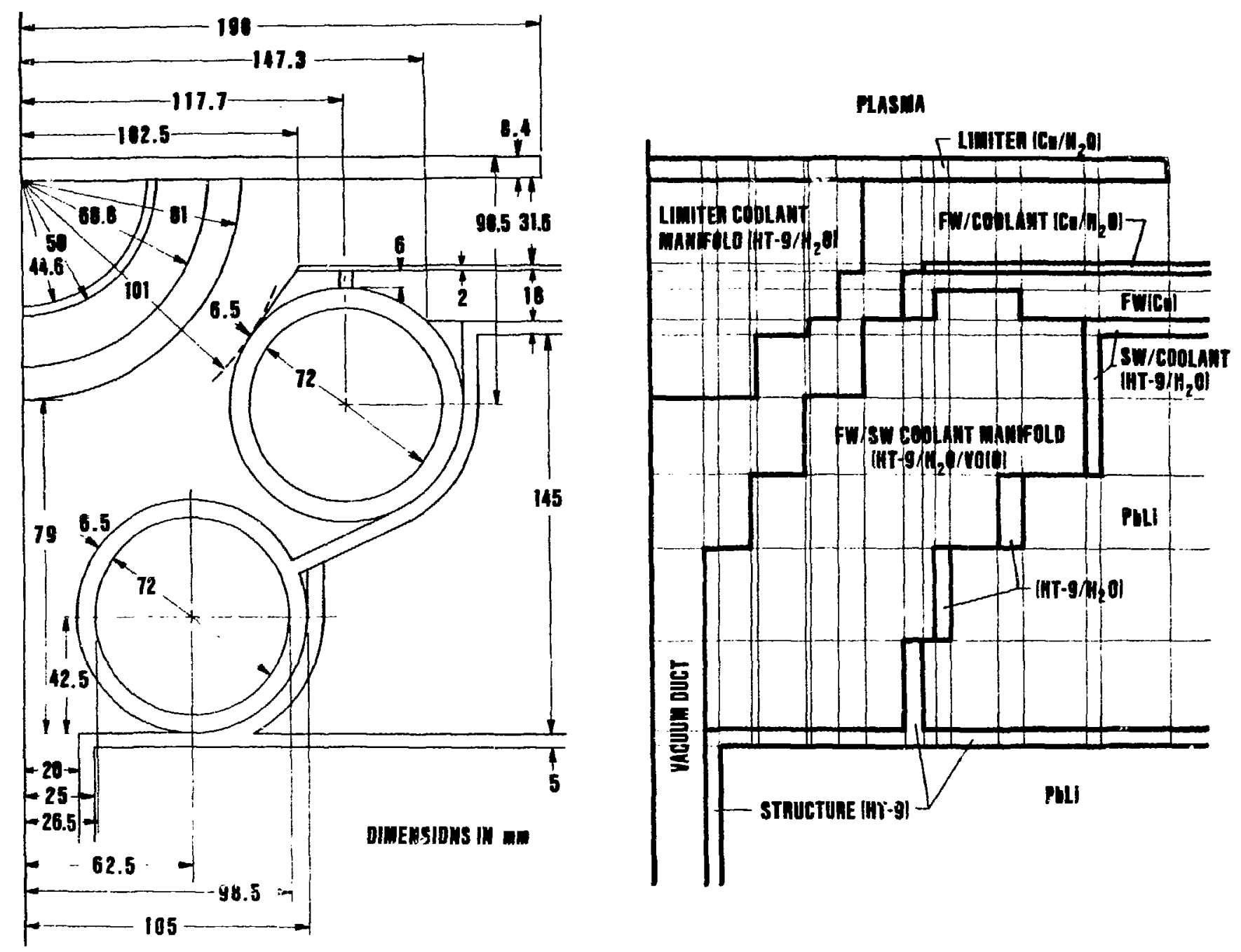

Fig. C-6. Coarse mesh of $\mathrm{FW} / \mathrm{B} / \mathrm{S}$ used in the TWODANT calculations in comparison to design geometry. 
materials or both would need to be changed. The TRIVENT-CTR calculation of the Sec. III.B. canonical blanket was used as a benchmark for a TWODANT calculation which gave a TBR of 0.786. A similar MCNP calculation was performed to provide an additional benchmark and gave a value of $0.778 \pm 0.006$. The fact that the discrete-ordinates and Monte Carlo results are the same, within statistics, gives credibility to the TRIDENT-CTR and TWODANT results for this system. Based on these calculations an interim reference $T B R$ of 0.78 was selected to demonstrate the dramatic effects of more realistic two-dimensional designs on this crucial parameter. The intent of the present study is to indicate means by which TBR can be increased above unity while (ideally) meintaining che essential elements of the FPC design evolved in Sec. III.L.

The effects of various design and material changes were subsequently examined with a series of TWODANT calculations. Selected TWODANT calculations were benchmarked with a TRIDENT-CTR calculation. The time investment for the longer-running calculations was minimized by restarting the code with the specified changes using the converged fluxes for the reference model.

The effect of thlckening the blanket was examined by replacing the 50-mm-thick vaculw region behind the blanket with PbLi. The ONEDANT calculations described in Sec. [II.B.2.b. examined the effects of first-wall thicknes: on TBR and indicated that a substantial enhancement would result by thinning the copper first wall (Table III.B-X and Fig. III.B-7). Based on this assessment, TWODANT and TRIDENT-CTR calculations were performed for the reference $20-\mathrm{mm}$-thick first wall decreased to $3.0 \mathrm{~mm}(2.0-\mathrm{mm}$-thick copper and water region followed by 1.0-mm-thick copper). The effects of relocating the FW/SW coolant manifold was estimated by shifting the homogenized material representing this manifold to the rear of the blanket, locating it in the shield region. The region previously occupled by the manifold was then replaced by PbLi, while conserving the volume of the regions. The shift of these manifold regions is depicted in $\mathrm{Fig}$. $\mathrm{C}-7$. In addition, the effects of using $\mathrm{D}_{2} \mathrm{O}$ as a $\mathrm{FW} / \mathrm{SW}$ and limiter coolant and increasing the $\mathrm{GLi}_{\mathrm{Li}}$ enrichment from $60 \%$ to $80 \%$ were investigated for the canonical $\mathrm{FW} / \mathrm{B} / \mathrm{S}(\mathrm{F} / \mathrm{g} \cdot \mathrm{C}-2)$. The values for the TBR calculated by TRIDENT-CTR and TWODANT for these design and material variations on the reference model are summarized in Table C-III.

With the exception of adding $50 \mathrm{~mm}$ of blanket, all of the design and material variations summarized on Table C-III yielded substantial improvement in the TBR. Only relocation of the FW/SW coolant manifold (Fig. C-7) or changing 


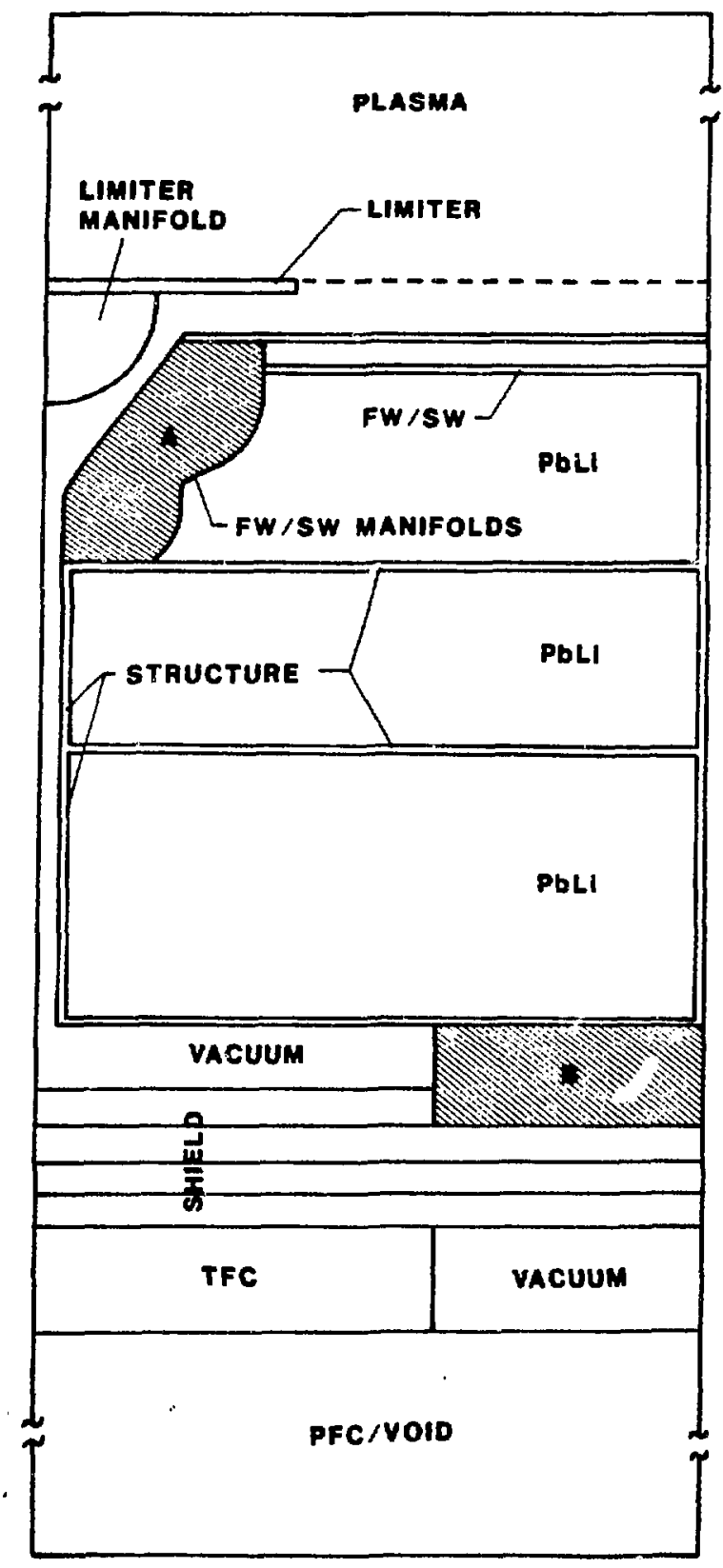
Fig. C-7. Two-dimensional FW/B/S model showing the shift of the
near-first-wall coolant manifold (location A) to the rear of the blanket (location B). 
to $\mathrm{D}_{2} \mathrm{O}$ coolant for the $\mathrm{FW} / \mathrm{SW}$ and 1 Imiter give TBR greater than unity, however. Both of these options were considered less desirable when compared with the more simple design modifications associated with thinning the first wall and using higher $\sigma_{1}$ enrichments. Specifically, use of $D_{2} \mathrm{O}$ coolant complicates the thermal cycle and is expected to add cost, whereas placement of the high-pressure FW/SW coolant manifolds in the shield region requires the use of tube sheets to deliver coolant to the FW/SW region, substantially increasing the number of individual tubes. Both design changes remain as possibilities but are ranked as less desirable relative to other cholces.

In each case where the first wall was thinned, an increase of about $14 \%$ in the TBR was calculated. It was decided, therefore, to perform a further parametric analysis for a modified reference model which included a thinner first wall.

b. Modifled Reference (Canonical) FW/B/S Model. The 5-mm-thick first wall was adopted for the modified reference model; the first wall is modeled as a region of 4-mm-thick copper and water following by a region of 1-mm-thick copper. The copper and water region adjacent to the plasma is consistent with the design for this portion of the first-wall presented in Fig III.D-3, but with a thinner bulk-copper region being used. Although this wall is thicker than that considered in the previous section, the copper volume fraction in the front region is lower, and the performance for the 5-mm-thick first wall is close to that calculated previously for the 3-mm-thick first wall.

The TKIDENT-CIR calculation for the modifled reference model gave a TBR of 0.899 , whtle a value of 0.892 was calculated by TWODANT. MCNP calculations were also performed for this model and yielded a TBR $0.870 \pm 0.010$ which agrees with the discrete-ordinates calculations. The calculations for both reference models are summarized in Table C-IV. A new interim reference TBR of 0.88 was selected for this modified reference model as compared with 0.78 for the base reference model.

Subsequent parametric calculations were performed with TWODANT and benchmarked with MCNP for selected calculations. To assess the self-shielding effects, a cross section library with self-shlelded cross sections for Cu and ET -9 was generated with TRANSX. A TWODANT calculation was performed for the reference using this library. Since the pure HT-9 and Cu cross sections are homogenized with water in the TWODANT calculation, the self-shlelding was overestmated, and the TBR calculated was 0.912 . This calculation did indicate 
TABLE C-III

TRITIUM-BREEDING RATIOS CALCULATED FOR DESIGN

AND MATERIAL VARIATIONS ON THE REFERENCE MODEL

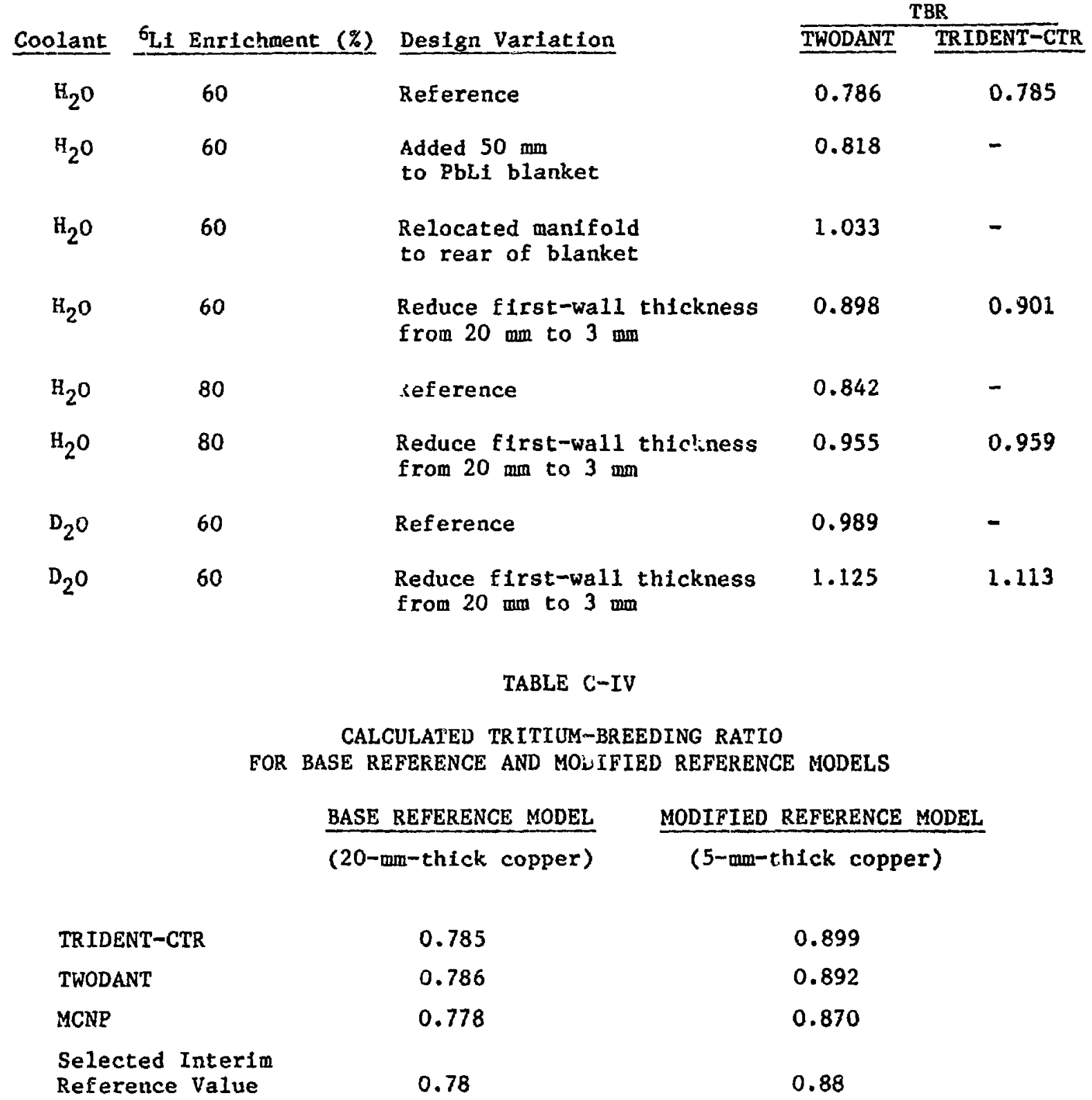


that the TWODANT calculations for a specific case would probably yield slightly higher TBR values if self-shielded cross-section sets were used.

In order to assess the effects of Gi enrichment, TWODANT calculations were made fo $80 \%$ and $90 \%{ }^{6} \mathrm{~L} 1$ enrichments. In the case that a larger TBR margin might be required, calculations were performed with $D_{2} 0$ coolant as well as 60 , 80 , and $90 \% \sigma_{\mathrm{L} 1}$ enrichments. The resulting TBRs are plotted in Fig. C-8. An approximate linear variation in the TBR with ${ }^{6} \mathrm{Li}$ enrichment for both $\mathrm{H}_{2} \mathrm{O}$ and $\mathrm{D}_{2} \mathrm{O}$ coolants is observed. The TBR approaches unity for $\mathrm{H}_{2} \mathrm{O}$ coolant only for $6_{\mathrm{L} 1}$ enrichments approaching $100 \%$, whereas TBR values calculated for a $\mathrm{D}_{2} \mathrm{O}$ coolant yield substantial marglns. The $\mathrm{H}_{2} \mathrm{O} / 90 \% G_{L}$ case was also calculated with MCNP, which gave a TBR of $0.939 \pm 0.005$. Th1s latter case appeared attractive for further parametric analysis in search of further TBR Improvement.

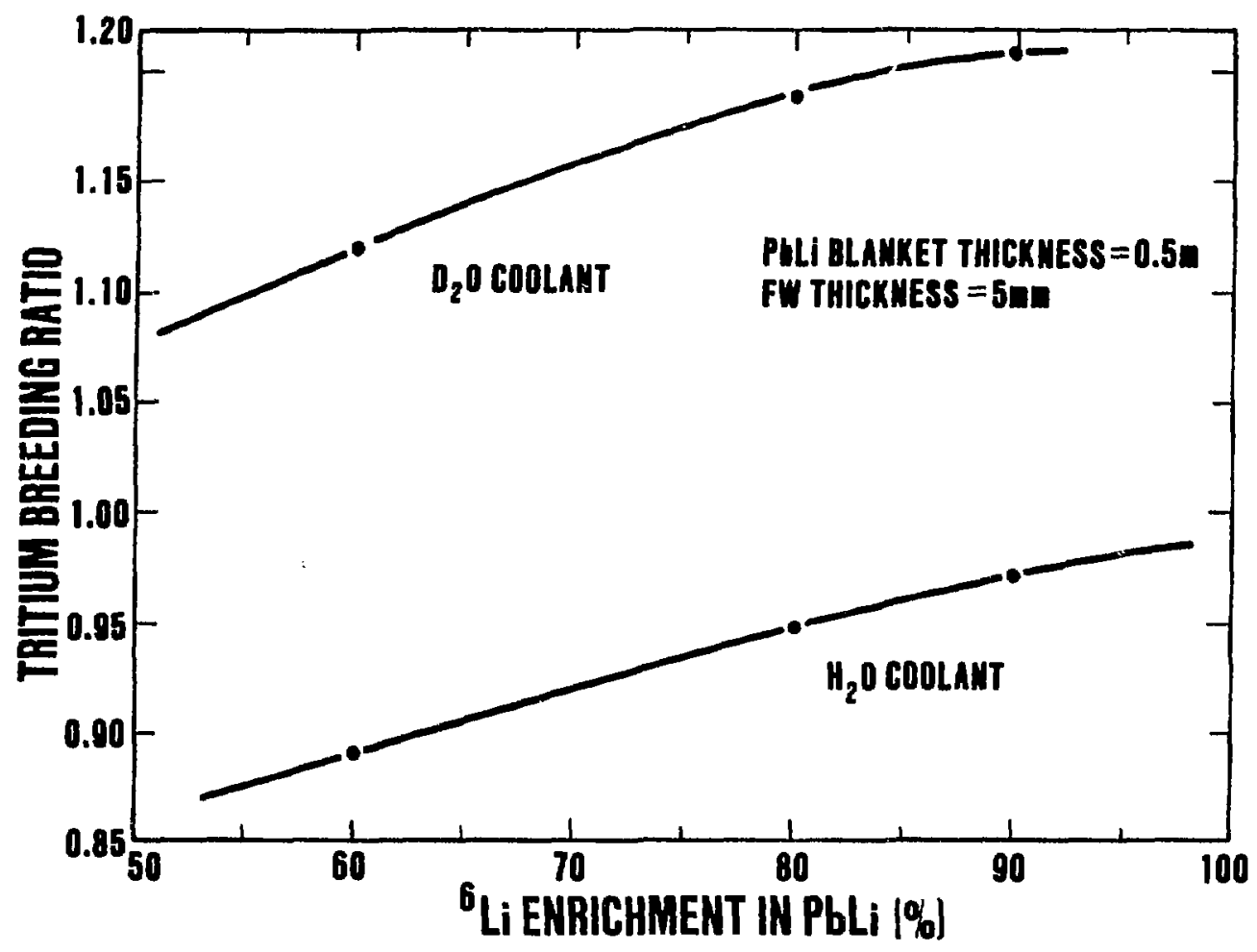

F1g. C-8. Dependence of tritium-breeding ratio on $6_{\mathrm{L} 1}$ enrichment in the modified reference $\mathrm{FW} / \mathrm{B} / \mathrm{S}$, showing effects of replacing $\mathrm{H}_{2} \mathrm{O} \mathrm{FW} / \mathrm{SW}$ and limiter coolant with $\mathrm{D}_{2} \mathrm{O}$. 
The $\mathrm{W} / \mathrm{B}_{4} \mathrm{C} / \mathrm{H}_{2} \mathrm{O}$ shield positions behind the blanket in the reference $\mathrm{FW} / \mathrm{B} / \mathrm{S}$ model (Sec. III.B.) was designed to optimize the total blanket energy recovery efficlency. This $0.10-m-t h i c k$ shield consists of alternating 25-mm-thick layers of $80 \%$ tungsten and $80 \% \mathrm{~B}_{4} \mathrm{C}$; each layer also contains $10 \%$ PCASs structure and $7.9 \% \mathrm{H}_{2} \mathrm{O}$ coolant. It appeared that a good reflector in this region could also enhance the TBR.

Calculations were performed with $90 \%$ enriched $6_{\mathrm{Li}}$ in which all four shield layers were a) taken as the $\mathrm{W} / \mathrm{PCASS} / \mathrm{H}_{2} \mathrm{O}$ mixture as described above; b) replaced with pure tungsten; or c) replaced with $90 \%$ PCASS and $10 \% \mathrm{H}_{2} \mathrm{O}$. The TBRs calculated using TWODANT for these three cases are 0.979, 0.992, and 1.006, respectively. The results for the PCASS reflector prompted a MCNP calculation; a TBR value of $0.978 \pm 0.009$ resulted. These results indicate that even high-enrichment, well-reflected blanket designs do not provide the TBR margtns required.

In order to investigate the possibility of providing a TBR margin with a slightly thicker blanket, TWODANT calculations were performed for the $90 \%$ enriched, PCASS-reflected model with $50 \mathrm{~mm}$ and $100 \mathrm{~mm}$ of additional PbLi in the blanket region. All radil behind the blanket were correspondingly increased. These calculations yielded TBR values of 1.033 and 1.058 , respectively, and are plotted in Fig. C.-9. An almost linear increase in the TBR with increased blanket thickness is observed, fndicating a design option for achieving the desired TBR margin. The latter case was also calculated with MCNP giving a TBR of $1.035 \pm 0.008$ which is consistent with previour results.

\section{C.5. Conclusions and Recommendations.}

The effects of more-detalled two-dimensional effects on the breeding potential of the canonical, one-dimensional neutronics model used throughout this franework study can be significant. A range of relatively minor $\mathrm{FW} / \mathrm{B} / \mathrm{S}$ material and design changes have been examined as means to increase the TBR above unfty using fully two-dimensional models. These changes include

- increase blanket thickness

- decrease first-wa11 thickness

- relocate $\mathrm{FW} / \mathrm{SW}$ water coolant manifold to rear of blanket

- Increase ${ }^{6} \mathrm{~L} 1$ enrichment in the self-cooled PbLi blanket

- replace $\mathrm{H}_{2} \mathrm{O}$ with $\mathrm{D}_{2} \mathrm{O}$ in the $\mathrm{FW} / \mathrm{SW}$ and limiter coolant loops 


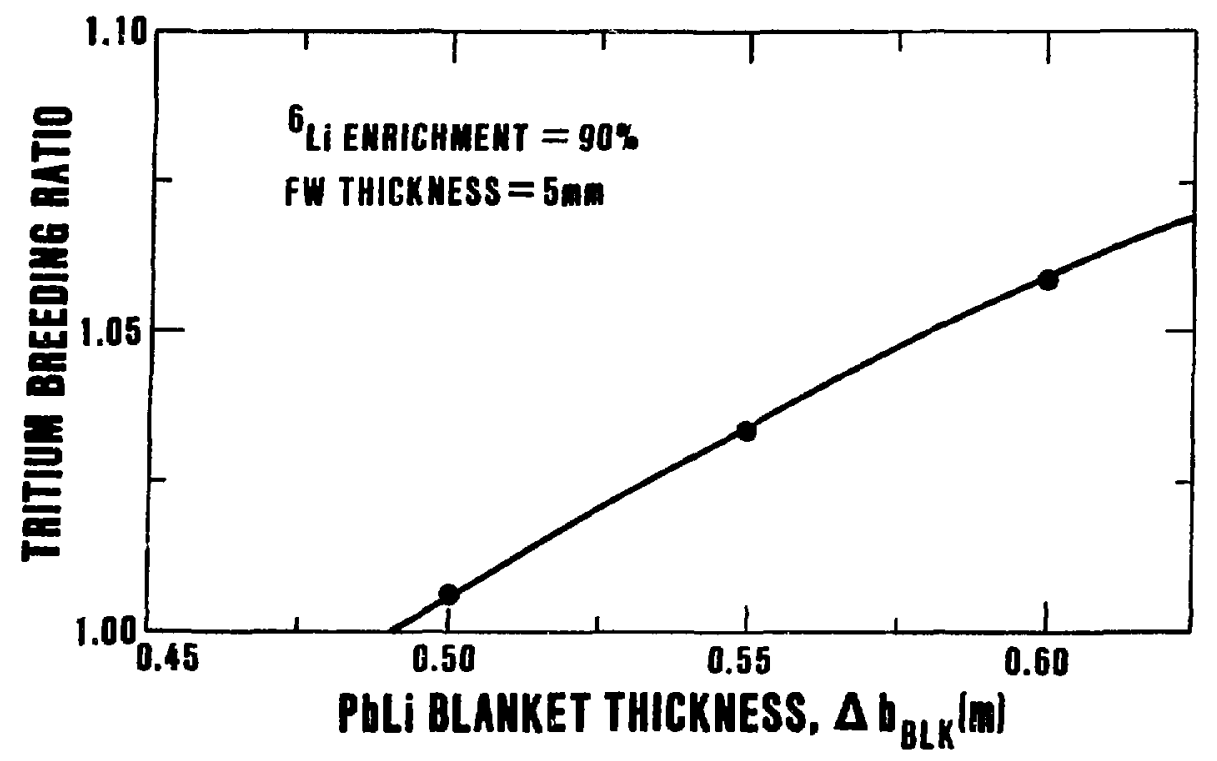

Fig. C-9. Dependence of tritium-breeding ratio on blanket thickness for the modified reference blanket with a PCASS/ $\mathrm{H}_{2} \mathrm{O}$ shield/reflector.

- emphasize reflecting role over shield role of thin shield/reflector placed between the blanket and TFC.

It appears that for a thinner copper first wall and high $\sigma_{L 1}$ enrichments $(\sim 90 \%)$, adequate tritium-breeding ratios could be achieved with the proper selection of reflector and a small addition to the blanket thickness. These changes preserve the FPC design suggested In Sec. III.L. and are considered minor. An alternative approach would relocate the FW/SW coolant manifold to the rear of the blanket. This option should provide large TBR margins and allow ${ }^{6} \mathrm{LI}$ enrichments below $90 \%$ should this present a cost issue. If further design modifications are anticipated which would have a strong negative impact on the TBR (1.e., increased FW or limiter thickness, increased FW/SW or 11miter manifold stze at the first-wall location, reduced ${ }^{6} \mathrm{~L} 1$ enrichment, etc.), changing the $\mathrm{FW} / \mathrm{SW}$ and limiter coolant from $\mathrm{H}_{2} \mathrm{O}$ to $\mathrm{D}_{2} \mathrm{O}$ presents an option with substantial TBR margins. Finally, if a thicker copper first wall should be required, an attractive combination of the above alternatives could be selected which would yield an adequate margin for the tritium-breeding ratio. 
1. R. D. O'Dell, Forrest W. Brinkley, Jr., and Duane R. Marr, "User"s Maisual for ONEDANT: A Code Package for One-Dimensional DiffusionAccelerated Neutral-Particle Transport," Los Alamos National Laboratory report LA-9184-M (February 1982.)

2. D. Garber (Ed.), "ENDF/B Summary Documentation," Brookhaven Natioral Laboratory report BNL-NCS-17541 (ENDF-201), 2d Ed. (ENDF/B-IV), National Neutron Cross Section Center, Brookhaven National Laboratory, Upton, NY (October 1975).

3. R. Kinsey (Ed.), "ENDF/B Summary Documentation," Brookhaven National Laboratory report BNL-NCS-17541 (ENDF-201), 3rd Ed. (ENDF/B-V), National Nuclear Data Center, Brookhaven National Laboratory, Upton, NY (Ju1y 1979).

4. J. Jung and M. Abdou, "Assessments of Tritium Breeding Requirements and Breeding Potential for the STARFIRE/DEMO Design," Nucl. Technol./Fusion 4, 361 (1983).

5. Thomas J. Seed, "TRIDENT-CTR User's Manua1," Los Alamos Scientific Laboratory report LA-7835-M (May 1979).

6. Ray E. Alcouffe, Forrest W. Brinkley, Duane R. Marr, and R. Douglas O'Dell, "User's Guide for TWODANT: A Code Package for Two-Dimensional, Diffusion-Accelerated, Neutral Particle Transport," Los Alamos National Laboratory report LA-10049-M (March 1984).

7. Los Alamos Monte Carlo Group, "MCNP - a General Monte Carlo Code for Neutron and Photon Transport," Los Alamos National Laboratory report LA-7396-M, Revised (Apr11 1981)

8. R. J. Barrett and R. E. MacFarlane, "The MATXS-TRANSX system and the CLAW-IV Nuclear Data Library," National Bureau of S.andards Special Publication 594 (September 1980).

9. R. E. MacFarlane, D. W. Muir, and R. M. Boicourt, "The NJOY Nuclear Data Processing System, Volumes I and II," Los Alamos National Laboratory report LA-9303-M, Vols. I and II (ENDF-324) (May 1982). 